\section{BEHAVIORAL SCIENCES AND HEALTH CARE}

1 THE SEQUELAE OF NEONATAL GROUP B BETA HEMOLYTIC STREPTOCOCCAL (GB-BHS) MENINGITIS. James R. Allen, Marilyn M. Dorsen, Dorrie L. Kanofsky and Robert H. Haslam.

(Intr. by Paul S. Lietman) Johns Hopkins Univ. Sch. of Med., Johns Hopkins Hosp., Dept. of Ped., Baltimore, Md. cause of neonatal meningitis. In some nurseries it is the most common organism in documented cases of neonatal meningitis and in most newborn units it is the leading gram positive isolate among infants with proven meningitis during the initial month of life. The long-term neurologic and psychological sequelae have not been systematically assessed in a significant number of survivors. A review was conducted of the medical records of all neonates with JHH or one of four affiliated community hospitals during a six year period between 1968 and 1974. 19 cases were identified. There were three deaths (mortality of $16 \%$ ) and two children with severe neurologic damage (obvious morbidity of $10 \%$ ). All 16 survivors were evaluated. This report will outline the results of a comprehensive evaluation of each survivor with particular emphasis on the 14 apparently normal children. The findings for each child will be compared to those obtained from a "normal" sibling or relative closest in age to the patient. Each child was evaluated by a developmental and sociological history, neurological examination, echoencephalogram, language assessment, audiologic evaluation, and a battery of psychological tests depending on the child's age. A skull radiograph and electroencephalogram were restricted to the study group. Finally, the eventual outcome will be correlated with the course of the disease.

2 ELIMINATION OF SELF-MUTILATION IN LESCH-NYHAN DISEASE Lowell T. Anderson, Lenora Herrmann, Murray Alpert \&

E. Ped., N.Y.

The effect of modifying the environmental consequences of the self-mutilatory behavior of Lesch-Nyhan disease has been studied in four children. At home the children react to different members of the family with varying types and intensities of selfmutilation. Painful stimuli interrupted the undesirable behavior but did not reduce the frequency of mutilatory attempts. Repeated prevention of undesirable behavior by the observers intervention caused a sharp increase in destructive behaviors. Response to mutilatory attempts by withdrawl of observer's attention (simply turning away of the eyes) produced a rapid reduction in frequency and an eventual elimination of the undesirable behavior. Restraining elbow splints, worn for years, have become unnecessary in all four children. The observations suggest a relation of the specific enzyme defect to the complex process of behavior development.
During the past several years the GB-BHS has become an important positive cerebrospinal fluid cultures for GB-BHS admitted to the

$\triangle$ LOW LEVEL LEAD AND SHORTENED RBC SURVIVAL. Carol $R$. Angle and Matilda S. McIntire. Univ. of Nebr. Col. of Med., Dept. of Ped.; Creighton Univ. Sch. of Med., Dept. of Ped., Omaha.

A hemolytic effect of lead at low levels of blood lead $(\mathrm{Pb}-\mathrm{B})$ was investigated in 100 urban students, ages 9-16, all with $\mathrm{Pb}-\mathrm{B}<40 \mathrm{mcg} / \mathrm{dl}$. G-6-PD and $6-\mathrm{PGD}$, rbc enzymes that are higher in the young cell, were significantly higher in the 21 students with $\mathrm{Pb}-\mathrm{B}>20$, although no differences were found for hemoglobin ( $\mathrm{Hgb})$, red cell indices, haptoglobin or reticulocytes.

\begin{tabular}{lrrrr} 
& $\mathrm{Pb}-\mathrm{B} \leq 20(\mathrm{~N}=79)$ & $\frac{\mathrm{Pb}-\mathrm{B} 20-40(\mathrm{~N}=21)}{12.4 \pm 2.9}$ & \multicolumn{1}{c}{} \\
G-6-PD & $10.2 \pm 2.3$ & & $9.2 \pm 1.6$ & $<.005$ \\
6-PGD & $7.8 \pm 1.6$ & & $5.4 \pm 0.8$ & $<.02$ \\
GSH & $6.0 \pm 1.1$ & & $5.4 \pm 1.7$ & n.s.
\end{tabular}

The increase of GSH is also consistent with a hemolytic effect, although suppression of GSH occurs at high levels of lead exposure. This data is consistent with evidence of altered rbc permeability and decreased membrane ATPase found at the low level lead toxicity of normal urban exposure.

\section{BLOOD LEAD OF IRON DEFICIENT CHILDREN -} INCREASE FOLLOWING IRON SUPPLEMENTATION. Carol $R$. Angle and Matilda S. McIntire. Univ. of Nebr. Col. of Med., Dept. of Ped.; Creighton Univ. Sch. of Med., Dept. of Ped., Omaha.

The effect of oral iron supplements $\left(\mathrm{FeSO}_{4}\right)$ on the blood lead $(\mathrm{Pb}-\mathrm{B})$ was evaluated in 30 urban children, ages $2-5$ years, with a serum $\mathrm{Fe}<80 \mathrm{mcg} / \mathrm{dl} . \mathrm{FeSO}_{4}, 200 \mathrm{mg} / \mathrm{d} \times 4$ months, was given to 14 low $\mathrm{Fe}$, low $\mathrm{Pb}$ children with $\mathrm{Pb}-\mathrm{B}<17.5 \mathrm{mcg} / \mathrm{dl}$ and to 9 low $\mathrm{Fe}$ children with $\mathrm{Pb}-\mathrm{B}$ of $17.5-40 \mathrm{mcg} / \mathrm{dl}$. The mean January to June increase of $\mathrm{Pb}-\mathrm{B}$ of 6.7 was similar for the two groups. In both groups, it was greater than the increase in $\mathrm{Pb}-$ $\mathrm{B}$ of 4.2 found in 7 low Fe children (initial $\mathrm{Pb}-\mathrm{B}$ of $17.5-40$ ) who were given placebo. The increase in serum $\mathrm{Fe}$ on therapy was unrelated to the $\mathrm{Pb}-\mathrm{B}$.

For a double blind study of the effect of $\mathrm{FeSO}_{4}, 200 \mathrm{mg} / \mathrm{d} x$ 4 months, vs placebo, 7 pairs of preschool children were matched for serum $\mathrm{Fe}( \pm 10 \mathrm{mcg} / \mathrm{dl})$ and $\mathrm{Pb}-\mathrm{B}( \pm 5 \mathrm{mcg} / \mathrm{dl})$. In 6 of 7 pairs the $\mathrm{Fe}$ treated had a greater increase in $\mathrm{Pb}-\mathrm{B}(\mathrm{p}<.05$ by Wilcoxen signed ranks test). Serum iron increased from 59 to 105 in the treated group and from 60 to 92 in the placebo group suggesting a seasonal increase in serum iron as well as in $\mathrm{Pb}-\mathrm{B}$. The data suggests facilitation of $\mathrm{Pb}$ absorption by $\mathrm{FeSO}_{4}$, at least at low levels of $\mathrm{Pb}-\mathrm{B}$.

\section{EARLY MOTHEK-INFANT RECIPDOCITY}

3 FROBLTIS OF RETAGE MOTHER.S, PUETR IMTANTS AND INFLUENCE OF FAMILY PLANMINC Billy F. Anárews, Univ. of Ioujisville sch. of lied., Louisville Gen. Hosp., Dept. Pec., Louisvilile pregnancy in the teenager has been considered high risk, and as a group teenaters have traditionally been late to seek medical care. our center cares primari-
ly for a. low socio-economic group. over the past few years 582 teenase nothers and their infants became participants in our comprehensive care center for High Risk Infants. Premature rupture of the membranes occurred in 8l, preeclampsia or eclampsia in 59 , uterine dystocia in 47 , infections in 29, first and/or thiro trimester bleeding in 24, and fetal aistress in 13. Four hundred twenty-six $(73 \%)$ had no recorded complications of lavor or delivery. Five major areas of problems of their progeny were: prematurity, asphyxia neonatorum, infections, hemolytic diseases, anc other causes for hyperbilirubinemia. AlI mothers were encouraced to seek family planning. over a 3-year period 512 continued to use oux centex. of 303 on oral contraceptives only 6 and of 94 who had intrauterine devices only 1 became pregnant. Thirtytwo of the 115 who cid not avail themselves of protection are known to have become pregnant. A $27 \%$ incidence of maternal and $30 \%$ incidence of fetal complications confirm the high risk status for teenage mothers and their infants. The value of bjrth control speaks for itself.

\section{T. Berry Brazelton, Edward C. Tronick, Lauren B. Adamson, Heidelise Als (Intr. by Mary Ellen Avery)} Harvard Univ. Med. Sch., The Children's Hosp. Med. Ctr., Dept. of Ped., Boston.

In a detailed analysis of early (2-20 weeks) mother-infant interaction from videotapes, we have been able to see that the infant's needs for homeostatic control over the psychophysiologic systems which are involved as he attends to his mother, serve to dominate their interaction. As she attempts to draw him into an intense playful interaction, he controls their interaction by looking away as much as several times per minute. A.s he greets her, attends to her behavioral displays, his excitement builds up to a peak, then he turns away as if to recover and digest the information, before he turns back for another brief period of attention. Plotted on a positivenegative matrix, the sequences form a homeostatic curve which is parallel to homeostatic curves of the autonomic and physiologic systems. If a mother is sensitive to his need for a rhythm of attention and non-attention, he becomes able to take in more and more information from her over the 20 weeks. If not, he learns to turn her off effectively, in order to protect himself. The significance of such a curve of attention which demands symchrony of attention from the mother suggests that this may be one of the environment's ways of influencing the infant's future psychophysiologic patterns. 
7 A RADICAL LDOK AT PEDIATRIC DERMATOLOGY. RObert L. Brent, Jefferson $N$

Phila., Pa. 19107

Over the past seven years 2800 dermatological evaluations of children's skin problems have been performed by our Pediatric staff with minimal outside consultation. This experience indicates that children with skin problems benefit by being cared for by a general pediatrician and that any residency program that does not include the basic science of clinical dematology and practical clinical dematology is deficient. One frequently quoted anecdote about the pediatrician's dermatologic ability is summarized in the concept that all skin diseases can be divided into two groups - "those that respond to cortisone and those that do not." Information will be presented to illustrate the following contributions of pediatrics to good dermatologic care: 1) the evaluation of whether the child is sick and then communicating this honestly to the parents; 2) the allaying of anxiety about skin manifestations that are evanescent or insignificant; 3) the willingness not to prescribe any medication when none is indicated; 4) the ability to discourage multiple visits by replacing followups with initial patient education; 5) the ability to feel comfortable even though one cannot ascribe two latin names to a dematological manifestation; 6) knowing when and how to biopsy. It is believed that these concepts can best be taught within the confines of a pediatric outpatient program by a pediatrician experienced in children's problems since several of these goals are contrary to the policies of many consultants including dermatologists.

PARENTAL REACTION TO REGIONALIZED PERINATAL CARE Barbara Chapleau, Jeannine Sacco, George A. Little, Alistair G. S. Philip, Jerold F. Lucey (Dartmouth Med. School, Hanover, N.H.; Univ. of Vermont Col. of Med., Burlington; Vt./N.H. Regional Perinatal Program)

Reactions of parents referred for tertiary perinatal care between $7 / 1 / 73$ and $7 / 1 / 74$ were studied by questionnaires and personal contact. The pximary purpose was to elicit comment and secondarily to acquire statistical data. Objectives included delineating (1) awareness of reasons for referral, (2) involvement before and after transfer, (3) reaction to separation, (4) channels of communications, (5) adjustment following discharge, and (6) recommendations.

\begin{tabular}{lcc} 
QUESTIONNAIRE GROUP & $\#$ Sent & \# Returned \\
\hline Mothers referred for delivery & 11 & 9 \\
Parents of neonatal deaths & 22 & 10 \\
Parents of surviving babies & $116^{*}$ & $60^{* *}$ \\
$* 6$ returned - inadequate address & &
\end{tabular}

* 10 random non-replies contacted by telephone, all

willing to discuss, replies similar to returns.

Approximately $90 \%$ of returns were interpreted as manifesting basic satisfaction with an aggressive program of communication and stress on parental involvement. Recommendations of import to primary, secondary, and textiary centers were derived. The 69 replies from parents with surviving babies were asked how they would advise a friend with a risk pregnancy:

39 would recommend delivery in a center

25 would recommend local delivery

COMPARISON OF HISTORICAL, QUALITATIVE, AND QUANTITA-

9 TIVE METHODS TO ASSESS LATERALITY. Charles C. Faust and Richard C. Juberg, Dept. of Biology, L.S.U. at Eunice, Louisiana, and Dept. of Pediatrics, L.S.U. School of Medicine in Shreveport, Louisiana.

We proposed to show that laterality is not a qualitative difference of right and left handedness but a quantitative characteristic estimable by history of side preferred in common functions, by observation of preference in certain habits, and by quantitative expression of comparative ability in tests of skill. Our 232 subjects were 54 white undergraduates, theix 108 parents, and 70 of their siblings. Each indicated hand or foot use for 22 different functions (handwriting...kicking a ball) and estimated her (128) or his (104) degree of handedness. Qualitative preference for upper extremity functions included pointing, clapping, sweeping, tying, folding arms, and clasping hands, and for the lower extremity stepping, hopping, and crossing legs. We timed or graded 5 tests of hand ability (grip strength, finger tapping, stereognosis, grooved pegboard, and dowel driving), I test of foot ability (tapping), and writing with each hand.

We computed a proficiency index for each subject by averaging the 5 hand functions (difference in right and left values/inferior value). History of hand use correlated closely with this quantitative estimate, but qualitative preference in habitual functions did not. History of foot use correlated with the proficiency index, and qualitative preference correlated less.

We concluded that these tests estimate quantitative degree of laterality, that history of use is less reliable, and that preference in some simple habitual functions is least reliable.
10 THE PRESCREENING DEVELOPMENTAL QUESTIONNAIRE (PDQ) William K. Frankenburg, William J. van Doorninck, of Colorado School of Medicine, Dept. of Ped., Denver, Colorado. To make mass developmental screening feasible, the authors developed a questionnaire which was answered by parents at the time they brought their children to the physician. Ninety-eight Denver Developmental Screening Test (DDST) items were formulated into questions to be asked at appropriate ages. Initially, answers to 1,430 questionnaires were validated through administration of corresponding DDST items. Agreement rate for the 98 items varies from $74 \%$ to $100 \%$ (mean 87 ). Of the disagreements, $70 \%$ were underestimations and $30 \%$ parental overestimations. To derive a screening cutting point, an additional sample of 1,033 parents answered 10 age appropriate questions, prior to having their children tested with the DDST. By defining suspect on the PDQ as 2 or more failures, it was possible to identify $86.2 \%$ of the abnorma1 DDSTs. The agreement rate between parents' responses on the PDQ and DDST findings in public health clinics and private pediatricians' offices did not differ significantly. Subsequent practical application of the PDQ in 8 clinic settings for the routine screening of 437 children resulted in $86 \%$ of children requiring no further screening and on $1 y$ 14\% referral for secondary DDST screening. Thus, through this two stage developmental screening procedure, which employs a questionnaire to screen all children and the DDST to rescreen those who are suspect on the questionnaire, it is possible to screen more children and more frequently at only a fraction of the usual
cost.

11 PSYCHOLOGICAL ISSUES IN BONE MARROW TRANSPLANTATION (BMT). G. Gail Gardner, Charles S. August and John H. Githens. Univ. of Colo. Sch. of Med, Denver.

Psychological services were available to five children who underwent BMT, their donors, and families. The patients' ages were 7 to 15 , with 2 females and 3 males. Three had acute lymphoblastic leukemia, one had congenital hypoplastic anemia, and one had aplastic anemia. The sibling donors were 10 to 19 years old. Both patients and donors underwent complete psychological evaluation prior to the transplants. Emotional disturbance ranged from mild to severe, including one donor who was borderline psychotic. All patients manifested significant anxiety and depression, related especially to fear of dying and to prolonged isolation. They were more likely to discuss specific fears with the psychologist and nurses than with the medical staff. One boy asked to see the psychologist and discussed his fears of death and his wishes regarding resuscitation only 5 hours before he had a cardiac arrest. The donors and other family members experienced exacerbation of underlying emotional problems and guilt, especially when post-transplant complications arose. One donor said she wanted to run away, while another began secretly fasting in hopes of being more helpful to her brother. The parents felt particularly guilty about pretransplant immunosuppression (large doses of cyclophosphamide or total body $\mathrm{X}$-ray), likening it to an execution to which they had knowingly consented. Our experience to date suggests that psychological problems arise frequently during BMT and that psychotherapy plays an integral part of total patient care.

12 NEUROLOGICAL ASSESSMENT OF NEWBORN INFANTS. Harris L Sreenwald, Arthur H. Parmelee, Univ. of Californ Dept. of Pediatrics, Los Angeles, Ca. 90024.

Several tools with which to assess the neurological status of full term newborns are now available. This neurological examination was designed to be administered in ten minutes, making it appropriate for the routine evaluation of large numbers of infants. It also can be administered with minimal stress to sick infants. This examination assesses active and myotatic reflex muscle tone, primary reflex behavior patterns, states of arousal, and spontaneous behavior and has been standardized for infants of 38 to 42 weeks gestational age to give a numerical score. Criteria have been established which result in separate subscores reflecting syndromes of hyperexcitability, apathy, coma, hypotonia, hypertonia, or hemisyndrome. 97 term infants between 39 and 41 weeks gestation were compared to 25 infants born at 38 weeks and 27 infants born at 42 weeks gestation. These infants were examined on the second day of life. There were significant group differences, suggesting the validity of assigning gestational age by mother's reported dates. The younger infants were more hypotonic and less excitable than the older infants. 27 ill newborns between 37 and 44 weeks gestational age were similarly studied and found to be more hypotonic and have greater occurrence of the hemisyndrome than any of the healthy newborn groups. 
13 THE EFFECT OF EARLY SKIN TO SKIN CONTACT ON MATERNAL BEHAVIOR AT TWELVE HOURS. Hales, D., Kennel, J., Klaus M., Mata, L., Sosa, R., Urrutia, J. CWRU Dept. of Pediatrics, Cleveland, Instituto De Nutrition De Centro America y Panama, Guatemala.

To determine the effects of maintaining mother-infant contact in the first hours of life, 9 Guatemalan mothers were given their healthy full term babies for 45 minutes in privacy immediately after they left the delivery room. Each mother-infant pair was nude, skin-to-skin under a heat panel, and most of the babies suckled. A control group of 10 mothers was separated from their babies shortly after delivery (routine at Hospital Roosevelt in Guatemala). Except for the early contact, the care of the two groups of primiparous mothers was identical. The babies in both groups were sent to the newborn nursery for the next 12 hours, after which they went to their mothers in a seven bed room for the first breast feeding. At 12 hours, each mother's interactions with her infant were observed by an investigator who did not know to which group the mother belonged. Observations of attachment behaviors such as fondling, kissing, looking "en face", gazing at and holding the baby close, as well as caretaking behaviors, were made for 15 seconds of every minute for 15 minutes. Mothers given early contact showed significantly increased attachment behavior $(p<.05)$ but no differences in caretaking. This study is further support for the concept that contact during the first minutes after birth may have a powerful influence on later maternal behavior and suggests a need for a reappraisal and rearrangement of delivery and post-partum procedures.

COMPARISON OF THREE YEAR AND FOUR YEAR MEDICA 4 STUDENTS IN A PEDIATRICS CLERKSHIP. JameS A. Hallock, Univ. of South Florida College of Medicine, Intr. by Lewis A. Barness, Tampa.

This study was designed to compare the clinical performance of two groups of medical students in a pediatric clerkship. The students were prepared in separate one year (Class II) or two year (Class III) basic science formats and were merged together to take the clerkships. There were four members from each class in groups of eight taking an eight week core clerkship. The students were evaluated according to a five category scoring system and by the NBME "mini" test in pediatrics. A five point grading system was used with 1 for failing and 5 for honors. The grades obtained were analysed via " $t$ " testing and interactional ratio analysis. The results were as follows: (1) Fund of Knowledge, Category, ClassiII 3.39 , and Class II, 3.15, with a P0.05, (2) NBME Class III, 77.41 and Class II, 63.89, with a $p<0.05$. (3) In the four other categories including medical skills, problem solving, patient rapport and professional standards, no differences were noted. The data demonstrates little discernible difference in clinical perfomance between the groups except as noted with regard to fund of knowledge. The possibility exists that a wide fund of knowledge in basic sciences may be necessary to learn facts in a pediatric clerkship. However, it appears that students can be prepared to accept and respond to the challenge of clinical performance with one year of preparation. The data do not answer the questions of the relative merits of a three year vs. four year curriculum, but suggest that there may be little difference in effect at a student
level.

15 DEVELOPMENTAL ASSESSYENT OF: CHILDIRLA WITH CYTOMEGALOVIRUS (CN) I giv ANTIBODY IN TTE COND BLOOD James B. llanshaw, Albert P. Scheiner, Ann Moxley, Lilly Gaev, and Vaudeen Abel, Dept. of Ped., Univ. Rochester Sch. Wied., Rochester, N.Y.

During the period 1967-70, 8644 consecutive cord sera were tested for CMV-IgM antibody. Fifty-three infants $(1: 163)$ had a titer of $1: 4$ or greater. Two infants died within 48 hours of birth. Six children were not available for testing and three families refused evaluation. The remaining 42 children, 4-6 years of age, underwent detailed pediatric, neurological, and psychometric assessment including Prechtl's examination for neurological function, Wechsler's Preschool and Primary Scale of Intelligence, and the School Readiness Survey. In the CMV-IgM positive group, $15 / 42(35.7 \%)$ had one or more developmental abnormalities precluding adequate performance in a normal school setting. All 15 affected children were born to 27 mothers in llollingshead's lowest socioeconomic groups (IV, V). Fourteen children had IQ levels of 91 or below; four of these were microcephalic. The remaining child had an IQ of 98 and was one of six children with sensorineural hearing loss. In contrast, only $3 / 39$ ( $7.6 \%$ ) control children (matched for socioeconomic group, age, sex, race, birthweight, gestational age at birth, and mother's marital status) and $3 / 46(6.5 \%)$ random controls had predictable school failure. These data suggest that a CMV-Igi positive child born to lower socioeconomic group parents has a significantly higher risk of school failure than CMV-IgM negative control children.
16 IS PARENTAL CONTACT WITH INFANTS IN THE IDEA? Rita G. Harper, Concepcion Sia and Sondra Sokal, Departments of Pediatrics and Ob:-Gyn. North Shore University Hospital, Manhasset, N.Y., and Departments of Pediatrics and Ob.-Gyn., Cornell Univ. Medical College, New York, N.Y. (Introduced by Fima Lifshitz).

To assess whether parents of infants in the Premature-Newborn Special Care Unit undergo prolonged stress and whether the stress is aggravated by the present policy of encouraging parental contact, the following study was done. One hundred parents of infants who stayed in the Premature-Newborn Special Care Unit three weeks or longer during 1974 were given a 63-item questionnaire regarding the medical, social, financial and psychological problems they experienced. Analysis of the data indicated that the degree of parental stress is directly related to the degree of the infant's illness and its outcome, and indirectly related to the nursery milieu, the quantity of personnel support and the extent of parentalinfant contact. The environment of the intensive care unit itself is a frightening experience to most parents. Although frequent parental-infant contact may indeed build long-term relationships, the parental emotional price tag is high. Policies regarding visiting should therefore be determined on an individual basis.

17 SUITABILITY OF THE DUBOWITZ IN ASSESSING MATURATION OF FULL-TERM BLACK INFANTS. Adadot Hayes and Alan Percy. Charles R. Drew Postgraduate Med. Sch., and Martin Luther King Hospital, Los Angeles, California 90059 one hundred full-term black AGA infants were examined according to the method of Dubowitz and Dubowitz to determine if this method for assessing gestational age is applicable in a non-white population. Up to 38 weeks, test scores were similax to those reported by Dubowitz. Beyond 38 weeks, black infants tended to score lower on some neurologic test items. Paradoxically, black infants appeared to be more advanced in items testing passive tone, causing them to score falsely low. Failure to exhibit passive tone ordinarily seen in full-term white infants is scored as tone not yet developed in the Dubowitz. This suggestion of greater neurological maturation was supported by more advanced reflex development (loss of Moro, grasp, etc.) items not scored on the Dubowitz. Some external characteristics (e.g., black infants have darker skin, less hair and fewer plantar creases) score either lower or higher but tend to cancel each other. It is suggested that in the assessment of gestational age, the neurological exam be modified to take these findings into account and a regression curve established for black inf ants.

COUNSELOR JUDGMENTS ABOUT REPRODUCTION ASSOCIATED WITH

18 HIGH RISK FOR INHERITED DISEASE. Verle E. Headings (Intr. by Roland B. Scott), Howard University College of Medicine, Dept. of Ped. and Ctr. for Sickle Cell Disease, Washington, D.C.

Two schools of thought exist regarding the intent of genetic and reproductive counseling; I) directive counseling. in which a professional judgment is given the client as to the best course of action for preventing occurrence of an inherited disorder, and 2) nondirective counseling in which comprehensive education is provided to the client as to the nature of the disorder, prognosis, risk of occurrence and available options for management and prevention. This study examines the hypothesis that the type or specialty of professional training is a significant determinant of which of these two counseling emphases in practiced. Seven problem situations involving reproductive decision-making by persons with sickle cell trait or sickle cell disease were designed in question form which called for a directive vs nondirective judgment by the respondent. Responses from 96 professional staff involved in delivering service at a number of Comprehensive Sickle Cell Centers and at Sickle cell screening and Education Clinics were included in this study ( 16 pediatricians, 20 internists, 6 geneticists, 14 nurses, and 40 persons in the social sciences). Pediatricians and internists were similar in the frequencies of judpments rendered on six of the seven problem situations. Geneticists, nurses and social. scientists were generally similar in their judgments. The frequencies of directive judgments were clearly lower among this latter group of professionals. 
19 MATERNAL DRUG ABUSE - INFANT PROGNOSIS. Reba M. Hill, Willie M. Verniaud, Lee B. McCulley (Intr. by L. Leighton Hil1). Dept. of Ped., Baylor Col. of Med., St. Luke's Episcopal Hosp., Newborn Res. Center, Houston, Texas. The somatic and mental development of 35 infants born to mothers with a history of drug abuse and 156 cohort infants (CI) have been followed for a 3 year period. Marijuana, hallucinogens, methaqualone and barbiturates were the most frequent drugs ingested by the mothers.

Twenty-one percent of the drug abuse infants (DAI) were fetal$7 y$ malnourished compared to $10.8 \%$ in the cohort population. Tweive percent of the DAI were reported to have some mild degree of CNS agitation in the first months of life. Irritability, hyperactivity or abnormal crying pattern was reported in $27 \%$, $15 \%$ and $15 \%$ respectful ly compared to irritability $(4 \%)$ and hyperactivity $(8 \%)$ in the CI group. Disfiguring or life-threatening anomalies occurred in $17 \%$ of the DAI compared to $4 \%$ in the $\mathrm{CI}$. Physical abnormalities observed in the DAI were: pyloric stenosis (2), dis location of the hips (1), hypospadius (1), mesocardia (1), ? congenital heart defect (1) and inguinal hernia (1). Only one infant had more than one anomaly.

Chromosome analysis were completed in 30 DAI. Two infants had $X X Y$ karyotype and one infant had an elongated short arm of the $D$ chromosome.

Psychometric testing after 21 months of age in $60 \mathrm{CI}$ and $12 \mathrm{DAI}$ demonstrated 19\% of the CI received DQ of 120 or greater compared to $8 \%$ in the DAI group. Equal numbers of infants $(8 \%)$ in both groups received DQ of less than 90. (Supported by NIH GM 16216)

20 A 10 YEAR FOLLOWUP OF THE FETALLY MALNOURISHED INFANT. Reba M. Hill, Willie M. Verniaud, Abbie L. Vorderman Tom Zion and Gail Rettig. (Intr. by L. Leighton Hi11) Dept. of Ped., Baylor CoT. of Med., St. Luke's Episcopal Hosp., Newborn Research Center, Houston, Texas 77025.

The growth and development of 38 fetally malnourished infants without chromosome abnormalities or laboratory evidence of intrauterine infection and 18 wellinourished cohort infants have been followed from birth to 10 years of age.

Psychometric testing (Gese11, WPPSI, WISC) revealed statistically significant differences in scores for fine motor ability $(p=<.05)$ at 9 months and gross motor $(p=<.05)$ at 18 months of age. At 3 years of age scores for all 5 areas tested showed a statistically significant difference $(p=<.05)$ between the fetally malnourished and the cohort population. Testing at later ages demonstrated continued lower verbal, performance and full scale scores for the fetally malnourished infant. Arithmetic Concepts, Comprehension, BTock Design, and Coding of Information were areas that were greatly altered. At 8 years of age, $25 \%$ of the fetally malnourished infants had a Full Scale IQ of 120 or greater compared to $33 \%$ of the wellnourished infants and $19 \%$ of the fetally malnourished infants had a Full Scale IQ of $<90$ compared to $0 \%$ in the wellnourished infant. Mental retardation ( $8 \%)$, special education (11\%), compensated learning disabilities (18\%) and late acquisition of ianguage skills (16\%) were more frequently observed in the fetally malnourished infant. (Supported by Doris Hebard Knapp Memorial Fund)

21 THE FATHER OF THE INFANT OF THE UNWED MOTHER Dorothy R. Hollingsworth, Kathryn T. Thompson, Jay W. Carlson and Alma T. Jackson. Univ. Ky, Med. Ctr., Dept. Ped., exington (1ntr. by dacqueline A. Noonan Scant attention has been given to fathers of infants of unwed
chers. In a study of 411 consecutive pregnancies in women age mothers. In a study of 411 consecutive pregnancies in women a
$12-18$ years, information was collected from mothers on social status, education, income, and relationship with father of infant. The population was unusual ( $46 \%$ white, $54 \%$ black) from low socioeconomic families (mean annual income $\$ 4,200$ ). Primary support was from parental earnings in $60 \%$ whites and $48 \%$ blacks.

Striking racial differences were: (1) $44 \%$ of black fathers were in school vs $14 \%$ whites. (2) Unemployment was $24 \%$ in whites vs $9 \%$ for blacks. (3) Before conception, $86 \%$ of fathers in both groups had a close relationship with the mother. By first
maternal clinic visit (mean 5.6 months gestation) $36 \%$ white fathers had abandoned patient vs 18\% blacks; $63 \%$ black fathers continued dating or planned marriage vs $34 \%$ whites. (4) Blacks were more concerned with infant plans: $73 \%$ fathers requested mother to keep child vs $41 \%$ whites; black fathers and/or their families offered infant support significantly more of ten than whites $(70 \%$ vs $40 \%)$. Post partum, $59 \%$ black fathers maintained a close relationship with mother and infant compared with only $36 \%$ whites. Conclusion: These data suggest adolescent black fathers are significantiy more interested and involved in the welfare of their illegitimate offspring than whites and have a stronger, more meaningful relationship with the infant's mother.
2 VALIDATION OF THE MINNESOTA CHILD DEVELOPMENT INVENTORY (MCDI) FOR DEVELOPMENTAL SCREENING OF NEONATAL ICU (NICU) SURVIVORS. Harold R. Ireton, Pi-Nian Chang, Carl E. Hunt. Univ. of Minnesota, Depts. of Fam. Pract. and Comm. Health, Psy., and Ped., Minneapolis, Minnesota. (Intro. by Rolf R. Enge1, Minneapolis).

The MCDI is a 320-item inventory which provides a maternal report of the child's developmental status. The MCDI has been evaluated as a means of screening for developmental status in NICU survivors by comparing MCDI results and psychological test results. To date, the MCDI has been obtained for 87 NICU survivors, all at 3.5-4.0 years of age. The MCDI results are classified as retarded (analogous to IQ $<70$ ), borderline, or normal. The study design includes direct psychological testing (intelligence, language and motor abilities) of all MCDI deviant children (borderline and retarded) and a random sample of MCDI normal children. To date, 43 children have been direct tested ( 28 of 33 MCDI deviants and 15 of 54 MCDI normals). Twenty chilldren were classified as deviant by direct testing. Of these 20,17 were detected by the MCDI; of the three "misses", one was associated with limited maternal education and two with mild speech or mild gross motor problems. The MCDI did "over-screen" in that 11 of the MCDI deviant children (all borderline deviant) were normal on direct psychological testing. Combining the MCDI and direct testing results, the overall incidence of deviant development was $29 \%$. The MCDI provides a practical means of obtaining developmental outcome data for large groups of NICU survivors; detailed psychometric testing can be reserved for those with maternal education below high school level and/or with a deviant MCDI.

THE QUANTITATIVE USE OF TESTS OF SKILL TO DEMONSTRATE THE SPECTRUM OF LATERALITY. Richard C. Juberg and Charles C. Faust, Dept. of Pediatrics, L.S.U. Schc

of Medicine in Shreveport,

I.S.U. at Funice, Louisiana.

Our objectives were to assess the utility of selected tests of skill to differentiate lateral orientation and to show that there is a continuous spectrum of laterality from one extreme of right handedness or footedness to the other of the left.

We timed or graded the ability of 217 right handed and 15 left handed white subjects on 5 tests with each hand (grip strength,

finger tapping, stereognosis, grooved pegboard, and dowel driving) and 1 test with each foot (tapping) and to write with each hand. There were 128 females and 104 males from 8 to 66 years in 54 families. We expressed the result of each test as proportionate superiority (difference in right and left values/inferior value)

with right as plus and left as minus and averaged the 5 hand tests

Values for the averaged hand tests ranged from -0.19, indicative of $19 \%$ left handed superiority, to +0.36 with mode between $+0.161 \rightarrow+0.200$ when the data were grouped by 0.04 intervals. They appeared to be continuously distributed in a normal curve. Handwriting values appeared normally distributed for the right handed. Values for foot tapping seemed normally distributed with mode between $+0.001 \rightarrow+0.050$ thus showing considerable left footed superiority but with more scatter than in other tests.

We interpreted these results as showing that laterality is a continuously distributed trait and not an all right or all left phenomenon. In this respect it resembles certain polygenically determined structural, physiological, and mental characteristics.

BEHAVIORAL ASSESSMENT OF INFANTS BORN TO NARCOTIC ADDICTED MOTHERS: COMPARISON BETWEEN SCORES ON THE BRAZELTON NEONATAL ASSESSMENT SCALE AND THE NEONATAL ABSTINENCE SCORING SYSTEM. S.L.Kaplan, R.E.Kron, M.Phoenix, and L.P. Finnegan (Intr.by M.Delivoria-Papadopoulos), Phila.Gen.Hosp, Depts. Ped.\& Psych., Univ. Penna.Sch.Med., Philadelphia, Pa.

In order to develop clinical methods for monitoring the behaviox of infants with narcotic abstinence, 28 infants born to nar cotic addicted mothers were examined between the $3 \mathrm{rd}$ and 5 th day of life using 2 independent methods for measuring newborn behavior:1) Brazelton Neonatal Assessment Scale(BNAS) which measures a wide spectrum of infant behaviors, 2) Neonatal Abstinence Scoring System(NASS) which records behaviors and physiological parameters that are clinically associated with neonatal narcotic withdrawal. Independent groups of raters performed the BNAS and NASS and were blind to each other's results. The 2 BNAS raters had high intexrater reliabilities $(r \geq .85)$, as did the 3 raters who performed the NASS $(x \geq .82)$. Behavioral items on the BNAS subdivide into 3 clinical groups:a) alertness(e.g.level of response to auditory stimulation), b) motor (e.g.level of motor maturity) and c) irritability (e.g.level of CNS excitement). BNAS alertness and motor scores did not correlate with the scores on the NASS but BNAS irritability items correlated significantly $(r=.499, p<.01)$. Brazelton irritability item reflect altered levels of CNS excitation which is characteristic of narcotic withdrawal. Data provide mutual support for the validity and reliability of Brazelton irritability scales and NASS in the study of passively addicted infants. These clinical scores may provide more accurate methods for monitoring course and treat ment of neonatal narcotic abstinence. 

25 THE EARLY DETECTION OF EDUCATIONAL DYSFUNCTION: A Melvin D. Levine, Judy Palfrey, Daniel Rosenn, Dorothea Johnson (Intr. by Dr. Mary Ellen Avery).

The Brookline Early Education Project is a demonstration model and a longitudinal development study with diagnostic and educational programs for moxe than 300 childxen from birth through 2nd grade. Critical to the study is the prospective documentation of early risk factors. A new set of instruments has been developed to record, weigh, and score perinatal medical factors associated in previous studies with later academic failure. The At-Risk Inventories take into account more than 100 forms of perinatal stress. Each factor is rated on a scale from 0 to 5 based on its educational relevance and severity. This is adjusted for duration of the stress when appropriate. 303 At-Risk scores and profiles have been obtained ranging from 0 to 254 points with a median score of 9 on the prenatal inventories (pregnancy and labor) and 10 on the postnatal inventory (delivery to 7 days). There have been significant correlations between these scores and findings on the Prechtl Newborn Neurologic Examination and the Bayley Mental and Motor Scales at 3, 6, and 14 months. Scores of over 25 points on the postnatal inventory showed high correlations with an assessment of infant activity level and a questionnaire on maternal satisfaction, but no correlation with a family adjustment inventory. It is suggested that these At-Risk Inventories may be useful tools for introducing more precise and relevant perinatal data into studies of developmental outcome.

REE-ESTAGL ISIIMEINT OF A FAMILY UNIT AFTER DEATH OF A

26 Michael A GROUP THERAPY APPROACH: Carolyn 3. Levitt, lark E iesbit Univ of ilinn, Cept. of Peds. Mpls. Mn and Children's Hospital, St. Paul, Mn.

After the death of a child from cancer a family can suffer greater emotional trauma and family upheaval than was evident throughout the child's illness. Many parents suffer acute incapacitating anxiety and depression with suicidal tendencies. Communication in the family unit breaks down, leading to separation and divorce. After years of medical treatment and emotional support for the chronically ill child the family is left devastated alone and without help. Standard supportive mechanisnis are often ineffective. 1 ith the insistence of parents, a group therapy program was organized in 1972. This consists of bimonthly sessions attended by parents and siblings, 2 social workers and the physician or nurse who cared for the child. He now have had experience with 20 families. In this group there were 2 suicidal attempts, I psychiatric admission and 3 separations. From the ses sions it was found that grieving parents need to keep talking about their child. They need to know how long they will feel anxious and find no joy in living. The distorted roles established during the child's illness need to be reassessed and parents helped to reinvest their energies. At this point 9 families have made a healthy readjustment and 8 families feel the need to continue in the program. This approach appears to be effective in re-establishing normal family relationships and uncovering situations that could have been corrected during the course of the
child's illness, possibly preventing the need for this therapy.

27 CARE OF THE LOW BIRTH WEIGHT AND SICK NEWBORN INFANT IN COMMUNTY HOSPITALS. M. Jeffrey Maisels, Sarah Fernsler, Kathleen Gifford, Richard J. Hildebrandt (Intr. by Nicholas M. Nelson). Penn State Univ. Col. of Med., M. S. Hershey Med. Ctr., Dept. of Ped., Ob/Gyn., Hershey, Pa.

A review of charts of all infants with birthweights $<2500 \mathrm{~g}$ and all neonatal deaths during the years 1972 and 1973 was performed in 11 community hospitals in Central Pennsylvania. 413 charts were reviewed to assess methods of care. In 7 hospitals no physicians' order was ever written for a specific incubator temperature which was recorded by nurses in $52 \%$ of infants in incubators. Orders for oxygen in liters or nonspecific instructions such as "keep baby pink" were used in $66 \%$. Although oxygen analyzers were present in every nursery, oxygen administration was recorded by nurses in liters $15 \%$ of the time. No ophthalmologic examination was recorded or scheduled after discharge in any infant receiving oxygen. Of 170 non-distressed low birth weight infants $55(32 \%)$ received their first feed after 16 hours of life. In 45 infants fed via a nasogastric tube only 1 had a recording of the residual volume of formula prior to subsequent feeding. $35 \%$ had no apgar score recorded on the infant's chart and no classification by weight and gestation was present in $74 \%$. In infants on antibiotic therapy, $74 \%$ had no blood, urine or spinal fluid cultures prior to therapy. Of infants having apnea, $58 \%$ had no laboratory investigations performed to determine the cause. This study suggested the urgent need for outreach educational programs to help physicians and nurses provide adequate care for newborn infants.
An attempt was made to explore the temperament of 24 SIDS victims retrospectively using a modification of WB Carey's questionnaire. Controls were $2-6$ siblings for each SIDS infant. SIDS victims differed from their siblings $(P<0.05)$ (a) less reaction ta strangers, loud noises, bright lights, bathing, changes in temper ature or food (b) less physical activity while sleeping \& during various waking activities (c) less vigorous suck, more breathless spells \& exhaustion during feeding (d) less differences between cries that express various needs \& moods. There were no differences between SIDS victims and siblings: (a) regularity of pattern of various waking activities or going to sleep (b) crying in response to various activities (c) frequency of coughing and associated dyspnea, color change or regurgitation (d) skin color when crying.

Some of the SIDS descriptions may be fantasies, the idealized memories of a yood or easy baby. However, some featues are unlikely to be fantasy, particularly observations associated with suck, exhaustion during feeding and abnormal cries.

Recent clinical and postmortem studies suggest that central neural mechanisms of respiratory and other homeostatic controls may be chronically abnormal in some SIDS victims. The current study indicates that SIDS victims may have other subtle brain abnormalities that manifest themselves by differences in infant temperament. (USPHS grant HL 14297-03 \& contract 1 HD-4-2817)
29 EEG AND SLEEP STATES IN NEWBORNS. Arthur H. Parmelee, Univ, of California, Sch. of Med., Center for the realization reflects the degree of development of cyber. State organization reflects the degree of development of cyber. netic nervous system controls in infants. Understanding of stat Three hour polygrams were recorded from 12 normal term newborns. Four previously described EEG patterns (Anders, Ende, Parmelee) identified independent of state were: Low Voltage Irregular (LVI); Mixed (M); High Voltage Slow (HNS); Trace Alternant (TA). The frequency of $20 \mathrm{sec}$. epochs of each pattern associated with Active, AS, Quiet, QS, and Transition, Tr, sleep states as identified by body and eye movements and respiratory pattern was determined. Distribution of EEG power by percent in the band widths Delta $1-3.5 \mathrm{~Hz}$, Theta 4-7.5 $\mathrm{Hz}$, Alpha $8-12.5 \mathrm{~Hz}$, and Beta 13-25 Hz for each EEG pattern was determined by power spectral computer analysis for five infants.

EEG State $\%$ Power by Frequency Bandwidth Pattern AS QS Tr

$\begin{array}{crrrrrrrr}\text { LVI } & \text { AS } & \text { QS } & \text { Tr } & & & & & \\ \text { M } & 63 & 5 & 32 & 72 & 13 & 8 & 7 & 16 \\ \text { HVS } & 42 & 10 & 48 & 77 & 12 & 4 & 5 & 23 \\ \text { TA } & 12 & 37 & 51 & 80 & 12 & 5 & 3 & 37\end{array}$

$\begin{array}{lllllllll}\text { HVS } & 12 & 37 & 51 & 80 & 12 & 5 & 3 & 37 \\ \text { TA } & 1 & 60 & 39 & 82 & 11 & 4 & 4 & 34\end{array}$

The EEG patterns and the distribution of power in each frequency band are ordered on a gradiant from AS to QS. The frequent occurrence of each pattern in transition periods makes the EEG less valuable in newborns than in adults for determination of states.

3 DEATHS RELATED TO CHILD ABUSE AND NEGLECT OVER A FIFTEEN-YEAR PERIOD. Irwin E. Redlener, Joseph Davis, Steve Morris, Fred Seligman, Karyl Morris, (Intr. by William W. Cleveland) Univ. of Miami Sch. of Med., Depts of Ped. and Path., Miami, Florida.

The real frequency of fatal outcome in child abuse and neglect is difficult to ascertain. In order to examine this problem we reviewed all cases under age 13 autopsied by the County Medical Examiner (M.E.) from 1960-1974. Criteria for the classification of definite and suspected abuse and neglect were developed. During the study period, 1897 deaths were studied by the M.E. Some $59(3.1 \%)$ of these were oxiginally stated to be related to child abuse or neglect. Our retrospective review found, however, that 323 cases $(17 \%)$ should have potentially been in this category. Estimates of the total child population in Dade County for each study year concluded that this group remained essentially constant in size because of a falling birth rate $(20.7 / 1000$ total pop. in 1960 vs. 12.6 in 1973) in spite of greater than a $35 \%$ increase in general population. Furthermore, the 323 abuse/ neglect deaths were distributed fairly evenly over each of the 15 study years. In contrast, the rate of agency reported child abuse cases rose to 3945 in 1974 from less than 100 in 1960.

We concluded: (1) The number of deaths related to child abuse per total child population has probably been unchanged in Dade County for the past 15 years in spite of an increased incidence of reported abuse and neglect. (2) A high index of suspicion is required to delineate all cases of abuse/neglect related deaths since our study yielded a five-fold greater mortality than previously reported. 
31 DIFFERENIIATION OF ENVIROMMENTAL FROM ORGANIC FAILURE TO THFIVE Daniel. W. Rosenn, Lorraine Stein, Martha B. Jura, (Intr. by Mary EIIen Avery) Harvard Univ. Med. Sch., The Children's Hosp. Med. Ctr., Dept. of Ped., Boston.

The traditional diagnostic evaluation of failure to thrive (FTT) infants in hospital can be prolonged, traumatic and costly. Preliminary findings of this study suggest that environmental FTT can be distinguished from organic FT during the first 7-10 days of hospitalization on the basis of easily documented and quantifiable behavioral changes. These changes are independent of caloric intake and weight gain. A 7 point Approach-Withdrawal scale was administered three times daily throughout hospitalization to 30 infants ages 6-15 months. The infants were subsequently grouped into 3 categories on the basis of medical evaluation: environmental FTT, organic FTT, and developmentally normal infants hospitalized with acute ilinesses. Environmental FTT infants seem to show significantly more positive behaviors to objects and distant social approaches, while organic and normal controls respond more positively to close, interpersonal interactions such as touching and holding. The Approach-Withdrawal scale has led to the development of "characteristic" behavioral profiles of hospital recovery for the three groups of infants and provides a method for recording and identifying the various patterns of coping that infants use in dealing with the stresses of separation, hospitalization and illness.

32 GENDER AND RACE-ETHNiC FACTORS IN HEALTH SERVICE PLANNING. F. Seligman, Univ. of Miami, Florida (Intr. by W. W. Cleveland)

More than 10,000 different patient records over a period of 6 years have been analyzed to determine the usage of health services in a comprehensive health care setting for children and youth by duration of time an individual patient is enrolled in the program and by race-ethnic grouping of the patient.

The data reflects that: 1) utilization tends to decrease during the first 3 years of care with a gradual increased utilization of services after this time, except for 2) Black patients who are more than 10 years old when registered. This category exhibits a decreasing rate of utilization with time, especially for Black boys. 3) Spanish patients utilize health services significantly more than Black patients. 4) Spanish boys utilize health services more than Spanish girls. 5) For Black patients brought into comprehensive health care after age 4 years, girls utilize services much more than boys.

Ramifications of this data include:

1) Race-ethnic groups value health differently.

2) Boys and girls are valued differently depending on raceethnicity.

3) Health service planning must take into account the gender and race-ethnic composition of the population serviced, in addition to age and duration of care provided.

33 INFANT'S SUCKING RESPONSES AS A CONJUGATE FUNCTION OF AUDITORY STIMULI. Shriner, T.H. (Intr. by R. S. Stempfel). Dept. of Ped., Univ. of Miami, School of Medicine, Miami, Fla.

The purpose is to summarize recent research on a relatively new method for recording the infants' sucking response as a conjugate function of changes in the intensity of auditory stimuli. In conjugate reinforcement the intensity of a continuously available stimulus varies directly and immediately with the rate of response. The method was recently evaluated by testing the auditory capabilities of 105 neonates, mean age 53 hours, range 24108 hours (Eisele, Berry, Shriner, 1974). In this project, three pulsed tones, 1,2 , and $4 \mathrm{kHz}$ were presented sound field. As long as the infant continued to suck at its base rate or higher the intensity of the pulsed tones would increase-when the infant ceased to suck the intensity of the tones would decrease. Results indicated that the infants responded differentially for the tones. They ceased to suck when the intensity levels were 59.2 , 62.0 , and $67.7 \mathrm{~dB}$ for 1,2 and $4 \mathrm{kHz}$, respectively. Based upon this finding it is hypothesized that the tones are mildly aversive. In another study, and by use of the same method, 37 infants with medically diagnosed problems were tested and are currently being followed. Of these 37, 7 of 9 (2 questionable) could not discriminate between speech-like and non-speech stimuli. Currently, these particular infants have delayed, medically determined motor development. Other infants who were diagnosed as medically normal and who also performed within normal limits on the discrimination task are currently developing normally.
34 HOTLINE - A BAROMETER OF COMMUNITY PROBLEMS M.S. Stern $\frac{\text { R.G. MacKenzie, }}{\text { Hosp. of L.A., Uoffberg, Dept. of Ped., Child. }}$ Hotline, a concept initiated in Los Angeles in 1968 has provided an easily accessible crisis intervention service for the adolescent community as well as a unique window on the problems in the community. Characterizing each of the 1,000 calls per month under 42 different headings has provided some quantitative information as to users needs and problems. Analys is of the data for nine months beginning in January, 1974 reveals the following: the average age of a Hotline user is 25 years, $28 \%$ of whom are over the age of thirty. Loneliness and depression accounts for $16 \%$ of calls from those $14-28$ years old while only $4.5 \%$ of these callers were seeking assistance for drug abuse problems. Alcohol was identified as a problem in less than $2 \%$ of callers between 15-28 years. Problems relating to sexuality, while few in number, had a significant increase in importance after age 15. However, problems surrounding pregnancy and contraception maintained significant importance for these young people 13 through 19 years of age. While the Hotline represents a limited view on the problems of the cormunity, we are able to appreciate some interesting trends. The caller tends to be older than in previous years, drawing on Hotline resources most in times of loneliness and depression. Despite current evidence of increasing alcohol abuse among the adolescent he either (1) does not perceive the. Hotline as a resource, or (2) more importantly does not perceive alcohol abuse as a problem. With continuous monitoring the "Hotline" can serve both for crisis intervention as well as a barometer of overall community problems.

PEER REVIEW OF AMBULATORY CHILD HEALTH CARE. Hugh C. Thompson and Charles E. Osborne, Dept. Ped., Univ. of Ariz. Col. of Med., and Acad. of Ped., Evanston,

IIlinois. (Introduced by Vincent A. Fulginiti)

The goal was to develop and test a methodology for Quality Assurance of Ambulatory Child Health Care. Criteria were developed by the Joint Committee on Quality Assurance. As the basic care given children should be the same from any provider the Committee was composed of pediatricians, family physicians and an osteopath. Areas studied were health supervision (foux age groups), tonsillopharyngitis, bronchial asthma and urinary infection.

Criteria were tested by self audit of charts by 130 primary care physicians. They were judged by 450 "Experts"-academicians and practitioners-for relevance to health outcome and desirability for use in Peer Review (PR). Resultant criteria were appraised by a representative sample of 2500 primary care physicians. Medical records personnel audited 10,500 charts in 166 of fices (100 pediatricians and 66 family physicians) to document recording of criteria performance.

Results: 1) Three specialties worked harmoniously in criteria development; 2) Academicians and "Expert" practitioners agreed very closely as to relevance and use in PR; 3) Overall recording of criteria was approximately $50 \%$; 4) Development and counseling items were very poorly recorded; 5) Audited physicians found the method acceptable and corroborated reviewer findings; 6) $50 \%$ said findings did not accurately represent their practice.

PR of ambulatory care by chart audit cannot be satisfactorily performed in 1975 because recording habits do not accurately represent physician performance.

36 ETHICAL ASPECTS OF PEDIATRIC EXPERIMENTATION, Grace S. Wolff, (Intr. by E. B. Hook), Dept. of Ped. Albany Med. Co1., Albany, New York.

The ethical dimensions of human experimentation are nowhere as problematic as in the realm of the infant and child.

Despite the fact that the infant and small child are not persons in the full sense of the definition, society has recognized the responsibility to protect them. To ensure such protection various codes have been formulated regarding human experimentation. Such codification has been necessitated, in great part, because of the conflict of interests between societal benefit and individual benefit. In the situation in which consent is by proxy and in which risk is unaccompanied by advantage, it is difficult to enlist ethical support for such experimentation. A utilitarian ethic may uphold such experimentation but it cannot do so without sacrificing a non-consenting individual to society. Can Society truly benefit by injustice and unfairness to its individual members? 
37 PATTERNS OF VISUAL FIXATION IN THE HIGH RISK INFANT. Judith Zarin-Ackerman, Michael Lewis and John M. Driscoll, Jr. (Intr. by L. S. James) Coll of Physicians and Surgeons, Columbia University, Div. of Perinatology, NYC $V$ isual attending behavior is an index of perceptual-cognitive function and provides a means of evaluating potential mental development at an early age before the full impact of environmental factors. It is assessed from the rate at which an infant becomes disinterested in an identical picture and shows renewed interest in a new picture and has been evaluated in 77 high risk infants, 32 of whom have suffered from severe respiratory distress syndrome (RDS) and 45 of whom have suffered from severe birth asphyxia, as well as a control population of 20 healthy term infants. Visual attending was measured at $3,6,9,12,18$ and 24 months of age. Results show less visual attention and lack of habituation in the high risk group as a whole compared to the control group infants. Furthermore, there was a poorer response in the asphyxiated infants than in those who had suffered from respiratory distress. With advancing age, habituation became more rapid in the RDS group than in the asphyxiated group.

These findings indicate that both severe RDS and birth asphyxia in general affect the infant's ability to attend to stimuli and may therefore significantly affect later learning ability. The harmful effect of asphyxia appears to be greater than RDS.

\section{CARDIOLOGY}

\section{CARDIAC MUSCLE: THE INTERACTION BETWEEN} INOTROPIC STATE, THE FORCE-FREQUENCY RELATIONSHIP, AND MUSCLE LENGTH. Page A. W. Anderson, Andres Manring, and Edward A.Johnson. Duke Med.Sch., Duke Med.Ctr., Dept. of Pediatrics and Physiology, Durham, N.C. (Intro. by M.S. Spach). Supported by grants from NIH (HL11307, HL5372, HL58305, HL12157 and HL50791) and North Carolina Heart Assoc.

Cardiac contractility in general depends on three functions; one a function of muscle length, $\lambda$ ( $F$ rank-Starling principle), a function of the rate and pattern of stimulation, $\phi$, and a function of environmental conditions, $\gamma$, (e.g., temperature and drug or ionic concentration). It has been previously demonstrated that $\phi$ is independent of $\lambda$ (and conversely) but is affected by environmental changes, e.g., changes in calcium concentration. An additional prerequisite for the complete description of cardiac contractility is an analysis of the interrelationship between $\lambda$ and $\boldsymbol{\gamma}$. This analysis was made for isolated cat and rabbit papillary muscle at $38^{\circ} \mathrm{C}$ by changing the muscle length and calcium concentration $(2.5-7.5 \mathrm{mM})$. These results demonstrate that if the maximum rate of rise of tension (although not the peak tension) is used as the measure of contractility, $\lambda$ is independent of $\boldsymbol{\gamma}$ (and conversely). Furthermore, the results demonstrate that contractility is a function of length times a function of the rate and pattern of stimulation which itself depends on environmental conditions.

EVALUATION OF LEFT VENTRICULAR FUNCTION IN PREMATURES

39 WITH PATENT DUCTUS ARTERIOSUS (PDA) AND PIJMMONARY DISEASE. Barry G. Baylen, Richard A. Heyer, Joan Korfhagen, and Samuel Kaplan, Children's Hosp., Cincinnati, Ohio. We cvaluated the percent shortening of internal diametex (\%SID) of the left ventricle in 157 serial echo studies of 41 prematures. Infants fell into one of several groups. Values for \%SID of 16 asymptomatic infants $(m=32.7 ; S . D .=3.18)$ and 15 with pulmonary disease $(P D)$ alone $(m=32.4 ; S . D .=4.49)$ did not differ significantly. These values served as controls and were comparable to those of asymptomatic adults. In infants with PDA with or without PD, values for $\% S I D$ and left atrial(LA) and left ventricular(LV) dimensions of 12 medically treated infants $(m=39.7 ; S . D .=6.6)$ and 14 surgically treated infants $(m=43 ; S . D .=9.2)$ were significantly increased, and as expected \%SID's of individual patients represented a wider range of LV function than those of controls. Upon spontaneous or surgical closure of the PDA, LV and LA and $\%$ SIO returned to the normal range.

Thus, significant left-right shunting through a PDA is associated with increased LA, LV, and \%SID. These values reflect increased LV volume and/or decreased peripheral vascular resistance. Decreasing \%SID in the presence of increased LA and LV size suggests deterioration of LV function. A readily available non-invasive method of evaluation of $1 \mathrm{eft}$ ventricular function in prematures with patent ductus arteriosus and pulmonary disease was demonstrated.
40 PRESSURE-FLOW RELATIONSHIPS IN THE UTERINE AND UMBILICAL CIRCULATIONS IN THE SHEEP. William Berman,

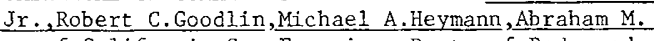
Rudolph. Univ, of California San Francisco,Dept. of Ped. and Cardiovascular Research Institute.

It has been suggested that uterine vascular pressures may regulate umbilical vascular resistance and thus,alter umbilical blood flow. This "waterfall"or"sluice"theory has been invoked to explain the supine hypotensive syndrome and fetal bradycardia during uterine contractions(Type I dips). We tested this theory in 13 standing, unanesthetized sheep. We continuously measured the following in chronic studies: umbilical and uterine arterial. blood flow with electromagnetic flow meters; pressures in maternal aorta, uterine vein, fetal aorta,common umbilical vein and amniotic cavity; and fetal heart rate. Balloon catheters were used to lower distal aortic and raise umbilical and uterine venous pressures. Elevation of umbilical venous or reduction of fetal aortic pressure decreased umbilical flow without affecting uterine flow. Reduction of maternal aortic or elevation of uterine venous pressure decreased uterine flow without affecting umbilical flow until the fetus became hypoxic;then fetal hypertension and bradycardia were associated with a fall in umbilical flow. Atropine to the fetus abolished the bradycardia of fetal hypoxia and prevented the fall in umbilical flow. These results indicate that the fetal umbilical-placental circulation obeys the Poisseuille-Hagen 1 aw and is not directly influenced by large changes in maternal vascular pressures. Supported by PHS Grant HL 06285 .

HEMODYNAMIC AND BIOCHEMICAL STUDIES IN SPONTANEOUSLY

41 HYPERTENSIVE RATS. Lawrence Berte, Anthony $\mathrm{F}$. Cutilletta, Peter Sodt, and Rene A. Arcilla. Univ. of Chicago, Department of Pediatrics, Chicago, Illinois. Hemodynamic and biochemical determinations were made on 40 spontaneously hypertensive Okamoto-Aoki rats (SHR) and 40 normotensive Wistar rats (NR) at age 4-6, 8-10, 20-24 and 40-44 weeks. LV systolic and aortic pressures were significantly elevated in the SHR than NR in all groups except at 4-6 weeks, as was systemic vascular resistance. Cardiac index (CI) measured with an electromagnetic flow probe at the ascending aorta was higher in SHR at 4-6 weeks $(165 \pm 42$ vs $141 \pm 22 \mathrm{~m} 1 / \mathrm{min} / \mathrm{kg})$, al though not statisti cally significant. After 8 weeks, CI of SHR and NR did not differ. Peak stroke power and stroke work were elevated in SHR. Maximal $\mathrm{dP} / \mathrm{dt}$ was higher in SHR than NR except at 4-6 weeks. However, $(\mathrm{d} / \mathrm{d} t) \mathrm{P}^{-1}$ did not differ. All. SHR had significant left ventricular hypertrophy (LVH). LV-body weight ratios were: $37,30,40$ and $73 \%$ above NR for each age group (all P<0.001). Total LV RNA of SHR was elevated at $20-24$ and $40-44$ weeks $(2.26 \pm .18$ and $2.67 \pm .28$ vs $1.95 \pm .07$ and $2.15 \pm .11 \mathrm{mg}, P<0.005$ and $P<0.05$, respective1y). Whi Te total LV DNA did not differ significantly between SHR and NR, LV hydroxyproline (OHP) was higher in SHR at 40-44 weeks than in NR, $1.22+.11$ vs $0.96+.08 \mathrm{mg}(P<0.05)$. When corrected for body weight, $\overline{R N A}$, DNA and $\mathrm{O} \overline{\mathrm{H} P}$ were significantly higher in SHR for all age groups. These data support the concept that LVH occurs in SHR before onset of hypertension. The high CI at 4-6 weeks suggest that factors other than elevated pressure play a role in LVH development in the young SHR. The increase in LV mass is accompanied by increase in connective tissue.

\section{THE PRE-AND POST OPERATIVE RESPONSE OF WITH CYANOTIC HEART DISEASE}

Marta Blesa, S. Lahiri, W. Rashkind and A.P. Fishman, Children's Hospital and Cardiovascular and Pulmonary Division of Department of Medicine, University of Pennsylvania School of Medicine, Philadelphia, Pa.

Adult subjects born and living at high altitudes have a blunted ventilatory response to hypoxia. Patients with cyanotic congenital heart disease also show a blunted response. It is not known when and how this phenomenon develops. The reversibility of this reaction in cyanotic patients following corrective surgery has been a matter of controversy. six children, aje 6 to 16 years, with cyanotic congenital heart disease were studied: their ventilatory response to hypoxia was blunted. Ten children who were cyanotic preoperatively were studied 2 months to 6 years postoperatively. They showed improved response to a degree directly correlating with their saO2. These results show that the blunted response of ventilation to hypoxia develops at least as early as within six years of persistent hypoxia during childhood; the blunting effect of chronic hypoxia diminishes rapidly after corrective surgery. Since the major change following surgery was an improvement in arterial blood oxygen saturation, the phenomenon of blunted response may be due in part to hypoxic depression. 
42 PRE AND POST OPERATIVE RIGHT VENTRICULAR FUNCTION STUDIES IN PATIENTS WITH TETRALOGY OF FALLOT Ramon V. Canent, Jr. and Rengasamy Gowdamarajan The Children's Mercy Hospital, Kansas City, Mo. Quantitative biplane angiographic analysis of right ventricular volume variables were done in 30 patients with Tetralogy of Fallot to assess right ventricular function. Right ventricular end diastolic volume (RVEDV) and end systolic volume (RVESV) were calculated by the Simpson's Rule and from these, the RV stroke volume (RVSV); RV ejection fraction (RVEF); RV systolic index (RVSI) were derived and compared statistically to normal data.

Preoperative data were obtained from 21 patients including 9 below 2 years (Group I), analyzed separately and compared with data from 12 children above 2 years (Group II). Group III showed analysis from 9 patients who had corrective surgery.

The RVEDV in Group I was $106 \pm 27 \%$ of $N$; Group III $114.5 \pm 23 \%$; but lower in Group II $(75+20 \%$ of $N)$. The RVEF was depressed in all groups, but more so in Group II. The RVSI was normal in Groups $I$ and III, and only $65 \pm 25 \%$ of $N$ in Group II.

The RV function was depressed in all patients before and after corrective surgery. However, following surgery most of the patients showed slight improvement in their depressed RVEF.

Full thickness RV myocardial biopsy were obtained during the corrective surgery from 11 patients and all patients showed endocardial fibroelastosis, but significant fibrous, elastic, and collagen tissue invasion of the myocardium occurred in 4 years and over age group. Extensive proliferation of fibrous and collagen tissue occurs around small and medium sized intramyocardial coronary vessels, including the central artery of the SA node.

RESULTS OF SYSTEMIC PULMONARY ARTERY SHUNTS IN TETRALOGY OF FALLOT Julie A. Clayman, Jerome Liebman, Jay
L. Ankeney, Univ. Hosp. of Cleveland, Dept. of Surg.

Recent enthusiasm for repair of Tetralogy of Fallot (TOF) during infancy raises the question as to what role palliative shunts should play and prompted us to review our experience with 68 patients who underwent shunts for TOF. Ascending aorta-pulmonary artery shunts (A-PA) were performed in 38 children whose ages ranged from 8 days to 4 years (median age 6 months). Indications for surgery were anoxic spells in 32 and arterial oxygen saturations $<70 \%$ in 6 . There were 9 operative deaths $(23.9 \%), 7$ of which occurred in the 28 patients $<1$ year of age. 5 deaths were due to congestive heart failure. 10 of the 29 survivors subsequently underwent total repair with 3 deaths $(30 \%)$, all shunt related. Subclavian-pulmonary artery shunts (S-PA) were performed in 30 patients whose ages ranged from 3 months to 27 years (median age $11 / 2$ years). Indications for surgery were anoxic spells in 22 , hematocrits $>70$ in 4 , and arterial oxygen saturations $<70 \%$ in 4. There were 2 deaths $(6.6 \%)$, both in infants $<6$ months of age and both $2^{\circ}$ to shunt thrombosis. 8 children were $<1$ year of age. 22 of 28 survivors subsequently underwent total correction with 2 deaths (9.9\%); neither were shunt related. Our experience indicates that S-PA shunts can be performed with a low operative risk without adding to the mortality of subsequent total correction. In contrast, A-PA shunts carry a significant risk and add to the mortality of subsequent repair. Therefore our current approach is to perform S-PA shunts in infants $<1$ year of age requiring palliar tion, reserving A-PA shunts for infants $<3$ months of age. Total correction is performed in children $>$ I year of age.

CARDIAC FUNCTION DURING DEEP HYPOTHERMIA. Anthony $\mathrm{F}$. $\frac{\text { Cutilletta, }}{\text { L. Replogle }}, \frac{\text { Chung Y. Lin, }}{\text { Univ. of Chicago, Dept. of Ped., Chicago, }}$

Illinois

Since 1971, 42 infants with congenital heart defects had surgery using surface cooling technics and total circulatory arrest followed by rewarming with cardiopulmonary bypass. Cardiac performance was measured in 10 infants prior to circulatory arrest, and during and after rewarming using electromagnetic flow probes and direct ventricular pressure recordings. Prior to circulatory arrest, body temperature averaged $21+0.4^{\circ} \mathrm{C}$. At this temperature, the following parameters were significantly decreased when compared to post-rewarming values: heart rate $53 \pm 3$ vs $145 \pm 6$ beats/min. ( $P<0.001)$; left ventricular systolic pressure $\overline{51}+4$ vs $99+8 \mathrm{mmHg}(P<0.001) ;$ cardiac index $0.45+0.08$ vs $1.99+0.35$ $1 / \mathrm{min} / \mathrm{M}^{2}(\mathrm{P}<0.005)$; stroke work $6.3+1.3$ vs $18.9+4.6 \mathrm{gm}-\mathrm{M} /$ beats $/ \mathrm{M}^{2} \quad(\mathrm{P}<0.025)$ and $\mathrm{dp} / \mathrm{dt} \max 363 \pm 46 \mathrm{vs} 1536 \pm 155 \mathrm{mmHg} / \mathrm{sec}$. $(P<0.001)$. Systemic vascular resistance and systolic ejection period were significantly increased during hypothermia, $96 \pm 12$ vs $34+5$ units $(P<0.001)$ and $0.47+0.02$ vs $0.20+0.02 \mathrm{sec}$ $(P<0.001)$, respectively. The decrease in cardiac output was related malnly to the fall in heart rate, since stroke volume did not differ significantly. This low cardiac output does, however, appeax to be adequate to meet the metabolic requirements during cooling since oxygen consumption decreased sharply, $14.6+2.5$ at $20^{\circ} \mathrm{C}$ vs $95.5 \pm 21.1 \mathrm{ml} / \mathrm{min} / \mathrm{M}^{2}$ at $37^{\circ} \mathrm{C}(\mathrm{P}<0.01)$. Blood $\mathrm{pH}$ remained normal or slightly elevated. These data support the concept that the reduction in cardiac performance during deep hypothermia is still sufficient to meet the metabolic needs of the body.
EVALUATION OF ATRIAL PACEMAKER SITE AND FUNC46 TION BY INTRACARDIAC RECORDING AND PACING. Domingo J. de la Fuente, Yaser Freij, Otto G. Thilenius and Rene A. Arcilla. Univ. of Chicago, Dept. of Pediatrics, Chicago.

Frontal plane $\mathrm{P}$ wave (FP-P) axis of $0^{\circ}$ to $30^{\circ}$ traditionally suggests left atrial enlargement in sinus rhythm. This was tested in 29 children during cardiac catheterization. With a multiple electrode catheter, intracardiac potentials in high right atrium-sinus node area (HRA-SN) and in low right atrium (LRA) were recorded simultaneously with ECG leads II and aVF, during spontaneous rhythm and during $\mathrm{HRA}-\mathrm{SN}$ pacing. Maximal pacing rate was $170 / \mathrm{min}$; post-overdrive recovery times were used as index of pacemaker function. In 22 subjects (group A) with sinus rhythm and FP-Paxis of +300 to 600 , HRA-SN activation preceded IRA by $22 \mathrm{msec}$ mean In 2 subjects (group $B$ ) with FP-P axis beyond $-30^{\circ}$, LRA activation preceded HRA-SN by 22 msecs. In 5 subjects (group C) with FP-P axis of +150 to 00 ( $P$ is oelectric or biphasic in $\mathrm{aVF}$ ), earlier activation of LRA was observed in all with mean LRA to HRA-SN interval of $26 \mathrm{msec}$. Post-overdrive recovery times in $A, B$ and $C$ were normal. In $B$ and $C$, the HRA-SN pacing produced positive $\mathrm{P}$ in II and aVF (similar to those in A) and FP-P axes of $+45^{\circ}$ to $60^{\circ}$. It is concluded that $\mathrm{P}$ axis of approximately $0 \circ$ (isoelectric $\mathrm{P}$ in aVF) most likely represents low-lying atrial pacemaker rather than sinus, and is not a valid criterion for left atrial hypertrophy. Atrial ectopic pacemakers appear to have normal pacing function.

\section{ELECTROPHYSIOLOGIC EFFECTS OF HYDROXYZINE ON CANINE HEART. Domingo $J$. de la Fuente and Craig} Willert (Intr. by Rene A. Arcilla). Univ. of Chicago, Department of Pediatrics, Chicago.

Hydroxyzine (Vistaril) is widely used as pre-medication for surgery including cardiac catheterization. This study was done to evaluate its assumed anti-arrhythmic properties using fundamental electrophysiological parameters. In 10 open chest, Nembutalanesthetized dogs with cut vagi and stellates, bipolar epicardial electrodes were placed in right atrium (RA) and right ventricle (RV) for recording, regular drive and test stimulation. An intracardiac multipolar electrode recorded His bundle potentials. Using a 5 -msec cathodal test pulse in diastole after regular RA drive, strength-interval curves were constructed. The same was done for RV. The functional refractory period of $A V$ conduction (FRPAV) was also determined. Hydroxyzine was given intravenously at 2.5 to $5 \mathrm{mg} / \mathrm{kg}$ and the measurements repeated. Results showed shift to the right of the strength-interval curves in both RA and RV indicating an increase in the refractory period, a measure of decreased excitability; and equivocal effect on FRPAV. Transmembrane potential studies with intracellular stimulation using microelectrode techniques showed similar effects. It is concluded that Hydroxyzine does have direct electrophysiologic effects on canine heart muscle qualitatively similar to quinidine.

18 INFLUENCE OF AGE ON CARDIAC REFRACTORY PERIODS IN MAN, Ira DuBrow, Elizabeth Fisher, Pablo Denes, Kenneth Rosen, Alois Hastreiter. Departments of Pediatric and Adult Cardiology, University of Illinois Abraham Lincoln School of Medicine, Chicago, Illinois.

Atrial (A) and A-V nodal (AVN) effective and functional refractory periods (ERP and FRP) were determined by atrial extra-stimulus technique in 70 patients, ages 7 mos. thru 77 yrs., with normal $P-R$ intervals and $Q R S$ durations. The patients were divided into six age groups: $<2$ yrs. $(N=10), 3-5$ yrs. $(N=15), 6-10$ yrs. $(N=14)$, $11-15$ yrs. $(\mathrm{N}=12), 16-30$ yrs. $(\mathrm{N}=8),>30$ yrs. $(\mathrm{N}=15)$. Refractory periods were determined at 1-4 controlled cycle lengths per patient, and each age group was divided into cycle length ranges (CL) in milliseconds (msec): CL $1800-600 \mathrm{msec}, \mathrm{CL}_{2} 599-460 \mathrm{msec}$, CL3 $<459$ msec. Statistical significance of data was obtained by analysis of variance. Cycle lengths within each CL for each age group were not significantly different $(F>.25->.05)$. AERP and FRP, and AVNERP and FRP within each age group at each CL were significantly different from each other $(\mathrm{F}<.025-<.001)$. Younger age groups had shorter refractory periods than older groups at equivalent cycle lengths. These differences were most marked between groups <2 yrs. and 6-10 yrs. A-V nodal full recovery times (FRT) were determined in age groups $<2$ yrs. thru 11-15 yrs. at $\mathrm{CL}_{2}$ and $\mathrm{CL}_{3}$. AVNFRT's were significantly different within age groups at $\mathrm{CL}_{2}(\mathrm{~F}<.05)$ and at $\mathrm{CL}_{3}(\mathrm{~F}<.01)$ - the younger groups having shorter values. These data are germane to understanding the maturation of electrophysiologic properties of atrioventricular conduction and help to explain differences in conduction disturbances at various ages in man. 
49 ELECTROCARDIOGRAM IN PROGENY AND SPOUSES OF FATHERS WITH PREMATURE MYOCARDIAL INFARCTION. Pedro L. Ferrer, Mary Jane Jesse and Sidney Blumentha1. Department of Pediatrics, University of Miami School of Medicine, Miami, Florida.

This case-control study, whose index cases were fathers who had a documented myocardial infarction prior to the age of 50 yrs, tried to determine if any ECG identifiable "pattern" was present in the progeny or spouses of affected individuals. Forty six families were studied ( 25 case families, 21 control families, 92 parents and 111 children, age 2 - 18 yrs).

Forty ECG parameters were evaluated including: heart rate and rhythm, mean $Q R S, T$ and $P$ axis, $P$ duration, $P R$ interval, $P$ voltages, QT index, detailed analysis of $S T$ segment, $T$ and U waves.

No significant differences were found in the progeny or spouses of fathers with prengture myocardial infarction, as compared with the progeny and spouses respectively of control families.
52 ACTIVE COMPLIANCE IN THE FETAL HEART by W.F. Friedman, R.A. McPherson, and J.W. Covell, University of Calif. San Diego, School of Med., La Jolla, CA. Our past studies indicate that fetal heart muscle is weaker intrinsically than the adult, whereas no or little age related differences exist in unloaded velocity of shortening. Although lesser contractile tissue per unit volume in fetal heart may explain its depressed force generation, an alternate explanation may be a more compliant series elasticity. Accordingly, the series elastic properties of ventricular myocardium isolated from fetal lambs and adult sheep were analyzed via quick releases using a hybrid computer and a closed-loop servo system and electromagnetic positioning device to control muscle length or tension. Data was normalized for the microscopically determined percent contractile tissue present in the muscles studied. Over a comparable range of muscle lengths, quick releases of adult and fetal muscle yielded an exponential relationship between change in stress and strain. Age dependent differences in active stiffness related primarily to the amount of muscle tissue present in fetal and adult myocardium. Correction of the raw data for muscle mass and system compliance demonstrated no significant differences in series elasticity between fetus and adult. Thus, a developmental difference in active compliance is an unlikely explanation for the attenuation of force generation in the fetal heart. Moreover, the findings validate the use in clinical studies of newborns of a modulus of elasticity similar to the adult when ventricular performance is assessed angiographically.

FUNCTIONAL PROPERTIES OF THE ATRIOVENTRICULAR NODE HEMODYNAMIC AND INTRACAVITARY ECG CHANGES WITH ISO0 PROTERENOL INFUSION IN DOGS WITH NORMAL AND HYPERTROPHIED RIGHT VENTRICLES. David E. Fixler, Charles B. Mullins Intro by Gladys Fashena, Dept. Ped \& Med, Univ of Texas Health Science Center, Dallas, Ix. Isoproterenol (I) was infused at $0.1 \mathrm{mcg} / \mathrm{kg} / \mathrm{min}$ into dogs with normal (N) and hypertrophied(RVH) right ventricles to examine differences in coronary and electrographic responses. The pulmonary artery(PA) was banded to a mean proximal PA pressure of $30 \mathrm{mrn} \mathrm{Hg}$ in 9 dogs and maintained for 6 months. Mean RV weights in $\mathrm{N}$ was $24 \mathrm{gms}$ and in RVH $51 \mathrm{gms}$. We measured RV and aortic pressures, coronary flow (radioactive microsphexes) and RV intracavitary electrograms. Response to I differed ( $p<.05$ ) between $\mathrm{N}$ and RVH groups in the following parameters: heart rate, up 43 beats/min in N vs 86 in RVH, RV systolic pressure up $6 \mathrm{~mm} \mathrm{Hg}$ in $\mathrm{N}$ vs $44 \mathrm{in} \mathrm{RVH,} \mathrm{RV} \mathrm{end-diastolic} \mathrm{pres-}$ sure down $.3 \mathrm{~mm} \mathrm{Hg}$ in $\mathrm{N}$ vs up $2.4 \mathrm{~mm} \mathrm{Hg}$ in $\mathrm{RVH}$. RV myocardial flow $(\mathrm{ml} / \mathrm{min} / 100 \mathrm{~g}$ ) with I increased from 58 to 86 in $\mathrm{N}(\mathrm{p}<.05)$ and from 93 to 159 in RVH $(p<.05)$. In $N$ dogs $R V$ coronary vascular resistance ( $\mathrm{mm} \mathrm{Hg} \cdot \mathrm{sec} \cdot 100 \mathrm{~g} / \mathrm{min}$ ) fell from 204 to 133 , and in RVH dogs from 121 to 33 . Therefore I stimulation increased hemodynamic factors affecting myocardial oxygen needs to a greater extent in dogs with RVH whose coronary vessels were more fully dilated at rest. On the $R V$ electrograms $T$ wave inversion with $\mathrm{I}$ increased more than $1 \mathrm{mv}$ in $6 / 9 \mathrm{dogs}$ with RVH but in none of $N$. The $T$ wave changes suggest ischemia occurred in some animals with RVH. Recording of RV electrograms during I stimulation may be useful in detecting altered coronary hemodynamics in hypertrophied right ventricles.

51 ECHOCARDIOGRAPHIC FEATURES OF SUPRAVAL VLLAR AORTIC STENOSIS. James W. French, James L. Bolen, $\frac{\text { Richard L. }}{\text { Popp. (Intro. by I. SchuTman) Departments }}$ of Pediatrics and Medicine, Stanford University Medical School, Stanford.

Six children with congenital supravalvular aortic stenosis (SVAS) of varied severity were studied by echocardiography and angiography. The left ventricular outflow tract, aortic root and ascending aorta were recorded in a continuous $M$ mode sweep.

The presence of SVAS was documented in each case by a diminution in aortic width as the transducer moved from aortic root to the ascending aorta. The degree of narrowing was quantitated by comparing aortic diameter at the level of minimum echocardiographic dimension in the ascending aorta with the width at the level of the aortic valve. These measurements were compared to angiographic measurements made at the level of the aortic sinuses and most severe narrowing of SVAS. Echocardiographic reduction in diameter ranged from $20 \%$ to $35 \%$ and angiographic reduction from $42 \%$ to $63 \%$. Although there was good qualitative agreement between the degree of echocardiographic and angiographic supravalvular narrowing, echocardiography consistently underestimated the degree of stenosis. This discrepancy may be explained by the obliquity of the echo beam as it passes through the ascending aorta or inconsistency in locating the maximum aortic diameter at the level of the aortic valve.

Seven children without aortic or aortic valve disease were a]so examined by this technique, and none were found to have significant diminution in aortic diameter. Thus, echocardiography appears to be a selective and semiquantitative noninvasive means for detecting SVAS.
53 IN INFANTS AND CHILDREN. Paul C. Gillette, CharlesE Mullins \& Dan G. McNamara. Department of Pediatri Section of Cardiology, Baylor College

Knowledge of the functional properties of the AV node (AVN) in children is important to the understanding and management of dysrhythmias. The purpose of this study was to define the properties of the AVN using His bundle electrography (HBE), atrial pacing and the extra stimulus method. Twenty-one children 9 months to 21 years of age were studied under meperidine, promethazine, and chlorpromazine sedation. Four had normal hemodynamics, ECG, and HBE intervals. Three had first degree AV block ( ${ }^{O}$ AVB) localized to the AVN by HBE. Ten had documented tachyarrhythmias. Eleven had undergone intracardiac surgery. The mean functional (FRP) and effective (ERP) refractory period of the AVN in those subjects without 1 AVB was shorter than that published for adults at comparable basic cycle lengths.

The FRP but not ERP of the AVN was prolonged in the subjects with $1^{\circ}$ AVB

The FRP but not the ERP of the AVN correlated with the atrial pacing rate at which $2^{\circ}$ block occurred.

Thus by measuring the functional refractory period of the atrioventricular node it may be possible to predict the maximum heart rate that can occur during supraventricular tachycardia in a particular patient.
5ECTORCARDIOGRAMS IN INFANTS AND CHILDREN WITH

54 PRIMARY MYOCARDIAL DISEASE. Ronald D. Greenwood, John F. Keane, Alexander S. Nadas, and Donald C. Fyler, Children's Hosp. Med. Ctr., and Harvard Med. Sch., Boston, Mass.

Vectorcardiograms performed in 27 patients ranging in age from $3 / 12$ to 17 years with primary myocardial disease have been evaluated. Thirteen ( $48 \%$ ) had left maximal spatial vectors consistent with left ventricular hypertrophy. The horizontal loop was counter-clockwise in $23(85 \%)$, clockwise in $1(4 \%)$ and Figure-oreight in $3(11 \%)$. The frontal 100 was clockwise in $13(48 \%)$ counter-clockwise in $9(33 \%)$ and figure-of eight in $5(19 \%)$. Four $(15 \%)$ of the frontal plane loops were counter-clockwise and superior. Abnormalities of depolarization were very frequent (77\%) in 21 vectors available for detailed examination. A conduction abnormality consistent with Wolff-Parkinson-White syndrome was present in $3(14 \%)$ and would not allow identification of depolarization abnormalities. In only $2(9 \%)$ was there no depolarization abnormality. The pattern of anterior and lateral infarct was noted in 5, lateral infarction in 1 and anterior and inferior infarct in 1 ; in 9 there was evidence of anterior infarct or absent initial anterior depolarization based on the $10 \mathrm{msec}$ horizontal loop vector. Two patients, one with anterior infarct and one with lateral infarct, had repeat vectors over a period of 1 and 7 months and showed mild improvement in infarct pattern. There was no relation between degree of depolarization abnormality or presence of infarct and outcome. The vectorcardiogram is a useful tool in quantitating left ventricular forces and identifying. abnormalities in depolarization. 
55 LEFT VENTRICULAR VOLUME DETERMINATIONS IN INFANTS AND CHILDREN WITH PRIMARY MYOCARDIAL DISEASE. Ronald D. Greenwood, John F. Keane, Alexander S. Nadas, Gert rude Scheer, and Donald C. Fyler, Children's Hosp. Med. Ctr., and Harvard Med. Sch., Boston, Mass.

Left ventricular volume determinations (end-diastolic volume (EDV), end-systolic volume (ESV), ejection fraction (EF) and mass (M) ], based on Simpson's rule, were made on 18 biplane angiograms from 16 patients with primary myocardial disease ranging in age from 2 weeks to 11 years at examination. The studies were normal (established normals ESV $10-35 \mathrm{ml} / \mathrm{m}^{2}$, EDV $50-80 \mathrm{ml} / \mathrm{m}^{2}$, EF $0.6-$ $0.75, \mathrm{M} 50-90 \mathrm{gm} / \mathrm{m} 2$ ) in four patients who had lost all significant evidence of their disease by the time of the examination. Twelve patients had abnormal studies. In these, the ESV ranged from 52 to $224 \mathrm{ml} / \mathrm{m}^{2}$ (mean 108) and was $200 \%$ of normal or greater in nine; the EDV ranged from 96 to $290 \mathrm{ml} / \mathrm{m}^{2}$ (mean 153) and was $\geq 200 \%$ normal in Pive; EF was 0.26 to 0.49 (mean 0.34 ) and was $<0.40$ in nine; $M$ was 93 to $294 \mathrm{gm} / \mathrm{m}^{2}$ (mean 176 ) and was $\geq 200 \%$ normal in five. Of the 12 with abnormal functions, only two now heve no disease and one is dead while the others have significant residual abnormalities. Residual disease resulted in all with $M$ $>180 \mathrm{gm} / \mathrm{m}^{2}, \mathrm{EDV}>125 \mathrm{mI} / \mathrm{m}^{2}, \mathrm{EF}<0.30$ or $\mathrm{ESV}>90 \mathrm{ml} / \mathrm{m}^{2}$. In Datients with primary myocardial disease, left ventricular volumes, $M$ and $E F$ offer valuable techniques for quantitative estimation of myocardial function.

56 THE CLINICAL COURSE OF CRITICAL CARDIAC DISEASE IN DOWN SYNDROME. Ronald D. Greenwood, and Alexander S. Nadas, Dept. of Cardiology, Children's Hosp. Med. Ctr., Dept. of Ped., Harvard Med. Sch., Boston, Mass.

Two hundred-thirty (628) of 369 patients (pts) seen between 1962 -1974 with Down syndrome (DS) had congenital heart disease (CHD). One hundred-nineteen of these (32\%) were catheterized, operated upon or died from their CHD. Fifty-three (44.58) had endocardial cushion defects (including 32 complete atrioventricular canals (AVC)). The next most frequent lesions were ventricular septal defect (VSD) (188), tetralogy of Fallot (168) and isolated patent ductus arteriosus (138). Catheterizations on those with AVC (mean age 10 mos) revealed pulmonary artery hypertension in all $(16 / 16)$ and pulmonary vascular obstruction (PVO) in over one half $(9 / 14)$. Patients over 3 months of age had PVO and those under this age did not. PVO was also present in 7 of 12 with other forms of cushion defects (mean 4 years of age at catheterization), and in over $1 / 2$ of those with other forms of shunts (11/16) including 8 of 11 with VSD. Twenty-two of the 31 pts with AVC and left-toright shunt did not undergo operation and 17 (778) died. Nine pts were operated upon and $5(56 \%)$ are dead. Seven underwent pulmonary artery banding and 3 of these required reoperation. Surgical intervention in the other acyanotic groups was accomplished with low mortality. Nineteen of 21 pts with diminished pulmonary blood flow (i9 with tetralogy) underwent 32 palliative and 3 corrective procedures; 13 are alive while 8 (38z) are dead including both unoperated pts. Of the $119,66(56 \%)$ are dead. In lesions with diminished pulmonary flow and AVC, surgical morbidity and mortality is very high.

57 ELECTROCARDIOGRAPHIC PATTERN OF MYOCARDIAL INFARCTION TV IUFANTS AND CHILDRN WTMH PRIMARY MYOCARDIAI DISEASE AND NORMAL COROMARY APTERIES. $\frac{\text { Ronald D. Greenwood, }}{\text { Dept. of Cardiologyander S. Hadas, }}$, and Donald C. Fyler, Harvard Med. Sch., boston, Mass.

Seven infants and children aged 1 day to $151 / 2$ years (median 13 months) at first encounter have exhibited tvoical os comolexes of myocardial infarction. These patients reoresent $4 \%$ of 166 vatients with primary myocardial disease seen over a thirty year period. All seven were first seen with congestive heart failure. Three were neonates and one of these also exhibited meningitis. The os complexes were present in the left precordium in six and in Lead I and AVL in one. The infarct pattern persisted to death in four disappeared in two and imnroved but remains in one. None of the patients had coronary artery anomalies. Five are dead (2 days to 6 months after first seen) and two are alive with residual cardiac disease (12 and 13 months after first seen). The etiology was proven at postmortem in four revealing nonobstructive cardiomyopathy in three and mocarditis in one. The best clinically determined etiolosy in the remaining three was felt to be myocarditis. Infants and chilaren with primary mocardial disease without anomalous coronary arteries may be seen with an infarct pattern. The prognosis is poor in this group (mortality $71 \%$ ) and probably represents severe myocarơial compromise and anoxia.
58 CELLULAR ADHESION AND ELECTRICAL COUPLING IN BEATINC EMBRYONIC MYOCARDIAL CELLS. Eva B. Griepp and Merton R. Bernfield. Stanford University Medical School, Dept. of Pediatrics, Stanford, California.

The relationship between cellular adhesion and electrical coupling was investigated using synchronization of beating myocardial cells as an index of coupling. Beating cell aggregates and layers prepared from single-cell suspensions of trypsin-dissociated 7-day chick embryo hearts were cultured for 24 hours. Aggregates were then added to cell layers: after $\frac{1}{2}$ hour, free aggregates were removed. $86 \pm 4 \%$ of aggregates were bound, and $73 \pm 3 \%$ of these were beating. Bound aggregates and the layer contiguous to them were observed at half-hour intervals under phase optics to assess synchrony. Results from several experiments were pooled; each \% (synchronously beating aggregate-layer pairs/total beating pairs) is the mean of at least 3 experiments (30 aggregate-layer pairs observed:

\begin{tabular}{cc} 
Time (Hours) & $\%$ Synchronous \pm Standard Error \\
\cline { 2 - 2 } 2 & $12 \pm 1$ \\
2 & $31 \pm 9$ \\
3 & $45 \pm 16$ \\
4 & $61 \pm 7$
\end{tabular}

Maximal synchrony, $88 \pm 4 \%$, was attained by 10 hours. Thus, after an initial $\mathrm{lag}$, a constant fraction of beating aggregate-layer pairs synchronized during a given time interval (first-order kinetics). These data indicate that although adhesion is undoubtedly vital in initiating functional cellular interactions, cell coupling is not an immediate consequence of adhesion.

59 ECHOCARDIOGRAPHIC STUDIES OF LEFT VENTRICULAR FUNCTION IN NORMAL CHILDREN. Howard P. Gutgesell, Desmond F. Duff, and Marc Pacquet (Intr. By Dan. G. MeNamara) Baylor Col. of Med., Texas Children's Hosp., Dept. of Ped., Houston

Echocardiograms were obtained from 37 normal children ranging in age from 1 day to 16 years. The patients were divided into 4 groups on the basis of age: Group $1-2$ years and under, Group $11-3$ to 6 years, Group $111-7$ to 12 years, and Group IV - over 12 years. The mean rate of circumferential fiber shortening $\left(V_{c f}\right)$, left ventricular ejection time (LVET), and percent change in left ventricular diameter with ejection (\% $\Delta$ LVD) were determined in all patients.

The calculated $V$ decreased with age $(1.70 \pm 0: 10$ SEM in Group 1, $1.46 \pm 0.09^{c}$ in Group $11,1.26 \pm 0.04$ in Group 111 , and $1.32 \pm 0.11$ in Group IV). This change appeared to be due to the increase in LVET which occurred with age. LVET increased from $0.21 \pm 0.008 \mathrm{sec}$. in Group 1 to $0.30 \pm 0.006 \mathrm{sec}$. in Group IV. The $\% \triangle L$ LV showed a slight but not statistically significant tendency to increase with age (from $34.6 \pm 1.1 \%$ in Group 1 to $38.8 \pm 2.7 \%$ in Group IV).

The $\% \Delta L$ LV was an easy to obtain index of ventricular function which appeared to be relatively independent of age and heart rate. Derived indices such as $V$ were altered by factors which change LVET, and did not appear to give additional information about ventricular function.

60 DYNAMIC ELECTROCARDIOGRAPHIC MONITORING IN INFANTS AND CHILDREN. Antonio Hernandez, Robert E. Kleiger and David Goldring. Washington University Medical School, Department of Pediatrics, St. Louis Children's Hospital, St. Louis, Missouri.

As a result of experience with arrhythmias, in adults, Holter long-term electrocardiographic monitoring, (HM), has proved its unique value in the discovery of arrhythmias, the correlation of arrhythmia with physical activity and symptomatology and the evaluation of therapeutic regimens. The HM was evaluated in 51 children suspected of having cardiac arrhythmias. There were 22 males and 29 females from one day to twenty years of age. Seventy-eight recording sessions were carried out. The types of arrhythmias encountered were the following: ventricular tachycardia - 8 patients, junctional premature beats - 8 , ventricular premature extrasystoles - 14, supraventricular tachycardia - 6 , complete heart block - 4, Wolff-Parkinson-White syndrome - 2 , congenital atrial flutter - 1. Holter monitoring documented that the stimulus for arrhythmias could be either physical activity or rest, correlated syncopal episodes or equivalents with serious dysrhythmias, and was the most effective means of evaluating anti-arrhythmic therapy especially in children with ventricular and supraventricular tachycardia. Significant negative data was also provided in 5 cases where arrhythmias were ruled out as the cause of symptoms. This was convincing support for the use of the Holter technique in pediatric patients with arrhythmia. 
61 EFFECTS OF PROCAINE AMIDE AND VERAPAMIL ON HUMAN ATRIAL FIBERS。 ALLAN J. HORDOF, RICHARD N。 EDIE, JAMES R。 MALM, MICHAEL R. ROSEN, DEPTS. OF PED., SURG., AND PHARM., COLUMBIA UNIN., NEW YORK (INTRODUCED BY WELTON M. GERSONY).

Clinical studies have shown that Verapamil (V) which blocks the inward $\mathrm{Ca}^{++}$current is more effective against suproventricular arrhythmias than drugs such as procaine amide (PA) that block the inward $\mathrm{Na}^{+}$current. We used standard microelectrode techniques to determine the effects of $V$ and PA on action potentials (AP) of human right atrial specialized conducting fibers (HAF) obtained at open heart surgery. HAF were divided into 4 groups (Grp). Grp. I and II, from normal sized atria, had mean maximum diastolic potentials (MDP) of $-75 \mathrm{mV}$ and $-67 \mathrm{mV}$. Grps. III and IV from dilated atria had MDP of $-55 \mathrm{mV}$ and $-48 \mathrm{mV}$. Grp. I and II had "fast response "AP due largely to an inward $\mathrm{Na}^{+}$current. Grp. III and IV HAF had "slow response" AP, due largely to an inward $\mathrm{Ca}^{++}$current. PA, 10-100mg $\Lambda$ had a significant concentration dependent depressant effect on AP amplitude and automaticity which was most pronounced in Grp. I-II. Verapamil, $0.1-1 \mathrm{mg} / \mathrm{L}$ had a significant concentration dependent depressant effect on AP amplitude and automaticity in Grp. III-IV. Verapamil at $1 \mathrm{mg} / \mathrm{L}$, had a small effect on Grp. II HAF and only depressed AP plateau in Grp. 1. Our studies suggest that diseased HAF have slow response AP more responsive to $V$ than PA. These results may explain in part why suproventricular arrhythmias are more sensitive to $V$ than PA.

SUSTATNED HYPERTENSION IN THE FETAL LAMB INDUCED BY

\section{2} RENAL ARTERY CONSTRICTION. Allen I. Hyman, Danie1 L.
Levin Abraham M. Rudolph, Michael A. Heymann. Univ. of Calif. San Francisco,Dept. of Ped. and Cardiovascular Res. Inst. Although renin is present in fetal kidney and infusion of exogenous angiotension II into the fetus causes a pressor response, it is not known if the renin-angiotensin system is induced and arterial blood pressure elevated by reducing renal blood flow chronically in the fetus. Therefore we studied 4 fetal lambs at $0.75-0.90$ gestation. Through a small hysterotomy, a fetal femoral artery (FA) and vein were catheterized,the left flank of the fetus incised, and left renal artery(RA)exposed. We placed a 4-5mm length of polyvinyl tubing(ID 0.05"and OD 0.09") around the RA to partially obstruct it. The twin of one fetus, only had its vesse1s catheterized and was a control to its experimental sibling. Beginning the day after surgery and daily thereafter we measured FA, central venous, and amiotic pressure of each fetus, while the mother stood quietly. The average mean FA pressure of the 4 experimental fetuses was lowest ( $56 \mathrm{mmHg}$, range $50-62$ ) the first day, rose steadily and within 8 days increased by $48 \%$. The average maximum mean blood pressure in the 4 animals ( $83 \mathrm{mmHg}$, range $75-90$ ) was $60 \%$ higher than that of a group of fetal lambs from our laboratory of comparable weight and gestational age. In the twins, the control animal's mean FA pressure remained $=50$ for 6 days after surgery, while its sibling's increased to $83 \mathrm{~mm} H \mathrm{~g}$. These findings indicate constriction of one RA can induce marked arterial hypertension in fetal lambs as early as 0.75 gestation. Although we have not yet confirmed the mechanism, it probably involves stimulation of the renin-angiotensin system. USPHS Grant HL 06285 .

63 EXERCISE ELECTROCARDIOGRAMS IN NORMAL AND HYPERCHOLESTEROLEMIC CHILDREN. F.James, C.J.Glueck, R.W.Fallat, F.Millett, S.Kaplan. Univ. of Cincinnati, Col. of Med. Children's Hosp. Med. Ctr., Dept. of Ped., Cincinnati, Ohio. Exercise electrocardiograms (E-ECG) and blood pressures (BP) during maximal bicycle exercise were analyzed in 73 children heterozygous for familial hypercholesterolemia (FHC) and in 97 normolipemic children (N), to reveal evidence of latent ischemic heart disease(IHD), All children had normal resting cardiac examinations. FHC and $N$ groups had similar ages (mean \pm SEM), (13.2 $\pm .5,13.1 \pm .5$ yrs.), resting systolic $\mathrm{BP}(117 \pm 2,115+1 \mathrm{~mm} \mathrm{Hg})$, and resting diastolic $\mathrm{BP}$ $(74 \pm 1,72+1)$. The criterion for a significant positive (+) E-ECG, readers blinded to lipid levels, was ST depression $\geq 1 \mathrm{~mm}$ in V5. Exercise programs were determined by body surface area (in $\left.\mathrm{m}^{2}\right)$. Results for + or negative (-)E-ECG for each subset and total follow:

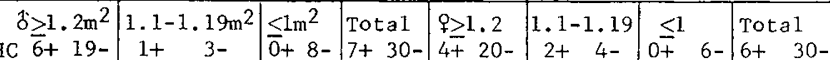

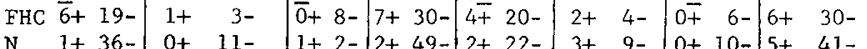
E-ECG was + in $13 / 73$ (18\%) FHC as compared to $7 / 97(7 \%) \mathrm{N}, \mathrm{X}^{2}=4.5$, $\mathrm{p}<.05$. FHC males $>1 \mathrm{~m}^{2}$ primarily accounted for the differences between the groups, with + E-ECG in $7 / 29(24 \%)$ as compared to $1 / 48$ $(2 \%) N, X^{2}=7.2, p<.01$. Mean + SEM systolic (S) BP during E-ECG in FHC was $166+3$, higher than $\mathrm{SBP}$ in $\mathrm{N}, 157+2.6 \mathrm{~mm} \mathrm{Hg}, \mathrm{p}<.05$. Maximal SBP along the regression line of SBP versus age was consistently 8-12 $\mathrm{mm}$ Hg higher in FHC, $\mathrm{p}<.025$. E-ECG reveals distinctive ST and maximal SBP changes in FHC as compared to $\mathrm{N}$ children, suggesting that IHD may have its genesis in children at biochemical risk for atherosclerosis.
64 plasma Cholesterol duRING infancy. Mary J. Jesse, Charles H. Hennekens, Barbara E. Klein, and Sidney Blumenthal. Dept. of Pediatrics, Univ. of Miami School of Medicine, Miami, Florida.

To determine whether plasma cholesterol levels in cord and neonatal blood specimens were predictive of subsequent values, blood samples were obtained from 72 pregnant women and their newborns. Subjects were followed longitudinally.

Eligibility criteria included that the mother be married, living with the father of the expected child, and attending the antepartum clinic of a local county hospital. Maternal levels were obtained during the third trimester of pregnancy, as well as 1,3 , and 6 months post-partum. Newborn levels were obtained on mixed cord blood at delivery, and peripheral venous blood on day 2 , and 1,3 , and 6 months of life.

Mean cholesterol values were (mg.\%):

$$
\frac{\text { Cord Blood }}{53.8} \quad \frac{\text { Day } 2}{72.1} \quad \frac{1 \text { mo. }}{107.1} \quad \frac{3 \text { mos. }}{114.0} \quad \frac{6 \text { mos. }}{124.3}
$$

There was no association of cord or neonatal plasma cholesterol with subsequent levels during the first months of life.

In these data, although mean cholesterol levels rise in the first 6 months of life, neither the cord blood nor neonatal values predict subsequent levels.

65 PRE AVI POSTOPERATIVE FCHOGRAPIIC, INVESTIGATION OF

65 LEFT VENTRICULAR FUNCTION IN AORTIC STINOSIS (AS) Gregory L. Johnson, Richard A. Yeyer, Joan Korfhagen and Sanuel Kaplan, Children's Hospital, Cincinnati, Ohio The purpose of this study was to investigate the serial non-invasive evaluation of the results of surgery upon left ventricular function in children with significant AS. Six grouns of patients were cvaluated: aortic stenosis (13 patients), coarctation of the aorta (9 patients), patent ductus arteriosus ( 4 patients), valvar pulmonic stenosis (4 patients), tetralogy of Fallot (9 patients) and ventricular septal defect (7 natients). Preoperative$1 y$ all patients with AS demonstrated shortening fractions greater than $40 \%$ (range $41 \%-60 \%$; mean $47 \%$ ), whereas of the 33 patients in the other groups studied, only four had shortening fractions of greater than $40 \%$ (range $22 \%-43 \%$ ). In addition, the patients with AS demonstrated septal and left ventricular wall thicknesses of $8 \mathrm{~mm}$ or greater, decreased left ventricular end diastolic volumes and stroke volumes for age and size, and prolongation of the left ventricular ejection time. Postoperatively patients with AS exhibited persistent abnormally high shortening fractions. The left ventricular end diastolic volumes and stroke volumes were unchanged or sightly increased and the septal and left ventricular wall thicknesses remained increased above normal. This study demonstrated that a shortening fraction of greater than $40 \%$ is strongly suggestive of $\mathrm{AS}$. In addition, parameters of left ventricular function were unaltered in postoperative patients with AS up to 4 years following adequate relief of the obstruction.

66 ECHO MEASUREMENT OF LEFT ATRIAL DIAMETER AS A GUIDE TO PULMONIC:SYSTEMIC FLOW IN PATENT DUCTUS ARTERIOSUS. W. Pennock Laird and David E. Fixler, (Intro by Gladys Fashena) Dept. Ped. Univ. of Texas Health Science Center, Dallas, Texas. Left to right shunting due to a patent ductus arteriosus (PDA) increases pulmonary flow (QP). In infants and children, a large PDA will produce left atrial enlargement as a result of increased Qp. The purpose of this study was to measure with ultrasound the left atrial diameter (LAD) as a guide to the magnitude of the shunt in pediatric patients with PDA. Using standard echocardiographic techniques the LAD was measured at the level of the aortic valve in 9 patients (ages 2 mos to 7 years) with the catheterization diagnosis of isolated PDA and no evidence of pulmonary disease. In all cases echocardiography was carried out within 24 hours of cardiac catheterization. Mean heart rates at the time of catheterization averaged $132.8 \pm 15.6$ (SD) and during echo examination $131.7 \pm 15.6$. Since LAD is known to vary with body size, this value was compared to body surface area (BSA) and to aortic root diameter (AO) in order to adjust for differences in body size. The LAD/BSA ratio showed poor correlation with the pulmonary to systemic flow ratio $(Q P: Q S)$ measured at catheterization $(r=0.02)$. However comparison of the LAD/AO ratio to $Q \mathrm{P}: Q \mathrm{Qs}$ revealed a 1 inear relationship $(r=0.84)$ where $Q p: Q s$ could be predicted from the regression equation $\mathrm{Qp}: \mathrm{Qs}=0.645(\mathrm{LAD} / \mathrm{AO})+0.394$. Therefore the ratio of left atrial diameter to aortic root diameter as determined by echocardiography may be used to estimate $Q p: Q s$ in uncomplicated PDA. 
SYSTOLIC TIME INTERVALS IN CHILDREN WITH CONGENITAL

67 AORTIC STENOSIS; Moo H.Lee, Oh B.Kwon, William B.Strong

(Intr.by Alex Robertson);Med.Col.of Ga., Dept.of Ped. Augusta, Ga. Systolic Iime Intervals (STI) were measured to e-
valuate the severity of congenital aortic stenosis. The measurements of the total electromechanical systole (Q-S2), the left ventricular ejection time (LVET), the pre-ejection period (PEP) and the systolic quotient (SQ) were then correlated with cardiac catheterization data. 70 normal children, 1-16 years of age, were studied as control and their values were: Q-S2 of $330 \pm 16 \mathrm{msec}$ (meant SD), LVET of $257 \pm 20 \mathrm{msec}$, PEP of $72 \pm 8 \mathrm{msec}$ and SQ(LVET/PEP) of $3.55 \pm 0.49$. In children (21) with congenital aortic stenosis (19 valvar and 2 subvalvar), mean LVET was $20 \mathrm{msec}$ longer than control, while mean PEP was $12 \mathrm{msec}$ shorter than normal. SQ was 1.25 greater than control. Cases were classified into 3 groups by the peak left ventricular-aortic pressure gradient: (1)mild (less than 50 $\mathrm{mmHg}$ ), (2) moderate $(50-100 \mathrm{mmHg})$, and (3) severe (greater than 100 $\mathrm{mmHg}$ ). In $13 \mathrm{mild}$ aortic stenosis, there was no significant difference in STI comparing with control. However, in 2 moderate and 6 severe aortic stenosis, LVET was abnormally prolonged and PEP was significantily shortened. In severe aortic stenosis, mean LVET was $27 \mathrm{msec}$ longer than normat, while mean PEP was $18 \mathrm{msec}$ shorter than control, thus increasing SQ to 1.67 greater than normal. Results indicated that LVET \& SQ were directly related to severity of obstruction while PEP was indirectly related. The SO appeared to best correlate with the hemodynamic status, being abnormal in all that the measurement of STI is a useful adjunctive non-invasive method for assessment of the child with congenital aortic stenosis. patients with moderate to severe aortic stenosis. It is concluded

70 THE INCIDENCE OF CONGENITAL HEART DISEASE WITH HEMIVERTEBRAE. M.C. MATUSTIK, R.F. MCCONNELL, and L.C. HARRIS. Univ. of Tex. Medical Branch. Dept. of Ped., Galveston, Tex.

The literature has documented the association of hemivertebrae with many congenital anomalies and syndromes, such as tracheo-esophageal fistulas, VATER association, hypoplastic lungs. The association of idiopathic scoliosis and cyanotic congenital heart disease has been reported by several authors. The association of hemivertebrae and congenital heart disease has not been reported in the available literature.

Of the patients under 15 years of age hospitalized during a 10 -year period, 48 were found to have hemivertebrae. The diagnosis of hemivertebrae was made on investigation of scoliosis or radiological investigation of other problems such as heart disease. Of these $15(31 \%)$ had congenital heart disease. The specific heart lesions were varied and often multiple but septal and conus defects predominated. The mortality rate in the group with heart disease was $5 / 15$ or $33 \%$. These were not surgically related. The other anomalies most commonly seen were rib and other types of spinal anomalies, renal and in the group without congenital heart disease, spina bifida occulta-meningomyelocele ( 9 cases).

The time of embryologic development of the septal-conus defects and hemivertebrae suggests a simultaneous teratogenic induction in the embryo. Associated anomalies and their diversity were consistent with an early fetal insult.

71 PHYSICAL TRAINING IN CHILDREN WITH CONGENITAL HEART DISEASE. William W. Miller, Damaris S. Young, C. Gunnar Blomqvist, Phillip S. Strange, Ladislav P. Novak, Robert L. Johnson, Jr., Jere H. Mitchell. Untr.

68 DISEASE. Aaron R. Levin, Jacobl. Haft, Mary A. Engle, and Kathryn H. Ehlers. The New York Hospital-Cornell Medical Center, Dept. of Pediatrics, New York, New York.

His bundle electrograms were recorded in 25 normal children and 178 patients with congenital heart disease aged 1 to 20 years. Normal intervals were $\mathrm{PH}=90.2 \pm 13.9 \mathrm{msec} ; \mathrm{AH}=68.1-14.5 \mathrm{msec} ;$ and $\mathrm{HV}=40.7-5.4 \mathrm{msec}$. Mean values for all parameters were within normal limits for patent ductus arteriosus ( $N=7$ ); mitral regurgitation ( $N=3$ ); tetralogy of Fallot (T.F.) ( $N=$ 16); aortic stenosis ( $N=20)$; and aortic coarctation ( $N=9$ ). Mean $\mathrm{PH}$ was significantly prolonged in secundum atrial septal defect (SASD) (N=15); ostium primum atrial septal defect (PASD) $(N=6)$; postoperative PASD $(N=4)$; pulmonic stenosis ( $N=22)$ and postoperative ventricular septal defect (VSD) $(N=12)$. The mean $A H$ was significantly prolonged in VSD $(N=24)$; postoperative PASD; postoperative VSD and idiopathic hypertrophic subaortic stenosis $(\mathrm{N}=4)$. Mean HV was normal in all groups except PASD;aortic valvular insufficiency ( $A I)(N=4)$; and postoperative VSD. Of the entire group, $18 \mathrm{had}$ $H V$ intervals above the normal range (32-50 msec); six of these had severe aortic valve disease, 6 were postoperative VSD or T.F. and 3 were PASD. Categorizing patients as right or left ventricular volume or pressure over-

load did not correlate with any of the parameters measured.
It is concluded that: (1) intra-atrial conduction, but not $A-V$ nodal conduction, is slower in patients with ASD; and, (2) mean HV interval was significantly affected only in patients with PASD and Al, al though among patients with severe aortic valve disease, postoperative VSD and postoperative T.F., a prolonged $H V$ was not uncommon.

60 LeFT ATRIAL RESPONSE tO VOLIME LOAD IN CHILDREN. Rajamma Mathew, Otto G. Thilenius, \& Rene A. Arcilla; Univ. of Chicago, Dept. of Ped., Chicago, Illinois Left and right heart volumes were analyzed from angiograms of 19 isolated patent ductus (PDA), and 20 isolated mitral regurgitation (MR). Maximal and minimal LA volumes (LAmax, LAmin), and ventricular end-diastolic (LVEDV, RVEDV), end-systolic (LVESV, RVESV) and stroke (LVSV, RVSV) volumes were compared with predicted normal (PN) using age, height, weight and heart rate. LVSV less RVSV=shunt volume (ShV) or regurgitant volume (ReV). Volume overload (VO) was expressed by ShV/RVSV in PDA, or ReV/RVSV in MR. Correlation coefficient between LAmax and LVEDV was 0.98 in PDA, and 0.85 in MR. Ratio of LAmax $/ \mathrm{M}^{2}$ to wedge pressure was 5.3 in PDA and 9.2 in MR ( $p<0.01)$. LAmax related to LVEDV showed: (in MR) LAmax $=0.9$ LVEDV + 134; (in PDA) LAmax=0.52 LVEDV + 70, where LAmax LVEDV $=\%$ PN. In $10 \mathrm{PDA}$ and $12 \mathrm{MR}$ with comparable vo ranging from 0.5 to 1.5 (mean, 0.95 and 0.96 , respectively) mean LAmax was $151 \%$ PN in PDA but $285 \%$ PN in MR $(p<0.005)$; however, mean LVEDV was $178 \%$ PN and $169 \% \mathrm{PN}$, respectively ( $\mathrm{p=ns}$ ). Data is summarized below $\left(* \mathrm{~cm}^{3} / \mathrm{M}^{2}\right)$. The kinetics of LA overload differ in both. In MR, total VO is handled by LA during LV systole on1y. In PDA, on $1 y$ a fraction of vo distends LA at this time. LA distensibility is increased in $M . R$.

\begin{tabular}{|c|c|c|c|c|c|c|}
\hline & & LAmax: & LVEDV* & RVEDV* & LA & LVEDV \\
\hline & vo & (a) (b) & (a) (b) & (a) & (a) & (b) \\
\hline $\mathrm{PN}$ & - & 37 & 58 & 56 & 0.62 & 0.57 \\
\hline PDA (a) & .73 & 54.2 & 91.9 & 68.5 & & 60 \\
\hline MR (b) & .99 & 114.6 & 128.5 & 75.3 & & 92 \\
\hline
\end{tabular}

72 CHANGES IN THE DISTRIBUTION OF CORONARY FLOW DURING PULMONARY ARTERY CONSTRICTION IN UNANESTHETIZED NEWBORN GOATS. Jim B. Norton, Jr. (Intr. by M. J. by James F. Marks). Department of Pediatrics, The University of Texas Health Science Center and Department of Anthropology, Southern Methodist University, Dallas, Texas.

Fifteen children with surgically treated congenital heart disease had studies of cardiopulmonary function before and after 5 weeks of physical training. Training consisted of heart rate to $80 \%$ of its maximum. Twelve children, 7.15 years old, completed at least $80 \%$ of the training. Tests of cardiopulmonary function were within 2 SD of normal in all children except for 2 in whom maximum oxygen consumption (Vंo2 max) was subnormat Significant differences were meassured only in hemoglobin $\{\mathrm{Hgb}\}$, one second forced expiratory volume $\left(\mathrm{FEV} \mathrm{V}_{1} / \mathrm{FV} \mathrm{C}\right)$, and single breath $\mathrm{CO}$ diffusion capacity $\left(\mathrm{D}_{\mathrm{L}_{\mathrm{co}}} / \mathrm{V}_{\mathrm{A}}\right)$.

$\dot{V}_{0}$ max increased significantly $(11 \%$ and $24 \%)$ in only 2 individuals. Maximum blood pressure decreased significantly in 8 children, was unchanged in 3 , and increased significantly in 1 . There were no significant changes in weight, lean body mass, hematocrit, or maximum heart rate. The changes in $\mathrm{FEV} / \mathrm{FVC}$ and $\mathrm{D}_{\mathrm{L}_{c o}} / \mathrm{N}_{\mathrm{A}}$ indicate training effects. In 21 normal adult males an identical training program produced significant increases in $\dot{V}_{0} 2$ max. The unchanged $\dot{V}_{0} 2$ max measured in 10 of the 12 children in this study suggests that either 1 ) the training requiremen Sweeney) Dept. of Ped., Univ. of Texas Health Science Ctr., San Antonio, Texas.

The purpose of this study was to evaluate changes in the distribution of coronary flow resulting from an abrupt elevation in right ventricular systolic pressure (RVSP) in young animals. Flow was measured with the microsphere technique in 12 awake and unsedated newborn goats, age 4-32 days having previously undergone thoracotomy for insertion of catheters, RV pressure microtransducer, and a circumferential balloon device around the pulmonary artery (PA). In 5 of 6 animals $<2$ wks, of age, RVSP could be raised to $>50 \mathrm{~mm}$. Hg. ( $72 \pm 18 \mathrm{SD})$ producing an average increase of $79 \%$. Though the resting RVSP in the 6 older animals was significantly lower than in the younger group, an average increase of $80 \%$ was still obtained with PA constriction. In 7 of the 12 , RV coronary flow increased, in 4 there was no significant change and in 1 there was a decrease. Yet in all 12 the proportion of total coronary flow delivered to the right ventricle increased significantly; from $27 \pm 7.2 \%$ (SD) of total coronary flow to $35 \pm$ $6.8 \%(p<.02)$. This pattern existed whether or not the cardiac output and total coronary flow increased or decreased. Coronary flow measurements during the control periods prior to PA constriction confirmed data previously obtained in open-chest anesthetized animals that suggested a steady reduction in RV coronary flow during the first 3 weeks of 1 ife corresponding to the fall in RVSP. 25 daily 30 minute sessions of stationary bicycle exercise at work loads adjusted to increase

$\begin{array}{lccc} & A V & S E & P \\ \text { Hgt (gm per } 100 \mathrm{ml} l & -0.6 & 0.2 & <0.01 \\ \mathrm{FEV}_{1} / \mathrm{FVC} & +6.25 & 1.50 & <0.01 \\ \mathrm{D}_{\mathrm{L}_{\mathrm{co}}} / \mathrm{V}_{\mathrm{A}}\left(\mathrm{ml} / \mathrm{mmHg}^{-1} / \mathrm{min}^{-1 / 1)}\right. & +0.33 & 0.10 & <0.01\end{array}$
cardiopulmonary adjustment to training is present long after successfut surgery. 

73 CONTINUOUS TELEMETRY OF CARDIORESPIRATORY AND TEMPERDROME (SIDS). Jim $\underline{B}$. Norton, Jr., $\underline{\text { H}}$. William Diserens, Robert L. Wilbur. (Intr. by M. J. Sweeney) Dept. of Ped., Univ. of Texas Health Science Ctr., San Antonio, Texas.

This pilot study was undertaken to establish the feasibility of continuous recording of heart rate, respiratory rate, and skin temperature over extended periods of time in infants using a miniature telemetry system. Two skin electrodes are used with a transmitter (range $70 \mathrm{ft}$., weight 37.9 grams) that fits comfortably into a pocket of the infant's diaper shirt. A receiver, demodulator, and a commercial 24-hour cassette recorder completes the portable system that accompanies the infant from hospital to home. Time signals are added to the recording by a digital clock in the demodulator. A cassette reproducer is used to retrieve the data at 32 times recording speed. Data is displayed in high density form in tachometer mode or in conventional mode for beat to beat analysis. Six normal infants and two infants thought to be at risk from SIDS have been studied. Three additional infants admitted after successful resuscitation following apneic periods at home have been studied and cardiorespiratory abnormalities documented during observation in the hospital. Data obtained thus far suggests but does not prove that a relationship exists between sleep and apneic spells. Long term recordings have demonstrated the value of this system in recognizing the frequency of arrhythmias in normal infants as well as suggesting a pattern of recurrent episodes of apnea in infants at risk for SIDS.

74 DISTRIBUTION OF FETAL CARDIAC OUTPUT-IMPORTANCE OF PaCeMaKer LOCATION by Paul T. Pitlick, Stanley E. Kirkpatrick and William F. Friedman, UCSD Sch. of Med. San Diego, CA. Important questions exist about the relative roles in regulating fetal cardiac output $(\mathrm{CO})$ of changes in heart rate vs. extent of myocardial shortening. Controversy exists because increases in heart rate created by left atrial (LA) pacing have been shown to increase right ventricular output (RVO) (UCSF), and also to decrease left ventricular output (LVO) (UCSD). Since the pacemaker site could importantly influence foramen ovale flow and hence, RVO and LVO, RA and LA pacing wires were implanted chronically in 8 fetal lambs. Ventricular outputs were determined by indicator dilution techniques. At rest RVO was $63 \%$ of total CO. LA pacing at 270 beats/min resulted in a substantially greater contribution of RVO to total CO. In contrast with RA pacing, LVO contributed significantly more to total $\mathrm{CO}(\mathrm{p}<0.005)$. Small increases were observed in total $\mathrm{CO}$ with pacing from either atria. Comparable reductions were observed in right and left ventricular stroke volumes with pacing of the ipsilateral atrium. These studies demonstrated clearly that the disparity that exists normally in the contributions of RVO and LVO to total $\mathrm{CO}$ is accentuated with left atrial pacing and minimized with right atrial pacing. The data clarify previous experimental differences which can be attributed to changes in the magnitude of shunting across the foramen ovale that depend upon pacemaker location. Moreover these findings suggest that important differences could exist in ventricular mass relationships in newborns with an ectopic LA pacemaker.

75 MYPERTROPHIC CARDIOMYOPATHY IN INFANTS OF DIABETIC MOTHERS. Ronald L. Poland, Laura J. Walther, Chung-Ho Chang, Wayne State Univ. Sch. of Med. Depts. Of Ped. and Path. and Children's Hosp. of Mich. (Intr. by Flossie Cohen)
Five infants of diabetic mothers presented with cardiomegaly, absent or inconstant cardiac murmuxs and signs of congestive failure. The class of maternal diabetes varied from $A$ to $C$ (poorly controlled). There were 3 males, 2 of whom had fetal distress and low Apgar scores and died. Foux were born by cesarean section and 3 were large for gestational age. Findings included: respiratory distress (5), hypoglycemia (4), hypocalcemia (2), positive blood culture (2) and hyperbilirubinemia (4). EKG's showed RVH (3), LVH (3), RAH (2) and mild S-T changes (2). Echocardiograms were normal except for chamber size. All received digoxin and diuretics and one received hydrocortisone for persistent hypoglycemia. Two underwent cardiac catheterization and angiocardiography. One showed a small ductus arteriosus and both had dilated ventricles with reduced contractility and no structural defect. At autopsy, both infants showed macrosomia, visceromegaly and hyperplasia of the islets of Langerhans. One showed anoxic brain damage and the other had evidence of sepsis. Both infants (BW 4.1 and $4.4 \mathrm{~kg}$ ) had hypertrophic pale hearts (51 and $50 \mathrm{~g}$ ). Microscopic sections showed a relative increase in cytoplasm in the muscle fibers and many central pale-staining areas or vacuoles. Stains for fat and glycogen were unremarkable. Electron micrographs revealed the vacuoles filled with proliferated mitochondria and some disruption of myofibrils. This picture is similar to that described in adult patients by Ferrans et al (in Cardiomyopathies, E. Bajusz
and G. Rona, eds, Univ. Paxk Press, Balti. 1973, pp. 23i-281).
76 EXERCISE CAPACITY OF NORMAL CHILDREN: TREADMILL PERFORMANCE. Marlene Rabinovitch, Joes R. Jacobsen, Jill H. Morriss, and Howard P. Gutgesell (Intr. by Dan G. McNamara) Baylor Col. of Med., Texas Children's Hosp., Dept. Of Ped., Houston

Multistage maximal exercise tests were performed on 73 normal children using a modification of the Bruce treadmill protocol. The measurements made included total work performed (TW)-vertical distance traveled times body weight, mean work to exhaustion (MWE)-TW'total treadmill time, maximal endurance index (MEI)-MWE/ body surface area (BSA), physical work capacity (PWC)-work level at which heart rate reached 170, and recovery pulse index (RPI)mean heart rate in the first 5 minutes post-exercise.

The TW increased from $2401 \pm 1004$ SEM kg-m to $25,945 \pm 3804$ $\mathrm{kg}-\mathrm{m}$, and MWE from $207 \pm 32$ to $1316 \pm 285$ as BSA increased from under $0.8 \mathrm{~m}^{2}$ to over $1.6 \mathrm{~m}^{2}$, showing that larger children could perform more work. The increase in MEI from $318 \pm 43$ to $765 \pm$ 156 with increasing BSA indicated that larger children were also capable of performing more work per unit of BSA. PWC likewise increased progressively with BSA, whereas RPI did not change significantly.

The results of this study are similar to previous studies employing a bicycle ergometer, and provide normal values of exercise capacity for children. The study also demonstrated the feasibility of treadmill testing of children as young as 5 and 6 years of age with equipment available in most hospitals.

\section{THE VENTRICULAR SEPTAL DEFECT IN TRICUSPID ATRESIA P. Syamasundar Rao, Medical College of Georgia,} Department of Pediatrics, Augusta, Georgia 30902

We have previously reported isolated cases of spontaneous anatomic and intermittent functional closure of the ventricular septal defects (VSDs) in tricuspid atresia (TA). To further study this phenomenon, clinical, angiographic and pathologic findings of 20 consecutive cases of TA seen at the Medical College of Georgia were reviewed. Diagnoses were made by angiographic and/or autopsy findings in 19 patients and by clinical features in 1.16 cases had normally related great arteries and 4 had transposition. Of these, 4 patients had evidence for closure of VSD; in 3 it was complete and in 1, partial. Increasing cyanosis and polycythemia or disappearance of a previously heard murmur were present in all patients. In the first patient, autopsy evidence for closure of VSD was present at age 6 months. Closure of VSO was angiographically shown between 2 and 12 years of age in the 2 nd case and between 1 and 12 months in the 3rd. In the 4 th case there was clinical and $x$-ray evidence for partial closure of VSD. All the closures occurred only in patients with normally related great vessels. The incidence of VSD closure in TA is $20 \%$ in the entire group, $25 \%$ in type I and $33 \%$ in type I cases with VSD. Since the VSD is the major (or the only) source of puimonary blood flow in TA, the high incidence of VSD closure is of great clinical importance. Clinical characterization of the VSO closure in TA, the mechanism of closure and its therapeutic implications will be discussed. Closure of VSD should be considered when cyanosis increases and a previousiy heard murmur disappears in a patient with TA.

78 SERIAL CARDIAC CATHETERIZATION IN SEVERE CHRONIC PULMONARY DISEASE OF PREMATURITY Elihu P. Rees, Douglas L. Roy, Thomas F. Ward (Intr. by Richard B. GoldbToom) DaThousie University, Department of Pediatrics and I.W.K. Hospital for Children, Hal ifax, N.S., Can.

Eight premature infants who developed moderate-severe chronic puimonary disease of prematurity (CPDP) each had two cardiac catheterizations during their clinical course. Gestational age was 28 to 36 weeks $(7 / 8<32$ weeks) and birth weight was 1020 to 2640 grams $(7 / 8<2000$ grams). Age at first catheterization was 14 to 82 days and at second catheterization 68 to 159 days.

At initial catheterization $5 / 8$ had patent ductus arteriosus, but only 3 had left-to-right shunt of $>3$ to 1 , which may have contributed to elevated mean pulmonary artery pressure (PAP). $7 / 8$ had elevated PAP $(>20 \mathrm{~mm} \mathrm{Hg})$, ranging from $22-65 \mathrm{~mm} \mathrm{Hg}$ with an ambient oxygen of $27 \%$. Left atrial saturation ranged from $40 \%$ $95 \%$ ( $7 / 8$ were $<80 \%$ saturation). In $100 \%$ oxygen left atrial saturation rose to $93 \%$ - $98 \%$ and PAP decreased markedly in 4 , achieving normal values in 2. There was no significant change in the 3 babies with large left-to-right shunts.

At the second catheterization, 4/8 had elevated PAP in $21 \%$ oxygen $(25-30 \mathrm{~mm} \mathrm{Hg})$, but with one exception, all lower than their values at first catheterization. In $100 \%$ oxygen $3 / 4$ showed significant decrease in PAP, 2 achieving normal values. None had left-to-right shunt.

We conclude that pulmonary hypertension associated with CPDP is largely related to hypoxemia and improves with time as the pulmonary disease improves. 
79 THE EXERCISE ELECTROCARDIOGRAM IN TRAINED AND UNTRAINED ADOLESCENT MALES. James H. Rogers, william B.Strong
and Herman K. Hellerstein;Med.Col. of Ga., Dept. OfPed,

The exercise electrocardiogram (EECG) is being used with increasing frequency in the pediatric population, but no quantitative data for the normal patient is available. This study was designed to quantitate the parameters of J-point displacement and ST segment slope in normal adolescent males. Three groups of boys were tested with maximum or near maximum exercise being performed on a modified von Dobeln bicycle ergometer. Groups I and II consisted of 43 and 17 subjects respectively, who were not engaged in organized athletics (untrained), while Group III consisted of 13 competitive swimmers (trained). $75 \%$ of the untrained subjects fell below the 50th percentile for physical fitness as described by Adams et al, while only $75 \%$ of the trained subjects were below the 50 th per centile. No subject was observed to have a negative $J$ point displacement or a sagging ST segment alone or in combination. There was no difference in J-point displacement or ST segment slope between the trained and untrained subjects. This study demonstrates the normal adolescent male EECG shows no J-point depression during maximum or near maximum exercise while studies in normal adult males accept a J-point depression of 0.5-1.5 millimeters as being within the range of normal. This study suggests that any J-point depression in the EECG be considered abnormal in the adolescent male. It further suggests that since no difference was seen in the J-point displacement and ST segment slope between trained and untrained subjects, the EECG is a valid test of myocardial function regardless of the activity level of the individual tested.

80 ISOMETRIC EXERCISE (IE) STUDIES IN CONGENITAL HEART DISEASE (CHD). Amnon Rosenthal, Michael D. Freed, and John F. Keane, Children's Hosp. Med. Ctr., and̆ Harvard Med. Sch., Boston, Mass.

Because rhythmic exercise cannot be performed during percutaneous femoral vessel catheterization (cath), we have investigated the response to, and application of, IE during cardiac cath in patients (pts) with CHD. Nineteen pts aged 8 to 29 years were exercised by the use of a hand dynomometer(Smedleys) at 50\% of maximal voluntary capacity (mean: $15 \mathrm{~kg}$ ) for 90 seconds. Heart rate (HR), intravascular pressures, oxygen consumntion $\left(\mathrm{VO}_{2}\right)$ and cardiac output ( $\mathrm{CO}$ ) were measured at rest, during the last 30 seconds of sustained handgrip $\mathrm{IE}$ and in the recovery veriod ( 2 minutes after Ik). Mean values are shown below.

HR (beats $/ \mathrm{min}$ )

Systemic) Systolic ( $\mathrm{mmHg}$ )

REST

86
111

111
82

138

VO2 $\left(\mathrm{ml} / \mathrm{min} / \mathrm{m}^{2}\right)$

$\mathrm{CO}(\mathrm{L} / \mathrm{min} / \mathrm{m} 2)$

$p$ (paired $t$ test $)<0.01$

Increase in $\mathrm{HR}$, systemic arterial pressure and $\mathrm{VO}_{2}$ was demonstrated during $I E$ in all pts, and $C O$ in $15 / 19$ pts. Values declined during recovery. Left to right shunt increased in 10/10 pts (mean Qp/Qs 2.3 to $2.4 ; p<.02$ ) with ventricular septal defect. Right to left shunt decreased in $3 / 3 \mathrm{pts}$ (mean 1.5 to $0.8 \mathrm{~L} / \mathrm{min} / \mathrm{m} 2$ ) with cyanotic CHD. Peak systolic gradient increased in $4 / 4$ pts (mean 43 to $65 \mathrm{mmHg}$ ) with pulmonary or valvar aortic stenosis. We conclude that $I E$ is a simple and useful stress test during cath in CHD pts.

ELECTROCARDIOGRAPHIC ABNORMALITIES IN NEONATES BORN TO HEROIN AND METHADONE DEPENDENT WOMEN, Peter Rothstein and Allan Goldblatt (Intr. by Joel J. Alpert). Boston Univ. Sch. of Med., Boston City Hosp., Dept. of Ped.; City of Boston Drug Treatment Program; and Harvard Med. Sch., Massachusetts General Hosp., Dept. of Ped., Boston.

Electrocardiograms (EKG's) of neonates born to drug dependent women were studied to see if there were any pathologic changes due to intrauterine exposure to heroin or methadone. This was undertaken following a report of sudden infant deaths in neonates born to methadone maintained women. During a 16 month period, 24 neonates were born to heroin or methadone dependent women at Boston City Hospital. Twenty-three of these neonates had serial EKG's taken, starting within 8 hours after birth. Tracings were obtained on all neonates during the first 4 days of life and then afterwards on those neonates whose initial tracings were abnormal. For analysis 3 neonates were excluded, 1 because of a patent ductus arteriosus and 2 because of incomplete tracings.

Thirteen neonates had abnormal EKG's. The predominant abnormality found was pathologic right ventricular hypertrophy. Other abnormalities found were left ventricular hypertrophy, prolonged QT intervals, $1^{\circ}$ block and enlarged $Q$ waves in the inferior leads. These findings were not related to the presence of drug withdrawal in the neonate or maternal usage of methadone. All neonates were asymptomatic and the abnormalities resolved within the first 4 weeks of life with one exception.

It is concluded that heroin and/or its contaminants may cause changes in the EKG's of neonates exposed to these drugs in utero.
FORMALIN INFTLTRATTON OF THE DUCTUS ARTERIOSUS : A NEW

82 METHOD FOR PALLIATION OF SELECTED CONGENITAL CARDIAC LESIONS. Abraham M. Rudolph, Michael A. Heymann, Noel Fishman and Jeffrey B. Lakier. Univ. of Calif.San Francisco, Dept. of Ped. and Surgery and Cardiovascular Res. Inst.

Persistent patency of the ductus arteriosus(DA) is crucial to survival of many infants with congenital cardiac lesions. Closure of the DA requires surgical establishment of an aortopulmonary shunt which is technically difficult and often not successfully maintained in neonates. We prevented postnatal closure of the DA by prenatal formalin infiltration of its wall in fetal lambs. We therefore infiltrated the DA with formalin in 4 infants( $2-5$ days) with severe hypoxemia and acidemia due to reduced pulmonary blood flow. The first had 3 previous unsuccessful attempts to increase flow; in the other 3 , it was considered impractical to perform a surgical shunt. At thoracotomy $0.4-0.6 \mathrm{ml} 10 \%$ buffered formalin was injected subadventially along the whole DA length and about $3 / 4$ of the circumference. Arterial $\mathrm{PO}_{2}$ increased dramatically in a1l (average 15-34 torr); the first baby died after 4 days, from pulmonary complications; the other 3 are well after 1,3 and 6 months and more definitive surgery has been performed. A 5 th infant ( 3 days)had aortic arch interruption and DA constriction, interfering with descending aortic flow. Surgical repair or an anastamosis was not feasible because of unusual anatomy. Formalin infiltration of the DA and bilateral pulmonary banding produced rapid improvement. At $2 \frac{1}{2}$ months the $\mathrm{DA}$ was widely patent and the infant well. Infiltration of the DA with formalin is a simple technique for palliation of certain congenital cardiac lesions in the neonate. USPHS Grant HL 06285.

83 MTTRAI VALVE PROLAPSE SYNDROME (MVP) IN CHILDREN ASSOCIATION OF AORTIC ROOT DILATATION (AOT) AS A MARKER OF A CARDIAC CONNECTIVE TISSUE DISORDER. David J. Sahn, Hugh D. Allen, Stanley J. Goldberg, and William F. Friedman, Depts. of Ped., Univ. of Arizona Med.

and Univ, of California-San Diego, Univ, Hosp.

The syndrome of MVP can be reliably diagnosed echocardiographically in children but its significance and prognosis are, as yet, unknown. The purpose of this study was to assess the prefindings of mitral murmur syndrome. Although 5 patients had criteria for Marfan's syndrome (MS) (body habitus, joint mobility, and ocular findings) none of the patients had evidence of other cardiac abnormalities. In all patients maximal mitral leaflet arching was easily visualized by real time two-dimensional echocardiography. Moreover, the findings of MVP could be confirmed by M-mode recordings derived from single elements within the multiple crystal array. Only those patients with severe MVP had increased left ventricular and atrial dimensions. A diagnosis of associated tricuspid valve prolapse could not be made in any patient. The major finding of the present study was the observation, in 21 patients, of a biconvex enlafgement of the aortic sinuses of Valsalva associated with significant Ao $\uparrow(>95$ th. $\%-i 1$ e). Ao $\uparrow$ was most marked in the patients with MS. The prevalence of Aot in young children with normal body habitus as well as those with MS suggests the presence in MVP of a generalized abnormality of cardiac connective tissue.

THE UTILITY OF SUPRASTERNAI NOTCH (SSN) ECHOCARDIOGRAPHY IN THE NONINVASIVE ASSESSMENT OF CONGENITAL HEART DISEASE (CHD). David J. Sahn, Hugh D. Allen, Stanley J. Goldberg, Theron Ovitt, and Barry B. Goldberg, Departs. of Ped. and Radiol., Univ. of Arizona Med. Ctr., Tucson, and Episcopal Hosp., Philadelphia.

Echocardiography from the SSN provides images of the transverse aortic arch (TAA) and right pulmonary artery (RPA). Nevertheless, the utility and accuracy of this technique has not been assessed in children. Accordingly, the present study was designed to validate measurements of these structures, define normal values and abnormal variations in patients with CHD. SSN echocardiograms were performed on 10 patients during cardiac catheterization. Correlation coefficients for TAA and RPA measured echocardiographically compared to cineangiographic measurements from the AP view were t0.96 for TAA and t0.95 for RPA. U1trasonic opacification by selective saline injection into either vessel validated structure identification. Twenty normal newborns (age $24-72 \mathrm{hrs}$.), weight $2-3.5 \mathrm{~kg}$, were found to have $\mathrm{RPA}=0.90+0.09 \mathrm{~cm}(\mathrm{SE}), \mathrm{TAA}=1.07+0.07 \mathrm{~cm} ;$ and $\mathrm{TAA} / \mathrm{RPA}$ ratio $=$ $1.16 \pm 0 . \overline{0} 4$. TAA/RPA ratio was not significantly different, 1.2 $\pm 0.0 \overline{4}$, in 50 norma 1 children (age 2-10 years) but was increased in patients with catheterization proven Tetralogy $2.21 \pm 0.3$ $(p<0.005) \quad(N=10)$ and aortic stenosis $1.55+0.18(p<0.05) \quad(N=$ $7)$. The ratio was decreased in patients with VSD $1.07+0.07$ $(p<0.05) \quad(N=16)$, ASD $1.01 \pm 0.06(p<0.005) \quad(N=13)$ and pulmonic stenosis $0.94 \pm 0.08 \quad(\mathrm{p}<0 . \overline{0} 05)(\mathrm{N}=8)$. These reliable noninvasive measurements of TAA and RPA are of significant utility in the diagnosis and management of CHD. 
85 EFFECT OF EXERCISE ON PREMATURE VENTRICULAR BEATS IN CHILDREN:M. Salehbhai, MooH. Lee, Willi iam B. Strong (Intr BY Alex Robertson);Dept.ofPed. ,Med.Col.ofGa.,

The purpose of this study was to evaluate the effect of exercise on children with premature ventricular contractions (PVCS). Six normal children with frequent PVCS were exercised to maximum on a treadmi11. A heart rate greater than $150 /$ min was obtained on each. All patients were continususly monitored electrocardiographically and recordings were obtained during the last $15 \mathrm{sec}$ onds of each stage of exercise and for 10 minutes after the cessation of exercise. The electrocardiograms were analysed for the frequency of PVCS, J-point and ST-T wave changes. The PVCS were abolished in all patients when a heart rate of $150 / \mathrm{min}$ or greater was achieved. In two patients the PVCS increased during early stages of exercise when the heart rate was less than $150 / \mathrm{min}$. In four patients there was an increase in frequency of PVCS during the immediate recovery period. In none of our patients was there any tachyarrhythmias, J-point depression, or ST-T changes. Our data explain the discrepancies noted in the study of adults with PVCS. The increase in frequency of PVCs noted during mild exercise may be speculated to be due to increased sympathetic tone with increased levels of circulating catecholamines causing increased automaticity of the ectopic focus. During the later stages of exercise the accelerated heart rate overdrives the ectopic focus causing suppression of the PVCs. In order to differentiate benign from pathologic PVCs children should have maximum exercise electrocardiography. This should help to avoid overtreatment and it will al so be psychologically beneficial for the
children and parents to observe their disappearance with activity. 86 EVALUATION OF IMPEDANCE CARDIOGRAM IN MITRAL AND R. Patel, Horst R. Jordan and Ronald M. L.auer. University of Iowa, Iowa City, Iowa.

If 2 electrodes in a high frequency circuit are placed on the thorax and the first derivative of the impedance to this current is recorded, a wave form related to events within the cardiac cycle results, the impedance cardiogram (ICG). Simultaneous electrocardiogram (EKG), phonocardiogram (PCG), echocardiogram (ECHO) and ICG were recorded from 10 control sub- ICO jects, 9 children with aortic regurgitation (AR) and 6 children with mitral regurgitation (MR). Measurements from
the ICG are as illustrated. In all

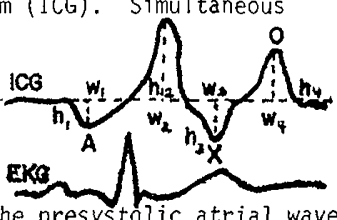
patients the $A$ point occurred during the presystolic atrial wave of the echo. The 0 point occurred simultaneously with the maximal anterior excursion of the echo of the mitral valve. The $X$ was synchronous with the PCG $A_{2}$. In patients with $M R h_{2} / h_{4}$ mean 2.5 (S.D.+1.6) was less than control 7.04 (S.D.+2.5); $p<0.001$ The (h4) WA was larger in MR 3.2 (S.D.t1.4) than control 1.4 (S.D. +0.09 ); $p<.005$. Patients with AR had increased ( $\left.w_{2}\right) h_{2} 74.8$ (S.D. F5.1) with control $7.2($ S.D. +2.7$)$ consistent with increased stroke volume. The area under the $X$ point $\left(w_{3}\right) h_{3}$ also increased in AR 2.7 (S.D.+1.1) versus control 2.0 (S.D.+0.85); p<.005. The ICG has a consistent wave form related to cardiac events as demonstrated by simultaneous ECHO events. AR and MR enhance the $A$ and 0 waves respectively.

87

CARD IOPULMONARY REFLEX RESPONSES IN THE PIGLET. Margo Schleman*, Norman Gootman, and Linda Crane*. Sch. of Med., State Univ. of N.Y. at Stony Brook and Long Island Jewish-Hillside Med. Ctr., Dept. of Ped., New Hyde Park, N.Y.

The purpose of this study was to investigate the piglet's response to stimulation of the J (juxtacapillary pulmonary) receptor in the lung. J receptor stimulation in the adult animal has been shown to produce reflex hypotension, bradycardia, and apnea. A recent investigation of newborn kittens has shown cardiopulmonary $\mathrm{J}$ receptor responses take 1 week to develop (M.Kahlia, 1974). we chose the piglet because of its physiological similarities to the human neonate. 14 piglets aged 1 to 23 days were given halothane $0.25 \%$ in a mixture of $\mathrm{N}_{2} \mathrm{O}$ and $\mathrm{O}_{2}$ via a tracheostomy. Respiration was spontaneous and blood gas determinations were made at hourly intervals. The following parameters were continuously recorded: heart rate (HR), arterial blood pressure (BP), EKG, expired $\mathrm{CO}_{2}$, and respiratory rate (RR). Phenyldiguanide (PDG), a drug which stimulates the J receptor, was injected into the right atrium in dosages of $60-80 \mathrm{mcg} / \mathrm{kg}$. The data indicate the piglet responds to $J$ receptor stimulation with changes in $B P$, $H R$ and $R R$. BP fell in all animals $30 \% \pm 4(p<.001)$. HR slowed in 8 animals $19 \%$ $\pm 0.9(p<.001)$. Cardiac standstill occurred in two. No change occurred in 4. In 9 animals in which RR was measured RR increased $28 \% 3.4(p<.001)$. 5 developed inspiratory apnea prior to the onset of rapid shallow breathing. The results suggest the piglet has a well developed response to $J$ receptor stimulation.

Supported by Rozenberg-Toner Heart Foundation.
88 DUCTAL CLOSURE IN UTERO BY A PROSTAGIAIDIIN SYNTHETASE INHIBITOR. George I. Shame Bertil Thalme and K. Sune Larsson (Intr.by A. Yao) Karolinska Inst. Dept. of Teratology, Karolinska Hosp. and Dept.of Ped., Huddinge Hosp. Stockholm, Sweden Closure of the Ductus Arteriosus was studied in 75 newbom rats using the whole-body freezing technique. In contrast to the effects of prostaglandin in redilat -ing the closing ductus ${ }^{1}$, the administration of a prostaglandin synthetase inhibitor $15 \mathrm{mg} / \mathrm{kg}$ indomethacin) to two grouns of 3 pregnant rats either 12 or $18 \mathrm{hrs}$. prior to delivery at term(22 d) was followed by intrauterine contraction of the fetal ductus arteriosus, (inner diameter $141 \mu \mathrm{m}$ ). Control rats born with ductal i.d. of $558 \mu \mathrm{m}$ showed normal closure from birth to 120 min. Newborms exposed to indomethacin had more lethargy, distressed breathing and cyanosis in the first 30 min after delivery but recovered thereafter. The indomethacin group showed a low $\mathrm{pH}$ and high pCO2 at $30 \mathrm{~min}$, returning to normal at $120 \mathrm{~min}$ after delivery. (the fetus and its postnatal adjustment.

1 Studies on Closure of the Ductus Arteriosus. X. In vivo effect of Prostaslandin: George I. Sharpe and Larsson,K.Sune. Cardiology (submitted)

89 THE ULTRASTRUCTURE OF THE DEVELOPING HEART Curtis Sheldon, Stanley E. Kirkpatrick, Harley Sybers, and William F.Friedman, UCSD Sch. of Med., San Diego, CA. Study of the changes in cardiac structure that accompany growth is a prerequisite to understanding pre and postnatal adaptation to cardiac disease. Accordingly, scanning electron microscopy (SEM) was employed to systematically analyze lamb myocardium from 90 days gestation to 36 days of age. Transmission electron microscopy (TEM) was utilized to assure adequate fixation and to confirm SEM observations. Important morphological changes were observed as a function of age. In young fetal tissue, prominent perinuclear spaces were seen containing long, branching mitochondria. These multilobular mitochondria decreased markedly in number with age and appeared to generate multiple small ovoid mitochondria in young tissue, which then enlarged to a size approximating I sarcomere length in adult tissue. Postnatally, the mitochondria arranged in characteristic parallel rows between adjacent myofibrils. The nuclear interior was exposed by tissue fracture of adult myocardium. In contrast, this process revealed the exterior surface of the fetal nucleus and allowed visualization of nuclear pores. These findings are compatible with a subsarcolemmal location and sparcity of myofibrils in early life. Division of myofibrils was observed in the fetus and prominent transversely oriented elevated ridges, presumably $T$ tubules, were seen coursing across myofibrils as early as 90 days gestation. These findings provide a new model for the subcellulor organization of myocyte content. POSITION OF THE GREAT ARTERIES. Laurence J. SlOSS, Ronald D. Greenwood, Michrel A. LaCorte, and Alexander S. Nadas, Children's Hosp. Med̉. Ctr., and harvard Med. Sch., Boston, Mass.

Cardiac arrhythmias were evaluated in 35 consecutive patients undergoing Mustard's operation for transposition of the great arteries, using routine electrocardiograms and Holter monitoring. Only eight patients (23\%) remained in sinus rhythm throughout the period of observation. Minor abnormalities (abnormal $P$ wave, coronary sinus rhythm) were detected in six (17\%). Conduction abnormalities were noted in eight (23\%) including first degree atrioventricular block, right bundle branch block, trifascicular block and complete heart block. Sick sinus syndrome was noted in five (14\%) of whom three had both tachy- and bradyarrhythmi as and two bradyarrhythmias onlv. Six patients $(17 \%)$ exhibited junctional rhythm, and seven $(20 \%)$ supraventricular tachycardia. HoIter monitoring frequently detected arrhythmias not noted on routine electrocardiograms. The high incidence of arrhythmias following Mustard's operation, presumably reflectine injury to the sinoatrial node and conducting pathways, may foreshadow a long-term problem in the management of these patients, and supgests the need for surgical techniques designed to avoid injury to pacemaker and conduction tissue. 
91 THE PROGNOSTIC SIGNIFICANCE OF LEFT ANTERIOR HEMIBLOCK COMBINED WITY RIGHT BUNDLE BRANCH BLOCK FOLLOWING INTRACARBFAC REPAIR OF, FALLOT TETRALOGY H. Sondheimer,$\frac{T}{\text { H. Lut Lukawa, }}$, Tor onto

Recent reports suggest that complete $x$ ight bundle branch block (RBBB) and left anterior hemiblock (LAH) following cardiac surgery carries a high risk of late complete heart block (CHB) and possible sudden death. Four hundred and one survivors of total correction for Fallot tetralogy who all had normal sinus rhythm (NSR) and right axis deviation pre-operatively were reviewed. Eighteen late deaths have occurred $(4.5 \%), 5$ due to dysrhythmias. Ten survivors $(2.5 \%)$, have required pacemakers either for permanent $\mathrm{CHB}(8)$ or intermittent block (2). Forty-seven patients $(11.7 \%$ ) had immediate post-operative (PO) $\mathrm{CHB}$ transiently (38) or permanently (9). Twelve of these $47(26 \%)$ have died of dysrhythmia or required permanent pacing. R:BB with LAH developed in $99(25 \%)$. Four died from non-dysrhythmic causes. Of the other 95,17 had had transient PO CHB of whorn died suddenly and 2 developed late onset CHB. The 78 patients without transient $\mathrm{PO} \mathrm{CHB}$ have had no $\mathrm{l}$ ate morbidity or mortality through 347 patient years. Only 2 of the 354 without PO CHB had dysrhythmic problems. $(0.6 \%$ V.S. $26 \% \quad \mathrm{p}<0.001)$ and neither had RBBB with LAH.

We conclude that transient $P O C H B$ is the most significant risk factor for predicting late onset $C H B$ in these patients. RBBB with LAH without transient $P O C H B$ is a less critical finding although its final significance will become apparent with further follow up.

92 HYPOTHERMTA AND REWARMING BY SURFACE AND He-02 INHALATE TEMPERATURE CONTROL, Donald R. Sperling,
Anthony $V$. Beran, and Kenneth G. Proctor (Intr. by T.L. Nelson), Univ. of CA, Col of Med., Dept. of Ped., Irvine,CA Hypothermia and circulatory arrest are clinically acceptable procedures for the correction of congenital heart lesions. The disadvantages of current methods are that noninvasive techniques are slow and difficult to control, while those which are fast and controllable are invasive and carry increased risk of complications. This study evaluated the efficiency of the respiratory tract as a heat exchanger for the production of hypothermia in rabbits. To maintain an optimal inhalate-respiratory tract temp gradient, inhalate temp was maintained at $-2.6 \pm 2.3^{\circ} \mathrm{C}$. To obtain optimal thermal conductivity, $80 \% \mathrm{He}-20 \% 0_{2}$ mixture was used. To maintain an inhalate-respiratory tract humidity gradient, inspiratory gases were dehumidified. To maintain satisfactory circulatory dynamics, hypercapnia and hypoxia were induced. The study population was divided into two groups. Group A-surface cooling and rewarming alone. Group B-surface plus inhalate $\mathrm{He}-\mathrm{O}_{2} \mathrm{COOl}_{\text {ing }}$ and rewarming. In group $A$ esophageal temp (ET) decreased from $39.4 \pm .5^{\circ} \mathrm{C}$ to $29.2 \pm 1.2^{\circ} \mathrm{C}$ in $146.2 \pm 26.8$ min representing a rate of cooling $(R)$ of $.07 \pm .07^{\circ} \mathrm{C} / \mathrm{min}$. In group $\mathrm{B} E T$ decreased from $38.3 \pm 1.6^{\circ}$ to $22.1 \pm .1^{\circ} \mathrm{C}$ in $123.8 \pm 27.1 \mathrm{~min}$, representing a significantly faster $\mathrm{R}$ of $.14 \pm .02^{\circ} \mathrm{C} / \mathrm{min}$. In addition, in group $B$ in the $38-29^{\circ} \mathrm{C}$ range, $R$ was $.17 \pm .03^{\circ} \mathrm{C} / \mathrm{min}$. The rewarming rate above $29^{\circ} \mathrm{C}$ for both methods was $.15^{\circ} \pm .02^{\circ} \mathrm{C} / \mathrm{min}$. All animals survived without signs of pulmonary, or CNS damage. Because of the efficiency, simpljcity and noninvasiveness of this technique, a

\section{COMPARATIVE CARDIOVASCULAR EFFECTS OF EXERCISE} AND ISOPROTERENOL IN CHILDREN WITH AORTIC STENOSIS. Carl N. Steeg, Nestor 1. Truccone, Welton M. Gersony, Columbia Presbyterian Medical Center, Dept. of Ped. , New Yark

The use of isoproterenol (1) has been advocated for simulation of exercise (E) in children with aortic stenosis. In order to evaluate the validity of this approach, cardiovascular dynamics were evaluated during a) rest (R), b) $(E)$, c) 1 infusions, in 8 children, ages 5 to 15 years, with mild or moderate aortic valve stenosis. Cardiac Output (CO) was measured by the green dye technique. Measurements of left ventricular (LV) pressures and $\mathrm{LV}(\mathrm{dp} / \mathrm{dt}) / \mathrm{p}$ were obtained with a catheter tip micromanometer. Mean results during the 3 states include:

\begin{tabular}{|c|c|c|c|c|c|c|}
\hline & $\begin{array}{l}\text { Heart } \\
\text { Rate }\end{array}$ & $\begin{array}{l}\text { LVSP } \\
\text { (mmHg }\end{array}$ & $\begin{array}{l}\text { peak Ao.P } \\
\text { g) }(\mathrm{mmHg} .)\end{array}$ & $\begin{array}{l}\mathrm{C.I} . \\
\mathrm{L} / \mathrm{m} / \mathrm{m}^{2}\end{array}$ & $\begin{array}{c}\text { Syst. Resist. } \\
\mathrm{U} / \mathrm{m}^{2}\end{array}$ & $\begin{array}{c}L V(d p / d t) / p \\
\sec -1\end{array}$ \\
\hline$R$ & 95 & 740 & 109 & 4.5 & 18.5 & 54 \\
\hline$E$ & 151 & 164 & 116 & 7.9 & 11.2 & 84 \\
\hline 1 & 148 & 162 & 77 & 6.6 & 7.9 & 111 \\
\hline$p=$ & NS & NS & $<0.01$ & $<0.01$ & $<0.01$ & $<0.01$ \\
\hline
\end{tabular}

Af comparable levels of H.R., E. and Tproduced a similar increase in $L V$
systolic pressure (LVSP). Peak systolic Ao-LV increased $54 \%$ with $E$ and $174 \%$ with 1 ( $p<0.01)$. LV $(\mathrm{dp} / \mathrm{dt} / \mathrm{p}$ increased $55 \%$ with $E$ and $105 \%$ with $1(p<0.01)$. These findings indicate that $I$ is a good predictor of exercise LVSP but results in lower peak Aortic Pressure (AOP). The finding of higher LV-Ao gradients with $I$ as compared to $E$ appears to be related to significant differences in LV $(d p / d t) / p$, systemic resistance, and $C . O$.
94 AORTIC COARCTATION (C) INI IHFANTS - THE ROLE OF HYPERTEISION (hyp).: Aluizio R. Stopa, Selwyn Uilner, Jennifer lill. Loggie, Samuel Kaplan, and James !lelnilsworth. Children's llospital ledical Center, cincinnati, chio

79 patients under 1 year of age vere seen with $C$ since 1060 . In the classical manner $6 C / 79$ ( $\varepsilon 3 \%$ ) presented in heart failure (HF) :Ith $19 / 66$ being operated on for IIF (mortality $42 \%$ ). 22/6E (33\%) died in $\| F$ without benefit of operation, usually with associated anolialies. then viewed from the aspect of presentation under 1 year of age with hypertension there were 39 cases. 29/39 hyper tensive patients had IIF. 9 (all with associated anomalies) were operated on primarily for liF (mortality 44\%). 2/23 hypertensive patients died from liF, and 1 died from cerebral vascular accident. 8 patients improved after initial lif hut later required cperation for hypertension ( 6 were between 6 weeks and 4 years of ace). At follow un $7 / 8$ asymptomatic patients operated on for hyn. are normotensive, while $7 / 9$ asymptomatic patients who were not operated on still have hypertension. Follow up blood pressure in the group who had hyp. and HF was variable. He conclude (1) hyp is common in infants with coarctation (2) hyp is alleviated by early surgery (3) byp. persists in the majority of natients who do not have surgery (4) :iedical therapy for $M F$ does not prevent the development of Hyp. and (5) Uncontrollable hyp. may be an indication for coarctectony in young children.

95 PERCUTANEOUS CARDIAC CATHETERIZATION IN INFANTS AND CHILDREN. Cecille 0 . Sunderland, Dale P. Henken, G. Michael Nichols, Fred Linstone, Martin H. Lees, Univ of Oregon Health Sciences Cénter, Portland, Oregon.

Percutaneous cardiac catheterization in pediatrics has been restricted by most groups to children over $10 \mathrm{~kg}$. We have evolved a simple technique applicable to all ages. Between November 1971 and July 1974 we have performed 520 of 557 (93\%) cardiac catheter izations percutaneously utilizing the femoral artery and vein on 413 patients ranging in age from 6 hours to 21 years and in weight from $.86 \mathrm{~kg}$ to $80 \mathrm{~kg}$. There were 113 patients less than $5 \mathrm{~kg}$ in whom 88 of 107 ( $82 \%$ ) venous and 26 of $29(90 \%)$ arterial studies were performed percutaneously; 47 patients between 5.1 and $10 \mathrm{~kg}$ in whom 49 of 54 ( $91 \%$ ) venous and 20 of 21 (99\%) arterial studies were performed percutaneously; 253 patients over $10 \mathrm{~kg}$ in whom 233 of 241 (97\%) venous and 104 of 105 (99\%) arterial studies were performed percutaneously. The complication rate for percutaneous venous studies was $0.27 \%(1 / 370)$, involving bleeding at the entry site controlled by pressure. Following percutaneous arterial catheterization, all children had normal capillary filling, temperature and color of the leg within 48 hours. The arterial pulse was absent at 48 hours in $6 / 26(23 \%)$ retrogrades in the under $5 \mathrm{~kg}$ group, $6 / 20(30 \%)$ in the $5-10 \mathrm{~kg}$ group and $8 / 104(8 \%)$ over $10 \mathrm{~kg}$. The percutaneous approach to cardiac catheterization in all infants and children has the advantages for right heart studies of virtually no complications, absence of wound infections and reusability of the vessel. For arterial studies there is a significantly lower complication rate than for the cutdown method of arterial entry often used in pediatric cardiology.

\section{BENEFICIAL HEMODYNAMIC EFFECTS OF PHENTOLAMINE IN EXPERIMENTAL VENTRICULAR SEPTAL DEFECT. David P.} Synhorst, Ronald M. Lauer and Michael J. Brody.

Univ. of Iowa Col. of Med., Depts. of Pediatrics and Pharmacology Iowa City, IA 52242

Reduction in the magnitude of left to right shunts by pulmonary artery banding has been shown to be beneficial in various cardiac defects. The possibility that similar hemodynamic changes could be effected by peripheral vasodilation was explored. Ventricular septal defects were created in $17-16 \mathrm{Kg}$ dogs by insertion of a $6 \mathrm{~mm} \mathrm{I.D.} \mathrm{Teflon} \mathrm{prosthesis} \mathrm{through} \mathrm{the} \mathrm{ventricu-}$ lar septum. Mean aortic $\left(\overline{A_{0}}\right)$, pulmonary artery $(\overline{P A})$ and left atrial (IA) pressures were recorded, aortic (QSS) and pulmonary (Q)p) flows were measured with electromagnetic flow probes and. total systemic (RS) and pulmonary (Rp) vascular resistances, $\mathrm{Qp} /$ $\dot{Q} \mathrm{~s}, \mathrm{Rp} / \mathrm{Rs}$ and $\overline{P A} / \overline{A_{0}}$ were calculated. After recovery from shunt placement, all hemodynamic measurements in control dogs remained unchanged throughout a $3 \mathrm{hr}$ period. Experimental dogs were given $1 \mathrm{mg} / \mathrm{Kg}$ phentolamine IV when stable after shunt introduction. Rs and $\overrightarrow{A_{0}}$ were reduced significantly $(p<.05)$ following phentolamine. During this period $Q p / Q s$ was reduced from 2.7 to $7.9(p<.01)$ and $Q p$ was reduced from 1.6 to $1.2 \mathrm{~L} / \mathrm{min}(p<.05)$. $\overline{P A}$ was decreased by $4 \mathrm{~mm} \mathrm{Hg}(p<.05)$. $Q \mathrm{QS}, \mathrm{Rp} / \mathrm{Rs}, \overline{P A} / \overrightarrow{A O}$ and $\overline{L A}$ were not changed significantly. These data suggest that in experimental left to right shunts beneficial hemodynamic effects including reduced $Q \mathrm{p}$, $\dot{Q} / \bar{Q}$ s and $\overline{P A}$ can be produced by peripheral vasodilation.

Supported in part by USPHS Grants HLP-14388, HL-12964 and HL-
T01-5577. 

97 ETIOLOGY OF THE rsR' ELECTROCARDIOGRAPHIC PATTERN IN
ATRIAL SEPTAL DEFECT. Dolores M. Tamer*, Ruey J. Sung*, AbduI S. Agha*, Agustin Castellanos*, Robert J. Myerburg*, Henry Gelband, Univ. of Miami School of Med., Fla. An rsR' pattern in the right precordial leads occurs in most patients (pts) with atrial septal defect (ASD). This pattern has been called incomplete right bundle branch (IRBB) block. These pts also have associated right ventricular overload (RVo). This study was carried out to determine whether IRBB block or RVO is the primary cause of the ECG pattern. His bundle $(H)$ and right ventricular apical (RVA) endocardial electrocardiograms were recorded in $15 \mathrm{pts}$, age 5 to 17 years, of whom 9 had ASD and 6 had mild aortic stenosis (AS). The H-RVA interval was assumed to approximate conduction time from the level of the His bundle along the $R B B$ to the site of earliest activation in the right ver tricle at the RVA. The $\mathrm{H}-\mathrm{V}$ interval was normal in all pts $(\because 40$ msec). All pts in the ASD group had an rsR' pattern in $V_{1}$ with a mean duration of $94.3 \mathrm{msec}$. (82 to $116 \mathrm{msec}$.); the mean duration of the H-RVA intervals was $53.6 \pm 6.3 \mathrm{msec}$. in this group. In pts with AS, the surface electrocardiograms were normal with a mean QRS duration of $82.7 \mathrm{msec}$ (70 to $95 \mathrm{msec}$ ) and the H-RVA intervals averaged $50.6+5.4 \mathrm{msec}$. The difference in the H-RVA intervals in the two groups was not significant $(p \times 0.20)$. Moreover, when functional RBB block was induced by premature atrial stimulation in the ASD group, the prolongation of QRS duration was accompanied by an increase in the H-RVA interval. It is concluded that since the H-RVA interval is normal in pts with ASD; the $\mathrm{rRR}^{\prime}$ pattern is more likely due to a manifestation of RVO
than to a true conduction delay in the $\mathrm{RBB}$.

\section{8} EFFECT OF VENTRICULAR ACTIVATYON SEQUENCE ON OUTFLOW OBSTRUCTION IN OBSTRUCTIVE CARDIO MYOPATHY (IHSS). Otto G. Thilenius, Domingo I. de la Fuente, Rabi Sulayman and Rene A. Arcilla. Univ. of Chicago, Dept. of Pediatrics, Chicago.

The etiology for the outflow obstruction in IHSS is unknown. Three children with IHSS and 6 with non-IHSS (discrete aortic stenosis, normal, other heart disease) underwent atrial and ventricular pacing using simultaneous recordings of left ventricular (LV) inflow and ascending aortic (AO) pressures with Millar microtransducers. Right ventricular (RV) pacing sites were outflow tract, apex and inflow tract; LV sites were outflow and apex. Pacing rates were approximately 10 beats more than sinus rate to allow capture. In IHSS subjects, outflow pacing of RV or LV produced $50 \%$ increase in pressure gradient while inflow pacing did not alter it. Apical pacing reduced it by $20 \%$ and $30 \%$ in 2 cases. Atrial pacing did not change the degree of obstruction. In the 6 non-IHSS, pacing at various sites did not alter the LV and Ao pressure relationships. The obstruction in IHSS is thus influenced by the sequence of ventricular depolarization. Early activation of the hypertrophied septum and outflow tract accentuates obstruction. Outflow tract pacing can be a diagnostic test in pre-obstructive cardiomyopathy. A-V block sometimes occur following myomectomy in IHSS requiring pacemaker therapy. Electrode placement in the latter should NOT be close to the outflow tracts.

90 CARDIOVASCULAR EFFECTS OF RAPID ATRIAL STIMULATION (RAS) IN ADULT DOGS AND IN PUPPIES.Nestor J. Truccone, $M D$, and Fhud Krongrad, $M D$, Department of Pediatrics, Columbia University, New York, N.Y. 10032

Previous studies defined the response of the cardiac A-V conduction system to RAS (Ped. Res. 7:299/71, 1973). The current study was undertaken in order to evaluate cardlovascular hemodynamics, (CV-hem) associated with RAS and $2: 1 \mathrm{~A}-\mathrm{V}$ block and its possible use in the treatment of supraventricular tachycardias (SVT). In each of 8 dogs (*) and 9 puppies (**) simultaneous EKG, cardiac index (CI), aortic pressure (Ao.P), pulmonary artery pressure (PAP), left ventricular (LV) systolic and end--diastolic pressures, LVAp/ $\mathrm{dt}) / \mathrm{p}$, were measured and pulmonary (RP) and systemic (RS) resistances calculated. Measurements were obtained at (1) control stata, (2) RAS with maximal 1:1 AV conduction, (3) RAS with 2:1 AV block. The following mean percentage changes from control (100\%) occurred.

\begin{tabular}{|c|c|c|c|c|c|c|c|}
\hline & $\begin{array}{l}\text { Atrial } \\
\text { Rate }\end{array}$ & $\begin{array}{l}\text { Ventricular } \\
\text { Rate }\end{array}$ & C.I. & Ao.P & $\begin{array}{c}\mathrm{LV} \\
(\mathrm{dp} / \mathrm{dt}) / \mathrm{p}\end{array}$ & RS & $\mathrm{RP}$ \\
\hline$\overline{*(1)}$ & 164 & 164 & $100 \%$ & $100 \%$ & $100 \%$ & $100 \%$ & $100 \%$ \\
\hline$\pi(2)$ & 328 & 328 & $-39 \%$ & $-15 \%$ & $+12 \%$ & $+45 \%$ & $+54 \%$ \\
\hline$\pi(3)$ & 436 & 218 & $-4 \%$ & $+3 \%$ & $+34 \%$ & $+15 \%$ & $+19 \%$ \\
\hline$* *(1)$ & 211 & 211 & $100 \%$ & $100 \%$ & $100 \%$ & & $100 \%$ \\
\hline$\pi *(2)$ & 344 & 344 & $-30 \%$ & $-16 \%$ & $-1 \%$ & $+19 \%$ & $+38 \%$ \\
\hline **(3) & 459 & 229 & $-6 \%$ & $-4 \%$ & $+15 \%$ & $-1 \%$ & $-10 \%$ \\
\hline
\end{tabular}

are significantly improved as compared to RAS with maxima 1 1:1 AV conduction. 2) This method may be useful in the management of some infants and children with rapid intractable SVT.
100 PERIPHERAL VEIN INJECTIONS AS A MEANS OF PRODUCINC Lilliam Valdes-Cruz, J-Michel Roland, Daniel Pieroni, P. Jacob Varghese, (Intr. by Catherine Neill) Johns Hopkins Hospital, Department of Pediatrics, Baltimore, Md.

Laboratory and clinical studies were designed to determine if peripheral vein injections (PVI) consistently produce venous microcavitations (MC) detectable by echocardiography(E). The effect of varying volumes, pressures and substances on $M C$ was also investigated. A 16 gauge needle catheter was introduced percutaneously into the right antecubital vein in 5 anesthetized dogs. The heart was exposed through a left lateral thoracotomy and the transducer positioned to visualize the left ventricular cavity, interventricular septum and right ventricular inflow area. Injection variables assessed were: blood and saline, volumes of 5 and $10 \mathrm{cc}$, hand and pressure injections of 100 and 200 psi. A strip chart E was recorded simultaneously with each injection and the result graded on a $0-4$ scale. Pressure injections, irrespective of volume, gave consistently high grades whereas similar results were obtained on hand injections only when using blood or larger volumes. Examination of the peripheral vein revealed no rupture, thrombus or endothelial damage. Clinical trials performed in 20 children, ages 5 to 15 , using hand injections of 2,5 and $10 \mathrm{cc}$ of normal saline and of blood gave results that corresponded to the laboratory observa tions. The pressure of the injection is the critical factor in consistently producing detectable MC. Thus PVI is a simple, safe and reliable method to produce venous $M C$ detectable by $E$ which can be used in the ambulatory patient to identify intracardiac
shunt.

101 ECHOCARDIOGRAPHIC ASSESSMENT OF VENTRICULAR DIMENSIONS AND MYOCARDIAL FUNCTION IN INFANTS OF DIABETIC MOTHERS. Gexald L. Way, Robert R. Wolfe, Gary P. Pettett, Gerald B. Merenstein, Michael A. Simmons, Richard D. Spangler, and James J. Nora. Univ. Colo. Sch. of Med. and Fitzsimons Army Med. Ctr., Dept. of Ped., Denver, Colorado. Cardiomegaly is noted radiographically in 5-30\% infants of diabetic mothers (IDM). In addition transient symptoms related to the cardiopulmonary system is seen in $30-50 \%$ of these infants. Etiologic considerations referable to apparent congestive heart failure are transient metabolic disturbance including hypoglycemia and hypocalcemia and infiltrative cardiomyopathy. In ordex to define an etiology of these findings the following study was undertaken. Fifteen infants with a mean gestational age of 38.5 weeks and all satisfying White's classification were evaluated echocardiographically. Two infants were eliminated from the study because of congenital heart disease. The velocity of circumferential fiber shortening (VCF), posterior wall thickness (PWT) and septal wall thickness (SWT) were measured and compared to normal infants. Normal infants $\mathrm{VCF}=1.51 \pm .34$. IDM's had significantly decreased VCF $1.04 \pm .28$ (p<.001). Only one patient had PWT or SWT greater than normal. All but one infant had apgar scores $>7$ at 5 minutes. No infants were hypoglycemic at the time of examination and only one infant was hypocalcemic. There was no correlation between the patients' clinical state and VCF, stroke volume and VCF ox birth weight and VCF. There was a good correlation between ejection fraction and VCF $(x=.886)$. Our data suggests that the cardiorespiratory symptoms are related to
SUPRABULBAR NERVOUS INHIBITION OF THE BAROREFLEX

102 IN THE CHRONIC FETAL LAMB PREPARATION. Robert Colle). McGili Univ.-Montreal Children's Hosp. Research Inst. and Dept. of Physiology, McGill University, Montreal

Experiments were performed to study mechanisms which might influence fetal cardiovascular reflexes which utilize the lower brainstem. Electrodes were implanted in vasoactive areas of the forebrain in 6 fetal lambs (gestational age from 112-124 days). Catheters were inserted into the amniotic cavity, the fetal trachea, carotid artery and jugular vein. Flentrocardiographic leads were sewn to the chest wall and a balloon catheter advanced from the femoral artery into the descending thoracic aorta. Studies were performed from 5 days after operation when the fetal heart rate was < 195 beats/min., arterial $\mathrm{pO}_{2}>20 \mathrm{mmlg}, \mathrm{pCO} 2<45 \mathrm{~mm} \mathrm{Hg}, \mathrm{plI}>7.32$ and there were no "respiratory-like" changes in tracheal pressure. Baroreceptors were stimulated by inflating the balloon for 5 seconds and the slope of the beat-to-beat relationship between carotid pulse pressure and the subsequent R-R intervalindicated baroreflex sensitivity. The prolongation of $R-R$ interval (slowing of heart rate) per $\mathrm{mmHg}$ rise in pulse pressure averaged $1.71 \pm 0.29$ (S.E.) msec per mmlg before, 1.14 \pm 0.20 during and $1.64 \pm 0.24$ af ter subthreshold 10 second electrical forebrain stimulation. There was no significant difference in resting heart rate or mean blood pressure between the 3 groups. These data suggest that neural pathways exist for active suprabulbar nervous inhibition of the baroreflex in late gestation fetal lambs. 

103 LCHOCARDIOGRAPHIC AND CINEANGIOGRAPHIC ASSESSIENT OF DIMENSION. Steven M. Yabek, Josephine Isabel-Jones, Rona1d Rosengart and Jay M. Jarmakani, UCLA School of Medicine, Department of Pediatrics, Los Angeles.

Echocardiographic (echo) LA dimension as an indication of $L \rightarrow R$ shunt has recently received much attention. To further assess this relationship, we obtained echocardiograms in 47 children (ages 1-18 years) undergoing cardiac catheterization and biplane cineangiographic LA volume (V) determination. Group $I(N=30)$ had normal LA V (70-130\% of normal predicted values); Group II $(\mathrm{N}=17)$ had large LA V (>130\%). Symmetrical LA enlargement with age and $\mathrm{V}$ overload, as measured angiographically, was indicated in both Groups by the following correlations: (1) LA anterior-posterior minor axis (mA) with LA major axis $(\mathrm{r}=.90)$; (2) LA mA with LA V $(r=.93)$. LA echo dimension showed good linear correlation with the cineangiographic LA MA $(r=67)$ and was smaller than the latter in all patients. There was, therefore, significant correlation between the LA echo dimension (y) and the cineangiographically determined LA V $(x),(y=0.15 x+14 ; r=.70 ; p<.001)$ enabling us to calculate LA $V$ from the echo dimension, but with limited accuracy. When corrected for body surface area, LA echo dimensions averaged 23 and $36 \mathrm{~mm} / \mathrm{m}^{2}$ for Groups $I$ and II respectively, with no patient in croup I having a LA echo $>34 \mathrm{~mm} / \mathrm{m}^{2}$.

In summary, although echocardiography cannot precisely predict LA volume, it can clearly separate those patients with marked LA volume overload and can satisfactorily be used to serially follow LA volume changes in patients with laxge $G \rightarrow R$ shunts.

104 SINUS NODE FUNCTION: FACTORS INFLUENCING ITS EVALUATION. Steven M. Yabek, Jay M. Jarmakani, Dilip Bhatt and Nigel K. Roberts, UCLA School of Medicine, Department of Pediatrics, Los Angeles.

Overdrive atrial stimulation is now widely used in adults as a method for evaluating sinus node function in the clinical situation. We are reporting observations obtained from 20 electrophysiologically normal children (ages 2-18 years) who were studied using atrial pacing at rates of $120,150,180$ and $200 / \mathrm{min}$ (Only two patients developed block at pacing rates less than $200 / \mathrm{min}$.) At $200 / \mathrm{min}$, the mean sinoatrial node recovery time (SANRT), measured as a $\%$ of the resting $\mathrm{R}-\mathrm{R}$ interval, was $102 \%$ $(1 S D=9)$. Significantly longer SANRTs $(136 \% ; 131 \%)$ were obtained at pacing rates of 120 and $150 / \mathrm{min}$. as compared to 180 and $200 /$ min. (p<.001). Parasympathetic blockade with atropine $(0.01 \mathrm{mg} . / \mathrm{kg}$. IV) abolished the pacing rate related differences and resulted in a lower maximal SANRT in all patients.

Because of the direct linear correlation found between the absolute recovery time and the resting $R-R$ interval $(p<.001$ ), we feel it is necessary to correct for age and heart rate by expressing the SANRT as a function of the resting interval. Our findings of greater overdrive suppression at relatively lower pacing rates indicate definite overdrive rate dependent sinus node function and stress the importance of interpreting the SANRT in relation to the rate of overdrive used.

These results on normal children have helped us to evaluate compromised sinus node function that has occasionally occurred either congenitally or following intracardiac surgery.

\section{MECHANISM OF TRANSIENT FETAL TACHYCARDIA, M.N.} Yeh, S.N.Caritis, H.O. Morishima, L.S. James, Coll. of

ogy, N.Y.C.

The cause of transient acceleration of the fetal heart rate (FHR) which is commonly seen during labor is not known. These transient acceleration patterns suggested that the cause might be partial occlusion of the umbilical vein resulting in a decrease in venous return and hypotension; the sympathetic response would be transient tachycardia.

This postulate has been investigated experimentally in 20 pregnant subhuman primates. Arterial and venous catheters and ECG electrodes were inserted into fetal vessels and two occluding devices were placed around the base of the intact umbilical cord (UC) and the intra-abdominal portion of the umbilical vein (UV). After surgery with the fetus intact in utero, the UC or UV were partially occluded for up to 7 minutes. Fetal BP, HR, ECG, were monitored continuously and arterial $\mathrm{PO}_{2}$ and $\mathrm{pH}$ intermittently. Two types of response were seen. In the well oxygenated fetus, partial occlusion resulted in a fall in BP and a rise in FHR. This response was abolished by pharmacologic blockade of the sympathetic nervous system with dibenzyline or propranolol. The same responses were seen with either partial occlusion of the UC or UV. In the hypoxic fetus, partial occlusion resulted in a smaller fall in BP and either no charge or a fall in FHR. Thus, transient acceleration of the FHR can be explained on the basis of diminished venous return and is an early sign of a potential cord complication during labor. Transient tachycardia will not be seen if the fetus becomes asphyxiated and
hypoxic.
106 ULTRASOUND DIAGNOSIS OF BICUSPID AORTIC VALVE ASSOCIATED WITH COARCTATION OF THE AORTA. Damaris $S$. Young and $W$. Pennock Laird, (Intr. by John D. Nelson) Univ. of Texas Health Science Center, Dallas, Texas. It has been comonly observed that a bicuspid aortic valve may be found in association with coarctation of the aorta. Recently it has been suggested that echocardiography may be a useful, accurate and non-invasive technique to identify the presence of a bicuspid aortic valve. Characteristically these valves can be recognized on the echocardiogram by an eccentric position of the aortic valve leaflets within the aortic lumen during diastole. Echocardiograms were performed on 40 patients with known coarctation of the aorta in order to diagnose the presence or absence of a bicuspid aortic valve. There were 18 females and 22 males. Their ages ranged from 2 days to 26 years ( $m=7$ years). Using the aortic valve echo, an index of eccentricity was calculated on each patient. Six patients $(15 \%)$ demonstrated a low eccentricity index (range 1.0 - 1.25) characteristic of a tricuspid aortic valve. Thirty-four patients $(85 \%)$ showed an eccentricity index indicating the presence of a bicuspid aortic valve (range 1.5 4.25). The cusps appeared clearly asymmetric in 18 of these patients. Recognition of the congenitally bicuspid aortic valve is of clinical importance because of the increased risk of developing infective endocarditis and because of the special predilection of the bicuspid valve to develop stenosis and/or incompetence. Echocardiographic evaluation of the aortic valve should be routinely performed in all patients with coarctation of the aorta.

7 ANGIOTENSIN II LEVELS DURING ACUTE HYPOXIC STRESS IN THE UNANESTHETIZED RABBIT. R. Zakheim, L. Mattioli
K. Mullis and A. Molteni. (Intr. by Cheng T. Cho) Depts of Pediatrics and Pathology, Univ. of Kansas School of Med., Kansas City, Ks. 66103

The response of the renin-angiotensin system to acute hypoxic stress was studied in 6 unanesthetized 2 to 3 lb female New Zealand white rabbits. Arterial angiotensin II (A2) levels were measured by the radio-immune assay technique in room air and after 10 minutes of ventilatory hypoxia $\left(\mathrm{FIO}_{2} 11 \%\right), 2 \mathrm{cc}$ of blood were required for the A2 measurements. Arterial blood gases, RV and aortic pressures were measured. Data were analyzed with the paired " $t$ " test. In 2 other rabbits the response of A2 to hypovolemic stress was measured. Results: (Average \pm SEM) A2 levels decreased in all 6 animals: from an average control value of $41 \pm 8 \mathrm{pg} / \mathrm{ml}$ to $16 \pm 6 \mathrm{pg} / \mathrm{ml}$ following 10 minutes of hypoxia $(\mathrm{p}<.001)$. The average $\mathrm{PaO} 2$ was $28 \pm 3.5, \mathrm{PaCO}_{2} 16 \pm 2$, $\mathrm{pH} 7.51 \pm 0.03$. RV systolic pressure increased in al1 animals: $21 \pm 2 \mathrm{~mm} \mathrm{Hg}$ to $25 \pm 2(p<.001)$. There was no significant change in aortic pressure during this period of alveolar hypoxia. In the 2 instances in which it was measured, $A 2$ returned to normal within 30 minutes after discontinuation of hypoxia. In 2 additional animals hypovolemic shock resulted in a $100 \%$ increase in A2 levels within 10 minutes after bleeding, with a return to normal levels 15 minutes after retransfusion.

These results show that acute hypoxic stress depresses arterial A2 levels in contradistinction to the stress of hypovolemic shock and that hypoxemia, at least to this extent $\left(\mathrm{PaO}_{2} 28\right)$, is not in itself a stimulus to A2 production.

\section{DEVELOPMENTAL BIOLOGY}

108 CARDIOVASCULAR AND RENAL FUNCTION IN THE PIGLET DURING THE FIRST SIX DAYS OF LIFE. Charles T. Alward, Michael. D. Bailie, Jerry B. Hook, and Thomas A. Helmrath. Michigan State Univ., Col. of Human Med., East Lansing.

Developmental studies of renal and cardiovascular function in the unanesthetized piglet demonstrate that this animal is a stable model for investigation of both effects of growth and stress in the newborn period. We have undertaken studies in animals ranging in age from 6 to 138 hours. Animals were anesthetized with nitrous oxide-oxygen and ketamine $(5 \mathrm{mg} / \mathrm{kg})$ and catheters placed in the femoral artery and vein, external carotid ar tery, jugular vein and urinary bladder. Animals were allowed to recover from anesthesia for I hour. Over 160 minutes cardiac output (CO), blood flow to kidneys (RBF) and 10 additional organs, blood pressure (BP), heart rate (HR), glomerular filtration rate (GFR), $\mathrm{Na}^{+}$and $\mathrm{K}^{+}$excretion, plasma renin concentration (PRC), arterial blood gases ( $A B G$ ), and hematocrit (Hct) were determined. $\mathrm{CO}$ was estimated by dye dilution, blood flow by radioactive microspheres, and GFR by inulin clearance. During the experimental period there were no significant changes in any of the variables measured except Hct which fell from an average of 27.5 to $26 \%$. Up to 138 hours of age $\mathrm{BP}, \mathrm{Na}^{+}$and $\mathrm{K}^{+}$excretion, $\mathrm{PRC}, \mathrm{AGB}$, and Hct were not correlated with the increasing age, weight or body surface area. GFR and RBF increased in relations to kidney weight and body surface area regardless of age. Increases in GFR and RBF appear to be directly related to increases in $\mathrm{CO}$. Since both GFR and RBF increased in proportion there was little change in filtration fraction. Glomerulotubular balance appeared well maintained. 
109 NOREPINEPHRINE (NE) UPTAKE IN RABBIT LUNG DEMONSTRATION OFAGE DEPENDENCE. Michael A.Berman, Kathleen Baker, C.N.Gillis, Yale Univ.Sch.Med., Dept.Ped.\& Anesth., New Haven. Intr. N.S.Talner

$N E$ uptake rates and deamination products were measured in isolated rabbit lung preparations in $\mathrm{g}$ roups aged $<20 \mathrm{hrs},, 2-3$ days, 10 days and at each 7 day interval unti1 70 days ( $n=7-11 / g$ roup). The technique included individual cannulation of the right and left pulmonary arteries (PA), perfusion of $\mathrm{NE} 20 \mathrm{ng} / \mathrm{ml}$ at $2 \mathrm{cc} / \mathrm{min} / \mathrm{lung}$, monitoring of PA pressures, and collecting pulmonary vein effluents at timed intervals for $\mathrm{NE}$ and metabolite analys is by column chromatog raphy. The \% uptake of NE increased from the newborn (1 $3 \%$ ) until 30 days when adult levels $(24 \%)$ were reached. Removal of NE per ml of inulin space (i.e.net removal corrected for perfusable space) however, showed higher values in newborns than in those $>30$ days ( 4.5 vs 2.4 $\mathrm{n}$ mole $/ \mathrm{ml})$. Similarly, the $\%$ deaminated products of monoamine oxidase rose from the newborn value $(7 \%)$ to adult level $(20 \%)$, while the $\%$ of catechol o-methylated product values were $3 \%$ for the newborn and $20 \%$ for the adult.

It is concluded that newborn rabbit lung has the capacity for uptake and deamination of $N E$. In addition, values of net removal obtained in the newborn, when corrected for perfusable space, are greate $r$ than adult levels and thus cannot be explained by an increas. ing number of lung units with age.

110 INTRAUTERINE CORTICOSTEROID INJECTIONS AND FETAL RAT ORGAN GROWTH AND DEVELOPMENT. Wil R. Blackburn, Gregg Weir, Elizabeth A. Barnes and Elaine T. Dark. University of South Alabama, Division of Growth and Development, Mobile.

Fetal rats injected IP at 17 and 18 days gestation with 1,2, and $4 \mathrm{jg}$ Dexamethasone (Cs) were assessed for organ growth (weight, DNA) at 24-hour intervals until birth. The effect on type II pneumocyte concentration and lung surfactant (SA) were also measured. Saline and noninjected littermates served as controls. Doses of 2 and $4 \mathrm{ug}$ increased lung SA-lipids 24 hours after injection and increased the number of cells recognizable as type II. By birth, these factors returned to normal. At 17 days gestation, $2 \mu \mathrm{g}$ Cs suppressed lung growth 48 hours post injection; by birth this effect disappeared. The $4 \mathrm{\mu g}$ dose decreased adrenal weight throughout the study period. 1 or $2 \mu \mathrm{g} C s$ at 18 days did not influence the weight of the heart, liver, kidney, placenta or adrenal. The $4 \mathrm{\mu g}$ dose decreased adrenal weight through out the study period. Overall fetal mortality was no greater for the IP injection of Cs than for saline. Cs injection led to a greater mortality at 17 (28\%) than at 18 days gestation (14\%). The highest mortality was observed with the $2 \mu \mathrm{g}$ dose $(41 \%)$. These data indicate that small doses of $\mathrm{Cs}$ induce lung maturation but that the ability of the lung to recover from depressed growth is dose dependent. Fetal mortality after intrauterine corticosteroid is related to both dose and fetal age.

\section{URINARY HYDROXYPROLINE/CREATININE RATIO AND TRINATAL GROWTH. Y. Brans, P. Bailey, M. Blake,} I. G. Cassady. Univ. Ala. Med. Sch., Birmingham. and Univ. Cnicago.

Serial 24h urine collections were obtained from 20 premature neonates for determination of protein-bound hydroxyproline and creatinine. Fourteen normally-grown infants (NG) had mean birthweight 1395g (range:980-1880) at mean $29 \mathrm{wk}(25-36)$ gestation; 6 intrauterine growth retarded neonates (IGR) had mean birthweight $1485 \mathrm{~g}(1000-1820)$ at mean $34 \mathrm{wk}(32-36)$ gestation. Hydroxyproline/creatinine ratio- $-\mathrm{H} / \mathrm{C}=$ weight $(\mathrm{kg}) \times \mathrm{H}(\mathrm{mM} / 24 \mathrm{~h}) / \mathrm{C}(\mathrm{mM} / 24 \mathrm{~h})-$ was calculated. No differences in mean $\mathrm{H} / \mathrm{C}$ were evident at any postnatal age between NG and IGR neonates:
Postnatal days: $0-6$
$7-13$
$14-27$

NG:

IGR:

$0.22 \pm 0.065(\mathrm{SE})$

$0.71 \pm 0.130$

$0.81 \pm 0.114$

NG + IGR:

$0.32 \pm 0.193$

$0.71 \pm 0.292$

0.73

$0.71 \pm 0.113 \quad 0.81 \pm 0.029$

For the whole group, mean $H / C$ at $0-6$ postnatal days was significantly lower than at $7-13$ days $(p<0.02)$ and remained stable during the second fortnight. These data suggest that neonatal $\mathrm{H} / \mathrm{C}$ does not reflect adequacy of fetal growth. Since the ratio has been shown to decrease during postnatal malnutrition, these results also imply that malnutrition may not be a satisfactory etiology for fetal growth retardation in the human. 112 THE NATURAL TNCIDENCE AND DISTRIBUTION OF DYING CELIS and Diana Schlesinger, Jefferson Medical College, Stein Research Ctr., Phila., Pa.

During the process of investigating the mechanism of embryonic death following irradiation of the rat zygote on the first day of development, the embryo was studied in the unirradiated and irradiated state. The eosin exclusion test was utilized to determine whether embryonic œlls were alive in embryos that were obtained on the first, second, third and fourth day of gestation. The irradiated zygotes were exposed to 150 or 200 rads and the controls were sham irradiated. Irradiation increased preimplantation and postimplantation death. The number and distribution of dead embryonic cells were detemined in the control and irradiated embryos. To our surprise there was no significant difference between the number of dead cells in the control and irradiated embryos at any stage studied. Even more important was the fact that there was a large number of dead cells in control embryos and that on the fourth day of gestation $43 \%$ of the control and irradiated embryos had one or more dead cells. If cell death is an important contributor to embryonic death following radiation on the first day, it is not manifested in the first four days of gestation. It is even more intriguing to question the significance of cell death in the preimplanted embryo and whether it has any significance in later embryonic development. (Supported by NIH Grant HD 370; NIH Contract 70-2306; AEC Contract 3268).

\section{Cell Turn-over, Whole Lung and Disaturated Lecithin Changes in Oxygen Adapted Rats George Brumley and Betty Tuggle Duke University, Durham, N.C.}

Juvenile male rats were exposed to $85 \%$ oxygen, sacrificed daily after injection with tritiated thymidine $\left({ }^{3} \mathrm{H} \mathrm{TdR}\right)$ and their lungs analyzed for histological changes and DNA incorporation of ${ }^{3} \mathrm{H} T \mathrm{TR}$. Other similarly exposed animal's lungs were analyzed for the quantity of whole lung lecithin (WLL) and disaturated lecithin (DSL) and their respective incorporation of ${ }^{32} \mathrm{P}$ and ${ }^{14} \mathrm{C}$ palmitate. The animals gained poorly or lost weight. Histologically the lungs showed minimal edema with increased alveolar wall thickness and increased numbers of type II alveolar cells. Less than $10 \%$ of the ${ }^{3} \mathrm{H}$ TdR present in the lung homogenate was in DNA. DNA specific activity (SA) increased 7 fold (day $5-6$ ) and subsequently was sustained at 2 fold. WLL concentration fell $50 \%$ (day 2-4) and return ed to baseline by day 10 . WLL-SA fell gradually to $50 \%$ of baseline by day 10 . DSL concentration fel1 $34 \%$ (d2) coincident with a $20 \%$ increase in DSL-SA and a return to baseline concentration by $d 4-6$. We have interpreted these results to indicate that: 1) WLL is more significantly effected by oxygen than DSL, perhaps reflecting the increased susceptibility of the type I alveolar cell to oxygen exposure, 2) oxygen exposure is associated with increased lung cell turn-over as reflected by an increase in DNA-SA and a return to baseline of WLL and DSL, and 3) Iung adaptation is associated with resumption of weight gain and normal appearance of the rats.

14 bilirubin binding Capactity in newBorn infants and THE EFFECT OF PHOTOTHERAPY. William J. Cashore, Edward J. Karotkin and Willian Oh. Brown University Program in Medicine, Women and Infants Hospital of Rhode Island, Department of Perinatal Medicine, Providence, Rhode Island. Serum bilirubin binding capacity (BBC) was estimated by Sephadex $G-25$ gel filtration in 29 infants of 26-42 weeks gestation, to characterize its changes during maturation. The BBC was lowest in 7 infants at $26-29$ weeks gestation $(13.7+2.1 \mathrm{mg}$ $\%$ ) and rose almost twofold to $23.0 \pm 3.6 \mathrm{mg} \%$ in 7 term infants. A direct correlation was observed bétween $\mathrm{BBC}$ and gestational age $(\mathrm{r}=0.675, \mathrm{p}<0.001)$. Previous in vitro study has shown that $B B C$ decreases significantly during phototherapy (Birth Defects, $6: 31,1970$ ). In the current series, $B B C$ was measured in 16 infants before and 24 hours after the initiation of phototherapy. During phototherapy, BBC remained within $10 \%$ of the pre-phototherapy values in 13 of the 16 infants. The net change in $\mathrm{BBC}$ due to phototherapy was less than $1.0 \mathrm{mg} \%$ and was not statistically significant $(p>0.05)$. Thus, bilirubin binding capacity increases with advancing maturity; and in vivo, $B B C$ is unaffected by phototherapy. 
115 CHANGES IN HEMOGLOBIN OXYGEN AFFINITY IN RELATION TO GESTATIONNAL AGE (GA)。A。Cornet and $\mathrm{H}_{\circ}$ Bard, Perinatal Service, Hốpital Ste-Justine, Dept. of Pediatrics, Univ. of Montreal, Montreal, Que., Canada.

In order to evaluate oxygen hemoglobin equilibrium curves in relation to GA, 20 samples of fresh cord or newborn blood were studied from newborns varying from 20 to 40 wks of GA with no evidence of perinatal distress. GA correlated with clinical evaluation. The following analyses were done: 2-3 diphosphoglycerate (DPG) levels (method of Keitt), $p_{50}$ (tonometry, using IL 217) and $\mathrm{HbA} \& \mathrm{HbF}$ concentrations (DEAE Sephadex chromatography). The P50 increased from 14.5 to 25.1 $\mathrm{mm} \mathrm{Hg}$ and correlated with GA $(2 \mathrm{p}<0.025)$. DPG varied from 0.6034 to $1.2880 \mathrm{~m} / \mathrm{m}$ Ho but there was no correlation with GA. There was a significant correlation between $\mathrm{P}_{50}$ and $\mathrm{HbA}$ concentration $(2 \mathrm{p}<0.025)$ but there was no correlation between P50 and DPG levels, nor between DPG and HbA. These results suggest that P50 increases inutero with GA and is mainly due to an increase in HbA. This is unlike what has been reported to occur postnatally where the increase in $P_{50}$ is due mainly to a rapid increase in concentration of red cell DPG。

MRENTAL AND POSTNATAL DEVELOPMENT OF BRAIN AND HEART 116 MITOCHONDRIA AND THEIR RESPONSE TO INCREASED OXYGEN HEMOGLOBIN AFFINITY. Maria Delivoria-Papadopoulos. Leena Mela, Cleon W. Goodwin, and Pedro D. Paez. University of Pennsylvania, School of Medicine, Philadelphia, Pennsylvania. Sequentiai measurements of brain and heart mitochondrial state 3 respiratory activity were made in 16 fetal lambs 90-145 days of gestation and 20 lambs and 30 puppies during the first 2 weeks postnatally. By the last week of gestation, State 3 respiratory capacity of lamb heart mitochondria was 80 , increasing to 140 nmoles $0_{2}$ utilized/min/mg protein at term. Postnatally two changes occurred: 1) an increase of respiratory enzymes, and 2) a decrease in mitochondrial respiratory capacity. Puppy heart cytochrome $c$ concentration increased from a value at term of 0.25 to 0.65 nmoles/mg protein and cytochrome ataz concentration from 0.14 to 0.21 nmoies/mg protein. State 3 respiratory capacity decreased from 1100 at birth to $200 \mathrm{moles} 0_{2} / \mathrm{min} / \mathrm{mole}$ of cytochrome a+a 3 at 4-7 days after birth. In the lamb heart, State 3 respiratory activity fell from 140 to 60 nmoles $0_{2} / \mathrm{min} / \mathrm{mg}$ protein during the same period. Similar changes occurred in brain mitochondrial respiratory capacity. An acute increase of oxyhemoglobin affinity for 1 hour was effected by administering fetal blood equilibrated with carbon monoxide ( $\mathrm{CO}$ ) through chronically implanted catheters to the fetal lamb in utero. The respiratory capacity of fetal brain and heart mi tochondria did not change, in contrast to our previous studies in newborn animals whose oxyhemoglobin curves were also shifted to the left using $\mathrm{CO}$. These data suggest that the fetus in utero does not have the same response to tissue hypoxia as the newborn animal.

117 CORRELATION OF FETAL LECITHIN SYNTHESIS AND THE NIH, Bethesda, Md.

AMNIOTIC FLUID (AF) L/S RATIO IN PRIMATES. Michael F

Rhesus monkey gestations were utilized to examine the relationship between the AF L/S ratio and fetal lung lecithin synthesis. In 24 fetuses (114-161 days, term 164) the rate of lung lecithin synthesis in vitro via choline incorporation (1) and phosphatidyl ethanolamine (PE) methylation (II) was determined by incubating lung slices with $2 \mu \mathrm{Ci}$ of $9{ }^{4} \mathrm{C}$-choline or ${ }^{4} \mathrm{C}$-methionine. Choline incorporation accounted for $90 \%$ of lung lecithin synthesis in all fetuses. Pathway I activity was stable from 114-148 days and then abruptly increased with peak activity ( $3 \times$ baseline, $p<.001$ ) at day 150 ( $90 \%$ term). The AF L/S ratio, determined in these and 60 additional gestations (70-161 days) showed a gestational pattern similar to that in humans with an abrupt increase at $90 \%$ of term. The correlation between lung choline incorporation and AF L/S ratio in the last $30 \%$ of gestation was significant $(r=.77, p<.001)$; lung $P E$ methylation showed no correlation with the L/S ratio. To confirm that lung choline incorporation into lecithin determines the AF $L / S$ ratio, $1 \mathrm{mC} i$ of $14 \mathrm{C}$-choline was injected into the maternal aorta (2 monkeys, 142 days gestation) to proyide labelled precursor for fetal lung $14 \mathrm{C}-1$ ecithin synthesis. Af $14^{4} \mathrm{C}-$ lecithin specific activity was determined every 48 hours over 2 weeks; it increased gradually until day 150 when an abrupt 2.5 fold increase $(5.6+12$ \& $2.8 \rightarrow 7.4 \mathrm{cpm} / \mathrm{hg}$ lecithin) occurred. This was coincident with a $30 \%$ and $120 \%$ increase in the $L / S$ ratio $(3.4 \rightarrow 4.4 \& 2.3 \rightarrow 5.1)$. It is concluded that the $A F$ L $/ S$ ratio accurately reflects developmental 118 ENZYMATIC SYNTHESIS OF PHOSPHATIDYLCHOLINE (PC) IN

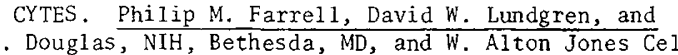
William H.J. Douglas, NIH, Bethes

Accelerated production of PC-rich pulmonary surfactant occurs following treatment with various hormones. To delineate pathways and regulation of $\mathrm{PC}$ biosynthesis, investigations are being conducted with diploid epithelial cells isolated from rat lung (clone L-2) which have been shown to contain osmiophilic lame11ar bodies (In Vitro 10, 1975). Studies to date have employed over 50 cultures of $\mathrm{L}-2$ cells grown in either a basal medium (control) or in the presence of hormones such as thyroxin and cortisol. The three catalysts of the principal de novo route for PC synthesis - the choline pathway - were readily detected in control cells and compared in terms of specific enzymatic activities with rat lung homogenates and fibroblasts from lung and skin. Results indicate that L-2 cells are enriched to as high as tenfold in choline kinase (CK), cholinephosphate cytidyl transferase, and cholinephosphotransferase (CPT). Cells continuously cultured in the presence of thyroxin showed markedly increased $\mathrm{CK}$ (mean $\pm \mathrm{SE}=$ $4815 \pm 353 \mathrm{cpm}$ product $/ \mathrm{min} / \mathrm{mg}$ protein; control $=2858 \pm 232$ ) but reduced CPT (both $\mathrm{p}<.001$ ). Although cortisol alone produced only slight enzyme elevations, its addition to thyroxin-supplemented cells led to a seven-fold increase in CPT activity (p<.001).

It may be concluded that L-2 cells in tissue culture are 1) capable of active PC production, and 2) respond to hormones in a manner consistent with observations on lungs of treated animals.

119 POSSIBLE ROLE OF SYMPATHETIC INNERVATION ON CONTROL OF CARDIAC MUSCLE THYMIDINE KINASE P.C. Gillette and W.C. Claycomb. Dept. of Cell Biophysics and Dept. of Pediatrics, Section of Cardiology, Baylor College of Medicine, Houston, Texas

Thymidine kinase (TK) is a key enzyme in DNA replication. TK activity declines progressively in rat heart during development reaching adult levels of almost zero by about the 17 th day of postnatal growth. Rat heart reaches adult values for contractility, sympathetic innervation, norephinephrine and cyclic AMP (cAMP) levels at about the same time. The present experiments were carried out to determine if CAMP might control TK activity in the developing heart. Isoproterenol (ISO) injected into neonatal rats was found to inhibit TK activity in a dosedependent manner (dose range: 5 to $75 \mathrm{mg} / \mathrm{kg}$ ). Theophylline potentiated the effect of small doses of ISO. Dibutyryl CAMP also depressed TK activity. We postulate that the sympathetic innervation of the heart may increase cAMP levels in the myocardial cell thereby depressing TK activity. This may limit avilability of deoxythymidine triphosphate needed for DNA synthesis in the developing heart.

Supported by USPHS HL 5925, 7906, 5435 and the American Heart Association 74-902.

120 THE STUDY OF ENDOCRINE CELLS OF THE HUMAN PANCREN MAINTAINED IN LONG TERM CULTURE. Hy Goldman and
Eleanor Colle. MCGill University - Montreal

Children's Hospital Research Institute, Montreal, Canada.

A cell culture system has been developed utilizing postmortem tissue for the long term maintenance of endocrine cells of the human fetal (11-18 wks) pancreas. These cells persist as monolayer and intact islets and maintain the morphologic characteristics of epithelial endocrine cells. Electron microscopy identified alpha, beta, delta and ductal epithelial cells. The substitution of pooled human cord ser (10\%) for fetal calf sera (10\%) in the culture media favors the preservation of the intact islets and results in prolonged secretion of immunoreactive insul in (IRI) immunoreactive glucagon (IRG) and immunoreactive gastrin (IRg) for periods of 1 month in monolayer cultures and over 2 months in intact islet cultures. IRG release continued after IRI release could no longer be detected. When replicate intact islet cultures were maintained in synthetic media containing high $(11.2 \mathrm{mM})$ and normal $(5.5 \mathrm{mM})$ glucose for 1 month, the former failed to release more IRI. The release of IRG was not suppressed in the high glucose cultures. Cells cultured in media containing $5 \mathrm{mM}$ glucose also failed to increase their rate of IRI or IRG release when glucose concentration in the media was increased acutely $(5.5 \mathrm{mM}$ to $16.5 \mathrm{mM})$ or when arginine $(5 \mathrm{mM})$ or leucine $(5 \mathrm{mM})$ was added to the media. 

121 LIPID SYNTHESIS BY DIETARY DEPRIVATION IN RAT LUNG Ian Gross, Seamus A. Rooney and Joseph B. Warshaw, Yale University School of Medicine, New Haven, Connecticut
Dietary deprivation in rats has been shown previously to result in elevated surface tension in lung extracts, lowered lung lecithin content and decreased lipid synthesis from glucose. We have investigated the effect of food deprivation on enzymes of lipid synthesis in rat lung. 21 day old rats were subjected to total food deprivation for 4 days but allowed water ad lib. Littermates were fed standard rat chow and served as controls. Enzyme activities were assayed in lung homogenates. Activities of acetyl-CoA carboxylase, fatty acid (FA) synthetase and microsomal FA elongation were measured to determine the effects on FA synthesis and choline phosphotransferase and glycerol phosphate phosphatidy 1 transferase (GPPT) to determine the effects on synthesis of the surface active phospholipids, lecithin and phosphatidy 1 glycerol respectively. Enzyme System Acety1-CoA carboxylase FA Synthetase

$\begin{array}{lcccc}\text { Control } & \text { Starved } & \frac{\text { Starved:Control }}{426} & \frac{p}{.50} & < \\ 750 & 450 & .00 & <.05 \\ 45.6 & 22.1 & .48 & <.001 \\ 82.3 & 45.9 & .56 & <.001 \\ 12.8 & 13.0 & 1.02 & \text { N.S. }\end{array}$
Choline Phosphotransferase GPPT

pmoles/mg protein/min. These findings support previous observations that surfactant production is inhibited by food deprivation and suggest a possible role for aggressive nutritional therapy in infants with respiratory distress syndrome.

122 CELLULAR CONTENT OF PLACENTAS OF METHADONEMAINTAINED ADDICTS. Anantham Harindranath, Ellen Feingold, Myron Sokal, Rita G. Harper \& George Solish, Depts.of Ped.\& Ob.-Gyn., North Shore Univ.Hosp. Manhasset, N.Y. \& Depts. of Ped.\& Ob.-Gyn., Cornell Univ.Med.Col.,N.Y.,N.Y., \& Depts.of Ped.\& Ob.Gyn., State Univ. of N.Y., Downstate Med.Ctr.,BkIyn,N.Y. (Intr. by Fima Lifshitz).

Babies born to narcotic-addicted mothers are often growth retarded in utero. Placentas from 12 methadonemaintained pregnant narcotic addicts and 7 non-addicted pregnant patients were analyzed for weight and RNA and DNA content to determine the effect of methadone on the cellular growth of the placenta. The patients and controls were healthy and comparable in age, race, parity and length of gestation. Frequent urines for drug-screening were obtained on all participants.

Mean birth weight of infants born to methadonemaintained mothers was similar to that of control patients $(3079 \mathrm{gm} \pm 448 \mathrm{gm}$ vs $3206 \mathrm{gm} \pm 695 \mathrm{gm}$ P> 0.05), as was mean placental weight (536 $130 \mathrm{gm}$ vs $532 \pm 104 \mathrm{gm})$. There was no significant difference in either RNA or DNA contents of the placentas of methadone patients and control patients (RNA $0.82 \pm$ $0.29 \mathrm{gm}$ vs $0.77 \mathrm{gm} \pm 0.29 \mathrm{gm} ;$ DNA $1.72 \pm 0.44 \mathrm{gm}$ vs $1.47 \pm 0.30 \mathrm{gm}$ ).

In our study methadone did not retard the intrauterine growth of the fetus or the cellular growth of the human placenta.

123

INSTANTANEOUS CHANGES IN UMBILICAL BLOOD FLOW IN FETAL LAMBS IN UTERO. Michael A. Heymann, William Berman Jr., Robert C.Goodlin and Abraham M.Rudolph. Univ of Calif.San Francisco,Dept. of Ped and Cardiovas.Res.Inst.

We developed a new technique for continuously measuring umbilical blood flow(Qua)in. fetal lambs in utero; this avoids major hemodynamic disruptions of methods reported previously. The umbilical arteries originate from a short segment as the terminal branches of the descending aorta. This common segment was encircled by a specially constructed electromagnetic flow transducer and flow measured on a Statham SP2202 meter. Fetal catheters were also placed, and the animal allowed to recover. Flow and pressures were continuously recorded $2-7$ days postoperatively in 12 animals (103-130 days gestation); fetal arterial blood gases were normal. Average Qua was $199 \mathrm{ml} / \mathrm{kg}$ fetal weight $/ \mathrm{min}(\mathrm{s.e.m} \mathrm{9)}$ and was stable over several days. Spontaneous variations in fetal heart rate and arterial blood pressure were associated with changes in Qua. An increase in heart rate of 1 beat $/ \mathrm{min}$ was associated with an average increase in Qua of $1 \mathrm{~m} 1 / \mathrm{kg} / \mathrm{min}$ at all heart rates. An increase in mean arterial blood pressure of $1 \mathrm{mmHg}$ was associated with an average increase in Qua of $6 \mathrm{ml} / \mathrm{kg} / \mathrm{min}$. These changes were similar at all gestations studied. Fetal grunting, associated with hypertension and bradycardia,was always associated with a fall in Oua. Beat to beat changes of arterial pressure related to fetal respiratory movement were associated with corresponding changes in phasic Qua. The importance of continuous measurement of Qua in studying physiologic and pharmacologic responses of the umbilical-placental circulation and placental transfer is stressed. USPHS Grant HL06285.
124

ENLARGEMENT OF THE HEART INDUCED BY GLUCOCORTICOIDS DURING EMBRYOGENESIS. D.C. Hicks, J.A. Lee, R. Clarke, E.R. Hughes, M.J. Elders. Ped. Dept., UaMC Little Rock, Ark., \& Univ. So. Ala., Mobile, Alabama.

Glucocorticoids are known to be essential for optimal cardiac function and mechanical efficiency of the heart. A direct effect of these hormones on the heart has not been demonstrated. The present investigation was undertaken to study the biochemical and morphological changes which may be induced by these hormones and to try and correlate these data with the appearance of stereospecific glucocorticoid receptors in the developing chick heart.

Stereospecific glucocorticoid receptors were demonstrated in the cytosol and nuclear fractions by 9 days of embryonic development and increased through 17 days of embryonic development. Wet and lipid-free dry heart weights were increased $16-54 \%$ in all aged embryos from day 9 to 19, following injection of hydrocortisone phosphate 48 hours prior to sacrifice. The body and other organ weights decreased 22-64\%. Analysis of the hearts for glycogen, lipids, proteins, uronic acids, DNA, and calcium as well as measuring the incorporation of radioactive precursors into specific products were all increased 18-64\%, suggesting increased biosynthetic activity. Assay of specific gluconeogenic and glycogen synthesizing enzynes were increased $33 \%$ to $48 \%$.

Demonstration of specific glucocorticoid receptors in the fetal heart and the association of hormone responsiveness as evidenced by specific biochemical changes suggest glucocorticoids may play a major role in the maturation and development of the embryonic heart, if the increased cardiac size and enzyme activity correlates with functional cardiac effectiveness.
125

THE INFLUENCE OF SEASON AND OTHER FACTORS ON SERUM 25-HYDROXY VITAMIN D (25-OHD) IN BLACK AND WHITE WOMEN DURING PREGNANCY, Laura S. Hillman and John G. Haddad (Intr. by P.R. Dodge) Washington Univ. Sch. Med., St. Louis Children's Hospital, Dept. of Ped. and Jewish Hospital of St. Louis, Dept. of Med., St. Louis, Missour1 63110.

Infant cord blood 25-OHD levels are directly correlated with maternal serum 25-OHD levels. The incidence of neonatal tetany is highest in late winter and non-caucasian groups. Therefore, random sera were obtained from last trimester pregnant women, black - $\frac{1}{2}$ white, under prenatal care during late Feb. $(n=56)$ and late Aug. ( $n=61)$ for determination of $\mathrm{CaH}, \mathrm{MgH}$, and 25-0HD.Ca1culated vit. D intakes were similar in Feb. and Aug. and for black and white women. The mean 25 -OHD level was $15.4+5.9 \mathrm{ng} / \mathrm{ml}$ (S.D.) $(6.5-33.6)$ in Feb. and $42.1+13.9 \mathrm{ng} / \mathrm{ml}(18-74)$ in Aug. No seasonal difference in Catt was noted (Feb. $9.40+.59 \mathrm{mg} / \mathrm{dl}$, Aug. $9.43+.64$ ) but $\mathrm{MgH}$ levels were lower in Aug. (Feb.1.50+.13meq/L, Aug. $1.43+.13 p<.005)$. No correlation existed between $25-0 H D$ levels and Catt or $\mathrm{Mgt+}$. There was no racial difference in 25OHD levels in Feb. (black, $14.5+6.5 \mathrm{ng} / \mathrm{ml}$; white $16.2+5.6$ ) or Aug. (black $39.7+14.8 \mathrm{ng} / \mathrm{ml}$; white $43.5 \pm 14.7$ ). The lower Feb. values showed only very weak correlations with vit. $D$ intake $(R=.32)$ and gestation $(R=.26)$. No correlation existed with parity, time since last pregnancy, maternal age, or social class. The higher Aug. values did not correlate with vit. D intake or other variables. It would appear from these data that ultraviolet exposure is the major determinant of maternal serum 25-OHD levels, in St. Louis, but that dietary intake and other factors may be important during periods of decreased ultraviolet exposure.

\section{COMPENSATORY HYPERPLASTIC GROWTH FOLLOWING LUNG COLLAPSE IN IMMATURE RATS. Laura S. Inselman,} Robert B. Mellins and Jo Anne Brasel. Cotumbia University, Co!lege of Physicians and Surgeons, Dept. of Pediatrics, New York, N.Y. To gain insight into the growth potential of the remaining lung tissue in young children with severe pulmonary disease, the effect of unilateral lung collapse on contralatera! compensatory lung growth was studied in developing rats. The left lungs of anesthetized three week old rats were either partially or totally collapsed by injection of dental plastic through a tracheostomy tube. The rats were killed four weeks later, 18 hours after injection of tritiated thymidine. Analysis of the right lung revealed increases over that of sham operated controls of DNA $(54 \%)$, RNA $(33 \%)$, protein content $(23 \%)$, and dry weight $(37 \%)$ ( $\mathrm{p}$ 0.05). There was no change in uptake of tritiated thymidine and no histologic evidence of inflammation. RNA/DNA and protein/DNA ratios were reduced by $13 \%$ and $20 \%$,respectively, of sham controls ( $p$ 0.05)。 Thus partial or total lung collapse in the immature rat stimulates growth of contralateral lung by cellular hyperp! asia, not hypertrophy, and this occurs prior to 4 weeks post-collapse. Further sudies will be necessary to delineate cell types involved in this growth response. The greater rise in DNA in these experiments as compared with similar studies following left pneumonectomy (Buhain and Brody, J.Appl. Physio!. $35: 898,1973$ ), may be attributed to differences in the magnitude of right to left shunting or of inflation or perfusion of the remaining lung. We conclude that the presence of a collapsed lung in the developing animal may be a more potent stimulus for lung growth than removal of the lung itself. 
127 ADIPOSE CELL DEVELOPNENT AS AN INDICATOR OF OBESITY. Jerome L. Knfttle, Fredda Ginsberg-Fellner and Roy E. Brown, Mt. Sinai School of Medicine, Dept. of Pediatrics, N.Y.C.

Previous studies in our laboratory have indicated that extreme childhood obesity is accompanied by increases in adipose cell number and size as early as age 2. However, the identification of the "obesity prone" child prior to the development of clinical obesity and apparent permanent increases in adipocyte number remains a problem. In the present report, we have prospectively examined adipose tissue cellularity in 77 children under 2 years of age for a perlod of 2 to 3 years. Since overt obesity is rarely encountered prior to age 2 , we postulated that variations in cellular development might be observed in those subjects who became obese. To date 8 subjects have become obviously obese and thelr cellular development was compared to a control group matched for age and sex. Increases in cell size were demonstrated in both groups during the first 12 months of life and no significant differences were found. From the 12 th to 24 th month nonobese subfects displayed a decrease in cell size while obese subjects continued to show an increase. Indeed by 24 months significant differences in cell size were noted. The data suggest that at 12 months of age alterations in metabolic function of the fat cell and/or hormonal control of fat cell metabolism occur in nonobese subjects which are not found in the obese child. Thus longitudinal studies of fat cell size and metabolic function during this time interval could provide a means for the prediction and prevention of obesity.

128 HISTIDINE DEGARBOXYLASE IN FETAL INTRAUTERTNE GROWTH-

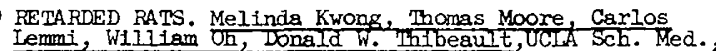

Harbor Gen. Hosp., Torrance, Calif.

Kahlson described an increase in excretion of histamine (from the fetuses) in the urine of pregnant rats beginning on day 15 with a sharp fall the day before term. The elevation of histamine (III) excretion coincides with rapid growth of the fetuses. Histidine decarboxylase (HDC) converts histidine to HI. It is anticipated that BDC would be increased during rapld growth of the fetuses and decreased in growth-retarded fetuses (IUGR). Experimental IUGR was induced by the procedure of Wigglesworth. On day 17 of gestation, the uterus was exposed and the uterine artery supplying one uterine horn was ligated. Degrees of IUGR of the fetuses on the Ilgated side resulted. Fetuses on the non-ligated side were the controls. On day 21 a cesarean section was performed, and the fetuses were weighed and anslyzed for total body HDC using Schayer's method. The arerage wt. of the fetuses on the ifgated side $(3.4+0.3 \mathrm{gr}$ ) was significantly smaller $(\mathrm{p}<.005)$ than those of the control side $(4.0+0.3 \mathrm{gr}$.$) . In contrast, the$

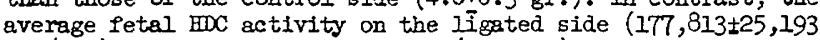
cpm/gr.) was signtficantly larger $(\mathrm{p}<.002)$ than the Floc activity of the controls $(72,727 \pm 13,965 \mathrm{cpm} / \mathrm{gr}$.). The HDC/gr. body wt. of all fetuses was related to body wt. ( $r=-0.48, p<.005)$ the HDC/ gram increasing as body wt. decreased. Why HDC increased with decreasing body growth is not known. With substrate deprivation, HDC activity may be increased to increase BII formation (which is a local vasodilator) and thus increase mutrient to the tissues.

12 DIFFERENTIAL BINDING OF EPIDERMAL GROWTH FACTOR TO NORMAL AND VIRALLY-TRANSFORMED HUMAN AND MOUSE FIBROBLASTS IN CULTURE. Roger Ladda and Robert Hoffman (Intr. by Nicholas Nelson). The Pennsylvania State Univ. Col. of Med., The Milton S. Hershey Med. Ctr., Hershey, PA 17033.

Cells in culture require certain macromolecular components of serum to sustain proliferation. These growth factors, of ten characterized by "insulin-like activity", bind to specific surface membrane receptors and presumably thereby initiate the chain of events leading to cell division. We have studied the binding of purified iodinated mouse epidermal growth factor (EGF) to normal and transformed fibroblasts and found that several transformed lines with infinite growth potential have 2 to 3 times the numbers of EGF receptors per $\mu^{2}$ surface area than do normal strains. Fibroblasts in very early passages derived from amniotic fluid bind EGF comparable to some transformed lines, but, in contrast, show marked reduction in subsequent passages. Normal diploid human strains grown from skin explants initially exhibit $50-75 \%$ of the EGF affinity of transformed derivatives. As these cells approach senescence less than $10 \%$ of the EGF receptors are detected. In a mouse $3 \mathrm{~T} 3$ and $\mathrm{SV} 403 \mathrm{~T} 3$ system EGF receptors are similar on a per cell basis, but $\mathrm{SV}_{40} 3 \mathrm{~T} 3$ cells have about $25 \%$ more receptors as determined by surface area measurement. EGF has no apparent affinity for exychrocytes, lymphocytes or lymphoblasts and normal and transformed chick fibroblasts. EGF binding appears to be related to the proliferative activity of cells with EGF specificity. Reduced serum requirements of transformed cells may be partly explained by increased numbers of serum growth factor receptors and more efficient utilization of these factors.
130 SODIUM RECOVERY AND EXTRAVASCULAR WATER IN THE LUNGS OF INTACT PIGLETS. O. Robert Levine and Erlinda Newark, N.J. Sunico. New Jersey Medical School, Dept. of Ped.,

It has been suggested that capillary permeability is greater in the immature than in the mature lung. In the present study sodium recovery and extravascular water volume ( $\left.V_{E L W}\right)$ were determined in the lungs of intact piglets using multiple singlecirculation indicator-dilution curves. The indicator bolus was delivered into the right atrium and consisted of T-1824, $10 \mathrm{mg}$, $22 \mathrm{NaCl} 10 \mathrm{uc}$, and THO $20 \mathrm{uc}$. Blood samples were collected at half-second intervals from an aortic catheter into test tubes mounted on a rotating turntable. Fifteen curves were obtained in 8 intact spontaneously breathing piglets 4 to 8 weeks old and anesthetized with pentobarbital. Using the samples before recirculation, ${ }^{22} \mathrm{Na}$ recovery expressed as a fraction of T-1824 recovery averaged $0.954 \pm 0.037$. This value is identical to the previously reported value in adult dogs and differs significantly from unity $(P<0.01)$. V VEW was $4.3 \pm 1.7 \mathrm{ml}$ per $\mathrm{kg}$ body weight as compared to $3.5 \pm 0.9 \mathrm{ml}$ per $\mathrm{kg}$ previously reported in adult dogs. Total lung water (TLW) determined in 16 piglets by drying the lungs and correcting for retained blood was $8.4 \pm$ $1.5 \mathrm{mI}$ per $\mathrm{kg} \mathrm{BW}$, or $6.2 \pm 0.3 \mathrm{~g}$ pex $\mathrm{g}$ dry lung as compared to $3.6 \pm 0.2 \mathrm{~g}$ per $\mathrm{g}$ dry lung previously reported in adult dogs. VELW averaged $51 \%$ of TLW in the piglets, as compared to $74 \%$ previously reported in adult dogs.

Piglet and mature dog lungs are equally impermeable to $22 \mathrm{Na}$, while lung tissue has an appreciably higher water content in the piglet than in the mature dog.

131 INTRAPARTUM FHR PATTERNS IN "SIDS" INFANTS. Chester B. Martin, Jr. Esther Doremus, Bruce B. Dept. of Obstetrics, Gynecology and Pediatrics, Univ. of So. Calif. Sch. of Med. and LAC-USC Medical Center.

Eleven SIDS victims have been identified who had electronic FHR monitoring intrapartum as part of maternal clinical care. The indications for fetal monitoring varied according to the obstetrical service. The most frequent abnormal feature in the records was a decrease in the amplitude of the 3-6 cycle/min. fluctuations in the baseline FHR, which was present in portions of 9 tracings. These fluctuations were quite regular (sinusoidal) in portions of 5 records. Episodes of increased beatto-beat FHR arrhythmia were present in 7 records. Variable decelerations occurred in 8 tracings, but the FHR returned promptly to the previous baseline in all but 2 of them. Nine fetuses exhibited accelerations with movements or uterine contractions. The magnitude of the accelerations was not unusual and the FHR returned to baseline promptly after the contractions. Typical early decelerations were present in 4 records, and late decelerations occurred in only one case.

A) though these SIDS infants exhibited reactive FHR patterns intrapartum, there were no clear indications of instability or impaired autonomic control of heart rate, and no characteristic features which would permit prior identification of these individuals.

132 Cellular Respiration in Abnormally Developing Embryos. M.L. Netzloff, M.D., O.M. Rennert, M.D., Dept. Ped. Univ, Fla. Gainesville, Fla.

Long-Evans rats were subjected to the teratogenic regimen of folic acid restriction and antagonism using the analogue 9-methyl pteroylglutamic acid on days 10, 11 , and 12 of pregnancy. This treatment has been shown to produce: 1) a $95 \%$ incidence of congenital thalformations in offspring at term and 2) elevated oxygen consumption by intact embryos on days 11,12 and 13 of gestation. Cells were dispersed on day 13 by passing the embryo twice through a $5 \mathrm{ml}$ Mohr pipette. Room air was used with the day 13 dispersed and day 11 intact, and 100\% oxygen with day 12 intact embryos. Oxygen consumptions were measured using the direct Warburg and oxygen electrode techniques.

Use of larger intact embryos in room air, and on day 13 even in $100 \%$ oxygen, is limited by restriction to oxygen diffusion imposed by thick layers of tissue. The use of dispersed embryonic cells or $100 \%$ oxygen obviates this difficulty. The means + standard errors for day 13 experimental and control respiratory rates are $12.3+0.7$ and $17.9+0.6 \mu \mathrm{l} / \mathrm{hr} / \mathrm{mg}$ protein, respectively, and differ at a $99 \%$ level of signtficance. Similar day 12 experimental and control data are $18.5+1.0$ and $18.8+1.0$, respectively. Preliminary day 11 data also suggest no difference between mean experimental and control uptakes. Because a day 13 embryo is dis tinct both developmentally and in duration of treatment from embryos earlier in gestation, the experimental and control oxygen uptakes may actually differ on this day, but not on days 11 and 12. However, the data also suggest the process by which the embryos are dispersed may itself introduce an antifact. 
133 IN VITRO CFIL GROWTH RATES IN PRIMARY CELLULAR GROWTH DEFICIENCY SYNDROMES. Donald Pious, Albert J.T. Millis and Kathleen Sabo, Univo of Washington Sch. of Med., Dept. of Pedo, Seattle

It has been suggested that genetically determined growth deficiency syndromes with onset in early fetal life may represent growth defects intrinsic to all cells of affected individuals ("primary cellular growth deficiency"). A consequence of this hypothesis is that cell cultures from affected individuals should manifest the growth defect. We therefore measured population doubling times (PDT) of cultured fibroblasts from donors with suspected primary cellular growth deficiency syndromes. We assayed the increase in cell number with time in replicate cultures initiated with a standard number of cells. The slope of the growth curve was determined by regression analysis, and, from it, PDT. PDT for 12 normal donors was $26.92 \pm .51$ hrs. (mean \pm SE). PDT for three Trisomy 18 donors was $39.50 \pm 1.13 \mathrm{hrs}$; the difference was highly significant $(p \times .001)$. Growth was also slow in cultures from one or two donors each with Trisomy 13, Roberts, Secke1 and Rothmund-Thomson syndromes. Growth was normal in cultures from the B4p- chromosomal deletion, Cockayne and RussellSilver syndromes. Cultures from 8 Down's syndrome donors grew normally (PDT $=26.28 \pm 1.1 \mathrm{hrs}$ ) ) under standard assay conditions, but reduction of the cell inoculum by 15 -fold resulted in significant growth retardation of Down's cultures compared to similarly treated controls. These findings indicate that there are intrinsic cellular growth abnormalities in some growth retardation syndromes of prenatal onset, and that cell culture will be useful in definition and in elucidation of basic mechanisms.

134 FETAL GROWTH RESTRICTION FOLLOWING MATERNAL NARCOTIC ADMINISTRATION: NUTRITIONAL OR DRUG EFFECT? John R. Raye, Joseph W. Dubin and Jack N. Blechner (Intr. by Martha L. Lepow) UConn Hea1th Ctr., Dept. of Ped and Dept. of ob/Gyn, Farmington

Intrauterine growth retardation has been observed in $25-45 \%$ of infants born to narcotic addicted mothers. The relative roles of maternal nutrition and narcotic drug effect are unclear. Rabbits treated with morphine (50 to $100 \mathrm{mg} / \mathrm{kg} / \mathrm{d} \mathrm{s.c.}$ ) 1 week prior to conception and throughout gestation were pair fed with weight matched controls. At $29 \mathrm{~d}$ litters were delivered surgically and measurements made of fetal weight, length, organ weight, protein and DNA. A 2 way analysis of variance was performed.

Significant reductions in mean fetal growth were seen in both the $50 \mathrm{mg} / \mathrm{kg}(\mathrm{p}<.01)$ and the $100 \mathrm{mg} / \mathrm{kg}(\mathrm{p}<.001)$ groups when compared to their pair fed controls. Decreases in forebrain (FB) weight were particularly striking. When compared to the $50 \mathrm{mg} / \mathrm{kg}$ group, the $100 \mathrm{mg} / \mathrm{kg}$ group showed significant additional growth restriction $(p<.001)$. Ad lib fed control offspring were not significantly larger than food restricted control of fspring.

(Mean $\pm 1 \mathrm{SD}) \quad 50 \mathrm{mg} / \mathrm{kg}$ Control $100 \mathrm{mg} / \mathrm{kg}$ Control Ad lib

$\begin{array}{llllll}\text { Weight }(\mathrm{gm}) & 30.3 \pm 8.2 & 35.6 \pm 7.4 & 26.9 \pm 7.2 & 40 \pm 9.3 & 35.4 \pm 6.5\end{array}$

$\begin{array}{llllll}\text { Length }(\mathrm{cm}) & 10.5 \pm .9 & 10.9 \pm .9 & 10.2 \pm .9 & 11.0 \pm .9 & 11.0 \pm .8\end{array}$

$\begin{array}{llllll}\text { FB Wt. (gm) } & .56 \pm .04 & .60 \pm .06 & .53 \pm .09 & .64 \pm .06 & .61 \pm .05\end{array}$

These data show that chronic maternal morphine administration in the rabbit leads to significant reductions in fetal growth that are not secondary to decreased maternal food intake. This effect appears to be dose dependent. (Supported in part by funds from SAPDAP, NIMH "DA00633-01)

135 DEVELOPMENTAL CHANGES IN SERUM AND KIDNEY POTASSIUM $(\mathrm{K}+)$ IN THE FETAL MOUSE. Jack S. Resnick, Robert L. Vernier, and David M. Brown, Dept. of Ped., Univ. of Minnesota, Minneapol is, Minn.

Previous studies of serum $\mathrm{K}+$ levels in developing mamalian fetuses have demonstrated marked elevations compared to maternal $\mathrm{K}+$ levels, even in the face of maternal $\mathrm{K}+$ depletion. This has led to the hypothesis that relative fetal hyperkalemia is essential for normal fetal development. Sequential studies of either serum or tissue $\mathrm{K}+$ levels from midgestation to term have not been reported. We have studied both serum and kidney $\mathrm{K}+$ levels in 32 day fetal mice through the newborn period. Serum K+ was increased in the newborn mean $(6.2 \mathrm{mEq} / \mathrm{L})$ over the maternal mean $(5.0$ $\mathrm{mEq} / \mathrm{L}$ ) and increased further with decreasing age until leveling at 14-1.6 days $(8.6-9.6 \mathrm{mEq} / \mathrm{L})$. A significant decrease was noted in both maternal and fetal values when ether anesthesia (above values) was used rather than cervical dislocation, probably due to hypoxia. Nat levels were similar in maternal and fetal serum (148-150 $\mathrm{mEq} / \mathrm{L}$ ). Anniotic fluid of 12-14 day fetuses had Nat levels similar to serum and $\mathrm{K}+$ levels slightly below maternal serum $(4.5 \mathrm{mEq} / \mathrm{L})$. Maternal kidney $\mathrm{K}+$ was $325 \mathrm{mEq} / \mathrm{kg}$ dry wt, newborn was $730 \mathrm{mEg} / \mathrm{kg}$ and fetal increased to a level of $950 \mathrm{mEg} / \mathrm{kg}$ at 12-14 days gestation. While both serum and renal $\mathrm{K}+$ levels are higher in the fetus and are highest at the youngest gestational age, the ratio of serum/kidney Kt remains stable. Maternal anesthesia has a significant effect on these values compared to previous studies. The role of increased serum and tissue $\mathrm{K}+$ upon cellular function in the fetus is uncertain.
136 Superoxide dismutase: an EnzYMe of maturation - ReLATIONSHIP TO IRDS - R. Roberts, L. Frank, A. Autor, The Toxicology Center, Depts. of Pharmacology and Pediatrics, University of lowa, lowa City, lowa 52242.

Superoxide dismutase (SOD) facilitates the reaction $20_{2}^{-}+2 \mathrm{H}^{+}$ $\rightarrow \mathrm{H}_{2} \mathrm{O}_{2}+\mathrm{O}_{2}$, thus scavenging and controlling the highly reactive and potentially destructive superoxide anion $\left(0_{2}^{-}\right) .0_{2}^{-}$is assumed to be generated in tissues during $0_{2}$ exposure. We have previously shown low SOD activity in whole blood and lung from infants with IRDS (Fed. Proc. 13, 1505, 1974). We have attempted further study of the maturation of SOD enzyme activity and its inducibility upon $\mathrm{O}_{2}$ exposure. From the mid-fetal period to adulthood in man specific activity of SOD in lung nearly doubles (257 to 445 units $/ \mathrm{gm})$. Fetal to adult lung SOD activity in rabbits was 78 to 344 units/gm, and in rats 215 to 520 units/gm. Lung SOD activity in premature rabbits was increased $38 \%$ above controls by exposure to $80 \% \mathrm{O}_{2}$ for 24 hours. In vitro experiments demonstrated an even more rapid response to hyperoxia with a $42 \%$ increase in rat lung SOD activity within $1-2$ hours of $100 \% \mathrm{O}_{2}$ exposure, the response being especially dramatic in lung tissue of premature and newborn rats compared to the adult. This capability of the newborn to increase 500 activity may serve to protect the lung (and other organs) against the relative hyperoxia of extrauterine life. We previously speculated that infants who succumb to IRDS are unable to respond to hyperoxia by an increase of SOD activity. Thus, the toxic pulmonary effects resulting from exposure to increased $0_{2}$ concentrations without the protective effect of SOD may be an important component of the IRDS disease picture. Supported by NIGMS 12675 and Trainee Grant 5701 HL5577-13.

137 PLACENTAL NUCLEIC ACIDS AND PROTEIN METABOLIS 1 DURING PROLONGED GESTATION. Rosso, P.,
Coll. of Phys.and Surg., Columbia Univ.,Dept. of Ped., Inst. of Human Nutrition, New York, New York It is generally assumed that the "Postmaturity Syndrome" is caused by placental insufficiency due to ageing of this organ, however, current knowledge on placental metabolism and function during prolonged gestation $(P G)$ is inadequate to define the characteristics of the ageing placenta. We studied nucleic acid and protein metabolism of the rat placenta during hormonally induced $\mathrm{PG}$. Rats were injected near term with $2.5 \mathrm{mg}$ of progesterone/100 g body weight and killed at day 23 . Placentas from day 20 through day 23 were compared. It was found that DNA and protein content remained constant. In contrast RNA/DNA ratio decreased from 2.0 at day 20 to 1.5 at day 23. This reduction may be due to increased RNA catabolism as Indicated by a steep increase in alkaline ribonuclease activity $(21,000 \mathrm{v} / \mathrm{g}$ to $35,000 \mathrm{v} / \mathrm{g}$ from day 20 to day 23 ). The rate of protein synthesis, measured by $14 \mathrm{C}$-leucine incorporation, decreases steadily. Values at day 23 were 30 per cent of those at day 20. Polyamine concentration changes as described in ageing tissues, with a reduced spermidine to spermine ratio. In addition putrescine concentration is reduced. These data suggest that $P G$ alters placental function by affecting RNA and protein metabolism without reducing cell number.

138 PLACFNTAL UTILIZATION AND CONVERSION OF GUUCOSE IN VIVO. Michael A. Simmons, Laurence I. Burd, James A. Lemons, M. Dougias Jones, Jr. Richard L. Schreiner, Giacomo Meschia, and Frederick c. Battaglia. Division of Perinatal Medicine, University of Colorado Medical Center, Denver.

Studies in sheep have shown that umbilical uptake of glucose supplies less than $50 \%$ of fetal catabolic substrate and that placental permeability to glucose is limited. This study presents the first quantification of placental glucose utilization in a mammalian fetus. Simultaneous determinations of fetal and placental utilization or production of gluoose, lactate, and pyruvate were carried out in chronic sheep preparations with gestations of 105 to 140 days. Total uteroplacental glucose uptake was $0.266 \mathrm{~mm} / \mathrm{min}$, of which $\sim 35 \%(.094 \mathrm{~m} / \mathrm{min})$ crossed directly to the fetus, and $\sim 65 \%(0.173 \mathrm{mM} / \mathrm{min})$ is utilized by the placenta. of this placental glucose utilization, $-60 \%$ is accounted for by lactate production $(0.198 \mathrm{~mm} / \mathrm{min})$, and $\sim 7 \%$ by pyruvate production $(0.011 \mathrm{~mm} / \mathrm{min})$. Placental $\mathrm{O}_{2}$ consumption was $4.86 \mathrm{ml} / 100 \mathrm{gm} / \mathrm{min}$ and fetal $\mathrm{O}_{2}$ consumption was $9.06 \mathrm{ml} / \mathrm{kg} / \mathrm{min}$. Glucose/oxygen quotient $\left(\mathrm{G} / \mathrm{O}_{2}\right)$ for the fetus was 0.55 and for the placenta was 1.08 . correcting the glucose utilization rate for production of lactate and pyruvate, the $\mathrm{G} / \mathrm{O}_{2}$ for the placenta was 0.37 , thus demonstrating significant non-glucose oxidative catabolism in the placenta. Of the total placental lactate production, $50 \%$ appears in the umbilical circulation and $50 \%$ in the uterine circulation. Approximately $50 \%$ of total uteroplacental glucose uptake ultimately appears in the umbilical circulation; $65 \%$ of this total carbon flow appears as glucose, $35 \%$ as lactate. 
139 EFFECT OF UNCYCLED LIGHT ON PLASMA HUMAN GROWTH HORMONE IN NEONATES. T.R.C. SISSON, G.

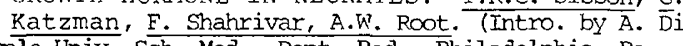
George). Temple Univ. Sch. Med., Dept. Ped., Philadelphia, Pa. The influence of a constant, uncycled nursery lighting environment upon plasma HGH was studied in 34 newborn infants 2 days of age, >38 wk. gestation, and 2500-3340 gm. birth weight. Infants were placed in 4 groups so that heel-stick blood sampling included each $2 \mathrm{hr}$. interval around the clock: Group A 0800, 1600, 2400 hrs; Group B 0200, 1000, 1800 hrs.; Group C 0400, 1200, 2000 hrs.; Group D 0600, 1400, 2200 hrs. The mean HGH for all observations was $43.7 \mathrm{mg} / \mathrm{ml}$. Peak values (range 46.8-53.2) occurred at $0200,0600,1200,1800$ and 2200 hrs. Minimal values (range 33.439.4 ) were found at $0400,0800,1600,2000$ and $2400 \mathrm{hrs}$.

These observations indicate that an ultradian rhythm of $\mathrm{HGH}$ is established by the 2nd day of life, as previously determined in this laboratory in neonates under a cycled light milieu. Appropriate analysis of the data from this investigation did not, however, demonstrate a circadian rhyth, in contrast to the prior study which had shown circadian rhythmicity under cycled light.

These results indicate that a constant nursery lighting environment, wherein a luminance of $100-130$ footcandles (daylight fluorescent lamps) either prevents or obliterates the cicadian rhythm of plasma HGH shown to be present in newborns under dark: light cycling, and suggest the impropriety of constant light in nurseries.

140 Placental transport systems for neutral amino acids. Carl H. Smith, Robert H. Enders, R. Michael Judd: Washington Univ. Sch. of Med., St. Louis Children's

Hosp., Dept. of Ped., 500 S. Kingshighway, St. Louis, Mo. 63110.

The human placenta is known to concentrate amino acids intracellularly for transfer to the fetus. To clarify the mechanism and regulation of this process we have determined the specificity of placental transport systems for neutral amino acids. Using competitive inhibition techniques three transport systems of overlapping specificity have been elucidated. These correspond approximately to the "A", "L", and "ASC" systems of Christensen. In the placenta their specificities are as follows: " $A$ " system glycine, alanine, serine, threonine, glutamine, a-aminoisobutyric acid, N-methylalanine and proline at physiologic concentrations and phenylalanine at high concentrations; " $L$ " system - leucine, phenylalanine, threonine, glutamine, and 2-aminobicyclo- $[2,2,1]-$ heptane-2-carboxylic acid (BCH); and "ASC" system - alanine, serine, threonine, and glutamine. Since glutamine is present in high concentration in maternal blood it may account for a major share of fetal amino acid uptake.

Placental AIB uptake has previously been shown to increase with preincubation of tissue in vitro. This preincubation increase has now been found to be limited to the "A" system. Activity of the other two systems is essentially unchanged, demonstrating that in the placenta, as in other organs, transport pathways are separately regulated. Knowledge of their specificity and regulation should contribute to understanding of the control of the transfer of amino acids to the fetus.

141 The Effect of Phenylketonuric and Other Metabolites Upon Sulfated Galactocerebroside Synthesis in Mouse Spinal Cord Cultures. T.J. Sprinkle*, Ph.D. and 0. M. Rennert**, M.D., *Department of Neurology, Johns Hopkins Univ., Baltimore, Md., **Department of Pediatrics, Univ. Florida, Gainesville, Fla.

Sulfated galactocerebroside synthesis was studied in vitro in mouse spinal cord cultures. This system permitted the study of the effects of Phenylketonuric and Maple Syrup Urine Diseaserelated metabolites upon synthesis of specific myelin components formed early in postnatal development. Significant lnhibition of sulfatide synthesis was observed when spinal cord cultures were grown in the presence of $1000 \mu \mathrm{M}$ Phe and $500 \mu \mathrm{M} \mathrm{PPA}$ ( $51 \%$ and $70 \%$ respectively). No effects were observed at concentrations of $300 \mu \mathrm{M}$ PLA or $250 \mu \mathrm{M}$ PA. The inhibitory effect of PPA was shown to be reversible. Intracerebral bilateral injections $(8 \mu \mathrm{g})$ of Phe, PPA, $\alpha-K B, \alpha-K I C, \alpha-K I V$, PLA, and PA in mice $8-15$ days old followed by $1 . p$. administration of radioactive sulfate resulted in significantly reduced sulfatide synthesis $(p<.05)$ with all compounds except PLA or PA. The effect of PPA as a structural analog of pyruvate yas examined in mouse brain and kidney homogenates measuring ${ }^{14} \mathrm{CO}_{2}$ release from $\left[1-{ }^{4} \mathrm{C}\right]$ pyruvate. The observed $\alpha-K I C$, PPA $>$ Phe $>\alpha-K B, \alpha-K I V$, PLA, PA reduction of sulfatide synthesis may be due to decreased ATP production, and decreased availability of substrate from acetate as a result of direct effects upon oxidative metabolism of pyruvate.
142 PERINATAL CHANGes OF ORGAN AND WHOLE bODY CAPACITIES OF GLUCOSE AND FATTY ACID UTILIZATION. Uwe Stave, Fels Research Inst., Yellow Springs, OH.

The determination of total amounts of specific key enzyme activities has been shown to be a suitable alternative for the measurement of substrate utilization rates in single organs. The enzyme activities represent metabolic capacities and can also be calculated for the entire organism; expressed per unit of body weight (BW) they facilitate age comparisons. Phosphof ructokinase (PFK) activity was selected to represent the upper segment of glycolytic reactions. Fatty acid oxidation is represented by 3-hydroxyacyl COA dehydrogenase (HAD). Both enzymes are rate limiting and both act unidirectionally. In fetal, neonatal and adult rabbits we have analyzed liver, kidneys, heart, lungs, brain, adipose tissue, intestines, skin, muscles, and bones. Organ weights and total protein distribution was determined in these organs. The share contributed by each organ to the total body key enzyme activity sheds light on the importance of the two metabolic capacities represented by PFK and HAD. The amount of muscle protein, calculated per unit of $\mathrm{BW}$, doubled from birth to maturity. The share neonatal muscle represents of total body PFK was $75 \%$, compared with $99 \%$ in adult rabbits. In the newborn organism $40 \%$ of total HAD activity was found in adipose tissue whereas only $3 \%$ of this activity was present in mature fat. The sum total of both PFK and HAD activities expressed per unit of $B W$ increased before birth. From birth to maturity the whole body PFK activity expressed per $g$ BW increased tenfold, and the HAD activity decreased with age to half of the neonatal value.

143 TAUR INE IN DEVELOPING BRA IN OF THE HUMAN AND MONKEY. John A. Sturman and Gerald $E$. Gaull, Dept. Ped. Res. N.Y., and Dept. Ped., Mt. Sinai Med. Sch. of CUNY, New York, N.Y. Taurine is one of the bodies most ubiquitous and abundant ninhydrin-positive compounds but takes part in few known biochemical reactions. The possibility that it may be involved in neurotransmission and epilepsy is the subject of much recent study.

We report the concentration of taurine in brain and liver from human fetuses (2nd trimester) and adults and from Rhesus monkeys from mid-gestation to maturity. The concentration of taurine in human and monkey fetal brain was $4-5$ fold higher than that in adult brain: In human fetal brain, the concentration decreased with increasing crown-rump length $(r=0.75 ; P<0.001)$. In monkey brain no correlation was found with gestational age; however, the concentration of taurine decreased in a linear fashion $(r=0.96$; $P<0.001)$ post-natally until values comparable to those found in the adult were reached 8-9 months after birth, approximately the end of weaning.

The concentration of taurine in liver both from fetal humans and fetal monkeys was approximately twice that in mature liver. Concentrations of taurine found in adult liver were reached within a few days after birth, compared to several months for brain. These results suggest that taurine may be associated with brain development, per se, in addition to any functional role it may play in the mature brain.

(Supported by the N.Y. State Dept. of Mental Hygiene.)

INSENSIBLE WEIGHT LOSS IN NEWBORN INFANTS ESTIMATED BY STABILIZED SKINFOLD THICKNESS. J. Sumners, B. Erwin, G. Cassady. Div. Perinatal Med., Univ. of Ala. in Birmingham, Birmingham, Alabama.

Insensible weight loss (IL) was measured on 42 occasions in 15 normally grown (NG) and 2 intrauterine growth retarded (IGR) infants cared for under a radiant warmer. Serial weighings for 2 to 3 hour periods were accomplished using a Brookline Metabolic Scale (Model 80-A). Birthweights ranged from $790 \mathrm{~g}$. to $3560 \mathrm{~g}$. with 5 infants weighing less than $1000 \mathrm{~g}$. at birth. Gestational age, corrected for postnatal age at time of study $\left(\mathrm{GA}_{\mathrm{C}}\right)$ ranged from 22 to 42 weeks.

IL was related inversely to weight at time of study in both NG $(r=-.70, p<.001)$ and IGR $(r=-.85, p<.005)$ infants. The same close relationship was seen when IL was compared with $\mathrm{GA}_{\mathrm{C}}(\mathrm{r}=$ $-.74, p<.001$ )

During 26 studies on $12 \mathrm{NG}$ and 8 studies on 2 IGR babies, skinfold thickness readings were taken 60 seconds after caliper application at left midtriceps (MT60) and subscapular $\left(\mathrm{SS}_{60}\right)$ areas. Both $\mathrm{MT}_{60}$ and $\mathrm{SS}_{60}$ were related to IL $(\mathrm{r}=-.53, \mathrm{p}<.005$ and $\mathrm{r}=$ $-.55, \mathrm{p}<.005)$; higher correlations were noted in studies performed at $<48 \mathrm{hrs}$. postnatal age $(r=-.64, p<.005$ and $r=-.74, p<$ .001 respectively). At $\geq 48 \mathrm{hrs}$. postnatal age, MT60 and $\mathrm{SS}_{60}$ correlated well with IL in IGR infants $(r=-.88, p<.02$ and $x=$ $-.92, p<.005)$.

These data reveal a relation between skin thickness and IL. In addition, they suggest that stabilized skinfold measurements may provide a useful clinical tool for prediction of IL. 
145 EFFECT OF UNDERNUTRITION ON THYMIDINE KINASE DURING RAT CEREBELLAR DEVELOPMENT. Morton E. Weichsel, Jx., (Intx. by Delbert A. Fisher), UCLA Sch. of Med. Harbor Gen. Hosp., Dept. of Ped., Torrance, Ca. and Mich. State Univ., Col. of Human Med., Dept. of Human Development, E. Lansing Michigan

Thymidine kinase (TK) activity in the developing rat cerebellum peaks in activity at 5 days of age and falls rapidly thereafter to disappear at around 19 days. To determine the effect of neonatal undernutrition on rat cerebellax DNA and TK activity, we studied 8 control litters of 8 pups each and 8 excessively large litters of pups expected to be undernourished at ages 5,12 and 19 days. Comparisons were made between values for DNA and TK in cerebella from the heaviest, medium weight, and lightest subgroups within the oversized litters as well as between the control litters and undernourished subgroups. Using cerebellar DNA as a measure of undernutrition, results showed that only the low and medium weight groups from the oversized litters at age 5 days contained significantly less DNA than controls. At ages 5 and 12 days, only the most undernourished subgroups showed a significant depression (74\%) and significant elevation (121\%) of TK activity respectively, compared to controls. Results were supported by comparisons between each of the three subgroups within the oversized litters. TK activity was not detectable in any group or subgroup at age 19 days. The data thus suggest that with increasing degrees of undernutrition during the critical period of brain development, a point may occur where key enzymes are selectively affected.

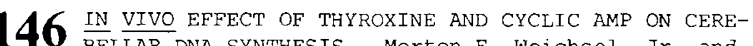
BELLAR DNA SYNTHESIS. Morton E. Weichsel, Jr. and James E. Trosko (Intr. by Delbert A. Fisher), UCLA Sch. of Med., Harbor Gen. Hosp., Dept. of Ped., Torrance, Ca. and Mich. State Univ., Col. of Human Med., Dept. of Human Development, E. Lansing, Mi.

Thyroid hormone administered from birth has been shown to induce cerebellar DNA synthesis in the rat from 2 through 6 days of age. We administered thyroxine, dibutyryl cyclic AMP, and a combination of both drugs to groups of rat pups within each of 5 litters for the first 5 days of life. At age 5 days, cerebellar DNA from cyclic AMP treated animals was significantly below control values (88\% of controls) while body weight showed less difference (92\%). DNA was significantly elevated to $117 \%$ of control values in thyroxine treated animals, while body weight was $94 \%$ of controls. The induction of cerebellar DNA synthesis by thyroxine was negated by administration of dibutyryl cyclic AMP ten minutes prior to thyroxine injection. This reversal of a thyroxine induced increase in cerebellar DNA synthesis by prior administration of dibutyryl cyclic AMP thus provides evidence that the early effect of thyroxine on cerebellar cell division may be mediated by cyclic AMP. These results support the hypothesis of Goldberg et al that induction of cerebellar cell division by thyroxine should involve an increase in the ratio of intracellular cyclic GMP to cyclic AMP, and suggest that this ratio was distorted by in vivo elevation of intracellular cyclic AMP.

\section{DEVELOPMENTAL CARDIOLOGY}

147

PHARMACOKINETICS OF GENTAMICIN DURING PERITONEAL DIAL YSIS IN CHILDREN. Tadla Baliah, Louise Gerbracht, Kyoung H. Kim, Sumner J. Yaffe, and William J. Jusko State Univ. of N. Y. at Bu

The pharmacokinetics of gentamicin were examined on two different occasions using intravenous and intraperitoneal route in 5 children ( $8-15$ yrs.) with chronic renal failure maintained on an inter mittent peritoneal dialysis program. Blood, urine, and dialysis fluid (DF) were assayed microbiologically for gentamicin and the data were subjected to computer analysis. Following i, v. inject ion of $1 \mathrm{mg} / \mathrm{kg}$ of gentamictn, the apparent volume of distribution averaged 23 (range 13 to 36 ) percent of body weight (similar to normal), the mean half-life was 21 (range 9 to 37 ) hours, (normal 2 hours) and the net dialysance averaged 4.0 (range 1.2 to 7.0 ) $\mathrm{ml} / \mathrm{min} / \mathrm{m}^{2}$. During peritoneal administration of gentamicin (15 mg 1. of $\mathrm{DF}, 0.71 . / \mathrm{m}^{2}$ administered in each cycle over 9 to 12 cycles serum concentrations increased towards extrapolated steady-state levels which averaged 46 (range 25 to 68 ) percent of DF concentration. The disposition half-lives following this regimen were 7.4 to 44 hours, similar to the values found in the i. y. studies The mean renal clearance of gentamicin was $1.6 \mathrm{ml} / \mathrm{min} / \mathrm{m}^{2}$ and averaged 71 percent of creatinine clearances. Total body gentamicin clearances ranged from 2.3 to $8.0 \mathrm{ml} / \mathrm{min} / \mathrm{m}^{2}$. These data have been found to be useful for designing initial therapy with gentamicin by either $i . v$. or peritoneal routes. The appreciabl variability in pharmacokinetics among adolescent patients with renal insufficiency necessitates dosage adjustments based on measurements of serum concentrations.
148 LUMBAR EPIDURAL ANALGESIA WITH BUPIVACAINE IN LABOOR. CONPINUOUS FETAL HEART MONITORING AND DRUG CONCENTRATION IN MATERNAL, FETAL AND NEONATAL BLOOD. Patrick Belfrage, Anita Berlin, Nils Raabe \& Bertil Thalme, Dept. of Obst.\& Ped., Karolinska Bospital, Stockholm, Sweden.

Lumbar epidural analgesia with bupivacaine was given to 33 women for labour. Serial determinations of $\mathrm{pH}$ and bupivacaine were made in fetal scalp-blood and matermal venous blood as well as continuous monitoring of the fetal heart rate.At birth and during the next 20 hours the bupivacaine concentration was determined in maternal and neonatal blood.

Fetal scalp-blood was within normal limits. A temporary decrease of the normal baseline fetal heart irregularity was seen in 6 cases.The fetal and neonatal drug concentrations were low and about $1 / 4$ of the maternal levels. The UA/UV drug concentration ratio increased with time after injection and approached one after about 60 minutes. During the first 20 hours after birth an initial rapid decline followed by a slower fall was observed in the neonatal drug concentration.All babies were in good condition during the period of the study.

ROLE OF $\mathrm{O}_{2}$ IN REGULATION OF CGMP AND CAMP IN HUMAN UMBILICAL ARTERY. Ronald I. Clyman, Adam S. Blacksin, Vincent C. Manganiello, Martha Vaughan. (Intr. by Howard Sloan) NIH, Bethesda.

In term (gestational) human umbilical artery segments incubated at $37^{\circ} \mathrm{C}$ in room air, agonists that cause contraction (bradykinin and ionophore A23187) cause accumulation of guanosine $3^{\prime}, 5^{\prime}-$ monophosphate (cGMP); prostaglandin $E_{1}\left(P_{G E}\right)$, which relaxes the artery, causes adenosine $3^{\prime}, 5^{\prime}$-monophosphate (CAMP) accumulation without affecting the cGMP content. Increasing oxygen tension enhances both constrictor activity and sensitivity of the umbilical artery to agents that induce constriction. Incubation of artery segments for $2 \mathrm{hr}$ under $100 \% \mathrm{~N}_{2}$, instead of room air, did not alter their cAMP content and the effects of PGE 1 on it. After incubation in $100 \% \mathrm{~N}_{2}$, however, the cGMP content of the artery segments was decreased by $40 \%$ and the effects of maximally effective doses of bradykinin and ionophore A23187 on CGMP were reduced to approximately $1 \%$ of those in room air. Changing the atmosphere from $100 \% \mathrm{~N}_{2}$ to $100 \% \mathrm{O}_{2}$ caused an increase in cGMP content from $0.46 \pm 0.08 \mathrm{pmol} / \mathrm{mg}$ protein (mean $\pm \mathrm{SEM}$ ) to $1.35 \pm 0.29$ within 2 min and also restored the effects of bradykininandionophore A23187 on CGMP. Inhibitors of oxidative phosphorylation (oligomycin $50 \mu \mathrm{M}$ or 2,4 dinitrophenol, $0.5 \mathrm{mM}$, for $40 \mathrm{~min}$ ) did not prevent the effect of changing the atmosphere from $100 \% \mathrm{~N}_{2}$ to $100 \%$ $\mathrm{O}_{2}$ on CGMP and, in fact, potentiated this response. These observations suggest that oxygen has a regulatory role in CGMP metabolism and that this effect is independent of oxidative phosphorylation. The relationship between effects of $\mathrm{O}_{2}$ on cGMP metabolism and on contraction remains to be elucidated.

150 ROLE OF Ca++ IN REgULATION OF CGMP AND CAMP IN HUMAN UMBILICAL ARTERY. Ronald I. Clyman, Adam S. Blacksin Jeffrey A. Sandler, Vincent C. Manganiello, Martha Vaughan (Intr, by Howard Sloan). NIH, Bethesda.

In term (gestational) human umbilical artery segments incubated in air at $37{ }^{\circ} \mathrm{C}$, histamine, acetylcholine, bradykinin, $\mathrm{K}^{+}$, and serotonin (agonists that cause contraction) cause accumulation of guanosine $3^{\prime}, 5^{\prime}$-monophosphate (cGMP) without altering the content of adenosine $3^{\prime}, 5^{\prime}$-monophosphate (CAMP); prostaglandin $E_{1}\left(\mathrm{PGE}_{1}\right)$, which relaxes the artery, causes CAMP accumulation without affecting the cGMP content. In other tissues $\mathrm{Ca}^{+t}$ is important in the regulation of cyclic nucleotides. In the umbilical artery the control of cAMP content by $\mathrm{PGE}_{1}$ was independent of $[\mathrm{Ca}++]$. After incubation in $\mathrm{Ca}^{++}$-free medium, the cGMP content of the artery segments was decreased by $50 \%$ and was unaffected by histamine, acetylcholine, bradykinin and $\mathrm{x}^{+}$. Basal cGMP content and the agonist effects on CGMP were partially restored by readdition of $\mathrm{Ca}^{++}(2.7 \mathrm{mM})$ or, to a lesser degree, by $\mathrm{Sr}^{++}(3.6 \mathrm{mM})$. Ionophores A23187 and X537A (agents that facilitate $\mathrm{Ca}^{++}$movement through membranes) mimicked the effects of these $\mathrm{Ca}^{++}$-dependent agonists on CGMP content. Incubation with the phosphodiesterase inhibitor 3-isobuty1-1-methyl xanthine $(0.1 \mathrm{mM})$ increased both the basal content of CGMP and the histamine-induced accumulation 3-fold. This effect was dependent on the presence of $\mathrm{Ca}^{++}$also. Accumuiation of CGMP induced by serotonin, on the other hand, was not diminished in $\mathrm{Ca}^{++}$-depleted arteries and, in fact, seemed to be inhibited by $2.7 \mathrm{mM} \mathrm{Ca}+$. We suggest the existence of two separate mechanisms for control of cGMP synthesis that are influenced differently by $\mathrm{Ca}+t$. 
151 CGMP AND CAMP CONTENT OF HUMAN UMBILICAL ARTERY: POSSIBLE ROLE IN PERINATAL PATENCY AND CLOSURE. Ronald I. Clyman, Jeffrey A. Sandler, Vincent C. Manganie110, Martha Vaughan (Intr. by Howard Sloan). NIH, Bethesda.

It has been suggested that guanosine $3^{\prime}, 5^{\prime}$-monophosphate (CGMP) plays a role in smooth muscle contraction and adenosine $3^{\prime}, 5^{\prime}-$ monophosphate (cAMP) in relaxation. This hypothesis has been evaluated in term (gestational) human umbilical artery segments incubated at $37^{\circ} \mathrm{C}$ and in room air. 1) The basal cGMP content ( $1 \mathrm{pmol} / \mathrm{mg}$ protein) of artery segments incubated in room air was almost twice that of CAMP. 2) Bradykinin, histamine, serotonin, acetylcholine and $\mathrm{K}^{+}-\mathrm{ion}$, which cause umbilical artery constriction, can increase the cGMP content of the artery segments within $30 \mathrm{sec}$ of exposure without altering the cAMP content.

3) Prostaglandin $E_{1}$, but not isoproterenol, caused accumulation of cAMP which is consistent with reports that umbilical arteries lack functional $\beta$-receptors and that only prostaglandin $E_{1}$ can bring about relaxation of umbilical arteries. 4) $1 \mu \mathrm{M}$ atropine blocked the effect of $100 \mu \mathrm{M}$ acetylcholine on CGMP content without altering the responses to histamine, bradykinin, serotonin, or $\mathrm{K}^{+}-\mathrm{ion}$. 5) Pyrilamine (an $\mathrm{H}-1$ antagonist), but not metianide (an $\mathrm{H}-2$ antagonist), blocked the effect of histamine on cGMP from which it is inferred that histamine causes accumulation of cGMP in umbilical artery via its interaction with $\mathrm{H}-1$ receptors. The results are consistent with the view that metabolism of the two cyclic nucleotides is independently controlled in the human umbilical artery and that cGMP is involved in contraction of the artery at birth.

152 ENDOCRINE EFFECTS OF CHRONIC ADMINISTRATION OF LSD TO PREPUBERAL MALE RATS. R. Collu, J. Letarte, G. Leboeuf and J.R. Ducharme. Dept. of Ped., Ste-Justine Hosp., and Univ. of Montreal, Montreal, Quebec.

No reports have appeared to our knowledge on the endocrine effects of the chronic administration of LSD to young animals. In this study, groups of Sprague-Dawley male rats were injected intraperitoneally 3 times a week for a month with either 100 or 500 ug/ $\mathrm{kg}$ of LSD or with the vehicle starting at 21 days of age. At the end of the experimental period, the animals were sacrificed by decapitation. Endocrine organ weights were recorded and plasma and pituitary levels of $\mathrm{GH}, \mathrm{LH}$ and $\mathrm{FSH}$ were measured by radioimmunoassay and brain levels of serotonin (5-HT), 5-hydroxyindoleacetic acid (5-HIAA), noradrenaline (NA) and dopamine (DA) were assayed fluorometrically. Animals injected with either dosage of LSD had smaller body weights than controls, while tail length was reduced in the high dosage group only. Plasma levels of $\mathrm{GH}$ were decreased in the high dosage group and pituitary levels in the low dosage group (Mean $\pm \mathrm{SE}$. Plasma GH: LSD $40 \pm 16$, controls $138 \pm 33 \mathrm{ng} / \mathrm{ml}$. $P<0.02$. Pituitary GH content: LSD $108 \pm 16$, controls $227 \pm 8$ ug. $P<0.001)$. Plasma and pituitary levels of $\mathrm{LH}$ and FSH were not modified by the drug. The low dosage of LSD decreased the brain levels of NA and increased those of DA, while the high dosage decreased those of 5-HIAA. These results suggest that LSD, when administered chronically to developing animals, can inhibit body growth probably by altering the secretion of $\mathrm{GH}$ through modifications of its neuroendocrine control.

153 RAPID DIPHENYLHYDANTOIN METABOLISM IN INFANTS.Richard G. Curless and Philip D. Walson (Intr. by J.J.Corrigan, jr.), Depts of Pediatrics, Pharmacology and Neurology, Univ. of Arizona, Tucson, Arizona.

The purpose of this study is to evaluate diphenylhydantoin (DPH) thetabolism in infants with poorly controlled seizure disorders and consistently low serum DPH levels. Three infants with severe seizure disorders refractory to a variety of medications including DPH were found to have serum DPH levels of less than 2 mcg. per $m$ l. 12 hours after a DPH dose. Each patient had been receiving DPH in a dosage of $10 \mathrm{mg} \cdot$ per $\mathrm{kg}$. given twice a day for a minimum of three months preceding the present study. To evaluate DPH kinetics, a half-life was determined on each child in the following manner. After obtaining a base line serum DPH (12 hours after the last DPH dose) $1 \mathrm{mg}$. per $\mathrm{kg}$. of DPH was injected through an intravenous line. Subsequent blood samples were drawn at one hour, two hours and four hours after injection. The DPH levels were determined by gas chromotography and DPH half-lives were determined using this data. The results indicate half-lives of 4.5 hours, 3.7 hours and less than 2 hours on the three patients. It is our conclusion that these three children must be treated with special consideration in terms of DPH therapy. The first two patients will require a DPH dosage interval of approximately 4 hours and the short half-life on the third patient indicates that DPH therapy is unlikely to produce adequate blood levels with any acceptable dosage regimen. We suggest that this mechanism of drug failure with DPH should be considered in any infant who has below therapeutic blood levels with an adequate oral dosage.
154 ACETAMINOPHEN DISPOSITION IN ADOLESCENTS. Michele Danish, Rafael Gorodischer, Steven Liebowitz, Luis Mosovich, Joseph Krasner and Sumner J. Yaffe. Dept. of Ped., State Univ. of New York at Buf falo.

Acetaminophen is a frequently used antipyretic and analgesic in infants and children. Dosage recommendations are empirically derived and not based upon pharmacokinetic studies in these age groups. We first investigated two groups of adolescents, 7 normal children, $10-18$ years old (average 13.4 years) and 5 cystic fibrosis patients, $13-21$ years old (average 15.6 years). The patients were given single oral doses of APAP, $1 \mathrm{~g} / 1.73 \mathrm{M}^{2}$, as the elixir. Serum and saliva samples were collected at hourly intervals for 6 hours following drug administration. Acetaminophen concentrations were determined by GLC. The serum half-lives of the normal adolescents ranged from 72 to $108 \mathrm{~min}$. (average 85 min.) and were significantly lower than literature reports of adult half-lives which range from 114 to $142 \mathrm{~min}$. The cystic fibrosis patients more closely resembled adults with a range of 100 to $132 \mathrm{~min}$., averaging $112 \mathrm{~min}$. This increase in average half-life in the cystic fibrosis patients may possibly be related to the older ages of this group. Saliva samples were collected simultaneously with !lood samples as a means of establishing a non-invasive technique for measuring APAP body levels. Saliva half-lives correlated well with the measured serum half-life. Saliva:serum concentration ratios averaged 1.04:1.0 for the normal adolescent and $1.14: 1.0$ for the cystic fibrosis patients. These data provide evidence of a shorter $\mathrm{T} 1 / 2$ in adolescents and justify alteration in dosage as well as study of younger age groups.

155 GENTAMICIN PHARMACOKINETICS IN PATIENTS WITH CYSTIC FIBROSIS. Michele Danish, Louise Gerbracht, Luis Mosovich, William J. Jusko and Sumner J. Yaffe. Depts. of Ped. and Pharmaceutics, State Univ. of N.Y. at Buffalo.

Gentamicin is frequently employed for the management of gramnegative pneumonia in children with cystic fibrosis. It is of major importance to determine the disposition characteristics of the antibiotic in diseased patients so that optimum dose for treatment may be established. Six cystic fibrosis patients, age range 12-24 years, who required intravenous gentamicin infusions for the treatment of pneumonia were studied. Doses ranged from 1.0 to $1.9 \mathrm{mg} / \mathrm{kg}$ with an approximate infusion time of 1 hour. Serum, sputum and urine samples were collected at hourly interfals for 8 hours after the initiation of the infusion. There was total urinary recovery of the drug within the 8 hour period in all patients. The average gentamicin half-life ( 2.0 hours) and volume of distribution ( $18.8 \%$ of body weight) correlated well with normal adult data. However ${ }_{3}$ gentamicin renal clearance averaged only $60.2 \mathrm{ml} / \mathrm{min} / 1.73 \mathrm{M}^{2}$, which was only $45 \%$ of the simultaneously measured creatinine clearance. Also significant was the lack of distribution of gentamicin to the sputum. The apparent sputum antibiotic concentration remained constant at $1 \mathrm{mcg} / \mathrm{ml}$ during the study period while the serum concentrations varied from $0.5-9.2 \mathrm{mcg} / \mathrm{ml}$. However, in sputum collected from cystic fibrosis patients not receiving any antibiotics, a natural inhibitory activity of $0.5 \mathrm{mcg} / \mathrm{ml}$ was observed. Thus, as measured in the sputum, no appreciable amounts of gentamicin can be found at the presumed site of infection.

PRENATAL AND POSTNATAL METHYLMERCURY POISONING IN MOUSE AND MAN. Richard A. Doherty and Allen H. Gates (Intr. by Robert $H$. Schwartz)

Univ. Rochester Sch. Med., Dept. Ped., Radiation Biology/Biophysics, Div. Genetics, Rochester, New York

Recently in Iraq, 6530 children $(34 \%)$ and adults $(66 \%)$ were hospitalized due to methylmercury poisoning from eating homemade bread prepared from seed wheat treated with a methylmercury fungicide (Science 181: 230, 1973). There were 459 hospital deaths. Long term follow-up studies of infants exposed in utero are in progress.

We are using a mouse model to aid in evaluating risk to the mammalian fetus and newborn. 24 hours after a pulse dose of methylmercury, maternal (M) and fetal (F) mouse blood, plasma and liver mercury concentrations are equivalent yet $F$ brain concentration is 3 -fold higher than $M$ brain. Mouse milk mercury concentration is highly correlated with plasma concentration $\langle r=0.94)$ throughout the wide range of blood methylmercury concentration ( 10 to $3000 \mathrm{ng} / \mathrm{ml}$ ) seen in the exposed Iraqi population. Normal human blood methylmercury concentration $=5 \mathrm{ng} / \mathrm{ml}$. Suck ling mice excrete mercury 35 -fold less rapidiy than adult mice: adult half-time of excretion $=7$ days; newborn (birth to 9 days) half-time of excretion $=240$ days.

Mouse and human data suggest that the fetus and suckling newborn may be at increased risk for toxic effects of methylmercury on developing brain. Prenatal and postnatal mouse and Iraqi human data will be used to discuss possible risk of human methy?mercury poisoning from ingestion of pelagic fish. 
157 DISPLACEMENT OF BILIRUBIN FROM ALBUMIN BY ANTIBIOTICS AND PRESERVATIVES. Peter Echeverria, Jean Loria, and Arnold $L$. Smith, Children's Hosnital Medical Center, Dept. of Med., Boston, Mass.

Sulfonamides were associated with an increased incidence of kernicterus in infants and have been shown in vitro to compete with bilirubin (BR) for binding sites on albumin (AL). Spectral absorption curves at 400-500 nanometers ( $\mathrm{nm}$ ), sephadex gel filtration analysis, and rate of transfer of $B R$ to cholestyramine (CS) were used to study the effect of antibiotics on BR-AL binding in human icteric serum.

The spectral absorption curves tested at BR concentrations from $10-68 \mathrm{mg} / \mathrm{al}$ were not significantly shifted until sulfisoxazole (SU) was $100 \mathrm{x}$ therapeutic concentrations (1670 ma/dl) ( $\mathrm{P}<$ .001 ). Gentamicin (GN) added at concentrations up to $1000 \mu \mathrm{q} / \mathrm{ml}$ (100x therapeutic concentration), did not produce a significant change. However the paraben preservatives, included in multiple injection Giv vials, produced a significant spectral shift at concentrations of $400 \mu \mathrm{g} / \mathrm{ml}(\mathrm{p}<.001)$. Fel filtration analysis of serum demonstrated a significant decrease in maximal BR binding capacity [drug free serum $61.8 \mathrm{mg} \pm 1.7 \mathrm{mg}(\mathrm{n}=18)$ ] at a Su concentration of $167 \mathrm{mg} / \mathrm{dl}(\mathrm{p}<.001$ ) but no effect was observed with GN at $833 \mu \mathrm{g} / \mathrm{ml}$. The rate of transfer of BR to CS was greater than the control of $0.36 \pm .02 \mu \mathrm{moles} / \mathrm{mg} \mathrm{CS} / \mathrm{min}$ ( $\mathrm{n}=$ 8) with SU at concentrations $>200 \mathrm{mg} / \mathrm{dI}$ and parahens at 1000 $\mu \mathrm{g} / \mathrm{ml}(\mathrm{p}<.001)$. GN un to $1000 \mu \mathrm{o} / \mathrm{ml}$ had no effect on the rate of uptake of BR by CS.

This study demonstrated that SU and parabens but not GN significantly reduce $B R-A L$ binding.

158 AGE DERENDENT CHANGE IN GENTAMICIN PHARMACOKINETICS Peter Echeverria, George Siber, David 4 . Smith, anc? Axnold L. Smith, Children's lospital Medical Center, Dept. of Med., Boston, Mass.

Peak concentration and half-lives of gentamicin were measured after $140 \mathrm{iv}$ and $13 \mathrm{im}$ doses in 60 children and 9 adults with normal renal function. Marked inter-patient variation was observed but individual patients tended to maintain similar values on repeated determinations. Age markedly affected the peak concentrations: a $1 \mathrm{mg} / \mathrm{kg}$ dose produced peaks of $1.58,2.03$ and $2.81 \mu \mathrm{g} / \mathrm{ml}$ in the 0.5 to 5,5 to 10 , and $>10$ year age groups respectively. Patients with a short $t 1 / 2$ and adults with a high extracellular fluid volume were noted to have low peaks. The mean $t 1 / 2$ after iv administration was 75.4 minutes. High serum creatinine and low creatinine clearance were associated with a prolonged $t 1 / 2$ and the $t 1 / 2$ was 28.6 minutes longer after im dosage. In the presence of fever $>100^{\circ} \mathrm{F}$ and a fall in hematocrit $>8 \%$ the $t \lambda / 2$ was 15 and 26.7 minutes shorter respectively. The dosage of gentamicin necessary to achieve mean peak concentrations between four and five $\mu \mathrm{g} / \mathrm{ml}$ is $2.5 \mathrm{mg} / \mathrm{kg} /$ dose for ages 0.5 to 5 years, $2.0 \mathrm{mg} / \mathrm{kq} /$ dose for ages $5-10$ years and $1.5 \mathrm{mg} /$ $\mathrm{kg} /$ dose for ages $>10$ years.

Presented in part at the 14 th Interscience conference on Antimicrobial Agents and Chemotherapy on Sentember 11-13, 1974 in San Francisco, California.

159

SOME PHYSICAL AND BINDING CHARACTERISTICS OF HUMAN ALBUMIN FROM CORD BLOOD, Saraswathy $K$. Ganapathy, and Sanford $N$. Cohen, Depts. of Ped. \& Pharmacol., NYU Sch. of Med., New York, N. Y., and WSU Sch. of Med., Detroit, Mi.

We have isolated the albumin fraction of cord blood and determined some of its physical and binding characteristics. We have also studied albumin isolated from adult blood to evaluate the suggestion that there may be a qualitative difference between plasma proteins from adults and neonates. Albumin for these studies was prepared under identical conditions from pooled cord blood and from fresh adult blood.

Disc gel electrophoresis and analytical ultracentrifugation identified both specimens as pure albumin with a molecular weight of 67,000 . Spectrographic studies of the samples revealed no difference in their spectroscopic properties. Circular dichroism studies yielded values of molar ellipticity of the cord and adult albumin of $1.2 \times 10^{7}$ and $1.3 \times 10^{7} \mathrm{deg} \mathrm{cm}^{2}$ dmole-1 respectively. Binding studies were carried out by equilibrium dialysis at four temperatures and four $\mathrm{pH}$ values with sulfadiazine as the small ion. The association constant (K) shows a slight increase as $\mathrm{pH}$ goes from 7.05 to 7.75 with both adult and cord albumin and a slight decrease with both at $\mathrm{pH} 8.0$. The average $\mathrm{K}$ for adult albumin was $0.334 \times 10^{5} \mathrm{M}$ and for cord albumin was $0.309 \times 10^{5} \mathrm{M}$. Standard free energy change $(\Delta \mathrm{F})$ was calculated for the two samples and showed $\Delta \mathrm{F}$ cord $=-7306 \mathrm{cal} / \mathrm{mol}$ and $\Delta \mathrm{F}$ adult $=-7347$ cal/mol.

We conclude that serum albumin at birth is not qualitatively different from that in the adult. Supported in part by USPHS Grant \# GM 17184.
160 DIGOXIN DISPOSITION (TISSUE DISTRIBUTION AND RENAL CLEARANCE) IN INFANTS. Rafael Gorodischer, William J. Jusko and Sumner J. Yaffe. Depts. of Ped, and Pharmaceutics, State University of New York at Buffalo.

Relative to size the therapeutic dose of many drugs is smaller in young infants than in older children and adults due mainly to decreased biotransformation and renal excretion. Digoxin is unique in that its therapeutic dosage is appreciably greater in infants than in adults. The pharmacologic reasons for the large dosage requirements are not clear. Tissue distribution (myocardium Skeletal muscle and erythrocyte) was studied in 8 infants (age $1-$ 20 mos.) and renal clearance in 11 infants (age 1-5 mos.) during digitalization and/or during the course of maintenance therapy. The mean myocardial digoxin concentration was $386 \mathrm{ng} / \mathrm{gm}$ (adults $78 \mathrm{ng} / \mathrm{gm})$. A linear correlation existed between myocardial and serum concentrations and over the serum concentration range of $0.5-8.6 \mathrm{ng} / \mathrm{ml}$. Erythrocyte:plasma concentration ratios of digoxin were 3 to 4 times greater than that found in adults. This was noted only during maintenance therapy. Samples obtained during digitalization had ratios of unity. Skeletal muscle:serum concentration ratios averaged 14.1 similar to that reported in adults. The renal clearances were low $\left(50 \mathrm{ml} / \mathrm{min} / 1.73 \mathrm{M}^{2}\right)$ at 1 month of age but increased progressively until the adult range was attained at about 5 months of age. The renal clearance of digoxin was $80 \%$ greater than the simultaneously determined creatinine clearance. This stands in marked contrast to adults where creatinine and digoxin clearances are similar. These data explain the larger dose needed in infants.

HEPATIC MICROSOMAL DRUG METABOLISM FOLLOWING ADMINISTRATION OF ENDOTOXIN TO RATS. Rafael Gorodischer, Joseph Krasner, James P. Nolan and Sumner J. Yaffe. Depts. of Ped. and Medicine, State Univ. of N.Y. at Buffalo.

Endotoxemia accompanying gram-negative bacterial infections is a serious life threatening condition that occurs in children with altered host responses. A key aspect of the therapeutic management of children with endotoxin shock is the administration of pharmacologic agents. Since most drugs are eliminated by hepatic metabolism prior to their excretion from the body, assessment of the capability of the liver to metabolize drugs in the course of endotoxemia is essential for the rational formulation of drug dosage regimens in this condition. Adult female rats received a single intraperitoneal injection of endotoxin and were sacrificed 24 hours later. The livers were examined histologically and in vitro activities of microsomal drug metabolizing enzymes were measured in liver homogenates. Control animals showed normal hepatic histology, whereas the livers of endotoxin treated rats disclosed damage of varying degree. Mean values for drug enzyme activities were lower in endotoxin treated animals than in the control rats. Significant decreases in enzymic activities were found for bilirubin uridine-diphosphoglucurony 1 transferase (30\%); aniline hydroxylase $(75 \%)$; benzpyrene hydroxylase $(50 \%)$; nitroreductase $(58 \%)$; azoreductase $(10 \%)$. The content of the rate-limiting electron acceptor, microsomal cytochrome $\mathrm{P} 450$ was $45 \%$ of normal. The relevance of these findings to endotoxemia in children is unknown but drug administration to these patients should be monitored carefully.

162 ZINC \& ACRODERMATITIS ENTEROPATHICA (AE). K. Michael Hambidge, Kenneth H. Neldnex, and Philip A. Walravens. Jniversity of Colorado Medical Center, Depts. of Ped. and Med., Denver.

Oral $\mathrm{Zn}$ has been reported recently to be effective in the treatment of AE. The present study was designed to evaluate $\mathrm{Zn}$ metabolism and to determine the efficacy of oral $\mathrm{Zn}$ therapy in 2 patients with AE. The first patient, a female aged 22 years, had been in prolonged remission on diiodohydroxyquin therapy. When off all therapy and in clinical relapse, her plasma $\mathrm{Zn}$ levels $(<20 \mu \mathrm{g} / 100 \mathrm{~m} 1)$, serum alkaline phosphatase ( a $z$ incdependent enzyme) activity and urine $7 n$ excretion rate (mean = $57 \mathrm{\mu g} / 24 \mathrm{hrs}$.) were all extremely low. Re-introduction of diiodohydroxyquin resulted in partial clinical remission, and was accompanied by a modest increase in plasma $\mathrm{Zn}(30 \mu \mathrm{g} / 100 \mathrm{ml})$. Introduction of oral $\mathrm{ZnSO}_{4} \cdot 7 \mathrm{H}_{2} \mathrm{O}$ therapy (100mg per day) was followed by rapid clinical remission and normalization of plasma zinc levels, serum alkaline phosphatase activity, and urine zinc excretion rate. The second patient, a boy aged 2 yrs., required 1 liter of human milk per day to maintain clinical remission. When human milk was discontinued he had a clinical relapse and plasma $\mathrm{Zn}$ levels fell from 60 to $39 \mu \mathrm{g} / 100 \mathrm{ml}$. Subsequently, clinical remission was achieved with $\mathrm{ZnSO}_{4} \cdot 7 \mathrm{H}_{2} \mathrm{O}$ $350 \mathrm{mg} / \mathrm{d}$. It is concluded that the inherited metabolic defect in $\mathrm{AE}$ results in a severe $\mathrm{Zn}$ deficiency state. Oral $\mathrm{Zn}$ provides a new and very effective means of treatment for this disease. 0 ther therapeutic approaches (diiodohydroxyquin and human milk) lead to a partial correction of the hypozincemia. 
163 DRUG ANALYSIS OF AMNIOTIC FLUID USING GC-MS-COM TECHNIQUES. Reba M. Hill, K. R. Sommer, Lee B. McCulley, M. G. Horning (Intr. by L. Leighton Hili). Dept
for Lipid Res., Baylor Col. of Med., St. Luke's Ped., Inst. for Lipid Res., Baylor Col. of Med., St. Luke's
Episcopal Hosp., Newborn Research Center, Houston, Texas 77025. Episcopal Hosp. administered to gravid females are rapidiy transferred to the fetus and are excreted by the neonate for variable periods of time in the neonatal period. Previous studies have demonstrated both parent drug and metabolites in maternal and neonatal blood and urine depending on the events surrounding labor and delivery. A method using gas chromatography-mass spectrometer computer system (GC-MS-COM) has been used to quantitate drugs in amniotic fluid obtained during labor and delivery. All samples analyzed contained caffeine in amounts ranging from .174-7.561 $\mu \mathrm{g} / \mathrm{m}$ l al though no drug containing caffeine was administered to the mother during labor and delivery. Theobromine (3,7-dimethy)xanthine) a metabolite of caffeine was found in amounts of 0.253 - $1.536 \mathrm{\mu g} / \mathrm{ml}$. Comparison of caffeine and theobromine levels in maternal plasma and cord blood with those of amniotic fluid showed a similar range of parent drug $(.382-6.586 \mu \mathrm{g} / \mathrm{ml})$ and metabolite $(0.118-1.486 \mathrm{\mu g} / \mathrm{mT})$. Samples of amniotic fluid collected after administration of secobarbital and phenobarbital to the mother revealed only the parent drug to be present. No metabolite of either drug was identified on repeat analysis. The absence of metabolites in amniotic fluid may be related to the different fetal methods for metabolism of the 3 drugs. (Supported by NIH GM 16216)

164 A CONVENIENT NEW METHOD FOR MONITORING ADRIAMYCIN BLOOD LEVELS IN CHILDREN. Robert R. Holcomb, Thomas D. Miale, and Kyle Popejoy (Intr. by Owen M. Rennert), Univ. of Florida Col. of Med., Dept. of Ped., Gainesville. Conflicting statements concerning ADR pharmacodynamics have appeared previously. Some authors have reported a consistent plasma half-life and disappearance pattern, while others have found considerable variation among patients. Some investigators have reported significant in vivo degradation of the parent compound to active metabolites; others have reported that ADR is not metabolized and is excreted unchanged in the urine. A reliable method specific for measurement of ADR blood levels has been developed in order to resolve the pharmacodynamics of this drug and to maximize its therapeutic potential. Venous blood samples were taken at timed intervals for 24-48 hours. The analysis consisted of extracting the ADR from a $1 M$ phosphate buffer-serum solution with n-butanol by a countercurrent distribution process. This process specifically extracted ADR and insignificant quantities of its metabolites. The specificity was demonstrated by partition characteristics and thin-layer chromatography. Preliminary results with this method indicate that the disappearance of this drug from the blood occurs in 2 phases. The first is a rapid tissue distribution phase and the second is a slower phase which is most likely due to metabolism, excretion, and redistribution. Correlation of therapeutic efficacy with rate of distribution and metabolism as reflected by ADR concentrations in timed blood samples appears feasible and convenient.

165 MATERRAL ALCOHOLISM AND FETAL ABNORMALITIES. Sylvia Iosub, Nesrin Bingol, Magdalena Fuchs, Intr by Eídward Wasseman, Department of Pediatrics, New York Medical College, New York, New York

A 38 year old $G_{7} P_{5}$ became a severe alcoholic when a teenager. She has a 22 year old normal daughter, born before the onset of alcoholism, a 20 year old son. with cleft palate, microcephaly and microphthaimia and a 17 year old daughter also with microphthalmia and microcephaly. The latter two children, conceived after several years of heavy alcohol intake, are in institutions for the mentally retarded. A fourth male child died at the age of 3 months with congenital heart disease. All children have different fathers.

After 2 previous first trimester abortions, at the age of 31 the mother delivered a female infant who exhibits all the characteristics of the fetal alcohol syndrome. She has some additional features not previously described in the offspring of alcoholic mothers: hypertoloriss, prominent metopic ridge, supernumerary digits, hyperextensible wrists and fingers, bifid pelvis, transient breast hypertrophy and advanced bone age. Laboratory studies including endocrine evaluation were normal. The child is hyperactive and has an I.Q. of 50.

The data suggest that the offspring of the alcoholic teenager is somewhat differently affected than that of the older mother and a direct relationship might exist between the duration (number of years) of heavy alcohol consumption and the severity of malformations in the products of subsequent pregnancies.
16 RAPID SCREENING FOR CHILDHOOD LEAD POISONING BY ZP (ZINC PROTOPORPHYRIN) ASSAY. M. Joselow,

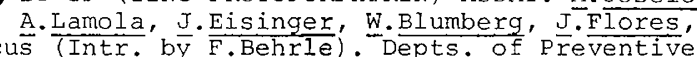

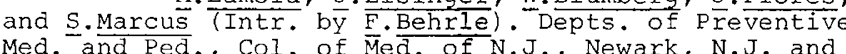
Med. and Ped. Murray Hill, N.J.

The interference of lead with heme synthesis, with subsequent build-up of protoporphyrins in erythrocytes, is well established, and forms the basis for the FEP (free erythrocyte protoporphyrin) tests now widely used for the detection of childhood lead poisoning. The predominant protoporphyrin of FEP, however, has recently been shown to be a chelate, zinc protoporphyrin (ZP), with specific spectral characteristics that permit its direct, rapid, and simple measurement. Using only a drop $(20 \mu \mathrm{l})$ of blood, ZP can be assayed fluorometrically on either the solution obtained by diluting the drop with detergent, or directly on the drop, without prior treatment, when it is smeared on a slide in a specially constructed fluorimeter. In field and hospital trials, this new procedure has proven to be a specific and sensitive screening tool for monitoring for the toxic effects of lead absorption. Applying appropriate cut-off values, more than $95 \%$ of children with lead levels over $40 \mathrm{\mu g} / 100 \mathrm{ml}$ can be detected; and, except for iron-deficiency anemia, other pathological conditions (e.g., erythropoietic protoporphyria, porphyria cutanea tarda, possibly other heavy metal intoxications) that vield high $\mathrm{ZP}$ values are rare.

167

INTERSUBJECT VARIATION OF BILIRUBIN BINDING IN NEONATAL AND ADULT HUMAN PLASMA AND THE DISPLACING EFFECT OF DRUGS. Gerhard Levy and Svein фie. (Intr. by Sumner J. Yaffe). State Univ. of N.Y. Sch. of Pharmacy, Dept. of Pharmaceutics, Buffalo.

The reaction rate method of Jacobsen (FEBS Letters, 5:112, 1969) has been modified to permit determination of bilirubin binding in 0.1 to $0.2 \mathrm{ml}$ undiluted plasma with a bilirubin: alburnin molar concentration ratio of less than unity (i.e., the usual physiologic conditions). Binding studies on plasma samples from 13 normal adults, with $12 \mathrm{mg}$ added bilirubin per $100 \mathrm{ml}$, yielded a range of free fraction values from 1.3 to $2.5 \times 10^{-5}$, with a mean of $1.9 \times 10^{-5}$ (representing $99.9981 \%$ protein binding) Similar studies on umbilical cord plasma from 6 neonates yielded free fraction values of 2.0 to $3.3 \times 10^{-5}$ and a mean value of 2.8 $x 10^{-5}$. This appreciable intersubject variation in the plasma protein binding of bilirubin in a small group of subjects suggests serious limitations in interpretations of the clinical significance of total (free and bound) unconjugated bilirubin concentrations in plasma. There is a strong, positive, linear correlation between the free fraction of bilirubin in plasma containing no drugs and the free fraction in the same plasma samples containing added $(10 \mathrm{mg} / 100 \mathrm{ml})$ sulfisoxazole or salicylic acid. This correlation permits quantitative prediction of the displacing effects of drugs for an individual patient prior to drug administration.

168 PHARMACOKINETICS OF INTRAVENOUS THEOPHYLLINE IN YOUNG CHILDREN. P. Loughnan, D. Sitar, R. Ogilvie, A. Eisen, Z. Fox, and A. Neims. McGill Univ., Montreal Children's Hosp., Depts. Ped., Med., and Pharmacol., Montreal, Canada.

Intravenous theophylline ( $T$ ) is commonly used in the therapy of acute asthma in childhood but we are not aware of any pharmacokinetic data relating to the use of this drug in children under 5 yrs of age. A single IV dose of $3.2 \mathrm{mg} / \mathrm{kg}$ of $\mathrm{T}$ was given over a 10-min period to 10 asthmatic children aged $1-4$ yrs. Serial plasma $T$ concs, were determined over the next 8 hrs by high pressure liquid chromatography. Data from the first 5 patients have been analyzed using the ASAAM-23 program according to a 2-compartment open system kinetic model and are summarized below.

$\begin{array}{lcccc} & \mathrm{Kel}\left(\mathrm{hr}^{-1}\right) & \beta\left(\mathrm{hr}^{-1}\right) & \mathrm{V}_{1}(\mathrm{l} / \mathrm{kg}) & \mathrm{V}_{1} \cdot \mathrm{Kel}(\mathrm{l} / \mathrm{kg} / \mathrm{hr}) \\ \text { Mean } & .365 & .201 & .289 & .087 \\ \text { Range } & .199-.632 & .155-.256 & .113-.569 & .057-.124 \\ \text { S.D. } & .194 & .042 & .169 & .031\end{array}$

Using mean values of $\mathrm{V}_{1} \cdot \mathrm{Kel}$ and $B$ the loading dose and maintenance infusion rate of aminophylline (A) to rapidly achieve and maintain a plasma $\mathrm{T}$ conc. of $10 \mathrm{ug} / \mathrm{ml}$ are $5.4 \mathrm{mg} / \mathrm{kg}$ (load) and $1.1 \mathrm{mg} / \mathrm{kg} / \mathrm{hr}$ (maintenance) ( $\mathrm{T}=0.8 \mathrm{~A})$. This dose of $\mathrm{A}$ is similar to that calculated for adults (load $5.6 \mathrm{mg} / \mathrm{kg}$, maintenance $0.9 \mathrm{mg} / \mathrm{kg} / \mathrm{hr}$, NEJM $289: 600,1973)$. The adult therapeutic range of $5-15 \mathrm{ug} / \mathrm{ml}$ is assumed to apply. In the above patients, this regimen would have achieved plateau $T$ concs. ranging from $7.0-15.3 \mathrm{ug} / \mathrm{ml}$. The data do indicate that in young children there is considerable individual variation in $T$ kinetics. The extent of this variation is similar to that reported in adults. 
169

PHARMACOKINETIC OBSERVATIONS OF DIPHENYLHYDANTOIN (DPH) IN THE NEWBORN AND YOUNG INFANT. Peter M. Loughnan, Gordon Watters, and Allen H. Neims. McGill Univ., Montreal Children's Hosp., Roche Dev. Pharmacol. Unit, Mtl. The elimination rate by newborns of transplacentally acquired DPH is initially slow but variable, and increases significantly in the first few days (Rane et al., Clin. Pharm. Ther., 15:39, 1974). Also, steady state plasma concs. of DPH (P.O. or I.M.) in young infants are unexpectedly low (Jalling et al., Pharmacol. Clin., 2: $200,1970)$. With use of radioimmunoassay (Cook et al., Res. Commun. Chem. Path. Pharm., 5:767, 1973), we confirm the latter finding in 8 infants aged $2.5,3,3.5,6,7,9,20$ and 21 wks who received DPH orally at a mean dose of $4.25 \mathrm{mg} / \mathrm{kg}$ q $12 \mathrm{~h}$. After at least 7 days of therapy, the mean minimum plasma DPH conc. was $2.3(0.5-3.8) \mathrm{ug} / \mathrm{ml}$. Four of these patients began therapy with an IV loading dose of $\mathrm{DPH}$; the log plasma conc.-time curve conformed to a first order one-compartment analysis. The plasma half-life was $6.9(5.5-8.7) \mathrm{hr}$ and apparent volume of distribution 0.75

(0.54-0.89) $1 / \mathrm{kg}$, predicting mean steady state concs. of $<5 \mathrm{ug} / \mathrm{ml}$.

Two full term infants, after termination of seizures with an initial IV loading dose of DPH $10 \mathrm{mg} / \mathrm{kg}$ over $20 \mathrm{~min}$, received oral $\mathrm{DPH}, 4 \mathrm{mg} / \mathrm{kg} \mathrm{q} / 2 \mathrm{~h}$. Plasma DPH conc., assessed daily, surpassed 20 $\mathrm{ug} / \mathrm{ml}$ in both infants 7 days after birth. One of these infants had an observed plasma half-life of $42 \mathrm{hrs}$ on day 2 and $15 \mathrm{hrs}$ on day 9. In both infants mean plasma DPH had fallen to less than 5 ug/ml on the same dosage by day 21. All patients described above received phenobarbital concurrently. These pharmacokinetic observations extend earlier reports and emphasize the rapidly increasing DPH dosage requirements in the first few weeks after birth.

170

MYOCARDIAL INACTIVATION OF NORADRENALINE (NA) AFTER 6-HYDROXYDOPAMINE (6-OHDA) TREATMENT IN YOUNG RATS. H. Nancy McCullough and Mark Nickerson (Intr. by B.S.L.Kidd) Hosp. for Sick Children, Dept. of Ped., Toronto and McGill Univ., Dept. of Pharmacology, Montreal, Canada.

The finding that 6-OHDA sympathectomy in young rats persists for several weeks provides the opportunity to study the role of nerve uptake in the inactivation of NA. The relative importance of mechanisms terminating inotropic responses to NA was compared in isolated papillary muscles from untreated and 6-OHDA treated rats. Diffusion of NA out of the tissues was prevented by immersing the preparation in mineral oil after responses to NA had reached a plateau. Intrinsic mechanisms of inactivation were inhibited by drug treatments. 6-0HDA slowed the inactivation of NA by intrinsic mechanisms 1.7 times, and inhibition of monoamine oxidase by iproniazid, catechol-0-methyl transferase by tropolone, nerve uptake by cocaine and extraneuronal uptake by phenoxybenzamine ( $\mathrm{POB}$ ) prolonged inactivation $1.8,0,2.5$ and 3.4 times rates measured in controls, respectively. Changes in responsiveness to NA resulting from treatment with the inhibitors were also measured. Cocaine and $\mathrm{POB}$ increased the sensitivity to NA 11.3and 13.1- fold in control and $0-$ and 2.1- fold in denervated papillary muscles, respectively. It was concluded that nerve uptake is less important than extraneuronal uptake in terminating the action of NA in rat myocardium, and that increased responsiveness to NA, often considered to reflect the ability of the inhibited mechanisms to dispose of the amine, may not be a reliable measure of termination of action.

171 KINETICS OF INTRAVENOUS THEOPHYLLINE IN CHILDREN CORRELATION WITH PULMONARY RESPONSE.

Keith N. Megathlin, Lorne K. Garrettson, Gilberto E. Rodriquez, John R. Howell, and Jordan L. Cohen. (Intr. by Harold M. Maurer) Departments of Pediatrics and Pharmacy \& Pharmaceutics, Virginia Commonwealth Univ., Med. Col. Virginia, Richmond, VA 23298 .

Theophylline ( $\mathrm{T}$ ) $4.7 \mathrm{mg} / \mathrm{kg}$, (as Aminophylline) was infused at a constant rate for $20 \mathrm{~min}$ in 8 children, age $7-16$. Serum was assayed for $T$ concentration by a liquid chromatographic method. Pulmonary function was assessed by FEV 1.0 and FVC measurements. A two-compartment open model was assumed. Kinetic parameters were estimated by best visual fit of the computer model to serum $\mathrm{T}$ values. Children with $\mathrm{T}$ half-lives as short as 1.5 hours was encountered. Kinetic parameters for the distributive (a) phase were 2-3 times larger than published values for adults (Mitenko, Clin. Pharmacol. Therap. 14:509, 1973). Simulation indicates that the peripheral compartment reaches peak $T$ concentration in 15 to $30 \mathrm{~min}$ after the end of infusion. Al1 children wheezing at the time of study demonstrated improvement in $\mathrm{FEV}_{1.0}$ by the end of infusion. Improvement reached a peak 30 to $60 \mathrm{~min}$ after the end of infusion. Response and peripheral compartment simulation curves are similar in shape and time course. This is interpreted as showing that the response behaves kinetically as being related to peripheral compartment concentration rather than central (serum) compartment concentration of $T$.
172 The VALUE OF THE MEASUREMENT OF FREE ERYTHROCYTE PORPHYRIN (FEP), RED CELL $\delta$-AMINOLEVULINIC ACID DEHYDRATASE (ALAD), AND BLOOD LEAD IN THE DETECTION OF PATIENTS WITH AN INCREASED LEAD BURDEN. Michael $L$. Weitzman, Ernest M. Post, Thelma R. Schneider, Barbara $F$. Oski and Frank A. Oski. S.U.N.Y., Upstate Medical Center, Department of Pediatrics, Syracuse, New York.

A total of 185 lead mobilizations with EDTA were performed in conjunction with measurements of red cell ALAD, FEP, and blood lead in an attempt to determine the sensitivity of these procedures in detecting patients with an increased body lead burden. In addition, 1282 simultaneous FEP and blood lead determinations were performed, of which 505 also had red cell ALAD assays. All parients with blood leads in excess of 60 ugms\% had abnormal FEP's (>200 $\mu \mathrm{gm} / 100 \mathrm{ml} \mathrm{RBC})$ and abnormal ALAD's (<15 units/100 ml RBC). In patients with blood leads between 40 and 60 , 90\% had FEP values in excess of 200 and $76 \%$ had ALAD values below 15 . Red cell ALAD activity most closely correlated with lead excretion following EDTA $(r=.73 ; p<.001)$, although blood lead $(r=.55 ; p<.001)$, and FEP ( $r=.47 ; p<.001$ ) also had significant predictive value. The use of FEP will produce many false positives while the use of the blood lead will miss patients with increased lead burden. The use of the FEP or the ALAD will identify virtually all children with an increased body lead burden (excretion of $>0.5 \mu \mathrm{gm}$ lead/mg EDTA). The FEP is far better suited for mass screening.

173 VARIATIONS IN THE BETA-ADRENERGIC RECEPTOR ACTIVITY WITH AGE. Myung K. Park, Antoni M. Dieh1 and John M. Sunderson. (Intr. by Cheng T. Cho) Univ. of Kansas School of Med., Dept. of Ped. and Ralph L. Smith Human Development Res. Ctr., Kansas City, Ks. 66103

Helically cut strips of thoracic aorta (Ao) and main pulmonary artery (PA) from rabbits of varying age and random sex were examined for their ability to relax with a cumulative dose of isoproterenol (LP) and sodium nitrite (SN) in the presence or absence of appropriate blocker(s) in isolated tissue baths. The bath contained modified Krebs' solution and was oxygenated with $95 \% 02-5 \% \mathrm{CO} 2$ at $37^{\circ} \mathrm{C}$. The relaxation induced by IP and SN was expressed as percent of maximal contraction produced by 5 -hydroxytryptamine $\left(3 \times 10^{-6} \mathrm{M}\right)$. The maximal relaxations (in percent) were as follows:

\begin{tabular}{lrr} 
Group I (I-2 days) & \multicolumn{1}{c}{ IP } & \multicolumn{1}{c}{ SN } \\
Group II (7-10 days) & 15.5 & 125.6 \\
Group III (1 mo.) & 53.5 & 115.2 \\
Group IV (4-5 mos.) & 63.4 & 101.6 \\
\end{tabular}

\begin{tabular}{rr}
\multicolumn{1}{l}{ IP } & PA \\
\hline 3.4 & SN \\
11.7 & 100.0 \\
57.8 & 101.0 \\
64.1 & 101.2
\end{tabular}

The magnitudes of relaxation produced by $\mathrm{SN}$ were the same in all four groups but those produced by IP were significantly less in the younger age groups ( $I \& I I$ ) than in the older age groups (III \& IV) in both Ao and PA $(p<0.01)$. These findings indicate that the beta-adrenergic receptor function is not fully developed in the younger age groups and it matures with age in both Ao and PA. Thomas F. Roe, Robert L.Podosin, Milan E. Blaskovics (Intr. by Maurice D. Kogut), USC School of Medicine, Childrens Hospital of Los Angeles, Dept. of Ped., Los Angeles. In 2 children treated for hypoglycemia and convulsions with oral diazoxide and diphenylhydantoin(DPH) therapeutic serum DPH levels $(10-20 \mu \mathrm{g} / \mathrm{ml})$ were not achieved despite DPH dosages of 17 and $30 \mathrm{mg} / \mathrm{kg} /$ day respectively, maintained for $>6$ months. The patient receiving $17 \mathrm{mg} / \mathrm{kg} /$ day became DPH intoxicated when diazoxide administration was discontinued. Subsequently, therapeutic serum DPH levels were maintained in both patients with DPH dosages of 6.6 and $10 \mathrm{mg} / \mathrm{kg} /$ day, respectively, in the absence of diazoxide administration. In one patient when diazoxide administration was restarted experimentally, serum DPH fell to undetectable levels over a 3 day period and seizures occurred; urine 5-( $p$ hydroxypheny 1)-5-phenylhydantoin, the major metabolite of DPH, increased 400 percent above the baseline. A similar fall in serum DPH levels occurred following diazoxide administration while DPH was given intramuscularly. ${ }^{14} \mathrm{C}-1$ abeled $\mathrm{DPH}$ was added in vitro to plasma samples obtained from a patient before and after $\frac{1 \text { days }}{5 \text { dat }}$ of diazoxide administration and to plasma samples from untreated age-matched controls, and plasma protein binding of DPH was measured. Significantly less $(p<0.0001)$ protein binding of DPH was found in the plasma obtained during diazoxide administration.

We conclude that diazoxide administration lowers serum DPH

levels in man. The low DPH levels are best explained by rapid DPH metabolism; decreased plasma protein binding of DPH may play a role. 

175 Dinetic ANALYSIS. Howard J. Rogers, Robert A. Haslam, James Longstreth, \& Paul S. Lietman, Depts. of Ped. and Med., Johns Hopkins Univ., Baltimore, Md.

DPH parahydroxylation is a saturable process obeying MichaelisMenten kinetics and is probably rate-limiting in drug elimination. Thus the plasma levels are not linearly related to dose and small changes in enzyme activity as might occur with concurrent drug therapy could markedly alter plasma DPH. High diazepam concentrations depress DPH metabolism by rat Iiver microsomes but effects in man are controversial. We report 2 cases where diazepam therapy produced DPH intoxication. The first, a $2 \frac{1}{2}$ year old white male, received $7.5 \mathrm{mg} / \mathrm{kg} /$ day DPH for post-meningitic seizures but became intoxicated when diazepam $(0.6 \mathrm{mg} / \mathrm{kg} / \mathrm{day})$ was added. DPH ( $15 \mathrm{mg} / \mathrm{kg}$ ) was given intravenously and repeated serum levels estimated by radioimmunoassay. " $\mathrm{K}_{\mathrm{m}}$ " and "Vmax", computed by an iterative program (NONLIN) were $\leqslant 0.2 \mu \mathrm{g} / \mathrm{ml}$ and $0.33 \mu \mathrm{g} / \mathrm{ml} / \mathrm{hr}$ respectively. Repetition of this dose 2 months after discontinuation of diazepam gave $" \mathrm{~K}_{\mathrm{m}} "=13.7 \mu \mathrm{g} / \mathrm{ml}$ and $" V \max "=1.1 \mathrm{l} \mu \mathrm{g} / \mathrm{ml} / \mathrm{hr}$ The second patient, a $5 \frac{1}{2}$ year old black male, received DPH (7.5$10 \mathrm{mg} / \mathrm{kg} /$ day) for over a year following neurosurgery. Adding diazepam $(0.4 \mathrm{mg} / \mathrm{kg} / \mathrm{day})$ was associated with toxicity. Intravenous $D P H$ was given as before yielding " $K_{m} "=\leq 0.1 \mu \mathrm{g} / \mathrm{ml}$ and "Vmax" $=0.24 \mu \mathrm{g} / \mathrm{ml} / \mathrm{hr}$ with diazepam and $" \mathrm{~K}_{\mathrm{m}} "=23.8 \mu \mathrm{g} / \mathrm{mI}$ and "Vmax"=0.65 $\mu \mathrm{g} / \mathrm{ml} / \mathrm{hr}$ without diazepam. These two patients illustrate the ability of diazepam to inhibit the elimination of DPH. Possibly this interaction extends to other benzodiazepines used concurrent ly with DPH.

176 PHARMACOKINETICS OF CEPHALOTHIN (C) AND DEACETYLCEPHALOTHIN (D) IN ADULTS AND CHILDREN: APPLICATION OF HIGH SPEED LIQUID CHROMATOGRAPHY IN CLINICAL PHARMACOLOGY. Thomas F. Rolewicz, Marion w. Anders, Mehroo Cooper and Bernard L. Mirkin. Univ. of Minnesota Hosp., Division of Clinical Pharmacology, Depts. of Ped. and Pharmacology, Minneapolis, Minnesota.

The extensive use of antibiotics in clinical practice, especially in the pediatric age group, has created a need for rapid, sensitive and precise methods of antibiotic analysis for the determination of antibiotic disposition. Presently most methods are based upon microbial bioassays. However, these methods do not allow for the differentiation between the parent compound and its biologically active metabolites. Utilizing ion pair extraction and high speed liquid chromatography, the disposition of $C$ and its major metabolite $D$ was carried out in adult and pediatric aged clinical subjects. Serial blood and urine samples were obtained and analyzed for $C$ and $D$ following the intravenous (I.V.) admin. of C. The half-life ( $\mathrm{T}_{\frac{1}{2}}$ ) for the disappearance of $C$ from the serum in pediatric subjects averaged $14.9 \pm 2.0 \mathrm{~min}$. This value was not significantly different from that obtained in adult subjects $(11.8 \pm 1.0 \mathrm{~min})$. Within 5 hours $70 \%$ of an I.V. dose of $C$ was excreted in the urine unchanged and another 208 as $D$. The $\mathrm{T}^{\frac{1}{2}}$ obtained by chromatographic methods of analysis is considerably shorter than that reported using microbial assays $\left(T^{\frac{1}{2}}=50-60 \mathrm{~min}\right)$. (Investigation supported by USPH Grant No. 08580).
178 PHENOBARBITAL DOSE AND PLASMA LEVEL IN THE NEONATE. T. S. Rosen and C. E. Pippenger, Coll. of Physicians and Surgeons, Columbia University, Dept. of Ped. and

Neurol., N.Y.C. (Intr. by L. S. James)

To investigate the relationship between phenobarbital dose, attainment of therapeutic plasma concentration $[\mathrm{Pb}]$ and urinary excretion, we studied 19 infants ages 1 day -2 mo., who were treated for seizures (asphyxia, meningitis or idiopathic) or for methadone and/or heroin withdrawal, with $\mathrm{Pb}, 5-15 \mathrm{mg} / \mathrm{kg} /$ day. Five capillary fubes of blood were drawn from each infant for the 1st 4-5 days of treatment and then every 2-3 days. [Pb] was measured by gas chromatography or EMIT Immunoassay System. Urine was collected for 24 hours for 3 days and then randomly every $2-5$ days. The therapeutic $[\mathrm{Pb}]$ was $20-30 \mathrm{ug} / \mathrm{ml}$. Infants given $\mathrm{Pb}, 5 \mathrm{mg} / \mathrm{kg} /$ day did not attain therapeutic $[\mathrm{Pb}]$ until day $7-8$ of treatment. Infants given $\mathrm{Pb}, 10-15 \mathrm{mg} / \mathrm{kg} /$ day attained therapeutic [ $\mathrm{Pb}]$ in $48-72$ hours. When this dose regimen was continued, toxic $[\mathrm{Pb}],>40 \mathrm{Ng} / \mathrm{ml}$ ) were reached by day $4-5$. At all dose levels, a decrease in $[\mathrm{Pb}]$, possibly due to liver enzyme induction or increased urinary. excretion occurred on day 17-19. $\mathrm{Pb}$ was excreted unchanged in urine until about day 10 when there were small amounts of $\mathrm{p}-\mathrm{OH}$ phenobarbital present. At toxic $[\mathrm{Pb}]$ unmetabolized urinary $\mathrm{Pb}$ was increased.

Our study suggests that for the conditions treated $\mathrm{Pb}, 10-15 \mathrm{mg} / \mathrm{kg} / \mathrm{day}$ results in therapeutic $[\mathrm{Pb}]$ in 2-3 days. Thereafter therapeutic $[\mathrm{Pb}]$ can be maintained with $\mathrm{Pb}, 5 \mathrm{mg} / \mathrm{kg} /$ day every $6-8$ hours. Close monitoring of $[\mathrm{Pb}]$ is necessary to assure therapeutic $[\mathrm{Pb}]$ and prevent $\mathrm{Pb}$ toxicity.

179 EFFECTS OF SYSTEMIC NOREPINEPHRINE (NE) ON DISTRIBUTTION OF UTERINE BLOOD FLOW (UBF) IN PREGNANT EWES. Charles R. Rosenfeld (Intr. by Chester Fink). Depts. of Ped. and Ob-Gyn, Southwestern Med. Sch. at Dallas.

Alterations in UBF may follow administration of pharmacoloaic agents employed in clinical obstetrics, adversely affecting fetal wel1-being. Development of a chronic preqnant sheep preparation has permitted evaluation of responses in magnitude and distribution of UJBF to these agents. We have studied the effects of a continuous systemic infusion of NE on the distribution of UIBF in seven pregnant ewes from 100 to 137 days gestation. Dose response curves were determined for each animal and a rate of infusion leading to a significant reduction in UBF without a significant change in systemic pressure was identified (mean $0.237 \mu \mathrm{g} / \mathrm{min} \cdot \mathrm{k} \eta \pm$ $0.035)$. Labeled microspheres were infused into the left ventricle prior to and during the constant infusion of NE at the previously identified rate. UBBF decreased from a mean of $1233 \mathrm{ml} / \mathrm{min} \pm 196$ to $817.4 \mathrm{ml} / \mathrm{min}+137(31.9 \% \pm 5.24)$. Reductions in blood flow occurred in each uterine tissue: myometrium decreased from $41.2 \mathrm{ml} / \mathrm{min} \pm 6.8$ to $22.4 \mathrm{ml} / \mathrm{min} \pm 4.1(-45 \%)$, endometrium from $196 \mathrm{ml} / \mathrm{min} \pm 24$ to 72.6 $\mathrm{ml} / \mathrm{min} \pm 10.9(-62 \%)$ and placental cotyledons from $996 \mathrm{ml} / \mathrm{min} \pm 174$ to $723 \mathrm{ml} / \mathrm{min} \pm 125(-25 \%)$. Decreases in blood flow also occurred in other reproductive tissues. Cardiac output decreased $17.8 \%+4.9$. These observations demonstrate that $N E$ is a potent vasoconstrictor in all tissues of the pregnant ovine uterus, that the response in each tissue is different, and that cardiac output and blood flow to other tissues may also be affected.
177 RED CELLLIEAD (RBC-Pb): USE OF THE CALCTUM IONOPHORE, PARTMENT Einstein Col. Med。, Montefiore Hospo \& MedoCtro, Dept。 Pedo, and The Rockefeller University, New York。

Previous studies in vitro demonstrated rapid displacement of RBC-Pb by physiological levels of $\mathrm{Ca}^{++}, \mathrm{Zn}^{++}, \mathrm{Cu}^{++}$, and $\mathrm{Fe}^{++}$. This fraction is likely membrane bound and accessible for physiochemical exchange and complex formation at the RBC surface.To characterize further $\mathrm{RBC}-\mathrm{Pb}$ compartments, forced entry of $\mathrm{Ca}^{++}$into RBC's was achieved by introducing $\mathrm{Ca}^{++}+\mathrm{A} 23187$ into the incubation medium. A23187 forms lipophilic complexes with $\mathrm{Ca}^{++}$, allowing their rapid entry into cells. $\mathrm{RBC}$ 's were collected from $\mathrm{Pb}$ intoxicated rats, washed, and incubated for $15 \mathrm{~min}$ 。 at $\mathrm{pH} 7.40$ in a balanced salt solution, to which $\mathrm{Ca}^{++}(0-2.50 \mathrm{mM})$ and/or A23187 ( $0-2.80 u M)$ were added.The results, as the mean change in $\mathrm{RBC}-\mathrm{Pb}$ (ug/dl) of the pre-vs。 post-incubation sample, were: (1) controls: $\pm 2.60 \pm .21$; (2).50mM Ca++: $-2.23 \pm .41$; (3) $.50 \mathrm{mM}$ $\mathrm{Ca}^{++}+2.8 \mathrm{O}_{4} \mathrm{M}$ A23187: $-9.83 \pm 1.02[\mathrm{p}<.001$ vs。(2)]。 Hence, enhanced displacement with ionophore was found at a suboptimal $\mathrm{Ca}^{++}$concentration.At an optimal level of $\mathrm{Ca}^{++}(2.5 \mathrm{mM})+\mathrm{A} 23187$, no further displacement was observed compared to $2.50 \mathrm{rmM} \mathrm{Ca^{++ }}$ alone: $-28.31 \pm 1.78$ and $-26.31 \pm 1.98$, respectively.

These data suggest that: 1 )The exchangeable $\mathrm{RBC}-\mathrm{Pb}$ compartment is separable into 2 components---one within or below the RBC membrane, and the larger one at its surface, 2) Metal-metal interactions $\left(\mathrm{Ca}^{++}, \mathrm{Zn}^{++}, \mathrm{Cu}^{++}, \mathrm{Fe}^{++}\right)$are regulators of $\mathrm{Pb}$ transport from RBC to plasma, and 3)Changes in levels of these metals in serum \& ECF may influence $\mathrm{Pb}$ transport and the toxic activities of $\mathrm{Pb}$.
CHANGES IN DRUG METABOLISM IN CHILDREN WITH

180 THYROID DISORDERS. P.Saenger,A.B.Rifkind,M.I New, Cornell Univ.Med.Col., Dept.Ped.New York,

The rate of disappearance of antipyrine from the plasma is a useful indicator for the in vivo capacity for hepatic mixed function oxidation. The half life of antipyrine(t1/2) was measured before and after treatment of 3 hypothyroid and 3 hyperthyroid children, ages 3 months to 14 years. The $t I / 2$ decreased in all 3 hypo thyroid subjects $\&$ increasedin all 3 hyperthyroid subjects after treatment. The mean $t 1 / 2$ decreased from 34.4 to $8 \mathrm{hrs}$ after treatment of the hypothyroia subjects and increased from 6.7 to 9.7 hrs after treatment of the hyperthyroid subjects. The most remarkable change was observed in a 3 month old cretin in whom the $t 1 / 2$ dropped from 80 to $11.5 \mathrm{hrs}$ after 2 weeks of treatment. The mean metabolic clearance rate (MCR) of antipyrine increased by $243 \%$ with treatment in hypo and decreased by 698 with treatment in hyperthyroid children. The apparent volume of distribution did not change significantly with treatment, thus changes in the $t 1 / 2$ were attributable alone to alterations in the MCR of antipyrine. These studies show that thyroid disordexs in childhood are accompanied by marked alterations in the rate of hepatic drug metabolism. physicians should be aware of such changes when administering drugs metabolized by the liver to children with thyroid disorders. 
181 EFFECT OF PHOTOTHERAPY ON HEPATIC MICROSOMAL DRUG METABOLIZING ACTIVITY OF RATS. Lester F. Soyka, William G. Hunt and Jerold F. Lucey. Depts. of Pharm. and Ped., Univ. of Vermont, Col. of Med., Burlington.

The widespread use of phototherapy for neonatal hyperbilirubinemia has raised questions regarding long-term safety. It is known that drug metabolizing capability is deficient in newborns and it has been proposed that its postnatal development might be influenced by phototherapy. Environmental lighting is known to affect the diurnal rhythm of hepatic drug metabolism of adult rats (Life Sci. 8:1291, 1969). Rats of different ages were exposed to ca. $150^{-} \mathrm{ft}$. candles of $11 \mathrm{ght}$ with maximum intensity in the blue range continuously for 5 days. Duration of sleep following admin. of hexobarbital was not affected by light exposure. Microsomal activity was assayed under optimum conditions by methods developed in our laboratory with aminopyrine, ethylmorphine, p-nitroanisole, aniline or benzo(a)pyrine (BP) as substrates. An increase in aminopyrine $\mathrm{N}$-demethylase activity was seen in rats exposed to light beginning at 17 or 30 days of age, and in rats exposed as newborns (2-7 days) and studied at 26-30 days of age. In no other assay was there ever a difference between lightexposed and control groups. In one experiment BP hydroxylation was doubled by phototherapy of 25 day old rats but this was not confirmed in a repeat experiment. Conclusion: Preliminary results indicate that phototherapy produces little or no alteration in microsomal drug metabolism, though further studies are needed to rule out a selective increase in aminopyrine demethylation. Supported in part by USPHS Grant HD 08708.

182 METABOLISM OF BENZO(a)PYRENE (BP) BY HUMAN FIBROBLASTS. Lester F. Soyka, William G. Hunt, Jenness B. Majeska and Richard J. Albertini. Depts. of Pharm. and Med., Univ. of Vermont Col. of Med., Burlington. $B P$ is a prototype for polycyclic aromatic hydrocarbons (PAH), chemicals known to cause cancer in experimental animals and found as pollutants of the atmosphere, soil, water and food chain. BP, itself inactive, is enzymatically oxygenated, often with epoxide intermediates, to proximate carcinogens and inactive metabolites. This enzymatic capability is found in many tissues, and is susceptible to induction upon exposure to PAH. Inducibility may be genetically determined and has been linked to carcinogenesis. We have incubated ${ }^{3} \mathrm{H}-\mathrm{BP}$ (25 ug/dish) with diploid, euploid fibroblasts from foreskins ( $2 \times 10^{6} \mathrm{ce} 1 \mathrm{~s} /$ dish) and determined formation of water soluble and alkali extractable metabolites. After $72 \mathrm{hrs}$ incubation three cell strains converted $6-17 \%$ of substrate to water soluble and $1.1-1.5 \%$ to alkaline extractable metabolites. These data confirm Huberman and Sachs (Int. J. Ca. 11:412, 1973) observation that fibroblasts, in contrast to hepatic microsomes, convert $\mathrm{BP}$ primarily to water soluble metabolites. Studies are in progress to define inducibility and to characterize metabolites.

183 POSTMORTEM TISSUE DIGOXIN IN INFANTS AND CHILDREN.

$183 \frac{\text { Lester F. Soyka, Pu Woong Kim, Richard W. Krasula and }}{\text { Alois R. Hastreiter. Dept. of Ped., Abraham Lincoln }}$ Alois R. Hastreiter. Dept. of Ped., Abrahan

School of Med. and Univ. of Illinois Hosp., Chicago, IL.

cokinetics of any drug and may be an age-dependent variable. Digoxin was determined by a radioinmunoassay developed in our laboratory ( $J_{-1}$ Ped.81:566,1972) using an antibody with affinity of $3.6 \times 10^{9} \mathrm{M}^{-1}$ and Sips index of heterogeneity of 0.94 .

Group $N$ Daily Dose L. Vent $\frac{\text { Kidney }}{n g / \mathrm{Nuscle}} \quad \underline{\text { Serum }}$ $\begin{array}{lcccccc} & & \mu \mathrm{gg} / \mathrm{kg} & \mathrm{ng} / \mathrm{g} & \mathrm{ng} / \mathrm{g} & \mathrm{ng} / \mathrm{g} & \mathrm{ng} / \mathrm{m} / \\ \text { Premies } & 7 & 9 & 191 & 73 & 37 & 1.9\end{array}$ $\begin{array}{lrrrrr}\text { Full-Term 4 } & 24 & 196 & 198 & 32 & 2.7 \\ \text { Children 4 } & 15 & 74 & 232 & 8 & 0.6\end{array}$ Atrial conc. were lower than ventricular in infants but equal in children. Liver, gal1 bladder, sm. and 1 g.intestine had mean conc. of 40-90 ng/g whereas other tissues (spleen, skin, lung, thyroid, Adrenal, etc) had mean conc. of 20-40. Fat always had the lowest conc. ( $4-8 \mathrm{ng} / \mathrm{g})$. Brain conc. in premies was significantly lower than in children ( 7 vs $21 \mathrm{ng} / \mathrm{g}$ ). Children's Rt. atrial levels were equal to those found in children at open heart surgery (Circ.49:1047,1974) providing some assurance of the validity of these postmortem data. Low kidney levels of premies may be responsible for their limited capacity to excrete digoxin. In infants skeltal muscle appears to conc. digoxin. Conclusions: High myocardial levels of digoxin in infants suggest that routine doses may be too high. In premies very low renal digoxin may explain their propensity to toxicity and high tissue levels despite treatment with relatively low doses.
GLUCURONIDE CON.JUGATES IN PREGNANT AND NON-PREGNANT RATS WITH AND WITHOUT PHENOBARBITAL TREATMENT.S.Vaisman and L.M.Gartner, Dept.Ped. Albert Einstein, N.Y. In a previous study we demonstrated that the stimulatory effect of phenobarbital (PB) on hepatic conjugation (CONJ) of bilirubin (B) with glucuronic acid decreases progressively during the course of pregnancy ( $p^{\prime} c y$ ) in the rat, from a $62 \%$ increase during days 5 through 9 to $19 \%$ on the 21 st day (Ped.Res. $8: 366,1974$ ). It has been reported that $1 / 3$ of the $B$ conjugates in the non-pregnant (NP) adult rat are with glucose (G) and xylose (X). We postulated that a change in this ratio occurs during $p$ 'cy either with or without $P B$ stimulation. A shift to non-glucuronide conjugates would then have accounted for the decrease in PB stimulated glucuronide CONJ. In this study the CONJ of $B$ with $G$ and $X$ during $p^{\prime} c y$ was examined in comparison with the NP rat with and without PB stimulation. The results are expressed in comparison to B glucuronide formation in the NP control group indicated as 100\%: NON-PREGNANT

Glucuronic Xylose Glucose Glucuronic Xylose Glucose

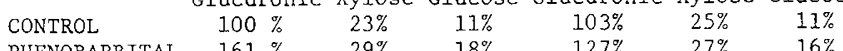

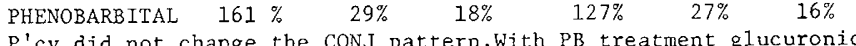
the $P$ rats. $X$ and $G$ CONJ in response to $\mathrm{PB}$ was not altered by $\mathrm{p}^{\prime} \mathrm{cy}$. In both NP and $\mathrm{P}$ the increase in $X$ CONJ was not significant, while the increase in $G$ coNJ was significant after $\mathrm{PB}$ treatment. A shift from glucuronide CONJ to $X$ or $G$ CONJ of $B$ does not occur during $p^{\prime} c y$. An alternate explanation must be sought to explain the decreased response to PB stimulation during $P^{\prime} c y$.

\section{DEVELOPMENTAL PHARMACOLOGY}

185 RE-EVALUATION OF HORMONAL REPLACEMENT P. L. Calcagno) Georgetown Univ. Sch. of Med., G.U. Hosp., Dept. of Ped., Washington, D. C.

Among various thyroid preparations, hormonal replacement therapy with thyroxine (T4) most closely resembles physiologic circumstances. Dosage is based on a previous concept advocating high levels of T4 to compensate for the lack of T3. Since a significant portion of $\mathrm{T} 3$ derives from extrathyroidal conversion of $T 4$, a re-evaluation of therapeutic dosage seems indicated. Twelve children ages 9-17 yrs. requiring chronic thyroid medication were placed on a daily a. $\mathrm{m}$. dose of T4. At various intervals blood was drawn for T4 assay by CPB method and for T3 and TSH by radioimmunoassay. Adequacy of replacement therapy was confirmed by clinical and biochemical parameters. The average daily dose of $\mathrm{T} 4$ was $3.64 \pm 0.64 \mu \mathrm{g} / \mathrm{kg} /$ day. T4 level 10. $5 \pm 2.2 \mu \mathrm{g} \%$ was slightly higher than controls $9.4 \pm 1.2$ but well within the normal range $5.4-13.4 \mu \mathrm{g} \%$. T3 level of $125 \pm 22 \mathrm{ng} \%$ was slightly but not significantly lower than $135 \pm 17.5 \mathrm{ng} \%$ in controls and TSH level $2,6 \pm 3 \mu \mathrm{u} / \mathrm{ml}$ was comparable to the controls of $1.9 \pm 2 \mu \mathrm{u} / \mathrm{ml}$. The administered dose of T4 $2.5-4 \mu \mathrm{g} / \mathrm{kg} /$ day is considerably less than the recommended dose $5-8 \mu \mathrm{g} / \mathrm{kg} /$ day. These observations indicate that physiologic requirements of T4 are much lower than those previously recommended and that new standards need to be established.

186 ENHANCED LINEAR GROWTH RATE, WEIGHT GAIN, AND INSULIN RELEASE IN HYPOPITUITARISM BY CYPROHEPTADINE (CP) PLUS HUMAN GROWTH HORMONE (HGH) VS HGH ALONE. $\underline{A} \cdot \underline{V}$. Agustin, M. deLevie, $N$. Hopwood, $\underline{A} \cdot \underline{\text { Drash }}, \underline{T} \cdot \underline{\text { Foley }}$, and E. Kenny (Intro. by T. K. Oliver, Jr.), Pittsburgh, Pa.

Because of the scarcity of hGH for treatment of hypopituitarism, we tested the hypothesis that $C P$ would increase appetite, thereby increasing the supply of amino acids and calories for incorporation during hGH therapy resulting in better growth than with hGH alone. Seven hypopit. patients received either hGH or hGH and CP for 6 -month periods with cross over. Both linear growth and wt. gain were significantly better on combined thexapy in this longitudinal study. The remainder recelved hGH or hGH and $C P$ for 1 year. Linear growth and wt. gain were greater on combined therapy. Data on all patients are sumarized in the table; $\star \star * x<0.025,{ }^{*} \mathrm{p}<0.05$.

\begin{tabular}{|c|c|c|c|c|c|c|c|}
\hline & $\mathrm{N}$ & First Six Mo & nths & & Seco & $\overline{d s i x}$ & Months \\
\hline $\mathrm{HH}+\mathrm{CP}$ & $\begin{array}{r}11 \\
9\end{array}$ & $\begin{array}{ll}\Delta \mathrm{Ht} & 4.7 \\
(\mathrm{~cm}) & 6.8^{\star *}\end{array}$ & $\begin{array}{l}\Delta W t \\
(\mathrm{~kg})\end{array}$ & $\begin{array}{l}2.1 \\
8.5^{*}\end{array}$ & $\Delta \mathrm{Ht}$ & $\begin{array}{l}3.5 \\
4.5\end{array}$ & $\Delta W t$ \\
\hline
\end{tabular}
The insulin responses to oral glucose tolerance were increased at each time interval on $\mathrm{hGH}$ and $\mathrm{CP}$ with $\mathrm{p}<0.05$ at $1+2 \mathrm{hrs}$, vs control or hGH alone. Since biological effects of insulin and somatomedin are additive in vitro (Rec. Prog. Horm. Res. 1974), the increases in insulin secretion, amino acid and caloric supply may account for the improved growth. Weight loss to previous percentiles occurred after stopping $C P$; there was no tendency to produce permanent obesity. 
187 INCOIPLETE DIGEORGE SYNDROME IN TWO MALE INFANTS . Arulanantham, M. Genel, Jo Dwyer, and F.R. Singer Depts. of Pediat. and Med., Yale Univ.Sch. of Med., New Haven, Ct. and Dept. of Med., LAC-USC Medical Center, USC Sch. of Med., Los Angeles, Cal. (Intr. by Joseph B. Warshaw)

DiGeorge syndrome ( $3 \mathrm{rd}$ and 4 th brachial arch syndrome) includes absent thymus and parathyroid glands, classically presenting with neonatal tetany and unusual susceptibility to infection, associated with abnormal facies and anomalies of the aortic arch and esophas We have observed two male infants who appear to have an incomplete form of this disorder. Though persistent, hypocalcemia improved with age in both, requiring only $\mathrm{Ca}^{++}$supplementation. Neither demonstrated an unusual propensity to infection.

Both presented with neonatal tetany, absent thymic shadows and in vitro $T$ cell dysfunction assessed by mitogenic stimulation and mixed lymphocyte culture. T cell numbers were persistently $<30 \%$ ( $S R B C$ rosette formation) with $B$ cell numbers normal or $\uparrow$ (fluorecein staining of surface immunoglobulins). PHA skin test was negative in both. One also had a Type IV truncus arteriosus defect plus esophageal dysfunction requiring gastrostomy feeding and expired from cardiac complications at 8 months of age. The other was restudied at 15 months of age, when he was retarded in development ( 6 months) and in growth $(-2$ S.D.). Parathormone was inappropriately low for serum $\mathrm{Ca}(7.6 \mathrm{mg} . \%)$ and failed to rise after EDTA infusion. Although PHA skin test became positive, in vitro $T$ cell abnormalities persisted, despite clinical absence of infection.

Incomplete forms of the DiGeorge syndrome may present with per sistent mild neonatal hypocalcemia, even without severe infection, and can be detected by sensitive tests of $\mathrm{I}$ cell function.

188 EFFECIS OF ACUTE AND CHRONIC METHYLPHENIDATE(M) ADMINISTRATION ON GLUCAGON-INDUCED GROWTH HORMONE SECRETION. Theodore W. AvRuskin, Shiu-C. Tang, and Christina S. Juan. Dept. Ped., The Brookdale Hosp. Med. Ctr., New York Univ. Sch. Med., New York.

Chronic methylphenidate (Ritalin) administration has been reported to decrease weight and retard linear growth. To investigate growth hormone secretion during $M$ therapy, five healthy short-statured children had IV glucagon tests $(G: 0.03 \mathrm{mg} / \mathrm{kg} ; \max .1 \mathrm{mg})$ before and after 3 days of $M(10 \mathrm{mg}$ twice daily), and serial blood samples were obtained for blood sugar (BS, mgs), insulin (IRI, $\mu \mathrm{U} / \mathrm{ml}$ ) and growth hormone(IRGH, ng/ml). Three males with hyperkinetic syndromes had IVG while on chronic $M$ therapy:one had tests at 2 days, 60 days, and 120 days while receiving $50 \mathrm{mgs}$. daily; two had tests aiter 1 month and 1 year of treatment. Acute studies revealed base line $\mathrm{BS}, 78 \pm 8.6(\mathrm{M} \pm \mathrm{SE})$; control and peak IRI, $9 \pm 2$ and $102 \pm 29$; control and peak IRGH, $1.1 \pm 0.3$ and $12.3 \pm 2.4$. After $M$ therapy, baseline BS, $84 \pm 6.0$, baseline and peak IRI, $14 \pm 4$ and $89 \pm 21$; baseline and peak IRGH were $1.4 \pm 0.2$ and $11.9 \pm 2.4$. No significant differences were noted in max. IRGH concentration, areas under IRGH curves, ortimes of maximum IRGH responses after acute $M$ administration. Peak IRGH responses in the patient tested 3 times while on chronic therapy were $12.6,12.0$, and $14.0 \mathrm{ng} / \mathrm{ml}$; while peak IRGH concentrations af ter IVG in the other two males were 12.5 and $16.8 \mathrm{ng} / \mathrm{ml}$. These data, while preliminary, would suggest that Ritalin administration has no inhibitory effect on acute growth hormone release, and that retardation in linear growth could be secondary to decreased caloric intake.

LH AND FSH RESPONSE TO LH-RH In NORMAL AND MAL-

189 NOURISHED CHILDREN. Dorothy J. Becker, Bernard L. Pimstone, and Shirley Kronheim, Dept. of Child Health and Medicine, Univ. of Cape Town, Observatory, Cape, South Africa (Intr. by Allan L. Drash)

The advent of the immunoassay of LH and FSH and the synthesis of LH-releasing hormone (LH-RH) has provided another tool to assess pitultary function, even in young children.

A total of 27 children aged 8-34 months were studied. Ten control children and 17 children with protein-calorie malnutrition (P.C.M.) were given LH-RH. It was found that normal children at a young age have measurable basal levels of both LH and FSH.

Their response to LH-RH showed a similar percentage increase to that found in adults, although absolute levels were lower. Both basal and stimulated levels of FSH were usually greater than LH, unlike the situation in older children and adults. Differences between the sexes were inconsistent. In P.C.M. the basal levels of LH and FSH were normal as was the FSH response to LH-RH. However, the LH response was statistically diminished and rose to normal on clinical recovery. This is in contrast with the other pitultary hormones with the exception of prolactin which have normal or increased basal levels and responses to stimulation.
190 LONG TERM THERAPY OF OSTEOGENESIS IMPERFECTA (O.I.) Salvador Castells, Downstate Medical Center, Dept. of Pediatrics, Brooklyn, N.Y. We have shown that in 0.1 . the $24 \mathrm{hr}$. exchangeable calcium (Ca) pool is two to three times the mean value of controls. Synthetic salmon calcitonin reduces the exchangeable $\mathrm{Ca}$ pool size (Curr. Therap. Res. 16:1,74). Calcitonin in 0.1 . may be inhibiting sket etal resorption, reduce skeletal turnover and increase Ca accumulation by the skeleton, (Clin. Orth. 93:239,73). 22 patients, 11 males and 11 females, ages from 22 yrs. to $6 \mathrm{~m}$. with $0 . x$. tarda and congenita of different types of severity have been receiving synthetic salmon calcitonin for periods of time ranging from 22 to $2 \mathrm{~m}$. Calcitonin was given subcutaneously at $2 \mathrm{MRC}$ units per $\mathrm{Kg}$. three days a week. $270 \mathrm{mg}$. of Ca were given daily as NeoCalglucon. Serum Ca, P, alk phosphatase and chemistries by SMA12 were not altered by calcitonin. Serum PTH, by immunoassay, showed no changes. Computed radiological bone mass and density assessed from the scanning of X-ray of the left wrist and hand (C.Colbert's method), obtained prior to and every three months during therapy indicated that in the patient with the longest period of therapy the rate of increase in bone density relative to the normal rate is such that about $36 \mathrm{~m}$. of therapy will be required for the bones to achieve normal density. Marked decrease in the number of fractures have occurred. The lack of adverse reactions and the clinical improvement, indicates that long term administration of calcitonin may improve the osteoporosis of 0.1 . Supported by the NIH Grant RR-318.

TESTOSTERONE (T) METABOLISM IN HYPOPITUITARY CHILOREN. Kathleen H. Chance, Thomas Moshang, Jr. and Alfred M. Bongiovanni Dept. of Hahnemann Med. Col. Both animal and clinical studies have suggested an interrelationship between pituitary hormones and androgen biologic action. Therefore, it was of interest to study the metabolism of $T$ in hypopituitary children. A single pharmacologic dose of $T$ was administered to 7 children with pituitary hormone deficiencies and urine collected for 3 days. Urinary $T$ glucuronide was determined by competitive protein binding assay. Urinary C-19 metabolites were evaluated by GLC. All of the hypopituitary patients excreted low levels of $T$ alucuronide and $\mathrm{C}-19$ steroids during the basal state. After a loading dose of $T$, the pattern of $C-19$ metabolites was similar to normal subjects with the predominant metabolites being androsterone and etiocholanolone. However, the hypopituitary children excreted not only less $T$ alucuronide than normal subjects but the excretory pattern differed. In the hypopituitary patients, the maximum $T$ alucuronide excretion was on the day of $T$ administration with a decline the following 2 days. In the normal subjects, $T$ glucuronide increased daily. These preliminary data suggest that $T$ metabolism is altered in the hypopituitary patient. However, inasmuch as the metabolic pathways appear to be intact, the abnormality may be a kinetic variation or an alteration of peripheral or intracellular binding of androgen.

192 CONTINUOUS OVERNIGHT GLUCOSE (COG) BY GASTROSTOMY IN PATIENTS WITH TYPE I GRYCOGENOSIS (GSD). John F. Crigler, Jr. , Lucienne A. Cahen, Danlel R. Fermaglich and M. Judah Folkman. Harvard Med. Sch., Children's Hosp. Med. Ctr., Departments of Pediatrics and Surgery, Boston.

Portacaval (PC) shunts in 2 brothers have resulted in marked improvement of growth in one but far less in the other. The difference in response is due to the frequent glucose intake (approx $2 \mathrm{~g} / \mathrm{kg} / 4 \mathrm{hr}$ ) of the brother who has grown. This treatment, however, has been associated with significant hypoglycemic reactions if glucose is not given every 4 hours. To overcome this nightly threat, the effect of COG (initially by nasogastric tube) at rates from 0.12 to $0.5 \mathrm{~g} / \mathrm{kg} / \mathrm{hr}$ on 30 minute blood sugar (BS), serum insulin (IRI) and growth hormone (IRGH) and on 8 am $\mathrm{pH}, 1 \mathrm{ac}-$ tate (L), triglyceride (TC), cholesterol (C) and uric acid (UA) levels were determined. During the day, he continued feedings as before. A 4-year old girl with GSD and hypoglycemic spells but without a PC shunt has been similarly studied. In both patients, COG at $0.25 \mathrm{~g} / \mathrm{kg} / \mathrm{hr}$ maintained $B S$ in the normal fasting range $(47-108, N=32)$ with somewhat elevated IRI levels (range 5-59 $\mathrm{uU} / \mathrm{m} 1$ ) without suppressing sleep-induced IRGH release. In the boy, serum $\mathrm{pH}, \mathrm{TC}, \mathrm{C}$ and $\mathrm{UA}$ were maintained in the normal range with a maximum $\mathrm{L}$ of $2.2 \mathrm{mM} / \mathrm{l}$. In the $\mathrm{g} 1 \mathrm{rl}, \mathrm{pH}, \mathrm{TC}, \mathrm{C}$ and $\mathrm{L}$ were reduced to normal values by $C O G$ but UA remained elevated. $C O G$ at $0.5 \mathrm{~g} / \mathrm{kg} / \mathrm{hr}$ was excessive and at $0.125 \mathrm{~g} / \mathrm{kg} / \mathrm{hr}$ was insufficient to maintain FBS levels. COG by gastrostomy is being continued at home to facilitate care, to determine long-term effects on growth and to evaluate the role of the PC shunt. 
193 ELEVATED URINARy ANDROSTANEDiOL AND TESTOS\& M.I. New, Cornell univ. Med. Col., Dept. ped., New York, N.Y

precocious adrenarche (PA) in girls was studied in the baseline state, after ACTH stimulation and dexamethasone suppression using a newly devised method to simultaneously measure urinary androstanediol (Adiol) and testosterone ( $T$ ). In the baseline state, four patients with PA between $48 / 12$ and $76 / 12$ years had significantly elevated excretion of Adiol and $T$ ( $p<.001$ ) compared to 12 normals aged 3-8. Adiol excretion was

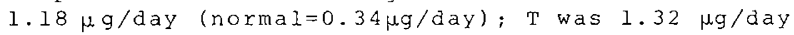
(normal $=0.30 \mu \mathrm{g} / \mathrm{day}$ ). In PA intravenous ACTH doubled urinary Adiol, T and $17 \mathrm{KS}$. Following dexamethasone administration urinary Adiol decreased to $1 / 2$ and $T$ to $1 / 3$ while $17 \mathrm{KS}$ decreased only to $4 / 5$ of baseline. The method includes glucuronide hydrolysis, methylene chloride extraction, 2 paper chromatography steps and radioligand assay. Intra-assay variation and interassay variation was $9.8 \%$ and $15.6 \%$ for Adiol; $11.1 \%$ and $14.5 \%$ for $T$. As little as 0.002 ug Adiol and 0.003 ug $T$ can be detected in $500 \mathrm{cc}$ of urine. In PA urinary Adiol and $T$ were more significantly elevated than the l7KS in the baseline state. Although all doubled with ACTH, Adiol and $T$ showed suppression with dexamethasone more regularly than $17 \mathrm{KS}$. This study points to an adrenal origin for increased urinary androgens in PA.

\section{4}

THE RANGE OF ACTIVITY OF MULLERIAN INHIBITING SUBSTANCE-Patricia K. Donahoe (Intr. by J. D. Crawford). Massachusetts Gen. Hosp., Dept. of ed, Surg., Boston

Dubstance has been studied in organ culture after the methods of Picon and Josso. In over 1000 organ culture experiments we have demonstrated that Mullerian Inhibiting Substance is produced by:

1.) The fetal rat testes from the ages of 14-21 days.

2.) The newborn testes.

3.) The testes of the rat hypophysectomized at weanling age.

4.) The testes of a young child with testicular feminization syndrome.

Mullerian inhibiting activity was not detected in the current system from:

1.) Normal testes after the newborn period.

2.) The testes of the rat hypophysectomized after 20 days of age.

3.) Placenta, muscle, ovary, or heart.

Media from beneath fetal testes incubated on an organ culture grid was cytotoxic to a monolayer of HeLa cells and to a monolayer of indifferent sex duct cells from the 14 day fetal rat. We are currently adapting these observations to a microtest system.

Using the organ culture assay and the testes of the rat hypophysectomized at weanling age, we are initiating experiments to biochemically identify the active Mullerian Inhibiting Substance.
196 COMBINED 21 AND 11 HYDROXYLASE DEFICIENCY AND XO/X: KARYOTYPE IN CONGENITAL ADRENAL HYPERPLASTA (CAH). J.W.Finkelstein, D.K.Fukushima, T.Nishima, R.HK Wu, J.Kochen, R.M.Boyar, Leon Hellman, Monteflore Hosp. and Med.Ctr., The Institute for Stero
trics, Bronx, New York.

The patient was the product of a full term, uneventful pregnancy and delivery. At birth an enlarged clitoris, urogenital sinus and labio-scrotal fusion were noted. There was no family history of intersex problems. Buccal smear at birth, 8 days \& 8 months of age showed $10 \%$ chromatin positive cells and karyotype on 11 occastons was XX/XO. Both parents had a normal karyotype. A vaginogram reverled the presence of a short urogenital sinus and a uterus and tubes ware also demonstrated. On day 8 prior to treatment $17 \mathrm{KS}$ were 1.5 , $17 \mathrm{KGS}$ were 6.0 and $17 \mathrm{OHCS} 6.0 \mathrm{mg} / \mathrm{day}$ al1 of which were elevated. Plasma sterolds were determined by specific radioimmunoassay and were:

\begin{tabular}{|c|c|c|c|}
\hline & lesoxy & Conpd $S \mu g / d l$ & $F \mu g / d l$ \\
\hline ase Day 1, Day & $10.3,11.5$ & $2.7,8.0$ & $3.0,3.0$ \\
\hline 1 oHase defect (2) & $<0.1,<0.1$ & $1.83,1.5$ & $3.1,2.6$ \\
\hline 21 OHase defect(2) & $1.2,2.7$ & $0.13,<0.1$ & 111.9 \\
\hline 1. Newborns 1-7 days & -0.44 & $<0.1$ & -22.6 \\
\hline Normal Adults & $<0.1$ & 0.1 & 5.4 \\
\hline
\end{tabular}
By day 10 serum $\mathrm{Na}$ was $120 \mathrm{mEq} / 1$ and $\mathrm{K}$ was $12.2 \mathrm{mEq} / 1$. This is the first reported case of combined $21 \& 11$ OHase deficiency. Six other cases of chromosomal abnormalities \& CAH have been reported and three had sex chromosome abnormalizies but the type of enzyine defect was only discernable in one case which had a 21 OHase deficiency.

197 Massive OBESity COMPLiCATING THERAPy OF CONGENitAL ADRENAL HYPERPLASIA (CAH) M. Firestone, M. Gene1, A.R. Enriquez, and B.F. Bower. Dept. of Pediat., Yale Univ. Sch. of Med., New Haven, Ct. and Dept. of Med., Univ. of Ct. $\mathrm{Sch}$, of Med., Farmington, Ct, (Intr. by Joseph B. Warshaw)

obesity (mean wt. $>200 \mathrm{lb}$.) developed during therapy of three girls with CAH, two salt-losing, accompanied by paradoxical Cushingoid appearance with lack of chemical control. In two, "Cushingoid obesity" appeared on hydrocortisone (HC) suppression of $12 \mathrm{mg}$. and $25 \mathrm{mg} . / \mathrm{m}^{2} / 24 \mathrm{hr}$. Both were overweight initially, the first from infancy and the other on presentation with simple $\mathrm{CAH}$ at age 5, and in both HC dosage was initially decreased on clinical suspicion of overdosage, then increased with further acceleration of obesity and Cushingoid habitus, but without chemical and clinical control. The third girl developed obesity at age 6 while receiving $56 \mathrm{mg} . / \mathrm{m}^{2} / 24 \mathrm{hr}$. of cortisone to maintain $17 \mathrm{KS}$ below 1-2 mg./day and then grew steadily heavier and Cushingoid on doses up to $90 \mathrm{mg}, / \mathrm{m}^{2}$ without control. All three achieved excellent control on one $0.25 \mathrm{mg}$. dose of dexamethasone and two on standard doses of $\mathrm{HC}$ or prednisone, provided at least $50 \%$ was given at night.

In a standard uniformly divided multiple-dose regimen, inadequate suppression of ACTH, especially at night, may occur in obese subjects, possibly due to more rapid clearance of cortisol. In such patients maximum night dosage of glucocorticoids or use of long-acting dexamethasone achieves satisfactory control without engendering total steroid overdosage.

Supported in part by NIH grant RR-125.

\section{$\Gamma 195$} NORMAL CYCLIC AMP RESPONSE TO PARATHORMONE WITH FAILURE OF PHOSPHATURIC RESPONSE IN A PATIENT WITH TURNER'S PHENOTYPE AND XO/X RING X MOSAICISM. David

Finegold, Louie Linarelli, and Frederic M. Kenny, Pittsburgh, Pa. Our patient at age 11 yrs had short stature, mental retardation, web neck, cubitus valgus, high arch palate, wide spaced nipples and short $4 \mathrm{th}$ and 5 th metacarpals. Since some of the stigmata are shared by both pseudohypoparathyroidism with Turner's syndrome, a three-day parathormone (pte) test 250 U I.M. q $12 \mathrm{hrs}$ was done without elevating serum $\mathrm{Ca}$ or depressing serum $P$ below several control values. Subsequently serum $\mathrm{Ca}$ and $\mathrm{P}$ were normal without therapy. When restudied at age 16 (pte 200 $U$ IV), her urine CAMP increased $(\triangle 22 \mathrm{Nm} / \mathrm{mg}$ Cr $)$ without change in phosphate clearance (ct1 $\bar{x} 15 \mathrm{ml} / \mathrm{m} 1 \mathrm{n}$, test $x$ 9) or $\%$ TRP (ct $1 \bar{x} 81$; test $\bar{x} 89$ ). Thus her defect lies in reception of the cyclic AMP signal. It appears to exist in both kidney and bone. Despite the defect, she maintains normal serum $C a$ and $P$ by an unknown mechanism probably involving endogenous hyperparathyroidism (laminas dura are undermineralized). If the biochemical defect is associated with the presence of the $X$ Ring $X$ chromosome, it is the first demonstration of metabolic activity of the $X$ Ring. To our knowledge, this is the 4 th reported instance of this form of renal unresponsiveness to pth; and the first instance of its occurrence in association with the above phenotype and karyotype.
198 OutPatient initiation of InSUlin therapy in JUVEnile DIABETES. Gordon E. Gibbs, Dept. of Ped., Col. of Med., Univ of Nebraska, Omaha.

Six diabetic children have been started on insulin without initial hospitalization, to determine the practicability of initial outpatient management in this condition. These have been cases in which the diabetes was detected by urine sugar, with little or no ketonuria and confirmed by hyperglycemia.

Such management is facilitated by a scheme of daily adjustment of insulin dosage at home, according to rules based on urine tests done 4 times daily. Such changes of insulin dosage have initially served to increase the insulin dosage gradually to meet the requirements. They have also taken care of the needed lowering of insulin dosage in the subsecuent critical period of rapidly decreasing insulin renuirement.

Such initiation of treatment reauires thorough teaching, in the outpatient setting on short notice, of various aspects of diabetes management. These are: (1) diet, (2) urine sugar and acetone testing, (3) use of the rules for changes of insulin dosage, (4) administration of insulin, (5) treatment of hypoglycemia. In the present series, such teaching has been done by the physician, but could also be done by other experienced personnel.

Initiation of insulin treatment in the new diabetic child without hospitalization appears to be practical. 
199

STEROID EFFECTS ON SERUM CALCIUM IN THE RAT.

M.A. Gottuso, C.T. Nervez, R.D. Jacobs, R.J. Shott, M.L. Williams and W.H. Bergstrom. Depts. of Pediatrics and Biochemistry, SUNY, Syracuse, N.Y.

Plasma estrogen is 20-30 ug/dl at birth; in severe RDS cortisol is similarly elevated. Since both steroids inhibit bone calcium release, they may contribute to hypocalcemia in stressed neonates. The effect of cortisol and estrogens on serum $\mathrm{Ca}$ was therefore studied in newborn $(6.5 \mathrm{gm})$ and adult $(350 \mathrm{gm})$ rats. Steroids were given IP, $0.2-1.0 \mathrm{ug} / \mathrm{gm}$. Changes in serum calcium in $6.5 \mathrm{gm}$ rats were as follows:

\begin{tabular}{|c|c|c|c|c|}
\hline \multirow[b]{2}{*}{ 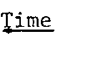 } & \multicolumn{2}{|c|}{$\Delta \mathrm{Ca}, \mathrm{mg} / \mathrm{dI}$ vs. controls } & \multicolumn{2}{|c|}{$0=\mathrm{n} . * 2=\mathrm{p}<.01$} \\
\hline & Estradiol & Estrone & Estriol & Cortisol \\
\hline & $\overline{-1.5(41)}$ * & $-0.8(9) *$ & $-0.3(14)$ & $-0.9(14$ \\
\hline $\begin{array}{r}6 \mathrm{hr} . \\
18 \mathrm{hr}\end{array}$ & $-1.0(30) *$ & & & \\
\hline
\end{tabular}

Serum 3H-cortisol and 3H-estradiol were 20-30 ug/dl. Parathyroid extract, $5 U$ IM, prevented $\Delta \mathrm{Ca}$ at 3 and 6 hours. Intact $350 \mathrm{gm}$ rats showed no $\triangle \mathrm{Ca}$; after adrenal and parathyroid ablation, estradiol lowered serum $\mathrm{Ca} 1.2 \mathrm{mg} / \mathrm{dl}$ in 3 hours $(\mathrm{p}<.05)$.

Estradiol, estrone and cortisol, at levels equal to those found in human newborns, lower calcium in the newborn rat and in parathyroidectomized adults. High levels of these steroids may contribute to hypocalcemia in functionally hypoparathyroid infants, particularly those subject to stress.

20 ELEVATED SERUM IMMUNOREACTIVE CALCITONIN (iCT) IN A CHILD WITH ACUTE LYMPHOCYTIC LEUKEMIA (ALL). Nasroliah Hakami, John S. Meyen, and Constantine S. Anast. Dept. of Child Health, Univ. of Mo and VA Hosp., Columbia, Mo., and Dept. of Fathology, Wash. Univ. Med. Scin., St. Louis, Mo.

Calcitonin (CT), a hypocalcemic polypeptide produced by thyroid $C$ cells is characteristically elevated in serum of patients with $C$ cell tumors. In addition, ectopic production of $C T$ has been reported in other malignancies, but not in acute leukemia. We observed a 9-yr-old boy with ALL who had an unusually high level of $\mathrm{i}$ CT. The child developed convulsions 3 days after ini tiation of chemotherapy (Prednisone, Vincristine and Methotrexate). Serum $\mathrm{Na}$ was $113 \mathrm{mEq} / \mathrm{L}$, serum osmolality $263 \mathrm{mosm} / \mathrm{L}$, and urine osmolality $487 \mathrm{mosm} / \mathrm{L}$. Serum Pitressin was $1.22 \mathrm{ng} / \mathrm{ml}$, a finding consistent with inappropriate secretion of ADH. The serum $C a$ was $7.9 \mathrm{mg} / \mathrm{dl}$, serum $\mathrm{P}$ was $3.0 \mathrm{mg} / \mathrm{dl}$, and serum $\mathrm{iCT}$ was $20,000 \mathrm{pg} / \mathrm{ml}$ (normal and in 9 children with ALL in remissio: <150 $\mathrm{pg} / \mathrm{ml})$. The chromatographic elution pattern of the patient's serum $i C T$ was similar to that of patients with $C$ cell tumors of the thyroid. The patient died of staphylococcal sepsis on the 7 th hospital day. There was no evidence of thyroid $C$ cell hyperplasia or tumor by light and electron microscopy, but large colonies of staphylocci were observed in thyroid. Inappropriate ADH secretion has been reported in association with Vincristine therapy. However, the reason for elevated $i c T$ in this patient is uncertain, and would include: l)release of thyroid CT induced by either drugs or products of staphyloccus sepsis, 2)ectopic pro-

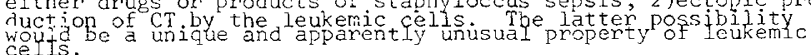

\section{THFROTROPIN (TSH) INDUCED NEONATAL, THMRN} A1berto Hayek, Lovola Univ. Stritch School of Med.,Dept of Ped., Chicago, Ill inois. (Intr.by:Johi: D. Crawford) Neonatal hyperthyroidism is generally attributed to transplacental passage of maternal LATS. The infant to be described sug gests a TSH dependent form may occur.

A 2800 gm female was delivered by section after a 38 week pregnancy complicated by hyperthyroidism. At diagnosis one month before conception the mother's $\mathrm{T}_{4}$ had been $20 \mathrm{ug} \%$ and LATS was po sitive. She was treated with PTU and received $200 \mathrm{mg} / \mathrm{d}$ during the last trimester. At delivery her $\mathrm{T}_{4}$ was $7.6 \mathrm{ug} \%$ and TSH $2 \mathrm{u} J / \mathrm{m} 1$. LATS was only weakly positive.

The baby was normal at birth except for unduly large fontanels and a palpable thyroid. Cord blood $\mathrm{T}_{4}$ was $3.7 \mathrm{ug} \%, \mathrm{~T}_{3} \mathrm{RUJ} 23 \%$ and TSH $99 \mathrm{uU} / \mathrm{m} 1$; LATS was undetectable. TSH at 15 and 30 minutes rose to 162 and $240 \mathrm{uU} / \mathrm{ml}$. For the ist five days of 1 ife the baby fed poorly and was constipated. On the 6 th day she developed diarrhea, tachypnea, tachycardia $(220 / \mathrm{min})$, and the $\mathrm{T}_{4}$ measured $34.7 \mathrm{ug} \%$ TSH was now undetectable. Propranolol and iodide were given. Five days later the baby was euthyroid with a $\mathrm{T}_{4}$ of $11.9 \mathrm{ug}^{\circ}$ and TSH $2 \mathrm{uU} / \mathrm{m} 1$.

This infant, born with well documented hypothyroidism, passed rapidly to a state of thyrotoxicosis requiring treatment. That thyrotoxicosis was precipated by TSH is suggested by the absence of measurable IATS in the infant's serm, the exaggerated postnatal TSH surge and the timing of the symmtoms of hypermetabo1 ism.
202 ATHEROSCLEROSIS, PUBERTY AND CASTRATION. FeIIX P. Heald, Mushtaq A. Khan, Theodore M. Farber, Francis L. Ear1, S. David Gertz, Marshal1 L. Rennels, M Hussain, and Edward J. Van Loon. Dept. Ped. \& Neurology, Univ. ville, Md. 20705.

This study was designed to determine if male sex hormones are one of the regulating factors in the process of atherogenesis. Male piglets were castrated or sham operated at 2 weeks of age and placed on an atherogenic (33\% of total calories from egg yolk) or control diet at 5 weeks of age. Serum cholesterol was determined at 5 week intervals up to 52 weeks of age. At one year, the animals were sacrificed and the aortas, carotids, and coronary arteries removed, fixed in formalin and stained with Sudan IV. The area involved in fatty streaking was expressed as percent of the total surface area of the vessel. Castration, accelerated the process of atherogenesis as evidenced by (1) significantly higher $(P<0.05)$ serum cholesterol in the castrates from 40-52 weeks of age as compared to shams at comparable ages in both egg yolk and stock diet groups and (2) castrated animals fed egg yolk diet have significantly greater $(P<0.05)$ surface area of the aorta involved in fatty streaking (12\%) as compared to $1 \%$ in shams. No appreciable fatty streaking was observed in the animals (castrates or shams) fed the isocaloric stock diet. These findings strongly suggest that castration of male miniature pigs fed an atherogenic diet results in increased susceptibility to the early stages of atherosclerosis. (Supported by Frank G. Bressler Research Fund \& NIH Research Grant 非HS NS 06779-07.)

203 CIRRHOSIS OF THE LIVER AND 'MATUR ITY-ONSET' TYPE DIABETES MELLITUS IN A GIRL. HILLEL I. HOCHMAN AND RAYMONDE DLMONT. (Intr. By Joel J. A1pert), Boston Univ. Sch. Med., Boston City Hosp., Dept. of Ped., Boston, Mass.

A 13 year old, non-obese, prepubertal girl with chronic active hepatitis, postnecrotic cirrhosis (biopsy) and evidence of portal systemic shunting (esophageal varices), developed persistent hyper glycemia, glucosuria and polyuria without ketoacidosis. She responded only to high doses of insulin. Inadvertent discontinuation of the latter for the period of two weeks did not result in ketoacidosis. Serum C-peptide immunoreactivity at 15 years of age was 10.9 - 11.5 before (overnight fast, $24 \mathrm{hrs}$. after a recent injection of $\mathrm{NPH}$ insul in, serum glucose: $340 \mathrm{mg} / 100 \mathrm{~m} 1$ ) and $11.2-$ $10.8-10.9-9.6 \mathrm{ng} / \mathrm{ml}, 1 / 2,1,2$, and 3 hours after an oral glucose load (serum glucose: 520 - 520 - 500 - $460 \mathrm{mg} / 100 \mathrm{ml}$ respectively), compatible with the presence and possibly hypersecretion of endogenous insulin. The association of hepatic cirrhosis and diabetes mellitus has been previously observed in adults. Impaired glucose tolerance and hyperinsulinemia have been shown in these patients, especially in those with portal-systemic shunting. The bypassing of the liver by glucose and insulin has been implicated among the pathogenetic mechanisms: Substantial fractions of glucose and insulin, normally reaching the liver via the portal vein, gain access to the systemic circulation. Glucose induced pancreatic hypersecretion may also contribute to the hyperinsulinemia. The latter may lead to insulin resistance. We believe that our data on a juvenile patient represent an experiment of nature and provide further evidence for the existence of hepatogenous diabetes as a nosological entity.

204 INCREASED INCIDENCE OF POST-ADRENALECTOMY NELSON'S SYNDROME IN PEDIATRIC VS ADULT CUSHING'S DISEASE : A NATIONWIDE STUDY. N. Hopwood and F. Kenny, (Intr. by T. K. Oliver, Jr.), Univ. of Pgh. Sch. of Med., Pittsburgh, Pa. The relative rarity of Cushing's disease in children disallows prospective randomization of therapies in a single center. When one of our two patients (ages 10 and 11 yrs) treated by total bilateral adrenalectomy (TBA), developed Nelson's syndrome (enlarged sella, optic nerve compression and hyperpigmentation), we polled The Lawson Wilkins Pediat. Endocrine Soc.: nfneteen respondents had 31 patients aged 10 mos to 16 yrs. Of these, 30 had TBA; one received 0 P'DDD only. Sixteen females and 15 males had pathological diagnoses of hyperplasia (29), and unstated (2). Post treatment hyperpigmentation was seen in 18 ; no change in 7 ; not specified in 6 . X-ray evidence of sella enlargement was detected in 8 after $1-5.5$ (mean 3 ) yrs post-adrenalectony. However, many patients were not serially $x$-rayed. This $8 / 31$, 1.e., $27 \%$ incidence of post-adrenalectomy Nelson's syndrome is higher than the adult figure of < $10 \%$ (Nelson,D.H., J. Clin. Endo. $26: 722,1966)$. All children with sella enlargement were hyperpigmented; two patients had visual field loss. Four had removal of pituitary adenomas. Our patient's visual fields became normal after transsphenoidal removal of a chromophobe adenoma, and her hyperpigmentation diminished. However, she lost TSH and GH responsiveness. A fifth patient with Nelson's syndrome has erosion of posterior clinotds with secondary hypothyroidism and hypogonadism. While TBA is the prevailing therapy, a safer treatment is required. 
20 APPLICATION OF SERUM 17OH-PROGESTERONE (17P) RADIO-

205 imMUNOASSAY TO DIAGNOSIS and MaNAGEMENT OF CONGENITAL ADRENAL HYPERPLASIA (CAH)。 leuan $A_{0}$ Hughes and Manitoba, Canada.

We have studied serum $17 \mathrm{P}$ serially through $24 \mathrm{hr}$ in 11 treated and untreated $\mathrm{CAH}(21-\mathrm{OH}$ deficiency) patients, correlating rehealthy children ( 1 day- 18 yr) $17 \mathrm{P}$ ranged from $5-250 \mathrm{ng} / \mathrm{di}$. In untreated CAH, I7P was markedly elevated (4500-54,000 ng/d1). Treatment with cortisol $\left(20-30 \mathrm{mg} / \mathrm{M}^{2} /\right.$ day in 3 doses) produced adequate mineralocorticoid. Even slightly inadequate mineralocorticold therapy (shown by high PRA with normal electrolytes) caused elevated 17P (to 78,000 $\mathrm{ng} / \mathrm{dl}$ ) in spite of adequate cortisol. Some patients with high pregnanetriol showed isolated I7P elevations (usually early AM) which responded to revision of dosage schedule without increased total cortisol dose. The data confirm the value of I7P assays in the diagnosis of CAH. Furthermore $17 \mathrm{P}$ appears to be the most sensitive index of therapeutic control; taken together with PRA, 178 can permit more exact control of CAH with possibly less glucocorticoid, and eventually better growth and reproductive potential. Jeremy S.D. Winter, Univ. of Manitoba, Dept. Ped., Winnipeg, sults with plasma renin activity (PRA), testosterone, FSH, LH and electrolytes, and urine $17 K S$ and pregnanetriol. In 431 normal I7P in 'non salt-losers' and 'salt-losers' receiving

208 AUTONOMOUS TESTICULAR AND ALRENAL TUMORS IN A PATIENT WITH $11-\beta$ HYDROXYLASE DEFICIENCY. Ann K. Kershnar, Robert D. Clemons, Maurice D. Kogut, Benjamin H. Landing, USC School of Medicine, Childrens Hospital of Los Angeles, Dept. of Pediatrics and Pathology, Los Angeles.

A $15 \frac{1}{2}$ year old male, with precocious sexual development since age 4 , had bilateral calcified testicular masses. Serum lldesoxycortisol(S) was $3.9 \mu \mathrm{g} \%$, serum testosterone(T), $819 \mathrm{ng} \%$, serum LH, $3.1 \mathrm{mIU} / \mathrm{ml}$ and $\mathrm{FSH}, 1.1 \mathrm{mIU} / \mathrm{ml}$. Urinary Tetrahydro-S (THS) of $3.6 \mathrm{mg} / 24 \mathrm{hr}$ failed to suppress with 1 year of hydrocortisone(HC) and testicular tumors did not regress. Blood cortisol(F) and $S$ and urinary 17-KS and THS levels did not change following ACTH infusion. Blood $S, F$ and progesterone(P) from left adrenal vein were $31.2 \mu \mathrm{g} \%, 38 \mu \mathrm{g} \%, 19756 \mathrm{pg} / \mathrm{ml}$, respectively; peripheral blood S, F, and $\mathrm{P}$ were $1.6 \mu \mathrm{g} \%, 12.4 \mu \mathrm{g} \%$ and 962 $\mathrm{pg} / \mathrm{ml}$, suggesting $11-8$ hydroxylation deficiency. In vitro adrenal incorporation of $\Delta 5$-pregnenolone, and desoxycorticosterone secretion rates, confirmed the block. Mean bilateral spermatic vein blood $\mathrm{F}$ was $9.75 \mu \mathrm{g} \%$ (peripheral vein (PV) $12.1 \mu \mathrm{g} \%$ ) and $\mathrm{S}$ was $15.5 \mu \mathrm{g} \%$ (PV, $1.7 \mathrm{\mu g} \%$ ). Right adrenalectony and left adrenal biopsy showed multiple cortical nodules. Orchiectomy, performed because of risk of malignant change with uncontrollable hyperplasia (autonomy) showed germ cell hypoplasia, Leydig cell adenomatosis and Leydig (or ? atypical Sertoli) cells arising in tubules.

The data are consistent with 11-8 hydroxylase deficiency and autonomous function of both adrenals and testes resulting from chronic ACTH stimulation. Lack of hypertension, and nonsuppressibility, may suggest that receptor deficiency is also present.

209 ESTRADIOL (E2) LEVELS IN UMBILICAL CORD PLASMA:

206 PSYCHOSOCIAL DWARFISM WITH DELAYED PUBERTY: REPORT OF A CASE WITH FEATURES OF ANOREXIA NERVOSA. Yozo Ichiba and Lytt I. Gardner. State Univ. of New York, Upstate Med. Ctr., Dept. of Ped., Syracuse, New York.

Delayed puberty as a late manifestation of psychosocial dwarfism (PSD) is a complication described only recently (Money, J. and Wolff, G.: Adolescence 9:121, 1974). We have documented a case of PSD in a 20 year old boy who had been under intensive psychiatric care because of anorexia nervosa in association with a complex mother-son relationship. Before age 11 his growth had been within normal limits; from age $116 / 12$ to $146 / 12$ he was described as having anorexia nervosa, and during this time he gained only $3 \mathrm{~cm}$. in height. In the home setting his mother fed him at a separate table. After the period of "active" anorexia nervosa he gradually gained wejght but did not exhibit any catchup growth. At age $166 / 12$ his height and weight were both at the 50 th percentile for 11 years, and his bone age was 11-12 years. Serum thyroxine was normal. There was no sexual development. At age 19 he entered college as a day student; during this year there was a striking increase in linear growth velocity $(5 \mathrm{~cm} /$ year). By age 20, still living at home, his height-age is 12 $4 / 12$, weight-age 12 , and bone-age 14 . A scattering of pubic hairs can be seen, but otherwise he is sexually infantile. He refused additional diagnostic procedures and androgen therapy. This case with dwarfism and delayed puberty illustrates possible interrelationships between PSD and anorexia nervosa.

EFFECTS OF GLUCOCORTICOID THERAPY ON GROWTH HORMONE

207 (HGH), GONADOTROPIN (FSH AND LH), AND 17-OH PROGESTERONE (17OH P) SECRETION IN CONGENITAL ADRENAL HYPERPLASIA (CAH). Robert P, Kelch, Martha L. Spencer, and George E. Bacon, Univ. of Mich., Dept. of Ped., Ann Arbor.

Short stature and abnormalities of sexual maturation are common problems in the management of children with $\mathrm{CAH}$. To investigate effects of glucocorticoid therapy, secretory patterns of HGH, FSH, LH and $170 \mathrm{H} \mathrm{P}$ were determined in 1 boy (9yr) and 4 girls (1219yr) (3 "salt-losers") both with and without maintenance hydrocortisone (HC). Patients were admitted to the Clin. Res. Unit on 2 occasions, 1-4 mos. apart. Treatment and non-treatment studies were randomized. HC $(20 \mathrm{mg} / \mathrm{m} 2 / \mathrm{d} \mathrm{p} . \mathrm{o}$.) was given as follows: 8 am $5,4 \mathrm{pm} \mathrm{5}$, and $12 \mathrm{MN} 10 \mathrm{mg} / \mathrm{m}^{2}$. On the $3 \mathrm{rd}$ day of each admission, blood samples were withdrawn at 20-30 min. intervals over $24 \mathrm{hrs}$. $\mathrm{HGH}, \mathrm{FSH}, \mathrm{LH}$ and $170 \mathrm{H} \mathrm{P}$ were determined by radioimmunoassay. HGH values were slightly, but not significantly greater during $\mathrm{HC}$ therapy. Nocturnal HGH was not altered by HC. While off $\mathrm{HC}$, mean LH was significantly greater in 4 patients and mean FSH was significantly lower in 4 patients. Episodic release of LH occurred in the 3 oldest girls during both studies, but was greatex in amplitude while of $\mathrm{HC}$. $170 \mathrm{H} P$ patterns during treatment correlated well with clinical estimate of each patient's control; while of $\mathrm{HC}, 170 \mathrm{H} \mathrm{P}$ was increased greatly but diurnal variation was present. These studies indicate that in $\mathrm{CAH}$ 1) glucocorticoid re placement does not decrease $\mathrm{HGH}$ secretion; 2) acute withdrawal of glucocorticoids exaggerates episodic secretion of LH and decrease the serum FSH/LH ratio; and 3 ) responsiveness to HC varies strikingly. (NICHD Prog. Proj. HD08333 \& USPHS \# SP 11GM 15559.)

\section{CORRELATION WITH MODE OF DELIVERY AND FETAL SEX.}

Klein, N. Radfar, and $\underline{F}$. M. Kenny, Univ. of Pgh. Sch, of Med. ittsburgh, $\mathrm{Pa}$.

Analysis of $E 2$ in umbilical vein (UV) in male vs female infants born by $\mathrm{C}$-section indicated that the mean for males was significantly higher than for females $(8.4 \mathrm{vs} 2.4 \mathrm{ng} / \mathrm{ml}$, $p<0.025$, df $=8$ ). This probably reflects $C-19$ precursors of testicular origin. In order to test the possibility that the fetus affects the onset of labor mediated by E2, we compared plasma $E 2$ in maternal, umbilical artery and $U V_{1 N} 23$ mother baby pairs (A) elective C-section, (B) spon, vaginal del., (C) Induced vaginal del, and (D) C-section post spontaneous onset of labor in pregnancies with female offspring (to exclude testis). Values in $\mathrm{Mg} / \mathrm{ml}$ Materna \begin{tabular}{l|l|l|l} 
Groups A, B, C, D & $19,19,25,23$ & $0.7,3.1,1.1,4$ & $2,9,6,12$
\end{tabular} E2 was lower in group $A$ vs $B$ in $U V(p<0.005)$ and UA $(p<0.01)$, whereas maternal values were equal. Since spontaneous labor, (groups $B+D$ ) were greater than induced (C), the data are consistent with the concept that spontaneous labor (rather than labor per se) results from, rather than causes, increased fetoplacental estrogen production. Studies in sheep implicate the fetal adrenals in initiating labor. Increased corticosteroid and/or estrogen production could be involved. The latter could stimulate prostaglandin F $2 \boldsymbol{C}$ synthesis with resulting uterine contraction. Our data support the concept that in the human the fetal adrenal may play a part in the onset of spontaneous labor.

THYROTROPIN (TSH) SCREENING FOR CONGENITAL HYPOTHYROIDISM Alan H. Klein, Amelia V. Agustin, Nancy J. Hopwood, Anita Perricelli, L. Johnson, Thomas P. Foley, Jr. Univ. of Pittsburgh Sch.

In a combined five year retrospective and prospective study at Magee Women's Hosp. (MWH), Pittsburgh, the incidence of congenita] hypothyroidism (CH) approximated 1 in 6000 . Between 8-13-73 and 12-13-74 we have determined TSH in all cord samples collected at MWH, and have detected $\mathrm{CH}$ in two of 8896 infants tested. A hypothyroid infant with a sublingual thyroid gland was detected at MAMC by TSH cord screening methods. Cord TSH Ievels were 302, 376 and $352 \mathrm{uJ} / \mathrm{m}$ ] in affected infants and were five-fold higher than our current recall leve? of $>60 \mathrm{uU} / \mathrm{ml}$. Follow-up T4 and TSH levels were normal on a? 1 infants with cord TSH between 60-85 uU/ml.

For comparison thyroxine (T/4) levels were measured in 200 normal infants. The mean normal T4 was $12.7 \pm 2.9$ (tSD) ug\%. Four infants had cord $T 4$ levels of $4.5,5.0,5.3$ and $5.4 \mathrm{ug} \%$. all of

To simplify samp?e collection we have devised an assay method to measure TSH eluted from blood on filter paper discs. Preliminary data indicate the lower limit of detectability at an equivalent serum TSH level. of $40 \mathrm{uU} / \mathrm{ml}$. Using this method one affected infant had a TSH level of $>180 \mathrm{uU} / \mathrm{ml}$ at three days of age. At a computed cost of $\$ 0.25 /$ test for large scale testing, this method will successfully screen for $\mathrm{CH}$ with 1) low recall rate, 2) collections simultaneous with the mandatory US screening program for phenylketonuria and 3) concomitant reduction in mental retardation caused by $\mathrm{CH}$. which were lower than two affected infants $(\mathrm{T} 4=5.6,6.7 \mathrm{ug} \%)$. 

FOR IMPAIRED CONVERSION OF 25-HYDROXYVITAMIN D TO $1 \alpha, 25-D I H Y D R O X Y V I T A M I N D$.

S.W. Kooh, D. Fraser, M.F. Holick,*H.F. Deluca.* From the Depts. of Paed. \& Physio1., Univ, of Toronto, and Hosp. Sick Child., Toronto, Canada; and the Dept. of Biochem., Univ. of Wisconsin, Madison, Wis. (PHP), pharmacological doses of vitamin $D$ correct hypocalcemia, but the mechanism is unknown. We studied 2 children with HP and 1 with PHP to test the hypothesis that these conditions cause a defect in synthesis of $1 \alpha, 25$-dihydroxyvitamin $D_{3}$, the principal active metabolite. Minute intravenous doses of $1 \alpha, 25$-dihydroxyvitamin $\mathrm{D}_{3}, 0.04$ to $0.08 \mu \mathrm{g}$ per $\mathrm{kg}$ per day, corrected hypocalcemia and increased intestinal calcium absorption in both conditions. On the other hand, the dose of 25-hydroxyvitamin $D_{3}$ to maintain normocalcemia in the patients was at least $5 \mu \mathrm{g}$ per $\mathrm{kg}$ per day, and with this agent the plasma concentration of this metabolite requirements of these 2 metabolites suggests that refractoriness is due to impaired conversion of 25 -hydroxyvitamin $\mathrm{D}$ to $1 \alpha, 25$-dihydroxyvitamin $D$. The response to $1 \alpha, 25$-dihydroxyvitamin $\mathrm{D}_{3}$ in PHP is consistent with experimental evidence that renal phosphate concentration may be a salient physiological determinant of $1 \alpha, 25$-dihydroxyvitamin $D$ synthesis.
211 RESPONSES OF HYPOPARATHYROIDISM AND PSEUDOHYPOPARA-

In hypoparathyroidism (HP) and pseudohypoparathyroidism greatly exceeded the normal range. The great disparity in the

214 STUDIES ON TRANSIENT HYPOMAGNESEMIA OF INFANCY AND REBOUND HYPERPARATHYROIDISM IN ONE PATIENT. Humberto Lattore, Louie G. Linarelli, Nancy Hopwood, Caroline M. Bobik, John R. Bobik, Frederic Kenny Univ. of Pgh., Chi Idren's, Allegheny General and Mercy Hosps., Depts. Ped. Pgh., Pa.

We studied two infants between the ages of 2-4 mos with seizures, hypomagnesemia and hypocalcemia associated with diarrhea. The purpose of these studies was to evaluate serum iPTH (bovine PTH responses to parathyroid extract (PTE) and calcium (Ca) infusion in these infants. While hypomagnesemic $(0.9-1.2$
meq/L) both infants had appropriate renal responses for age to PTE infusion (5unit/kg) wi th a $\Delta$ Mean \%TRP of 30 and $\Delta$ Mean cyclic AMP of 12.2 Nmoles/mg creat. There was insignificant Ca rises $12 \mathrm{hrs}$ post PTE infusion $(<0.3 \mathrm{mg} \%$ ). Magnesium therapy was found important in correcting hypocalcemia. One infant whose iPTH was $535 \mathrm{pg} / \mathrm{ml}$ (Ca $5.8 \mathrm{mg} \%$ ) at 2 mos of age slowly developed hypercalcemia (peak $13 \mathrm{mg} \%$ ) over a 6 mo period which then spontaneously resolved. This infant's serum iPTH level fell from 467 to $271 \mathrm{pg} / \mathrm{ml}$ and urinary cyclic AMP was reduced from 6.7 to 5.3 Nmoles/ing creat in response to Ca infusion ( $15 \mathrm{mg} / \mathrm{kg}$ ) at 9 nos of age. The other infant in response to $\mathrm{Ca}$ infusion at 3 mos of age had a fall in iPTH from 362 to $273 \mathrm{pg} / \mathrm{ml}$ ( $\mathrm{Ca} 9.1 \mathrm{mg} \%$ ) and urinary cyclic AMP from 14.2 to 7.1 Nnoles/mg creat. In summary, two infants with hypomagne semia in infancy showed renal cyclic AMP and phosphate responses but lack of Ca rise to PTE infusion. Hypercalcemia and secondary hyperparathyroidism may develop following successful management of hypomagnesemia and hypocalcemia in infancy. Ca infusions are effective in reducing serum iPTH and urinary cyclic AMP in infants.

215 GLUCAGON RESPONSE TO ARGININE INFUSION IN GROWTH HOR-

212 ADRENAL ANDROGENS IN PRECOCIOUS ADRENARCHE. S. KorthMed. Col., Dept. Pediatrics, New York.

The contribution of adrenals and ovaries to the androgen levels in precocious and normal adrenarche has been studied, Radioirmunoassays were used to measure levels of androgens in 27 girls with precocious adxenarche (PA). Mean levels of testosterone (T), dihydrotes tosterone (DHT), androstenedione (A), dehydroepiandrosterone (D), and dehydroepiandxosterone sulfate (DS), wexe significantly higher $(p<.005)$ than in normal prepubertal children $(\mathrm{NC})$ :

\begin{tabular}{|c|c|c|c|c|c|}
\hline & $\mathrm{T}$ & DHT & A & D & DS \\
\hline$P A$ mean $\pm S E$ & $\begin{array}{c}10.2+0.7 \\
(2 \overline{7})\end{array}$ & $\frac{5.6+0.7}{(19)}$ & 28. $\frac{2+2.5}{(27)}$ & $\begin{array}{c}325.3+38.3 \\
(2 \overline{1})\end{array}$ & $\begin{array}{c}(1 \mathrm{gg}) \\
73.9+13.3 \\
(10)\end{array}$ \\
\hline$\underset{(n)}{C}$ mean $\pm S E$ & $\begin{array}{l}5.9+0.5 \\
(22)\end{array}$ & $\begin{array}{c}3.0+0.4 \\
(17)\end{array}$ & 17. $\frac{7+2.4}{(22)}$ & $\begin{array}{c}41.9+8.0 \\
(11)\end{array}$ & $23.8+5.7$ \\
\hline
\end{tabular}

The contribution of ovaries and adrenals to androgens was assessed in 7 girls with $P A$ and in 4 agonadal patients with adrenarche(AA) under stimulation and suppression:

\begin{tabular}{|c|c|c|c|c|c|c|c|c|c|}
\hline \multirow[b]{2}{*}{ Treatment } & \multirow{3}{*}{$\frac{T}{T}$} & \multicolumn{4}{|c|}{$\mathrm{PA}$} & \multicolumn{4}{|c|}{$\mathrm{AA}$} \\
\hline & & DHT & $\bar{A}$ & $\bar{D}$ & $\overline{\mathrm{DS}}$ & $\mathrm{T}$ & DHT & A & D \\
\hline none & & II & $\overline{36}$ & 336 & $8 \overline{9}$ & $\overline{15}$ & 7 & 58 & 454 \\
\hline АСТH & 19 & 10 & 79 & 525 & 140 & 27 & 7 & 93 & 866 \\
\hline $\operatorname{dex}$ & $<2$ & 5 & $<2$ & 125 & 17 & 3 & 4 & 4 & 126 \\
\hline dex + Norlutin & 7 & $<4$ & $<2$ & 72 & 14 & 3 & $<4$ & $<2$ & 95 \\
\hline dex + HCG & 7 & 5 & 30 & 129 & 18 & 2 & 3 & 4 & 89 \\
\hline
\end{tabular}

The results indicate that PA is associated with precocious secretion of androgens which are of adrenal origin. The ovaries in PA contribute androgens only under stimulation with HCG.

213 CLINICAL STUDIES UTILIZING TIMED URINARY GONADOTROPIN 3 MEASUREMENTS. Howard E. Kulin, Patricia M. Bell Richard J. Santen, The M.S. Hershey Med. Ctr., The Pa. State Univ., Div. Endocr., Depts. Ped. \& Med., Hershey, Pa. Gonadotropin $(G)$ determinations on single blood samples are frequently inaccurate due to problems of assay sensitivity and fluctuating levels. To circumvent these difficulties, we deve1oped a method of measuring timed urinary excretion. The method was validated in 71 patients by comparing $G$ results from multiple blood samples with FSH and LH levels in 3-6 hr urine collections. The following data were obtained (mIU/hr of 2nd IRP, hMG):

\begin{tabular}{|c|c|c|c|c|c|}
\hline Category & №. & Age & $\underline{\text { Sex }}$ & FSH $(x \pm S E)$ & $\mathrm{LH}(\mathrm{X} \pm \mathrm{SE})$ \\
\hline Newborn & $\overline{8}$ & $\overline{2-3}$ wks & M\&F & $15 \pm 9$ & $32 \pm 12$ \\
\hline Prepubertal & 40 & 1 mo-9 yrs & M\&F & $79 \pm 14$ & $47 \pm 7$ \\
\hline Adult & 16 & $22-73$ yrs & M\&F & $820 \pm 279$ & $1300 \pm 424$ \\
\hline Delayed adolescence & 11 & $14-18$ yrs & M & $122 \pm 24$ & $162 \pm 45$ \\
\hline Craniopharyngioma & 3 & $8-15$ yrs & $\mathrm{M} \& \mathrm{~F}$ & $1 \pm 0.3$ & $12 \pm 11$ \\
\hline Precocious adrenarche & 4 & $7-9$ yrs & F & $105 \pm 41$ & $33 \pm 8$ \\
\hline Precocious puberty & 3 & $2-9$ yrs & M\&F & $305 \pm 107$ & $247 \pm 99$ \\
\hline Anorexia nervosa & 1 & 14 & $\mathbf{F}$ & 20 & 33 \\
\hline Intra-abdominal testes & 1 & 10 & $\mathbf{M}$ & 325 & 360 \\
\hline Agonadal & 1 & 11 & M & 4230 & 1610 \\
\hline Vanishing testes & 1 & 16 & $\mathbf{M}$ & 5400 & 3600 \\
\hline
\end{tabular}

Six girls with gonadal dysgenesis ( $1 / 2$ to 17 yrs) excreted $11,600 \mathrm{mIU} / \mathrm{hr}$ of $\mathrm{FSH}$ and/or LH. Luteinizing hormone releasing factor (LRF) caused a 75-400\% increment in urinary FSH and/or LH (3-hr samples) in 3 boys with undetectable blood levels of G.

accurate means of assessing circulating G levels.
215 MONE DEFICIENT CHILDREN BEFORE AND AFTER GROWTH HOR-
MONE ADMINISTRATION. Lynne L. Levitsky, Jane A. Uehara and JoAnn Marchichow, Dept. Ped., Univ. Chicago at Michael Reese Hosp. Med. Ctr., Chicago, IIl. (Intro. by S. P. Gotoff)

Increased glucagon levels have been demonstrated in acromegaly and after short-term growth hormone administration in normal adults. The role of hyperglucagonemia in the pathogenesis of the glucose intolerance seen with increased levels of growth hormone is unknown. Following intravenous arginine infusion $(0.5 \mathrm{~g} / \mathrm{kg}$ over $30 \mathrm{~min}$ ), growth hormone deficient children had a prolonged glucagon response compared to normal short children. However, after 5 days of growth hormone administration, 10 growth hormone def icient children displayed elevated plasma glucose levels, but glucagon response to arginine resembled that in normal short children.

Time (min)

$\begin{array}{ccccc}\text { Time (min) } & 0 & 30 & 45 & 60 \\ \text { Glucose pre GH (mg\%) } & 78 \pm 5 & 98 \pm 8 & 85 \pm 8 & 79 \pm 9 \\ \text { post GH } & 94 \pm 8 * & 132 \pm 20 * & 115 \pm 17 * & 98 \pm 9 * * \\ \text { Glucagon pre GH (pg/ml) } & 155 \pm 14 & 309 \pm 38 & 234 \pm 23 & 198 \pm 22 \\ \text { post GH } & 133 \pm 13 & 270 \pm 17 & 184 \pm 17 * & 152 \pm 24 *\end{array}$

$\begin{array}{ccccc}\text { post } \mathrm{GH} & 133 \pm 13 & 270 \pm 17 & 184 \pm 17 * & 152 \pm 24 *\end{array}$

Normalization of the glucagon response to arginine following short-term growth hormone administration makes it unlikely that hyperglucagonemia plays a role in the hyperglycemic effect of growth hormone in these children.
HYPOTHAITMTC-PTTUITARY DYSFUNCTTON IN A MALE WITH MC

216 CUNE-ALBRIGHT SYNDROME Fadil. Helene LeBlanc and S.S.C. Yen. (intr. by Grant Morrow, III) Dept. of Ped., Univ. of Arizona Medical Center, Tucson, $\mathrm{Az}$, and Dept. of $\mathrm{Ob}$. and Gyn., Univ. of California, San Diego School of Medicine, La Jolla, Callfornia.

Prior study of a $5 \mathrm{yr}$. old boy with McCune-Albright syndrome and elevated growth hormone (G.H.), which was non-suppressible both with glucose and chlorpromazine, Ied us to speculate that hypothalamic dysfunction might be responsible for his autonomous G.H. secretion and for his pubertal gonadotrophin levels and patterns. Sequential studies at age $6 \frac{1}{2}$ yrs. with arginine and direct evaluation of pituitary function with thyrotrophin releasing factor (TRF) and luteinizing releasing factor (LRF) showed: (a) subnormal thyroid stimulating hormone (TSH) response to TRF; (b) elevated G.H. ( $>100 \mathrm{ng} / \mathrm{ml}$ ) and prolactin (PRL) (>125ng/m1) which were unresponsive to arginine but which responded aberrantly to TRF; (c) no response of luteinizing hormone (L.H.) or follicle stimulation hormone to LRF; (d) hyperresponsiveness of glucose, glucagon (IRG) and insulin (IRI) to arginine. Somatostatin (SRIF) ( $I$.V. bolus plus constant infusion) given prior to the second administration of arginine, TRF, and LRF induced only a moderate fall in G.H. and no fall in PRL. SRIF lowered fasting levels of glucose, IRG and IRI and altered theix prior response to arginine. Sleep studies revealed (a) no pubertal L.H. rise; (b) an aberrant G.H. pattern; (c) no rise of PRL. These latest studies indicate multiple abnormalities of hormonal function, possibly mediated through both abnormal hypothalamic input to, and abnormal hormonal interaction within, the pituitary gland. 
217 PITUTTARY GIGANTISM: EFFECT OF SOMATOSTATIN AND TREAT. MENT WITH TRANSPHENOIDAL MICROSURGERY. Robert D. Lovinger, Felix A. Conte, Charles B. Wilson, and Melvin M. Grumbach, Dept. Pediatrics \& Neurosurgery, University of California San Francisco, San Francisco, California.

A 14 year old pubertal male with an excessive linear growth rate of $28 \mathrm{~cm}$ in 30 months, an enlarged sella turcica, and baseline plasma growth hormone (GH) levels consistently above $150 \mathrm{ng} /$ $\mathrm{ml}$ was studied. Somatostatin (100 $\mathrm{\mu g}$ stat and $3 \mu \mathrm{g} / \mathrm{min}$ for $2 \mathrm{hr}$ ) caused a decrease in plasma GH from 148 to $71 \mathrm{ng} / \mathrm{ml}$ by 45 minutes with no further decline. Sequential infusion of arginine and insulin evoked a paradoxical decrease in plasma GH from 206 to 118 $\mathrm{ng} / \mathrm{ml}$; however, oral glucose reduced plasma GH from 256 to $79 \mathrm{ng} /$ ml. The LRF response was pubertal; plasma $T$ was $131 \mathrm{ng} / \mathrm{dl}$. The rise in plasma TSH induced by TRF was blunted, although serum Th was $5.3 \mu \%$. Baseline prolactin was $9.2 \mathrm{ng} / \mathrm{mI}$ and declined on oral L-dopa to $<1 \mathrm{ng} / \mathrm{ml}$. A small eosinophilic adenoma was removed by transphenoidal microdissection. Only "little GH" was found in both plasma and a tumor extract by gel filtration.

Six months later the growth rate had decreased to $7.5 \mathrm{~cm} /$ year. Basal GH was $7.2 \mathrm{ng} / \mathrm{ml}$ and rose to $12.2 \mathrm{ng} / \mathrm{ml}$ during an arginine infusion. Somatostatin decreased plasma GH from 12.2 to $4.8 \mathrm{ng} / \mathrm{ml}$. other pituitary-hypothalamic function tests were unchanged. Thus (a) somatostatin effectively lowered but did not suppress plasma GH levels, implying partial resistance; (b) transphenoidal microsurgery appears to be a promising, low-morbidity approach to removal of intra-sellar tumors; and (c) removal of the tumor by this method results in return of normal $\mathrm{GH}$ levels without apparent loss of other pituitary functions.

218 OSTEOMALACIA IN A PATIENT RECEIVING DISODIUM ETIDRONATE (EHDP). Paul T. McEnery, R.C. Tsang, (intr. by Clark D. West, Children's Hospital Research Fdn., Cincinnati, Ohio.

Side effects of EHDP treatment in patients with ectopic calcification are reported to be minor. This report concerns a girl with nephrocalcinosis secondary to hypervitaminosis $D$ who developed rickets while receiving EHDP. At age 2 yrs. a mildly rachitiform metaphyseal lesion was observed. Serum calcium measured $9.0 \mathrm{mg} \%$; phos. $4.5 \mathrm{mg} \%$ and alk. phos., 18 BU. Metaphyseal dysostosis did not improve while receiving as much as $150,000 \mathrm{u}$ of vitamin $D$ daily for several years. Urinary amino acid excretion and renal function were normal as were tests of parents and male sibling. At age 8 yrs. hypervitaminosis $D$ developed with serum Ca of $16 \mathrm{mg} \%$; P, $5.1 \mathrm{mg} \%$; alk. phos. $9.4 \mathrm{BU}$ and creat. $3.9 \mathrm{mg} \%$. Ectopic calcification was noted on skull roentgenograph. Nephrocalcinosis was observed in renal biopsy tissue. EHDP therapy ( $5 \mathrm{mg} / \mathrm{kg}$ daily) started $12 / 71$, age $8-9 / 12 \mathrm{yrs}$, with cerum Ca level $12.6 \mathrm{mg} \% ; \mathrm{P}, 3.9 \mathrm{mg} \%$; and $\operatorname{Ccr} 27.9 \mathrm{ml} / \mathrm{min} / 1.73 \mathrm{M}^{2}$. On follow-up examinations serum P ranged 2.2 to $3.5 \mathrm{mg} \%$, no change in $\mathrm{Ca}$ or alk. phos. levels nor improvement of heterotopic calcification. Ccr increased to $55 \mathrm{~m} 1 / \mathrm{min} / 1.73 \mathrm{M}^{2}$. Tubular reabsorption of phosphate (TRP) measured $76 \%$. 11/72 EHDP was increased to $10 \mathrm{mg} / \mathrm{kg}$. 4/73 roentgenographs revealed florid rickets of knees and wrists. Serum Ca measured $9.9 \mathrm{mg} \%$; P, $2.5 \mathrm{mg} \%$; alk. phos. 14.4 BU and no change in TRP or renal function. parathormone leve1 was norma1. No improvement occurred with unrestricted calcium diet, $1000 \mathrm{u}$ of vitamin $\mathrm{D}$ daily or discontinuation of EHDP, but did occur following vitamin D 50,000 u daily.

219 SOMATOMEDIN AND GROWTH RESPONSE TO HGH REPLACEMENT Steven Milewski, Myron Gene1 and Raymond L. Hintz UConn. Health Ctr., Farnington and Yale Univ. Medical School, New Haven (Intr. by Martha L. Lepow)

Thirteen children with documented HGH deficiencies were treated with NPA HGH at $0.15 \mathrm{U} / \mathrm{kg} /$ week. All patients increased their growth rates while on therapy, and only $2 / 13$ did not reach normal or above growth rates for age. Somatomedin (SM) levels determined by porcine bioassay in this group prior to treatment were 0.22 $\pm 0.16 \mathrm{U} / \mathrm{ml}$ (mean \pm S.D.). This was significantly lower $(p<\otimes .001)$ than SM levels of 25 normal children, $0.65 \pm 0.15 \mathrm{U} / \mathrm{ml}$. While the $\mathrm{HGH}$ deficient children were on treatment their SM levels rose to $0.69 \pm 0.12 \mathrm{U} / \mathrm{m} 1$, indistinguishable from the control group. However, SM levels did not correlate with growth responses within the treatment group. The change in growth rate (rate during treatment minus rate before) did negatively correlate $(\mathrm{R}=0.52)$ with the growth rate before treatment. Thus, the slower growing children responded with greater increases in growth rates following HGH therapy. This implies that these children can use a dose of HGH more effectively, perhaps due to less endogenous HGH, than faster growing children. The change in growth rate also negative1y correlated $(R=0.51)$ with the age at the start of replacement therapy. Thus, the younger a child is at the onset of therapy, the better his response in terns of increase in growth rate. Early diagnosis and treatment of $\mathrm{HGH}-$ deficient dwarfism is therefore of great importance. This study confirms the normalization of SM levels with HGH therapy. However, factors other than SM levels alone may be important in responsiveness to HGH therapy.
220 MATERNAL SMOKE EXPOSURE: QUANTITATIVE EFFECTS ON NEONATAL HCS, T3 RIA AND TSH. RUSsell J. Moser, Dorothy R. Hollingsworth. Univ. Ky. Med. Ctr., Dept. Ped., Lexington (Intr. by Charles C. Mabry).

Maternal smoking exposure has a dose related effect on neonatal human chorionic somatomammotropin (HCS), triiodothyronine (T3) and thyrotropin (TSH), but not as expected. 44 normal pregnant women were studied ( 23 smokers and 21 nonsmokers). High and low exposure groups were determined by butt analyses (tar, nicotine, butts returned, average butt length), smoking questionnaire (cigarettes reported smoked), and puff profile (volume inhaled, puffs/cigarette, interval between puffs, puff duration, and smoking time/cigarette). Mean Tevels of HCS, $T_{3}$ RIA, and TSH were determined in infants in first hour. Infants of all smokers had higher mean levels of HCS $(11.4 \mathrm{ng} / \mathrm{d}$ l) than infants of nonsmokers $(10.6 \mathrm{ng} / \mathrm{d} 7)$. In $8 / 10$ smoking categories, infants of heavier smokers had higher HCS levels than infants of lighter smokers $(p<.01$ in 2 categories). Infants of all smokers had higher mean T3RIA levels $(136 \mathrm{ng} / \mathrm{dl})$ than infants of nonsmokers (117 $\mathrm{ng} / \mathrm{d} \mathrm{l})$. Infants of heavier smokers had higher mean levels of $T_{3}$ RIA than infants of lighter smokers in $6 / 10$ smoking categories $(p<.7$ in 5 categories). Infants of all smokers had lower mean TSH levels $(82 \mathrm{uU} / \mathrm{ml})$ than infants of nonsmokers $(94 \mathrm{uU} / \mathrm{ml})$. In $9 / 10 \mathrm{smok}-$ ing categories, infants of heavier smokers had higher mean levels of TSH than infants of lighter smokers ( $p<.09$ in 4 categories).

Conclusions: (1) Infants of mothers who smoke have higher HCS and TRRIA Tevels, and lower TSH values. (2) Levels of all 3 hormones were lower in lighter than heavier smokers.

221 A DIFFERENCE IN MATERNAL HUMAN CHORIONIC GONADOTROPIN (HCG) CONCENTRATIONS, BUT NOT IN CORD SERUM HCC CONCENTRATIONS, AS RELATED TO THE SEX OF THE FETUS. Robert Penny, N. Olatunji Olambiwonnu, S. Douglas Frasiex. Univ. So. Calif. Sch. Med., Los Angeles County-USC Med. Ctr., Dept. Ped., Los Angeles.

Using a radioimunoassay specific for the beta subunit of HCG, HCG concentrations were determined in paired maternal and cord sera. The sera of 46 mothers and 46 infants, 24 female and 22 male, were assayed.

Mean ( \pm SD) serum HCG concentration of mothers giving birth to female infants $(8.8 \pm 5.2 \mathrm{IU} / \mathrm{m} l)$ was significantly $(p<0.005)$ greater than that of mothers giving birth to male infants $(4.9 \pm 2.0 \mathrm{IU} / \mathrm{ml})$. Cord serum mean ( \pm SD) HCG concentration of female infants $(0.09 \pm 0.04 \mathrm{rU} / \mathrm{ml})$ was not significantly different from that of male infants $(0.09 \pm 0.02 \mathrm{IU} / \mathrm{ml})$. All cord serum HCG concentrations were less than maternal serum HCG concentrations. Poor correlation, on an individual basis and on a statistical basis, was observed between maternal and cord serum HCG concentrations.

These data axe in agreement with the results of previous studies employing less specific methods which suggested that maternal serum HCG concentrations were lower when the sex of the fetus was male than when it was female. It is also consistent with a placental barrier effect with regard to HCG transfer to the fetus.

222 Luteinizing Hormone (LH) and Follicle Stimulating Hormone (FSH) Excretion in Isosexual Precocity. Robext Penny, N. O. Olambiwonnu, S. Douglas Frasier. Univ. So. Calif. Sch. Med., Ios Angeles County-USC Med. Ctr. Dept. Ped., Los Angeles.

The 24-hour urinary excretion of LH and FSH was determined by radioimmunoassay of acetone extracts of urine for 30 days in an 8.3 year old girl with isosexual precocity and for 25 days in a normal 11.9 year old girl. The pattern of daily variation in urinary LH and FSH excretion observed in the girl with sexual precocity was similar to that of the noxmal menstrual cycle. LH and FSH midcycle peaks were $132.5 \mathrm{IU} / 24$ hours and $26.3 \mathrm{IU} / 24$ hours, respectively. Excluding the midcycle peak, the daily excretion of LH was $28.4 \pm 9.3$ (SD) IU/24 hours, and the excretion of FSH was $8.9 \pm 1.9$ (SD) IU/ 24 hours, values comparable to those of normal adult females. In contrast, the daily excretion of LH in the normal 11.9 year old girl was $6.9 \pm 1.1$ (SD) $I U / 24$ hours and FSH excretion was $3.9 \pm 0.9$ (SD) IU/24 hours. No LH or FSH surge was observed. The data are consistent with early maturation of the hypothalamic-pituitarygonadal axis in idiopathic isosexual precocity. 
223 EFFECT OF CONSTAANT INFUSTON OF LUTEINIZING HORMONERELEASING HORMONE (LH-RH)UPON GONADOTROPIN SECRETION IN CHILDREN. E.O.Reiter, G.E.Duckett and A.W.Root. Univ. of So.Fla., Tampa and All Children's Hosp., St.Petersburg.

In order to evaluate the effect of prolonged pituitary exposure to LH-RH in children, the secretory patterns of LH and FSH were determined in 7 prepubertal(PreP) and 15 pubertal(Pub) subjects during constant infusion of $\mathrm{LH}-\mathrm{RH}$. After a $2 \mathrm{hr}$. control period, $100 \mu \mathrm{g}$ synthetic LH-RH was infused over $3 \mathrm{hrs}$. with sampling at 15 intervals. In PreP mean serum LH rose $(\mathrm{p}<.001)$ from $3.1 \pm 0.3(\mathrm{SE}) \mathrm{mIU} /$ $\mathrm{m} 1$ (2nd IRP-hMG) to $7.0 \pm 0.5$ during the infusion. In Pub LH rose ( $p<.001$ ) from $6.0 \pm 0.4$ to $28.2 \pm 1.5$. The peak LH level in Pub $(46.9 \pm$ $7.8)$ was greater $(p<.001)$ than in PreP(11.5 \pm 2.5$)$. The integrated $3 \mathrm{hr}$ area response during $L H-R H$ infusion was greater $(p<.01)$ in Pub $(4825 \pm 605$ units) than in PreP $(1209 \pm 255)$. Both immediate and delayed increases in LH levels were recorded in Pub during LH-RH infusion, whereas only an immediate rise in LH to a plateau occurred in PreP. Pulsatile secretion of LH,i.e.LH values differing from nadir to peak by $>20 \%$, occurred during the basal period in Pub without observable change in amplitude or frequency during infusion, but rarely during the basal period. Pituitary FSH reserve was greater in girls than in boys and did not increase with pubescence. These data demonstrate:(1)A maturity-related increase in LH reserve and sex-specific FSH release; (2)The presence of two releasable pituitary LH pools in Pub; (3) That LH-RH infusion does not alter episodic LH release when present basally but does permit its demonstration when previously inapparent.

\section{DELAYED PUBERTY AND ANOSMIA: COINCIDENCE OR KALLMANN} VARIENT? Iraj Rezvani, Ange lo M. DiGeorge, Joseph Reitano, and Peter J. Snyder, St. Christopher's Hosp. for Children, Dept. Ped., Temple Univ. Sch. Med. and the Endocrine Section, Univ. Pennsylvania Sch. Med., Philadelphia, Pa.

A $17 \frac{1}{2}$ year old male with anosmia, lack of spontaneous sexual development and prepubertal levels of serum gonadotropins which were further suppressed by clomiphene was believed to have the classic Kallmann syndrome. Other pituitary functions were normal and bone age was 13 years. The patient failed to develop any signs of puberty during the ensuing 15 months of observation. Testicular biopsy revealed a prepubertal structure with neither leydig cells nor spermatogenesis. During treatment with methyltestosterone ( $10 \mathrm{mg} /$ day) for $1 \frac{1}{2}$ years, he developed secondary sexual characteristics and, unexpectediy, an increase in the size of testes to $4.5 \times 2.5 \mathrm{~cm}$., bilaterally. At $20 \frac{1}{2}$ years of age, therapy was discontinued. Three months later, he was still anosmic by formal olfactory testing, but his serum testosterone level was normal (535 and $512 \mathrm{ng} / \mathrm{di}$ ). A sperm count was $28 \times 10^{6} / \mathrm{ml}$ with normal morphology and mobility, and serum LH response to an I.V. bolus of 50 ug Gn RH (Abbott) was normal (Table). This patient could represent the rare chance association of delayed puberty and represent the rare chance association of delayed puberty and Regardless, misdiagnosis would lead to unnecessary prolonged treatment and erroneous counseling concerning fertility.

$\begin{array}{lllllllllll} & -30^{\prime} & 0^{\prime} & 10^{\prime} & 20^{\prime} & 30^{\prime} & 45^{\prime} & 60^{\prime} & 90^{\prime} & 120^{\prime} & 180^{\prime} \\ \text { FSH (mIU/m1) } & 7.5 & 7.3 & 9.0 & 10 & 9.8 & 9.8 & 9.8 & 10.1 & 10 & 8.9 \\ \mathrm{LH}(\mathrm{mIU} / \mathrm{ml}) & 16 & 11^{3} & 68 & 52 & 50 & 50 & 41 & 30 & 20 & 17\end{array}$

225 RADIOIMMUNOASSAY OF HYPOTHALAMIC GONADOTROPIN RELEASING HORMONE (GN-RH) : EFFECT OF CASTRATION AND STARVATION IN ADULT MALE RATS. A.W.Root, E.O.Reiter, G.E. Duckett and M.Sweetland, Univ. of So.Fla.Coll.of Med., Tampa and A1l. Children's Hospital, St. Petersburg.

A non-equilibrium, double antibody radioimmunoassay for Gn-RH has been established. The mean $B_{0}$ is $30.4 \%$; the mean $B_{50}$ is $95 \mathrm{pg}$ and the least detectable amount of $\mathrm{Gn}-\mathrm{RH}$ is $5 \mathrm{pg}$. Serial dilutions of methanol extracts of rat median eminence-hypothalamus cross react in a parallel manner to the standard. This assay was applied to the measurement of hypothalamic $\mathrm{Gn}-\mathrm{RH}$ content in adult male rats, who were castrated or sham operated and sacrificed by decapitation 7 days later. Groups of intact or castrated animals were either fed ad lib or starved for 7 days before sacrifice.

\begin{tabular}{|c|c|c|c|}
\hline & & Fed & Starved \\
\hline $\begin{array}{l}\text { Gn-RH } \\
\text { (ng/hypo) }\end{array}$ & $\begin{array}{l}\text { Intact } \\
\text { Castrated }\end{array}$ & $\begin{array}{l}1.6 \overline{8 \pm} 0.62 ! \\
0.67 \pm 0.25\end{array}$ & $\begin{array}{l}1.82 \pm 0.60 \\
0.69 \pm 0.30\end{array}>$ \\
\hline $\begin{array}{l}\text { Serum LH } \\
(\mathrm{ng} / \mathrm{m} 1)\end{array}$ & $\begin{array}{l}\text { Intact } \\
\text { Castrated }\end{array}$ & $\begin{array}{l}25.3 \pm 17.8 \\
94.9 \pm 37.4\end{array} ; * *$ & $\begin{aligned} 27.6 & \pm 16.8 \\
182.0 & \pm 99.8\end{aligned}>*$ \\
\hline $\begin{array}{l}\text { Serum FSH } \\
(\mathrm{ng} / \mathrm{ml})\end{array}$ & $\begin{array}{l}\text { Intact } \\
\text { Castrated }\end{array}$ & $\begin{array}{l}379.7 \pm 135.0 \\
893.9 \pm 240.4\end{array}>*$ & $\begin{array}{r}194.4 \pm 83.9 \\
1173.4 \pm 110.4\end{array}$ \\
\hline
\end{tabular}

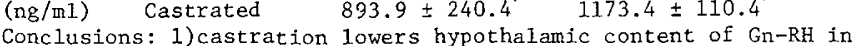
both fed and starved rats; 2) short term starvation does not alter hypothalamic Gn-RH levels in either intact or castrated animals.

$$
: \overline{\mathrm{X}} \pm 1 \mathrm{SD} \quad{ }^{*} \mathrm{p}<0.01
$$

226 REVERSE $T_{3}\left(R T_{3}\right), T_{4}$ AND T3 IN THE HUMAN NEWBORN. Joseph Sack, Inder J. Chopra and Delbert A. Fisher. Med. and Ped., Los Angeles, Cal ifornia.

Thyroid hormone metabolism is different in the fetus than in the adult; fetal serum $\mathrm{T}_{4}$ levels are higher and $\mathrm{T}_{3}$ lower than in aduit serum. In addition, serum RT3 (3, $3^{\prime} 5^{\prime} \mathrm{T} 3$ ) levels are higher in fetal than in adult serum. To get some insight into the metabolic processes responsible for the elevated fetal RT3 le vels, we studied serum $\mathrm{T}_{4}, \mathrm{~T}_{3}, \mathrm{RT}_{3}$ and $\mathrm{TSH}_{\mathrm{H}}$ in normal newborns during the first 30 days of life.

At four hours of age the serum RT3 concentration (mean + SE) $(165 \pm 13 \mathrm{ng} / 100 \mathrm{~m} 1)$ was not significantly different from that in paired cord sera $(194 \pm 25 \mathrm{ng} / 100 \mathrm{ml})$ whereas the serum $\mathrm{T} 3$, T4 and TSH levels $(175 \pm 29 \mathrm{ng} / 100 \mathrm{ml} ; 17.8 \pm 2.7 \mu \mathrm{g} / 100 \mathrm{ml}$; and 27 $+5 \mu \mathrm{U} / \mathrm{ml}$ ) were significantly higher at 4 hours than at birth (47 $\pm 5 \mathrm{ng} / 100 \mathrm{ml} ; 15.2 \pm 1.2 \mu \mathrm{g} / 100 \mathrm{ml}$; and $11 \pm 2 \mu \mathrm{U} / \mathrm{ml}$, respectively). The mean serum RT3 in one to four day old newborns did not differ significantly from the mean cord serum concentrations, but all values were significantly lower after 5 days and after 7 days were similar to mean adult serum concentrations. Mean serum $T_{3}$ levels between one and 30 days were higher than or comparable to the mean concentration of nomal adults. Mean serum 74 levels between birth and 30 days of age were significantly higher than in adults. These results suggest 1) the early TSH surge does not influence serum RT3 in the newborn, 2) the high RT3 in the fetus falls to adult levels after one week, 3) there are separate enzyme systems for alpha and beta iodothyronine ring deiodination.

227 A SCREENING TEST FOR GROWTH HORMONE (GH) DEFICIENCY. Bonnie Shanis and Thomas Moshang, Jr. Dept. of Ped., Hahnemann Med.Col., Phila, Pa.

There is a need for a safe, inexpensive and yet reI iable screening test for GH function. Exercise has been utilized for this purpose but has a "false negative" response (non-GH release) in $10-33 \%$ of normal children. Beta adrenergic blockade has been effective in enhancing GH release in response to several pharmacologic agents. Propranolol ( 20 to $40 \mathrm{mg}$ ) was used in conjunction with exercise $(15$ minutes of easy running in place, followed by 5 minutes of hard running) in 28 non-GH deficient children. All responsed to propranolol-exercise with serum GH levels exceeding $7 \mathrm{ng} / \mathrm{ml}$. One child who had classic clinical findings of emotional deprivation dwarfism and abnormally low GH responses to insulin and L-Dopa, responded normally to propranolol and exercise. Two GH deficient patients showed no response to propranolol-exercise, insulin and L-Dopa. Results in 2 other $G H$ deficient patients are pending. Non GH Def. $\frac{\text { Basal }(\mathrm{ng} / \mathrm{ml})}{5.01 \pm 0.68} \frac{\text { Post-Propranolol }}{18.12 \pm 2.63} \frac{\text { Post-exercise }}{26.37 \pm 2.55}$ $\begin{array}{ccc}\text { GH Det. } & 1.61 .67 \\ \text { There were no instances of hypoglycemia. This test, }\end{array}$ because of the moderation of exercise, can be : it: ized in young children, the youngest child tested being age $3 \frac{1}{2}$ years. Propranolol plus exercise appears to be an effective screening test for GH function, being safe, convenient and at present, no false negative responses.

\section{8} EFFFCTS OF ORAL HYPOGLYCFMIC AGENTS ON C.ARBOHYDRATE METABOLISM AND CAPILLARY BASEMENT MEN!BRANE TIITCKNESS
(BMT) IN CHILDREN WITH EARLY DIABETES MELLITIS (DM) Bagher $M$. Sheikholislam, Julian J. Irias, George $H$. Lowrey and lung-Jung Lin. Department of Pediatrics, School Medicine, University of California, Davis, California 95616 .

This is a prospective study of the effects of phenformin (P)
pal tolazamide (T) on BMT, glucose disappearance rate $(K)$, and plasma insulin and growth hormone responsiveness during intravenous glucose tolerance testing (IVGTT) in 11 children with early DM.

Changes in these parameters after treatment with $P$ alone were variable and generally small. Addition of $T$ within 6 to 8 weeks decreased plasma glucose concentrations to values which were $27 \%$ to $91 \%$ of the pretreatment values. In most instances $\mathrm{K}$ values increased. These effects of the combined therapy were not uniformly sustained during longer observation ( 7 months to 4 years).

Responses of plasma insulin and growth hormone during IVCTT were quite variable. Patients who exhibited a poor insulin response before treatment had more severe manifestations of diabetes and in most instances became insulin dependent.

Changes in BMT and in $K$ value were considered collectively and were found to be inversely related $(P<0.001)$. Increases in BMT appeared to be reversible provided that carbohydrate tolerance could be improved. When the patients were considered individually, it was found that every patient whose BMT increased significantly had persistent or worsening glycosuria, but the converse was not true.

The findings suggest that glucose intolerance may be a prein BMT, and that the latter may be reversible if glucose tolerance can be improved. 
229 DISPROPORTIONATE ACCELERATED SKELETAL MATURATION FROM CORTISONE ACETATE. Edna H. Sobel Albert Einstein Col. of Med., Dept. of Ped. New York

Corticosteroids are being used to prevent or ameliorate respiratory distress syndrome. An evaluation of possible deleterious effects was undertaken in Caeserian-derived Sprague-Dawley rats by giving $1.25 \mathrm{mg}$. of cortisone acetate I.M. on the 4th day of life to 20 pups; 25 controls received $0.05 \mathrm{ml}$ saline. pups were reared in litters of 8 (all treated or all control); terramycin was added to the drinking water. At age 21 days body weight, stem-length and tibia length were all less in the cortisone treated animals than the same measurements in the controls, but skeletal maturation was advanced in the treated animals.

$\begin{array}{lccl} & \text { Treated } & \text { Control } & \mathrm{P} \\ \text { Body weight, gm. } & 51.14 & 63.04 & <0.001 \\ \text { Stem length, mm. } & 112.55 & 125.32 & <.001 \\ \text { Tibia length, mm. } & 16.88 & 20.35 & <0.001 \\ \text { Ossif. center, no. } & 118.0 & 107.56 & <0.05\end{array}$

These findings have implications for the future growth of infants given corticosteroids because reduced linear growth and accelerated skeletal maturation will eventuate in short stature.

23 THE PARATHYROID HORMONE (PTH) RESPONSE TO EDTA INFUSIONS IN INFANTS AND CHILDREN, Reginald C. Tsang, I-Wen Chen, Paul T. McEnery, David R. Brown, Kikuo Iataka, Arun Pramanik \& MaryAnn Friedman. Univ. of Cincinnati. Improved understanding of parathyroid function can be learned from changes in PTH production in response to decreases in ionized calcium. Intravenous EDTA (ethylenediaminetetraacetate) infusions result in decreased serum ionized $\mathrm{Ca}$ and can serve as parathyroid function tests. Such tests have not been reported in infants and children. EDTA infusions in normal adults increase serum PTH (assay recognizing predominantly 9500 molecular weight PTH) to $142 \%$ (peak value compared with pre-infusion value) and $260 \%$ in parathyroid adenoma patients (Chen, et a1 J Nucl Med 15: $763,1974)$. In this study 6 relatively normal PTH responses were elicited in 5 patients; one phosphate induced infantile tetany (124 and $108 \%$ PTH response), a mother of infants with idiopathic hypercalcemia (142\%), a 9 year old with hypophosphatemic (hypoP) rickets $(175 \%)$ and 2 persons with healed familial hypop rickets $(174,141 \%)$. Five hyperactive PTH responders included 4 children with hypop rickets: ages one month $(317 \%), 4$ and 8 months (one infant-296\% and $621 \%), 9$ year $(490 \%)$ and 10 year $(1013 \%)$. Four relative hyporesponders included 3 children ages 1,2 and 4 with idiopathic hypercalcemia $(50 \%, 78 \%, 106 \%)$, and a 14 year child with "cast syndrome" hypercalcemia (100\%). The maximum falls in serum ionized $\mathrm{Ca}$ during all tests ranged from 0.3 to $1.3 \mathrm{mg} \%$ and did not correlate with PTH responses. There were few side effects and inconsistent changes in serum $\mathrm{Mg}$, total $\mathrm{Ca}$ and $\mathrm{P}$. The EDTA infusion test may be useful in assessing the functional capabilities of the parathyroids in infants and children.

231 IODINE TRAPPING DEFECT AND ATHYREOTIC CRETINISM IN SIBLINGS. H. Lawrence Vallet, Dorothy Barnard, Ieuan Hughes, Lesley Baldwin, N.Y.S. Birth Defects Inst.* and Dept. Ped., A.M.C., Albany and Dalhousie Univ. Halifax, N.S. (Intro. by Richard J. Pickering).

A 3 mo. old presented with athyreotic cretinism which was confirmed by a $\mathrm{T}_{4}$ of $0.6 \mathrm{ug} \%$, a $3.0 \%$ RAI uptake at 6 and 24 hours and negative technecium scan. TSH was $>400 \mathrm{uI} . \mathrm{U} . / \mathrm{m} 1$. A neonate sibling, at one day of age had a $\mathrm{T}_{4}$ of $1.7 \mathrm{ug} \%$ with mild clinical hypothyroidism and a small goitre. TSH was $>600$ UI.U./ml. Technecium scan was negative; RAI uptake was $9.3 \%$ at $6 \mathrm{hrs}$, and $7.0 \%$ at $24 \mathrm{hrs}$.

131. I Salivary-plasma (S/P) with calculated salivary clearances $\left(S_{131_{F}}\right)$ were studied in sibling (A) and goitrous sib (B), one parent $f(C)$ and control (D) from 15 to 155 min. after administration of IV $131_{I}$. S/P ratios approach unity in trapping defects and are $>20$ in normals. The $\mathrm{S} / \mathrm{P}$ ratios for (A), (C) and (D) were normal with means of $38.14,31.38$ and 29.33 respectively. Goitrous (B) was abnormal being 7.3. $\mathrm{S}_{131}$ Ias abnormal in (B) being $0.5 \mathrm{~m} 1 \mathrm{~s} / \mathrm{min}$. These findings confirm an iodine trapping defect in goitrous sib (B). Biopsy of the goitre revealed marked hyperplasia and smal1 acini without inflammation. Agglutinating and C.F. antibodies were negative.

The presence of two hypothyroid states of different etiologies in these siblings suggests the need for screening all sibs at risk and defining the etiology of the hypothyroidism in each case before genetic counseling is undertaken.

Support in part by MRC DG-79.

*Unit of N.Y. State Department of Health.
232 THE PITUITARY - THYMIC AXIS. H. Lawrence Vallet, Catherine Kyong, Vernon W. Krause, Richard Pickering, Ped., A.M.C., Albany and Dept. Path., I.W.K. Hospital, Halifax, N.S.

Because of hyperpigmentation and radiological bone abnormalities a patient with combined immuno-deficiency (CID) and absent $\mathrm{RBC}$ adenosine deaminase (ADA) underwent endocrinologic evaluations between the ages of $5 \mathrm{mo}$. and $1 \mathrm{yr}$.

Water deprivation tests were normal as were the $T_{4}$, Lh and FSH. Appropriate response to metapirone was present. Three abnormal responses to tests for GH release were seen; to arginine, glucagon and insulin the peak levels were $2.0,2.8$ and $4.0 \mathrm{ng} / \mathrm{ml}$ respectively. Fasting hypoglycemia $(26.0 \mathrm{mg} \%$ ) was present with persistent insulinopenia $(6.0 \mu \mathrm{U} / \mathrm{ml})$ following an OGTT. Ten days of hGH therapy normalized the fasting glucose but insulinopenia persisted. At post-mortem a markedly hypoplastic anterior pituitary was found. Rib sections showed blunted growth plates and narrow zones of provisional calification, as seen in GH deficient rats. Since similar radiological bone abnormalities have been observed in other children with ADA deficiency and CID, disturbances of $\mathrm{GH}$ should be sought in such children.

Pituitary-thymus relationships have been suggested by the Sne11-Bagg mouse mode1; the presence of 1 ymphocyte growth hormone receptors in man may further support the existence of this "axis". It is suggested that ADA may be necessary for specific hypothalamic-pituitary function and when absent could be responsible for this clinical picture.

233 THYROID FUNCTION IN CHRONIC RENAL FAILURE (CRF). Steven J. Wassner, Bruce A. Buckingham, Ann K. Kershnar, Mohammad H. Malekzadeh, Alfred J. Pennisi and Richard N. Fine. Univ. of Southern California Sch. of Med. Dept. Ped. and Childrens Hosp. of Los Angeles, Dialysis \& Transplant Program and Div. Endocrinology and Metabolism, Los Angeles. Thyroid function was studied in 24 patients aged $4-18$ years, with CRF; 15 prior to hemodialysis and 9 after $>3$ months of hemodialysis. Two, 1 with radiation induced thyroiditis and 1 with cystinosis, were hypothyroid; the other 22 were clinically euthyroid. The mean values for patients either before or after chronic hemodialysis were: free thyroxine index (FTi) $(5.8$ vs. $5.3 \mu \mathrm{g} \% \mathrm{p}<.5)$ RIA $T_{3}\left(T_{3}\right)$ (85 vs. $\left.108 \mathrm{ng} \% \mathrm{p}<.025\right)$ and free $\mathrm{T}_{4}\left(\mathrm{FT}_{4}\right)(2.0 \mathrm{vs} .1 .7$ $n g \% p<.1)$. Except for the 2 hypothyroid patients whose TSH values were 82 and $84 \mu \mathrm{U} / \mathrm{ml}$ respectively, and 1 adolescent girl with TSH $=20 \mu \mathrm{U} / \mathrm{ml}$ and positive fluorescent antithyroid antibodies (ATA), all other patients had TSH values $<8 \mu \mathrm{U} / \mathrm{ml}$ and negative ATA. Although mean values for $\mathrm{FTI}, \mathrm{T}_{3}$ and $\mathrm{FT}_{4}$ in clinically euthyroid patients were normal, there were only 11 patients in whom all tests were normal. Nine of $22 \mathrm{FTI}$ values, 5 of $22 \mathrm{~T}_{3}$ values and 6 of $22 \mathrm{FT}_{4}$ values were in the hypothyroid range. Five patients had both low FTI and FT4, 4 had low FTI and normal FT4 and 2 had low $\mathrm{T}_{3}$ only. All 6 patients with low $\mathrm{FT}_{4}$ values had normal $\mathrm{T}_{3}$ levels.

It is apparent that: 1) most patients with CRF are euthyroid. 2) either a normal $\mathrm{FT}_{4}$ or normal $\mathrm{T}_{3}$ can suppress TSH in patients with CRF; 3) other than TSH, no single test of thyroid function gives consistent results in patients with CRF, and; 4) TSH is a reliable indicator of primary hypothyroidism in patients with CRF.

234 EVALUATION OF GROWTH HORMONE (GH) RELEASE IN CHILDREN USING ARGININE AND L-DOPA IN COMBINATION. Virginia V. Weldon, William L. Clarke, Georgearna Klingensmith, Stephen C. Duck, Morey W. Haymond and Anthony S. Pagliara. Washington Univ. School of Med., Dept. of Ped., St. Louis, Mo.

L-dopa (125-500 mg) and arginine monochloride $(0.5 \mathrm{G} / \mathrm{kg})$ were given simultaneously to 56 children with short stature. The response to combination testing was compared to arginine ( 46 patients) and L-dopa (54 patients) alone with results expressed as mean peak $\mathrm{GH}$ in $\mathrm{ng} / \mathrm{m} 1 \pm \mathrm{SEM}$. Patient nos, are in ( ):

\begin{tabular}{|c|c|c|c|c|}
\hline & L-dopa & & Arginine & Combination \\
\hline ficiency & $1.3 \pm 0.3$ & (16) & $2.2 \pm 0.4 \quad(11)$ & $2.8 \pm 0.4$ (16) \\
\hline $\operatorname{Re}$ & $14.5 \pm 1.8$ & & $4.6 \pm 2.7$ & $\begin{array}{l}13.8 \pm 1.0 \\
21.5 \pm 2.4\end{array}$ \\
\hline
\end{tabular}

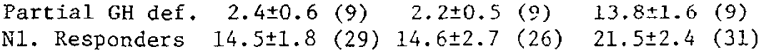
Sixteen children were diagnosed as having GH deficiency ( $\mathrm{GH}<6 \mathrm{ng} /$ $\mathrm{ml}$ ). Of the remaining 40,9 passed the combination test and failed to respond to L-dopa and arginine alone. These results suggest that the combined use of arginine and L-dopa may identify patients who have partial GH deficiency. However, if currently accepted normal responses are applied to the combination test, failure to identify patients with partial GH deficiency may result. Mean integrated secretion rates above basal release in the 31 children without GH deficiency were determined by planimetry with the following results expressed in $\mathrm{ng} / 120 \mathrm{~min} \pm \mathrm{SEM}$ : L-dopa, $259.3 \pm 141.7$; arginine, 405.8 \pm 127.1 ; combination, $844.8 \pm 174.7$ (p $0.02)$. These results suggest that the effects of L-dopa and arginine are additive, implying different mechanisms of action for these agents. 
235

HERMAPHRODITISM WITH ANORCHIA. R.HK Wu, J.W

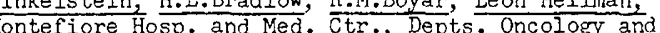
Pediatrics, The Institute for Steroid Research, Bronx, New York. An 18 year old female had primary amenorrhea and no breast development. She had a small amount of sexual hair. She had a slightly enlarged clitoris with normal labia and no posterior fus$i$ on. Her vagina was of normal length but no cervix was palpable. At laparoscopy no gonads, uterus or tubes were seen. Buccal smear was chromatin negative and the karyotype was $46 \mathrm{XY}$. There was normal adolescent episodic secretion of gonadotrophins in plasma with a higher concentration of LH during the sleep vs. wake periods although the absolute concentration was in the castrate range. Plasma testosterone was $37 \mathrm{ng} / \mathrm{ml}$ and urinary estrogens were 15 and $19 \mathrm{mg} / \mathrm{day}$. The ratio of androsterone to etiocholanolone recovered in urine following the administration of labelled testosterone was 1.4 which is well above the normal adult female range and is in the normal adult male range. These data suggest that the patient was a genetic male in whom the gonaductal system began to mature in a normal male fashion. The Mullerian system atrophied and the phallus and Wolffian systems began to develop, but were probably not completed because of destruction of the gonads early in gestation. This resulted in complete agenesis of the gonaductal system as far as can be determined by laparoscopy and almost normal female external genetalia. This represents the second case of this variation of pseudohermaphroditism. The patient has responded to estrogen replacement therapy and is making a good psychological adjustment to her disease.

236 STUDIES OF BIOCHEMICAL AND HORMONAL MARKERS OF RICKETS IN CHILDREN RECEIVING ANTICONVULSANT DRUGS (ACD). Helen Yeager, John Winnacker, John Saunders, Barbara Russell and Constantine Anast. Depts. Child Health and Medicine, Univ. of Mo. and VA Hosp., Columbia, Mo.

Previous reports reflect conflicting opinions regarding the significance of ACD in relation to $\mathrm{Ca}$ metabolism. In an effort to better characterize alterations in $\mathrm{Ca}$ metabolism, we studied serum $\mathrm{Ca}, \mathrm{P}$, alk phos, 25-OH-vit $\mathrm{D}(25-\mathrm{OH}-\mathrm{D})$ and immunoreactive parathyroid homone (iPTH) in 39 control children and 41 epileptics, ages 2-16, treated with combinations of phenobarbital (PH), diphenylhydantoin (DPH) and Primidone (PRIM). Treated and untreated children received comparable vit D:

\begin{tabular}{|c|c|c|c|}
\hline & & All ACD & ACD Group \\
\hline rum values & & Children(4I) & Receiving PRIM(1I) \\
\hline $\mathrm{Ca}(\mathrm{mg} / \mathrm{dl})$ & $10.2 \pm .06$ & $10.0 \pm .07 *$ & $9.8 \pm .15 *$ \\
\hline$P(m g / d l)$ & $5.0 \pm .07$ & $4.8 \pm .07 *$ & $4.9 \pm .16$ \\
\hline Alk Phos $(\mathrm{mU} / \mathrm{ml})$ & $20.1 \pm 10$ & $270 \pm 21 *$ & $235 \pm 38$ \\
\hline $25-\mathrm{OH}-\mathrm{D}(\mathrm{ng} / \mathrm{ml})$ & $22.7 \pm 1.0$ & $20.7 \pm 1.3 *$ & $17.5 \pm 2.9 *$ \\
\hline $\mathrm{iPTH}(\mu \perp \mathrm{Eg} / \mathrm{ml})$ & $3.4 \pm 0.4$ & $4.2 \pm 0.6$ & $4.6 \pm 0.9$ \\
\hline Treatment (mos.) & & $65 \pm 8$ & $94 \pm 12$ \\
\hline
\end{tabular}

$*$ Significantly different from controls by rank sum test The lower serum 25-OH-D, Ca, and $\mathrm{P}$ and higher alk phos in the $\mathrm{ACD}$ groups is consistent with altered vit $D$ metaholism. The magnitude of difference of each of these parameters and the smali nonsignificant increase in serum iPTH in the ACD groupsindicates that the metabolic defect is mild. The distinctly lower serum $\mathrm{Ca}$ and 25-OH-D in children receiving PRIM may be due to the PRIM itself or to the longer treatment period.

\section{EPIDEMIOLOGY}

237 GROUP B STREPTOCOCCI (GBS) IN PERTIATAL NNFECTIONS: NATURAL HISTORY OF MATERNAL AND NEONATAL COLONIZATION. Bascom F. Anthony, Donald Okada, and Calvin J. Hobel, Depts. of Ped. and Ob-Gyn., UCLA Sch. of Med., Harbor Gen. Hosp., Torrance, Ca.

In a prospective study, 187 women and their infants have been followed through pregnancy, postpartum and neonatal periods. GBS were isolated from $17 \%$ of the pregnant women at the first prenatal visit but, with repeated cultures, from $33 \%$ at some time during pregnancy or the postpartum period. Urethral cultures contained more colonies and were more often positive (15\%) than cervical cultures (II\%). The prevalence of GBS did not vary significantly among the 3 trimesters, intrapartum or postpartum periods. GBS were more frequently isolated in the last half of the calendar year. Type III, followed by type II, was the most comon serotype isolated from both mothers and infants.

Twenty-four women were transiently, 13 intermittently and 23 persistently positive for GBS. Colonization was more frequent $(35 \%)$ in infants of these women than in those of negative women (9\%). However, the most significant determinant of infant colonization was GBS in the birth canal at delivery; GBS of the maternal serotype was recovered in the first week of life from almost $60 \%$ of infants borm under these circumstances.

GBS were recovered from nose, throat, cord or combinations of these sites in newborms. Approximately 1/3 of colonized infants examined at 4-8 weeks still harbored GBS, usually in nose or throat. No serious infections related to GBS have been observed in these infants under study.
238 NOSOCOMTAL EPIDEMICS OF NEONATAL NECROTIZING ENTEROCOLITIS. Nirmala S. Desai, M. Douglas Cunningham, and H. David Wilson. (Intr. by Charlton Mabry). Albert B Chandler Medical Center, Univ. of Ky., Dept. of Peds., Lexington.

observations of 2 outbreaks of necrotizing enterocolitis (NEC) in a neonatal intensive care unit suggest the epidemic nature of this syndrome. The outbreaks, 1 year apart, involved $6 / 15$ and $12 / 17$ infants. Average birth weight was 1590 gms; mean gestational age was 31.5 wks. The mean age of onset was 28 days of age. A11 18 infants ( 9 males/9 females) had guaiac positive stools, abdominal distention and bile stained gastric aspirates. 12 had pneumatosis intestinalis, including 4 with $x$-ray findings of air in the portal venous system. All the affected infants and cohorts were isolated from new admissions. At the onset of symptoms feedings were withheld, stomachs decompressed, and antibiotics given. 4 infants developed bowel perforation, all underwent surgery, and 3 died; 1 in the first outbreak and 2 in the second. The fatality rate was $16.6 \%$ for each outbreak. $50 \%$ of the cases in the first outbreak and $75 \%$ in the second occurred within the first 3 days. In 3 instances symptoms developed within 4 days of admiss ion to the unit. Cases ceased to develop after 24 days in the first outbreak and 17 days in the second. No new cases occurred when the unit was re-opened. No correlation could be seen with respect to umbilical catheters, formula, Apgar scores, acidosis, hypotension or apneic spells, in infants with and without NEC. Blood, cerebrospinal fluid and urine cultures were negative. Stool cultures revealed enteric flora. Representative E. coli were non-invasive and non-enterotoxigenic. NEC outbreaks were epidemic in nature with distinct clinical and communicable features.

239 GROUP B BETA STREP (GBS) COLONIZATION UF MOTHERS AND (t) Eidelman and George Szilagi. (Intr by: Lawrence Gartner) Dept of Pediatrics, Albert Einstein College of Medicine, Bronx, New York.

903 deliveries were studied (Mar.-July) to ascertain the route and incidence of $G B B S$ colonization and disease. Single vaginal or cervical maternal cultures were obtained during labor. Admission neonatal throat and umbilical cultures were obtained on all 903 maternal-infant pairs, and in 539 infants at age 4-days. Maternal colonization was $89 / 1000$. Of 80 GBS positive mothers, if infants $(17.5 \%)$ were colonized at birth compared to only 9 infants $(1.1 \%)$ born to 823 GBS negative mothers $(0 .<.001)$. Twelve $(33 \%)$ previously GBS negative infants born to 35 GBS positive mothers compared to only $14(2.6 \%)$ infants born to 504 GBS negative mothers $(p .<.001)$ became GBS colonized during nursery stay. The total colonization rate (admission plus nursery) in infants born to $r_{3} B S$ positive mothers was $470 / 1000$ compared to $41 / 1000$ in GBS negative mothers $(p .<.001)$

Two infants developed early onset GBS sepsis for an attack rate of 2.2/1000. In both cases the maternal and newborn admission cultures were GBS positive for an attack rate of $87 / 1000$ infants colonized at birth. No infants GBS negative at birth developed early or late onset GBS disease. The above confirms that neonatal GBS colonization results either from contact with colonized mothers antenatally or during nursery period while neonatal GBS infection relates primarily to antenatal colonization. Screening programs that identify maternal GBS carriers and colonized infants will thus allow physicians to
identify infants with the greatest risk for GBS disease.

240 ORRELATES OF NEONATAL TRANSPORT VARIABLES IND MORTALITY. PRHDICTVE VALUE. Angelo Ferrara, Rajagopalan Indra, (Intr. by Joseph Dancis) NYU Sch.Med.-Bellevue hosp.Ctr., Dept.Ped.

In a 33 month period (4/71-12/73), the NyC-ITS moved 2759 neonates from $>60$ hosps. to 15 NICUs. Weight,age, 5. Angar, tenperature at pickup, were variables analyzed. Neonates were placed in 5 weight groups (in grms $<1000$, 1001-1500,1501,2000,2001-2500,>2500). A 20\% random sample of survivors was used. Rerression curve (1972 data) of neonatal mortality (Yc) on weisht(x) yielded $Y C=125.39-.106 x+(2.325)\left(10^{-5}\right) x^{2} . F_{2}^{2}$ test $=50.01 \& P<.05$. $98 \%$ of mortalify was explained by weight. Tn add to this finding, $x^{2}$ analyses were done.Results: (1) Age at pickup did not reveal sisnificant differences in survival rates in 3 years in all weights. (2) Hipher Apsar ( $>7)$ was a good nredictor for increased survival, mostiy in $<2000 \mathrm{gm}$. (3) Higher temperature at pickup $\left(236^{\circ} \mathrm{C}\right)$ was related to increased survival in all weiphts.

\begin{tabular}{|c|c|c|c|c|c|c|c|c|c|}
\hline Variable & in $=17$ & $70-10$ & 71 & & $255-19$ & 772 & $N=29$ & $4-1$ & $7 \overline{3}$ \\
\hline & $A$ & $B$ & $C$ & $\bar{A}$ & $B$ & C & $A$ & $\bar{B}$ & C \\
\hline $3-6 h r$ & 2.8 & 1.2 & .4 & .1 & .1 & 0 & .4 & .01 & 03 \\
\hline $5^{\prime} \operatorname{spgar}, \leq 6, \geq 7$ & $4_{;}^{4}$ & 3.8 & .1 & $\begin{aligned} 32.2 \\
* * * *\end{aligned}$ & 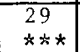 & .03 & \begin{tabular}{|l|}
81.44 \\
$* * *$
\end{tabular} & $\begin{array}{l}10 \\
* *\end{array}$ & $4^{4.6}$ \\
\hline $\begin{array}{l}\text { Temperature } \\
\leq 35.6^{\circ} \mathrm{C}-\geq 36^{\circ} \mathrm{C}\end{array}$ & $A \vee \Lambda T$ & $\begin{array}{l}\text { NOT } \\
\text { I } L A B I\end{array}$ & & $\begin{array}{r}32.1 \\
* * x\end{array}$ & $\begin{array}{l}28 \cdot 3 \\
* * * *\end{array}$ & $\begin{array}{c}8.6 \\
* *\end{array}$ & $\begin{array}{l}60 \\
* * *\end{array}$ & $\frac{4}{4} \cdot 2$ & 3.9 \\
\hline
\end{tabular}

$A=A 1 l$ weishts, $B=<2000 \mathrm{gths}, C=>2000 \mathrm{gms}$ 
241 EPIDEMIOLOGY OF PESTICIDE POISONINGS IN NORTH CAROLINA, Stephen $\underline{H}$. Gehlbach \& Wilton A. Williams. Intr. by Floyd W. Denny, Univ. North Carolina Sch. of Med., Wake Mem. Hosp., Dept of Ped., Raleigh, N. C.

In four years, 208 pesticide "poisonings" of children under 10 years of age have been reported to the North Carolina Pesticides Program. Investigations revealed 48 symptomatic cases and 7 fatalities. Mean age of exposed children was 27 months; males outnumbered females almost 2 to 1 . Most episodes occurred in summer months when pesticides are in heavy use. Agents most of ten implicated were anticoagulant rodenticides (16\%) and organophosphate insecticides $(16 \%)$. None of the children who ingested anticoagulants was symptomatic, but $76 \%$ of those in contact with organophosphates became ill and 3 died.

Exposure histories emphasize three special poisoning hazards of pesticides. 1) Dermal absorbtion of ten occurs and followed contact with contaminated soil, "empty" containers, or sprayed rooms in 8 symptomatic cases. 2) Poisons are frequently applied as food baits making them especially attractive to children. 3) Petroleum distillates in many household pesticides present greater danger than the "active" ingredients and accounted for one of the fatalities in our series.

Despite the extreme toxicity of many pesticides, and the added hazard created by petroleum distillate carriers, none of the pesticides currently marketed are required to be in safety packaging. Federal agencies have failed to create packaging standards although they possess the authority to do so.

242 MENingoCOCCAL CARRIER STATE IN INFANTS AND CHILDREN.

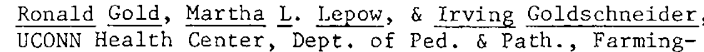

ton, Ct.

The meningococcal carrier state was studied in infants and children. Pharyngeal cultures were obtained and plated on ThayerMartin agar. Neisseria meningitidis (Mgc) was identified and serogrouped by standard methods and differentiated from $N$. lactamica (Lac) by sugar fermentations. Cultures were obtained from 202 infants at $3,4,7,8,12$ and 13 months of age (and from 127 of these same infants at 24 and 25 months of age), from 693 1st and 2nd grade children every 8 weeks and once from 7606 th grade and 4009 th-12th grade children (during 1 school year). At $3,7,12$ and 24 months of age, prevalence rates of $\mathrm{MgC}$ were $1.0,0.7,0.7$ and $0.7 \%$ and of Lac were $3.4,7.9,10.9$ and $17.8 \%$ respectively. $31 \%$ of infants had acquired Mgc and/or Lac during the first year of life and $50 \%$ by age 2 years. Acluisitions were evenly distributed over the 8 sampling points, were equal in boys and girls, and were not affected by the number of older siblings. The prevalence of Mgc in lst and 2nd grade children varied between 1.4 and $1.9 \%$ and of Lac from 4.9 to $8.5 \%$. $23.5 \%$ of children acquired Mgc and/or Lac during the school year. Peak prevalence occurred in April. $3.2 \%$ of 6 th graders carried $\mathrm{Mgc}$ and $5.1 \%$ Lac. Prevalence of $\mathrm{Mgc}$ and $\mathrm{Lac}$ in 9 th and 12 th grade children were 4.5 and $1.3 \%$ respectively. The reversal in the ratio of ilgc:Lac carriage rates which occurred in the older children was significant. Acquisition of nontypable Mgc and of Lac strains may be a major source of natural immunization against meningococci.

243 PROGESTINS AND CYANOTIC CONGENTTAL HEART DISEASE: A CASE-CONTROL STUDY. James W. Hanson, W. Jere Houseworth, and Godfrey P. Oakley, Jr., Bureau of

Epidemiology, Ctr. for Disease Control, Atlanta. (Intr. by David W. Smith, Univ. of Washington Sch. of Med., Dept. of Ped., Seattle.)

Recent studies suggest that some types of congenital heart disease, particularly transposition of the great vessels, might be related to maternal exposure to progestins early in pregnancy. To test this hypothesis we studied the frequency of exposure to such agents among mothers of children with serious types of cyanotic cardiac malformations and a matched control series of mothers of normal infants. AlI cases of transposition of the great vessels (58), tetralogy of Fallot (34) and hypoplastic heart (43) born during a 6 year period in metropolitan Atlanta were ascertained through a registry maintained by the Birth Defects Section of the Center for Disease Control. Four normal controls matched for race, maternal age, time of birth and obstetrician were chosen for each case. Private obstetrical records of each set of women were reviewed in a randomized blina fashion for evidence of exposure to oral contraceptives, hormonal pregnancy tests or therapeutic progestins during the cardiac-sensitive period of organogenesis. We found no evidence of a positive association between maternal exposure to progestins and any of the 3 categories or with total cases of cyanotic congenital heart disease. Our results suggest that if there is an increased risk of cyanotic congenital heart disease associated with maternal exposure to progestins, it must be relatively small.
244 Infections in a Newborn Intensive Care Unit (NBICU). V.G. Hemming, M.R. Britt, J.C. Overall, Jr., B. Epstein, and A. Nordquist. Dept. of Ped. U. of Utah, Col1. Med. Sait Lake City, Utah. 84132

Little data is available regarding hospital acquired infection rates in the NBICU. Surveillance (1970-1974) by a nurse epidemiologist in our NBICU provided data concerning infection rates by type of infection, birth weight, etiologic agent, presence of respiratory distress (RDS) and/or congenital anomalies. Infection rates per 100 discharges from the NBICU as compared with rates in general hospital patients and normal nursery infants compiled by the National Nosocomial Infection Study (NNIS) of the Center for Disease Control are listed below:

\begin{tabular}{|c|c|c|c|}
\hline Type & Utah NBICU & NNIS Hosp & NNIS Nursery \\
\hline acteremia & 2.5 & $0.1-0.2$ & $0.1-0$. \\
\hline tory & & $0.2-1.3$ & $0.1-0.2$ \\
\hline & 1.4 & $1.0-2.5$ & $0.1-0.2$ \\
\hline rinary Tract & 0.9 & $1.0-2.5$ & $0.1-0.2$ \\
\hline 11$]$ Infections & 26.3 & 3.0 & \\
\hline
\end{tabular}
E. coli, group B streptococci and staphylococci were the common organisms causing bacteremia, meningitis and pneumonia. Fortyeight of $92(51 \%)$ babies with bacteremia and 12 of $19(68 \%)$ with meningitis died. Low birth weight, RDS, and congenital anomalies were significant risk factors. The data indicate that NBICU hospital acquired infection rates are much higher than those reported from NNIS hospitals and nurseries, and mortality from bacteremia and/or meningitis in this high risk group of infants is higher than previous reports concerning newborn infants.

245 COMMUNICABILITY OF H. INFLUENZA b FROM

245 EPIGLOTTITIS. EdwardB. Lewin, Sharon Fra1onardo, Richard G. Robertson and Sheri Simon (Intr. by M. Klemperer) Univ. of Roch. Sch. of Med. and Dent., Strong Mem. Hosp., Depts. of Pediatrics and Microbiology, Rochester, New York.

During the early symptomatic stage of his illness, a 3 y.o. child with H. influenza b ( tis spent 1 hour with 60 other children in a single room day care center. 4 days later, blood and N/P culture were obtained from 56/60. 3 wks after exposure, $51 / 55$ had studies repeated. $3 / 55(5.5 \%)$ had $\mathrm{NP}+$ for H.f b at $4 \mathrm{~d} ; 3 / 51(5.9 \%)+$ at 3 wks (Table). None of these children demonstrated a significant antibody titer. $A \geqslant 2$-fold $\uparrow$ titer (radioimmunoassay) was seen in $4 / 51$ ( $7.8 \%)$ other children. $2 / 4$ of these children experienced a febrile illness over the $2 \frac{1}{2}$ wk observation period. There were no secondary cases of epiglottitis or meningitis.

\begin{tabular}{|c|c|c|c|c|c|c|c|}
\hline & & GMT & $(n g / m 1)$ & $\geqslant 2 x \uparrow t$ titer & & $+\mathrm{Cu} 1 \mathrm{tu}$ & \\
\hline Age & No. & $4 d$ & $3 \mathrm{wks}$ & & $4 \mathrm{~d}$ & $3 \mathrm{wks}$ & $\underline{B} \circ \mathrm{th}$ \\
\hline$< 2 \longdiv { y r s }$ & $\overline{10}$ & $2 \overline{6.3}$ & 22.5 & 0 & $\overline{0}$ & 0 & 0 \\
\hline $2-\leq 3 y r s$ & 11 & 54.0 & 60.0 & 1 & 0 & 0 & 1 \\
\hline$>3 y$ rs & 30 & 305 & 308 & 3 & 1 & 1 & 1 \\
\hline
\end{tabular}

These data suggest that, with epiglottitis as the source and under the epidemiologic conditions described, $\underline{H}$. influenza b is not highly communicable.

246 FIVE YEAR'S EXPERIENCE WITH EPIDEMIC AND ENDEMIC BACTERIAL INFECTION IN A LARGE NEWBORN NURSERY. Ellen $\underline{F}$. Monkus, George W. Counts, and Vichien Lorch (Intr. by William $\bar{W}$. Cleveland), Univ. of Miami Sch. of Med., Dep. of Ped. and Path., Miami, Florida.

Since 1970 four epidemics of bacterial infection have been observed on the newborn services at Jackson Memorial Hospital, designated in 1973 as a Regional Perinatal Center: (1) 1970-71, Serratia, kanamycin resistant; (2) 1972, Staphylococcus aureus; (3) 1973, enteropathogenic E. coli; (4) 1974, Klebsiella, kanamycin and gentamicin resistant.

The peaks of these outbreaks can be related temporally to a yearly increase in bixth rate in the Fall, with consequent increased nursery census. Endemic bacterial disease continued, due to a number of different organisms such as beta-hemolytic strep-
tococcus and several gram negative bacilli. During each epidemic, increased carrier rate in asymptomatic infants or increase of the specific organism in the environment could be documented. The pathogenic $E$. coli was introduced by a transferred infant thought to have milk intolerance; specific source of the other epidemics was not found. The last three outbreaks could only be brought under control by strict cohorting and improved techniques in patient contact. Liberal use of broad spectrum antibiotics for suspected hospital acquired infection has been the rule: with penicillin-kanamycin until Fall, 1970, and with penicillin-gentamicin subsequently. Our experiences reinforce the need for a cautious use of antibiotics and for strict surveillance and control of infection when large numbers of sick and premature infants are brought together in regional centers. 
247 AMNIOTIC FLUID INFECTIONS \& PREMATURE RUPTURE OF THE MEMBRANES, EPIDEMIOLOGIC DIFFERENCES, Richard Naeye, Penn State Univ Col Med, Dept Pathology, Hershey, Pa. Non-iatrogenic premature rupture of the membranes (PRM) common1y causes amniotic fluid infections but the latter often develop with intact membranes (AFI). There are important epidemiologic differences between the two disorders. In the 55,908 cases of the Collaborative Study of the NINDS there were 183 cases of PRM that led to fetal or neonatal death and 386 fatal cases of AFI. Slultivariate analysis was used to determine the interaction of 28 mat-ernal \& infant factors in the genesis of each disorder.

PRM was strongly associated with increased parity, maternal obesity, gestational anemia, cigarette smoking and premature delivery. It significantly increased $(\mathrm{P}<.05)$ with increasing maternal age and it resulted in abnormally long labors.

AFI with intact membranes was also associated with premature delivery. However, by contrast with PRM it was significantly increased in young mothers, blacks and those who had few or no prenatal clinic visits. It was more common in very short mothers and strikingly increased in those who had prior fetal \& neonatal losses. It too resulted in abnormally long labors. It was somewhat increased with low socioeconomic status.

The strong associations of PRM with increasing parity, advancing maternal age and obesity raise the possibility that structural changes in the cervix may contribute to its genesis. Since AFI was increased in the young, blacks, the very short and those with prior losses, genetic influences should be considered. (Supported by USPHS contract N01-3-2311)

248 FATAL ACCIDENTS AND RESPIRATORY TRACT INFECTIONS OF INFANCY, CONTRASTING EPIDEMIOLOGIES, Richard Naeye, Penn State Univ Col Med, Dept Pathology, Hershey, Pa.

Respiratory tract infections and accidents are common causes of death in infancy. There were 107 of the former and 73 of the latter in the 55,908 cases of the Collaborative Study of the NINDS who were followed through gestation to 8 years of age. Multivariate analysis was used to determine the interaction of 28 maternal and infant factors in the genesis of infant death.

Fatal respiratory tract infections were significantly more common $(\mathrm{P}<.05)$ in males, blacks, Puerto Ricans and families of low socioeconomic status. They were also more common when mothers were married, underweight and made few or no clinic visits for prenatal care. There was no association with housing density but the incidence of such infections increased significantly with the number of older siblings. The most striking association was with mother's smoking habits. With each increase in cigarette smoking there was a corresponding increase in fatal infant infections.

Epidemiologic features of fatal accidents were very different. They were as common in females as in males and more common viten there was none or only one vicier sibling. They were significantly more common when mothers were markedly underweight or overweight. They had a positive association with low housing density and high socioeconomic status.

The most surprising finding of the analys is was the striking association of fatal pneumonia in infants with mothers' smoking habits. (Supported by USPHS contract NO1-3-2311)

240 FETAL hYPOXIA, A PROSPECTIVE STUDY, Richard Naeye, Dept Pathology, Penn State Univ Col Med, Hershey, Pa. Hypoxia led to 570 fetal and neonatal deaths in the 55,908 gestations of the Collaborative Study of the NINDS. These and other deaths were reviewed in detail and classified by a single observer. 204 of the hypoxic deaths were due to premature separation of placenta, 114 to large placental infarcts, 100 to other known causes and 152 to unknown causes. Multivariate analysis was used to determine the interaction of 28 maternal and fetal. factors in the genesis of death.

Premature separation of placenta was significantly increased in mothers who were anemic, delivered before 34 weeks gestation and had prolonged labor. It was significantly decreased in first pregnancies, overweight and very tall mothers. Fatal placental infarcts significantly increased with maternal age, hematocrit, socioeconomic status, late gestational age, low prepregnancy maternal body weight, low gestational weight gain and subnormal placental weights. Fatal hypoxia of unknown cause was greatly increased in anemic gestations, those with subnormal sized placentas, first pregnancies, single mothers and those with prior fetal and perinatal losses. Its peak was at 28-34 weeks gestation, earlier than placental infarction \& later than premature separation.

The incidence of fatal antenatal hypoxia might be reduced by maintaining optimal maternal body weights, gestational weight gains, preventing anemia, prolonged labors and discouraging pregnancy at older maternal ages. The events of labor \& delivery, including mode of delivery and anesthesia played only a small role in the hypoxic deaths (Supported by USPIS contract N01-3-2311)
250 SUDDEN INFANT DEATH SYNDROME, A PROSPECTIVE STUDY Richard Naeye, Joseph Drage, Dept. Path., Penn. State Univ. Col. Med., Hershey, Pa. \& NIH, Bethesda, Md.

Recent studies have given evidence that some SIDS victims are chronically hypoxemic before death. The current study analyzed brainstem structures controlling respiration in 34 SIDS victims and 19 controls from the Collaborative Study of the NINDS. Sections were stained with Holzer technique since Dr. Richard Lindenberg recently found astroglial proliferation in brainstems of some SIDS victims. Using point counting, abnormal astroglial proliferation was found in the reticular formation (respiratory control area) of $24 / 34$ SIDS victims and $4 / 19$ controls. Myelin was quantitated in the same area by computer assisted microfluorescence. Abnormal astroglial proliferation was associated with retarded myelination.

Clinical comparisons were made between all 124 SIDS victims of the Collaborative Study and 372 matched controls. Anesthesia and many other factors of labor and delivery were similar in SIDS victims and controls. By contrast, SIDS victims had a $\times 1.4$ greater incidence of low Ap dence of abnormal reflexes, hypotonia, jitteriness and weak suck. Future SIDS victims also had an increase of : neonatal resuscitat ion $x 2.1$, oxygen $\times 1.8$, first bottle feeding after 4 th day $x 2.3$, feeding by gravage $\times 2.6$, abnormal body temperature $x 1.5$ and respiratory distress syndrome $x 6$. Mean body weight decreased from the 40 th to the 20 th percentile between birth and 4 months in future SIDS victims. Body length and head circumference exhibited a similar retardation. (USPHS contract N01-NS-3-2311)

251 AN EXAMINATION OF THE PRE-TEST. Frank A. Oski. S.U.N.Y., Upstate Medical Cente Pediatrics, Syracuse, New York.

The pre-test (PT) examination of the American Board of Pediatrics is a widely employed measure of cognitive learning used for the evaluation of pediatric house officers. The performance on this examination can be compared with the performance of applicants taking the American Board of Pediatrics Examination for certification. During the past 3 years, all pediatric house officers in our program have taken the PT in each year of their training. Prior to the PL-1 year, $45.1 \%$ (14 of 31 ) house officers already achieve a mintmum passing grade; after one year of training, $75 \%$ achieve a passing grade; and after 2 years of training, all have achieved a passing grade. Year-to-year comparisons reveal that all house officers improve their performance but their rank order within their cohort of peers remains virtually unchanged. Faculty prediction of performance is excellent with a discrepancy score of only 12 out of a possible 70 in predicting rank order. After the PL-1 year, greatest gains in scores occur in the areas of neonatology and general information, while between the first and second year the greatest gatns are observed in the area of general knowledge. It is evident from this small sample that this form of written exam is insufficient by itself in measuring over-all competence in pediatrics. Other means of evaluation must supplement this exam or else it must be assumed that residency training is unnecessary for certification in pediatrics in almost one-half of our trainees and one year is sufficient in most.

252 TREATMENT UF IRON DEFICIENCY IN YOUNG CHILDREN WITHOUT ANEMIA by George $M$. Owen and Philip J. Garry. Univ. of $\overline{N e w} \bar{M}$ exico Sch. of

Iron deficiency without anemia is common among young children. Associations exist between iron deficiency, physical growth and attentiveness but the clinical significance of iron-deficiency without anemia is not known. To what extent a subnormal transferrin saturation or elevated free erythrocyte protoporphyrin will in the absence of therapeutic intervention, allow prediction of hemoglobin concentration some months later is not known. On the assumption that levels of serum iron in young children ( $1-3$ yrs.) have predictive value for levels of hemoglobin at older ages (46 yrs.), data pertaining to 107 pairs of siblings (Anglos) in the preschool nutrition survey were examined. Younger sibs were divided into two groups according to level of trasferrin saturation, one group (LTS) with levels less than $16 \%$ and another group (HTS) with lerels equal to or greater than $16 \%$. Mean hemoglobin concentration of older siblings (mean age 55 mos.) of LTS group was 12.5 (SD 0.7 ) gm/dl and that of older siblings (mean age 57 mos.) of HTS group was 13.3 (SD 0.6 ) $\mathrm{gm} / \mathrm{dl}$, the difference being significant $(p<.001)$. Serial data collected on infants followed longitudinally are needed to help decide which iron deficient, non-anemic youngsters are most likely to
develop anemia and might be started on iron supplement. 
253 EARLY NUTRITION AND OBESITY AT NINETEEN SEARS. Ravelli, Gian-Paolo, Stein, Zena, A. , Public Health, 600 West 168th Str., New York 10032.

Prenatal and postnatal levels of nutrition are examined as determinants of obesity in young adults. Early nutrition has been thought to influence adipose cell number and, in turn, adult obesity (H1rsch and Knittle, 1970). Th1s epidem1ological study provides one test of these causal relations.

Nutritional levels are defined in terms of rations available to the Dutch population, in different parts of the country at aifferent times, before, during, and after the 'hungerwinter' of 1944-45. The place of birth and the date of birth of 400,000 nineteen year old men examined for military induction in 1964 to 1967 has been used to infer relationships to famine exposure in early $11 \hat{f e}$. Helght and welght measures are avallable on $93 \%$ of the population. Obesity in these young rien is defined in teriils of a weleht $\vec{i}$ or helght scale (Jelliffe, 1966); approxinately $2 \%$ of men are obese by the criterion used.For those born in the period following the famine, the rate of obesity was similar across the country. For those born during the famine period, men born in the famine areas showed lower rates of obesity, at age 19 years, than those born in other areas.

254 ASTHMA: SOCIAL AND FAMILY INFLUENCES AS DETERMINANTS. Sadow, Mark, Susser, Mervyn, W., Stein, Zena, A. Columbia Univ. Sch. Public Health, 600 west l68th st., New york 10032 . efected in some way by the social environment of the child, the specific elements that might be implicated have rarely been examined in a systematic way. Epidemiological studies were carried out to test the relationship of three elements of the social environment; social status, urban/rural origin and family size and position, on the prevalence of reported asthma in young men.

The population base includes 223,000 Dutch men, born during the years 1944-1946 and examined in their nineteenth year for military induction. The diagnosis of asthma was made in 3,656 cases, based on the history and confirmed by physician's letters. Social status (based on occupation of the father at the time of the military examination) rural or urban origin (based on the place of birth) and family position (according to number of sibs, and birth rank of the subject) are systematically recorded in all cases.

The prevalence of reported asthma is consistently higher in sons of fathers in non-manual occupations, and in those from urban backgrounds. Family position was shown to have no effect on the prevalence.

255 SCREENING FOR ALPHA-1-ANTITRYPSIN DEFICIENCY Robert H. Schwartz, Janice D. Van EsS, Klaus Roghmann, William J. Hall, Richard W. Hyde and John C. Vance Univ. of Rochester School of Medicine, Rochester, New York

Population screening for severe alpha-l-antitrypsin (AAT) deficiency ( $P$ iZZ and $S Z$ ) has been considered since both are associated with inherited chronic obstructive lung disease and/or familial juvenile cirrhosis. Our laboratory has determined protease inhibitor types $(\mathrm{Pi}$ ) on 4000 blood samples from various sources. Groups include:(I) randomly selected adults from Monroe Co., N.Y., (II)adults seeking general care, (III)adults referred for pulmonary function tests, (IV) ambulatory asthmatic children, (V) institutionalized asthmatic children, (VI)children with cystic fibrosis, (VII) newborns, (VIII) patients considered as possibly deficient by their physicians. The following $P i$ prevalences $(\%)$ have been found:

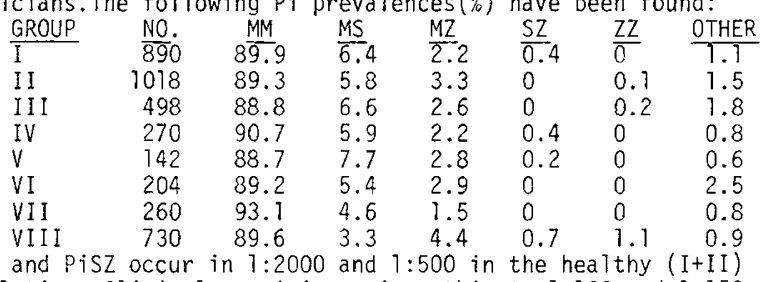
PiZZ and PiSZ occur in $1: 2000$ and $1: 500$ in the healthy (I+II)
population. Clinical suspicion raises this to $1: 100$ and $1: 150$. (VIII). Until prevention of AAT associated disease can be found, general population screening does not seem warranted.
256 THE EFFECT ON THE OFFSPRING OF MATERNAL HEPATITIS B SURFACE ANTIGENEMIA. Robert McNair Scott, Dumrong Chiewsilp and William H. Bancroft, (Intr. by

Franklin H. Top, Jr.) SEATO Medical Research Laboratory, Bangkok Thailand.

During one year 1625 samples of maternal and cord blood were collected at Women Hospital Bangkok. Hepatitis B surface antigen (HBsAg) was detected by Counterelectrophoresis (CEP) in $93(5.7 \%$ ) maternal bloods. There were no differences between HBsAg positive mothers and time matched negative controls for any demographic variable including parity and number of surviving offspring. No differences were seen in the birth weight, or perinatal survival among infants born of HBSAg positive and control mothers. CEP did not demonstrate HBsAg in any cord blood but antigen was detected in $9(9.3 \%)$ of the infants of positive mothers by the more sensitive radioimmune assay. There were no differences in IgM levels between 38 infants of positive mothers, 9 HBsAg positive infants and 74 negative control infants. A one year followup showed HBsAg in over $50 \%$ of the infants of HBsAg positive mothers, a higher prevalence than among the control group. These data suggest that (1) the maternal carrier state does not adversly affect pregnancy or the newborn clinically (2) infants born of HBsAg carriers are likely to be infected by HBV (3) vertical transmission may partially account for high prevalence of HBsAg carriers in Bangkok. In addition lack of elevated IgM levels in $\mathrm{HBSAg}$ positive infants suggests that transmission of the HBV from carriers to their offspring probably does not occur long before delivery.

257 MATERNAL CLINICAL INFECTIONS AND EFFECTS ON THE

Perinatal infections are preventable causes of damage to children. For most infections we are unaware of the true frequency of the disease nor the complete spectrum of associated clinical findings. To obtain this information in detail we have analyzed the prospectively collected data and specimens from 58,828 pregnant women from twelve Institutes in the Collaborative Perinatal Study. Most children have now been observed for at least seven years.

Influenza/"flu" occurred at a rate of 270 cases per 10,000 pregnancies. Specific infections such as mumps, rubella, chicken pox/zoster and measles occurred at rates of 2 to 19 cases per 10,000 pregnancies. Gonorrhea was reported at the rate of $32 / 10,000$ pregnancies.

For the children with congenital rubella, we now find that deafness is the most frequent problem, with congenital heart disease second, microcephaly-low IQ, third. Maternal mumps was associated with several cases of mental retardation, one case of diabetes and one child with endocardial fibroelastosis. In all there were 8,180 clinical maternal infections and data for these children are now available.

258 THE ANATOMY OF THE DIAGNOSTIC PROCESS. Mark P. Shampain, H. James Brown, Walter W. Tunnessen, Jr., and Frank A. Oski. S.U.N.Y., Upstate Medical Center, and St. Joseph's Hospital, Syracuse, N.Y.

In at tempting to establish the optimal situation for use of a computer assisted diagnosis, it was recognized that no facts were available concerning the temporal sequence of the diagnostic process and its impact on hospital stay. For this reason, 472 consecutive admissions to a university hospital (UH) were contrasted with 100 at a community hospital $(\mathrm{CH})$ with these results:

$\begin{array}{lll}\text { Correct diagnosis at admission } & \frac{\mathrm{UH}}{11.5 \%} & \frac{\mathrm{CH}}{72 \%} \\ \text { Correct diagnosis by } 48 \text { hours } & 90.0 \% & 79 \% \\ \text { Correct diagnosis at discharge } & 94.9 \% & 85 \%\end{array}$

The type of patients differed at the 2 hospitals. Over $60 \%$ of patients at $\mathrm{CH}$ had acute respiratory or gastrointestinal problems while $40 \%$ of patients at UH had either cardiac or hematologic disease. Patients that took longest to diagnose at UH included those with seizures, metabolic errors, or fallure to thrive, while at $\mathrm{CH}$ those with prolonged fever, arthritis, or chronic gastrointestinal disease produced the greatest diagnostic difficulty. Patients admitted to UH with a diagnosis were hospitalized a mean of 6.3 days; patients not diagnosed until after 48 hours, 12.1 days $(p<.001)$. This study confirms the subjective impression that more patients are diagnosed and quicker at a UH and that the establishment of a diagnosis reduces length of hospital stay. 
259 CXS:IC FIBROSIS - A NEN NUMLOOK. IIarry Shwachman, School, Chilcren's Hospital Medical Center, Dent. of Vedicine, Boston, "ass.

our clinic has haci an active and continuous interest in pts.with $\mathrm{CF}$ and related diseases for a neriod of over 37 vrs. Farlv and accurate diannosis, continuous supervision and intensive therany has resulted in the prolongation of life and reduction in morbiditv. of nearly 3000 pts. with CF seen durina this neriod, annroximatelv 800 axe still living. This report deals with our lonq-texm survivors which number $7 n$ who have rone hevond their 25 th hirthdav: 14 pts. are over 30 yrs. of are and 5 pts. are over 35 vrs., the old est is 44 yrs. Males nredominate, 47 males to 23 females. 16 pts do not have nancreatic insufficiency. 22 males an'? females are married, 6 women have 7 children. Two women in cood nhysical condition had an interruption of preanancy. None of the males have childxen and all those examined vere asnermatic. 13 were diacnosed as a result of family screening. 6 of the nts. were born with meconium ileus and 5 had surgery; in 2 , the diannosis of CP was made late, at 6 mos. and $41 / 2$ vrs. The diamnosis of $C F$ was made ovex $10 \mathrm{vrs}$. Of aqe in $20 \mathrm{nts}$. and over $20 \mathrm{vrs}$. of are in 10 nts. The severity of disease at diaqnosis varied considerably and not all cases were mild. Over 42 pts. attended college and $4 n$ graduated; 13 have masters deqree, 1 is a M.D., 2 lawvers, 3 nurses, 1 physiotherapist, 1 encineer, 2 research fellows in nhysics and $1 \mathrm{Ph} . \mathrm{D}$. in physics. $4 \mathrm{pts}$, were never hosnitalized. The complications in order of frequency are sterilitv in male 100\%, nasal polyposis (over 508), fecal imnaction (15\%), diabetes (15\%), pneumothorax (108), and salt depletion (108).

260 EPIDEMIOLOGIC INVESTIGATION OF PRIMARY EPSTEIN-BARR VIRUS (EBV) INFECTIONS IN CHILDREN. Ciro V. Sumaya (Intr, by James D. Cherry), UCLA Sch. Med., Dept.

Ped., Los Angeles.

Antibodies against EBV-early antigen (EA) and EBV-specific IgM (both of which denote a current or recent EBV infection) were determined on sera collected from 209 children in 73 families living in a rural community. Thirteen children had anti-EA and 10 of these also had EBV-specific IgM. There was no EBV-specific IgM detected in sera which was anti-EA negative. Neither antibody was found in the sera of the 0-6 mo age group. The prevalence of anti-EA or EBV-specific IgM peaked in the 7-12 mo group, decreased slightly in the 2-5 $\mathrm{yr}$ and 6-10 $\mathrm{yr}$ groups and decreased significantly in the 11-15 yr group. There were no antibody differences by sex or race. The prevalence of anti-EA and EBVspecific IgM antibody in parents or adult siblings of children with primary infections was not significantly different from that noted in similar adults in households of children without primary infection. Three familfes had 2 siblings with anti-EA or EBVspectfic IgM reactivity suggesting an important role of children in the horizontal transmission of infection. Seven of the 13 children with primary infections had been asymptomatic 2-3 mo prior to the serum sampling; 5 had experienced a recent "cold;" 1 had a "cold" and diarrhea. Stmilar clinical manifestations were present at a lower rate in the children without evidence of a primary EBV infection.

261 EPIDEMIOLOGIC AND IMMUNOLOGIC ASPECTS OF NONPARENTERAL TRANSMISSION OF HEPATITIS B INEECTION. M.L. Tiku*, R.I. Ramirez $z^{*}$, D. Kerr Grant** and P.L. gra. Depts. Peds. and Microbiol., SUNY at Buffalo and West Seneca State School, West Seneca, N.Y.

The resident population of an institution for the mentally handicapped was carefully followed by frequent determinations of SGPT activity and hepatitis $\mathrm{B}$ antigen ( $\mathrm{HB}_{\mathrm{S}} \mathrm{Ag}$ ) and antibody to $\mathrm{HB}_{\mathrm{S}} \mathrm{Ag}$ (anti-HBS ) activity in serum, urine and nasopharyngeal secretions, employing the techniques of counterelectrophoresis, radioimmunoassay and passive hemagglutination. Initially $9 \%$ of subjects were found to have $\mathrm{HB} \mathrm{S}_{\mathrm{SAg}}$ in serum. Of these, $20 \%$ were found to contain $\mathrm{HB}_{S} \mathrm{Ag}$ in nasopharyngeal secretions. $35 \%$ of the resident population contained anti-HB $\mathrm{H}$ in serum and the remaining $56 \%$ were seronegative for both. Subsequent surveys carried out 9 and 18 months later indicated that of the initially seronegative residents, $5 \%$ developed $\mathrm{HB}_{\mathrm{S}} \mathrm{Ag}$ activity, and $20 \%$ developed anti-HBS activity in serum. The interval between admission and seroconversion ranged between 16 to 20 weeks, and seroconversion was frequently associated with transient elevations in SGPT levels. The highest incidence of seroconversion was observed in the contacts of those serum $\mathrm{HB}_{S} \mathrm{Ag}$ positive cases who had detectable $\mathrm{HB}_{\mathrm{S}} \mathrm{Ag}$ in the external secretions. These observations suggest. that $\mathrm{HB}_{\mathrm{s}} \mathrm{Ag}$ infection occurs regularly in susceptible institutionalized population, the incubation period of non-parenteral infection may range from 16-20 weeks, and the source of such infection may be the $\mathrm{HB}_{\mathrm{S}} \mathrm{Ag}$ present in the external secretions.
262 CHILDREN HETEROZYGOUS FOR ALPHA-1-ANTITRYPSIN John C. Vance, Robert H. Schwartz, Rhea Fox, William J. Ha TI, Janice D. Van Ess, Donna Speers, KTaus Roghmann Univ. of Rochester School of Medicine, Rochester, New York

86 of 442 randomly selected families from Monroe County, N.Y. were found to have at least one parent heterozygous for Alpha-1antitrypsin:protease inhibitor(Pi) types-53MS, 18MZ,7IM, 2SS, IFS, 2SZ, IMS-MS, TMZ-MS, TMZ-SZ. 214 children from 72 of these families were studied to determine; (1) Pi types, (2)abnormalities on physical exam, and(3)respiratory historical abnormalities by questionnaire. The frequency $(\%)$ of $\mathrm{Pi}_{i}$ types in children were; $M M(50.6)$, MS (30.7), MZ (17), IM(1.7). Males=Females. Mean age(yrs.):MM(13.4), MS(13.2), MZ(13.9), IM(13.3). Heterozygous children were compared to the PiMm sibling group. No significant differences at this age were found in respiratory and medical histories (neonatal respiratory distress, as thma, hayfever, bronchitis, pneumonia, and frequency of URI's). These conditions did not occur more commonly than in the general child population of Monroe County. These groups of children have been identified for prospective studies of the relationship of alpha-1-antitryps in to the development of chronic respiratory diseases.

(Supported by NHLI contract N01-HR1-2221)

263 ZINC NUTRITIONAL STATUS OF HEAD START CHILDREN. Philip A. Walravens, $\frac{K \text {. Michael Hambidge, Monty L. }}{\text { Roth, Sarah B. White }}$ Roth, Sarah B. White and Mary L. Anthony. Universit of Colorado Medical Center, Dept. Ped., and Denver Head Start,

Dietary $\mathrm{Zn}$ deficiency has previously been reported amongst young children from middle- and upper-income families in the U.S. This deficiency was associated with impaired taste acuity, anorexia and poor growth. Since typical low income diets provide relatively little $\mathrm{Zn}$, a study was designed to evaluate the $\mathrm{Zn}$ nutritional status of low-income children at the time of enrollment in the Denver Head Start Program. Seventy-four children, aged 3-5 yrs., were selected because of short stature in comparison to classmates. Ethnic distribution comprised 53 Spanish-American, 16 Caucasian, and 5 other. Heights: 42 were $\leq 3 \mathrm{P}, 23$ were between 3 and $10 \mathrm{P}$, and $9>10 \mathrm{P}$. The mean plasma $\mathrm{Zn}$ level $(74.5 \mu \mathrm{g} / 100 \mathrm{ml})$ was lower than normal $(\mathrm{p}<0.001)$ and 25 children $(34 \%)$ had hypozincemia $(<68 \mu \mathrm{g} / 100 \mathrm{ml})$. The mean hair $\mathrm{Zn}$ level $(86.2 \mathrm{ug} / \mathrm{g})$ was lower than that of middle income children of the same age $(p<0.001)$. Forty-nine percent of the Head Start children had low hair $2 n$ levels $(<70 \mu g / g)$. Serum vit. A levels were below $20 \mathrm{\mu g} / 100 \mathrm{ml}$ in 26 children (35\%) and below $10 \mathrm{\mu g} / 100 \mathrm{ml}$ in 8 of the latter $(11 \%)$. No direct correlation existed between hair or plasma $\mathrm{Zn}$ and vitamin $\mathrm{A}$ levels, although low vitamin $\mathrm{A}$ levels have been reported in $\mathrm{Zn}$-deficient rats. In conclusion, of the 74 Head Staxt children, 69\% demonstrated low levels of $\mathrm{Zn}$ in plasma and/or hair, thus suggesting that suboptimal $\mathrm{Zn}$ nutrition is common in young children from low-income families.

\section{GASTROENTEROLOGY AND ENZYMOLOGY}

264 NITROGEN SPARING EFFECT OF ESSENTIAL AMINO ACIDS IN UREMIA, Carolyn Abitbol and Malcolm Holliday. Univ. of California San Francisco, Dept. of Pediatrics.

This study was designed to determine whether essential amino acids administered during night time fasting would improve the nutritional status of uremic children. Seven children (8-14 yrs) with mean SUN=107 mgn\% were given $10 \%$ glucose and essential amino acid nitrogen (EAAN) to provide $0.25-0.5 \mathrm{gm} / \mathrm{K} / \mathrm{night}$ for 5 nights following a control period of one night. Ad libitum diet provided a mean of $69 \mathrm{Kca} / \mathrm{K} /$ day (Range 26-109) and $0.68 \mathrm{gm}$ Protein $/ \mathrm{k} /$ day (Range $0.12-1.37$ ). Urea production (UrP) per 24 hours was determined from change in urea nitrogen pool and urinary excretion. Apparent protein synthesis (PrS) was determined as the difference between total nitrogen intake (NI) and UrP. Cumulative data comparing the control and study periods follows: $\begin{array}{cccc}\text { NI } & \text { Cal. Intake } & \text { UrP } & \text { PrS } \\ (\mathrm{Gm} / \mathrm{K} / \mathrm{d}) & (\mathrm{Kcal} / \mathrm{K} / \mathrm{d}) & (\mathrm{Gm} / \mathrm{K} / \mathrm{d}) & (\mathrm{Gm} / \mathrm{K} / \mathrm{d})\end{array}$ $\begin{array}{lllll}\text { Control (5) } & .107(+.07) & 68.9(+21.2) & .093( \pm .06) & -.028( \pm .036)\end{array}$ Infusion (28) $\quad .184( \pm .05) * \quad 56.1( \pm 23.2) \quad .101(\underline{ \pm} .05) \quad .082(\underline{ \pm} .082)^{*}$ *difference significant $\mathrm{p}<.05$

Results were evaluated by testing the correlation of nitrogen intake with urea production and apparent protein synthesis:
NI vs UrP

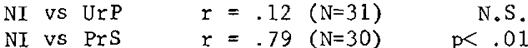
N.S.

We conclude that infusion of EAAN in uremic patients may promote protein synthesis without increasing urea production. 
265 NEPHROLITHIASIS IN A PATIENT ON TOTAL PARENTERAL

265 NUTRITION. Raymond $\mathrm{D}$. Adelman, Charles H. Hlalsted, Steven B. Abern (Intro, by Eli Gold) Depts. of Ped

and Med., School of Med., Univ. of Calif., Davis, California. and large bowel Crohn's disease of 3 months duration was begun on $2000 \mathrm{cc} /$ day of total parenteral nutrition (TPN). Each $1000 \mathrm{cc}$ contained 10 grams of protein as Freamine, 100 grams of dextrose, $10 \mathrm{mEq}$ of $\mathrm{Ca}, 8 \mathrm{mEq}$ of $\mathrm{Mg}$ and $20 \mathrm{mEq}$ of $\mathrm{P}_{4}$. Protein and dextrose intake were increased by 10 grams and 100 grams respectively every 2 days. On day 10 of TPN, the patient developed abdominal pain, hematuria, and renal calcifications on $x$-ray, a finding absent on $x$-rays taken 5 days before. By day 12 , renal calcifications had increased and calculi partially obstructed both ureters. Elevated urinary Ca $17.2 \mathrm{mg} / \mathrm{kg} /$ day [normal $1-4 \mathrm{mg} / \mathrm{kg} /$ day] fell to $2.5 \mathrm{mg} / \mathrm{kg} /$ daywhen Ca was removed from the TPN solution. Renal calcifications decreased in 1 week and disappeared in 1 month. A large calculus (100\% apatite) and a few small calculi were passed. Urinary $\mathrm{P}$, oxalate, uric acid and serum $\mathrm{Ca}$, alk p'tase, $\mathrm{Mg}$, creatinine, uric acid, BUN and bicarbonate were normal. Average urine pH was 6.6 and specific gravity 1.012. Bowel diversion, renal tubular acidosis, dehydration, cystinuria, bone disease and urinary tract infection were absent.

The rapid appearance of nephrolithiasis shortly after initiation of TPN and the rapid dissolution after cessation of TPN calcium was associated with a change from hypercalcuria to normocalcuria. Hyperalimented patients, especially if bedridden, may be at risk for development of nephrolithiasis. Patients should be monitored for hypercalcuria so calcium intake can be adjusted.

66 STUDY ON :ECONIUM: ENZYME ACTIVITIES IN MECONIUM

266 FROM INFANTS WITH CYSTIC FIBROSIS AND IEALTIIY CONTROLS, Irena Antonowicz, Sotoo Ishida, Haryy Shwachman, Harvard hedical School, The children's Mospital Medical Center, Dept. of Medicine, Boston ileconium ileus represents the earliest nandfestation of cystic fibrosis. ilumerous abnormalities have been found in the composition of meconium from newborns with cystic fibrosis such as increase in proteln, mucoprotein, glycoprotein content and the decrease in water and total reducing sugars. These alterations might be of significance relative to the pathogenesis and early diagnosis of cystic fibrosis. We studied the enzymatic activity of disaccharidases (lactase, sucrase, maltase and palatinase) and several lysosomal enzymes. We found marked increase in the specific activity of all studied disaccharidases in the meconium from infants with cystic fibrosis. Among the lysosomal enzymes some were increased $\alpha-$ and $\beta-g$ lucosidase, $\alpha$ - and $\beta$-fucosidase and $N$-acetyl-R-glucosaminidase) some were decreased $(\alpha-$ mannosidase, $\beta$-glucuronidase) in cystic fibrosis mecontum. Thero was no difference in the activity of acid phosphatase between the two groups. Alkaline phosphatase was significantly increased in meconium from cystic fibrosis infants. The increase was not uniform in $\alpha$ - and $/ /$-forms, being much higher in $\beta$-forms, especially for $\beta$-fucosidase (no activity in the meconium from healthy infants, $457 \mathrm{u}^{\circ} / \mathrm{qP} / 60^{\circ}$ in meconium from cystic fibrosts). This pronounced difference $\ln \beta$-fucosidase activity could be of special value as an alternative test for screening newborns for cystic fibrosis.

RADIOTMMUNOASSAY (RIA) OF SERUM CONJUGATES OF CHOLIC ACID IN INFANTS AND CHILDREN. William F. Balistreri, Melvyn G. Korman, Jacqueline Turcotte, and Alan F. Hofmann (Intr. by ijilifiam $\mathrm{K}$. Schubert). Mayo Clinic, Gastroenterology Unit, Rochester, Minnesota.

$\Lambda$ sensitive, rapid and reliable RIA specific for cholyl conjugates, a class of primary bile acids, has been developed and validated by showing identical results by RIA or gas chromatography (courtesy of N.B. Javitt). RIA of cholyl conjugates, using $0.1 \mathrm{ml}$ serum, was carried out in nornal children and children with liver disease. Iligher values in infants may reflect recent feeding, since in adults, the level of cholyl conjugates rises several fold after meals.

\begin{tabular}{|c|c|c|c|}
\hline \multirow{5}{*}{ Irormals } & & No. & Mean + SEM \\
\hline & (Birth-12 mo) & $\overline{18}$ & $0.81 \mp 0.2$ \\
\hline & ( $1-4$ years) & 42 & $0.50 \mp 0.1$ \\
\hline & ( $5-15$ years) & 80 & $0.38 \mp 0.03$ \\
\hline & (16 or over) & 19 & $0.42 \mp 0.1$ \\
\hline Acute viral & hepatitis & 22 & $9.80 \mp 1.8$ \\
\hline Chronic act & tive liver dis. & 5 & $10.70 \mp 4.7$ \\
\hline Cholestatic & disease $(C D)$ & 14 & $22.10 \mp 8.3$ \\
\hline Reye's synd & trome & 13 & $0.69 \mp 0.2$ \\
\hline
\end{tabular}

Consistently elevated values were seen in the various forms of

liver injury. RIA of serum cholyl conjugates is a sensitive indicator of liver disease in children. Further studies appear indicated to determine whether this new test has clinical value for detection or management of liver disease in infants and children. Supported by NIH grant AM 16770 .
268 NUTRITTONAL MANAGEMENT OF NEONATES WITH VTVONEX. James Betzhold, Univ. WI Sch. of Med., Dept. Fed., Madison, WI, John V. Hartline, Bronson Mem. Hosp., Kalamazoo, MI (Intr. by Richar D. Zachman).

Intragastric infusion of Vivonex has been effective and safe in providing nutrition in infants with intractable diarrhea of infancy ( $\mathrm{J}$. Sherman, Personal Communication). We describe the use of Vivonex in four neonates (two cases of gastroschisis) omphalocele, one case of congenital volvulus and one case of intractable diarrhea). In the first two patients,. Vivonex was used sequentially with intravenous feedings to decrease the duration of hyperalimentation. Vivonex was given by constant nasogastric infusion controlled by a iolter Pump. The concentration and rate of infusion was progressively increased from $\frac{1}{2}$ strength at $50 \mathrm{cc} / \mathrm{Kg} / \mathrm{da}$ initially to $2 / 3$ strength at $165 \mathrm{cc} / \mathrm{Kg} / \mathrm{da}$. This final rate and strength provided $110 \mathrm{cal} / \mathrm{Kg} / \mathrm{da}$ and approxi. mately 4 grams protein $/ \mathrm{Kg} / \mathrm{da}$. The technique was used for $17-48$ days in these 4 patients. CBC, electrolytes, BUN, weight and head circumference were monitored. Weight gains averaged from 24 grams/da to 37 grams/da. Head circumference also progressed along standard curves. No abnormalities in electrolytes, CBC, or BUN were noted.

Early institution of Vivonex feedings in these neonatal patients has supported adequate growth and has the additional advantage of avoiding the hazards of prolonged intravenous alimentation.

269 ABNORMAL LIPID ACCUMULATION IN THE JEJUNAL MUCOSA OF CHILDHOOD DIABETICS. Robert C. Bobo, John C. Partin, William K. Schubert, Children's Hospital Research

Foundation, Cincinnati, Ohio.

Most studies have claimed that the intestinal mucosa is normal in diabetics, including most of those suffering from protracted diarrhea, with or without steatorrhea (diabetic enteropathy) Four diabetic children, ages $8,12,12$ and 16 presented with abdominal pain; none were ketotic or acidotic. Three of four had Type IV hyperlipidemia and moderate hepatomegaly. None had measurable steatorrhea. Fasting jejunal biopsies, from all four, examined by serial paraffin section and serial frozen sections stained for lipid demonstrated expansion of the villus core from the muscularis mucosa to the villus tip with large amounts of triglyceride. Electron microscopy (EM) showed the triglyceride to be mainly lipoprotein like particles. One child, biopsied at age 8 and again at age 10, had numerous granulomas surrounding veinules at the level of the crypt-villus junction; these macrophage nests contained much birefringent crystalline material, possibly cholesterol. Electron microscopy revealed these crystals to be within macrophage lysosomes. Two diabetic children without gastrointestinal complaints had no excess lipid accumulation.

Triglyceride accumulation could result from endogenous epithelial, very low density lipoprotein synthesis. Alternatively mechanically defective chylomicron clearance, a possible early expression of diabetic visceral enteropathy, may account for the lipid retention.

Support: NIH \#IF-22-AM-03204-01 and CRC Grant \#RR-00123.

EFFECTS OF ESSENTIAL FATTY ACID (EFA) DEFICIENCY ON

270 MEMBRANE FUNCTION. H.J. Bohles, W.C. Heird, and J.F. Nicholson. Columbia Univ. Col. of Phys. and Surgs. Dept. of Peds. New York.

The effects of EFA-deficiency on membrane function were studied in erythrocytes (RBC) and in liver mitochondria (LM) of male rats fed a fat-free diet for 150-200 days. Although the RBCs of EFA-deficient rats had reduced linoleic and arachidonic acid as well as increased palmitoleic, oleic and eicosatrienoic acid, no significant difference was demonstrated between these cells and control cells with regard to osmotic fragility or intracellular $\mathrm{Na}^{+}$and $\mathrm{K}^{+}$concentrations. However, the $\mathrm{I}$ minute uptake of $14 \mathrm{C}$-Leucine at $15^{\circ} \mathrm{C}$ was greater in EFA-deficient RBCs and correlated inversely with the membrane content of arachidonic + linoleic acid ( $r=-0.66$ ) but not with the membrane Trienel Tetraene ratio $(r=-0.04)$. Uptake of ADP and ATP by mitochondrial inner membrane was determined in EFA-deficient and control IM at $00^{\circ}$. At 20 seconds, ADP uptake of EFA-deficient LM was $54 \%$ and ATP was $146 \%$ as related to control LM. Neither EFA-deficient nor control LM showed evidence of ADP leakage when the inner membrane translocation mechanism was blocked with atractyloside. In addition, nucleotide uptake of control LM pretreated with 2,4dinitrophenol was identical to that of EFA-deficient LM. Thus, these studies lend further support to the concept that EFAdeficient mitochondria behave as if uncoupled with regard to oxidative phosphorylation. They also demonstrate a heretofore unobserved abnormality of EFA-deficient RBCs. 

271 EXTRACELLULAR WATER SPACE IN PARENTERALLY SUPDweck, P. Bailey and G. Cassady. Univ. Ala. Med. Sch., Birmingham.

Ten neonates with mean birthweight $1250 \mathrm{~g}$ (range:800-1980) and mean gestational age 31 wks $(24-38)$ were randomly assigned to oral $20 \mathrm{cal} / \mathrm{oz}$ formula feeding (PO) or oral $30 \mathrm{cal} / \mathrm{oz}$ formula feeding plus parenteral supplementation with dextrose and synthetic amino acids (SUP). Two of 4 infants assigned to $P O$ and 3 of 6 babies assigned to SUP were intrauterine growth retarded. Extracellular water space was estimated as corrected bromide space (CBS) within $19 \mathrm{~h}$ of birth, before initiating feedings, and between the 7th and 28th postnatal day. Mean CBS in PO was $366 \mathrm{ml} / \mathrm{kg}$ (SD 73. 8) on day 1 and $346 \mathrm{ml} / \mathrm{kg}(82.7)$ on days $7-28$ (difference non significant). Mean CBS in SUP was $397 \mathrm{ml} / \mathrm{kg}$ (107.3) on day I and $464 \mathrm{ml} / \mathrm{kg}(60.2)$ on days 7-28 (non significant). Whereas on day 1 no difference in mean CBS was evident between PO and SUP, the latter regimen was associated with increased mean CBS on the latter days $(p<0.05)$. These data suggest that parenteral supplementation results in water retention and/or shifts from the intracellular to the extracellular space. Previously reported earlier and greater mean daily weight gains in SUP babies may be related to water accumulation rather than tissue accretion.

272

INTESTINAL DRUG ABSORPTION IN UNDERNOURISHED SUCKLING RATS. Charlotte Catz, Claude Chanez and Alexandre Minkowski. (Intr. by Sumer J. Yaffe). State University of New York at Buffalo and Fac. Port-Royal, Paris, France.

Intrauterine growth-retarded IGR (E) pups were obtained at birth from dams fed a low protein diet starting on the 5 th day of gestation. Postnatally, pups were ralsed by normal mothers. The intestinal absorption of $\mathrm{C}^{14}$ salicylate was studied at 10 and 21 days of age using an in-vivo perfusion technique. The effluent from the distal ileum was collected in 10 minutes aliquots for five periods. In the first 30 minutes the rate of absoxption was equal in $E$ and control (C) pups, but a significant slower rate in E existed in the next 20 minutes. $(20.5 \mathrm{nM}$ vs. 25.0$)$. The percentage of the dose absorbed over 50 minutes was significantiy higher in the C. ( 86 vs. $69 \%)$. Since the wet weight of the intestine is different (E $0.49 \mathrm{gm}$ and $C 0.77$ ) the total absorption expressed per $10 \mathrm{mgm}$ weight is apparentiy higher in E. $(0.51 \mathrm{nM}$ vs. 0.35$)$. Simultaneousiy a greater amount of drug (nM/gm wet tissue) was recovered from the intestinal wall of E pups. These differences seem to disappear at weaning. The pattern is reversed if IGR pups are due to ligation of uterine vessels (17th day of gestation). The slower absorption was recorded in the first 30 minutes. These results in $E$ animals warrant studies in malnourished infants receiving drugs by the oral route.

\section{5-HWROXYVITAMMN $D_{3}$ (25-OHD ) METABOLISM IN CHIDREN}

273 WITH BIIIARY ATRESIA (BA). Fred Daum, John F. Rosen, Martin Roginsky, Michael I. Cohen, and Iaurence Finberg. Albert Einstein Col. Med., Montefiore Hospo and Med. Ctr., Dept. Ped., and Nassau County Med. Ctr., New York.

Children with uncorrected biliary atresia often develop osteoporosis and/or rickets. Despite normal serum calcium and phosphorus levels, plasma 25-0HD levels measured by radioassay were notably depressed in 4 such patients $(3.8 \pm 2.7$, normal $=27 \pm$ $5 \mathrm{ng} / \mathrm{ml})$. Vit. D IM resulted in a normal $25-\mathrm{OHD}$ level $(5-34)$ and skeletal repair in one child, while, in the 2nd, similar therapy led to only transiently normal $25-0 \mathrm{KD}$ concentrations $(6-18-15)$ suggesting a partial 25-hydroxylation defect. In a third patient receiving oral $25-\mathrm{OHD}_{3}$, the $25-0 \mathrm{HD}$ level became normal $(2-30)$ ana rickets resolved. A fourth child challenged with a loading dose of oral $25-0 \mathrm{HB}_{3}$ had a subnormal rise but adequate absolute $25-0 \mathrm{HD}$ value after 24 hours $(15-35)$. A fifth patient with low plasm 25-OHD had a 15-fold increase after surgical repair and a normal response to an oral loading dose of $25-\mathrm{HD}_{3}(32-95)$.

These data indicate: (1)direct measurement of $25-0 \mathrm{HD}$ is currently the only method of documenting sub-clinical Vit. D deficiency (2)in uncorrected biliary atresia, Vit. D may be inadequately hydroxylated at $\mathrm{C}-25$ (3) though submaximally absorbed in children with uncorrected biliary atresia, oral $25^{\circ}-\mathrm{FD}_{3}$ therapy results in a normal 25-OHD level and is effective in preventing or healing rickets (4)absorption of $25-\mathrm{OHD}_{3}$ is related to the degree of biliary obstruction.
274 EFFECT OF GLUCOSE AND WATER TRANSPORT BY A GLUCOSB

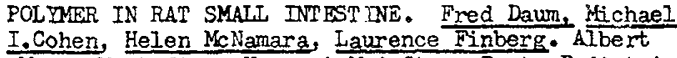
Einstein Col.Kedo, Montefiore Hospo \& Med.Ctr., Dept. Pediatrics, Bronx, New York.

When children with diarrhea are fed oral $5 \%$ glucose solutions, exacerbation of symptoms may recur. An orally administered synthetic polymer consisting of anylose and amylopectin will have a lower intraluminal intestinal solute content than $5 \%$ glucose and thus might avoid this problem. To evaluate this hypothesis in 53 adult rats, a $5 \mathrm{~cm}$ length of isolated duodenum in continuity with its blood supply was utilized to instill equivalent amounts of polymer or 5\% glucose. A saline control was also used. The succus entericus ( $\mathrm{SE}$ ) was renoved at $3^{\prime}, 15^{\prime}, 30^{\prime}$ and $60^{\prime}$ after instillation and the duodenal mucosa excised. At $3^{\prime}, 15^{\prime}$ and $30^{\prime}$, the osmolality of the SE after polymer was significantly lower than after $5 \%$ glucose solution or saline. At $60^{\prime}$, this difference disappeared despite a persistent elevation in SE glucose after 5\% glucose. A four-fold increase of intra-mucosal glucose over SE glucose was apparent after polymer but not with $5 \%$ glucose or saline, suggesting enhanced absorption. The latter Pind ing may be related to the observed stimulatory effect of the polymer on pancreatic amylase secretion. Evaluation of intramucosal water and electrolytes revealed no major mucosal to luminal shifts in flufd. These observations of reduced luminal solute and enhanced glucose absorption with a synthetic polymer suggest advantages to such a preparation over $5 \%$ glucose solution in the per oral nutritional management of children with upper gastrointestinal dysfunction。

ABNORMAL SERUM AMYLASE ISOENZYME PATTERNS IN CHILDREN WITH SHWACHMAN'S SYNDROME AND THEIR RELATIVES, ROIf R. Fngel and Michael D, Levitt, Univ, of Minnesota,

Depts. of Fed and Med. Minneapolis, Minnesota.

Evaluation of pancreatic amylase production entails a cumbersome collection of duodenal aspirates. Interpretation of total serum amylase levels is limited by the multiple organs of origin of this enzyme. The present study of a family with Shwachman's syndrome, employed isoelectric focusing on a colum to separate organ specific isoamylases which were then quantitated utilizing Phadebas reagent. Normal serum has 3 major and several minor isoamylase peaks. The peak with an isoelectric point of $\mathrm{pH} 6.9-7.2$ is specific for serum pancreatic isoamylase (SPIA) as evidenced by its disappearance following surgical pancreatectomy, and its exclusive elevation in acute pancreatitis. In contrast parotid secretions and the serum of patients with mumps have peak amylase activity at a lower isoelectric $\mathrm{pH}$ range. The concentration of SPIA in 12 normal subjects ranged from 33 to 145 IU/L. Two siblings with Shwachman's syndrome were readily identified by their very low SPIA of 1.5 and $2.5 \mathrm{IU} / \mathrm{L}$. The healthy parents and two asymptomatic siblings had intermediate SPIA from 19 to $29 \mathrm{IU} / \mathrm{L}$. This study demonstrates that isoelectric focusing of serum isoamylases permits identification of patients with parotitis, pancreatitis and pancreatic exocrine insufficiency. The finding of negligible SPIA in children with Shwachman's syndrome provides a new diagnostic aid and the abnormally low SPIA values in their asymptomatic relatives suggests that they may also have reduced pancreatic amylase production, thereby providing a new tool for investigating the familial occurrence of this disease.

The Association of Alcaptonuria and Atypical

276 Sucrase-Isomaltase Deficiency in Three Siblings. A. Garnica, M.D., H. Preiser, M.D.*, R.K. Crane,M.D.* O. Rennert, M.D., J.J. Cerda, M.D.**. Department of Pediatrics, Univ. Fla., Gainesville, Fla., *Department of Physiology, Rutgers Medical School, Piscataway, N.J., **Department of Medicine, Univ, Fla., Gainesville, Fla.

Three siblings, two males and one female, ages 13,10 , and 6 years, with alcaptonuria and a life-long history of diarrhea have been shown to have sucrose malabsorption. Two other siblings and both parents exhibit no sign of either condition. Homogentisic aciduria of greater than $5 \mathrm{gm} / 24$ hours was demonstrated only in the three affected individuals. Sucrose absorption was deficient while lactose and glucose absorption were normal. Assay of jejeunal dissacharidase activity showed no sucrase-isomaltase, maltase approximately one-third normal, and increase lactase and trehalase. On acrylamide gel electrophoresis of brush border membranes the protein band usually associated with sucrase-isomaltase activity was absent. Sucrose, glucose, and lactose absorption were normal in unaffected family members. This represents the first documentation of (1) alcaptonuria associated with sucrase-isomaltase deficiency, and (2) sucrase-isomaltase deficiency with no demonstrable protein complex. The segregation of both of these rare, autosomal recessive conditions in three of five siblings and the absence of either alone in the other two siblings suggests that the loci determining them might be closely linked. 

277 Malnutrition, Diarrhea, and Transient HypollpoproFrias, M.D., Department of Pediatrics, University of

Diarrhea, failure to thrive, and acanthocytosis in an infant usually herald the diagnosis of abetalipoproteinemia, the BassenKornzweig Syndrome, an autosomal recessive disorder of 1ipoprotein metabolism. The appearance of these signs usually precedes the development in early childhood of atypical retinitis pigmentosa and ataxic neuropathy, and typically implies a natural course marked by recurrent complications and early death. Transient acanthocytosis and hypolipoproteinemia have recently been reported in children with malnutrition and diarrhea. This report documents the association in an infant of protracted diarrhea, acquired hypolipoproteinemia, acanthocytosis, and failure to thrive, which was completely remediable by nutritional therapy. Two other infants with chronic diarrhea treated with total parenteral alimentation manifested hypocholesterolemia and acanthocytosis without hypobetalipoproteinemia. These observations suggest that significant but transient hypolipoproteinemia and acanthocytosis may be frequently overlooked sequelae of diarrhea with malnutrition. Florida, Gainesville, Florida.

280 INTESTINAL CALCIUM BINDING PROTE IN (CABP) IN VITAMiN D RESISTANT RICKETS (VDRR). W. A. Gleason and D. H. Alpers. (Intr. by A. E. McElfresh). St. Louis Univ. and Washington Univ. Sch. of Med.; Cardinal Glennon Mem. and Barnes Hosps.; Depts. of Ped. and Med.; St. Louis, Mo.

To determine intestinal end organ responsiveness to vitamin $D$ in familial hypophosphatemic VDRR, biopsies taken from the duodenojejunal junction of 5 patients were assayed for activity and concentration of $\mathrm{CaBP}$, a mucosal protein previously shown to be vitamin $D$ responsive in man. All patients had received no vitamin D supplement for at least 4 weeks. All biopsies were histologically normal. Six normal adults served as controls. The $10^{5} \mathrm{xg}$ supernate of homogenates of a portion of each biopsy was assayed for activity (Chelex-100 assay) and CaBP concentration (electrophoretic assay). Activity and CaBP concentration in controls were $117.2+12.9 \% / \mathrm{mg}$. protein and $4.8+0.9 \%$ of total protein respectively.

Activity

$(\% / \mathrm{mg}$. protein) Patien

1

Concentration

(\% of total protein)

99.5

99.5

2

21.4

21.4

3

158.3

346.5

\section{$3.7 \quad 11.1$}

2.7

$\left(\%\right.$ of total protein) $\begin{array}{ccccc}3.7 & 11.1 & 2.7 & 10.1 & 11.2\end{array}$
Normal or supernormal values were noted in 4 patients $(1,3$,

355 5). Vitamin D resistance may thus be due to normal amounts of CaBP in gut before vitamin $D$ treatment. One patient (2) had a twice normal CaBP concentration, but low activity suggesting synthesis of an abnormal protein. The variability of results suggests a heterogeneity of intestinal calcium absorptive mechanisms in VDRR.

278 INHIBITION OF FATTY ACID OXIDATION AND UREAGENESIS Chase. Univ. Colo. Med. Ctr., Dept. Ped. Denver

Among the unexplained features of propionic acidemia axe hyperammonia and fatty degeneration of the liver. 4-Pentenoic acid (PE), an inhibitor of fatty acid oxidation and ureagenesis, produces fatty degeneration of the liver and hyperammonemia in rats and has been proposed as an animal model for Reye's syndrome (Pediatr. Res. $8: 432,1974$ ). We have now studied the effects of propionic acid (PR) on fatty acid oxidation and ureagenesis.

In studies of fatty acid oxidation, both PR and PE (100 $\mu \mathrm{M}$ and above) significantly ( $\mathrm{p}<.05$ ) inhibited ${ }^{14} \mathrm{CO}_{2}$ production from ${ }^{14} \mathrm{C}$ palmitate in both control and methylmalonic fibroblasts. At $1 \mathrm{mM} P R$ or PE the inhibition was 30-50\%. The inhibition by 100 $\mu M$ PR or PE was reversed by $1 \mathrm{mM}$ DL carnitine in that palmitate oxidation was higher than without carnitine.

Ureagenesis from $\mathrm{NH}_{4}{ }^{+}$in rat liver slices was inhibited $43 \%$ $(p<.02)$ by $5 \mathrm{mM} P R$. There was no inhibition or ureagenesis with aspartate and citrulline as substrates, suggesting the block precedes the formation of citrulline. PR had no direct effect on carbanyl phosphate synthetase or ornithine transcarbamylase activities, and the block was not reversed by adding ornithine. The inhibition of fatty acid oxidation and ureagenesis by PR could explain the fatty degeneration of the liver and hyperammonemia in propionic acidemia.

279 COLONOSCOPIC REMOVAL OF JUVENILE COLONIC POLYPS $\frac{\text { Gleason, W. A., Goldste in P. D., Shatz, B. A.2 and }}{\text { Tedesco, F. J. (Intr. by A. E. McE 1fresh). St. Louis }}$ Univ. and washington Univ. Sch. of Med. F Cardinal Glennon Mem. Hosp. for Children, St. Louis Children's Hosp., Jewish Hosp. of St. Louis, and Barnes Hosp.; Depts. of Ped. and Med., St. Louis, Mo.

Six children underwent colonoscopic polypectomy between May 1972 and December 1974. Five had recurrent hematochezia and the sixth had colo-colonic intussusception, which had been hydrostatically reduced. In five cases, the polyps were beyond the reach of the sigmoidoscope. Ketamine anesthesia was used in the first two cases. More recently, intravenous diazepam has proven satisfactory. The polyps were removed by electrocautery. One patient developed fever and left lower quadrant tenderness after polypectomy, which subsided in 24 hours. No patient had rectal bleeding after polypectomy. Our experience suggests that colonoscopic polypectomy is a safe, non-invasive technique applicable to children with juvenile colonic polyps beyond the reach of the sigmoidoscope.
281 Richard J. Grand, John D. Wojcik, Daniel V. Kimberg Center, Beth Israel Hospital, Departments of Medicine, Boston. The mechanism of cholinergic stimulation of parotid secretion is unknown. To characterize intracellular effects of cholinergic and adrenergic stimulation, amylase secretion (AMY-SECR) and levels of cyclic GMP (CGMP) and AMP (CAMP) were studied in rabbit parotid slices incubated in vitro with autonomic agonists and inhibitors. CGMP rose 8-fold 5 min after exposure to $0.01 \mathrm{mM}$ carbachol(CAR) without change in CAMP; AMY-SECR rose significantly: effects completely inhibited by atropine(ATR). Epinephrine(EPI) $(0.04 \mathrm{mM})$ gave brisk increases in CGMP, CAMP and AMY-SECR: the CGMP rise inhibited $\alpha$-adrenergic blockade with phenoxybenzamine $(P B Z)$, the CAMP rise prevented by propranolol ( $\beta$-block). Q-adrenergic stimulation with $0.4 \mathrm{mM}$ methoxamine produced modest elevations in CGMP and AMY-SECR, effects blocked either by ATR or PBZ. At $4 \mu \mathrm{M}$ isoproterenol (ISO) ( $B$-agonist) did not af fect cGMP but promptly increasec CAMP and AMY-SECR. CAR and EPI used together gave additive effects on CGMP and AMY-SECR, but no rise in CAMP beyond that achieved with EPI alone. When CAR and ISO were used together effects on CGMP and AMY-SECR were potentiative. The data show that cholinergic and $\alpha$-adrenergic agonists stimulate ANY-SECR by mechanisms involving CGMP. Intracellular events effected by $\alpha$-stimulation are ATR-sensitive and, therefore, dependent on endogenous generation of acetylcholine. Responses achieved by CAR+ISO suggest that ISO may increase sensitivity of parotid to cholinergic stimuli. This model is uniquely suited to studies of cell surface receptors and their intracellular transducers.

28 MEDICAL TREATMENT OF TYPE I GLYCOGEN STORAGE DISEASE.

282 (GS-1). Harry L. Greene, $\frac{\operatorname{lan} M \text {. Burr }}{\text { Ross }}$, Alf E. Slonim, derbilt Univ. Sch.of Med., Vanderbilt Hosp., Dept. Ped., Nashville, Tn. Most metabolic anomalies associated with GS-1 are reversed by portacaval shunt or total parenteral nutrition(TPN). That this effect is mediated by modulation of hormonal effects on 1 iver and therefore attainable with continuous intragastric high carbohydrate feeding $(1-G)$ was tested on two patients. Also, the effect of clofibrate(c) an inhibitor of adenylate cyclase, has been tested in two patients on the assumption that glucagon mediated cyclic AMP production in liver induced by chronic hypoglycemia is a major mechanism for generation of the metabolic anomalies in GS-1. Representative data in one patient are summarized: Period Day. Uric A. SGOT Lactate Triglycer$\mathrm{mg} \% \quad \mathrm{IU} / \mathrm{L} \quad \mathrm{mM} / \mathrm{L} \quad \mathrm{mg} \%$ $\begin{array}{llrrrr}1-G \text { Feeding } & (9-28) & 8.9 & 30 & 2.1 & 1244 \\ \text { Control } & (29-49) & 18.5 & 154 & 4.3 & 1848\end{array}$ Clofibrate $\quad(50-64) \quad 8.4 \quad 35 \quad 2.4 \quad 1196$

Some confirmation of the mode of action of $C$ was obtained. In one patient $C$ slightly reduced the basal hepatic cyclic AMP levels (from 54 to 37 ) and markedly reduced glucagon stimulated cyclic AMP (from 538 to 264 pmoles/mg protein). These changes correlated with similar changes in hepatic size, triglyceride content, glycolytic and gluconeogenic enzyme activities. These data indicate the possible usefulness of continuous $1-G$ feeding and/or
clofibrate in the management of GS-I.
20 CONTROL OF PAROTID SECRETION BY CYCLIC GMP AND AMP. Harvard Medical School, Children's Hospital Medical

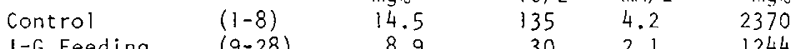



283 FRESH HUMAN MILK: BACTERIAL FLORA IN URBAN U.S. Fanaroff. (Intr. by Marshall Klaus) Dept. Of Ped., Case Western Reserve University School of Medicine. Cleveland To establish standards for the collection and the safety of refrigerated expressed breast milk, we cultured the nipple and performed serial cultures on milk samples from 20 mothers of neonates in the Intensive Care Nursery. All were healthy mothers between 1 \& 22 days (mean 8.7 ) post delivery. The milk was expressed using a sterile Loyd-B breast pump after handwashing and cleansing the nipple with sterile water. Milk was cultured before refrigeration at $4^{\circ} \mathrm{C}$ and serially for 48 hours. The same organisms were cultured from nipple and milk, staphylococcus albus being present in all specimens. Hemolytic streptococci were also cultured from 3 specimens; non-fermenting gram negative bacilli from 2, and staphylococcus aureus, Enterobacter Klebsiella, Serratia marcescens, non-group A hemolytic streptococcl and diptheroids each from 1 sample. These organisms represent normal maternal skin flora, and al though potentially pathogenic, produced no disease when fed within 24 hours of expression.

Colony counts ranged from $10^{2}-10^{6} / \mathrm{ml}$. with no increase during the 48 hours of refrigeration possibly indicating the bacteriostatic properties of breast milk. These preliminary results indicate that clean collection of milk and refrigeration produces no change in bacterial flora for up to 48 hours. Refrigeration, unlike freezing or autoclaving, does not destroy potentially beneficial factors in milk. This suggests the feasibility of home expression of breast milk for use in the
neonatal nursery.

284 VITAMIN A DISAPPEARANCE IN TOTAL INTRAVENOUS FEEDING SOLUTIONS. John V. Hartline, Bronson Memorial Hosp. Kalamazoo, Mich., and Richard D. Zachman, Univ. of WI Sch. of Med., Dept. Ped., Madison, Wis.

The stability of vitamin A in a total intravenous feeding solution was studied. Vitamin A was added to Aminosol-DextroseElectrolyte total intravenous feeding solution in the form of Multivitamin Infusion $(R)$ to give an initial concentration of 3 micrograms vitamin $\mathrm{A} / \mathrm{ml}$. In glass one liter bottles protected from light the vitamin A concentration fell to half the initial value within 24 hours and remained stable at that level for 3 to 5 days. In the intravenous Soluset $(R)$ chamber, vitamin A levels fell to 2 micrograms/ml in $5-6$ hours. When the solution was pumped through the tubing at rates commonly used for newborn infants $(7-17 \mathrm{ml} / \mathrm{hr})$, the vitamin A concentration of the effluent dropped to 1 microgram/ml within $2-4$ hours. Shielding the intravenous feeding solution from light did not alter the rapid disappearance of vitamin A. Repeated washing of the tubing with hexane recovered $50 \%$ of the vitamin A "lost" during passage through the tubing.

Vitamin A delivery to patients receiving total intravenous feedings is considerably less than expected from amounts added to the solution. A significant factor in this loss is adherence to the container and tubing. Photodegradation could not be proved.

285 ASSESSMENT OF ADEQUACY OF AMINO ACID INTAKE IN TOTAL PARENTERAL IIUTRITION (TPN) USING MOLAR RATIOS OF PLASMA AMINO ACIDS. William C. Heird, John F. Nicholson, Lewis D. Stegink, Ralph B. Dell and Robert W. Winters. Depts. Ped., Columbia Univ. Col. Of Phys. \& Surg., N.Y., and Univ. of Iowa, Sch. of Med., Iowa City, Iowa.

One of the important problems in TPN is the formulation of the "ideal" I-V amino acid (AA) mixture. Graham and Placo (Hopk. Med. J. $126: 19,1970$ ) suggested that the plasma molar ratio of each individual essential amino acid (Ind.EAA) to the total EAA and/or the plasma molar ratio of TEAA to that of all amino acids (TAA) may be useful indices of oral AA adequacy.

We used this approach in analyzing plasma AA data obtained on infants on TPN using one of 6 different AA solutions (FreAmine, Abbott AA soln., Cutter AA soln., Aminosol, Amigen or NeoAminosoD. Correlation coefficients ( $r$ ) were computed for the plasma molar ratio for each Ind.EAA/TEAA versus the comparable ratio in the $I-V$ AA intake. Values for $r$ of 0.56 to 0.75 for Ind.EAA/TEAA were found for THE, VAL, LEU, MET, CYS \& PHE, while $r$ values of 0.36 to -0.15 were found for HIS, ILEU, LYS \& TYR. The plasma molar ratio of TEAA/TAA vs intake TEAA/TAA showed $r$ to be 0.56 .

Although the biological significance of these correlations between the various molar ratios in plasma and those in the I-V AA intake are unclear, they may be of considerable empirical value in defining optimal intake of Ind.EAA and TEAA in AA solutions for TPN, e.g., by comparison of plasma ratios observed in TPN with post-prandial values observed in normal orally fed infants.
286 NASAL JEJUNAL (N-J) FEEDING TUBES-CATHETER STIFFNESS RELATED TO TIME. Richard W. Henderson and Errol R. Alden, Depts. Ped., Madigan Army Medical Ctr. Tacoma, WA, (intro. by w. Alan Hodson) Univ. of Wash., Seattle, WA.

Nasojejunal feeding tubes (polyvinyl chloride) are used for feeding small premature infants. The length of time feeding tubes should be left in place without changing is not known. Eleven catheters $\# 5$ French used for $N-J$ feedings of varying lengths of time (1-9 days) were compared with 17 controls for changes in catheter stiffness and energy loss in the distal (jejunal) portion. An Instron mechanical testing machine \#TT-BML. was used to record loading and unloading cycles forming hysteresis loops with stress, $\sigma=\frac{\beta}{\mathrm{A}} \mathrm{gm} 2$ as the abcissa; and strain, $\varepsilon=\frac{\mathrm{L}}{\mathrm{L}} \mathrm{cm}$ cm the ordinate. Youngs modulus, $\mathrm{C}=\frac{\sigma}{\mathrm{cm}} \mathrm{gm} 2$ representing the material stiffness was measured. Also measured was the hysteresis loop area (representing energy loss) which denotes change in viscous properties.

In Vivo, Distal Portion of PVC N-J Catheters Stiffness (E) $\frac{\text { Control (17) }}{2 \pm 0.4} \frac{1-4 \text { Days (5) }}{\star \star 3.2 \pm 0.5}$ 3SD $\frac{5-9 \text { Days (6) }}{\star \star 4.7 \pm 0.73 \mathrm{SD}}$ $\mathrm{gm} / \mathrm{cm}^{2} \times 10^{5}$

\% Energy Loss $33+4 \quad 250 \quad * 38+10 \quad 2 \mathrm{SD} * \star 50 \pm 10$

There is an increase in catheter stiffness and progressive change in physical properties with time. Catheter sections in the jejunum were morc than twice as stiff after 4 days. These data suggest that PVC $\mathrm{N}-\mathrm{J}$ feeding catheters should be replaced prior to 5 days in the jujunum. * $p<.01,{ }^{* *} p<.001$

287 SEQUENTIAL CHANGES IN METABOLIC BALANCES IN PRETERM INFANTS IN THE NEONATAL PERIOD. SZE K. Ho and Paul Y.K. Wu. Dept. of Ped., Univ. of So. Calif. Sch. of Med., LAC-USC Medical Center.

Little data is available on sequential metabolic balances in preterm infants fed on formulas currently used in nurseries. Twenty-seven well preterm infants, b. wt. range 1015-1980 g. gestational age 30-35 weeks, who were appropriate in size for their gestation were divided into 2 groups and placed on 1 of 2 isocaloric milk $(24 \mathrm{cal} / 30 \mathrm{mT})$. Group I was fed on a milk containing $22.1 \mathrm{~g} / \mathrm{L}$ of protein and $14.3 \mathrm{meq} / \mathrm{L}$ of $\mathrm{Na}$ (Similac 24 ). Group II was fed on a milk containing $18.6 \mathrm{~g} / \mathrm{L}$ of protein and $8.9 \mathrm{meq} / \mathrm{L}$ of $\mathrm{Na}$ (Similac PM 60/40, $24 \mathrm{cai}$ ). Twenty-four hour metabolic balances were performed on the $7,14,21$ and 28 postnatal days. Results: There were no differences in rates of weight gain between the 2 groups. Serum osmolarity, serum water and corrected serum $\mathrm{Na}, \mathrm{K}$, and $\mathrm{Cl}$ levels were similar in both groups during all the study periods. Serum protein levels decreased in all infants during the neonatal period, but the decrease was greater in Group II. N retention was greater in Group I but $N$ retention as percentage of intake and C.A. N\% were similar in both groups. Na intake was greater in Group 1 but Na retention was similar in both groups due to greater Na excretion in Group I. $\mathrm{K}$ and $\mathrm{Cl}$ retention were similar in both groups. Fat retention and C.A.Fat\% was higher in Group II. The pattern of balances in each group in subsequent weeks was similar to that in the first week.

COURSE AND PROGNOSIS AFTER SURGERY FOR CROHN'S DISEASE

288 Daryl R. Homer, Richard J. Grand, Arnold H. Colodny. Harvard Medical School, Children's Hospital Medical Center, Departments of Medicine and Surgery, Boston.

Long-term effects of surgical resections for Crohn's disease in childhood and adolescence have not been carefully studied. Accordingly, 37 such patients(pts) (25 males, 12 females, ages 7-18 yrs at diagnosis) were followed from 6 mos to 26 yrs postoperatively. $27 \mathrm{pts}$ had disease involving both colon and terminal ileum; in 10 , disease was 1 imited to small intestine. At surgery, 30 pts were $\leqslant 3 \%$ for weight, and only 17 pts had achieved puberty; 17 pts (15 pre-puberty, 2 post-puberty) had severe impairment of linear growth (height $\leq 3 \%$, or no growth for $>1 \mathrm{yr}$ ). Surgery was performed within 2 yrs of diagnosis in 24 pts, within 4 yrs in 10 , and within 6 yrs in 3 . There were 51 resections; 10 pts had more than 1 procedure. Of $37 \mathrm{pts}, 19$ have had no recurrence (average follow-up, $6.1 \mathrm{yrs}$ ); 16 have had recurrences (11 within 2 yrs, 5 from 2.5 to $10 \mathrm{yrs}$ ); and 2 pts who had active disease left in at surgery have had persistent symptoms. Site of recurrence was neo-terminal ileum in 12 pts, colon in 3, small bowe 1 in 2 . Of the 15 pre-pubertal pts with preoperative linear growth failure, only 4 had catch-up growth after surgery ( 3 of these had no recurrence); the remaining $11 \mathrm{pts}$ have failed to grow (2 without active disease, 2 with persistent disease, 7 with recurrences). Catch-up weight gain (to $>25 \%$ ) occurred in only 18 of the 30 pts initially $\leqslant 3 \%$. These data show that: 1) recurrence rate is nearly $50 \%$; 2) most recurrences occur within 2 yrs; 3) postoperative improvement in linear growth occurs in only $25 \%$ of pts; and 4) catch-up growth is best in those lacking a recurrence. 
289 TOXIC MEGACOLON OF ULCERATIVE COLITIS IN INFANCY, $\frac{\text { Manouchehr Karjoo and Brian McCarthy (Intr. by }}{\text { Frank Oski) State Univ. of New York, Upstate Med. }}$ Frank Oski) State Univ.
of Ped., Syracuse, N.Y.

Ctr., Dept. of Ped., Syracuse, N.Y. arrhea, fever, failure to thrive, and rectal bleeding. Studies revealed findings consistent with the diagnosis of ulcerative colitis. Investigation included bacterial cultures and parasitic sampling, sigmoidscopy, barium enema, upper gastrointestinal and small bowel series, hematologic, metabolic, digestive and absorptive studies. Barium enema showed shortening. lack of haustration, edema and ulceration of entire colon. Sigmoidscopy revealed erythematous, ulcerated, friable rectal mucosa with bleeding. His diarrhea and rectal bleeding responded to prednisone (2 mg./ $\mathrm{kg}$ ). However, he developed toxic dilatation of the transverse colon while on azulfidine (250 mg daily) one week after discontinuation of prednisone. His toxic megacolon disappeared after close medical observation, readministration of prednisone and avoidance of repeated abdominal examinations. Eighteen months later the barium enema still shows the presence of ulcerative colitis but with remarkable improvement from the initial study. Sigmoidscopy shows only friable mucosa. Currently he is on 375 mg. azulfidine daily. Growth and development are normal and he is free of diarrhea or rectal bleeding.

To our knowledge this is the first time that toxic megacolon has been reported in early infancy as a result of ulcerative colitis.

290 SERUM ALPHA-1-ANTITRYPSIN DEFICIENCY AND CHILDHOOD

LIVER CIRRHOSIS. Manouchehr Karjoo and Paul Mintz (Intr, by Frank Oskî) SUNY, Upstate Med. Ctr., Dept. of Ped. Syracuse, N.Y.

A 6 year old male presented with jaundice, failure to thrive, abdominal distention and hepatosplenomegaly of 3 year duration. Studies revealed findings consistent with the diagnosis of alpha1-antitrypsin (A-1-A) deficiency. Liver biopsy showed advanced liver cirrhosis with scattered inflammatory infiltrates, liver cell necrosis, cholestasis and finely granular PAS positive intracellular material. His serum (A-1-A) was less than $10 \mathrm{mg} \%$ $\mathrm{m} 1$ and alpha-1-globulin fraction $0.11 \mathrm{gm} / 100 \mathrm{~m} 1$. He is free of pulmonary involvement. His parents have decreased alpha- 1 -globulin fraction and (A-I-A) (father 80 , mother $70 \mathrm{mg} \% / 100 \mathrm{ml}$.), and are carriers. Two sisters (15 years and 9 months) are healthy and their serum protein electrophoresis and (A-1-A) is normal. An oldex brother presented with jaundice in his seventh week of life in 1962. Laparotomy at three months of age showed a hard and nodular liver. The gallbladder was normal in size and filled with golden yellow bile. The cystic duct was normal but the patient had an atretic common bile duct. A cholecysto-duodenostomy was performed. He remained jaundice and died at the age of 3 years of liver disease. We strongly suspect that this patient may have also had (A-1-A) deficiency. (A-1-A) deficiency should be suspected in any infant with unexplained prolonged neonatal jaundice or childhood liver disease. This deficiency may result in advanced liver cirrhosis in infancy and early childhood.

\section{1} IN VIVO HEMOLYSIS IN NEONATAL LIVER DISEASE Aubrey J. Katz, Richard J. Grand, John B. Watkins and Edward E. Lawson, Harvard Medical School, Children's lospital Hedical Center, Department of Nedicine, Boston.

significant hemolysis is said to be a rare concomitant of neonatal liver disease(NLD). To verify this observation, evidence of hemolysis (reticulocytosis and abnormal smear) was searched for retrospectively in 75 patients (pts) ages 2-4 months with NLD in whom blood group incompatibility, congenital infection, septiceria, GCPD deficiency, metabolic and $\mathrm{HB}-\mathrm{Ag}$ associated disease were excluded. Uf 35 pts with idiopathic nconatal hepatitis(iv) 26 had both reticulocytosis $(4-30 \%$ mean $10 \%$ ) and abnormal peripheral ulood smears (fragmentation, burr cells, target cells); 10 of these also had low serum haptoglobin levels. In the other 9 pts data was incomplete although 3 had reticulocytosis and 3 abnormal smears. $20 / 35$ pts had anemia (IIct $<28$ ). In 21 pts with extrahepatic biliary atresia( $B A) 10 / 21$ had no hemolysis, 6 had mild reticulocytosis $\leq 4 \%$ and 5 had reticulocytosis $(6-16 \%)$ and abnormal smears, together with an intense hepatic inflammation histologically. $5 / 19$ pts with $\alpha-1$ antitrypsin deficiency ( $\alpha$ lAT) had mild hemolysis (reticulocytes $3-10 \%$ ). Serial observation in 7 pts witi $\mathrm{NH}$ revealed persistent hemolysis for 2-10 months despite adequate tocopherol replacement. Conclusions: In vivo hemolysis is clearly established as a common complication of $\overline{\mathrm{NLD}}$ and is more common and severe in NH than BA or alAT. The lack of response to tocopherol questions the role of vitamin $E$ in the etiology of the hemolysis. In addition, there is excellent correlation between the degree of reticulocytosis and severity of hepatic inflammation.
292 The intestinal response to intrajejunal perfusion of the young piglet with a nutrient hypertonic load. Lorne Katz, and J.R. Hamilton. Res. Inst., Hosp. for Sick Children, Dept. of Ped., Univ. of Toronto, Toronto, Canada.

The effects of a constant short term intrajejunal perfusion of Vivone ${ }^{R}$ into young piglets were examined. Eight piglets were operated on at 2 weeks of age, having triple lumen perfusion tubes inserted into the proximal jejunum. At 3 weeks of age each piglet was perfused randomly on 4 days with one of four solutions:

(1). physiologic iso-osmotic solution $(320 \mathrm{mosm} / \mathrm{L})$, (2) Vivonex, (3) Vivonex with the carbohydrate replaced by Mannitol, and (4) Vivonex with the amino acids replaced by Mannitol. Solutions (2), (3), and (4) were of the same osmolality (500 mosm/L). The solutions were perfused at the slowest rate necessary to achieve a steady state, this being $1.8 \mathrm{ml} / \mathrm{min}$ for solution (1) and $.74 \mathrm{ml} / \mathrm{m} 1 \mathrm{n}$ for solutions (2), (3), and (4). The conventional diet (2) elicited a net secretion of electrolytes $(-.050+.058 \mathrm{mM} / \mathrm{hr} / \mathrm{cm}$ for sodium) and water $(-.437+.641 \mathrm{ml} / \mathrm{hr} / \mathrm{cm})$ over the $30 \mathrm{~cm}$ of test segment. The isotonic solution (1) caused net absorption of electrolytes $(+.018 \pm .084 \mathrm{mM} / \mathrm{hr} / \mathrm{cm}$ for sodium) and water $(+.681 \pm .575$ $\mathrm{ml} / \mathrm{hr} / \mathrm{cm}$ ). Solutions (3) and (4) also elicited a net secretion of electrolytes and water(sodium: $-.186+.136$ and $-.128+.106$ $\mathrm{mm} / \mathrm{hr} / \mathrm{cm}$; water: $-1.534+1.204$ and $-.93 \overline{2}+.935 \mathrm{ml} / \mathrm{hr} / \mathrm{cm} \mathrm{for}$ solutions (3) and (4) respectively). Compared with Mannitol, the carbohydrate significantly enhanced net absorption of sodium and water, whereas amino acids did not significantly affect the fluxes. This data suggests that a secretory state is related to the osmotic load which is compensated by transportable nutrients.

THE STGNTFICANCE OF INTESTINAI SURFACE AREA IN INFANTS WITH ACQUIRED MONOSACCHARIDE INTOLERANCE (AMI). W. J. Klish, J. Udall, J. T. Rodriguez, D. B. Singer ană B. L. Nichols. Section of Nutrition and G.I., Texas Children's Hospital, Baylor Col. of Med., Houston, Texas 77025.

AMI is a clinical syndrome of chronic acidic diarrhea seconaary to the malabsorption of all carbohydrates including glucose. Five infants with this synorome, aged 2 to 10 months, have been evaluated for carbohydrate digestion and absorption at the time of diagnosis and during recovery on total intravenous nutrition. Glucose absorption was measured by perfusion of a $10 \%$ glucose, $1 \%$ PEG solution with samples taken from a collecting point $30 \mathrm{~cm}$. distal. Intestinal biopsies were obtained for disaccharidase and histologic study. Glucose absorption (calculated by method of Fordtran) ranged from 11.2 to $38.9 \mathrm{mg} / \mathrm{min}$ for $30 \mathrm{~cm}$ of jejunum. Multiple histologic sections were subjected to planographic surface measurements over $1 \mathrm{~mm}$ segments of submucosa. The mucosal surface ranged from 2.7 to $21.1 \mathrm{~mm}^{2} / \mathrm{mm}^{2}$ of submucosa. A significant linear relationship exists between glucose absorption and mucosal surface area $(R=0.8099, p<0.005)$. Lactase, sucrase and maltase activities were within normal range in all biopsy samples.

Carbohydrate malabsorption in infants with AMI could be due to a defect either in transport and digestive enzyme function or decreased surface available for absorption. Our data shows that glucose absorption is directly related to surface area. Since disaccharidase activity is present, intolerance to disaccharides must also be due to reduced surface area.

Work supported by: David Underwood Trust, NASA Contract 90059
USPH RR-00188 and AM-0572I.

294 PLASMA RNase IN MALNUTRITION. Ingeborg Krieger, and Qamar Taqi. Children's Hosp. of Michigan, Detroit. Plasma RNase is considered a good parameter of grade I - III malnutrition in developing countries. Plasma RNase was therefore measured in 20 infants over 10 weeks of age ( 12 with grade III, and 8 with grade II malnutrition), and in 2 chil= dren with anorexia nervosa. Four infants and 1 child had obvious signs of weight loss, i.e. loosely redundant axillary and gluteal skin folds. BUN was normal. Comparison was made with 8 normal age controls, 27 nomal adults, and 34 infants and children with a variety of illnesses. In accordance with observations of others there was no difference between normal infants, children, and (young) adults, range $2580-6200 \mathrm{U}$, mean $4802 \pm 1048 \mathrm{U}$. of the malnourished infants, 5 had values outside the normal range. Ele= vations were not related to the degree..of malnutrition, based on predicted weight for age (Gomez), or weight for height. However, all 4 infants with signs of weight loss had high values. RNase was elevated in all cases of renal disease and elevated BUN (15), kidney transplant and norma1. BUN (2), endstage neuroblastoma (1), $5 \%$ second degree burn ( 1 ), and malnutrition due to cystic fibro= sis (1) or cystic fibrosis with liver cirrhosis (1). Values were normal in febrile illnesses, incl. diarrhea and dehydration (6), sickle cell crises (4), Wilms tumor (1), and cystic fibrosis with active pulmonary disease (2). All 10 adults over 45 years of age had elevated values, $6460-3740 \quad(\mathrm{p}=0.001)$. Conclusion: There are differences in malnutrition that are not described by parameters of body weight. RNase elevations in malnutrition and other conditions seem to reflect rates of cell breakdown. 
FECAL BILE ACID (BA) AND NEUTRAL STEROLS IN A GNOTO-

295 BIOTIC CHILD. G. S. G. Krishna, C. T. L. Huang, J. T. Rodriguez, W. J. Klish, B. L. Nichols and $R$. Wilson. Section of Nutrition and Gastroenterology, Baylor Col. of Med., Houston, Texas 77025.

Sterol metabolism in the intestine is a function of gut microflora. The primary bile acids, cholic and chenodeoxycholic, are subject to deconjugation, dehydroxylation and dehydrogenation, whereas cholesterol is changed to coprostanol and other secondary neutral sterols. Thus, bile acid and neutral sterol assays of the feces, form an indicator of the bacterial metabolism in the gut of persons with intact intestine and normal liver function.

Fecal samples from healthy children and adults on a regular diet were analyzed for bile acids and neutral sterols by the gas liquid and thin-layer chromatography methods of Grundy. In adults, there is an increase in the secondary BAs. They also excrete less unmodified cholesterol and more bacterial metabolites such as coprostanol. The reverse is true in children. With increasing age, children achieve the adult pattern.

The fecal BAs and neutral sterols were studied in a 3 year old child with combined immune deficiency, living in a germ-free isolator, since birth. Fecal BAs of this cnotobiotic child are essentially the same as at 1 year of age, and comparable to that of a lis year old child. His fecal neutral sterol shows almost $100 \%$ unmodified cholesterol with coprostanol content lower than that of normal children under $1 \frac{1}{2}$ years. This suggests an alteration in normal gut microflora with maturation.

057Cl. wip suported by NASA Grant \#90059, USPH RR-00188 and AM-

29 CHOLESIATIC STNDROME OF INFANCY: QUANIITATTIVE ASSESMENT OF DEFECTS IN BIIE ACID METABOLISM AND EXCRETION. UXi Lavy \& Normen B. Javitt, New York Hosp.-Cornell Univ. Med. Col., Dept. of Ped. \& Gastroenterology, New York, N.Y. 10021 (Introduced by Maria New, M.D.)

Bile acid (B.A.) balance studies in 6 children (2-7 yrs. of age) with persistent cholestasis and patent bile ducts were done during control, phenobarbital and cholestyranine (QUES.) treatment periods. Quantitative analysis of B.A. in serum, urine and feces were dore before and after solvolysis by gas liquid chromatocraphy. The response of these children to medication was compared to two children with extrahepatic biliary atresia. As expected, fecal B.A. excretion in bables with atresia was below $0.4 \mathrm{mg} / \mathrm{kg} /$ day when treated with QUES. Although this drug increased fecal B.A. excretion in all children with patent bile ducts, a marked quantitative difference was observed. In one group, with bilirubin levels of $1-2$ mgo and relatively normal livers, B.A. excretion rose from 1.5 to $20 \mathrm{mg} / \mathrm{kg} /$ day. In the other group with clinical cirrhosis the rise was from 1 to $2 \mathrm{mg} /$ $\mathrm{kg} /$ day on QUES. Although phenobarbital tends to reduce serum bilirubin levels there was no increase in fecal or urinary B.A. excretion. Urinary excretion of B.A. was generally related to serum levels. As reported before, chenodeoxycholic acid is excreted mainly as the sulfate conjugate, and cholic acid as the Iree acid. Monohydroxy B.A. constitute $1-4 \%$ of the total. The study established objectively a group of QUJS. responsive children and supports the concept of primary B.A. transport defect in these children.

JUVENILE $\chi_{1}$ ANIITRYPSIN DEFICIENCY: INEVITABLE CIRRHOSIS

297 FOLLOWS NEONATAL HEPATITIS. Edward E. Lawson, Rłchard J. Grand. Harvard Medical School, Children's Hospital Medical Center, Department of Medicine, Boston.

The course and long term prognosis of symptomatic neonatal hepatitis $(\mathrm{NH})$ associated with $\alpha_{1}$ antitrypsin deficiency (k $x_{1}$ ATD) is unknown. Accordingly, we have surveyed 23 infants and children with homozygous $X_{1}$ ATD $(21, \mathrm{PiZZ} ; 2, \mathrm{PiSZ})$. Eighteen of the 23 pts were found among 53 infants with idiopathfe NH presenting at this hospltal, and all 18 had obstructive jaundice and persistently in creased SGOT, SGPT and alkaline phosphatase values. The other 5 $p$ ts presented insidiously at age $1 \mathrm{~d}-18$ yrs, I asymptomatically, 2 with hepatomegaly and 2 with increased alkaline phosphatase. Histologic data were available for 21 of the 23 pts. Most biopsy specimens showed diastase-resistant PAS+ granules in the hepatocytes, inflammatory infiltrates, and fibrosis. Nine operative cholangiograms were performed before age 4 mos; 6 showed abnormalities of either extra- or intrahepatic radicles. Nine pts received corticosteroid thexapy. Of the 18 pts with $\mathrm{NH}, 15$ have been. followed for greater than 2 yrs or until death; 3 have died from hepatic failure ( 2 by age 7 mos, 1 at 3 yrs) and 12 have evidence of cirrhosis and/or ongoing hepatic damage. Four of the 5 pts with variable presenting features have cirrhosis at ages 2 to 18 yrs. These data show that at our hospital juvenile $\alpha_{1}$ ATD represents $34 \%$ of pts with idiopathic NH. In such pts with $\alpha_{1}$ ATD, prognosis cannot be predicted by severity or longevity of neonatal liver disease; however, all $\mathrm{NH}$ patients presenting with $\mathrm{PIZZ} \propto_{1} \mathrm{ATD}$ had evidence of cirrhosis at the time of follow-up.
298 T. CFILL FUNCTION AND PROTEIN TURNOVER IN PRIMARY AND SECOIIAARY INTESTINAI, LYMPFLANGIECTASIA. Emanuel Lehenthal, John Gatien, Lawrence Button, Harry Shwachman, Harvard Medical School, children's Ilospital Medical Center, Dept, of Medicine, Boston, Mass.

Intestinal I,varhangiectasia(IL), may vary widely in its manifestation and severitv. The purpose of this study was to determine if it is a single disease entity with a wide spectrum, or separate diseases, one heing a congenital disorder and the other a secondary disease. It was proven histologically in 15 patients nresenting with: diarrlea 14 , vomiting 7 , lymphedema 4 ; chylus effusions 5, abdominal swelling 4; hypoproteinemia and hypogammaglohulinemia 12, hypocalcemic seizures 5, lymphopenia 6 and growth retardation 7. Two distinct groups were separated. The first yxoup included 6 natients with associated peripheral iymphedema and chylus effusions, prolonged iymphopenia, family history in two patients and recurrent exacerbations. Three of these pts.125 in clinical remission revealed shortened half-life of plasma I albumin 28 hours-3 dagys. Four day stool collections demonstrateo gastrointestinal $\mathrm{Cr}^{51}$ albumin loss $1.7-6.38(0.2-0.78$ in normals4 These patients showed a depression of circulating $\mathrm{T}$ cells $\mathrm{S}_{3}$ (Erosetting cells), 150,289 and $345 / \mathrm{mm}^{3}$ (normals $450-600 / \mathrm{mm}^{3}$ ), and norma? numbers of cells showing $\mathrm{B}$ cell markers. The second grour consisted of 9 patients with no peripheral lymphed ma, no lymphopenia and only one attack. Two patients studied in clinical remisston revealed normal half-life of plasma $I^{125}$ albumin, no gastrolntestinal protein loss, no lymphopenia and normal ahsoluta numbers of circulating $T$ cells and cells showing $\mathrm{B}$ cell markers.

T CELI DEFICYENCY, HIGH PRO' INTESTINAL PROTEIN LOSS ASSOCIATED WITH INTESTINAL LYMPIOID NODULAR HYPERPLASIA (ILNI). EmanueI

Lebenthal, John Gatien, Stuart Rhein, Robert Parkman, Harry Shwachnan, harvard Medical School, Children's Hospital Medical Center, Dept. of "ledicine, Boston, Mass.

Three patients presenting with diarrhea, malabsorption, hypoproteinemia and unusual susceptibility to infections had ILNH. They were studied for albumin turnover, immunoglobulins, IgG subclasses and $T$ and $B$ cell function. Cr51 albumin turnover studies of these patients revealed a short half-life of $6-17 \mathrm{hrs}$. (normal 4-7 days). A foux day stool collection revealed severe GIPL $12.7-18.4 \%$ (normal $0.2-0.7 \%$ ). One natient studied in remission showed $a$ short half-life of 1.2 days and slight GIPL (2.38). Sexum Igr levels were decreased. I g' normal to high, and IgA was normal. Igr suhclasses clenonstrated a low gama-G $G_{2}$ of the total IgG $(0-10 \%$ vs $18-25 \%$ in normals). Immunofluorescent studies. in two patients revealed nomal numbers of IgG, IgA, Ig"1 and IgD in the lamina pronria. Two natients studied exhibited a moderate to severe depression of circulating $x$ cells (E-rosetting cells), 127 and $2.07 / \mathrm{mm}^{3}$ (normals $450-600 / \mathrm{mm}^{3}$ ). The patients had normal numbers of cells showing $B$ cell markers. Mother of one patient revealed a slight depression of circulating $T$ cells, $388 / \mathrm{mm}^{3}$. She also had a short serum half-life of Cr51 albumin -1.9 days with no GIPL. ILNH is similar to intestinal lymphangiectasia in that it is associated with $T$ ccll deficiency, high protein turnover and GIPL. It seems probable that ILNH causes degradation or loss of $\mathrm{T}$ lymphocytes and serum proteins.

\footnotetext{
300 KASAI'S OPERATION FOR BILIARY ATRESIA. John R. Lilly, 300 John $\frac{\text { o. Taubman, Robert }}{\text { Battaglia, Univ. Shikes, Intr. by Fred C }}$
} Ped. Surg., Dept. of Radiol., and Path., Denver.

The Japanese operation for biliary atresia (Kasai procedure) is just beginning its evaluation in this country. The following investigations were undertaken to determine if a true functioning biliary-enteric communication can be created by the operation and if the procedure alters the fundamental disease process.

Six of 24 infants who have had Kasai's operation were studied by a special type of transhepatic cholangiography (Chiba needle). Liver biopsy was done concomitentiy.

Cholangiography demonstrated: 1) unequivocable visualization of a patent biliary-intestinal anastomosis in those infants having relief of jaundice; 2) in patients still jaundiced, strikingly enlarged lymphatic channels draining the liver via the surface of the surgically interposed bowel segment, and; 3 ) the intrahepatic biliary tree in infants with biliary atresia to be grossly abnormal irregardless of the relief of biliary obstruction. These cholangiographic interpretations were entirely consistent with the hepatic histological findings.

The results of this study suggest that although bile drainage can be obtained following Kasai's operation, in biliary atresia bile obstruction is only one component of the basic hepatobiliary disease process. Consequently, the effect of the operation on the long term outlook of infants with biliary atresla is still to be determined. 
FEPATIC FFFECTS OF ISONIAZID IN ADOLESCENTS AND

301 RODENTS. Iris FoLitt, Michael I.Cohen, Helen McNamara. Albert Einstein Col. of Ked., Monte

Med. Ctr., Dept。 Pediatrics, Bronx, New York。
Isoniazid (INH) is recommended for adolescents with positive uberculin reactions because of the risk of activation of tuberculosis. This drug causes transient elevations in serum of hepatic enzymes in adults. To evaluate the hepatic effect of INH when used in this prophylactic manner, adolescents 11 to 16 years of age with a positive PPD were studied over 24 months. Liver function tests and enzyme levels were obtained on all patients prior to institution of daily oral therapy and at regular intervals thereafter. 178 adolescents were entered into the study. Initial SGPT determinations were normal in 155 patients. After INF therapy, SGPT values became abnormal in 10\%. Jaundice was noticeably absent in these 15 teenagers prompting investigation into the possible effect of INH on bilimubin metabolism. Accordingly, 30 Sprague Dawley rats were injected intraperitoneally, with $250 \mathrm{mg} / \mathrm{kg}$ of $\mathrm{TNH}$, for 4 days, sacrificed and hepatic bili rubin glucuronyl transferase activity determined. 27 control rats were given saline. Transferase activity was found to be ele vated in the INH treated animals $(p<.001)$ suggesting stimulation of hepatic smooth endoplasmic reticulum with consequent enhanced glucuronyl transferase activity. These studies demonstrate that INH is possibly hepatotoxic in $10 \%$ of adolescents treated as evidenced by increased SGPT levels and suggest further that this drug may enhance production of glucuronyl transferase and thus partially explain the absence of hyperbilimbinemia in these same subjects. POSITIVE NITROGEN BALANCE IN SEVERELY BURNED CHILDREN 302 Lubin, A.L. Caffo, R.D. Pearson, L.C. Lewis (Intr. by Bruce D. Graham). Ohio State Univ., Ped. Dept., Columbus, Ohio.

Early supplementation with a "chemically-defined" elemental diet (Vivonex-HN) within $72 \mathrm{hrs}$. following burn injury \& continua tion of this supplement during convalescence is suggested as a successful therapeutic method to achieve balanced nutrient intake $\&$ ample caloric supply in severely burned children. Data from a comparison of the efficacy of a "chemically-defined" diet with a milk-based formula supplement \& without a dietary supplement performed on a group of pediatric burn patients (scald or flame burns involving $20 \%$ body surface area) indicate that energy \& protein requirements of a burned child need not be supranormal (2-3 times those recommended for a well child), early achievement \& careful maintenance of normally recommended caloric intakes is the crucial consideration. Nitrogen balance studies correlated with clinical, biochemical, \& dietary information demonstrate that individuals supplemented with the "chemically-defined" diet (Vivonex-HN) have achieved positive nitrogen balance within $48-72$ hrs. post burn while unsupplemented patients have remained in negative nitrogen balance for 2 wks. or longer. Supplemented children have experienced a marked decrease in no. \& volume of stools, and prolonged anorexia demonstrated by many extensively burned children has been circumvented. Age comparison of individuals with the same severity of burn involvement confirm the adolescent burned child is more prone to prolonged negative nitrogen balance than the younger child \& requires equally intensive concern regarding nutrient intake. Sponsored by Grant 71-230 from Eaton Laboratories.

OBESITY IN CHILDREN WITH PHENYLKETONURIA: DIETARY, 303 AHYSICAI, ANT HOCHEMICAL PARAMETERS. A. Lubin, Antoinette Eaton, and Charlene Doane (Intr. by Bruce D. Graham). Ohlu stale viser., Ped. Dept., Columbus, Ohio.

Eight children with classical phenylketonuria, now 2 to $3 \mathrm{yrs}$. old and followed since their lst month of 1 ife, have continued to grow in wt. and ht. at a rate within or above the normal range, (compared to accepted norms for the average American child). Carefully monitored diets within accepted parameters for phenylalanine restriction and appropriate for age in caloric intake per $\mathrm{kg}$. per day, averaged $55 \%$ tota 1 intake as carbohydrate, $12.5 \%$ as protein, and $32.5 \%$ as fat. Parental ht. averaged $65.7 \mathrm{in}$. and wt. averaged 129 lbs. Bone age films correlated well with chronological age, but skinfold measurements were above age and sex-appropriate norms. Serum phenylalanine levels during treatment in all individuals were elevated above normal, as expected, but were consistent with good biochemical control. Carbohydrate sensitivity evaluated by glucose tolerance testing was within nor mal limits, however, measurements of lipid sensitivity were in the high normal or elevated ranges: cholesterol $166 \pm 12 \mathrm{mg} \%$, triglyceride $104 \pm 28 \mathrm{mg} \%$, free fatty acids . $7 \pm .3 \mathrm{MEQ} / \mathrm{L}$ and total lipids $394 \pm 42 \mathrm{mg} \%$. Lipoprotein gel electrophoresis in 5 of 8 children was consistent with type II hyperlipoproteinemia. Inability to efficiently utilize fat may have been responsible for previous reports of retarded growth in PKU children managed on carefully restricted diets, and additional evaluation of lipid metabolism correlated with dietary intake is advocated. Recent modifications in dietary management should permit normal rates of growth throughout childhood. Supported by CHRF GRS Grant \#81-756.
304 EFFECT OF UNEVEN PROTEIN TO CALORIE DISTRIBUTION ON PLASMA FREE AMINO ACIDS, William C. MacLean, Jr。\& George G. Graham. Johns Hopkins Univo Sch。 Med。 (Ped。), Sch。 Hygiene \& Public Heal th (Intern. Hlth。), Baltimore. Benefits of child feeding programs might be maximized by providing all of the daily protein requirement in the single meal usually given. Adequate $N$ balance and linear growth continue on diets with uneven protein/calorie distribution $(125-150 \mathrm{Kcal} / \mathrm{kg} / \mathrm{d}$ evenly divided in 5 meals; all protein, $6.4 \%$ of calories, in $3 \mathrm{rd}$ bottle). To elucidate mechanisms whereby amino acids ingested as a bolus seem available for protein synthesis throughout the day, plasma free $A A^{\prime}$ 's were analyzed at various times. Fasting ( $\left.8 \mathrm{am}\right)$ samples in 10 infants after a minimum of 9 days on the diet showed either normal aminograms or slight elevation of essential $A A^{\prime}$ 's (EAA). Post-prandial samples in 6 infants showed a drop in EAA, especially branched chains, thr $\varepsilon$ met, 4 hrs after the 1 st and, more so, after the 2 nd protein-free meal. In samples 3 \& 4 hrs after the 3 rd (protein-containing) meal, total AA's (TAA) increased $50-100 \%$ \& EAA increased $100-200 \%$, most prominently branched chains, thr and met. Fasting TAA and EAA, despite marginal level and uneven distribution of protein intake, were not indicative of protein deficiency. The marked elevation of EAA after the protein load, with persistence in some cases up to 16 hrs, suggests that a supply of EAA may have been selectively conserved for subsequent use in protein synthesis as additiona energy became available from the non-protein-containing meals. (U.S.P.H.S.Grants AM-09980-10, I F-22 AM-03252-01, and Nutrition Foundation Grant No. 486 .)

305 COMPARATIVE VALUE OF THREE WHEAT VARIETIES IN iNFANT NUTRITION. William C。 Maclean, Jr, \& George G. Grahamo Johns Hopkins Univ. Sch. Med. (Ped。), Sch. Hygiene \& Public Health (Intern. Hlth.), Baltimore.

Although U.S. infants eat cereal grains primarily as a filler to decrease milk consumption, millions of infants and children depend on them for protein and energy. Efforts to increase protein supplies through improvement of protein quality and content by genetic or other means require knowledge of digestion and utilization by infants. Two high protein wheats (Atlas 66, $17 \%$ and $\mathrm{NapHal}, 18 \%$ ) and U.S. ordinary wheat $(13 \%)$, as whole wheat (WW) and white (Wh) flour, were compared isonitrogenously and isocalorically to casein at $6.4 \%$ protein calories. Mean apparent $\mathrm{N}$ absorptions and retentions (\% of intake) were: US-WW $77 \& 26$, Wh 86 \& 31; AT-WW 79 \& 29, Wh 83 \& 26; NH-WW 77 \& 26, Wh $82 \& 22$; Casein $86 \& 41$. Stool wet wt, a measure of CHO digestibility, was higher with wheat than with casein, or, from previous experience, pasta-based diets. Serum albumin concentration decreased and rate of wt, gain was less while consuming wheat. Milling of U.S. increases digestibility without loss of protein quality whereas milling of AT and NH leads to loss of high quality protein not compensated for by increased absorption. Because of increased protein content and higher biologic value, AT offers advantages in infant nutrition when consumed as WW. Human evaluation, with knowledge of the form in which cereals are to be consumed, is essential prior to increasing production and distribution of new cereal varieties in the developing world. (U.S.P.H.S. Grants AM-09980-10, 1-F22 AM-03252-01, and A.1.D。

PROPHYLACTIC ORAL ANTIBIOTICS IN NECROTIZ-

306 ING ENTEROCOLITIS. Mary A. MeCaffree, Anne B. Fletcher, Gordon B. Avery, George Washington University Medical Center and Children's Hospital National Medical Center, Department of Pediatrics, Washington, D.C. 20009.

To investigate whether prophylactic oral administration of broad spectrum antibiotics will decrease the incidence of necrotizing enterocolitis (NEC), a high-risk group was identified by perinatal asphyxia, hypovolemia, sepsis and prematurity. All infants were given the intensive care which is usual in our treatment group, who received oral gentamicin at twice the parenteral dose for at least 4 days prior to feeding by mouth, and a control group whose care was otherwise similar. Data thus far fajl to show significant benefit for the prophylactic routine. of 23 babies, 2 developed enterocolitis: 1 in the treatment and 1 in the control group. Both infants were less than $1500 \mathrm{gm}$.

In the treatment group, gentamicin blood levels were obtained on the lst and $3 \mathrm{rd}$ day. Absorption was minimal, and even in infants receiving parenteral gentamicin for other indjcations, serum levels never exceeded $6 \mu \mathrm{gm} / \mathrm{ml}$. 
307 NEONATAL JAUNDICE AND BREAST FEEDING. M. Jeffrey Maisels, Kathleen Gifford (Intr. by Nicholas $M$. Nelson). Penn State Univ. Col. of Med., M. S. Hershey Med. Ctr., Dept. of Ped., Hershey, Pa.

Total serum bilirubin (TSB) determinations were performed on 264 infants consecutively delivered term infants (excluding Caesarean sections). TSB was determined in every case on the third hospital day and was repeated if clinically indicated. 41 infants (15.5\%) had TSB $>12 \mathrm{mg}$. \% at some time in the first 5 days of life. A cause for this was found in 18 infants (ABO incompatibility, high hematocrit, cephalhematomas, bruising, asphyxia) and no cause in 23, a1though in 3 of these infants the subsequent course suggested the diagnosis of "true" breast milk jaundice.

Excluding the 18 infants with obvious causes for jaundice the remaining 246 infants were studied. At a mean age of $67.6 \pm 7.8$ hours (SD) the TSB in these infants was $6.7 \pm 3.4 \mathrm{mg} . \%$ (SD) with a range of $0.5-14.0$. In 115 totally breast fed infants the mean TSB at $68 \pm 7.8$ hours (SD) was $6.9 \pm 3.6 \mathrm{mg}$. \% (SD). In 129 bottle fed infants TSB at $67 \pm 7.8$ hours (SD) was $6.5 \pm 3.2$ $\mathrm{mg}$. \% (SD) ( $\mathrm{p}>0.1$ ) (Two infants both bottle and breast fed.) 8 infants ( $3.2 \%$ ) ( 4 bottle, 4 breast) had TSB $>12 \mathrm{mg} . \%$ in the initial ( $3 \mathrm{rd}$ day) samples, but a total of 23 infants ( 18 breast. 5 bottle fed $p<0.01$ ) had TSB $>12 \mathrm{mg}$. \% at some time in the first 5 days. These studies suggest that ( 1 ) of infants diagnosed as having hyperbilirubinemia (TSB $>12 \mathrm{mg} . \%$ ) about $50 \%$ have no obvious cause. (2) Breast feeding has no effect on TSB in the first 3 days of life but may be associated with an increased incidence of hyperbilirubinemia subsequently.

308 CORRELATION OF SGOT AND SGPT WITH SALICYLATE LEVEL IN JUVENILE RHEUMATOID ARTHRITIS. John J. Milier III and David B. Weissman, Stanford Univ. Sch. Med., Children's Hosp. at Stanford, Rheumatic Disease Service, Palo Alto, Calif.

Recent reports of the hepatotoxicity of aspirin in juvenile rheumatoid arthritis (JRA) prompted a 13 month prospective study of all children receiving aspirin in an arthritis clinic. The patients studied included 7 with systemic JRA; 49 with polyarticular JRA; 32 with pauciarticular JRA; and 1 with ankylosing spondylitis. The SGOT, SGPT, serum salicylate level (ASA), and eosinophil count were measured at each visit. $41 \%$ had an elevated SGPT, and $59 \%$ an elevated SGOT at some time during the study. $11 \%$ of patients had either SGPT or SGOT concentrations or both reater than $100 \mathrm{IU} / \mathrm{L}$ for short periods. When data from al patient visits were analysed, significant correlations $(p<0.05)$ were found between the ASA and the SGOT and SGPT concentrations in every type of JRA. However SGPT was elevated in $16 \%$, and SGOT in $48 \%$, of instances when ASA was less than $25 \mathrm{mg} / \mathrm{dl}$, and normal concentrations of SGPT and SGOT were present in $67 \%$ and $38 \%$ respectively of instances when ASA exceeded $25 \mathrm{mg} \%$. Among 8 patients with 8 or more visits, correlations between ASA and SGPT existed in only 3 and between ASA and SGOT in only 2. There were no differences when patients receiving gold and aspirin were compared with those receiving only aspirin. No correlation between eosinophil count and ASA was found. While the data supports the hypothesis that salicylates are hepatotoxic, the dose effect was unpredictable for individuals. Clinically evident liver disease was not seen in any patient.

309 METABOLIC RESPONSES TO CONTROLLED SUBSTRATE CHALLENGE DIURING PARENTERAL ALIMENTATION FOR GASTROINTESTINAL PROBLEMS. Charles E. Mize, Gerald R. Faloona, and Zaven $\underline{H}$. Chakmakjian. Tiniv. Tex. Health Sci。 $\bar{c} t r$. (Southwestern); Depts of Ped. Biochem, and Med., Dallas.

24 patients who required complete parenterai nutrition either for medical or surgical causes were randomly assioned to receive minimal or greater $L$-amino acid $(A A)$ mixture concentrations. They received $1.5,3.0$, or $4.5 \mathrm{gm}$ Freamine $/ \mathrm{kg} / \mathrm{d}$, and an incremental, standardized caloric input $(60,90,120,750 \mathrm{cal} / \mathrm{kg} / \mathrm{d})$ adjusted with glucose-HOH. Plasma pancreatic glucagon was high $(170-255 \mathrm{pg} / \mathrm{ml})$ and plasma insulin normal to low $(2.3-6.2 \mu \mathrm{U} / \mathrm{ml})$ at the initiation of alimentation, yielding a low insulin/glucagon molar ratio $(0.4-$ 1.8). The effect of introducing a glucose challenge was to lower the glucagon level incrementally as the caloric input increased; this was not affected by the different AA loads. Blood glucose ranged random7y (40-195 $\mathrm{mg} \%$ ) throughout the sequences; plasma insulin increased slightly with increasing glucose load at low AA (5-9 $\mathrm{uU} / \mathrm{ml})$, but was increased significantly at high $A A$ load (2l$24 \mu \mathrm{U} / \mathrm{ml})$. Free fatty acid concentration was highest at $4.5 \mathrm{gm} A A$ $\mathrm{kg} / \mathrm{d}(1.4-1.7 \mathrm{meq} / \mathrm{l})$. Plasma cortisol values varied slightly, but growth homone consistently fell for all AA levels during increasing glucose challenge $(19-21 \mathrm{ng} / \mathrm{ml}$ to $2-5 \mathrm{ng} / \mathrm{ml})$. Urinary excretion of methyl-histidines $(0-49 \mu \mathrm{g} / \mathrm{d}$ ) was very low during progression from measured negative to positive nitrngen balance, suggesting that muscle proteolys is is being suppressed. These data support the thesis that hormonal homeostasis is responsive to substrate-level control by $A A$ and carbohydrate. (Supported by USPHS Grant 5R01 HD07707). ON THE PLASMA AMINOGRAMS IN INFANTS DURING TOTAL PARENTERAL NUTRITION (TPN), John F, Nicholson, William C. Heird, Lewis D. Stegink, Ralph B. Dell, and Robert W. Winters. Depts. Ped., Columbia Univ. Col. of Phys. \& Surg., N.Y. and Unív. of Lowa, Sch. of Med., Iowa City, Towa.

Plasma amino acid (AA) concentrations were measured in surgical neonates and in infants with chronic intractable diarrhea during clinically steady-state conditions of TPN in which the AA intake $(2.5$ to $3.25 \mathrm{~g} / \mathrm{kg} / \mathrm{d}$ ) was provided by one of 6 different $A A$ solutions (FreAmine, Abbott AA soln., Cutter AA soln., Aminosol, Amigen or NeoAminoso1). Compared to either normal fasting or 2 hour post-prandial values, the most significant abnormalities were: (a) Iow VAL with Cutter and Aminosol; (b) low LEU with all except Aminosol and NeoAminosol; (c) high MET with FreAmine containing D, L-MET, but normal MET with FreAmine containing only LMET; (d) low TYR with all solutions; (e) low CYS with all solutions (although CYS was not optimally evaluated).

The distinctive AA composition of each AA solution permitted construction of curves and computation of correlation coefficients ( $r$ ) relating plasma composition of each AA to intake of that AA. For THE, VAL, MET, CYS \& PRO, $\mathrm{r}$ varied from 0.54 to 0.70 ; for LEU, ILEU, PHE \& ARG, $\mathrm{x}$ varied from 0.36 to 0.43 , while for LYS, HIS, TYR, ALA \& SER, r varied from 0.26 to -0.22 . These relationships not only raise a number of theoretical biochemical questions but also provide a potentially practical and useful approach for rational optimization of parenteral AA intake during TPN in infants. TESTINE OE THE RAT. J.E. Robillard and M.K. Younoszai. University of Iowa, Iowa City, Iowa.

The threshold to reabsorb bicarbonate in the proximal renal tubule is lower in newborn than in adult animals. The present study was designed to measure net transport of bicarbonate $\left(\mathrm{J}_{\mathrm{HCO}_{3}}\right)$ in the developing rat intestine, using an in vivo onepass perfusion technique. In suckling (14 to 15 day), weanling (21 to 22 day) and adult rats, 15 to $20 \mathrm{~cm}$ segments of the proximal jejunum and distal ileum were cannulated, flushed with $5 \mathrm{ml}$ of 0.85 percent sodium chloride solution and $10 \mathrm{~m} 1$ of air. They were then perfused in situ at $0.35 \mathrm{ml} / \mathrm{min}$ for 2 hours with a solution containing $25 \mathrm{mEq} / 1$ of sodium bicarbonate, $115 \mathrm{mEq} / 1$ of sodium chloride, and $50 \mathrm{\mu g} / 1$ of phenol red (as nonabsorbed indicator for volume change). Mannitol was added to attain 300 mosmoles/l. $\mathrm{J}_{\mathrm{HCO}}$ was determined from changes in $\mathrm{pH}, \mathrm{pCO}_{2}$ and total $\mathrm{CO}_{2}$ content of the solution perfused during the last hour of perfusion. $\mathrm{J}_{\mathrm{HCO}_{3}}$ in the jejunum was positive (absorption) and negative in the ileum (secretion) in the majority of the rats studied. In the jejunum $\mathrm{J}_{\mathrm{HCO}}$ (mean \pm S.E., $\mu \mathrm{Eq} / \mathrm{hr}$ per $\mathrm{g}$ seg-

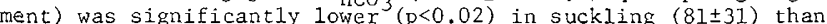

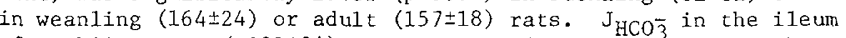
of suckling rats ( $-101 \pm 24$ ) was not significantly different than those in weanling $(-40 \pm 31)$ or in adult $(-53 \pm 20)$ rats. Thus, the maturation of bicarbonate absorption in the developing rat jejunal mucosa seems to parallel the maturation of bicarbonate reabsorption in the developing proximal renal tubule noted in other animals.

312 EFFECT OF BILE AND TAUROCHOLATE ON THE CELL DYNAMICS OF THE SMALL INTESTINE. C.C. Roy, G. Laurendeau*, G. Doyon*, L. Chartrand* and M.R. Rivest*, Dep

Pediatrics, Ste. Justine Mosp. and the Univ. Or Montreal. has received ittle attention. Forty eight hours after the creation of a bile fistula in 10 Sprague-Dawley rats, 3-thymidine ( $1 \mu \mathrm{c} / \mathrm{g}$ body weight) was given I.V. and the animals were sacrificed one hour later. The DNA specific activity of mucosal scrapings was decreased $(P<.05)$. The labeling index, unchanged in the jejunum, was $5.8 \pm .2$ as compared to $11.6 \pm .8$ in the ileum of 10 sham animals. Cell loss was studied after 48 hours of biliary diversion by measuring the DNA of cells exfoliated during a $2 \mathrm{hr}$ period of saline perfusion of the entire small intestine. DNA loss ( $\mathrm{\mu g}$ ) in 8 experimental animals (104.9 \pm 7.2 ) was lower $(P<.001)$ than in controls $(143.0 \pm 3.9)$. After creating a bile fistula, the intraduodenal perfusion of an amigen-sucrose solution with or without added $\mathrm{Na}$ taurocholate $(30 \mu \mathrm{M} / \mathrm{ml})$ was started at a rate of $1.6 \mathrm{mI} / \mathrm{h}$. After 48 hours, $3 \mathrm{H}$-thymidine was given I.V. and the duodenal perfusate was continued. Groups of 4 rats were sacrificed $1,12,24$ and 48 hours following injection of the tritium label. The slope of the regression lines, obtained by plotting the leading edge of labeled cells along the crypt-villus units, showed that the bile acid perfused animals had a faster rate of cell migration in both the jejunum $(P<.05)$ and ileum $(\mathrm{P}<.01)$. These data suggest that bile and bile acids constitute important regulatory factors influencing enterocyte proliferation, migration and loss. 
313 RESPONSE OF RAT INTESTINE TO AN ACUTE HYPEROSMOTIC FEEDING. S. Teichberg, F. Lifshitz, R。Pergolizzi and R. A. Wapnir, Dept. of Peds. \& Labs., North Shore Univ. Hosp. Manhasset, NY 11030 and Dept. of Peds., Cornell Univ. Med. Coll NY io021.

Effects of orally administered hyperosmostic loads on rat small intestine were examined. Male Wistar $80-100 \mathrm{gm}$ rats were force-fed $(5 \mathrm{ml} / 100 \mathrm{gm}$ body wt.) $1400 \mathrm{mLsm} / \mathrm{kg}$ mannitol (M) solution containing $100 \mathrm{ul}$ of $5 \mathrm{uCi} /$ $\mathrm{ml}{ }^{3} \mathrm{HM}$ and $600 \mathrm{mg} \%$ polyethylene glycol; and killed after 0,20,40 and $120 \mathrm{~min}$. Intraluminal osmotic pressure, water, $\mathrm{Na}^{+}$and $\mathrm{K}^{+}$fluxes, mucosal disaccharidases, alkaline phosphatase and ultrastructure were determined. The stomach retained most of $M$ for $120 \mathrm{~min}$., osmolal ity decreased from 1300 to $900 \mathrm{mOsm} / \mathrm{L}$. The jejunal osmotic pressure equilibrated at $40 \mathrm{~min}$ $\left(900 \mathrm{mOsm} / \mathrm{L}\right.$ to $300 \mathrm{mOsm} / \mathrm{L}$ ) and $\mathrm{Na}^{+}$and water influx was doubled. The duodenum and ileum showed similar changes. All jejunal disaccharidases decreased 50 to $65 \%$ from basal levels by $20 \mathrm{~min}$. $(p<0.05)$ and then rose back at $120 \mathrm{~min}$. Alkal ine phosphatase significantly increased. The brush border showed transient morphological alterations. By $20 \mathrm{~min}$ microvilli of many epithel ial cells were decreased in number and fused ot their bases with occasional filament-like aggregates in their terminal web region. All other organelles were unchanged. At $120 \mathrm{~min}$ microvilli of most epithelial cells appeared normal in number and morphology. Reduced disaccharidase activity may be due to washing of enzyme molecules off the glycoprotein matrix surrounding microvilli during the intraluminal water and $\mathrm{Na}^{+}$fluxes. Microvilli may be able to reversibly retract towards the cytoplasm during stress.

314 EFFECTS OF THE NUTRITIONAL DISTURBANCES, INFLAMMATORY BOWEL DISEASE (IBD) AND ANOREXIA NERVOSA (AN) ON HORMONAL SECRETIONS. Alfred Tenore, John S. Parks, Rebecca T. Kirkland, Michael Ranke, Martin Silink, and Alfred M. Bongiovanni. Univ. of Penna. Sch. Med. Children's Hosp. of Phila. Dept. of Endocr., Phila., PA.

IBD ranks second to acquired hypothyroidism as a cause of growth failure in our population of 8-14 year old children. Growth may slow years before abdominal symptoms appear. Seven children with a history of decreasing growth velocities had no specific abdominal complaints but were found by $x$-ray to have IBD One child had overt abdominal symptoms when studies. Investigations of growth hormone (GH) reserves in these children showed excessive rather than impaired responses as had been previously reported. The mean peak sleep-related $\mathrm{GH}$ value was 26 vs $16 \mathrm{ng} / \mathrm{ml}$ in 25 controls $(P<0.01)$ and the mean peak value after propranolol and glucagon (PG) was $48 \mathrm{vs} 17 \mathrm{ng} / \mathrm{ml}$ in controls ( $\mathrm{P}<0.0005$ ).

Since $A N$ and $I B D$ have in common some degree of protein-calorie malnutrition, 8 females with AN were also studied. In contrast to reports of elevated levels of $\mathrm{GH}$ in children with kwashiorkor the patients with AN had significantly blunted GH responses to PG (mean peak 9 vs $17 \mathrm{ng} / \mathrm{ml}$ in controls - $\mathrm{P}<0.005$ ); whereas, their sleep-related $G H$ levels were normal to elevated compared to the controls (22 vs $16 \mathrm{ng} / \mathrm{ml}$ - P<0.05).

On the basis of the altered pituitary functions, a possible explanation is offered for the differences in the hormone responses in the 2 groups of patients and for the growth failure in patients with IBD.

FAILURE OF EPINEPHRINE TO INDUCE HYPERGLYCEMIA AFTER FRUCTOSE INJECTION IN YOUNG MICE. Jean HolowachThurston, Elizabeth $M$. Jones, Richard E. Hauhart, Wash. Univ. Sch. of Med., Dept. of Peds., St. Louis, Mo.

In young mice fructose injection inactivates liver phosphorylase, reduces $\mathrm{Pi}$, and increases fructose-1-phosphate to markedly inhibitory levels. At the concentrations of $\mathrm{P}_{i}$ and fructose-1phosphate found in liver after fructose liver phosphorylase activity in vitro was inhibited $88 \%$ (Soc. Ped. Res., Atlantic City, 1971). These findings may be causally related to the hypoglycemia and the lack of response to glucagon seen in patients with hereditary fructose intolerance af ter fructose ingestion. Since enzyme inhibition is not necessarlly reflected in reduced function, an in vivo study of the hypergiycemic effect of epinephrine in fructose-injected mice was undertaken. In 23 control animals a 2 -fold increase in liver phosphorylase activity 10 min after epinephrine was associated with a $55 \%$ increase in plasma glucose ( $p<0.001)$; at 20 min plasma glucose was $247 \%$ of the control value $(p<0.001)$. In 23 experimental animals 11 ver phosphorylase activity was reduced $74 \%, 20$ min after fructose injection ( $p<$ 0.001 ). Although phosphorylase activity increased 5-fold within 5 min of epinephrine injection, no increases in plasma glucose concentration over the level found in fructose-injected animals which did not recelve epinephrine was seen at either 5,10 or 20 min after epinephrine injection. The data confirm inactivation of liver phosphorylase after fructose injection and support inhibition of the epinephrine-activated enzyme by the reduction in $\mathrm{Pi}$ and elevation of fructose-1-phosphate levels in liver produced by NASOJEJUNAL FEEDING IN THE SMALL PREMATURE INFANT: A
CONTROLLED TRIAL, Ricardo Uauy, Sherry Loo, Ian GrosS and Joseph Warshaw, Yale Sch. Med。, New Haven, Conn.

The effect of high volume nasojejunal (NJ) feeding on the growth of premature infants weighing less than 1500 gm has been compared to 2 gavage-fed groups. The data for the NJ group and Contro1 Gavage Group I (CGI) were obtained from a prospective study in which the aim was to administer $150 \mathrm{cals} / \mathrm{kg} /$ day. The data for Gavage Group II (GII) was obtained by retrospective analysis of patients, one year prior to the controlled study, when a far less aggressive feeding policy was practiced. The groups were matched for weight and gestational age. There were approximately 20 patients in each group, and $20 \mathrm{cal} / \mathrm{oz}$ formula was used.

\begin{tabular}{|c|c|c|c|c|c|}
\hline \multirow{2}{*}{\multicolumn{2}{|c|}{ Group }} & Hospital & Days to Regain & $\%$ Weight Loss & $\%$ Weight Gain \\
\hline & & Stay & Birth Weight & First Week & For 3 weeks \\
\hline$\longdiv { 7 2 0 0 }$ & NJ & 32.7 & 8.6 & 2.1 & 24.1 \\
\hline $\mathrm{gm}$. & CGI & 32.0 & 13.0 & 5.8 & 20.6 \\
\hline & GII & 36.8 & 14.6 & 11.6 & 11.9 \\
\hline$<1200$ & $\mathrm{NJ}$ & 49.5 & 8.7 & 1.3 & 20.1 \\
\hline $\mathrm{gm}$, & CGI & 54.3 & 12.0 & 4.6 & 19.7 \\
\hline & GII & 59.0 & 14.9 & 13.9 & 14.2 \\
\hline
\end{tabular}
The NJ group had a greater increase in length over the first 3 weeks, but the growth in head circumference was the same in the NJ and CGI groups. There was no increase in the incidence of necrotizing enterocolitis in the NJ group. The greatest benefit of NJ feeding was in the sickest infants who could not be fed adequately by the gavage method. NJ feeding is a safe and effective method of providing optimal nutritional support to small premature
infants during the critical first weeks of life.
317 INFLUENCE OF THYROXINE ON PHENOBARBITAL STIMULATION OF BILIRUBIN CONJUGATION IN PREGNANT RATS.S.Vaisman and L.M.Gartner, Dept.Ped. Albert Einstein, N.Y.

In a previous study we demonstrated that the stimulatory effect of phenobarbital (PB) on hepatic conjugation of bilirubin (B) with glucuronic acid decreases progressively during the course of pregnancy ( $p^{\prime} c y$ ) in the rat, from a $62 \%$ increase during days 5 through 9 to $19 \%$ on the $21^{\text {st }}$ day (Ped.Res.8:366,1974). In an earlier study we had reported that thyroid hormone ( $T$ ) administration to rats decreased hepatic $B$ glucuronide formation. Elevated $T$ concentrations in sera have been observed late in p'cy, suggesting that the decreased response to $P B$ may result from enhanced $T$ activity. In this study the hepatic conjugation of $B$ with glucuronic acid following 1-thyroxine and/or PB administration was examined in nonpregnant (NP) and 21 day pregnant (P) rats. The results are express ed in comparison to B glucuronide conjugation in the NP control group indicated as $100 \%$ :

$\begin{array}{lrrcc} & \text { CONTROL } & \text { THYROX. } & \text { PHENOB } & \text { THYROX.+PB } \\ \text { NON-PREGNANT } & 100 \% & 90 \% & 163 \% & 136 \% \\ \text { PREGNANT } & 98 \% & 81 \% & 111 \% & 118 \%\end{array}$

Without $\mathrm{PB}$ treatment, $\mathrm{P}$ and $\mathrm{NP}$ rats showed no difference in B conjugation. In both groups thyroxine administration decreased conjugation. Combined $\mathrm{PB}$ and thyroxine therapy to NP rats resulted in significantly less enhancement of activity than $P B$ alone; an effect which appears to mimic the response to $P B$ stimulation during $p^{\prime} c y$. Combined therapy in $P$ rats resulted in no decrease as compared to $P B$ alone, suggesting that in $P^{\prime} c y$ the $T$ inhibitory effect may have already been maximal and that $T$ may be a significant mediator of reduced hepatic conjugation response to $P B$ during $P^{\prime c y}$.
318 INRALUMINAL PHASE OF TNTESTINAL ANTIBODY FUNCTION: first step in control of antigen absorption. W. Allan Walker and Stephen N. Abe1. Harvard Medical Center, Mass. Gen. Hosp., Pediatric GI Unit, Boston. In previous studies, we have demonstrated that antibodies present in the mucous coat (glycocalyx) of the gut interact with antigens to form complexes which axe readily broken down by local proteases and therefore less available for absorption ("membrane" phase of intestinal antibody function). In this study, we examined the possible role of antibodies within the intestinal lumen in controlling antigen uptake. To simulate intraluminal antio body activity, we prepared soluble, radiolabeled antigenaantibody complexes in antigen and antibody excess; these complexes were exposed to gut sacs from rats in vitro. There was a significant decrease in absorption of antigen by gut sacs exposed to antigenantibody complexes compared to antigen alone. However, no difference in labeled antigen breakdown occurred in both groups of experiments. In addition, less radiolabeled antigen-antibody complex could be demonstrated within the mucous of gut sacs. Samples of incubation medium containing antigen-antibody complexes were analyzed on sucrose gradients; radioactivity appeared at the bottom of the gradients suggesting the presence of large antigen-antibody aggregates. These studies suggest that antibodies present within the intestinal lumen can interact with antigens to form complexes and/or aggregates. These cannot be taken up onto the intestinal surface and therefore will not be available for absorption. This phenomenon may represent the "intraluminal" phase of intestinal antibody control of antigen ingestion. 

319 PROWTH OF INFANTS FED A $2 N$-SUPPLEMENTED MILK FORMULA of Colorado Medical Center, Dept. of Ped., Denver.

The present study was designed to evaluate the effects of supplementing an infant milk formula (Similac with iron) with $\mathrm{zn}$. The formula was prepared and coded by Ross Laboratories for a double-blind controlled study. Each of the normal, appropriate for gestational age, full-term infants received, with informed parental consent, sufficient formula to cover total milk requirements from 4 days to 6 months of age. Other food items were inreceived a formula containing $1.8 \mathrm{mg} \mathrm{Zn} / \mathrm{l}$; test infants (19M \& $16 \mathrm{~F}$ ) received the same formula supplemented with $4 \mathrm{mg} \mathrm{Zn} / 1$. The 2n-supplemented formula was well tolerated and not associated with increased gastro-intestinal problems. At 3 months the mean plasma $2 \mathrm{n}$ level of test infants $(70.3+1.8 \mu \mathrm{\mu} / 10 \mathrm{cml})$ was higher than that of controls $(61.4+2.1 \mu \mathrm{g} / 10 \overline{\mathrm{m}} \mathrm{m}) \quad(\mathrm{p}<0.005)$. From $0-6$ months of age the mean growth velocity (length and weight) of the male test infants was greater than that of male controls $(p<0.05)$ 0-6 month increment in: Height $(\mathrm{cm})$ Weight $(\mathrm{g})$ Zn-supplemented: $\quad 19.42+0.52 \quad 4497+116$ Control: $\quad 17.49 \mp 0.78 \quad 4047 \mp 267$ Growth velocity of female infants was the same for test \& control infants. There was no difference in serum cholesterol levels between test and control groups, though $\mathrm{Zn}$-supplementation of the formula increased the $\mathrm{Z}_{\mathrm{n}}$ : $\mathrm{Cu}$ ratio from $1: 5$ to $1: 17$. It is concluded that $2 \mathrm{n}$-supplementation of Similac is associated with an increase in growth velocity of male infants from $0-6$ months,
and is not associated with any evidence of toxicity.

\section{0}

13C-TRIOCTANOIN: A SAFE AND SENSITIVE TEST FOR MALABSORPTION. John B. Watkins, Dale A. Schoeller, Donald G. Ott and Peter D. Klein. Harvard Med. Sch.,

Children's Hosp. Med. Ctr., Dept. Ped., Boston, MA. and Argonne Nat'l Lab., Argonne, Ill. and Los Alamos Scientific Lab., Los Alamos, NM.' (Intr. by Richard J. Grand).

Gastrointestinal and hepatic function has been assessed by measuring excretion of $14 \mathrm{CO}_{2}$ from $14 \mathrm{C}$ trioctanoin. This sensitive, non-invasive test is limited by radiation hazard to the adult population. In order to study malabsorption in children we have initiated the use of trioctanoin labeled with carbon -13, a stable non-radioactive isotope. Preliminary studies with unlabeled trioctanoin indicate that the endogenous $13 \mathrm{CO}_{2}$ production was constant over six hours (standard error $\pm 0.6 / \mathrm{mil}$ ). To evaluate malabsorption $1-13 \mathrm{C}$ labeled trioctanoin was then administered to six patients in conjunction with $72 \mathrm{hr}$. fat balanced studies. Peak excess production of $13 \mathrm{CO}_{2}$ by 2 hours in three pts. without steatorrhea ( $<3 \%$ intake excreted/day) was 60,55 and 43 per mil. In three pts. with steatorrhea $20.6 \pm 5.1$, (avg. \pm SD) of intake excreted/day, peak excess $13 \mathrm{CO}_{2}$ production was 5,10 and $\mathrm{l} / \mathrm{mil}$. Two of these pts. had cystic fibrosis. Pancreatic enzyme replacement reduced fat excretion to $10.6 \%$ of intake and increased $13 \mathrm{CO}_{2}$ production 5 fold. Cumulative $13 \mathrm{CO}_{2}$ excretion by 2 hrs. was $30.5 \pm 10 \%$ of the administered dose in normals and $0.4 \pm$ $0.12 \%$ in pts. with steatorrhea.

Conclusion: 1. Fat malabsorption may be accurately detected with $13 \mathrm{C}$ trioctanoin. 2. These data indicate that stable isotope labeled substrates are useful for sensitive and safe metabolic studies in children.

321

NEONATAL INTRAHEPATIC CHOLESTASIS. D. Wirtschafter, R. Ermocilla, H. Harris and G. Cassady. Univ. of Ala. in Intrahepatic cholestasis is a relatively unusual finding in neonates. During a $2 \mathrm{yr}$. period, all 10 infants with Stage IIIIV bronchopulmonary dysplasia at autopsy were found to have cholestasis with dilated canaliculi containing plugs of bile and no evidence of liver cell damage or inflammatory reaction. In 2 of these, fibrosis and centrilobular congestion were present. Mean birth weight was $1725 \mathrm{~g} .(4 \leq 1200 \mathrm{~g}$.) and mean gestation was 32 wks. $(4 \leq 30$ wks.). Perinatàl asphyxia $(7 / 10)$, shock and/or renal failure $(8 / 10)$, and prolonged mechanical ventilation $(10 / 10$ $\bar{x}=260 \mathrm{~h}$.$) were common. Systemic infection was proven in 5$; all had received antibiotics. All but one baby died 5-18 days af ter birth. Serum conjugated bilirubin ranged from $1.0-1.6 \mathrm{mg} \%$ in 5 babies (Group I) and from $3.0-17.4$ in 5 others (Group II). Babies in Group Ir tended to have smaller birth weight $(\bar{x}=1305$ vs $2150 \mathrm{~g} ; \mathrm{p}^{<.10)}$. Cumulative exposure to $\mathrm{FiO}_{2}>0.6$ was longer in Group II $(\tilde{x}=121 \mathrm{~h}$.$) than in Group I (x=15 \mathrm{~h} . ; p<.005)$; all babies in Group II had $\geq 40 \mathrm{~h}$. FiO $2 \geq 0.6$ while all Group I babies had $<40 \mathrm{~h} . \mathrm{FiO}_{2} \geq 0.6(\mathrm{p}<.005)$. Àsphyxia, shock, infection and total duration of ventilation were similar in both groups. Umbilical venous catheters were present in $3 / 5$ infants in each group. Intravenous synthetic amino acid mixtures were given in only 5 infants ( $4 / 5$ Group I; $1 / 5$ Group II). The histologic severity of the process was unrelated to the degree of conjugated hyperbilirubinemia. These findings suggest that factors which lead to pulmonary oxygen toxicity may also result in intrahepatic cholestasis.
322 CHANGES IN PANCREATIC SECRETIONS (PS), AMINO ACID TRANSPORT (AT), AND ENTEROKINASE ACTIVITY (EA) AFTER PROXIMAL INTESTINAL RESECTION (IR) IN THE RAT. Micheline van Caillie, Claude L. Morin, Victor Ling and Nicole Deschênes, Dept. of Ped., Ste. Justine Hospital, Univ. of Montrea1, Montrea1, Canada. (Introduced by C.C. Roy).

To define the changes characteristic of functional adaptation after IR, we studied PS, EA and AT in 80 young male SpragueDawley rats, 1,4 and 12 weeks after removal of $50 \%$ of the proximal intestine (IR) or sham operation (SH). The results of PS were as follows (mean \pm SD):

\begin{tabular}{|c|c|c|c|c|c|c|c|c|}
\hline \multirow[b]{2}{*}{ Wk } & \multicolumn{3}{|c|}{ Secretion rate $(\mathrm{ml} / \mathrm{hr}$} & \multirow[b]{2}{*}{$\left(\mathrm{N}^{\mathrm{O}}\right)$} & \multicolumn{3}{|c|}{$\mathrm{HCO}_{3}$-output $(\mu \mathrm{M} / \mathrm{hr})$} & \\
\hline & $\mathrm{SH}$ & $\left(\mathrm{N}^{\circ}\right)$ & IR & & $\overline{\mathrm{SH}}$ & $\left(N^{O}\right)$ & $\overline{\mathrm{IR}}$ & $\left(\mathrm{N}^{\mathrm{O}}\right.$ \\
\hline . & $.45 \pm .05$ & (3) & $.18 \pm .15 *$ & (5) & $8.86 \pm 1.1$ & (3) & $5.12 \pm 2.4 \%$ & $(5$ \\
\hline 4 & $.41 \pm .06$ & (6) & $.54 \pm .05 *$ & (7) & $10.18 \pm 2.2$ & (6) & $12.3 \pm 2.9$ & (7) \\
\hline 12 & $.42 \pm .11$ & (7) & $.33 \pm .16$ & (9) & $9.13 \pm 3.1$ & (7) & $6.29 \pm 3.7$ & (9 \\
\hline
\end{tabular}

* p statistically significant.

Enterokinase remained similar in both specific and total activity/segment throughout. Intestinal uptake (mMole/segment) of alanine, lysine and cycloleucine in IR was increased $(p<.01)$ at 4 weeks and then decreased at 12 weeks ( $p<.05)$. In summary we observed a transient fall in PS, then a peak to above normal levels followed by a decline to subnormal values. The intestinal uptake of amino acid followed a similar pattern. Our study underlines the importance of periodic shifts in $a b-$ sorptive mechanisms following IR.

32 EFFECT OF N-(PHOSPHONACETYL)-L-ASPARTATE(PALA) ON 323 INTESTINAL SUCRASE ACTIVITY IN RATS. Takashi Yoshida (Intr. by Norman Kretchmer), Stanford Univ. Sch. Med., Dept. Peds., Stanford, Cal

Rapldly growing cells have elevated requirement for de novo pyrimidine nucleotide biosynthesis. Impairment in supply of pyrimidine nucleotides should disturb not only proliferation, but also developing enzyme systems. We studied the effect of PAIA, a specific and potent inhibitor of aspartate transcarbamylase, on the development of sucrase activity in rat intestine. Three groups of male swiss rats of 19 days (A), 22 days (B) and 6 weeks (C) were used. All were fed a balanced synthetic diet with sucrose as a sole carbohydrate source. PALA was added to the drinking water $(720 \mathrm{mg} / \mathrm{L})$ to one-half of each group of rats. Rats were killed 8 days later, and sucrase activity was assayed in the mucosal celi homogenate. Normally, sucrase appears around 17 days after birth. In groups $A$ and $B$, PALA inhibited development of sucrase markedly (A: $-82 \%, \mathrm{~B}:-62 \%$ ), while in group $\mathrm{C}$, no effect of PALA on sucrase activity was observed. There was a marked dilatation and watery stool in the entire gut of weanling rats given PALA. In the adult rats, intestinal content was less watery but no solid stool was present in the colon. In all animals, the activity of $\mathrm{Na}^{+}, \mathrm{K}^{+}$-ATPase, but not $\mathrm{Mg}^{+}$-ATPase, was signiffcantly reduced. It is likely that de novo pyrimidine biosynthesis is essential for the development of sucrase in weanling but not in adult rats.

IN VIVO HEXOSE ABSORPTION IN THE JEJUNUM AND ILEUM OF SUCKLING AND WEANLING RATS. M. Kabir Younoszai. Ped., Iowa City, Iowa.

During in vivo perfusion of the whole jejunum + ileum, rate of hexose absorption was lower in suckling than in older rats (Younoszai, Gastroenterology 64:A-190, 1973, J. Nutr. 104:671, 1974) The present study was undertaken to compare the proximal to distal gradient of glucose absorption in the intestine of suckling (14 to 15 day) and weanling ( 21 to 22 day) albino rats. In anesthetized rats approximately $15 \mathrm{~cm}$ segments of the proximal jejunum $(J)$ and distal ileum (II) were flushed with $5 \mathrm{ml}$ of 0.85 percent sodium chloride solution and $10 \mathrm{mI}$ of air. The segments were then cannulated and perfused in situ for 2 hours at $0.36 \mathrm{ml} /$ min with solutions containing D-glucose, phenol red $(25 \mu \mathrm{g} / \mathrm{ml}$, as nonabsorbed indicator) and sufficient sodium chloride to achieve 300 mosmoles/liter. The concentration of D-glucose in the solutions perfused varied in different groups of rats and were 3,5 or $10 \mathrm{~g} / 1$. Disappearance of $\mathrm{D}$-glucose from the lumen during the last hour of perfusion was taken as absorption. In both suckling and weanling rats at all three glucose concentrations, rate of absorption (meantS.E.; $\mathrm{mg} / \mathrm{hr}$ per $\mathrm{g}$ segment) was greater in the jejunum than in the ileum $(\mathrm{p}<0.05)$. Suckling rats: $3 \mathrm{~g} / 1 \mathrm{~J}$ $24 \pm 6$, I1 $14 \pm 4 ; 5 \mathrm{~g} / 1 \mathrm{~J} 21 \pm 3$, I1 $13 \pm 2 ; 10 \mathrm{~g} / 150 \pm 4$, I1 $15 \pm 6$. Weanling rats: $3 \mathrm{~g} / 1 \mathrm{~J} 30 \pm 3$, Il $24 \pm 3 ; 5 \mathrm{~g} / 1 \mathrm{~J} 48 \pm 6$, Il $38 \pm 4 ; 10 \mathrm{~g} / 1 \mathrm{~J}$ $63 \pm 4$, Il $45 \pm 4$. At all three glucose concentrations the rate of absorption was lower in segments of suckling than in corresponding segments of weanling rats. 
325 CHYLOMICRON CLEARANCE IN A TYPE I CHILD FOLLOWING LOW DENSITY LIPOPROTEIN INFUSION. James H. Zavoral, Kanta Kuba, Franklin Zieve, William Krivit. Univ. of Mn. Sch. of Med., Dept. of Ped.\& VA Hosp., Dept. of Med., Minneapolis. Type I hyperlipidemic patients have hyperchylomicronemia with a deficiency in lipoprotein lipase and low and high density lipoproteins (L.D.L., H.D.L.) which are cofactors necessary for the clearance of chylomicrons by glyceride hydrolysis.

Plasma with increased L.D.L. was given to an $8 \mathrm{yr}$. old child who has documented type I hyperlipidemia to provide a cofactor for the clearing of chylomicrons. The plasma infusion in our patient represents the first successful in vivo report of chylomicron clearance. Chylomicrons were measured as the triglycerides in the top fraction of plasma spun for 30 minutes at 19,000 rpm's. Chylomicron leveIs did not change following 10 units $/ \mathrm{kg} / \mathrm{IV}$ heparin or $10 \mathrm{cc} / \mathrm{kg} / \mathrm{IV}$ of normal plasma. Following $10 \mathrm{cc} / \mathrm{kg} / \mathrm{IV}$ of L.D.L. rich plasma the chylomicrons decreased from $1210 \mathrm{mgm} \%$ at $.0^{\prime}$ time to $996 \mathrm{mgm} \%$ at $10^{\prime}$ and $268 \mathrm{mgm} \%$ at $30^{\prime}$. The plasma after $24 \mathrm{hrs}$. also demonstrated a dramatic decrease in the creamy top layer of chylomicrons. Agarose electrophoresis of the plasma indicated decreased chylomicrons. No change was seen in the plasma free fatty acids or lipoprotein lipase levels.

This in vivo method of chylomicron clearance suggests: 1) potential therapy for type I patients crises and 2) that the L.D.L. provided sufficient cofactors for glyceride hydrolysis and chylomicron clearance.

\section{GENETICS}

26 T LYMPHOCYTE DEPLETION IN FAMILIAL LEUKEMIA. Renner

326 s. Anderson, John H. Kersey, Peter F. Coccia, Mark E. Krivit. Univ, of Mn. Sch. of Med., Depts. of Ped., Lab Med.\& Oral Path., Minneapolis, Mn.

When multiple cases of acute lymphatic leukemia occur in the same family, immunologic abnormality may be a predisposing factor and is a testable hypothesis. The proband, a deceased sib and paternal aunt were affected with acute lymphoblastic leukemia. T 1ymphocytes were: Per Cent Absolute Number Father

Sib $J$.

$\begin{array}{ll}\text { Per Cent } & \text { Absolute Number } \\ 32.1,44 . & 576,1169 \\ 46.5,21.0 & 1670,554 \\ 19.0,26.0 & 744,810 \\ 64+6.9 \text { (S.D.) } 1910+590 \text { (S.D. }\end{array}$

Sib L.

$19.0,26.0$
$64+6.9$ (S.D.) $1910+590$ (S.D.)

Normal
The individuals with low circulating $T$ cells and the proband shared the same HL-A haplotype, 2-5. Mother and unaffected paternal aunt had normal $T$ lymphocytes but did not have 2-5 haplotype. $B$ lymphocytes and imnunoglobulins $G, M, A$ and $E$ were within normal limits. Mitogen studies which included PHA and Con A were normal, but sib L. had an abnormal response to SK/SD. MLC data indicated lack of stimulation between sibs L. and J. Analysis of chromosomes of 6 members of the family by G-banding did not reveal any intrinsic structural abnormalities but a tetraradial figure was found in sibs $J$, and $L$. The results are consistent with hypothesis that the apparent predisposition to develop leukemia may result from an abnormal clone of $\mathrm{T}$ cells. An immune response gene defect closely linked to the HL-A locus might cause a decreased responsiveness in affected family members against environmental leukemogens such as viruses.

327 NTERACTION OF ANTBODY TO HGPRT WITH 327 EXTRACTS FROM NORMAL INDIVIDUALS AND PATIENTS WITH THE LESCH-NYHAN SYNDROME. Bohdan Bakay, Michael Bazaral, and William L. Nyhan, Dept. of Ped., Univ. of California, San Diego, La Jolla, CA.

The cross-reaction of antibody to HGPR T derived from normal human erythrocytes with cell extracts containing the enzyme was analyzed by polyacrylamide gel disc electrophoresis. In this system, free HGPR T migrated to its normal location (peak A), while HGPRT antibody complexes formed at low concentrations of antibody, migrated a notably shorter distance (peak B). Complexes formed at high concentrations of antibody were unable to enter the gel. No precipitable enzyme-antibody complexes were formed. It was concluded that complexes which entered the gel and appeared in peak B, and those which did not enter the gel, were of different molecular size. Enzymatic activity of the complexes appearing in peak B was about the same as that of free enzyme. The enzyme activity, saturated with large quantities of antibody, was about 50-60\% that of the free enzyme. Antibody prepared to partially and highly purified HGPRT and to a highly purified single isoenzyme, reacted with each of the four HGPRT isoenzymes found in erythrocyte lysates. Thus, all four isoenzymes of HGPRT appear to be immunochemically identical. In contrast, no cross-reacting material (CRM) was detected by this assay in extracts of red cells and fibroblasts of patients with the Lesch-Nyhan syndrome. 328 PFFECT OF CYSTIC FIBROSIS (CF) SALIVA ON INORGANIC CHONDRIA. Gerald J. Bargman, James E. Changus, Mary Sukup, Ann Vale and Henry C. Pitot (Intr. by J.A. Mangos) Depts. of Ped, and Path., Univ. of Wisconsin, Sch. of Med., Madison.

As part of our continuing studies of respiratory energy production in $C F$ we have investigated IPE by rat liver mitochondria in the presence of mixed fresh saliva (MFS) from CF homozygotes (HMZ), obligatory heterozygotes (HTZ) and normal control subjects (NC). Rat liver mitochondria were used because they are a well-defined system for studies of oxidative phosphorylation. IPE was measured as $\mu \mathrm{M} \mathrm{P} / \mathrm{hr} \cdot \mathrm{mg}$ mitochondrial protein without knowing the origin of the samples. In the absence of saliva, the rat liver mitochondria showed a mean IPE of 24.22+S.E.1.41 in 19 patients. In the presence of MFS from NC a decrease in IPE was observed with a mean value of 18.15+S.E.1.15 in 20 patients. In the presence of MFS from 4 HTZ the mean IPE was $16.80+S . E .1 .13$ which was not different from that induced by MFS from $\overline{\mathrm{N} C}$. MFS from $6 \mathrm{HMZ}$ eliminated or caused a marked decrease in IPE $(1.67 \pm S . E .0 .97)$. However, MFS from 6 other HMZ had an effect on IPE $(16.93 \pm S . E .1 .38)$ which was not different from that induced by MFS from $\bar{N} C$ or HTZ. These studies demonstrate that within a group of patients with classical $\mathrm{CF}$ we have found at least two biochemically separable populations, suggesting heterogeneity in this disease.

Supported by grants from NIAMDD 非AM-13660 and the Human Growth Fndn.

329 DIAGNOSIS OF $\alpha$-MANNOSIDASE DEFICIENCY: Arthur L. $\frac{\text { Beaudet and }}{\text { of Medicine, }} \frac{\text { Buford L. Nichols, Jr. }}{\text { Depts. Ped. and Med., Houstor. }}$

The enzymic properties of a-mannosidase activity from fibroblast extracts of a patient with mannosidosis and controls were studied to define conditions for accurate diagnosis. Mannosidosis was suspected in a male product of a consanguineous mating because of hepatosplenomegaly, coarse features, short stature and mucopolysaccharidosis-like bony changes without mucopolysacchariduria, but initial enzyme assays were normal. Using $12.5 \mathrm{mM}$ 4-methylumbelliferyl $\alpha$-D-mannoside substrate in pH 4.0 sodium acetate buffer, patient activity was normal, but at $0.5 \mathrm{mM}$ substrate patient activity was $10 \%$ of controls, with an apparent $\mathrm{Km}$ for substrate of $25 \mathrm{mM}$ versus $0.79 \mathrm{mM}$ for controls. When extracts were treated at $53^{\circ} \mathrm{C}$ for 15 minutes, patient

$\alpha$-mannosidase was $15 \%$ and control extract $160 \%$ of the respective unheated values. While altered $\mathrm{Km}$ and heat stability suggested a mutant enzyme activity in the patient, the apparent residual mannosidase activity could be due to an interfering glycosidase hydrolysing the mannosidase substrate or to an impurity in the substrate. Heat inactivation studies suggested that the activity was not due to neutral mannosidase $C$. When assayed in citrate-phosphate buffer at $\mathrm{pH} 4.0$, the kinetic properties of patient enzyme were altered, but the deficiency was partially evident even at high substrate concentration. Thus, the use of low substrate concentrations, heat inactivation and citrate-phosphate buffer all facilitate the diagnosis of $\alpha$-mannosidase deficiency.

330 CARRIER DETECTION IN FUCOSIDOSIS, N.G. Beratis, B.M. Turner, and $K$. Hirschhorn. Mount sinai School of $\alpha-\mathrm{L}$-Fucosidase deficiency is the basic defect in fucosidosis. We have utilized the newly available fluorogenic substrate 4methylumbelliferyl $\alpha-L-f u c o s i d e$ and we have compared the findings with those using the p-nitrophenyl derivative. pH curves of fucosidase in cultured skin fibroblasts (SF), lymphocytes (LY) and polymorphonuclear leukocytes (PMN) showed 2 peaks of activity with different specificities to these substrates. The fucosidase activity in SF, leukocytes (LK), LY and PMN was determined under optimal assay conditions for each cell type in 22 members of a family in which fucosidosis has occurred and in 10 controls. In both of the patients' parents, in one of each of the grandparents and 8 other potential carriers the activity generally fell between the homozygote (SF:0.0, LK:6.4, LY:5.6 PMN:4.8 nmoles 4-methylumbelliferon/mg prot./hr) and normal values (SF-mean \pm SD-: $186.0 \pm 61.8, \mathrm{LK}: 274.4 \pm 60.8, \mathrm{LY}: 196.8 \pm 48.2, \mathrm{PMN}: 285.6 \pm$ 74.0). There was no overlap between control subjects, carriers and patients when LY were used. In the mixed LK of an obligate heterozygote the activity (188.6) was at the lower range of the normal values (190.8-353.4). Occasional overlap between heterozygotes and controls was observed when PMN or SF were used.

Although it has been suggested in the past that carriers may be detected only by fucosidase/mannosidase ratios in LK, our findingls indicate that detection of heterozygotes for ducosidosis is possible by direct fucosidase determinations and that the best system is purified LY. 
RADHOLOGIC AND BIOCHEMICAL ABNORMALITIES IN

MANNOSIDOSIS, Carol W. Booth, Kathy Chen, and

Henry L. Nadler, Northwestern Univ. Med. Sch. Children's Mem. Hosp., Dept. of Peds., Chicago.

Mannosidosis is a lysosomal storage disease characterized biochemically by storage of mannose-rich oligosaccharides and a deficiency of acid $\alpha$-mannosidase activity. Seven patients, als under 10 years of age, have previously been described. We report here three affected siblings, now 13, 16 and 26 years old. All had slow development from the first year of $1 \mathrm{i}$ fe and now have moderately severe mental retardation requiring institutionalization. Significant medical problems have included hearing impai rment ( 2 siblings), kyphoscoliosis (2), chronic osteomyelitis (1), inguinal hernias (1), and a psychotic break responsive to phenothiazine therapy (1). On physical examination, all three showed coarse facial features and joint laxity but no organomegaly, corneal changes, gibbus, or cardiac murmurs. Skull $x$-rays of the three patients revealed thickening of the cranial vaults with complete obliteration of the diploic spaces in the older two siblings. No pneumatization was noted in the mastoid or frontal sinus regions. The phalanges and metacarpals were elongated with little tubulation of the bones. Biochemical analysis of normal liver, cultivated skin fibroblasts and white blood cells demonstrated two acid $\alpha$-mannosidases, one resistant to and one inhibited by $1.25 \mathrm{M} \mathrm{NaCl}$. When fibroblasts and white blood cells from these patients were analyzed, both forms of the enzyme were found to be deficient. The diagnosis of mannosidosis should be considered in patients with undiagnosed mental retardation and minimal physical abnormalities.

332 GLYCOSAMINOGLYCANS (GAG) OF EDTA SOLUBLE AND INSOLUBLE DENTIN IN AUTOSOMAI DOMINANT OSTEOGENESIS IMPERFECTA. David M. Brown, John J. Sauk, Kendall W. Corbin, David S. Bradford, and Carl J. Witkop, Dept. of Ped., Oral Genetics, Ortho. Surg., Univ. of Minnesota, Minneapolis.

Three subjects with autosomal dominant osteogenesis imperfecta (O.I.) were investigated, as were a comparative group of agematched controls. The dentin was demineralized and extracted with 0.5 M EDTA pH 7.4 after the method of Carmichael (1971). GAG of EDTA soluble dentin and EDTA insoluble dentin were determined after cetylpyridinium chloride (CPC) precipitation and elution from CPC cellulose columns aftex the method of Antonopoulos (1964). Further chromatography on Dowex $50 \mathrm{~W}$-XB identified specific hexosamines in the various GAG fractions. Dentin phosphoprotein, which may have a critical role in regulating matrix calcification, was determined by the method of veis, 1967. Results indicate that patients with O.I. possess significantly higher levels of GAG in EDTA soluble dentin and significantly lower values of GAG in EDTA insoluble dentin as compared with controls. Keratan sulfate was increased in O.I. soluble dentin, whereas hyaluronic acid was increased in soluble and decreased in insoluble dentin. Chondroitin sulfate was decreased in O.I. insoluble dentin, whereas dermatan sulfate was increased in O.I. soluble dentin. Dentin soluble phosphoprotein in patients with O.I. was approximately 408 that of age-matched controls. These data suggested that differences in GAG and phosphoprotein may be. responsible for the observed decreased aggregation and increased

solubility of collagen from patients with $O . I$. and may account
for several clinical aspects of O.I. ABSENCE OF A SERUM FACTOR IN PATIENTS WITH CYSTIC FIBROSIS. Liang Chou and Henry Nadler. Northwestern
Univ. Med Abnormalities in cellular transport mechanlsms at the plasma membrane (PM) level have been proposed to explain elevated ion concentrations in secretions of patients with cystic flbrosis (CF). To evaluate the potential abnormalities of $P M$ in $C F, 3 H-L-$ fucose incorporation into cultured skin fibroblasts from normal individuals $(N)$ and $C F$ was assayed as previous studies in mammalian cells have shown that L-fucose is directly incorporated into the PM fraction. Skin fibroblasts from $N$ and CF were grown in MEM wi th $15 \%$ fetal calf serum. At confluency, cells were incubated with $3 \mathrm{H}$ - $\mathrm{L}$-fucose in $0.01 \mathrm{M}$ sodium phosphate buffer, $\mathrm{pH} 7.0,0.15 \mathrm{M} \mathrm{NaCl}$ and $0.5 \%(\mathrm{w} / \mathrm{v})$ bovine serum albumin. The amount of fucose incorporated into PM was determined by measuring the radioactivity in TCA-precipitable material. Results revealed no significant difference between the amount of fucose incorporated into fibroblasts from $N$ and $C F$. When serum from $N$ was added to the reaction mixture, a linear increase in the amount of fucose incorporation could be demonstrated. In contrast, serum from 10 $C F$ patients failed to enbance fucose incorporation. The serum factor in $N$ was found to be: (1) non-dialyzable, (2) inactivated with heating at $50{ }^{\circ} \mathrm{C}$ for 30 minutes, and (3) present in the noneuglobulin fraction. These studies demonstrated the absence of a serum factor in patients with CF which enhances the incorporation of fucose into cultured human skin fibroblasts. In addition, the similarity in the amount of fucose incorporated into skin fibroblasts from $N$ and $C F$ patients suggests the plasma membranes in CF patients may not be altered.

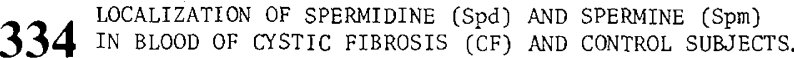
Lawrence F. Cohen, Philip M. Farrell, Jane W. Willison, and David W. Lundgren, NIH, Bethesda, MD.

This study was prompted by a recent finding in our laboratory that whole blood samples from 47 . CF homo- and heterozygotes $(\mathrm{Hz})$ show lower Spm and a higher Spd/Spm ratio than 27 matched controls. Although blood polyamines (PA) represent the first chemically definable components consistently abnormal in $\mathrm{CF}-\mathrm{Hz}$, their localization as to the specific cell type remained to be defined in normal and pathological metabolism. Accordingly, seven (3 control, 4 CF) blood fractionations have been performed by a modification of Rabinowitz's glass bead technique and Ficoll-Hypaque gradients resulting in essentially pure fractions of plasma, RBCs, lymphocytes (Ly), and granulocytes (Gr). PA were extracted with perchloric acid, separated and quantitated via an amino acid analyzer. Results indicate that plasma contains $<0.5 \mathrm{nmole}, \mathrm{PA} / \mathrm{ml}$ $(<5 \%$ of whole blood PA) and that the cellular distribution of Spd and Spm, respectively, is as follows (nmole $/ 10^{9}$ cells): RBC 1.65 $\pm .34,0.74 \pm .13$; Ly $149 \pm 21,452 \pm 101$; Gr $<5,254 \pm 94$. Despite the higher cellular content in WBCS, conversion of Spd and Spm levels to a unit blood volume basis reveals that red cells account for $>90 \%$ of circulating PA. CF blood was again found to have a higher $(\mathrm{p}<.01)$ Spd/Spm ratio than control samples $(2.06 \pm .34$ vs. $.82 \pm$ .1). Of great interest and in keeping with the distribution data, purified RBCs from CF patients also show an elevated ratio (CF = $3.02 \pm .44$; control $=1.53 \pm .39$ ). It is concluded that the major portion of blood $\mathrm{PA}$ is present in the RBC fraction and that blood $\mathrm{Spd} / \mathrm{Spm}$ abnormaitities are reflected in $\mathrm{CF}$ red cells.

EVALUATION OF 4-METHYLUMBELLIFERYLSULFATE (4-MUS) AS SUBSTRATE FOR ARYLSULFATASE A (ARA). E.E. Delvin, A. Pottier and F. H. Glorieux. (Intr. by K.N. Drun Genetics Unit, Montran a Shachromatic leucodystrophy patients (MLD) ARA, deficient he Melachromatic Leucodys trophy patients (MLD), is usually measured colorimetrically using 4-Nitrocatecholsul
(4-NCS) as sutstrate. The abnormal kinetic behaviour of ARA toward 4-NCS and the low sensitivity of the method leading to wide variations in the normal values, a more sensitive fluorometric method, using 4-MUS as substrate, has been evaluated with both rat liver and liuman cultured fibroblasts as sources of enzyme. Total activities (nnoles of substrate cleaved/min/g of tissue for the former and /mg Prot. for the latter) were in all cases lower with 4-MUS and a higher percentage was found in the rat liver microsomal fraction. When unfractionated cultured fibroblasts from 2 MLD patients were assayed for ARA using 4-NCS, a severe deficiency of activity was observed contrasting with the results obtained when 4-MUS was used as substrate (see table).

\begin{tabular}{ccccc}
\multicolumn{7}{c}{$4-$ NCS } & $\%$ & $4-$ MUS & $\%$ \\
rat liver Total homogenate & $507 \pm 15$ & & $150 \pm 5$ & \\
$(n=4) \quad$ Lysosomal fraction & $379 \pm 11$ & $75 \pm 2.5$ & $56 \pm 3$ & $38 \pm 2.5$ \\
Microsomal fraction & $50 \pm 8$ & $10 \pm 1.5$ & $63 \pm 3$ & $42 \pm 2.0$ \\
Hum. Fibrob. Norma? ( $n-6)$ & $12.9 \pm 3.8$ & & $0.11 \pm 0.4$ \\
MLD - case 1 & 0.4 & 3 & 0.05 & 45 \\
MLD - case 2 & 0.5 & 4 & 0.06 & 54
\end{tabular}
The low specific activity toward 4-MUS, the different subcellular distribution and the activity retained in MLD fibroblasts, all indicate that 4-MUS measures preferentially a microsomal arylsulfatase isoenzyme probably not affected by the MLD mutation.

336

ENTYME REPLACEMENT THERAPY: IN VIVO FATE OF NATIVE, ERYTRROCYTE- AND LIPOSOME-ENTRAPPED B-GLUCURONIDASE. Robert J. Desnick, Morris B. Fiddler, Lynn D. Steger, Dale A. Cumming, Carolyn D. Dullum and Suzanne R. Thorpe, Dept. of Ped. and Lab. Med., Univ. of Minnesota Med. Sch., Minneapol is. Enzyme therapy for inherited metabolic diseases requires delivery of the excgenous enzyme to the sites of pathologic substrate deposition. Therefore, the tissue and subcellular distributions of native, erythrocyte- and liposome-entrapped bovine B-glucuronidase administered to $\beta$-glucuronidase deficient mice were investigated to determine optimal therapeutic strategies for enzyme delivery and protection prior to human trials. Following IV administration of native enzyme, the activity was rapidly cleared from the plasma ( $T 1 / 2 \sim 3 \mathrm{~min}$ ) and almost exclusively recovered in the liver $(70 \%$ of dose at $30 \mathrm{~min})$; hepatic activity linearly decreased to non-detectable levels by $24 \mathrm{hr}$. In marked contrast, administration of enzyme entrapped in either murine erythrocytes or liposomes significantly prolonged the intracellular half-life of recovered activity. Following erythrocyte or liposome administration, maximal activity (approx. $75 \%$ of dose) was recovered in liver by $2 \mathrm{hr}$, retained for 12 and $48 \mathrm{hr}$, and gradually decreased to non-detectable levels by 5 and 9 days, respectively; $<15 \%$ was found in spleen or kidney. Subcellular fractionation of liver at early and late intervals revealed that $>80 \%$ of recovered activity was localized in the lysosomal-enriched fraction following native and entrapped enzyme administrations. Chemical modification of vesicle surfaces should further enhance the efficient delivery of enzymes for increased substrate metabolism in specific pathologic sites, especially for selected lysosomal storage diseases. 
GENETIC DEFTCIENCY OF THE SECOND COMPONENT OF HUMAN

337 COMPLEMENT (C2): DEMONSTRATION OF THE BIOSYNTHETIC

DEFECT IN VITRO. L. Peter Einstein, Chester A. Alper, Kurt J. Bloch, John T. Herrin, Fred S. Rosen, John R. David, and Harvey R. Colten. Harvard Med. Sch., Boston, MA. 02115 .

The biochemical basis for a genetically determined deficiency of the second component of complement (C2) was studied in a previously unreported $\mathrm{C} 2$ deficient family using a method for prolonged culture of human monocytes in vitro. C2 was absent in sera of the proposita, a 13 year old with a history of anaphylactoid purpura, and her asymptomatic homozygous deficient brother.

Evidence was obtained that in vitro, monocytes from homozygous deficient individuals were unable to synthesize and/or secrete C2 under conditions that did support $\mathrm{C} 2$ synthesis by heterozygous deficient and normal monocytes. This defect in biosynthesis appeared to be specific, since total protein synthesis, morphology, phagocytosis of $5.7 \mu$ diameter latex beads, killing of $L$. monocytogenes, and capacity to form rosettes with IgG or C3 coated erythrocytes were similar in monocytes of normal and affected individuals. The biochemical defect persisted in culture up to eight weeks after isolation of the monocytes. No inhibitor of $\mathrm{C} 2$ production or function was identified. These data indicate that the decrease in serum $\mathrm{C} 2$ in patients with genetically determined $C 2$ deficiency is probably not a result of hypercatabolism and can be accounted for by a specific abnormality of synthesis and/or secretion of the gene product.

This method permits in vitro studies of other monocyte functions as well as inherited protein deficiency states.

NORMAL AND ABNORMAL CYSTATHIONINE SYNTHASE (CS)

338

Longhi, H. Tallan and G. Gaull, Dept. Ped. Res., N.Y. State Inst. Res. Ment. Retard., Staten Is., N.Y., and Dept. Ped. and $\mathrm{Cl}$ in. Genet. Ctr. Mt. Sinai Med. Sch. of CUNY.

Since CS deficient patients may possess a more labile enzyme than normal, we investigated the thermostability of CS from cultured skin fibroblasts. The effect of pyridoxal phosphate (PLP) also was studied, since some patients respond to vitamin $B_{6}$. When crude lysates of normal fibroblasts were preincubated at $55^{\circ}$ before substrate addition, CS activity increased with time of preincubation, reaching maximum "activation" at $2 \mathrm{~min}$. Addition of $0.4 \mathrm{mM}$ PLP to the preincubation mixture resulted in greater activation, maximal at 3 min. Inactivation followed the activation period, with a $60 \%$ loss of activity at $10 \mathrm{~min}$ without PLP and $35-40 \%$ with PLP, suggesting a protective effect of PLP. Of 3 patients: 2 (1 B6-responsive, 1 not) had very low CS activity, precluding judgement about heat stability. A third (B6-responsive with $6-7 \%$ mean control activity) showed no activation; inactivation proceeded faster than normal, with no observable PLP effect. Three obligate heterozygotes showed no activation without PLP, although one of them showed evidence of activation with PLP. CS of a clinically and biochemically atypical patient and his parents reacted normally to heat. Extracts of normal cultured lymphocytes and of liver also showed activation with heat which was increased by the addition of PLP. Thus, heat-induced activation followed by inactivation is a property of normal CS which is altered in individuals with mutant CS genes; different patterns of response suggest genetic heterogeneity.

339

AN ANIMAL MODEL FOR LYSOSOMAL ENZYME REPLACEMENT STUDIES. Harry A. Frankel, Janet Glaser, and of Ped. and Med., St. Louis Children's Hosp., St. Louis, Missouri.

Previous work on $\beta$-glucuronidase deficiency mucopolysaccharidosis suggested this disorder as a good model to test the feasibility of lysosomal enzyme replacement therapy in man. However, an animal model system would be desirable to answer questions about the uptake, distribution, and fate of infused enzyme that cannot be answered in man. Human fibroblasts in culture can distinguish between "high-uptake" and "low-uptake" forms of human B-glucuronidase. We have done a comparitive study of uptake of human $\beta$-glucuronidase by fibroblasts established from primary explants from cow, sheep, rabbit, hamster, pig, rat, and the AKR mouse. Thermostability of human $\beta$-glucuronidase allowed us to inactivate endogenous $\beta$-glucuronidase in extracts and to demonstrate the amount of human enzyme taken up by the animal fibroblasts. Cow, rat, and hamster cells show high enough levels of uptake of human enzyme to suggest that their mechanisms for lysosomal enzyme recognition and uptake are similar to those of human cells. Because human high-uptake B-glucuronidase is recognized by rat fibroblasts, is taken up with kinetics similar to those of human fibroblasts, and is stable in rat cells once taken up, the rat appeared a favorable model for in vivo studies.

Heat inactivation of endogenous $B-$ glucuronidase in rat organs after infusion of high uptake human enzyme showed that the fibroblast studies could be extended to the whole animal to determine the uptake, distribution, and fate of infused human B-glucuronidase.
340 CHROMATID EXCHANGE RATES IN ATAXIA-TELANGIECTASTA: Ernest B. Hook, Bermard Pollara, Norma H. Hatcher, Dept. of Health, and Dept. of Ped., Albany Med. Col., Albany, N.Y.

Chromatid exchanges (CE) and chromosome translocations and rearrangements (CTR) are of similar origin in that both result from breakage and remion of chromosomal material. Ataxia telangiectasia (AT) is a disorder associated with increased rate of CTR, but no study of CE has yet been done. Two affected siblings (AT+) already known to have increased rates of CTR (Amer. J. Hum. Gen, 26: 39A, 1974) were compared with 2 unaffected siblings and an unaffected, unrelated adult (AT-). Latt's technique using Hoechst 33258 was modified in that deoxycytidine (dC) was added (in 3 fold excess over BrdU) to diminish cell toxicity. In 25 PHA stimulated lymphocytes from each person, the rates (m \pm S.D.) of CE "events" were: 7 y.o. P ATt: $4.36 \pm$ $2.16 ; 13$ y.0. $0^{7} \mathrm{AT}+; 6.36 \pm 2.46 ; 9$ y.o. $\sigma^{7} \mathrm{AT}-: 4.12 \pm 2.11$; 10 y.0.0 AT-: $5.00 \pm 2.57 ; 25$ y.0. $\%$ AT-: $7.92 \pm 2.77$. (Cultures were not exposed to light until harvest which may account for the relatively low rates of $\mathrm{CE}$ events in all. In a separate study, dC was found not to lower rates of CE.) Thus, despite the increase of CTR in AT, there is no total increase in CE. A study of rates in specific chromosomes showed that, unlike CTR, CE did not occur preferentially in D chromosomes of ATt individuals.

341 THREE SUCCESSIVE RRENATAL DIAGNOSES OF $47, \mathrm{XY},+21$ Mount Sinai School of Medicine, Department of Pediatrics, New York, New York.

Within three working days in September, 1974, we made three prenatal diagnoses of $47, X Y,+21$ from 3 women of advanced maternal age. Two were 37 and 38 years old and nulliparous. One was 42 years old and had 4 nomal children. The possibility of cell contamination arose when the second diagnosis of trisomy 21 and a male fetus was made. This suspicion increased when the third case was found within three working days. All three cases were then studied with both $Q$ and $G$ banding for identification of individual chromosome markers. Fortunately, the distinction was clear by $Q$-banding. Case 1 showed a) two 21 chromosomes with brightly fluorescent satellites and one 21 with dull satellite and b) bright fluorescent bands in the centromeric regions of both chromosomes No. 3 . Case 2 showed a) one 21 chromosome with bright satellites and two with dull satellites and b) brighter fluorescence in the centromeric region of one of the two nos. 3 chromosomes. Case 3 showed all three 21 chromosomes with faint fluorescent satellites and absence of bright fluorescence in the centromeric regions of both nos. 3 chromosomes. Thus, it was clear that these were three separate cases. All three couples requested therapeutic abortion. All three abortuses were males and had typical pathologic features for Down's syndrome. Further cytogenetic confirmation was possible in the third case. Problems relating to diagnosis of two or more successive identical trisomies of the same sex can be resolved by banding techniques.
TEN YEARS OF EXPERIENCE WITH AN AUTOSOMAL RE-

342 CESSIVE MALFORMATION SYNDROME OF LIMBS \& CRANIUM IN CREE INDIANS. Elizabeth J. IVes, $C$ Stuart Houston, (Intr. by J.W.Gerrard), Depts.of Ped. \& Saskatoon, Sask. Canada.

In 1967 experience was reported (abstract-Am. Soc. Hum.Gen.ann.meeting) with a multiple malformation syndrome of 1 imbs and cranium first encountered in 1965 in a consanguineous predominantly native northern Saskatchewan community. Analysis of 7 affected infants in 3 families indicated autosomal recessive inheritance and an attempt was made to forecast the probable future contribution of this gene to overall fetal wastage. By 1974 at least 7 further affected infants have been bo$r n$ to the same and 4 additional mothers. The phenotype consists of microcephaly, microphthalmia, prominent flat nose,brain and limb anomalies. The latter consists commonly of a single curved forearm bone fused to humerus at elbow and bearing 2-4 digits distally. Legs are occasionally similarly involved. Prematurity and stillbirth are common and maximum postnatal survival has been 6 days. Additional affected infants has allowed more accurate definition of skeletal anomalies and the range of gene expression. Genetic counselling has been of little value in the face of socioeconomic circumstances.Updated estimates of the contribution of this gene to the high perinatal mortality of the area are made. 
343 FAMiLIAL transLocation WITH DOUBLE PARTIAL TRISOMY OF 13 AND 22. Hyon J. Kim, Lillian X.F. Hsu, Lawrence c. Goldsmith, and Kurt Hirschhorn, Department of Pediatrics and Pathology, Mount Sinai School of Medicine of the City University of New York, New York.

A newborn male infant with multiple congenital anomalies was found to have an extra acrocentric chromosome of a size between some was identified as a translocation, $t(13 ; 22)(13 \mathrm{qte}-r) 13 \mathrm{q} 22:$ : 22 q12 22 pter). Therefore, the patient was trisomic for the distal $1 / 3$ of the long arm of no. 13 and the short arm and the proximal region of the long arm of no. 22. Subsequent autopsy findings and the clinical features showed a combination of both trisomy 13 and trisomy 22 syndromes. The features of trisomy 22 were preauricular sinus, finger-like and malopposed thumb and deformed lower extremities; features of trisomy 13 were absence of olfactory buib, micropolygyxia of frontal lobes, microcephaly with wide sagittal suture and fontanelles, epicanthal folds, bilateral microtia, capillary hemangiomata, multiple cardiovasculax anomalies including absence of lt. umbilical artery, genitourinary tract and gastrointestinal tract anomalies; the features common to both trisomy 22 and 13 were microcephaly, micrognathia, cleft palate and atrial septal defect. It appears that the presence of the extra proximal unbanded, euchromatic region of no. $22(22 \mathrm{ql} 1)$ and the presence of extra chromosomal material of the distal $1 / 3$ of the long arm of no. 13, especially the unbanded euchromatic regions, are essential to produce trisomy 22 and trisomy 13 syndrome respectively. The patient's phenotypically nor-

PRENATAL DIAGNOSIS OF MUCOLIPIDOSIS IV BY ELECTRON

MICROSCOPY. Gertrude Kohn, Nelly Livni and Yoram Beyth, Departments of Human Genetics, Pathology and Obstetrics and Gynecology, Hadassah-Hebrew University Medical Center, Jerusalem, Israel.

Mucolipidosis IV (ML IV), a recently recognized metabolic storage disease, is characterized by corneal opacity and psychomotor retardation in infancy. Electron microscopy (EM) examination of a conjunctival biopsy from a 2 year old affected girl revealed multiple cytoplasmic storage bodies compatible with the diagnosis of ML IV. Her mother was four months pregnant at the time and following parental counselling and agreement as to the possibility of a prenatal diagnosis, transabdominal amniocentesis was performed. Four weeks following initiation of the amniotic fluid cell culture, EM examination of the cells revealed the presence of storage bodies identical to those observed in the conjunctival biopsy of the proposita. On the basis of these findings, the pregnancy was interrupted and the diagnosis was confirmed in cells obtained from the culture of the amnion. This case demonstrates the possible efficacy of EM investigation in the prenatal diagnosis of those metabolic storage diseases in which the biochemical defect is yet unknown. the $D$ and $G$ group. With both $Q$ and $G$ banding, this extra chromomal mother was found to be a balanced translocation carrier. 346 DEFINITIVE CHARACTERIZATION OF THE GLYCOSAMINOGLYCANRobert E. Hurst, and R. C. Cezayirî, C.D.L.D., Dept. of Ped., Univ. of Ala. in Birmingham, Birmingham, Ala. Lorincz (1960) reported a naturally occurring heritable disorder of mucopolysaccharide metabolism occurring in brachycephalic "snorter" dwarf cattle. Upon the basis of the then best available crude mucopolysaccharide (glycosaminoglycan, GAG) biochemical screening tests, a 20 - 30 fold increase of GAGS of affected animals over normals was reported. The clinical features shared by the autosomally recessive determined dwarfism in cattle and its comparisons to the human mucopolysaccharidoses were delineated. Subsequent studies by various laboratories using different genetic lines and definitions for the "snorter" dwarf state focused on controversies whether there was specifically elevated excretions of dermatan sulfate (CS-B) or heparanmonosulphate (HS) which would correspond with the mucopolysacchariduria of the then known human mucopolysaccharidoses.

The definitive isolation and identification of urinary glycosaminoglycans of brachycephalic "snorter" dwarf cattle and control cattle is reported here. The urinary glycosaminogiycans were isolated and preparatively fractioned by ion-exchange chromatography. The fractioned glycosaminoglycans were qualitatively identified and quantiatively measured using gas-liquid chromatography of hydrolyzates, enzymatic digestion, electrophoresis, and infrared spectroscopy. The "snorter" dwarf cattle were found to excrete a 30 -fold excess of sulfated glycosaminoglycan, most of which was found to be chondroitin 4-sulfate (CS-A).

CHARACTERIZATION OF 25 CHROMOSOMAL ABNORMALITIES

347 WITH R BANDING. Herbert A. Lubs, Ram S. Verma and Arthur A. Robinson. Univ. Colo. Med. Ctr., Dept. Ped. and Dept. Biophysics and Genetics, Denver.

A series of 25 cytogenetic abnormalities ascertained by trypsin $G$ banding were further studied using acridine-orange (AO) $\mathrm{R}$ banding. The theoretical advantages of this technic include clear delineation of many telomeric and centromeric regions which are dim with $Q$ and $G$ banding as well as characterization of the entire karyotype in a spectrum of colors ranging from bright red to pale green rather than in black and white. Because of the wide variation in colors in the short arms of the acrocentric chromosomes the detection of dicentric Robertsonian fusions should be more accurate with AO R banding. Three Robertsonian translocations were re-evaluated but were not found to be dicentric. In both a ring 21 and $1 \mathrm{p}$ duplication, however, the details of the abnormality were more convincingly documented in color than in the black and white. Abnormalities involving both the $\mathrm{X}$ and chromosome 8 were particularly well demonstrated with AO $\mathrm{R}$ banding. This technic has proven reliable,

reproducible and suitable for routine use. Definition of the 25 abnormalities was at least as good as with trypsin and the technic deserves consideration as a routine cytogenetic diagnostic technic.

\section{8}

MAROTEAUX-LAMY SYNDROME: A DEFICIENCY OF N-

345 CHROMATID BREAKS IN FANCONI'S ANEMIA. $\frac{\text { Dubin, }}{\text { Med. Sch., Ghildren's Hosp. Med. Ctr., Dept. of Ped., }}$ Boston, Massachusetts

Sister chromatid exchanges, which reflect chromosome repair in response to certain forms of DNA damage, provide a means of investigating the increased chromosome fragility characteristic of Fanconi's Anemia (F.A.). Both exchanges and breaks, the latter representing incompletely repaired chromosome damage, have been measured in lymphocytes from three patients with F.A. whose clinical course had been stabilized by prednisone and androgen therapy. The baseline frequency of sister chromatid exchanges in these F.A. lymphocytes, detected by a recently developed technique ( 33258 Hoechst-BUaR), differs little from that in normal lymphocytes. However, addition of mitomycin $C$ to the F.A. cells during the last day of culture induces less than half the increase in exchanges found in identically treated normal cells. This reduced increment in exchanges is accompanied by a partial suppression of mitosis and a marked increase in chromatia breaks and rearrangements. Many of these events occur at sites of incomplete chromatid interchange. These results suggest that chromosomal breaks and rearrangements in Fanconi's Anemia may reflect a defect in a form of repair of DNA damage.
ACETYLGALACTOSAMINE 4-SULFATE SULFATASE. Reuben Matalon and Albert Dorfman. Univ. of Chicago, Dept. of Pediatrics, Chicago, Illinois 60637.

Maroteaux-Lamy syndrome is an autosomal recessive mucopolysaccharidosis, characterized by severe skeletal deformities without mental retardation. Dermatan sulfate is the major mucopolysaccharide excreted in urine and deposited in tissues. Stumpf et al (Am. J. Dis. Child. 127, 747, 1973) and Fluharty et al (Biochem. Biophys.Res.Commun. 59, 455, 1974) have reported a deficiency of arylsulfatase $B$ in tissues and fibroblasts of patients with Maroteaux-Lamy syndrome. Cultured skin fibroblasts were utilized to elucidate the sulfatase deficiency with regard to the specific sulfate linkage in dermatan sulfate. When extracts of skin fibroblasts were incubated with $[35$ S $]$ chondroitin 4 -sulfate prepared from rat chondrosarcoma, normal, Hurler, Hunter, Sanfilippo $A$ and Morquio fibroblasts released $13-20 \%$ of ${ }^{3} 5 \mathrm{SO}$ while MaroteauxLamy fibroblasts released less than $1.5 \%$. Similar results were obtained when a heptasaccharide GalNAc ${ }^{35} \mathrm{SO}_{4}$ - (GlcUA-GalNAc $\left.35_{\mathrm{SO}_{4}}\right)_{3}$ prepared from chondroitin 4-sulfate was incubated with extracts of the various fibroblast strains. These findings indicate that Maroteaux-Lamy syndrome is due to a deficiency of N-acetylgalactosamine 4-sulfate sulfatase which is the natural substrate for arylsulfatase B. (Supported by USPHS Grant Nos. AM-05996, HD-04583, AM-05589 and RR-00305.) 
349 AN 18/21 TRANSLOCATION IN AN INFANT WITH PARTIAL TRISOMY 18. Richard L. Neu, Claudio Ortega, Lytt I. Gardner, Mark Merkens and Gale Barg. State Univ. of New York, Sch of Med, Upstate Med Ctr, Dept of Ped, Syracuse, NY

To our knowledge, no translocations between the short arm of a number 21 chromosome and the long arm of an 18 have been descr ibed, although 18q material translocated to other chromosomes in the complement has been reported. The case presented here is that of an infant with clinical features suggestive of trisomy 18 associated with a translocation of most of the long arm of an 18 to the short arm of a 21 chromosome, identified by G-banding. The $21 \mathrm{p}$ satellite stalks are apparent between the $21 \mathrm{p}$ and the 18q material composing the translocation chromosome, thus making it easy to identify. Special staining techniques are in progress to determine if the satellite material is also present in the translocation chromosome. The mother and father were 34 and 38 years of age when the propositus was born; both parents have normal chromosome complements. The patient was first evaluated at 7 mos. of age. The clinical findings were failure to thrive, developmental retardation, micrognathia, elongated skull, hirsute forehead, low set ears, short neck with redundant skin on nape, left inguinal hernia, terminal hypospadias, cryptorchidism, wrist deformity with radial deviation of left hand, short fifth finger bilatera1ly, hypoplastic toe nails, finger tips all with arch pattern, a seizure disorder, and an abnormal EEG. Cardiac catherization revealed a ventricular septal defect.

350

DOWN'S SYNDROME: LOCALIZATION OF LOCUS TO DISTAL POR TION OF LONG ARM OF CHROMOSOME 21. James J. O'Donnell Bryan D. Hall, Felix A. Conte, Judith C. Romanowski, and Charles J. Eostein. Univ. of California, San Francisco, Dept. of Ped., San Francisco.

Although the association of Down's synarome (D.S.) with trisomy 21 has been known since 1959, a few reports have suggested that complete trisomy is not necessary and that there may be a smalle "Down's syndrome locus." Investigation of a family of three children with typical D.S. has substantiated this conclusion and has shown that trisomy for the distal half of the long arm of 21 (219) produces the full phenotype. On the other hand, trisomy for the remainder of 21 does not cause D.S. and is compatible with noxmal physical and mental development. Because the extra distal half of 2lq was inserted into chromosome 15, its presence, prior to the use of banding techniques, had previously been missed. This indicates that other cases of D.S. with a "normal" karyotype may actually be the result of small, undetected, and possibly transmissible translocations. The unique distribution of chromosome 21 abnormalities in the reported family will aid in the fire mapping of genetic loci on this chromosome. Cytogenetic data:

Mother: $46, \mathrm{XX}$, ins (15;21)(q11;q21q22), del(21)(q21) - carrier Children: 46,XX (and XY), ins $(15 ; 21)$ (q11;q2lq22) - D.S., partial trisomy of distal $21 q$

47, xX, ins $(15 ; 21)(q 11 ; q 21 q 22),+\operatorname{del}(21)(q 22)-$ D.S., complete trisomy 21

$47, X Y,+\operatorname{del}(21)(\mathrm{q} 21)-$ normal, partial trisomy of $21 \mathrm{p}$ and of proximal $21 q$

351 G6PD AND HEREDOFAMILTAL BILATERAL MICROPHTHALMIA: ABSENCE OF EVIDENCE FOR LINKAGE IN MAN. Olu O. Ogunye, Robert F. Murray, Jr., and Ted Osgood (Intr. by Melvin E. Jenkins). From the Dept. of Pediatrics and the Medical Genetics Unit, Howard Univ. Col. of Med., Washington, D.C.

G6PD phenotype studies were done on a black family with Xlinked Heredofamilial Bilateral Microphthalmia (HBM).

Three crossovers and three non-crossovers were detected in three informative matings of four generations yielding a recombination value $(\emptyset)$ of 0.5 . The odds for complete linkage is zero and there is a maximum likelihood if the two loci are not linked. Lod Score $z=-2.163$ for $\emptyset=0.05$

These findings do not provide evidence for linkage between the G6PD and HBM loci suggesting either that the G6PD and HBM loci are far apart on the $X$ chromosome or that Heredofamilial Bilateral Microphthalmia in this family is inherited as an autosomal dominant sex-limited trait. STUDIES OF THE ISOENZYMES OF PHENYLALANINE HYDROXYLASE Rebecca Newhouse, Samue1 Bessman; Childrens Hosp. of L.A., Univ. of Southern California,Sch. of Med., Los Angeles, Calif. We have demonstrated three isoenzymes of phenylalanine hydroxylase. They have been named $\mathrm{P} i$, Kappa and Upsilon respectively. We have studied the phenylalanine hydroxylase isoenzymes in two families. Family " $A$ " was a sibship of four in which three of the siblings had classical phenylketonuria and the fourth showed no abnormality of phenylalanine metabolism. Family " $B$ " consisted of a boy with classical phenylketonuria and his parents.

Tissue specimens are obtained by open liver biopsy. They are placed directly in a buffer solution and homogenized. The supernatent is then put onto a chromatographic column of $\mathrm{Ca} \mathrm{PO}_{4}$ gel. The enzymes are eluted using a linear $\mathrm{KCl}$ gradient. Alternate fractions are incubated with and without phenylalanine and assayed for tyrosine using a semiautomated modification of the method of Udenfriend and Waalkes.

All persons affected with phenylketonuria whom we have biopsied have shown a total phenylalanine hydroxylase activity of less than $5 \%$ accompanied by a change of the phenylalanine hydroxylase isoenzyme pattern. The normal sibling of family " $A$ " had $60 \%$ pheny1alanine hydroxylase activity but demonstrated only two isoenzymes, $\mathrm{Pi}$ and Upsilon. The heterozygote parents of the boy with classical phenylketonuria in family " $B$ " each had less than normal total phenylalanine hydroxylase activity. The mother, with $40 \%$ activity, demonstrated all three isoenzymes in about half quantity while the boy's father with $78 \%$ activity demonstrated a small peak of $\mathrm{Pi}$ isoenzyme and a large peak of Upsilon.

353 CYSTATHIONASE IN LONG TERM LYMPHOID CELL LINES: EVIDENCE FOR ALTERED ENZYME PROTEIN IN CYSTATHIONINURIA. T. Pascal, G. Gaull, N. Beratis, B. Gillam, H. Tallan, Mirschhorn and C. Parker, Dept. Ped. Res., Inst. Basic Res. Mental Retdn., S.l., N.Y.; Dept. Ped. and Ci inical Genetics Ctr., Mt. Sinai Med. Sch. CunY; Child. Hosp., L.A., Cal.

Cystathionase in lymphoid cell lines has been measured with a sensitive procedure utilizing optimum conditions established for these cells. 15 normal cell lines had a mean specific activity of $29.5 \pm 1.95$ (SE) nmoles cysteine formed $/ \mathrm{hr} / \mathrm{mg}$ protein at $0.25 \mathrm{mM}$ pyridoxal phosphate (PALP); no increase in activity was observed at $1.0 \mathrm{mM}$ PALP. Without added PALP, normal cells retained $75 \%$ of their activity. Cell lines from a patient with B6-responsive primary cystathioninuria had an activity of only 5.1 at $0.25 \mathrm{mM}$ PALP, increasing to a maximum of 12.0 at $1.0 \mathrm{~mm}$. Without added PALP, the patient's cells had no activity. A single culture of lymphocytes from an obligate heterozygote had activity intermediate (19.9) between mean control and affected lines. No appreciable activity has been found in normal cultured skin fibroblasts.

Immunological studies in conjunction with a stain specific for cystathionase revealed the presence in the mutant cells of an immunoreactive protein without catalytic activity, which exhibits antigenic identity with the enzyme from normal cells and from liver.

These studies provide evidence for an inherited synthesis in the cystathioninuric of an altered cystathionase protein with greatly diminished activity and an increased requirement for PALP. They also show the value of these lines for studies of enzymes of internal organs which are not detectable in skin fibroblasts.

354 CULTURfD AMNIOTIC CELls. David C. Peakman, Marilyn F. Moreton, Arthur Robinson. Univ, of Colorado Med. Ctr., Dept. of Biophysics and Genetics, Denver.

Trio of the probiems facing laboratories involved in prenatal diagnosis are the idertification of mosaicism ane the possirilitv that maternal cells may contaninate the amiotic fluic sample. In situ processing of cultured amniotic fluid cells greatly enhances the possibility of identifying true and fseudonosaicism since mitoses can be analyzed from multiple individual colonies. of 223 fluids processed in this manner, 4 showed apparent pseudomosaicism, only a single colony being found with the abnormal chromosome complement. Of these, three samples yielded a trisomy 2 complement and the fourth domonstrated an isochromosome of a D group chromosome. Screening of amriotic fluid cell samples for fluorescent $Y$-chroratin can be of help in indicating the genotypic sex of the primary ceils. This was found to be accurate in $95 \%$ of the 223 fiuids checked. In one case with a $Y$-chromat in positive finding a colony with an XX complement was found initially, but in a repeat culture only XY cells were identified. In cases where an XX complement is found, Q polymorphism comparisons can be made between the amniotic fluic cell and maternal lymphocyte mitoses. Of 17 such studies, 9 demonstrated marked differences in their polymorphism constitution. The combined findings from the three different techninues can yield a result in which there can be increased confidence that the cells analyzed were fetâl in críg:n. 

355 part, Shun Ling*, Charles Brinkman*, Elton Lassila ${ }^{*}$, Klaske Zeilstra* and Helen Roberson*. Mental Retardation Center, University of California, Los Angeles.

An affected fetus with I-Cell disease has been detected previously on the basis of increased hydrolase activities in the amniotic fluid and abnormal histochemical reactions in cultured and uncultured amniotic cells (Warren, et al., Ped. Res. 7:343, 1973). Although the primary defect is not known a dozen hydrolases are deficient in fibroblasts and amniotic cells while they are significantly increased in most body fluids. Cultured fibroblasts are stuffed with inclusion bodies, which led to the recognition of this disorder. Amniotic fluid was oitained from a woman at risk. The $600 \mathrm{~g}$ supernate was used to assay 4 hydrolase activities, $\beta$-galactosidase $(22 \mathrm{nmole} / \mathrm{hr} / \mathrm{ml}), \beta$-glucosaminidase (729), $\alpha$-glucosidase (119) and $\beta$-glucuronidase (36). These values fall in the normal range while a 10 to 50 -fold increase is observed in body fluids from I-Cell patients. Amniotic cells grew well and did not exhibit the I-Cell phenomenon at any time. Hydrolase activities in cultured cells were normal. The pregnancy was brought to term and a healthy boy was delivered. Plasma hydrolase levels at 6 weeks were normal. Lipid and mucopolysaccharide turnover in cultured cells did not reveal the failure to degrade macromolecules, which is characteristic of I-Cells. I-Ce11 disease can apparently be safely monitored during pregnancy.
358 GLYCOLIPID DISTRIBUTION IN FIBROBLASTS FROM PATIENTS

358 WITH LYSOSOMAL STORAGE DISORDERS. Miche1 Philippart, Seiji Nakatani* and Helen Roberson*. Mental Retardation Center, University of California, Los Angeles.

Lipids were extracted from confluent cultured skin fibroblasts containing at least $6 \mathrm{mg}$ protein. Results, expressed as nmole/mg/ protein are as follows. In control lines $(\mathrm{N}=6)$, which included only $l$ child, total glycolipid (GL) concentration was $4.2 \pm 2.8$, glucosy1 (Glu) ceramide (Cer) $1.0 \pm 0.9$, lactosyl (Lac) Cer $0.7 \pm 0.3$; and hematoside $\left(\mathrm{GM}_{3}\right) \quad 0.4 \pm 0.2$. Traces of disialyl Lac Cer $\left(\mathrm{GD}_{3}\right)$ were generally present but too low for accurate quantitation. In Farber's disease and Fucosidosis there was no abnormality. In Batten's disease $\mathrm{GM}_{3}$ was increased to 1.7. In Niemann-Pick A, on line was normal, another had increased GL (13.5) T Cer (6.7), Tet Cer $(1.7)$ and $\mathrm{GM}_{3}(1.5)$. Two siblings with Niemann-Pick $\mathrm{C}$ had even higher GL $(22.8,22.4)$, Glu Cer $(6.1,3.2)$, Lac Cer (3.1, 1.2), T Cer $(12.4,9.9)$, Tet Cer $(3.4), \mathrm{GM}_{3}(6.0,3.9)$, and $\mathrm{GD}_{3}$ $(1.7,0.7)$. Mannosidosis: GL (10.8), T Cer (7.2), $\mathrm{GM}_{3}(2.1)$; no mannose was detected in these fractions. Fabry's disease (2 males 1 female): GL (11.3-25.2), T Cer $(6.3-20.7)$. T-Ce11 disease (Mucolipidosis type II and III): GL (24.8-41.9), Glu Cer (1.8-7.2), Lac Cer $(0.8-2.2)$, T Cer $(10.8-23.7)$, Tet Cer $(3.2-3.7)$, GM 3 (5.6$6.9), \mathrm{GD}_{3}(0.4-1.5)$. One obligatory I-Cell carrier had a significant increase of the different GL fractions, to about half the homozygote level. This, if confirmed in other families, may help in identifying $\mathrm{I}-\mathrm{Ce} 11$ heterozygotes. GL distribution in fibroblasts is thus frequently abnormal in lysosomal disorders.
356 MULTIPLE SULFATASE DEFICIENCY PRESENTING AS SANFILIPPO A DISEASE. Michel Philippart, Elsa Kamensky*, Gerald Sugarman*, and Seiji Nakatani*. Mental Retardation Center, University of California, Los Angeles.

A 2-yr-old boy had microcephaly, delayed psychomotor development, hypotonia, weakness, decreased hearing and organomegaly. Corneas were clear. Bone survey was non-contributory. Urine contained excess mucopolysaccharides, mostly heparan sulfate. Cultured skin fibroblasts did not correct the defect in $35 \mathrm{SO}_{4}$ turnover in an authentic Sanfilippo A line (Dr. Neufeld's gift). Liver ultrastructure was compatible with mucopolysaccharidosis. The diagnosis of Sanfilippo A was made. At age 3 the child had decreased vision, retinal pigment clumping, myoclonias, and pyramidal symptoms. At age 4 he became hyperactive and developed ichthyosis. This course was at odds with the original diagnosis. Since arylsulfatase (AS) B activity in liver and fibroblasts was about $50 \%$ normal, a multiple sulfatase deficiency was felt to be ruled out. AS A was decreased in urine, fibroblasts, and liver, but not much lower than in Sulfatidosis carriers or typical Sanfilippo A patients. At age $51 / 2$ the child was blind and deaf, had continuous myoclonias, upward rolling eyes, distal amyotrophy, and polyneuropathy. CSF protein was $277 \mathrm{mg} / \mathrm{dl}$. Numerous Alder granulations were present in neutrophils. Nerve conduction was markedly reduced. AS A and B were barely detectable in urine; sulfatase $C$ and heparin sulfamidase were absent in fibroblasts. Urinary sulfatide and cholesterol sulfate were much increased. This established the diagnosis of multiple sulfatase deficiency, which may masquerade as "ganglioside storage" or Hurler's disease combined with Sulfatidosis.

357 MUCOPOLYSACCHARIDOSIS VI: EARLY DIAGNOSIS. Miche1 Philippart, Earl L. Semones*, Elton Lassila*, and Seiji Nakatani*. Mental Retardation Center, University of California, Los Angeles.

An 18-month-old female had frequent upper respiratory tract infections since the age of 3 months when Alder granulations in neutrophils were first noticed. Somatic and psychomotor development were normal. Physical examination revealed a thick skin, slightly hazy corneas and increased nasal secretions. There was no coarseness of features, joint limitations, claw hands, or organomegaly. Upon X-ray, multiple bone changes suggested Hurler's syndrome. Urinaxy uronic acid was $35 \mu \mathrm{g} / \mathrm{ml}$. Dermatan sulfate accounted for more than $90 \%$ of purified mucopolysaccharides. Most lysosomal hydrolase activities, including $\beta$-glucuronidase, were high or increased in cultured skin fibroblasts. Arylsulfatase A (282 nmole/hr/mg protein) and $\alpha$-L-iduronidase $(6.5 \mathrm{nmole} / \mathrm{hr} / \mathrm{mg}$ protein) were low, whereas arylsulfatase $B$ was not detectable.

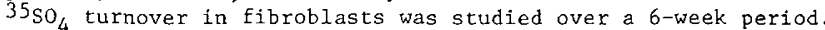
The half-life of the total counts was increased ( 20 days) although the initial incorporation was normal. The proportion of insoluble ${ }^{35} \mathrm{SO}_{4}$ (half-life 36 days) increased from $40 \%$ to $80 \%$ during the experiment. This is usually a minor fraction in mucopolysaccharidoses, including two other lines with arylsulfatase $B$ deficiency. Pediatricians have a major responsibility in detecting metabolic disorders in infants while there is still time to offer families at risk the option of monitoring future pregnancies by amnicentesis.

\section{CELL. CYCLES IN PATIENTS WITH SEX CHROMOSOME ANOMALIES, Ian H. Porter and Betty Paul, New York State Health Dept., Birth Defects Inst., and Dept. of Ped., Albany Med. Col., Albany, N.Y.}

Last year we reported that patients with the autosomal trisomies 13,18 and 21 have a significant increase in the length of the cell cycle as measured by pulse labeled cultured fibroblasts and this has now been confirmed by others. This year we report our findings in patients with sex chromosome anomalies.

Fibroblasts obtained from skin biopsies from patients with sex chromosome anomalies $(45, X ; 47, X X X ; 47, X X Y$; and $45, X / 46, X X$ mosaics) were cultured and the cell cycles determined by puise labeling and counting the proportion of labeled metaphases every 3 hours for 36 hours. Our results indicate that the presence or absence of an $X$ chromosome also influences the length of the cell cycle and it appears that extra heterochromatic material retards the rate of cell division as has been observed in root tip. 

361 EXPANDED HEALTH CARE THROUGH REGIONAL GENETIC COUN-
SELING Vincent M. Riccardi, (Intr. by A. Robinson), Dept. of Biophysics \& Genetics, Univ. of Colorado Med. Ctr., Denver

The Colorado-Wyoming Regional Genetic Counseling Program has served as a model for health care delivery by providing genetic counseling services, professional training $\mathcal{E}$ public education on a routine widespread basis. Although more than $20 \%$ of hospitalized pediatric patients have genetic disorders and birth defects and genetic counseling can improve the management of affected patients and families, it has of ten been restricted to larger medical centers. By providing our services at regular and frequent intervals over 201,000 sq. mi., hundreds of families yearly obtain care they otherwise would not receive. In addition a means for effectively preventing many such disorders has been thereby established. In the first 18 months 9 communities held clinics more than 100 times, directly serving 410 families in 614 visits. A questionnaire survey of serviced families and physicians demonstrates that such a program is both necessary and ef fective: of 182 patient responses $(62 \%)$ 98\% felt the counseling was helpful but $52 \%$ would not have travelled to the Medical Center for it; $53 \%$ were influenced to alter their attitudes about procreation. In addition, many were seen for assistance in longterm management. A sampling of the 700 involved physicians ind $i-$ cated that at least $66 \%$ were aware of the Program and $33 \%$ were directly benefited. Family practitioners, pediatricians and obstetricians were most involved. The need for additional similar Programs and the means for their establishment and implementation will be presented in detail.

INCIDENCE, GROHTH, AND DEVELOPMENT OF CHILDREY WITH

362 A $45, X$ KARYOTYPE IDENTIFIED AT BIRTH. Arthur Robinson, Katherine H. Tennes, Mary H. Puck, Dolores Orfanakis. Univ. Of Colorado Med. Ctr., Dept. Of Biophysics

In an epidemiological study of sex chromosomal aneuploidy in consecutive births at two Denver hospitals, 11 out of $19,705 \mathrm{fe}$ males $(0.06 \%)$ had a $45, \mathrm{X}$ karyotype. Of these 11 newborns, 3 died at birth with multiple anomalies, and 3 had none of the stigmata associated with the Turner's phenotype. Six of these patients were enrolled in a long-term developnental study. At six months they were all irritable, hypertonic, and slept poorly. At one year they were quick, active, and socially responsive. These characteristics were neither as marked nor as consistent in 5 girls with $45, X / 46, X X$ karyotypes or in a group of 11 girls with 47, XXX karyotypes. The latter two groups were followed in the same way as the $45, X$ group and hence can be considered as a type of control. Edema of the hands, one of the commonest findings, impaired fine motor development during the first year. During the preschool years, high activity and good verbal development combined with small stature tend to elicit positive responses from adults and creates a favorable emotional climate.
364 CORTICOSTEROIDS AND MUCOPOLYSACCHARIDOSES (MPS): CLINICAL AND BIOCHEMICAL STUDY. Norio Sakuragawa, George Constantopoulos and Anatole S. Dekaban. (Intr. by Paul A. di Sant Agnese). NIH, Bethesda, MD. 20014

Administration of corticosteroids ( $\operatorname{CoS}$ ) to patients with MPS leads to a modest amelioration of their clinical status. To understand the mechanism of this effect, we have studied changes (on and off corticosteroids) in urinary, plasma, cerebrospinal fluid, and leukocyte glycosaminoglycans (GAG) in selected patients with MPS Types I, II, IITA and V. In general, during the first few days on $C o S$, there is a small increase in urinary excretion of GAG, followed by a gradual but persistent decline of GAG below baseline, and return to baseline values after $\operatorname{CoS}$ were discontinued. The cetyl pyridinium chloride non-precipitable degradation products of GAG parallel the diphasic change in urinary GAG. A patient with Type V MPS (Scheie) showed a sharp increase in urinary GAG (200\% over the baseline) about 2-3 days preceding menstruation on two different occasions; this was followed by a return to baseline beginning at about the $3 \mathrm{rd}$ day of menstruation. These results suggest that in addition to corticosteroids, estrogens also exert a considerable effect on the metabolism of GAG in patients with mucopolysaccharidosis. Tentative interpretation of the results: initial increase in urinary GAG may be related to the change in body fluid volume while the sustained decrease may depend on decreased tissue synthesis of GAG.
365 BILATERAL GONADOBLASTOMA IN A 6YEAR OLD CHILD WIT

365 AMBIGUOUS GENITALIA AND 46,XY KARYOTYPE: GONADAL HISTOPATHOLOGY BEFORE AND AFTER DEVELOPMENT OF THE TUMOR. João A.M. Santana, Lytt I. Gardner, John P. Russell and R.L. Neu. State Univ. of New York, Upstate Med. Ctr., Depts, of Ped. and Path., Syracuse, N.Y.

This report presents observations on the ontogeny of gonadoblastoma (GB) in an infant with ambiguous genitalia and a $46, \mathrm{XY}$ karyotype. Patients with ambiguous genitalia and a $Y$ chromosome in their karyotype are at risk for this rare tumor; only a few patients under age 7 have been so diagnosed. In our case diagnostic laparotomy was done at age 6 weeks, and in both inguinal canals gonadal masses were found; these were connected by tubular structures to a mid-1ine organ. Both gonads, the mid-line structure and skin all were $46, \mathrm{XY}$. At age 6 , due to concern about neoplastic change, both gonads were removed and found to contain GB. The 1 . gonad (larger) had shown well-developed immature testicular tissue with Leydig cells at age 6 weeks; at 6 years no testicular tissue was seen, but GB was present with well-developed wolffian structures. The $r$. gonad (smaller) had shown poorly organized stroma with cords at 6 weeks, resembling a streak; at 6 years there was immature testicular tissue, with only a few recognizable germ cells. GB was present, with some germinomatous infiltration of stroma. These observations support the postulate of Boczkowski that GB arises in dysgenetic testicular tissue, and reinforce the need to be on the alert for this neoplasm in infants. We axe indebted to Dr. Robert E. Scully for his aid.
363 AMNIOCEITESIS: ITS IMPACT ON MOTHERS AND INFANTS. Jean R. Robinson, Katherine H. Tennes, Arthur Biophysics and Genetics and Dept. Of Psychiatry, Denver.

In this one year follow-up study, we assessed the effects of amniocentesis for genetic diagnosis upon 22 infants and thein mothers. The infants were tested at one year of age on the Bayley Infant Scale of Mental and Motor Development. No deviations from normal mental or motor development were found.* The mothers were interviewed at the same time to better understand the emotional aspects of the experience. Homen between ages 35-39 with no previously affected child tended to accept amniocentesis easily, as an appropriate part of prenatal care. Mothers with a previous defective child tended to have more painful emotional reactions, including higher anxiety over the test and more conflict about a possible abortion. They needed more intensive and extended counseling. We suggest that the issue of abortion is important even if manageable by most women, and that a chance to discuss it may be significant. * Scores of Study Group at One Year of Age (Bayley Scale)

\begin{tabular}{|c|c|c|}
\hline Number: & Girls 12 & Total 22 \\
\hline & Mental Scale & Motor \\
\hline & 105 & 105 \\
\hline ange & $89-124$ & $80-124$ \\
\hline
\end{tabular}

Stand. Dev.

10
THE DEFTNITION OF A CELLULAR DEFECT IN TWO PATIENTS

366 WITH COCKAYNE SYNDROME. Roy D. Schmicke1, Ernest H. Sch., Depts. of Ped. and Human Genetics and Michigan State Univ. Dept. of Human Development. Ann Arbor and East Lansing.

Cockayne Syndrome is a well recognized and severe form of dwarfism which is accompanied by mental retardation, retinitis pigmentosa, deafness, photosensitive skin and early death. We have shown that the fibroblasts derived from two patients with Cockayne Syndrome are defective in the repair of DNA after irradiation with ultraviolet light. These cells do not show an abnormal sensitivity to $\mathrm{X}$ irradiation. Abnormal sensitivity to UV light was determined by demonstrating decreased cloning efficiency following irradiation. The DNA of the cells was examined after the following steps: 1) labeling growing cells with ${ }^{14} \mathrm{C}$ thymidine, 2) irradiating the confluent cells with UV light, 3) relabeling the cells with $3_{\mathrm{H}}$ thymidine and 4) analysis of the double labeled DNA in alkaline sucrose gradients. The procedure showed that ${ }^{14} \mathrm{C}$ thymidine could be incorporated into normal size DNA under normal growth conditions. The ${ }^{3} \mathrm{H}$ DNA formed after irradiation was not part of the high molecular weight cellular DNA. The defect in DNA repair synthesis was also demonstrated by radioisotope quantitation of DNA synthesis in cells inhibited for de novo DNA replication. Normal cells incorporated ten times more $3 \mathrm{H}$ thymidine into DNA after UV irradiation than Cockayne cells. This demonstration of a specific cellular defect should lead to an understanding of the molecular basis of this growth and developmental abnormality. It will also provide a means for the diagnosis and genetic recognition at the cellular level. 
ORIGIN OF EXTRA 21 CHROMOSOME IN PATIENTS WITH

367 DOWN SYNDROME. Rina Schmidt, Hanna Dar and Harold M. Nitowsky. Dept. Ped, , Albert Einstein Col. Med.,

New York. families-both parents and affected child-were examined using the fluorescence banding technique. 19 children had simple trisomic Down syndrome while one child had a de novo $21 / 21$ translocation trisomy. Maternal age ranged from 16 to 44 years; 11 mothers were 30 years or younger. In all cases it could be demonstrated that the trisomy resulted from an error in chromosome segregation during the first meiotic division. Meiotic non-disjunction had occurred in 9 "younger" mothers (including In 6 families the findings were inconclusive as to the parent in whom an abnormality had occurred during the first meiotic division. This is due to the resemblance of one of the 21 chromosomes in the affected child to a member of this pair in both mother and father. However, in no case was a paternal origin for the trisomy demonstrated. These findings suggest that an error in chromosome segregation during meiosis in the female plays a major role in the origin of 21 trisomy in infants with Down syndrome, irrespective of maternal age. (Supported by NIH Grant GM 19100).
The chromosomes in samples of peripheral blood from members of 20 the mother of the infant with the translocation) and in 5 "older" mothers.

370 CHROMOSOMAL STUDIES OF METHADONE-MAINTAINED MEN AND THEIR NEWBORNS. George SOlish, Ellen Feingold, \& Rita G. Harper, Depts. of Ped.\& Ob. -Gyn.,State Univ. of N.Y.,Downstate Med.Ctr., Bkıyn,N.Y.\& Depts.of Ped.\& Ob.-Gyn., No.Shore Univ. Hosp., Manhasset,N.Y. \& Depts. of Ped.\& Ob.-Gyn.,Cornell Univ.Med.Col., N.Y.,N.Y. (Intr. by Fima Lifshitz).

The effect of methadone on chromosomal structure has not been sufficiently studied. We assessed the peripheral blood chromosomes in: (1) 12 pregnant women maintained on methadone doses of 10 to $60 \mathrm{mg}$ per day throughout pregnancy; (2) 4 pregnant women maintained on methadone until the 28 th week of gestation, then becoming drug-free until delivery; (3) 9 pregnant women matched for age, race, and parity, who took no drug during pregnancy. Frequent urines for drug-abuse screening were obtained from all women. No mother was included with medical or obstetrical complications. Cord blood chromosomes were studied in all 25 infants. Twenty metaphase spreads per subject were analyzed for chromosome number and aberrations (gaps, breaks, fragments, dicentrics, deletions, additions, rings). No significant difference was found between the 3 groups of mothers or between the 3 groups of infants, either in chromosome number or total number of chromosomal aberrations. These data allow us to infer that methadone taken during pregnancy does not alter the cytogenetic status of the mother or infant.

$\beta$-GALACTOSIDASES ( $\beta$-GaI) IN GM GANGLIOSIDOSIS (GM 1 ). Emmanuel Shapira, Anne David, Ron DeGregorio, and Henry $L$. Nadler. Northwestern Univ. Med. School, Children's Mem. Hosp., Dept. of Pediatrics, Chicago.

$G M$ types I and II are autosomal recessive disorders related to deficlent activity of the acidic $\beta$-gal isoenzymes. In the present work, the $\beta$-gal isoenzymes were studied in liver and skin fibroblasts of normal and $G M$ patients. Three distinct peaks with enzymatic activity towards 4-methylumbelliferyl $\beta-D$-galactopyranoside $(4-M U \mathcal{B}$-gal) were revealed by chromatography of normal human liver homogenates on Bio-Gel A-0.5 m columns. The first two eluted peaks had an acidic pH optimum ( 4.2 and 4.6 respectively) whereas the thi rd had a broad neutral $\mathrm{pH}$ optimum of 4.9-6.6. The first two peaks had negligible activity with 4-MU B-glucopyranoside (4-MU $\beta$-glu) (4-MU $\beta$-gal to 4-MU $\beta$-glu activity ratio $40: 1$ ) whereas the third peak had identical activity towards both substrates. Liver homogenates from a GMI type $1 /$ patient revealed the same elution pattern on chromatography but the enzymatic activity of the first two peaks was only $7 \%$ of normal whereas the third was increased to nearly twice normal. Monospeclfic antiserum against $\beta$-gal from the second peak cross-reacted immunologically with the other two normal forms as well as with the GM Iype II enzyme.

Four lines of normal fibroblast lysates revealed on Bio-Ge only the two acidic forms: the neutral form was undetectable. In 2 fibroblast lines from $G M_{1}$ type 1 and 3 from type 11 , the residua) 4-MU $\beta$-gal activity was $0.6-3.6 \%$ of normal but the ratio of 4-MU $\beta$-gal to 4-MU $\beta$-glu activity was 2.8 as compared to 223 for the normal lines. No significant differences in the elution pattern and $\mathrm{pH}$ optima were observed between normal and GMI lysates.

PERMANENT LYMPHOID LINES FROM GENETICALLY

369 MARKED LYMPHOCYTES: SUCCESS WITH MAILED BLOOD SAMPLES AND FROZEN LYMPHOCYTES. William S. Sly, Rerer Kennett, Walter Bodmer, and Julia Bodmer, Genetics Lab, Dept . Biochem., Oxford Univ. and Washington Univ. Sch. of Med., Depts. Ped. and Med., St. Louis Children's Hosp., St. Louis, Mo.

George Miller and coworkers described techniques to produce permanent cell lines from human lymphocytes by exposing them to culture fluid from an EB virus-producing marmoset cell line B95-8. We asked whether these techniques could be used to produce permanent human lymphoid cell lines from mailed blood samples or from lymphocytes which had been separated and stored frozen.

Permanent lymphoid lines were successfully established in 5 of 5 trials on lymphocytes from blood drawn in the U.S. from one $B$-glucuronidase deficient and four mucolipidosis III patients, and separated on a Ficoll-Triosil gradient 4-7 days later when received by mail in Oxford. Cells were exposed to transforming agent and grown initially over a feeder layer of irradiated fibroblasts.

Similar treatment of lymphocytes which had been separated 3 years earlier, and stored frozen in straws for HL-A studies, produced lymphoid lines in 2 of 2 trials. These lymphoid lines were of interest because they came from sisters whom family studies had shown to be homozygous at both HL-A loci

Separation of lymphocytes and freezing them for possible future use requires a relatively small investment. This method of preserving cells from patients with interesting genetic disorders or other jiochemical markers, and the possibility of propagating them years later, could be important to many types of investigation.
A BASTC DEFECT OF MUCOPOLYSACCHARIDE METABOLISM IN

371 OSTEOGENESIS IMPERFECTA FIBROBLASTS AND POSSIBLE USE FOR INTRAUTERINE DIAGNOSIS. C. C. Solomons, E. M. Handrich, E. A. Millar, and G. Pettett, Department of Pediatrics, University of Colorado Medical Center, Fitzsimmons Army Medical Center, Denver, and Shriners Hospital, Chicago.

Osteogenesis Imperfecta (O.I.) is an inherited metabolic and connective tissue disorder of variable clinical expressivity characterized by fragile bone, blue sclera, deafness, laxity of joints, dental abnormalities, excessive bruising, hyperthermia and increased diaphoresis. A hypermetabolic state, abnormal proline tolerance, decreased growth rate and disturbances in leukocyte and platelet metabolism are present. Increased serum pyrophosphate and an increased resistance to malignancy has been reported. Previous studies of Osteogenesis Imperfecta indicat an impaired collagen synthesis and histochemical abnormalities of the acid mucopolysaccharide ground substance.

In the present investigation, the sulfate metabolism of cultured 0.I. skin fibroblasts was studied. A 45-55 percent reduction was observed in the net incorporation of [35S] $\mathrm{SO}_{4} \mathrm{com}-$ five $0 . I$. cell lines. The fifth $0 . T$. congenita line showed an even greater reduction ( $90 \%)$ of [35S] uptake. This severelyaffected patient died at 11 months of age. This technique clearly distinguishes $0 . I$. cells from normal fibroblasts and is being evaluated as a means of prenatal diagnosis and further characterization of the basic defect.

372 and Edward Bradel (Intr. by Henry Nadler). Ohio State Univ., Children's Hosp. Dept. of Peds., Columbus and Northwestern Univ. Med. Sch., Children's Mem. Hosp., Chicago. Hereditary mucolipidoses are systemic storage diseases characterized by abnormal mucopolysaccharide (MPS) metabolism and absence of MPSuria. A 10 -month old infant of consanguinous parents had typical findings of this group of disorders. Examination revealed motor and mental retardation, severe hypotonia, coarse facial features, corneal clouding, optic atrophy, cherry red macula, protruding tongue, hepatosplenomegaly, dysostosis multiplex. Urinary MPS excretion was normal. GMI gangliosidosis was ruled out by normal $\beta$-galactosidase $(\beta$-gal) activity in white blood cells and liver. Electron microscopy of a liver blopsy showed many abnormal single membrane-bound vacuoles and membranous lamellar bodies free in the cytoplasm in hepatocytes and Kupffer cells. Lysosomal enzyme levels (arylsulfatase, $\beta$-gal, $\beta$-glucuronidase, $\alpha$-mannosidase, $\alpha$-fucosidase, and hexosaminidase) were normal in fibroblasts. Kinetics of radioactive sulfate accumulation were markedly abnormal, indicating defective MPS metabolism in the cells. These findings suggest a new mucolipidosis, clinically similar to GM gangliosidosis but biochemically characterized by normal $\beta$-gal activity and abnormal MPS metabolism. pared to normal into intracellular mucopolysaccharides in four of

372 MUCOLIPIDOSIS " $X "$ ". Annemarie Sommer, Carol Booth, umbillical hernia, spade-like hands and radiological evidence of 
373 CHROMOSOMAL STUDTES ON YOUNG MOTHERS OF DOWN'S SYNDROME. Rawatmal B. Surana and Patrick E. Conen (Intr. by Melvin E. Jenkins). Howard Univ. Col. of Med., Dept. of Ped, Washington, D.C., and The Hosp. for Sick Children, Dept. of Path., and Res. Inst., Toronto.

Twenty-one mothers, under the age of 30 years, when their child with primary trisomy 21 was born, had chromosomal studies done on peripheral blood lymphocytes and skin fibroblasts. In most of these cases, 100 metaphases were analyzed from each culture for structural and numerical aberrations.

The pedigree analysis failed to show increased incidence of Down's syndrome, congenital anomalies or mental retardation. None of the mothers were mentally retarded and were not exposed to radiation or viral infections during the pregnancy in question

A total of 1,911 cells were analyzed from 20 blood cultures and none of them showed any degree of mosaicism or structural aberrations. A total of 1,430 cells were analyzed from 18 fibroblast cultures and mosaicism was not found as far as trisomy 21 ce11s were concerned. However, four mothers who had normal karyotypes in blood, had a number of cells in their fibroblast cultures with structural aberrations which were of different kind in each case. The findings could be as a result of (a) mosaicism not detectable in blood, and (b) or due to de novo translocations arising in fibroblast cell culture.

Spot checks of cultures for mycoplasm contamination were negative. Structural chromosomal aberrations in four mothers might have influenced meiotic nondisjunction for chromosome number 21 . However, this is speculative.

374 TEN YEAR EXPERIENCE WITH THE SWEAT TEST Paul M. Tocci, Laura Martinez and Robert M. McKey, Jr. Univ. of Miami Sch. of Med., P.0. Box 520006, Miami, Florida, 33152. Intr. by M. Novak.

The quantitative analysis of sodium and chloride in sweat samples is a simple procedure that has gathered accretions of myth and fallacy.

Experience with 1210 individuals at risk for cystic fibrosis reveals that iontophoresis of pilocarpine and application of heat is essential to get sufficient sweat for analysis (over $100 \mathrm{mg}$ ). The sweat is collected on weighed filter paper, sealed on the site of pilocarpine application. Specimens are then sealed in vials, weighed and diluted. Sodium and potassium are analyzed by flame photometry and chloride by silver titration.

Potassium levels did not correlate with either sodium or chloride levels. Chloride determination by specific ion electrode on the skin did not correlate with silver titration results. The electrode gave satisfactory results only when used directly on the weighed filter paper.

$\begin{array}{lll}\text { Na vs C1 } & -1 \text { arm } & \text { Correlation Coefficient } \\ \text { Na \& C1 vs Na \& Cl } & -2 \text { arms } & 0.97 \\ \text { C1 vs C1 } & \text { - different days } & 0.94 \\ \text { Na \& C1 vs Cl } & \text { - meter } & 0.94 \\ \text { The most variable and therefore the most important part of }\end{array}$

The most variable and therefore the most important part of
the procedure for reproducibility is the collection and prepthe procedure for reproducibili
aration of the sweat sample.

375 COLOR VARIATIONS IN THE SHORT ARMS OF D AND G CHROMOSOMES BY ACRIDINE ORANGE R BANDING. Ram $S$. Verma and Herbert A. Lubs (intr. by Donough $0^{\prime} B r i e n$ ). Univ. Colo. Med. Ctx., Dept. Ped., Denver.

Reliable characterization of variation in the short arms of acrocentric chromosomes has many important uses in clinical medicine and genetic investigation. These include detection of markers for linkage and cell fusion studies, paternity exclusion or inclusion and certain epidemiological and population studies. Other cytogenetic technics permit characterization of these regions only in terms of size and intensity of staining. With this technic, which is in press in the American Journal of Human Genetics, a wide range of colors ranging from red through green were observed in group $D$ and $G$ short arms. The present study was designed to estimate the frequency of such variation in a normal population. The color of each short arm was recorded from projected color transparencies using a standard series of 6 colors (present in other regions of the karyotype) as withincell standards. In addition, the area of the short arm of chromosome 18 was used as a basis for classifying the size of each short arm into one of 5 categories. Five cells were studied in each of 25 normals both by this technic and Q banding. Except for chromosome 13, more variation in the short arms of all acrocentric chromosomes was found than with $Q$ banding, but the combination of $Q$ and $R$ banding resulted in more variant chromosomes than either technic alone. The technic should prove extremely useful in the type of studies described above.
376 HURLER'S SYNDROME: HETEROZYGOTE IDENTIFICATION EMPLOYING LEUKOCYTES. Rebecca S. Wappner and Ira K. Brandt, Indiana Univ., Sch. Med., Indiana Univ. Hosp. Dept. Ped., Indianapolis.

Ten families in which Hurler's Syndrome has occured were

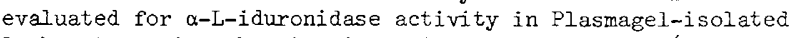
leukocytes using phenyl- $\alpha-i$ duronide as a substrate (gift of Dr. Neufeld and Dr. Weissmann, NIH-NIAMDD 73-2205).

Significant differences $(P<0.001)$ were found between the specific activities of obligate heterozygotes and normals.

\begin{tabular}{lcccc} 
& & \multicolumn{3}{l}{$\begin{array}{l}\text { Specific Activity } \\
\text { (nmoles/18h/mg protein) }\end{array}$} \\
\cline { 3 - 5 } Normals & (15) & $\frac{\text { Mean }}{236}$ & $\frac{\text { SD }}{25}$ & $\frac{\text { Range }}{197-286}$ \\
Heterozygotes & (15) & 103 & 25 & $45-141$ \\
Affecteds & (8) & 2 & & $0-7$
\end{tabular}

Comparison of the iduronidase activity to other lysosomal acid hydrolases did not help to further differentiate between heterozygotes and normals. The data suggests that heterozygosity for Hurler's Syndrome may be determined with confidence employing leukocytes. 377 TLTRASTRUCTURAL INVISTIGATIONS OF SKIN FIBROBLASTS I. WEISS and S. NOONAN Henry Ford Hosp. and Wayne State Univ. Med. School Depts. of Pediatrics and Anatomy. Detroit In 1973 we reported the clinical and radiographic findings in a mother and child both of whom had frontometaphyseal dysplasia (Clinical Delineation of Birth Defects, 1973.) Danks et al (1972) found metachromatic material within cultured fibroblasts from a patient with frontometaphyseal dysplasia. Cultured fibroblasts from both the mother and child with frontometaphyseal dysplasia contained toluidine blue positive cytoplasmic granules by light microscopy. Ultrastructural investigations revealed fibroblast containing single membrane bounded inclusions. The contents of the inclusions were variable. Some inclusions contained a ring of electron dense amorphous material condensed around the periphery of the inclusion membrane, while other inclusions contained various amounts of melin-like material or membranous whorls embedded in an amorphous matrix. The size, shape, number and distribution of the inclusions varied within individual cells. Dilated rough endoplasmic reticulum and Golgi complexes and lack of lipoid droplets were also observed. The remaining cellular ultrastructure was consistent with normal fibroblasts in confluent culture. The morphology of the inclusions reported here are different from inclusions observed in the mucopolysacchridoses. The presence of metachromatic material in cultured fibroblasts from a mother and child and its absence in cultured and uncultured lymphocytes and in the Rebuck skin window suggests that this dominantly inherited disorder is a generalized connective tissue disease.

378 THE POSSIBLE FALSE-POSITIVE DIAGNOSIS OF KRABBE'S by D. O'Brien). Univ. of Colorado Med. Ctr., Depts of Pediatrics, and Biophysics and Genetics, Denver 80220.

The biochemical diagnosis of Krabbe's disease is based on the deficiency of a B-galactosidase which degrades galactosyl ceramide (gal-cer), lactosyl ceramide (lac-cer), psychosine and monogalactosyl diglyceride. We have studied 2 families wherein heterozygotes for a mutant $\beta$-galactosidase have enzyme levels in leukocytes that overlap with those of Krabbe's disease homozygotes. The values for gal-cer and lac-cer B-galactosidase activity for two healthy women were about $5 \%$ of control values. This value is the same found in patients with Krabbe's disease. While refining a more sensitive assay for the diagnosis of Krabbe's dis. using lac-cer as substrate, Family 1 was coincidently discovered in the control group. The proband's leukocytes had both gal-cer and laccer $\beta$-galactosidase activity indistinguishable from those of Krabbe patients. However, her level of this enzyme in fibroblast culture was about $20 \%$ of controls. Her heterozygosity was demonstrated by family studies showing normal levels in her son, her mother and a sister, but deficient levels in her daughter and othex sister. Deficient activity was not found for other enzymes and mixing experiments failed to demonstrate a soluble inhibitor. In Family 2 the heterozygous father of a Krabbe dis. child also had a gal-cer and lac-cer $\beta$-galactosidase activity similar to that seen in the proband of Family 1 . However in culture this man did not overlap with homozygous affected children. As diagnostic amniocentesis becomes increasingly available, the recognition of 


\section{HEMATOLOGY}

379 LHVER IN NEONATE

Arturo J. Aballi, M.D., Avelina Maralit, M.D. Marvin Lieber, M.D., Fernando Costales, M.D., Dept. of Ped. Queens Hospital Center, Affiliation of the Long Island JewishHillside Medical Center, Jamaica, New York

Before routine administration of vitamin $K$ to the newborn cases of subcapsular hematoma of the liver (SCHL) in large full term babies were not rare. Usually these infants appeared normal at birth and developed alarming symptoms between 1 and 4 days of age. Typical changes of vitamin $K$ deficiency, tncluding very low levels of factors II, VII, IX and $X$ and normal factor $V$, were seen in 3 such cases studied previously. We have not observed this clinical form after 1959. On the other hand two cases with slowly developing bleeding have been seen which presented at 17 days and 7 weeks as abdominal masses. Both had received vitamin $\mathrm{K}$ and it appeared that it had attenuated a pre-existent hemostatic defect. SCHL is still a common lesion ( 38 or $9.5 \%$ of 400 autopsies in our service). Most are small and devoid of clinical significance. All cases with larger lesions had experienced severe fetal distress. Most of these ( 8 of 10 ) were extremely immature and appeared in critical condition at birth. Coagula tion studies in 8 of the 38 cases of SCHL showed alterations suggestive of disseminated intravascular coagulation. This is the usual form observed at the present time. Therefore, it appears that vitamin $K$ prophylaxis has contributed to modify the clinicopathological pattern of SCHL.

380 ANTENATAL DETECTION OF B-THALASSEMIA ( $\beta$-THAL). Blanche P. Alter, C. Bernadette Modell, Henry Chang, Dennis Fairweather, John C. Hobbins, Fredric D. Frigoletto, and David G. Nathan. Harvard Med. Sch., Children's Hosp. Med. Ctr., Dept. of Ped., Boston Hosp. for Women, Dept. of Obst., Boston; Univ. Col. Hosp. Med. Sch., Depts. of Ped. and Obst., London; and Yale Med. Sch., Dept. of Obst., New Haven.

Antenatal detection of $\beta$-thal requires fetal expression of the thal genes, and safe fetal blood acquisition. The normal fetal $B / \gamma$ synthetic ratio, using ${ }^{3} \mathrm{H}$-leucine, rises from 0.07 at 5 weeks to $0.11( \pm 0.02)$ at 20 weeks. The first trimester diagnosis of $\beta$-thal trait in abortuses has been reported. Recently we have studied an 11 week abortus at risk for homozygous disease. The $\beta / \gamma$ ratio was 0.007 , indicative of $\beta$-thal major.

Blood samples were obtained in utero from two fetuses at risk for $\beta$-thal major, without disturbing the pregnancies. Case 1: At 23 weeks, the Dyonics fetoscope was used to nick a fetal vessel on a posterior placenta. The ammiotic fluid had $4 \%$ fetal red cells; the $B / Y$ ratio was 0.156 . Case $2: \beta$-thal trait mother was transfused to suppress her globin synthesis. At 19 weeks, using ultrasound guidance, blood was aspirated from a posterior placenta. A sample with $45 \%$ fetal red cells had a $\beta / \gamma$ ratio of 0.06 . A sample with $4 \%$ fetal red cells had a ratio of 0.07 . The first fetus appears to be normal; the second to have $B$-thal trait.

The $\beta$-thal genes are expressed in utero. Fetal blood can be obtained for the prenatal detection of hemoglobinopathies without interruption of the pregnancy.

381 Rh SENSITIZATION OF Rh NEGATIVE INFANTS BORN OF Rh POSITIVE MOTHERS. Frank W. Bowen and Marilyn N. Renfield (Intro. by Gordon B. Avery), Dept. of Pediatries, George Washington University Medical Center, Washington, D.C. and William Beaumont Army Hospital, El Paso, Texas.

There is approximately a $20 \%$ incidence of Rh erythroblastosis in the first pregnancy of Rh negative women whose own mothers were Rh positive. Such an incidence correlates with the known occurrence of maternal-fetal transfusion at birth and suggests sensitization of the infant at birth. To confirm this, 63 cord blood samples of Rh negative infants from Rh positive mothers were obtained and tested for Anti-D by automated technique. Also, the 63 infants had blood sampled and tested for Anti-D at various intervals up to one year. Of the 63 cord bloods, none were positive for Anti-D. Seven of the 63 infants subsequently became positive between $1-3$ months of age, suggesting sensitization at birth due to maternal-fetal transfusion. Further studies are ongoing to confirm this finding and test the efficacy of Anti-D human globulin in prevertion of $R$ h sensitization in the neonate at risk.
382 DETECTION OF ANTI-NEUTROPHIL (PMN) ANTIBODIES IN DRUG INDUCED NEUTROPENIAS AND OTHER DISORDERS BY THE METABOLIC RESPONSE OF HUMAN LEUKOCYTES TO SENSITIZED PMN. Laurence A. Boxer, Betty W. Chang, and Thomas P. Stossel. (Intr. by Bertil Glader). Harvard Med. Sch., Children's Hosp. Med. Ctr., Boston.

A quantitative assay was devised in which normal human PMN purified by gradient centrifugation were incubated in test sera in the presence of a metabolic inhibitor 2-deoxyglucose for 30 minutes, washed and added to normal leukocytes (indicator cells)

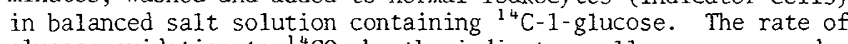
glucose oxidation to ${ }^{14} \mathrm{CO}_{2}$ by the indicator cells was measured over 30 minutes. The following quantities of ${ }^{14} \mathrm{CO}_{2}$ (counts/minute $/ 10^{7}$ indicator cells) evolved from mixtures of indicator cells and PMN treated with test sera.

Test serum

39 normals, nonimmune neutropenia,

ITP, anti-Rh

3 drug related neutropenia

4 neonatal isoimmune neutropenia

3 of 14 idiopathic neutropenia

${ }^{14} \mathrm{CO}_{2}$ evolution

$1732 \pm 652$ (mean \pm S. D.) $6767 \pm 2387$

$5224 \pm 1644$

$3412 \pm 743$

The anti-PMN activity was isolated in the IgG fraction. The measurement of metabolic activity elicited in normal leukocytes by antibody-PMN complexes appears to be a sensitive screening technique with potential for investigating neutropenias of unknown cause and neutrophil transfusion compatibility.

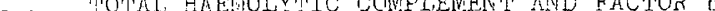
LEVEIS IV CYILIREN VITH THALASSAEMIA MAJOR K.P. Carlson, D.Iaski, J.0. Ninta, .D. Biggar Hosn. for Sick Children and Univ. of Toronto Depts. of Ped., Immunolory and Pathology. Toronto, Canada

Children solenectomised for thalassaemia major have a hi.gher incidence of nyogenic infections than children splenectomised tor some other reasons. A decrease in one or more of the complement components may be respon sible for the increased susceptibility to infection. An abrormalit.y of the altemate pathway of complement has been surcested as a reason for the himher incidence of pneumococal infection in natients with sickle cell anaemia and with nenhrotic symdrome. Decause children with these conditions and chijdren with thalassaemia are affected hy similar orcanisms, it was decided to assess comnlement activity in children with thalassaemia major. In this study, levels of total haemolytic complement, $\mathrm{C} 3$ and Factor $\mathrm{B}(\mathrm{C} 3 \mathrm{P} \Lambda$ of the altemate pathway)vere measured in twenty-seven children with thalassaenia major, ten of whorn vere splenectomised. Con trols included seven norma? children and six children snlenectomised for other reasons. Total haemolytic complement, $C 3$ and Factor B levels, as determined by haemolytic assays, were similar in each rroun. These results sugrest that some mechanism other than a decrease in these narameters of comolement fixnction may be responsible sur infection in splenic thalsssaemics.
384 FAMILIAL NEUTROPENTA, MONOCYTOPENIA, AND NERVE DEAFNESS WITH DIMINISHED COLONY STIMULATING FACTOR ACTIVITY. Robert R. Chilcote, W. Joseph Rierden, and Robert L. Baehner, Dept. of Ped., Indiana Univ. Sch. of Med., and the James whitcomb Riley Hosp. for Children, Indianapolis, Ind. Colony stimulating factor (CSF) promotes maturation of bone marrow (BM) stem cells into granulocyte and monocyte/macrophage colonies in vitro. The peripheral blood (PB) monocyte is a potent source of CST. This report describes 3 siblings, (JW, KW, and $\mathrm{CW})$ with monocytopenia $(<1 \%)$, neutropenia $\left(<1000 / \mathrm{mm}^{3}\right)$, and congenital nerve deafness. One girl, $J W$, died of staphylococcal septicemia at age $18 \mathrm{yrs}$., one girl, $\mathrm{KW}$, has recurrent skin infections, and a $17 \mathrm{yr}$. old boy, $\mathrm{CW}$, is asymptomatic. In 4 separate studies, feeder layers (fl) prepared either from mononuclear enriched Ficoll-Hypaque gradients or from unseparated white cell suspension from $\mathrm{CW}^{\prime} \mathrm{S}$ PB promoted growth of fewer colonies than did normal PB when the same BM sample was overlayered in $1.6 \%$ methyl-cellulose. CW's BM produced diminished numbers of colonies when cultured at the usual concentrations $\left(2-8 \times 10^{5}\right)$ cells but produced large numbers of predominantly granulocyte colonies when $3.5 \times 10^{6}$ cells were used. In each case, normal fl stimulated growth of CW's BM to a greater extent than did his own PB fl which completely failed to stimulate colonies in 3 separate studies. CW's plasma incubated at $10 \%(\mathrm{v} / \mathrm{v})$ produced a further stimulation of colony growth similar to that observed with 2 control plasmas indicating that his plasma lacked CSF inhibitor. These studies suggest that the cause of the neutropenia in this family was due to diminished production of BM monocytes leading to monocytopenia with lack of subsequent monocyte derived CSF. 
385

FACTOR VIII Chapel Hi11: A VARIANT OF FACTOR VIII. A. Myron Johnson, and Campbell $\mathrm{N}$. Dept. Ped., Chapel Hill, N.C.

Anomalous plasma factor VIII activity has been documented in a 15 year old white male whose coagulation functions were initially studied because of massive gastric hemorrhage following ingestion of aspirin. This anomaly is characterized by persisting discordance between results of partial thromboplastin time (PTT) and thromboplastin generation test (TCT) methods for measuring plasma factor VIII activity. His PTT is abnormal with a mean of $118 \mathrm{sec}$ (normal $\leqq 60 \mathrm{sec}$ ) and factor VIII activity in a PTT assay system is low with a mean of $6 \%$ (normal $=50-150 \%$ ). On the other hand, his TGT is normal and factor VIII activity in a TGT assay system is also normal with a mean of $102 \%$ (normal $=60-160 \%$ ). Factor VIII-related antigen, measured by electroimmunoassay, is approximately twice normal. Other clotting functions and the Ivy bleeding time are uniformly within normal limits. All immediate family members - both parents, a brother, and a sister - are normal with the exception of the mother; in her case, factor VIII activity by TGT assay is $105 \%$ but by PTT assay is $35 \%$. These findings are interpreted to indicate that the proband has a sex chromosome-related molecular variant of factor VIII, not previously described; the clotting defect is completely corrected by addition of aged serum. This variant is apparently harmless since subsequent major abdominal surgery was well tolerated by the patient without factor VII replacement therapy.

386 ERYTHROID STEM CELL STUDIES IN THE DIAMOND-BLACKFAN SYNDROME, Melvin H. Freedman, Dominick Amato and E. Fred. Saunders, Dept. of Pediatrics, Hosp. for Sick Children, Univ. of Toronto, Toronto, Canada.

The pathogenesis of erythropoietic failure in the DiamondBlackfan Syndrome (congenital hypoplastic anemia) was studied in 4 children. Growth of erythroid colonies from marrow in response to added erythropoietin (EPO) was assessed in a plasma clot tissue culture system. Each colony arose from a committed erythroid stem cell that differentiated to progeny of hemoglobin-producing erythrocytes. Studies of 30 control marrows with normal erythropoiesis revealed a linear increase in colony numbers with increas ing concentrations of EPO from 0.5 to 2.5 units $/ \mathrm{ml}$, and with numbers of cells plated from $10^{4}$ to $10^{5}$ per culture. Optimal growth of $158 \pm 79$ colonies per $10^{5}$ cells plated was obtained with 2.5 units/ml EPO at 7 days of incubation. Marrows of all 4 patients grew colonies. Two patients on prednisone in partial remission had normal colony numbers; 2 not on therapy and with no recognizable marrow erythroids had low numbers. In contrast, marrows from 4 children with Fanconi's aplastic anemia produced no colonies. When marrows from patients or controls were cultured with patients' sera, colony growth was increased or unchanged but not inhibited. We conclude that in the Diamond-Blackfan Syndrome: exythroid stem cells are present, even when in relapse; there are no inhibitors to EPO or erythroid progenitors; there may be decreased stem cell responsiveness to EPO which can be modified with corticosteroids.

387

VARIATIONS IN GLOBIN CHAIN SYNTHESIS IN HEREDITARY PERS ISTENCE OF FETAL HEMOGLOBIN (HPFH) IN BLACKS. Shlomo Friedman, Elias Schwartz, Edgar Ahern, and Valda Ahern, Univ. of Pennsylvania Sch. of Med. and the Children's Hosp. of Philadelphia, and the Univ, of the West Indies, Kingston, Jamaica.

In $\mathrm{Hb} \mathrm{S}-\mathrm{HPFH}$ or $\mathrm{Hb} \mathrm{A}-\mathrm{HPFH} \beta$ and $\delta$ genes are inactive $\mathrm{cis}$ to the HPFH gene. The benign clinical course in these disorders is believed to be the result of compensatory synthesis of $\gamma$ chains. However, since $\mathrm{Hb} \mathrm{A}$ or $\mathrm{Hb} \mathrm{S}$ account for more than $60 \%$ of the total hemoglobin and the $\mathrm{MCH}$ levels are normal or slightly decreased, there has to be increased $\beta^{A}$ or $\beta^{S}$ synthesis trans to the HPFH gene. Globin synthesis in peripheral blood (PB) and bone marrow (BM) was studied to compare this compensatory synthesis of $\beta$-chain to that found in $\beta$-thalassemia trait. The $\beta / \alpha$ specific activity (SA) ratios in $\mathrm{PB}$ were close to 1.0 in 5 individuals $(0.98-1.10)$, indicating balanced globin synthesis. In 3 persons the $\beta / \alpha$ ratios were slightly low in one with $\mathrm{Hb} A-$ $\mathrm{HPFH}(0.84)$ and decreased in two with Hb S - HPFH $(0.71,0.75)$. The BM globin synthesis in the latter two persons and in one with $\mathrm{Hb} A-\mathrm{HPFH}(\mathrm{PB} B / \alpha=0.99$ ) revealed $\beta / \alpha$ SA ratios of $1.04,0.99$, 0.99 and $(\beta+\gamma) / \alpha$ radioactivity ratios of $0.92,1.05,1.09$, respectively, indicating balanced globin synthesis. The decreased SA $B / \alpha$ ratios in $3 / 8 \mathrm{HPFH}$ heterozygotes are similar to those found in $50 \%$ of blacks with high $A_{2} \beta$-thalassemia trait. In patients with compensatory synthesis of $\beta$-chain in $B M$ erythroblasts, including those heterozygous for $B$-thalassemia, Hb Lepore or HPFH, the synthesis of $\beta$-chain in PB reticulocytes may decrease more
rapidly than that of $\alpha$-chain.
388 HEMOLYTIC ANEMIA SECONDARY TO INHERITED IDIOPATHIC David Tukey, William Krivit. Univ. of Mn. Sch. of Med

Primary familial splenomegaly as a cause of hemolytic anemia has been reported by Rao, Shahidi and Opitz (Clin. Genetics 5: 1974). Our kindred with a similar syndrome included 9 affected members of 3 successive generations with hemolytic anemia associated with splenomegaly. Extensive investigations of the red cells being reported for the first time in this new syndrome support the concept of a primary spleen defect rather than an intrinsic red cell abnormality. The morphology of the red cells was norma by indices, Coulter counter sizing, light microscopy, and scanning E.M. No membrane abnormality could be demonstrated by the following studies: unincubated and incubated osmotic fragility, filterability, intracellular $\mathrm{Nat}, \mathrm{K}+$, and water, Nat flux, $\mathrm{Ca}+$ flux, ATP'ase, protein electrophoresis, spectrin phosphorylation, cholesterol, and phospholipids. Hemoglobin was normal by electrophoresis, isoelectric focusing, heat stability, protoporphyrin, globin synthetic rates, and glycolytic enzymes. Coombs and Donnath-Landsteiner tests were negative. Total hemolytic complement was normal. Liver function tests and histology were unremarkable. Unlike the histology of the spleens reported by Rao et al with prominence of lymphoid follicles, the architecture in the 8 spleens which we examined had a marked paucity of lymphoid follicles as well as dilation of venous sinuses. The complete amelioration of the hemolysis and normalcy of the red cells up to 23 yrs subsequent to splenectomy supports the concept that the spleen is the primary source of this hemolytic anemia.

389 IRREVERSIBLY SICKLED CELLS (ISC'S) : A CONSEQUENCE OF CELLULAR DEHYDRATION. Bertil E. Glader and Aixa Muiller. Dept. Ped., Harvard Med. School, Children's Hosp. Med. Ctr., Boston, Mass.

It is generally agreed that ISC's are a result of irreversibie membrane injury, unrelated to the physical state of intracellular hemoglobin. ISC's can be produced by metabolic depletion of deoxvgenated SS-RBC's in vitro. In addition we have observed that ISC's are generated in ATP-replete cells following deoxygenation and incubation with propanolol $(0.5 \mathrm{mM})$ and calcium $(1.5 \mathrm{mM})$.

The common denominator of these ISC models is a calcium-dependent $\mathrm{K}$ and water loss. Chlorpromazine (CPZ) is known to displace membrane-bound calcium. In both models of ISC production, preincubation of sickle RBC's with $50 \mu \mathrm{M} \mathrm{CPZ}$ prevented $\mathrm{K}$ loss, dehydration, and ISC formation. In order to evaluate the relative roles of calcium and dehydration in ISC production, additional experiments were done in a high K-buffer (similar to intracellular cation content) in order to prevent the propanolol-induced changes in $\mathrm{K}$ and cell water. Under these conditions, the maintenance of nornal cation composition and cellular hydration prevented ISC. formation associated with calcium-propanolol. These studics suggest that cellular dehydration per se can induce irreversible membrane injury, and thereby lead to the production of ISC's, independent of cellular energy status or calcium content.

390 ERYTHROPOIETIN LEVELS IN RESPIRATORY DISTRESS SYNDROME D. GTaubiger, J.F. Garcia and T.J. Gribble Dept. of Pediatrics, Stanford Univ., Stanford, California and Lawrence Berkeley Laboratory, Berkeley, CA (Intr. by P. Sunshine).

Infants with a variety of conditions including hemolytic diseases, cyanotic congenital heart disease and placental insufficiency have been shown to have elevated levels of erythropoiet in in the neonatal period using a mouse bioassay method. Using a new radioimmunoassay technique for the measurement of erythropoiet in developed by J.F. Garcia (The Radioimmunoassay of Human Plasma Erythropoietin, in Regulation of Erythropoiesis, published by Il Ponte Press, Milan 1971), erythropoietin levels were determined in 20 infants with respiratory distress syndrome and/or bronchopulmonary dysplasia from 4-30 days of 1 ife. Levels were also determined in two other groups of infants without pulmonary disease. Eight normal infants had erythropoietin levels of $6.4+2.6 \mathrm{milli}$ units per milliliter $(\mathrm{mu} / \mathrm{ml})$. Wine infants with a variety of conditions that included cyanotic heart disease and hemolytic anemia had erythropoietin levels of $17.4 \pm 6 \mathrm{mu} / \mathrm{m}$. Twenty infants ( 32 determinations) with respiratory distress syndrome and/or bronchopulmonary dysplasia had erythropoietin levels of $5.2+2.7 \mathrm{mu} / \mathrm{ml}$ All the infants in this group required oxygen therapy and/or assisted ventilation and had a mean arterial $\mathrm{pO}_{2}$ of $52+13 \mathrm{~mm} \mathrm{Hg}$. The results of this study indicate that infants with respiratory distress syndrome and/or bronchopulmonary dysplasia do not respond with elevation of erythropoietin despite sustained hypoxia. These findings may explain the poor hematological response observed in infants with respiratory distress syndrome. 

391 AN ORAL EVON-CHELATING DRIJG IN B-THALASSEMIA MAJOR. Joseph H. Graziano, Charles M. Peterson, Robert W. Grady, Robert L. Jones, Albert de Ciutis, Virginia C. Canale, Denis R. Miller and Anthony Cerami, Cornell Univ. Med. Col., Dept. of Pediatrics and The Rockefeller Univ., Lab. of Med. Biochemistry New York City.

2,3-Dihydroxybenzoic Acid (2,3-DHB) has been identified as a potentially useful iron-chelating drug (JPET 190:570, 1974). A clinical trial of 2,3-DHB in patients with $\beta$-thalassemia major who have secondary iron-overload due to multiple transfusions has therefore been undertaken. Patients were maintained on a fixed, low-iron diet, and urinary and fecal iron excretions determined. The administration of $25 \mathrm{mg} / \mathrm{Kg}$ of 2,3-DHB for eight days to five patients, ages 18-24, resulted in an average increase in iron excretion of $5 \mathrm{mg} /$ day. The drug was well tolerated, and there were no changes in the excretion of $\mathrm{Ca}, \mathrm{Mg}, \mathrm{Cu}, \mathrm{Zn}$, Na or $\mathrm{K}$. Three patients (age 18) given $25 \mathrm{mg} / \mathrm{Kg}$ of 2,3-DHB q.i.d. for 21 days excreted $13.0,10.6$ and $4.5 \mathrm{mg}$ of iron per day above baseline. Once again, no adverse effects were noted. The increase in iron excretion in one patient was $4.3 \mathrm{mg} /$ day at a dosage of $25 \mathrm{mg} / \mathrm{kg}$ ( 8 days) and $10.6 \mathrm{mg} /$ day at $25 \mathrm{mg} / \mathrm{Kg}$ q.i.d. (21 days). The possibility that the increase in fecal iron was due to gastrointestinal bleeding was ruled out by measuring fecal $51 \mathrm{Cr}$ following the infusion of $51 \mathrm{Cr}$ labeled red cells. An evaluation in younger patients (ages 9-14) is in progress; preliminary results sugzest that the younger age group does not respond as well as the older group, who are more heavily iron-overloaded.

392 RON DEFICIENCY ANEMIA AND GASTROINTESTINAL IRON ABSORPTION. Gross, S., Stuart, M.J., Swender, P. Syracuse, N.Y. and Oski, F.A. Upstate Medical Center, SUNY,

In children with Iron Deficiency Anemia (I.D.A.), failure to absorb oral iron is considered to be rare. This hypothesis was tested by performing an oral iron absorption test in 2 groups of children with documented I.D.A. Group I consisted of 25 children with I.D.A, who had not received iron therapy. Group II comprised 7 children with "chronic" I.D.A. who had not responded to therapeutic doses of oral iron, and in whom chronic infection and malabsorptive disease had been excluded. Following a fasting serum iron level a test dose of $1 \mathrm{mgm}$ of elemental iron per $\mathrm{kgm}$ was administered to both patient groups, and serum iron levels measured at one, two, and four hours. Results in mcgms\% ( \pm 1 SD) were as follows:

\begin{tabular}{llrlrl} 
& Baseline & 1 & Hour & 2 Hours & \multicolumn{1}{c}{ Hours } \\
\cline { 2 - 4 } Group I & $30( \pm 16)$ & $247( \pm 93)$ & $304( \pm 126)$ & $277( \pm 140)$ \\
Group II & $37( \pm 8)$ & $83( \pm 29)$ & $90( \pm 36)$ & $66( \pm 31)$
\end{tabular}
Due to the abnormal "flat" absorptions ( $p$ value) .0005) manifested by children in Group II, these patients were treated with Imferon with therapeutic success. In 3, absorption studies 6 to 8 weeks following Imferon were repeated. All had improved; two were normal. Those in Group I were treated with therapeutic dosages of oral iron with predictable response. One patient in this de novo group demonstrated a "flat" absorption, necessitating Imferon therapy. In I.D.A. malabsorption of oral iron does occasionally occur and must be considered in children not responsive to oral therapy.

393

DEPRESSION OF CLOTTING FACTORS WITH ANTICONVULSANT THERRPY. Margaret W. Hilgartner, G. E. Solomon and H. Kutt, Cornel1 Univ. Med. Col., New York Hospitât, Depts. of Ped. and Neurology, New York. Intr. by D.R. Miller

Neonates born to mothers receiving a hydantoin anticonvulsant during pregnancy have shown defects in the vitamin $K$ dependent clotting factors with or without clinical bleeding in the first few days of life. Diphenylhydantoin (DPH) induced coagulation defects have been well documented experimentally on a dose related basis in cats. Milder defects have been noted with Phenobarbital. Recently high levels of DPH have been observed to produce depression of clotting factors in older children as well as neonates. Four children, ages 8 months to 7 years who had ingested high doses of DPH alone or in combination with Diazepan or Phenobarbital developed abnormal vitamin $x$ dependent clotting factors. They were all on normal diets and had normal liver function tests. The DPH values ranged between $40-53 \mu \mathrm{g} / \mathrm{ml}$ and the Phenobarbital $45-74 \mu \mathrm{g} / \mathrm{ml}$ in two children with poorly controlled generalized seizures. An 8 month male with Down's syndrome was on prednisolone and Diazepan along with DPH for infantile spasms and generalized seizures. His DFH Tevel was also $53 \mathrm{\mu g} / \mathrm{ml}$. The fourth child ( $7 \mathrm{yr}$ old male, $23 \mathrm{~kg}$ ) had an acute ingestion of 1 gram DPH over a $36 \mathrm{hr}$ period with resultant DPH level of $39 \mathrm{\mu g} / \mathrm{ml}$. Nystagmus and mild drowsiness were noted in the younger children, ataxia in the older children. Clotting Factors II, V and X decreased to $30 \%$ of normal when levels of DPH were above $20 \mu \mathrm{g} / \mathrm{ml}$. By lowering the level of DPH the clotting factors returned to normal. No clinical bleeding was seen in these patients.
394 PLATELET DYSFUNCTION OURING CRISIS AND INFECTION IN SICKLE CELL ANEMIA. Marilyn A. Hruby, Victoria Villesca and Karen Costello. (Intr. by George Honig)

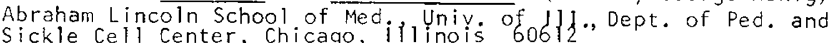
platelet function studies were performed in 21 children (age 1 to 15 years) with SS anemia or $S-\beta$ thal who were admit ted to the tologic evaluation, platelet counts, glass bead retention and platelet aggregation using collagen, epinephrine and $1 \mu M$ and 20 MM adenosine diphosphate (ADP) were performed within 72 hours of the onset of symptoms and every second day during hospitalization. None of the patients had received any medication known to affect aggregation were compared to results obtained in 30 normal subjects and 26 asymptomatic children with SS anemia.

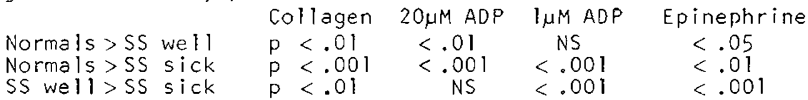
The impaiced aggregation response in sick $\$ S$ patients improved
within 2 to 8 days and corresponded to overal1 improvement in clinical status.

These results confirm other observations of impaired aggrega$t$ ion response to low-dose ADP during vaso-occlusive crises in SS nemia. In addition, they demonstrate that a reduced platelet re the well ss mediated by collagen and epinephrine is present the well SS patient, with further significant impairment during to the hypothesis that platelets are activated or utilized in the course of these acute manifestations of sickle cell disease.

395 HEMATOLOGICAL STUDIES IN G-6-PD DEFICIENCY AND JAUNDICE IN FULL TERM AMERICAN NEGRO NEONATES. Wen-Hsuing Huang and $A$. Leonard Luhby. Deparfment of Pediatrics, New York Medical College, New York, N.Y.

Although there appears to be an increased frequency of jaundice in full term caucasian and Asiatic newborns with $G^{-} 6^{-} P D$ ( $B^{-}$type) deficiency in Mediterranean and Far East countries, reports of hyperbilirubinemia due to $G^{-6-P D}$ (presumably $A^{-}$type) deficiency in full term American Negro neonates have been conflicting. This report presents statistical and hematological data which do not support such a relationship in the American Negro newborn. Among 22 black and hispanic G-6-PD deficient newborns in whom other causes of jaundice were ruled out, the frequency $(60 \%)$ mean bilirubin $(13 \mathrm{mg} \%$ ) and the pattern of jaundice resembled that in physiological jaundice. Hyperbilirubinemia in the neonate is believed owing to either increased hemolysis or reduced liver clearance of a normal bilirubin load. Since hepatic cell G-6-PD in $A^{-}$type deficients is normal, evidence for increased hemolysis was sought. Findings among 212 newborns in whom known causes for hemolysis or increased jaundice were ruled out: Jundice

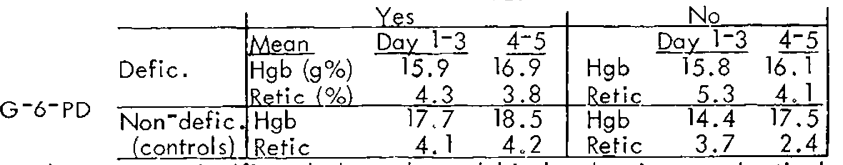

There was no significantly lower hemoglobin level or increased reticulocytosis among the jaundiced G-6-PD deficient newborns as compared to non-jaundiced deficients, or jaundiced or non-jaundiced G-6-PD normal infants. RBC morphology was similar in all groups.

396 INACCURACIES IN ELECTRONTCALLY DETERMINED HEMATOCRITS (HCT) IN POLYCYTHEMIC CYANOTIC CONGENITAL HEART DISEASE (CCHD). Marsha $R$, Jones, Harold M Maurer Carolyn M. McCue, Charles L. Johnston, Nancy B. MCWilitiams. Medical College of virginia, Depts. of pediatrics and Pathology, Richmond, Virginia.

The degree of polycythemia is an important factor in determining the course in children with CCHD. We have noted a striking difference in hcts measured by micro- and Coulter counter (Model "S") methods in polycythemic children with CCHD. In the present study, these differences were correlated with blood viscosity, $M C V$, and $R B C$ count in 7 children during phlebotomy with plasma exchange $(15-20 \mathrm{ml} / \mathrm{Kg})$ for symptomatic severe polycythemia. Viscosity was determined with a Brookfield cone-plate viscometex at $42 \mathrm{sec}^{-1}$ at $37^{\circ} \mathrm{C}$. Within the hct range of $53-74 \%$, Coulter values were up to 10.2 "units" lower than micro-hct values; the differences were most marked when the MCV was $<86 u^{3}$. As viscosity fell with phlebotomy, the gap between hct values narrowed, but micro-hcts and viscosity were still high even when Coulter hats were normal. A more linear relationship existed between micro-hct and viscosity than Coulter hat and viscosity. Viscosity varied directly with $M C V$ at a given $R B C$ count. A nomogram converting Coulter to micro-hct values was devised. These data show the superiority of the micro-hct in relation to blood viscosity. We, therefore, recommend its use as a more accurate overall measure of the polycythemic state in following children with CCHD. 
397 GLOBIN MESSENGER RNA IN HUMAN DEVELOPMENT AND THE THALASSEMIA SYNDROMES. Haig H. Kazazian, Ir., Gordon D. Ginder, pamela G. Snyder, and Rebecca J. Van Beneden, Johns Hopkins University School of Medicine, Department of Pediatrics, Baltimore.

By formamide gel electrophoresis globin messenger RNA (mRNA) of human adult reticulocytes separates into two RNAs with migratory rates identical to the $\alpha$-and $\beta$-globin mRNAs of the rabbit and mouse. In contrast, human fetal reticulocytes $(10-20$ weeks gestation) contain a new mRNA, presumably encoding the $\gamma$ chain of $\mathrm{Hb}$ $F$, which has a smaller size than $\beta$ mRNA. Moreover, by gel electrophoresis, $B$ mRNA is strikingly deficient in fetal cells. When mRNA preparations from fetal reticulocytes are added to a cell-free system, $\alpha$ and $\gamma$ chains are synthesized. Thus, regulation of the $\gamma$ to $\beta$ chain switch in human development occurs during transcription or processing of the mRNA. Globin mRNA content in reticulocyte polyribosomes of patients with hematological disorders was also examined. Individuals with either hemolytic anemia without hemoglobinopathy or sickle cell anemia had $\beta$ mRNA to $\alpha$ mRNA ratios of $\cong 1$, while a patient with Hb S- $\beta$-thalassemia had a $\beta / \alpha$ mRNA ratio of 0.75 ; and two subjects with homozygous $\beta$-thalassemia had severe deficiencies of $\beta$ mRNA. Conversely, a patient with $\alpha$ -

thalassemia ( $\mathrm{Hb} \mathrm{H}$ discase) had a $B / \alpha$ ratio of 6 . The se latter data confirm the existence of a quantitative deficiency of chain-

specific globin mRNA in patients with the thalassemia syndromes.
400 LEAD BINDING BY TRANSFERRTh. Joseph Kochen and Yigal Greener.Albert Einstein Col. Ked., Konted
Med.Ctr., Dept. Ped, Bronx, New York

Little is known about the factors which determine the distribution of lead $(\mathrm{Pb})$ in the body. We have previously show that saturation of the total iron binding capacity (TIBC) markedly suppresses the uptake of plasma Pb by red blood cells and increases the uptake of $\mathrm{Pb}$ by the liver. This finding suggests that transferrin ( $\mathrm{Tf}$ ) may interact with plasma $\mathrm{Pb}$ and affect its distribution. In the present study the binding of iron and lead to $T f$ was determined by the incubation of heparinized plasma with $i^{5} \mathrm{Fe}$ ferric chloride and/ori:10 $\mathrm{Pb}$ ? lead acetate followed by the quantitative precipitation of If with anti-human if goat serum (provided by $\mathrm{U}$ o viller-Eberhard). At concentrations of added $\mathrm{Pb}$ ranging from tracer amounts to $200 u g_{\%}^{\infty}, 50$ to $6 \gamma_{\circ}^{\circ}$ of the plasma $\mathrm{Pb}$ was foumd to be bound to Tf. Similarly, over $95 \%$ of added tracer amounts of iron was bound to $\mathrm{Tf}$. Saturation of TIBC prior to the addition of $2{ }^{\circ} \mathrm{Pb}$ or ${ }^{5} \mathrm{Fe}$ resulted in a tope by Tfo The addition of increasing concentrations of iron after $\mathrm{Po}$ binding by $\mathrm{if}$ resulted in an increasing displacement of $\mathrm{Pb}$ which was completed at saturation of the TIBC.Increasing concentrations of $\mathrm{Pb}$ did not displace iron bound to $\mathrm{Tf}$. Preferential binding of $\mathrm{Pb}$ by a specific plasma protein has not previously been shown.These results indicate that plasma $\mathrm{Pb}$ is to a large extent bound to $\mathrm{Tf}$; that $\mathrm{Pb}$ may occupy the same binding sites as iron and that $\mathrm{Pb}$ can be displaced by iron.The failure of red cell uptake of $\mathrm{Pb}$ from plasma and the increased uptake of $\mathrm{Pb}$ by liver when the PIBC is saturated suggests that the transferrin-Pb complex plays an important part in the tissue distribution of lead。
398

GLOBIN SYNTHESIS IN CLINICALLY SIGNIFICANT DISORDERS WITH HB S or HB C. Haewon C. Kim, Robert G. Weierbach, Shlomo Friedman and Elias Schwartz, Univ. of Pennsylvania Sch. of Med. and the Children's Hosp. of Philadelphia, Philadelphia, Pennsylvania.

There is a wide range of globin synthesis ratios reported in patients with sickle cell disease, with $\alpha / \beta^{S}$ from 0.89 to 1.57 . These data cast doubt on whether the presence of genes for $\alpha$ - or $\beta$-thalassemia in combination with $\mathrm{Hb} S$ can be detected by globin synthesis studies. We have studied globin synthesis in peripheral blood (PB) of 20 patients with Hb SS, 12 with $\mathrm{Hb}$ S- - -thalassemia, 4 with $\mathrm{Hb} \mathrm{SC}, 3$ with $\mathrm{Hb} \mathrm{CC}$ and $2 \mathrm{Hb} C-\beta$-thalassemia, in order to determine the range of ratios in these patients. In 19 patients with $\mathrm{Hb} S \mathrm{~S}$, the $\alpha / \beta \mathrm{S}$ specific activity ratios were $0.85-1.02$ $(0.92 \pm 0.04)$. The $\alpha$-chain radioactivity in the $\mathrm{PB}$ free $\alpha$-chain pool in 5 patients was $14.0-17.5 \%(16.1 \pm 1.6)$. The remaining Hb SS patient had an $\alpha / \beta S$ ratio of $0.82, \alpha$-chain pool radioactivity of $3.5 \%$, and an MCV of 72 without iron deficiency, suggesting the presence of $\alpha$-thalassemia. In $\mathrm{Hb} S-\beta$-thalassemia the $\left.\left(\beta S_{+\beta}\right)\right) / \alpha$ ratios were $0.50-0.95(0.74 \pm 0.13)$. In Hb SC $(\beta S+\beta C) / \alpha$ was $0.91-1.01(0.94 \pm 0.04)$, in $\mathrm{Hb} C \mathrm{CC} \beta C / \alpha$ was 0.99 , 1.07 and 1.10 , and in $H b C-\beta$-thalassemia $\left(\beta^{C}+\beta^{A}\right) / \alpha$ was 0.74 and 0.91 . These studies show that the variance of globin synthes is values in $\mathrm{Hb} S \mathrm{SS}, \mathrm{Hb} \mathrm{SC}$ and $\mathrm{Hb} \mathrm{CC}$ is similar to that in controls, that the PB radioactive $\alpha$-chain pool size in Hb SS may be precisely measured and may be of use in detecting the presence of thalassemia, and that synthesis ratios in $\mathrm{Hb} \mathrm{S}-\beta$-thalassemia and $\mathrm{Hb} C-\beta$-thalassemia vary from $0.50-0.95$, similar to those seen Hb C- - -thalassemia vary from 0.50
in $\beta$-thalassemia trait in blacks.

399 INTERACTION OF FERRITIN WITH LEAD(PB)AND CADMIUM(Cd). Joseph Kochen and Yigal Greener, Albert Einstein Col. Med. Montefiore Hosp.\& Med。Ctro,Dept。 Ped. New York. Ferritin (Fn) limits the absorption and facilitates the storage of unneeded iron. This study indicates that Fn may fulfill similar functions with $\mathrm{Pb}$ and $\mathrm{Cd}$ by binding these metals. Interaction of $\mathrm{Pb}$ or $\mathrm{Cd}$ with $\mathrm{Fn}$ results in displacement of hydrogen ions and precipitation of a complex with a maximum binding capacity of 400 atoms of $\mathrm{Pb}$ or 200 atoms of Cd per molecule of Fn. At reactant concentrations below those required to produce an insoluble complex, binding of $21{ }^{2} \mathrm{~Pb}$ or ${ }^{109} \mathrm{Cd}$ to purified $\mathrm{Fn}$ is shown by the crystallization or immuno-precipitation of isotope-containing Fn. Similar binding to $F n$ in vivo is demonstrable by the isolation of isotope-containing $\mathrm{Fn}$ from rat liver after oral ${ }^{210} \mathrm{~Pb}$ or ${ }^{109} \mathrm{Cd}$ In iron supplemented rats, 24 hours after a tracer dose of $\mathrm{Pb}$ or $\mathrm{Cd}, 50 \%$ of liver $\mathrm{Pb}$ and $15 \%$ of liver $\mathrm{Cd}$ is bound to $\mathrm{Fn}$. In iron deficient rats, only $6 \%$ of liver $\mathrm{Pb}$ and $2 \%$ of liver $\mathrm{Cd}$ is $\mathrm{Fn}$ bound. Intestinal absorption of $\mathrm{Pb}$ and $\mathrm{Cd}$ is diminished in iron treated rats when compared to iron deficient rats。At 30 minso after an oral dose of $\mathrm{Pb}$ or $\mathrm{Cd}$,liver $\mathrm{Pb}$ is $6 \%$ and $\mathrm{Cd}$ is $41 \%$, and at $24 \mathrm{hrs}$, liver $\mathrm{Pb}$ is $26 \%$ and $\mathrm{Cd}$ is 19\%. The decrease in $\mathrm{Pb}$ and $\mathrm{Cd}$ absorption after iron supplementation is associated with decreased toxicity indicated by improved weight gain and survival. The binding of $\mathrm{Pb}$ and $\mathrm{Cd}$ by $\mathrm{Fn}$ in intestinal mucosa cells and their exfoliation may explain the decreased absorption of $\mathrm{Pb}$ and $\mathrm{Cd}$ following iron supplementation. Oral administration of $\mathrm{Fn}$ similarly diminishes $\mathrm{Pb}$ absorption. Further binding of $\mathrm{Pb}$ and $\mathrm{Cd}$ by $\mathrm{Fn}$ in other tissues may also contribute to decreased $\mathrm{Pb}$ and $\mathrm{Cd}$ toxicity in iron supplemented animals.
401 IDIOPATHIC THROMBOCYTOPENIC PURPURA IN CHILDHOOD. Jeanne M. Lusher, Rathi Iyer, A. Samy Khalifa and Wolf W. Zuelzer. Wayne State Univ.Sch.Med. and Children's Hosp.of Michigan, Detroit. Dept. of Ped.

Idiopathic thrombocytopenic purpura (I.T.P.), as traditionally defined, is a syndrome of unknown etiology and greatly variable severity and duration. The question whether acute and chronic forms are variants of the same disease process has not been resolved and difficulties remain in distinguishing one from the other at onset. These uncertainties are reflected in the frequent use of corticosteroids in childhood I.T.P., despite its predominantly acute self-limited character. This report will present a series of 325 newly diagnosed cases of I.T.P., analyzed with respect to apparent etiologic factors, course and outcome. The majority did not receive steroids. $90 \%$ recovered completely, with platelet counts generally returning to normal within 3 weeks. In this group there was a high frequency of antecedent infections. A few children had recurrent episodes of I.T.P., while in the remainder the course became chronic. In the latter group IgA levels were significantly below normal $(p<0.001)$. Reducing the risk of intacranial hemorrhage (ICH) is usually given as the chief therapeutic rationale for the use of steroids; however, ICH seems sufficiently rare in childhood I.T.P. to raise the question whether it ever occurs in the absence of vascular lesions. In our series the dur ation of bleeding and of thrombopenia in the untreated group compared favorably in that in the steroid-treated group. Moreover in the untreated group all remissions were permanent, whereas several treated patients had a transient relapse when steroids
were withdrawn, suggesting that they may actually delay recovery.

102 ALPHA-GaMta $(\alpha-\gamma)$ THALASSEMIA: A CAUSE OF HEMOLYTIC

402 ANEMIA OF THE NEWBORN. Michael K. McCormack, Gary R. Geller, Solomon Zak, William Krivit. Univ. of Mn.

Sch. of Med., Dept. of Ped., Minneapolis, Mn.

A previously unrecognized hypochromic, hemolytic anemia associated with marked normoblastemia during the newborn period has now been characterized as an $\alpha-\gamma$ thalassemia. This is in contrast to a previously reported complex of gamma-beta thalassemia of the newborn. (Kan et al, NEJM 286:1972) One male and 2 female siblings had hemolytic anemia and marked normoblastemia (300-900 $\mathrm{nb} 2 / 100 \mathrm{WBC})$ at birth. Globin chain synthesis studies on peripheral blood indicated $\gamma$-thalassemia and $\alpha$-thalassemia. The globin synthetic ratios obtained on the proband and controls are:

$\begin{array}{lllll}\text { Normal cord (3 controls) } & \beta / \alpha & \gamma / \alpha & \alpha / \beta+\gamma & \beta / \gamma \\ \text { (3) } & .39 \pm .03 & .63 \pm .05 & .99 \pm .08 & .60 \pm .02\end{array}$ Proband

Globin chain synthesis done on the father, 2 older affected siblings of the proband, and the proband at 1.5 yrs. of age, revealed $\alpha / \beta$ synthetic ratios between 0.45 to 0.54 , compatible with $\alpha$-thalassemia trait. Thus, only $\alpha$-thalassemia can be demonstrated subsequent to the switch from $\gamma$ chain to $\beta$ chain production. This new complex $\alpha-\gamma$ thalassemia provides the basis of a model for regulation of globin chain synthesis. Theory: this single gene defect which affects the expression of 2 unlinked structural genes suggests the presence of a common regulator gene which may be defective in $\alpha-\gamma$ thalassemia as illustrated:

$\delta, B_{1} \gamma, Y=$ "Regulator" $\longleftarrow \alpha$ 
403 DIFFERENTIATION OF CONGENITAL hyPOPLASTIC ANEMIA FROM TRANSIENT ERYTHROBLASTOPENIA. William C. Mentzer, Winfred C. Wang, \& Louis $K$. Diamond. University of

California, San Francisco Medical Center, Department of Pediatrics The onset of transient erythroblastopenia of childhood (TEC) may resemble that of congenital hypoplastic anemia (CHA). In an attempt to develop methods for distinguishing these conditions, we have evaluated 6 patients with TEC and 9 with CHA ( 3 in remission and 6 in varying degrees of relapse). TEC and CHA red cells differed with respect to size, content of fetal hemoglobin and presence of i antigenicity:

$\begin{array}{cccc} & \text { MCV }>90 \mu^{3} & \text { HgbF }>2 \% & \text { i antigen } \\ \text { TEC } & 0 / 6 & 0 / 5 & 0 / 2 \\ \text { CHA } & 9 / 9 & 7 / 9 & 3 / 9\end{array}$

Patterns of red cell glycolytic enzyme activity were also different. CHA resembled cord red cells in that the activity of 6PGD, LDH, enolase, and G3PD was almost always above the normal range, while in TEC the same enzymes were at low or normal levels. Conversely, glutathione peroxidase activity, low in cord blood and TEC, was normal or high in CHA. PFK activity was low in TEC and high in CHA. These abnormalities in CHA red cells persisted during remission or relapse. If found at the onset of an erythroblastopenic anemia, they may be useful in differentiating CHA from TEC. This is an important consideration in deciding about steroid therapy, unnecessary in TEC but indicated in CHA and in offering a prognosis which in TEC is favorable but in CHA is
guarded because of chronicity and possible relapse.

404 CORRELATION OF FETAL HEMOgLOBIN (FH) DETERMiNations WITH THE CLINICAL SEVERITY OF SICKLE CELL ANEMIA (SSA). Thomas D. Miale, John F. Kelleher and Daniel L. Lawson. (Intr. by Owen M. Rennert), Univ. of Florida Col. of Med., Dept. of Ped., Gainesville.

Previous studies have presented conflicting evidence concerning the correlation of FH levels with the severity of symptoms in SSA. In order to determine whether FH levels may be a factor influencing severity, the quantity and distribution of FH in 20 American black patients with SSA were evaluated. Quantitative FH values were obtained by alkali denaturation, corrected for methemoglobin formation. The distribution of $\mathrm{FH}$ among erythrocytes was determined by the acid-elution staining technique, analyzed both manually and by computerized image analysis.

Two patient groups were recognized: 12 mildly-affected individuals aged $3-18$ years; and 8 severely-affected individuals, aged $4-16$ years. Frequency and duration of crises, growth and development, and severity of anemia were the parameters utilized in patient selection. The mean and range of quantitative $\mathrm{FH}$ levels were $17.3 \%(6.0-35)$ in the severely-affected group and $13.8 \%(4.8-29.3)$ in the mildly-affected group.

A difference in the distribution pattern of $\mathrm{FH}$ was recognized in these 2 groups. Severely-affected individuals had greater percentages of erythrocytes lacking stainable FH than did the mildly-affected patients. These findings suggest that FH distribution pattern may be a more significant factor than the FH level in determining the course of SSA.

405 THROMBOCYTOPENIA IN ALPHA-1-ANTITRYPSIN DEFICIENCY (AmD). Thomas D. Miale, Charles T. Dellinger, Ralph McWhorter and Saba Demian. (Intr. by Owen $M$.

Rennert), Univ. of Florida Col of Med., Dept, of Ped.,

Gainesville.

Thrombocytopenia has been detected occasionally in association with alpha-1-antitrypsin deficiency and was present in a 12-yearold boy with moderate secondary lung and liver disease. Platelet counts ranged from 50,000-85,000 with normal platelet morphology. Megakaryocytes contained an abnormal tubular structure visible only on electron micrographs. Platelet aggregation was normal after addition of both ADP and epinephrine. Platelet survival time was normal as measured by $\left[{ }^{51} \mathrm{Cr}\right]$-tagging and by post-salicylate aggregation studies. External counting over lungs, liver and spleen demonstrated no site of platelet sequestration. The results indicate that the thrombocytopenia in this case of ATD was a probable result of a deficit in platelet production rather than increased destruction or sequestration. This may have been related to a defect observed in the ultrastructure of the patient's megakaryocytes.
406 HENE SYNTHESIS IN HEREDITARY HEMOLYTIC ANEMIAS. Denis R. Miller and Gerald B. Kolski, Cornell Univ. The synthesis of heme and globin are interdependent: disorders of the synthesis of one will affect the production of the other. The rate-limiting step in heme synthesis, the formation of $\delta$-aminolevulinic acid (ALA) from succinyl COA and glycine is catalyzed by ALA synthetase (ALA-S). In $\beta$-thalassemia major the synthesis of structurally-normal $\beta$-globin chains is decreased or absent and decreased heme synthesis has been reported. In Hemoglobin $(\mathrm{Hb}) \mathrm{Köln}$ disease ( $\beta^{98 \mathrm{val}-m e t}$ ), defective and deficient synthes is of $\beta$ Köln chains occurs, and in a Puerto Rican family with $\mathrm{Hb}$ Koln disease, the $\beta^{A} /{ }_{\beta} K_{0}$ in ratio was $5: 1$, as determined by ${ }^{3} \mathrm{H}$ leucine incorporation and globin chain separation by coluimn chromatography. ALA-S activity in peripheral blood reticulocytes was assayed with a direct radioisotopic micromethod. A significant correlation $(r=0.973, p<0.001)$ of ALA-S activity with reticulocyte count was observed in neonates (268.114 pmole/ immature RBC/hr), pyruvate kinase deficiency (249 \pm 88 ), sickle

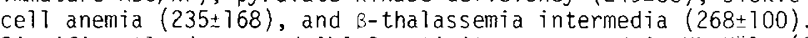
Significantly decreased ALA-S activity was noted in $\mathrm{Hb}$ Köln ( $97 \pm$ $39, \mathrm{D}<.01, N=5)$ and $B$-thalassemia major $(114 \pm 58, \quad p<.02, N=5)$. ALA dehydratase activity was not decreased and did not correlate with cell age. The decreased synthesis of globin chains in these disorders may be related to feedback inhibition of ALA-S, caused by excess free heme derived from denatured BKoln chains or excess $\alpha$ cnains. The primary site of inhibition (membrane, translation or direct enzyme inhibition) has not been determined.

407

HYPONATREMIA IN BETA-THALASSEMIA. Donald I. Moel, Virginia C. Canale, and John E. Lewy. Cornell Univ.

Severe hyponatremia has been noted in the later stages of iliness in some patients suffering from homozygous beta-thalassemia. In order to evaluate whether a renal functional defect in sodium handling is responsible for the hyponatremia, renal function studies were performed on 11 patients with thalassemia major aged 7 to 25 years, 3 patients with thalassemia intermedia, and 2 normal controls. GFR's as determined by inulin and creatinine clearances and RPF's as determined by PAl clearances were normal in all. Maximal urinary osmolality attained after an overnight fast ranged from 800 to $1154 \mathrm{mOsm} / \mathrm{Kg}$. Following hypotonic saline loading all patients diluted their urines to less than $100 \mathrm{~m} 0 \mathrm{sm} / \mathrm{Kg}$. Infusion of $3 \%$ saline resulted in urinary osmolalities ranging between 450 and $665 \mathrm{mosm} / \mathrm{kg}$ in the beta-thalassemic groups and 734 and 722 $\mathrm{mosm} / \mathrm{Kg}$ in the two control patients. After 4 days of salt deprivation ( $10 \mathrm{mLq} \mathrm{Na} / \mathrm{day}$ ), all patients excreted less than $10 \mathrm{mEq}$ of sodium per 24 hours. A sodium balance study was conducted on one 20 year old male with thalassemia major, hyponatremia (serum $\mathrm{Na}$ $117 \mathrm{mEq} / \mathrm{L}$ ) and congestive heart failure. His urinary sodium was less than $1 \mathrm{mEq} / \mathrm{L}$ during this period except when he received furosemide. The hyponatremia often noted in older adolescents with thalassemia major cannot be explained by the mild defects in sodium handling observed. It is more likely that the treatment of chronic congestive cardiac failure with salt restriction and diuretics is responsible for the hyponatremia and thus is potentially preventable by careful electrolyte monitoring and management of these patients.

408 THROMBOTIC AND HYPERCOAGULABLE SYNDROMES IN CHILDHOOD Robert R. Montgomery, Toni Servoss, and Wm. E. Hath-

Thrombosis, known as an important cause of morbidity in adults, has been infrequently emphasized in children. 109 pediatric patients with diseases considered to have an increased tendency to intravascular coagulation underwent detailed coagulation analyses to look for laboratory evidence of hypercoagulability. Patients with laboratory evidence for a consumptive coagulopathy were excluded from this study. The disease categories were vasculitis, inflammatory bowel disease, solid tumors, liver disease, congenital heart disease, sickle cell disease, Henoch-Schönlein purpura, renal disease, nephrosis, and hemolytic uremic syndrome. The studies included partial thromboplastin time (PTT), prothrombin time (PT), thrombin time (TT), fibrinogen, platelet count, thrombelastogram (TEG), fibrin split products (FSP), Factor VIII, Factor VIII antigen, Factor $\mathrm{V}$, and antithrombin III (AT III).

The routine coagulation studies (PTT, PT, TT, fibrinogen, and platelet count) were not reliable in predicting which patients were hypercoagulable. Further evaluation revealed abnormally short TEG (61\%), low AT III (36\%), and elevated Factor VIII (65\%), Factor VIII antigen $(47 \%)$ and FSP $(35 \%)$.

Various pediatric diseases were found to be associated with hypercoagulability. Routine coagulation studies were found to be unreliable in delineating these hypercoagulable patients. The spectrum of hypercoagulability in these diseases includes localized intravascular coagulation within an organ, large vessel thrombosis, and chronic intravascular coagulation without evidence for a consumptive coagulopathy. 
409 AN EVALUATION OF THE ROLE OF HEMOLYSIS IN NEONATAL JAUNDICE. Thomas $F$. Necheles, Uma tology, New England Medical Center, Boston and the Institute of Child Health, Athens. (Intr. by S.S.Gellis) The role of hemolysis in non-specific neonatal hyperbilirubinemia(NSNH) and in severe neonatal jaundice ( $S N J$ ) of various etiologies was assessed by measuring $\mathrm{CoHb}$ levels. In a series of 250 consecutive normal newborns (Boston) third day S.Bil. correlated with the third day CoHb level $(r=0.3293, p<0.001)$. Of the infants with third day CoHb up to $0.75 \%$, $63 \%$ had trird day $S . B i l$. values $\leq 8.0 \mathrm{mg}$ and $1.6 \%$ values $>16.0 \mathrm{mg}$. The corresponding percentages for the infants with. CoHb above $0.75 \%$ was $30 \%$ and $6 \%$. There was a significant correlation between the cord and third day values for reticulocytes, for S.Bil. and for $\mathrm{CoHb}$ (infants of non-smoking mothers). It is concluded that increased hemolysis existing even in utero and due to as yet unspecified cause(s)plays an important role in NSNH. High levels of CoHb (over $1 \%$ ) were found in 61 out of 75 infants with SNJ (mainly from Athens) of various etiologies including prematurity, G-6-PD def. and undetermined causes. COHb measurement proved a valuable method for determining the relative contribution of hemolysis and deficient bilirubin removal in the production of hyperbilirubinemia.

410 B TOCHEMICAL CHARACTERISTICS OF "YOUNG" AND "OLD" ERYTHROCYTES OF THE NEWBORN INFANT. Frank A. OSki. S.U.N.Y., Upstate Medical Center, Department of Pediatrics, Syracuse, N.Y.

Most attempts to characterize the unique features of the newborn's erythrocytes have been confined to an examination of the entire population of erythrocytes. Because the older erythrocytes, present at birth, may be more representative of biochemical processes present in cells produced earlier in gestation, several features of young and old erythrocytes from newborns were contrasted with similar fractions obtained from adults. The oldest cells from the infants had significantly greater phosphoglycerate kinase and enolase activity than did even the youngest cells of adults. In contrast, the phosphofructokinase activity was only $50 \%$ of that observed in the young populations and was significantly less than that observed in the oldest cells of normal adults. Glucose consumption in the "old" cells from infants was less than that of the "old" cells from adults despite the fact that the se fetal cells were only 60 to 80 days of age rather than the 100 to 120 days of age of adult red cells. Analysis of glycolytic intermediates suggested that the decreased phosphofructokinase activity in the newborn's red cells had become rate limiting. These findings indicate that the cells produced early in gestation possess the developmental characteristics of fetal blood to a much more significant degree and are primarily responsible for the differences observed when unfractionated blood of newborns is studied.

411 LACK OF IRON DEFICIENCY IN INFANTS EXCLUSIVELY BREAST FED FOR PROLONGED PERIODS OF TIME. Frank A. Oski and Kathaleen C. Perkins. S.U.N.Y., Ups

Center, Department or Pediatrics, syracuse

It has been stated that prolonged feeding with milk alone will produce iron deficiency anemia because of the low iron content of the diet. Physicians have claimed that tron deficiency anemia is unusual in breast fed infants. In an attempt to clarify these conflicting assertions, studies were performed on 11 infants who had been exclusively breast fed for periods ranging from 6 to 18 months of life (mean 8.1 months). Infants had increased their birth weight from 2.1 to 3.17 times (mean 2.52 times). The mean hemoglobin in this group of infants was $11.5 \mathrm{gm} \%$. Only one infant had a hemoglobin value of less than $11.0 \mathrm{gm} \%(10.6)$. In only one infant was the transferrin saturation less than $16 \%$. In only one infant was the level of free erythrocyte porphyrin elevated or the MCV decreased below a value of $76 \mathrm{u}^{3}$. Calculations indicate that net iron gain in those infants who had grown sufficiently ranged from 8 to 98 milligrams. This suggests that perhaps 20 to $40 \%$ of the one $\mathrm{mg} / \mathrm{L}$ of iron normally present in breast milk may be absorbed. This is much higher than the iron absorption reported to occur with cow's milk. Prolonged feeding with breast milk does not generally result in iron deficiency. Iron deficiency seen with early introduction and exclusive use of cow's milk is probably a result of a combination of factors which include: inadequate dietary iron, reduced absorption, and accelerated iron losses from gastrointestinal bleeding.
412 GRANULOPOIESIS IN CHILDHOOd APLASTIC ANEMIA. Abdelsalam H. Ragab, Ellen Gilkerson, William M Crist, Elna $T$. Phelan and Martha Myers. (Introduced by Philip R. Dodge). Washington University Medical School, St. Louis Children's Hospital, Department of Pediatrics, St. Louis, Mo.

The pathophysiology of aplastic anemia is not completely understood. Extrapolation from animal models suggests that the basic defect may reside either in the bone marrow microenvironment or the pluripotent stem cell. We have investigated various factors in the regulation of granulopoiesis by the soft agar technic in 8 children with aplastic anemia (A.A.). This included 3 children with Fanconi's anemia, 1 post-hepatitic A.A. and 4 idiopathic A.A. It was observed that children with A.A. had markedly reduced granulocytic colony forming cells (CFC) in their bone marrow (0-9 colonies $/ 10^{5}$ nucleated cells plated) when compared to "control" children (15-135 colonies/10 $0^{5}$ nucleated cells plated). The peripheral blood CFC was also decreased when compared to normal children. Colony stimulating activity (CSA) was also decreased in 5 of the 8 children tested. Serum from 6 children with A.A. was added to normal bone marrow cells in culture on normal underlayers. No inhibition of colony formation was observed.

It may be concluded that the defect in A.A. resides in the stem cell, with involvement of the CSA producing cells in some cases. The serum of these patients does not contain an inhibitor of granulopoiesis. Bone marrow transplantation in these cases could replace a defective stem cell with a normal one.

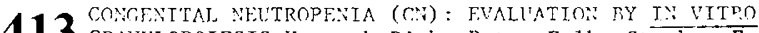

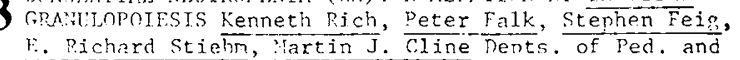
"ed., vCl. school of "ed., ios Anseles, Califonia.

$C:$ renresents a diverse spectrum of clinical and hematolocic abnormalities. "e studied 7 neutronenic natients and 4 parents from 5 families. The patients' aces ranged from 7 mos. to 18 yrs. One patient had immunodeficiency with hyner-I wh whe the remainder had no underlving, disorders. All patients vere severely neutronenic: monocytosis and eosinonhilia were variable. Progranulocytes or promonocytes were present in the $39 !$ aspirates of $7 / 7$ but mature Pins were decreased or absent in 5/7. Fpinephrine stimulation tests failed to provole neutrophilia in $5 / 6$ and piss vere diminished or absent in $4 / 5$ Rebuck skin windos prenarations. To study the mechanism of abnormal rranulonoiesis, Fy: and hlood vere cultured in apar and diffusion chambers and assayed for colony forming stem cells (CFCa) and colony stimulatino factor (CSF). $6 / 7$ patients manifested defective PMon maturation in vitro: $1 / 7$ had defective mononuclear nharocute develonment. CFCa were generally present but only mononuclear colonies were formed. No patient studied had impaired CSF production and addition of exogenous C.SP did not reverse the abnormality in oranulopoiesis. In 2 families the parents had normal marrow differential counts: in $3 / 4$ PM. maturation in vitro was ahnomal and $<5 \%$ Pro colonies were observed in 7 day cultures (nomal $>30 \%$ ). This surgests that culture of $P: M$ of phenotypically normal parents may he used to demonstrate a heterozyote defect in granulopoiesis in some families. These studies indicate that the Cin sundromes can be reclassified on the basis of in vitro granulopoiesis as well as clinical observations.
414 SICKLE CELL ANEMIA: NONINVASIVE CARDIOVASCULAR PROFILE. Judy J.Rigby, William B. Strong, Moo H.Lee, Priscilla Gilman,,$\left(\frac{\text { Intr. by Alex Robertson);Dept. of }}{\text { Int }}\right.$ Ped. Med.Cot.of Ga

Exercise electrocardiography(EECG), systolic time intervals (STI), echocardiography (ECHO), electrocardiography(ECG) and vectorcardiography (VCG) were performed on 12 children with sickle cell anemia. Ages ranged from 5 to 12 years, 7 were male and 5 were female. Hematocrit values ranged from $20 \%$ to $34 \%$. The purpose of the study was to obtain baseline information on cardiovascular performance. Results of EECG demonstrated that all were able to increase their heart rate greater than $100 \%$ of resting values. $25 \%$ $(3 / 12)$ had $J$ point depression and negative slope to the ST segment during exercise. Significant abnormalities of the STI were noted in $2 / 12$ children who had prolongation of the preejection period, left ventricular ejection time and systolic quotient. Sixtysix percent $(8 / 12)$ of children had abnormalities of ECHO measurements: $5 / 12$ left ventricular posterior wall thickness, $8 / 12$ septal thickness, 2/12 LA diameter enlargement and aortic diameter were increased. Left ventricular end diastolic and end systolic volumes were increased in many of these children. This noninvasive profile provides data indicating the hearts of children with sickle cell disease to be dilated and hypertrophied. Subendocardial ischemia was present in $25 \%$ of this series and depression of myocardial contractility occurred in $2 / 12$ patients. In addition to providing insight into myocardial mechanics, this data provides an ideal baseline from which to evaluate therapeutic interventions in these children. 
415 PROMETHAZINE IMMUNOSUPPRESSION OF Rh.ERYTHROBLASTOSIS Arye Rubinstein, Harold Schulman and Arthur I.Eidelman Albert Einstein College of Medicine, Bronx, New York.

Previous studies have indicated that promethazine (PROM) can alter human fetal phagocyte function. Maternally administered PROM immunosuppressive therapy to modify the course of $R h$ hemolytic disease was studied in two mothers whose clinical and laboratory data indicated moderate to severe intrauterine for periods of 5 and 24 weeks in Cases 1 and 2 respectively. Both infants were born non-hydropic at 38 weeks gestation. Case 1 had a cord hct. of 37, required 2 exchange transfusions and developed Group B strep sepsis. Case 2 had cord hct. of 27, a peak bilirubin of 16 and required only simple transfusions at age 1 and 3 days and 6 weeks.

Immunological data revealed a significant decrease in cord blood $T$ cells (E rosettes) 9\% (Case 1) and 6\% (Case 2); control $(n=12) 31-50 \%$. T cell number approached normal by 6 weeks. Mixed lymphoctye culture reactivity (MLR) was normal in Case 1 but absent in Case 2. MLR returned to normal at 4 weeks. Cord lymphocytes EAC 3 rosettes and mitogenic response to PHA, PWM and $C$ on $A$ were normal in both. PROM immunosuppression of $R h$ disease may avoid need for intrauterine transfusion or early induction. The above data, however, indicates that the effects of PROM are not limited to suppression of in utero hemolysis but have broader effects on neonatal immunologic competence. (Intr. by: Lawrence Gartner), Depts.of Ped and Ob/Gyn, hemolysis. PROM was administered in doses of 75 and $150 / \mathrm{mgm} /$ day

418

ASPIRIN (ASA) INDUCED HEMOLYSIS: THE NEED FOR CONCOMITANT OXIDANT $\left(\mathrm{H}_{2} \mathrm{O}_{2}\right)$ CHALLENGE. Stockman, J.A. III, Oski, F.A., Lubin, B.H. State University of New York, Upstate Medical Center, Syracuse, New York, and th Children's Hospital Medical Center, Oakland, California.

Although ASA is not an oxidant compound, it has been implicated as one of the agents capable of inducing hemolysis in G-6-PD deficient subjects. Studies were conducted in an attempt to determine its hemolytic potential at doses employed in clinical practice. ASA (25 mg\%) did not impair red cell G-6-PD, glutam thione peroxidase or catalase activity, glutathione stability or hexose monophosphate shunt activity. Prolonged incubation of cells (Hct. $5 \%$ ) in the presence of either ASA or its oxidant metabolite, gentisic acid, failed to produce hemolysis. Hemolysis was readily produced, however, when red cells were suspended in both ASA ( $25 \mathrm{mg} \%$ ) and $\mathrm{H}_{2} \mathrm{O}_{2}(2.4 \%)$. Hemolysis did not occur with $\mathrm{H}_{2} \mathrm{O}_{2}$ alone but was directly proportional to the concentration of the ASA present in the system. Hemolysis was preceded by lipid peroxidation and could be inhibited by the presence of the antioxidant, thymo1. With $\mathrm{ASA}$ and $\mathrm{H}_{2} \mathrm{O}_{2}$ a marked reduction in membrane phosphotidyl ethanolamine occurred with oxidation of 20 and 22 carbon polyunsaturated fatty acids. No effect on phospho lipids or fatty acids was noted with either $\mathrm{H}_{2} \mathrm{O}_{2}$ or ASA alone. Salicylic acid, singly or with $\mathrm{H}_{2} \mathrm{O}_{2}$, does not exhibit these effects, suggesting the importance of acetylation in the hemolytic process. It is proposed that ASA does not induce hemolysis in G-6-PD deficients except in the presence of concomitant oxidative stress and that hemolysis may be mediated via membrane lipid peroxidation.

419 Eercise tOLERAYCe AS RELATED TO MEMOGLOBIA LEVEL IN SÜBJECTS WITH THALASSEMIA MAJOR. James A. Stockman, EFFECT OF EXCHANGE TRANSFUSION WITH FRESH "SETTLEO" EXCHAC TRASSFUSIONG WTH FRESH "SET LED" BIRTH WEIGHT INFANTS. S. David Rubenstein, Ann R.
K. Anday, and Maria Delivoria-Papadopoulos. Stark, Endla K. Anday, and Maria Delivoria-Papadopoulos. Univ.

Low birth weight infants after exchange transfusion with fresh adult blood show increased $P_{50}$ and $P_{v} 0_{2}$, suggesting facilitation of $\mathrm{O}_{2}$ transport, but decreased $\mathrm{O}_{2}$ content, reflecting a post exchange anemia. The present studies examine the importance of improving the whole blood $\mathrm{O}_{2}$ content by exchange transfusion with settled cells in 11 infants (mean birth weight $980 \mathrm{~g}$ ) in the first 6 hours of life. Donor blood cells were allowed to settle in the bag prior to exchange transfusion, raising the Hct to $60-65 \%$. Infants exchange transfused with whole blood served as controls. Blood gases, $\mathrm{P}_{50}, \mathrm{HbO}_{2} \%$ saturation, and $\mathrm{Hb}$ were measured before and after exchange transfusion, and $\mathrm{O}_{2}$ content and arterial-venous $\mathrm{O}_{2}$ difference (AVD) were calculated. ${ }^{P_{5}} \mathrm{P}_{2}$ rose from 18 to 2.3 and $27 \mathrm{~mm} \mathrm{Hg}$ and mean $\mathrm{P}_{\mathrm{v}} \mathrm{O}_{2}$ rose from 35 to 42 and $46 \mathrm{~mm} \mathrm{Hg}$ at 3 and 24 hours post exchange. Mean Hb rose from 12.5 to $15.5 \mathrm{~g} \%$ post exchange. Mean arterial $0_{2}$ content rose from 16.3 to $21 \mathrm{ml} 0_{2} / 100$ $\mathrm{ml}$ blood after settled cell exchange, while it remained either unchanged or decreased following whole blood exchange. AVD increased from 1.6 to 2.8 and $4.0 \mathrm{ml} 0_{2} / 100 \mathrm{ml}$ blood, reflecting. the decrease in $\mathrm{O}_{2}$ affinity and the increase in $\mathrm{O}_{2}$ content in the settled cell infants. Only 1 infant died, in the settled cell group. These results indicate that the beneficial effects of an early exchange transfusion in low birth weight infants are greatly enhanced by using settled cells, which results in increased $\mathrm{O}_{2}$ content as well as decreased $\mathrm{HbO}_{2}$ affinity.

417 COMPLEMENT LEV'ELS IN TRANSFUSED PATIENTS WITH HOMOZYGOUS Ó-THALASSEMIA. Emily A. Schmalzer, Denis R. Miller, Virgiria C. Canale, Babette B. WeksTer and Noorbibi K. Day, Cornel1 Univ. Med. Col., New York Hospita T, Dept of Ped., and Memorial-Sioan Kettering Cancer Center, New York. Because of the splenic dysfunction and recurrent immune hemolysis seen in transfused thalassemic patients (pts), serum complement $(C)$ and $C$ components were studied in 24 transfusion-dependent pts with $\beta$-thalassemia. Blood was drawn immediately before transfusion. Of these pts, 4 were found to have elevated $\mathrm{CH}_{50}$ with normal immunochemical $\mathrm{C} 3.8 \mathrm{pts}$ had significantly depressed or unmeasurable levels. Of these, 3 had abnormal $\mathrm{CH}_{50}$ and $\mathrm{C}_{3}, 3$ had low $\mathrm{CH}_{50}$ alone and 2 had low $\mathrm{C}_{3}$ alone. $\mathrm{Clq}$ was markedly elevated in 15 pts and normal in the rest. 7 pts were selected for analysis of functional $C$ components. The 4 with unmeasurable $\mathrm{CH}_{50}$ had very low values for $\mathrm{C} 4, \mathrm{C} 2, \mathrm{C} 3, \mathrm{C} 5$ and $\mathrm{C} 6$. In contrast, $\mathrm{Cl}$ was only mildly affected. In the one patient with low but measurable total $\mathrm{C}, \mathrm{C} 2$ was also spared. In the 2 pts with normal $\mathrm{CH}_{50}, \mathrm{C} 2$ was markedly elevated and the remaining components normal. Depression of components did not appear to correlate with age, hepatic function, frequency of transfusion, or presence or size of spleen. There does appear to be a relationship with Coombs' positivity at the time the sample was drawn. These findings suggest that activation of the classical pathway occurs in the subject with Coombs' antibody after transfusion, and that depletion of complement factors may be consequent to tinis.
N.Y., Depts. III, J. liowland Auchincloss and Frank A. Oski. S.U.

llemoglobin levels (Hb) in thalassemia may be maintained in the normal range or at lower values depending on the design of the transfusion program. To study the effects of different $\mathrm{Hb}$ on exercise tolerance, 4 subjects with transfusion dependent thalassemia were exercised at varying llb, a total of 27 times. With obtained in each subject which would consistently raise the heart rate (H.R.) to 150 . The Hb was then varied from 5 to $13 \mathrm{gra}$ and repeated determinations were made of resting and exercise H.R., venous lactate and pyruvate and RBC 2,3-diphosphoglycerate. Fetal $\mathrm{Hb}$ was also obtained prior to each session. Although individual variations were noted, in general raising the $\mathrm{Hb}>10 \mathrm{mg} \%$ did not decrease the maximal exercise H.R. ( $\mathrm{Hb}=10-13 \mathrm{gm} \% \mathrm{H} . \mathrm{R} .=152 \pm$ 10), but at $\mathrm{Hb}<9 \mathrm{gm} \%$, maximal exercise $\mathrm{H} . \mathrm{R}$. began to rise, recovery of baseline $H . R$. increased markedly. The increment rise of lactate post-exercise was relatively constant between 9-13 gm\% $\mathrm{Hb}$ but rose progressively at $\mathrm{Hb}<9 \mathrm{gm} \%$. Non-thalassemic subjects with increased $\mathrm{P}_{50}$ values despite $\mathrm{Hb}$ of $6-8 \mathrm{gm} \%$ seemed to perform well in comparison to subjects with thalassemia. These studies suggest that maintenance of $\mathrm{Hb}>9 \mathrm{gm} \%$ seems to be associexercise tolerance may be useful in the long-term evaluation of subjects with thalassemia major.

420 THE ALternative Pathway OF COMPLEMENT Ronald G. Strauss, Judith Forristal and Clark D. West, Univ. of Cincinnati College of Medicine, Dept. of Ped. (Intr. by Joseph V. Simone). explain the increased susceptibility to overwhelming bacterial infections present in this disorder. In an attempt to identify this abnormality, the competence of the APC was assessed in sera from 31 children with SS. Concentrations of properdin, C3 proactivator (C3PA), C3 and C3 inactivator were equal to controls when measured by radial immunodiffusion using monospecific antisera. Classical hemolytic activity (CH50) was normal. Activation of the APC by reacting serum with inulin was equal to controls when assessed by shift of C3PA mobility from that of a beta- to a gammaglobulin, cleavage of $\mathrm{C} 3$ (decrease in $\mathrm{B}$ antigen concentration and increase in anodal mobility) and consumption of lytic components (decrease in $\mathrm{CH} 50$ ). Function of the APC, indicated by lysis of erythrocytes treated with reduced glutathione, was equal to controls in the presence of inulin or cobra venom factor. Although the APC was normal in whole serum, an rendering serum $0.01 \mathrm{M}$ with respect to ethyleneglycoltetraacetic acid (EGTA). This abnormality probably was an artifact of chelation because complement functions were normal when the same EGTA sera were supplemented with $\mathrm{Mg}^{++}(0.004 \mathrm{M})$. The opsonic defect in SS could not be explained by an abnormality in the APC. The $\mathrm{Mg}^{+*}$ content of EGTA sera must be considered in future studies before confirming the presence or absence of an alternative pathway defect in SS. graded treadmill exercise, at $10 \mathrm{gm} \% \mathrm{Hb}$, a standard work load was reaching $H . R .=175+15$ at $5-7 \mathrm{gm} \% \mathrm{Hb}$. At $\mathrm{Hb}<9 \mathrm{gm} \%$, time to ated with optimal exercise performance. Simple determinations of ACTIVATION (APC) IN SICKLE CELL ANEMIA (SS).

The previously reported defect of the heat labile opsonins in SS may abnormality similar to that previously reported could be produced by 
421

NHIBITION OF THE PLATELET RELEASE REACTION BY HYDROCEN PEROXIDE. Marie J. Stuart, Holm Holmsen Dept. of Pediatrics, Syracuse, N.Y., and Thrombosis Research Center, Temple University lospital, Philadelphia, $\mathrm{Pa}$.

The in vivo generation of hydrogen peroxide $\left(\mathrm{H}_{2} \mathrm{O}_{2}\right)$ by wite blood cells may effect platelet function. A previous study has demonstrated that micromolar concentrations of $\mathrm{H}_{2} \mathrm{O}_{2}$, although unable to cause detectable platelet aggregation, does cause enhancement of both first phase platelet aggregation by Adenosine Diphosphate (ADP) and subsequent disaggregation. We have studied the effect of $\mathrm{II}_{2} \mathrm{O}_{2}$ on platelet aggregation and metabolism by the pre-exposure of platelets to $\mathrm{H}_{2} \mathrm{O}_{2}$ followed by the addition of the aggregating agent ADP in concentrations normaliy sufficient to induce biphasic aggregation and release. The experiments were performed using platelet rich plasma (PRP) previously labelled with tritiated Adenine. $\mathrm{H}_{2} \mathrm{O}_{2}(200$ to $500 \mathrm{NH}$ final concentration) when incubated for 3 minutes with PRP caused inhibition of ADP induced biphasic aggregation and the release of adenine nucleotides from the nonlabelled storage pool when compared to control platelets. This inhibition of release was associated with a mean fall of $58 \%$ in the level of platelet metabolic adenosine triphosphate, concommitant with increases in inosine monophosphate and hypoxanthine to approximately four times basal levels. Platelet-granulocyte interaction may therefore be important in the regulation of in vivo thrombus formation.

422 DEVELOPMENTAL CHANGES OF RED CELL GLYCOLYTIC ENZYMES AND II:TERMEDIATES IN NORMAL TERM INFANTS DURING THE FIRST YEAR OF LIFE. Susan F. Travis, Joan M. Solow, Bakulesh Patel, Pedro D. Paez, and Maria Delivoria-Papadopoulos. Univ. of Pennsylvania and Thomas Jefferson Univ., Phila., Pa.
Previous studies of erythrocyte glycolytic enzymes and inter mediates in normal term infants on day 1 of life show decreased activity of phosphofructokinase (PFK); increased activity of enolase and phosphoglycerate kinase (PGK); increased glucose-6phosphate (G6P), fructose-6-phosphate (F6P), and dihydroxyacetonephosphate (DHAP); and decreased phosphoenolpyruvate (PEP). In the present study, 8 normal term infants were studied sequentially to determine at what postnatal age red cell glycolytic intermediates approach normal adult levels. Mean values of PFK activity increased significantly from 139.4 (day 1) to 174.3 (8-9 weeks) and slowly thereafter to 188.8 units/min/100 ml RBC (11-12 months). Enolase activity decreased from 341.3 (day 1) to 288.0 (3-4weeks) and continued to decrease to 185.0 units/min/100 ml RBC $(14-16$ weeks). PGK did not decrease significantly until 14-16 weeks, from 4409 (day 1) to 3397 units/min/100 ml RBC (14-16 weeks). Changes in glycolytic enzymes occurred despite the persistence of a young red cell population, as shown by elevated levels of pyruvate kinase. Changes of glycolytic intermediates did not parallel those observed for enzymes, e.g., G6P progressively increased to 86.1 at $8-9$ weeks from $54.4 \mathrm{nmoles} / \mathrm{ml} R B C$ on day 1 . These data suggest that developmental changes in glycolytic enzymes coincide with the disappearance of fetal $\mathrm{Hb}$, and those in glycolytic intermediates may be influenced by other intra- or extracellular control mechanisms.

423

TRANSIENT PYRUVATE KINASE (PK) DEFICIENCY IN NEWBORNS. Timos Valaes, Kalliopi TsochatzisYiannopoulos, Aris Karaklis, and Thomas F. Necheles. The Institute of Child Health, Athens and Hospital, Boston (Intr. by S.S. Geliis).

Cord blood from normal term newborns (NTN) (297 in Athens and 250 in Boston) and blood from 222 Greek infants with severe neonatal jaundice (SNJ) was tested with a battery of screening tests for the detection of red cell enzyme defects. An abnormal PK screening test was obtained in 2.6\% of the Greek NTN and in $2.2 \%$ of the SNJ. In Boston the percentage was 1.2 and among infants of South Luropean origin 3.3 ( 2 out of $60)$. In both series NTN with FK abrormality showed no evidence of increased hemolysis. All 5 infants of the SNJ group and 4 of the 8 Greek NTN with neonatal abnormal $F K$ screening test were re-examined at the age of 9-17 mo. Normal PK screening, normal values for PK activity and normal blood picture was found in all of them. In one of the SNJ infants DK activity (measured) on the 4 th day was $50 \%$ of that found at 9 mo. These data indicate that a transient PK deficiency exists in a small percentage of otherwise normal newborns and that this deficiency is not related to neonatal jaundice. Previous reports, particularly from Asian populations, of a high incidence of PK deficiency among jaundiced newborns should be re-examined in the light
424 THE EFFECT OF INTRACELLULAR CAICIUM ON DEFORMABILITY AND MORPHOLOGY OF NORMAL AND SICKLE RBC'S. Thomas R. Walters, Patricia N. Farnsworth, Patricia Burke and Suzie King. (Intr. by Franklin Behrle). New Jersey Med. Sch., Dept. of Ped., Med. and Physiol., Newark.

Intracellular Calcium $\left(\mathrm{Ca}_{i}^{2+}\right)$ accumulation as well as ATP-depletion in human RBC's is considered an important factor in the loss of cellular elasticity, its filterability and therefore its survival time. This is of particular significance in sickle RBC's since these cells have elevated $\mathrm{Ca}_{1}^{2+}$ levels, decreased deformability and decreased survival times. In our experiments, normal and sickle RBC's were incubated in Trinitrocresol (TNC) containing either $5 \mathrm{mM} \mathrm{Ca}^{2+}$ or none, according to Dunn (BBA 352: 97 , 1974). This technique has been shown to alter intracellular $\mathrm{Ca}^{2+}$ without conconmitant cellular ATP-depletion. As Ca2t was varied by TNC incubation, normal and sickle RBC deformability and morphology were studied by cone-plate viscometry and scanning electron microscopy. In the control experiments, sickle cells contained a mean $\mathrm{Ca}_{i}^{2+}$ of $0.373 \mathrm{mM} / \mathrm{L}$ cells whereas normal $\mathrm{RBC}^{\mathrm{s}}$ contained a mean of $0.014 \mathrm{mM}$. Following $\mathrm{Ca}^{2+}$ loading, sickle cell increased their $\mathrm{Ca}_{1}^{2+}$ 2-6 fold; in contrast with normal cells, there was a 20-40 fold increase. Increased $\mathrm{Ca}_{i}^{2+}$ resulted in decreased cellular deformability. Sickle cells and normal $\mathrm{RBC}^{\prime} s$ with comparable $\mathrm{Ca}_{1}^{2+}$ were found to have similar deformability even though their morphology, as seen by SEM, differed considerably. These data suggest that in addition to the presence of abnormal hemoglobin, an increase in intracellular $\mathrm{Ca}^{2+}$ in sickle cell anemia is an important factor
ity and consequentiy cell survival time.

425 THE TNFLUENCE OF FORMULA IRON FORTIFICATION AND FAT COMPOSITION ON VITAMIN E DEFICIENCY INDUCED HEMOLYTIC ANEMIA OF THE PREMATURE INFANT. Margaret L. Williams, Roger J. Shott, Patricia L. O'Neal and Frank A. Oski. S.U.N.Y., Upstate Medical Center, Dept. of Pediatrics, Syracuse.

The incidence of a vitamin $E$ associated hemolytic anemia anang premature infants differs from nursery to nursery. This suggests that other dietary variables may contribute to its clinical expression. To test the role of 2 dietary factors - the quantity and nature of the polyunsaturates of the formula and the presence of iron fortification - in the development of a vitamin $\Sigma$ deficiency hemolytic anemia in infants weighing less than $1500 \mathrm{gms}$ at birth, infants were fed Similac ( $32.4 \%$ linoleic acid) or SMA (12.8\% linoleic acid) with or without iron fortification. Eight infants were fed each of the 4 diets. At age 5 wks., infants fed the low unsaturate, with or without iron, and the infants fed the formula rich in unsaturates, but without iron, had simflar mean $\mathrm{Hb}$ ( 9.6 to $10.6 \mathrm{gms} \%$ ), while infants fed a formula containing both the increased unsaturates and iron had a significantly lower mean $\mathrm{Hb}$ ( 8.1 gms\%). These infants also had higher reticulocyte counts $(5.2 \%$ vs. $2.3 \%$ ). Infants fed the high unsaturate milk were functionally $\mathrm{E}$ deficient as reflected in their $\mathrm{H}_{2} \mathrm{O}_{2}$ hemolysis tests ( 53 and $62 \%$ vs. 1.8 and $3.2 \%$ in the low unsaturate group) but only the iron supplemented group had hematologic abnormalities. E deficiency hemolytic anemia in prematures requires at least 3 factors: 1) lack of sufficient antioxidant; 2) increase in peroxidizable substrate, i.e., diet polyunsaturates; and 3 ) the presence of an oxidant such as iron.

126 ERYTHROCYTE PORPHYRIN ASSAYS IN THE DETECTION AND EVALUATION OF LEAD POISONING IN CHILDREN: COMPARISON OF METHODS. H. Zarkowsky, D. Dietzler, S. Gupta, C. Smith, T. Tinker, V. Jonsson, D. Jones. Washington Univ. Sch. of Med., St. Louis Children's Hospital and St. Louis City Health Division. (Intr. by D. Goldring).

Free erythrocyte porphyrin determination has been recommended as a simple rapid alternative to blood lead determination in screening for lead poisoning. To select a procedure for general use, three methods for micro-measurement of erythrocyte porphyrins were evaluated using a general purpose laboratory filter fluorometer: 1) double extraction with ethyl acetate/acetic acid and HCl (Piomelli, J. Lab. Clin. Med. 81:932,1973); 2) single extraction with acidified acetone (Chisholm, J. Ped. 84:490, 1974); and 3) single extraction with $94 \%$ ethanol (Murphy, pers. comm.).

The double extraction procedure gave complete recovery of the porphyrins. It is suitable as a reference procedure, but lacks the overall simplicity of single extraction methods. With the acetone procedure, recovery of porphyrin was low $(9-14 \%)$ and quite variable. The ethanol procedure yielded a higher $(60 \%)$ and more consistent recovery of porphyrin over a wider range of values and was adopted for routine use. With this procedure, patients with blood lead concentrations greater than $50 \mathrm{\mu g} / 100 \mathrm{ml}$ invariably had erythrocyte porphyrin concentrations of greater than $100 \mu \mathrm{g} / 100 \mathrm{ml}$. $\mathrm{RBC}$. Since the erythrocyte porphyrin assay measures the effect of lead on a sensitive physiological process, it has the potential to be useful not only in screening but also in the evaluation of the disturbance of function in patients with elevated lead concentrations in blood. 


\section{IMMUNOLOGY}

427

EETAL THYMUS TRANSPLANTS IN CELLULAR IMMUNODEFICIENCY OISORDERS Arthur J. Ammann, Diane W. Wara, Nancy E. Immunology Section, Dept. of Peds. Dept. of ob-Gyn.

Five patients with cellular immunodeficiency were evaluated prior to and following fetal thymus transplantation. Three patients had thymic hypoplasia with abnormal immunoglobulin synthesis, one ataxia-telangiectasia and one Wiskott-Aldrich syndrome. All had various degrees of abnormal antibody and cell mediated immunity assayed by delayed hypersensitivity skin tests, lymphocyte response to mitogens and allogeneic cells, T-cell rosettes, immunoglobulins and antibody response. Transfer factor was given prior to transplantation. Fetal thymus was obtained from 12 to 20 wk. gestational age prostaglandin $F 2 a$ abortions and transplanted intraperitoneally. Reconstitution of cellular immunity occurred in 3 of 5 patients. Some reconstitution of antibody immunity occurred in 2 of 5 patients. Two patients ( 1 died shortly after transplantation) failed to show evidence of reconstitution. Reconstitution occurred within weeks and was temporary (1 month to 2 years). Repeated reconstitution could be achieved by additional transplantation. Cell chimerism or graft versus host reaction was not observed. These studies suggest that past failure of thymic transplants may have been related to technical approaches and that transfer factor-thymus transplantation may provide complete but temporary reconstitution. The lack of chimerism and graft versus host reaction, the temporary nature, the onset within weeks, suggest that reconstitution was by a hormonal mechanism distinct from that achieved in the Di Ceorge syndrome or severe combined immunodeficiency.

428 IMMUNOGLOBULINS IN RESPIRATORY TRACT SECRETIONS. Ranjit K. Chandra, B.M. Abrol, S.K. Kacker, J.R. Talwar and $N$. Gopinath (Intr. by P.L. Ogra) Depts. of Ped., ENT, and Thoracic Surg., All India Inst. of Med. Sci., New Delhi, India.

Immunoglobulins $\mathrm{G}, \mathrm{A}, \mathrm{M}$ and $\mathrm{E}$ were estimated in the serum and respiratory tract secretions of patients with a variety of infective and allergic conditions. By determination of serum concentrations and the levels of albumin and a 2 -macroglobulin, the amounts of immunoglobulins produced locally and through passive vascular permeability, were calculated. Locally derived IgE was found in a large quantity in patients with atopy, and correlated with IgE-producing plasma cells and eosinophilia. Local IgA synthesis was pronounced in the nonatopic group with infection. It is suggested that the estimation of locally derived immunoglobulins may help in etiopathogenetic diagnosis.
430 IMMUNOLOGICAL FINOINGS IN PATIENTS WITH RECURRENT E PERSISTENT RESPIRATORY PROBLEMS. Flossie Cohen, Rukmani Raghunath, \& Barbara Rowe. Wayne State Univ. Sch. of

The Clinical Immunology Service at CHM tested host-defense nechanisms associated with recurrent \& persistent respiratory problems in 105 patients referred by practising pediatricians $\varepsilon$ allergists. Patients divided into 4 groups, based on onset $\varepsilon$ duration of problem:Gr.I(19) onset<12mo., duration<12mo. (infants312mo.); Gr.II(63) onset<12mo., dur. $>12 \mathrm{mo}_{0}$; Gr.III(11) onset $12-24$ mo., dur.>12mo.; Gr.IV(12) onset $>24 \mathrm{mo}$., dur.>12mo. Of the 105 patients, $34 \%$ were atopic; $46 \%$ had alveolar involvement (X-ray), $\xi$ $54 \%$ had pre-alveolar involvement (URI, bronchitis, bronchiolitis).

The tests reveal four main points: First: $60 \%$ of al1 patients had immunodeficiency (IO). This high percentage results from the large number of tests performed:a) humoral immunity(B-cells, Igs, viral \& bact, antibody), b) cellular immunity (T-cells, DHR, in vitro response to mitogens $\varepsilon$ antigens), $\varepsilon$ c) PMNL function (NB reduction, bactericidal, phagocytosis, chemotaxis, \& $\left.C^{\prime} 3\right)$. I0 types were: humoral $70 \%$, cellular $26 \%$, \& PMNL $20 \%$. Second: IgA was absent in $8 \%$ \& 1 ow in $20 \%$. Third: In Gr.I, $27 \%$ of the IDs had atopic stigmata suggesting future bronchial asthma, $E$ their longterm outcome should afford clues to early identification \& therapy. These patients were given gammaglobulin, with early favorable short-term response. (of the entire group, $80 \%$ had ID, largely humoral \& transient; $26 \%$ had alveolar involvement not associated with atopic features). Fourth: In Gr.III there was a significant association between atopy $\&$ ID $(p<0.05)$. In Gr. IV,

431 DEFECTIVE LEUKOTAXIS IH A CHILD WITH RECURRENT INFECTIONS. A. Todd Davis, Pamela G. Grady, Emmanuel Shapira, Lauren M. Pachman. Northwestern Univ. Med.Sch. Children's Mem. Hosp., Dept. of Ped., Chicago.

This ten year old girl with a long history of recurrent bacterial infections and eczema was found to have an abnormal Rebuck skin window test. In vitro chemotaxis assay tests showed a total absence of chemotactic activity of her polymorphonuclear leukocytes (PMN's) when suspended in autologous serum or plasma. Heterologous sera or plasma restored chemotactic activity to varying degrees, ranging from 0 to $100 \%$ of normal. Conversely, $25 \%$ patient plasma had variable effects on PMN's from 11 normal donors; 2 were not affected by patient plasma, 4 were partially inhibited and 5 were completely inhtbited. A reciprocal effect independent of $A B O$ group existed between a given normal and the patient. If $25 \%$ patient's plasma totally inhibited the donor's PMN's, the donor's plasma totally restored chemotactic activity to the patient's PMN's. If the patient's plasma had no effect on the donor's PMN's the donor's plasma did not restore activity to the patient's PMN's. The inhibitor fraction of the patient's plasma was non-dialyzable. The patient's plasma did not inhibit bacterial chemotactic factor, and the serum exhibited normal zymosan activation of chemotactic activity. Other immunologic studies-random mobility of PHN's, phagocytosis and killing of bacteria, plasma and sera complement levels, latex fixation testwere normal. Immunoglobulin levels were: IgG-1750mg\%, IgA-1060 $\mathrm{mg} \%, \mathrm{~g} M-390 \mathrm{mg} \%$ and a normal $1 \mathrm{gE}$. It seems likely that the patient's predisposition to bacterial infection could be attributed
to her leukotactic inhibitor. (NIH GrantsMOI-RROOI9985SOIRRO547512)

432 ROLE OF MICROTLBULES AND MICROFILAMENTS IN COMPJ,EMEN -MEDIATED RELEASE OF HISTAMINE. Elton Dupree, J. Andrew Grant, Armond S. Goldman, Depts. of Pediatrics and Internal Med., Univ. of Tex. Med. Branch, Galveston, Tex.

We have previously shown that activation of the classical or alternate pathway of complement (C) produces in vitro release of histamine from basophils of normal or allergic humans. The generation of this histamine-releasing factor can be blocked by antibodies to $\mathrm{C} 3$ or $\mathrm{CS}$ or by heating of serum at $56^{\circ} \mathrm{C}$ and is independent of IgE-mediated histamine release. However, like allergen-mediated histamine release, the C-mediated process is dependent upon divalent cations, but it is much more rapid and is virtually complete by one minute.

Recently, we have conducted in vitro studies regarding the role of microtubules and microfilaments in $\mathrm{C}$-mediated release of histamine. Colchicine and vinblastine, drugs that prevent assembly of microtubules, inhibited C-mediated release of histamine. Deuterium oxide $\left(\mathrm{D}_{2} \mathrm{O}\right)$, an agent that promotes assembly of microtubules, enhanced histamine release by more than $50 \%$ and reversed the inhibition of release by colchicine. Cytochalasin $B$, a drug which disrupts microfilaments, markedly enhanced $C-$ mediated histamine release at a concentration of 5 micrograms/ml, but inhibited release at higher concentrations.

This study demonstrates that microtubules and microfilaments are involved in the $\mathrm{C}$-mediated release of histamine from human basophils, a process that may be a central mechanism in inflammatory and hypersensitivity reactions. 
433 CELLLULAR IMMUNE RESPONSES OF BRONCHIAL ASTHMA PATIENTS TO THEIR SKIN POSITIVE ANTIGENS. Charles $\mathrm{H}$. Feldman*, Rajesh M. Bhatnagar*, John B. Zabriskie*, Bernard Feldmant, Wiiliam Davis *The Rockefeller University,

The celi-mediated immune response of eighteen bronchial asthma patients has been investigated by means of two lymphocyte function tests, blast transformation (BT) and migration inhibition (MI), and compared to conventional skin tests (ST) in these patients. The in vitro responses of these patients were tested with six antigens utilized for in vivo skin-testing evaluation. The best correlation was noted in those patients with positive skin tests. $60 \%$ of these individuals had positive transformation indices to four of the six antigens studied $(\mathrm{P}<.01)$. Only three instances of positive transformation indices were seen in 43 negative skin reactors. An examination of the duration of hyposensitization treatment with respect to skin testing and transformation indices reveal ed the following: a much higher frequency of patients on long-term therapy ( 1.8 years) had positive ST and negative BT when compared to a recently treated $(0.8$ years) group in which ST and BT were both positive. Almost identical results were obtained with direct leucocyte migration inhibition tests. These parameters of cellular immunity show good correlation with ST results and may provide an effective means of evaluating the status of asthmatic patients. This quantitative in vitro measure of function may thus provide more accurate criteria for assessing the efficacy of immunotherapy in atopic diseases. A long-term study is currently being performed.

\section{4} DIABETES AND NEUTROPHIL CHEMOTAXIS. Senih M. Fikrig, Churku M. Reddy, Eduardo orti, Llewellyn Herod, Kamala Suntharalingam. Downstate Med. Ctr., Dept. of Ped., Brooklyn, N.Y.

Impaired polymorphonucleax (PMN) chemotaxis and deficiency in generating chemotactic activity from sera or plasma of diabetic patients have been reported. Since objective clinical studies do not show an increase in the incidence of infections in well controlled diabetics, present study was undertaken to re-evaluate the above findings. Chemotaxis was studied in modified Boyden's chambers and chemotactic activity was generated from fresh serum by incubation with zymosan. Final evaluation was made by taking the average measurements in five random fields a) of the distance travelled from the top of the filter to the furthest plane by at least 2 cells and $b$ ) of the number of neutrophils found on the lower surface of the filter. Similar measurements were made in filters obtained from control chambers without chemotactic factors. The difference between the two values were reported as final chemotaxis. The results demonstrated no difference in chemotaxis between the PMN of adult, juvenile diabetics and appropriate controls. Furthermore the chemotactic activity generated by diabetic serum was no different than the one generated by normal serum.

\begin{tabular}{lccc} 
& \multicolumn{2}{c}{ Distance Travelled } & Number of PMN \\
\cline { 2 - 3 } & Mean \pm S.D. (Range) & Mean \pm S.D. (Range) \\
Adult Diabetics & $41 \mu \pm 12.3(19-72 \mu)$ & $86 \pm 17(10-229)$ \\
Adult Controls & $39 \mu \pm 13.1(18-71 \mu)$ & $52 \pm 13(19-119)$ \\
Juvenile Diabetics & $50 \mu \pm 9.5(31-67 \mu)$ & $49 \pm 11(11-128)$ \\
Juvenile Controls & $53 \mu \pm 13.2(25-72 \mu)$ & $36 \pm 7(11-66)$
\end{tabular}

435 ABNORMAL CHEMOTAXIS IN BURNS. Senih M. Fikrig, seung C. Karl, Greg Rauscher, Kamala Suntharalingham, Llewellyn Herod. Downstate Med. Ctr., Depts. Of Ped. and Surgery, Brooklyn, N.Y.

Disturbances of cellular defense mechanisms following severe thermal injury have been only partially analysed. Present investigation was undertaken to study polymorphonuclear (PMN) chemotaxis in burns and to try to correlate the findings with the development of infection and "burn toxemia". Chemotaxis was studied in modified Boyden's chambers using a $5 \mu$ filter. Normal WBC as well as WBC from patients with burns were tested. Chemotactic activity was generated from fresh and burn serum with Zymosan and compared with the chemotaxis in chanbersi containing plain TC 199. Final evaluation was made by measuring and averaging in five random fields the furthest distance travelled by at least 2 cells and then counting the total number of PMN at that distance in additional five random fields. Three patients with $3^{\circ}$ burns of 5,24 and $30 \%$ and one patient with $22 \%, 20$ burns were found to have abnormal chemotaxis. The abnormality except for the $2^{\circ}$ burn was associated with clinical signs of "burn toxemia" and positive bacterial cultures taken from the burned axeas. Systemic blood cultures remained negative. Serum from the burned patients had no adverse effects on the generation of chemotactic activity. Improvement of clinical signs in each case was associated with the return of chemotaxis to normal values. Transient abnormality of PMN chemotaxis associated with burns may undermine the ability of these patients to combat local or systemic infections.
436 BEHCET'S SYNDROME IN CHILIIIOOD. L. I). Frenkel, H. Bauer, and J.A. Bellanti. Departments of Pediatrics and Pathology, Georgetown Lniv. Sch. of Med., Washington, I). C. Jehcet's syndrome, a systemic inflammatory condition usually characterized by the triad of aphthous stomatitis, uveitis, and genital ulcers is rarely diagnosed in the lnited States and has not been reported in young children. A 14-year-old white girl with onset of symptoms at age 3 years demonstrated progressively more frequent and severe oral and perineal lesions with the onset of puberty, There was no evidence of colitis, ar thritis, ocular or CNS lesions, Biopsy of a perineal lesion demonstrated a nonspecific vasculitis consistent with this diagnosis.

Pertinent laboratory data include the following abnormal results: elevated WBC $(13,000-16,500)$ with a prominent polymorphonuclear response; elevated $\alpha 2\left(2.1 \mathrm{gm}^{\prime}\right)$, elevated fibrinogen level of $500 \mathrm{mg} / ;$; reticulocyte count $2.6 \%$ in the absence of anemia. In spite of a prior history of exposure to herpes simplex virus and immunization with trivalent oral polio vaccine, titers to HSV-1, HSV-2, poliovirus I and poliovirus II were not detected and a titer of 1:32 to poliovirus III was demonstrated.

Immunologic evaluation included normal valucs for IgG, IgA, Ig M, and IgF. Tests of delayed dermal reactivity were positive using SK-SI) and mumps and negative with candida in spite of persistent oral and genital candida colonization. Tests of lymphocyte function including PHA stimulation and E-rosette formation were normal as was NBT dyc reduction by neutrophils. Azathioprine therapy was ineffective but partiai improvement has been noted with steroids and transfer factor.

437 STUDIES ON THE FIFTH COMPONLNT UF COMPLEMENT(CS). COMPARISON OF HEMOLYTIC AND OYSONIC ACTIVITLES IN BOVINE MILK ANU HIMMAN PLASMA. R.G. Ganges and M.E. Millex, Dept. of Peds., Charles Drew Sch., Los Angeles 90059 .

We nave previously demonstrated $C 5$ dependent opsonisation of yeast particles in human plasma and functionally similar activities in human, animal,proprietary and soy milks. We now compare complement dependent functions in milk and plasma, in order to determine whether the opsonic activity of milk is identical to that of human plasma(i.e.-C5 dependent) or merely analagous and dependent upon a material with complement-like activity.Effective molecule titration of human plasma and bovine milk was carried out by lysis of EAC140xy23 ce1ls in che presence of a human serum source of C6-C9 (KCNS treated). Over a wide range of concentrations, no hemolytic C5 activity of milk was demonstrated. Despite this, concentrations of milk as low as $1 \%$ of normal had full yeast opsonic activity in reconstitution of either dysfunctional human C5 or C5 deficient mouse serum. By contrast, in no instance did human plasma devoid of hemolytic activity demonstrate opsonic activity. These observations are significant for the following reasons: 1) The demonstration of full yeast opsonic activity but absent hemolytic activity in bovine milk differs from studies of purified human $C 5$ in which some preparations which are opsonically deficient may have normal hemolytic activity but not the converse. This suggests that the opsonically active fragment in milk is not identical to $C 5 ; 2$ ) Since we have previously shown inhibition of milk yeast opsonic activity with anti-c5, it follows that the active principle in milk is structurally similar to human c5.

13 EFFECTS OF CORTICOSTEROID HORMONES ON EOSINOPHILO-

438

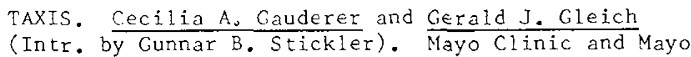
Foundation, Rochester, Minnesota.

Corticosteroids have long been known to greatly ameliorate allergic inflammatory reactions but the mechanisms of these beneficial effects are largely obscure. We tested the effects of hydrocortisone and methylprednisolone on eosinophilotaxis using the eosinophil chemotactic factor of anaphylaxis (ECF-A) and zymosan-treated guinea pig serum ( $\mathrm{C} 5 \mathrm{a}$ ) as attractants for guinea pig eosinophils in a modified Boyden chamber. ECF-A was obtained by homogenizing human lung tissue. Both chemotactants had the expected molecular weights when tested by gel filtration. ECF-A and Cya induced brisk migration of cosinophils. Addilion of and Cfa induced brisk miglation of cosinophils. Addition of dose-dependent inhibition of chemotaxis. With doscs of hydrocortisone and methylprednisolone of more than $1 \mathrm{mg} / \mathrm{ml}$, eosinophilotaxis was essentially suppressed whereas $0.1 \mathrm{mg} / \mathrm{ml}$ did not exert any inhibitory effect. Mixing hydrocortisone with the chemotactant yielded no inhibition, suggesting that the effect of this drug is directly on the cell, Another anti-inflammatory agent, chloroquine, also caused a dose-related inhibition of chemotaxis. In a concentration of $10^{-5} \mathrm{M}$, this drug caused $50 \%$ inhibition of eosinophil migration in response to ECF-A and $70 \%$ inhibition of eosinophil migration in response to $\mathrm{C5}$ a. These results indicate that corticosteroids and chloroquine exert a suppressive action on the migration of eosinophils, and they may explain, in part, the mechanism by which these drugs alter allergic inflamation. 

439 SERUM IgE LEVELS IN CAUCASIANS IN CENTRAL
AND IN METIS(NORTH AMERICAN INDIANS) IN NORTHERN SASKATCHEWAN. John W.Gerrard, Patty $\frac{\text { Hansen, Carol Geddes. Col. of Med., Univ.of Saskatchew- }}{\text { an, Univ. Hosp., Dept.of Pediatrics, Saskatoon, Sask. Canada }}$ an, Univ. Hosp., Dept. of Pediatrics, Saskatoon, Sask. Canada. of 176 Caucasian (C) families living in central Saska tchewan and in 275 of 58 Metis (M) families living in an isolated community in northern Saskatchewan. Mean IgE levels, adjusted for age, were $85 \mathrm{u} / \mathrm{ml}$ in the $\mathrm{C}$ and $275 \mathrm{u} / \mathrm{ml}$ in the $M$. Even though IgE levels were much higher in the $M$ than in the $C$, the prevalence of asthma $(P<0.05)$, eczema $(P<0.001)$ and urticaria $(P<0$. $001)$ was significantiy less. (See Table).

TABLE. PREVALENCE OF ASTHMA, ECZEMA AND URTICARIA IN CAUCASIAN AND METIS POPULATIONS.

\begin{tabular}{lrrrrrr}
\hline & \multicolumn{2}{c}{ Asthma } & \multicolumn{2}{c}{ Eczema } & \multicolumn{2}{c}{ Urticaria } \\
\hline No. & $\%$ & No & $\%$ & No. & $\%$ \\
\hline Caucasian & $38 / 858$ & 4.4 & $149 / 858$ & 17.0 & $82 / 858$ & 9.5 \\
Metis & $7 / 418$ & 1.6 & $10 / 418$ & 2.4 & $9 / 418$ & 2.1
\end{tabular}

IgE levels in the $C$ and $M$ populations are highest
in the 3-13 year-old age groups, ages at which many infections are encountered for the first time. We suggest that IgE plays an important role in the body's defences against such infections, and when primed repeatedly by such infections atopic diseases are less i ikely to develop subsequently.

440 ANTIBODY PRODUCTION BY HUMAN COLOSTRUM CELLS, Randall M. Goldblum, Stephan Ahlstedt, Barbara Carlsson, and Lars A. Hanson, Dept. of Pediatrics, The University of Texas Medical Branch, Galveston, Texas and Institute of Medical Microbiology, Goteborg, Sweden (Intr. by Armond S. Goldman).

Human colostrum and milk contain leukocytes which may be important in host defense. Among these are lymphocytes which produce $\lg \mathrm{A}$. In the present study, the production of antibacterial ( $E$. coli) antibodies by these cells was investigated using the hemolysis-in-gel technique In this assay, human RBCs coated with a pool of antigens from common strains of $E_{0}$ coli are lysed by the antibody produced by single cells when appropriate antiimmunoglobulin and complement reagents are added.

Only cells producing $\lg A$ antibodies were found. The number of the cells was greatest on the third day postpartum. When plaques were developed with an anti-secretory component serum, most of the IgA produced was shown to be of the secretory type $(s \lg A)$.

In order to examine the mechanism of sensitization of colostrum lymphocytes to $\mathrm{E}$. coli, the gastrointestinal tracts of three pregnant women were colonized with an unusual strain of $E_{\text {. coli }}(083)$. In each case, cells producing $s \lg A$ antibodies to the $08 \overline{3}$ bacterio appeared in the colostrum within one week of ingestion of the bacteria. As there was no change in the serum antibody titer, antigenemia was unlikely. Therefore, it would appear that sensitized cells from the gastrointestinal tract migrated to the breast. This suggests a system for transferring specific antibacterial antibodies and lymphocytes from the mother to the infant.

441 DEFECTIVE SERUM OPSONIC ACTIVITY AND DEFECTIVE NEUTROPHILS IN A CHILD WITH SEVERE INFECTIONS. William H. Greene, Jonathan M. Gerrard, Harry R. Hill, William $\frac{\text { Krivit and Paul Quie. }}{\text { Peds., Minneapolis. }}$

Studies of immune function were done in a $2 \frac{1}{2}$ year old oirl with recurrent pneumonia, E. coli sepsis, D. preumoniae meningitis, splenectomy and nodular lymphoid hyperplasia of the GI tract. Normal values include $T$ and $B$ cells, mitogen stimulation with PHA, Con-A: IgG, IgM, IgA, IgE; Rebuck skin window; $\mathrm{CH}_{50}, \mathrm{C}_{3}, \mathrm{C}_{3} \mathrm{PA}$ and properdin. Normal neutrophil studies include NBT reduction, phagocytosis and killing of staphylococci and $\mathrm{E}$. celi using the patient's cells and control sera. Neutrophil chemotaxis using a Boyden chamber was persistently abnormal. The patient's chemotactic index was $27 \%$ of control on 8 determinations over $1 \frac{1}{z}$ years (range $9-50 \%$ of control). Patient's sera and plasma did not inhibit control cell chemotaxis. An in-vivo correlation to the abnormal chemotaxis was observed when CSF cultures were positive for D. pneumoniae for 8 days, yet during this time, her spinal fluid had only 0-7 monocytes and 0-2 PMNs; glucose was $54 \mathrm{mg}$ and protein 7-9 mg. The patient's serum opsonic activity (Malbe method) was deficient for $\underline{E}$. coli and normal for $\underline{S}$. aureus 502A. This serum defect was corrected with $0.12 \%$ control serum. Previous studies have shown that this E. coli K12 strain is opsonized by the alternate complement pathway and $\mathrm{S}$. aureus 502A by the classical pathway. Therefore this patient appears to have two abnormalities in immune function: a defect in opsonic function of the factors of the alternate pathway and a cellular defect in neutrophil chemotaxis.
442 INTERACTION OF BACTERIAL PATHOGENS WITH IMMUNOGLOBULINS AND COMPLEMENTS IN SPUTUM. Suhung Hann, Douglas S. Holsclaw. (Intr. by Robert Kaye). Hahneman $\bar{n}$ Medica1 Coljege, Department of Pediatrics, Philadelphia.

Persistent pulmonary infections by $P$. aeruginosa (PA) and $S$. aureus (SA) in cystic fibrosis (CF) have been postulated to be due to a local defect in phagocytosis. On the basis of our preliminary findings that IgG, IgA, IgM and complements, $C_{3}, C_{4}$ and $\mathrm{C}_{5}$, are present in sputum of $\mathrm{CF}$ patients, the interaction of the bacterial pathogens with immunoglobulins and complements in sputum was investigated by an immunofluorescent technique on 32 sputum specimens from $21 \mathrm{CF}$ patients. The bacteria separated from fresh sputa were incubated with fluorescein conjugated antisera to human IgG, IgA, IgM, $C_{1 g}, C_{3}$ and $C_{4}$. All strains of $P A$ and $S A$ tested were found to be coated with IgG, IgA, IgM, $C_{1 g}, C_{3}$ and $C_{4}$. Fluorescence with IgA was most intense (+++ to ++++$)$, followed by IgG (++ to ++++), IgM (+ to ++++) and complements (t to ++++). Specific blocking of fluorescence was shown by prior incubation of bacteria with unconjugated antisera to human immunoglobulins and complements. Also, the fluorescein antisera were shown to be free of antibodies against subcultured bacterial isolates. When subcultured bacteria were incubated with eluates of sputum, the fluorescence reappeared, indicating that antibody and complement coating can take place in sputum in situ, without invasion of bacteria into tissue. Specificity of antibody coating was demonstrated by absorption study with the use of subcultured PA and $S A$. These observations suggest that PA and SA in sputum are well prepared for phagocytosis, and possibly for extracellular lysis.

443 Defective Neutrophil Chemotactic Responses in Patients with Recurrent Episodes of Otitis Media. Harry R. Hill, Linda S. Book, Val G. Hemming, and John J. Herbst. Univ. of Utah Col. Med. Dept. of Ped. and Pathol. Salt Lake City, Utah

Fifteen patients who had suffered repeated episodes of acute otitis media were studied to determine if abnormal neutrophi] function might contribute to the unusual incidence of infections. Eleven of the patients were under 2 years of age and each had suffered at least 4 episodes of acute otitis media. In addition to recurrent ear infections, twelve of the patients had symptoms suggestive of allergic gastroenteropathy, three had atopic eczema, and three had recurrent asthmatic episodes. The patients with recurrent otitis media had a marked defective in neutrophil chemotactic responsiveness. The mean chemotactic index of the 15 patients was $20 \pm 6$, while that of 25 controls was $62 \pm 10$. Neutrophil random migration, phagocytosis, bactericidal activity, NBT reduction and lymphocyte $T$-cell populations were normal in the patients. Serum IgG, IgA, and IgM concentrations were normal to slightly depressed. In contrast to previous patients we have studied with atopic eczema, recurrent abscesses and defective neutrophil chemotaxis, the present patients did not have markedly elevated concentrations of immunoglobul in $\mathrm{E}$. There was, however, clinical evidence suggestive of allergy and several had an increased number of eosinophils in intestinal biopsies. Abnormal neutrophil granulocyte chemotaxis may contribute to the recurrent middle ear infections in these patients. Such abnormalities may in turn be secondary to chemical mediators of the allergic res-
ponse.

444 PRELIMINARY RESULTS OF A CLINICAL TRIAL WITH AN OCTAVALENT PNEUMOCOCCAL VACCINE IN THE PREVENTION OF OTITIS MEDIA IN INFANTS. Howie, Virgil M., Ploussard, John H., Sloyer, John L., Ir.: and Cate, Charles C.:

An octavalent vaccine containing purified pneumococcal polysaccharide (PPP) Types $1,3,6,7,14,18,19,23$ was administered s.c. to 60 infants $2-19$ months of age whose clinical histories were closely followed for an additional year. Of 34 children who had no previous history of otitis media and who were immunized at ages 2-5 months, 10 experienced vaccine type pneumococcal otitis during the observation period as compared to 5 of 8 age-matched saline injected subjects. In a second group of 26 older children (immunized at 6-19 months), whose clinical histories showed a range of from 0-7 prior bouts of otitis of mixed etiologies, only one child suffered a breakthrough episode of vaccine type otitis media. The difference in incidence of vaccine type otitis among the two groups of vaccinees was significant at $p=.02$. The elicitation of apparent protection against type-specific pneumococcal otitis media as demonstrated in the older vaccinees suggests that a definitive efficacy trial in that age group is justified. 

445 STRONIC ACTIVE HEPATITIS FO OF SEVERE COMBINED IMMUNODEFICIENCY. JECONH. Hutto, Stanford T. Shulman, and Elia M. Ayoub, Univ, of Florida Col. of Med. Dept. of Ped., Gainesville.

Sherlock has suggested that chronic active hepatitis (CAH) may be due to a partial T-1ymphocyte response to a virus infecting the liver. Such a response may result in incomplete eradication of the virus and in consequent chronic low-grade tissue destruction. Possible support for this hypothesis was encountered during our experience in reconstituting a patient with severe combined immunodeficiency (SCID). An eight-month-old girl, whose brother died with findings of SCID, presented with Pneumocystis carinii pneumonia and candidiasis. Immunologic evaluation confirmed the diagnosis of SCID. Administration of transfer factor was followed by clearance of candidiasis and, shortly thereafter, by a marked but transient elevation of serum hepatic enzymes. Subsequent bone marrow transplantation from an HL-A and mixed-1ymphocyte-culture compatible sibling was followed by recurrence of a more pronounced elevation of hepatic enzymes (peak SGOT $=2360$, $S G P T=1040$ ) followed by a rapid fall to pre-transplant levels and persistence of moderately abnormal values (SGOT $=100-150$ ). No evidence of graft-versus-host disease was noted. The child developed evidence of portal hypertension and liver biopsy demonstrated severe CAH with piecemeal necrosis and cirrhosis. Although the patient had received potentially hepatotoxic agents, the histologic appearance of $\mathrm{CAH}$ more likely represents a possible untoward effect of immunologic reconstitution in a previously immuno-incompetent patient harboring a latent hepatitis virus.
448 SUCCESSFUL RLTRANSPLATTATIU: OF IIUMAN BONE MARROW: TWO IMMTHOSUPPRESSIVE REGIMEXS, John H. Kersey, James Roloff, Steve Hokeness, F. Leonard Johnson, Nark Nesbit, William Krivit. Depts. of Pediatrics, Univ. of Minn. Minneapolis, Minn. and Univ. of Wash., Seattle, Wash.

A 15 year old boy with aplastic anemia who was refractory to androgens and adrenal steroids was placed in a laminar flow room and treated with non-absorbable oral and topical antioiotics. Prior to bone marrow transplant from his matched (I:LC, HL-A) sister, he received cyclophospharide (CY) $50 \mathrm{mgm} / \mathrm{Kilo}$ for 4 days. Marrow engraftment ensued and karyotype analysis indicated that about $90 \%$ of cells were of donor origin. Lymphocytosis anci return of host cells, as indicated by karyotype analysis, heralded graft rejection. The second inmunosuppressive regimen consisted of procarbazine, antithymocyte globulin, and $C Y$. The same donor was used for retransplantation. Successful engraftment accompanied by mild chronic graft versus host reaction continues eight months following transplant. Host cells are not detectable in blood or bone marrow after the second imnunosuppressive regimen.

Following the first imrunosuppressive regimen and prior to transplantation, host $\mathrm{T}$ lymphocytes, as determined by spontaneous rosette formation with SPHCs, continued to be detectable in peripheral blood. Following the second immunosuppressive regimen I lynphocytes became undetectable in peripheral blooc until engraftmert with donor cells was successful. 446 ABNORMAL NEUTROPHIL PHAGOCYTOSIS IN SICKLE-CELL Patricia D. Hecht, and John H. Githens. Univ. Colo. Med. Ctr., Depts. Biophysics/Genetics and Pediatrics, Denver. The abnormal susceptibility of sickle-cell disease (SCD) patients to Salmonella infections has not been elucidated by studies of humoral host defense factors and is compatible with dysfunction of phagocytic cells. Neutrophil (PMN) functions were thus studied in 10 children with SCD, including one who had developed severe salmonella sepsis 3 years earlier. Phagocytosis and bactericidal power towards Staphylococcus aureus 502A were simultaneously studied by Tan's method which permits differentiation between surviving intracellular (IB) and extracellular bacteria (EB). Results are expressed as percent $I B$ and $E B$, compared with the initial bacterial inoculum, and correlate respectively with bactericidal power and phagocytosis.

values $(X \pm S E M)$ for 20 normal adults were: $4+0.5, \mathrm{~EB}$ $6.7 \pm 0.8$. In $S C D$ patients, IB values were all normal $12.7 \pm$ $0.8)$ while EB values were significantly abnormal ( $12.2 \pm 4.6$, $P<0.05) ; 4$ of 9 patients with SCD had EB values more than 2 SD above the control mean (range 13.6-45.8). The most abnormal value belonged to the patient who had had salmonella sepsis. We concluded that most SCD children have normal PMN bactericidal power. In a significant number of them, PMN phagocytosis is abnormal and may contribute to their increased susceptibility to bacterial infections, particularly when salmonella organisms are involved.

447 EFFECT OF BREAST FEEDING ON ANTIBODY RESPONSE TO ORAL POLIO VACCINATION. T. Jacob John, (Intr. by Dr. G. Morrow, III) Univ. of Arizona Col. of Med., Dept. of Ped., Tucson.

Colostrum and early breast milk may inhibit polio vaccine virus take and antibody response in newborn infants. However, apart from two retrospective analyses, there has been no direct investigation of the effect of breast feeding in older infants in whom vaccinations are routinely given.

Three hundred south Indian infants between 6 and 52 weeks of age were divided into six groups and given three doses of trivalent oral polio vaccine (OPV) of known adequate potency. One group was given unrestricted breast feeding with a mandatory breast feeding during the interval between 30 minutes before and 15 minutes after each dose. In four groups of infants breast feeding was withheld for $3,4,5$ and 6 hours both before and after each dose of OPV. The sixth group of infants was bottle fed. Samples of blood were collected from all infants before vaccination and 4 weeks after the first and third doses of OPV. Antíbody responses to poliovirus types 1,2 and 3 were determined following one dose and three doses of OPV and the rates of response were found to be approximately equal in all groups of breast fed infants irrespective of their breast feeding schedules, as well as in bottle fed infants.

Thus breast feeding is shown to have no inhibitory effect on oPv. 449 DEFECTIVE NEUTROPHIL CHEMOTAXIS IN DOWN'S SYNDROME
A.J. Khan, H.E. Evans, L. Glass, Y.H. Shin, A.J. Khan, $\frac{\text { H.E. Evans, }}{\text { D. Almonte, }}$ Department of Pediatrics, Jewish Hospital \& Medical Center of Brooklyn, Brooklyn, New York

Chemotactic migration of neutrophils from 10 non-infected in fants and children with Down's syndrome (DS) was compared with that of age matched healthy controls $(C)$, utilizing a modification of Boydens technique.

The subjects' cells were placed in the upper chamber, and tested against each of the following attractants in the lower chamber: 1) normal $A B$ serum (ABS) + E. coli endotoxin $(E)+$ Hanks solution (HS) ; 2) Down's syndrome serm (DSS) + E + HS 3) HS alone. Qualitative NBT tests and serum inmunoglobulin levels (IgG, IgA and Igif) were carried out on cases with DS. The chemotactic index (CI) of DS cells was significantly decreased when compared to PMNS of normal controls. Chemotactic activity of DSS was similar to that of ABS.
ATTRACTANTS CI DS CI C ATTRACTANTS CI $C$

\begin{tabular}{|c|c|c|c|c|c|}
\hline & Median & (Range) & Median & (Range) & P value \\
\hline$\overline{\mathrm{ABS}}+\mathrm{E}+\overrightarrow{\mathrm{HS}}$ & 125.5 & $(54-194)$ & 210.5 & $(86-345)$ & 0.01 \\
\hline$\overline{\mathrm{DSS}}+\mathrm{E}+\mathrm{HS}$ & 121 & $(42-187)$ & 199 & $(89-331)$ & 0.05 \\
\hline H & 65 & $(45-101)$ & 89 & $(30-132)$ & NS \\
\hline
\end{tabular}

The similarity of indices within each group, whether using
S This defect, which was unrelated to either the NBT test or serum immunoglobulin levels, may be partially responsible for the increased incidence of bacterial infection observed in individuals with DS.

PLASMA CELI ANTIGEN BEARING LYMPHOCYTES IN PRIMARY IMMUNODEFICIENCIES. Bobby Q. Lanier, Armond S. Goldman and Nick S. Harris, Departments of Pediatrics and Surgery, The University of Texas Medical Branch and Shriners Burns Institute, Galveston, Texas.

An antisera to human plasma cells which does not cross react with immunoglobulins recognizes a surface antigen on a special population (12\%) of human blood B lymphocytes (Nature 250:508, 1974). In the present study, blood lymphocytes from patients with primary immunodeficiencies were examined for the presence of the plasma cell antigen (PC), as well as surfoce immunoglobulin $(\mathrm{lg})$ bearing lymphocytes and T lymphocytes. The relative frequency of lymphocytes with plasma cell antigen was dependent upon the type of immunodeficiency. Blood lymphocytes bearing plasma cell antigen were deficient in $x$-linked a-y-globulinemias (mean of $4 \%$ vs 11.5 $\%$ in normal controls) but were normal or increased in severe combined immunodeficiency, selected $\lg A$ deficiency, $x$-linked dys-y-globulinemia and other immunoglobulin deficiencies. The levels of PC bearing lymphocytes correlated with the number of $\mathrm{lg}$ bearing cells in some of these disorders, but not in others. These studies suggest that the measurement of the plasma cell antigen on lymphocytes is an additional tool for the study of abnormalities of immunodeficiency diseases, and may aid in further understanding and categorizing the primary immunodeficiencies. (Supported in part by NCI 1-ROI CA 15278-01). 
451 ABNORMAL T AND B CELL FUNCTTON WITH ASSOCLATED DYS GAMMACLOBULINEMIA--POSSIBLF T CELL HELPER DEFECT William E. Larter. James J. Corrigan Jx. and David Lucas, Dept. of Ped, and Microbiology, Univ. of Arizona Medical Center, Tucson, Az

A 4 year old female with recurrent bacterial infections and failure to thrive was noted to have deficiencies involving $T$ and 13 lymphocyte functions and abnormal endotoxin induced NBT uptake by neutrophils. Lymphocyte counts have been greater than $1700 /$ $\mathrm{cmm}$; by rosetting technique $56 \%$ were T-cells and $34 \% \mathrm{~B}$-cells. Representative in vitro lymphocyte stimulation indices were: PHA 6.1 control 69.7); Con A 1.9 control 14.0); PPF 1.1 (control 56.0); and PWM 2.0 'control 6l.1). Skin tests with DNCB and SK/ SD have been positive. Immunoglobulin studies have revealed IgG $200 \mathrm{mg} / \mathrm{dl}$, IgA $0 \mathrm{mg} / \mathrm{dl}$, IgM greater than $1000 \mathrm{mg} / \mathrm{dl}$, and IgE 94 $\mathrm{IU} / \mathrm{ml}$. Iso-agglutinins and polio antibody (functional antibody) were present as was secretory IgA. Neutrophil Ni3'T uptake was $23 \%$ with an endotoxin stimulated value of $26 \%$ (normals, less than $15 \%$ and greater than $60 \%$, respectively). Normal plasma did not improve her stimulated NBT uptake. Addition of patient plasma to normal neutrophils suggested a suppressive effect on the endotox-1 in stimulation. While on oral D-penicillamine the serum IgM level decreased, but the clinical picture did not improve. Weekly fresh frozen plasma infusions have decreased the frequency and severity of infections and also decreased the $\mathrm{IgM}$ to $150-250 \mathrm{mg} / \mathrm{dl}$. The data suggest that this patient has an immune deficiency syndrome characterized by defects in $T$ and $B$ cell function and in neutrophil phagocytosis. Although no single abnormality appears to explain all the data, a T-cell helper defect may be involved.

452 CYCLIC SHIYTS TN THYMUS (T) NND BONE: MARROH (B) DERIVID LYMPIOCYYTES FROM A SEVERE, COMBINED IMNHODERICIINCY (SCTD) PATIENT. B.P. Mackler, N. Mukhopadhyay, J.R. Montgomery and L. Richie. Dental Sci. Inst.; Baylor Col. of Med.; M.D. Anderson llosp., Univ. of Texas Health Science center, Houstom, Texas 77025

The thymus (T) and bone marrow (B) derived lymphocytes from a severe combined immunodeficiency (SCID) patient maintained in a gnotobiotic environment were evaluated longitudinally for 10 months. High B cell percent ages (70-90\%) were initially observed with only $(1-10 \%) \mathrm{T}$ cells for the first 30 months of life. Subsequently cyclic fluctuations were observed in which the levels of $E$-rosette forming ( $T$ ) cells increased to $35-60 \%$ while $B$ cell percentages concomitantly decreased. Several such cyclic fluctuations have been seen at 60-90 day intervals.

Although lymphocytes from the S(II) patient bound PHA and con $\Lambda$ through membrime receptors, they underwent no blastogenic responses. Inmunofluorescent studies of mitogen binding by SCID lymphocytes showed only $52 \%$ of the cells binding PIA and $88 \%$ bjunding Con $A$; control cells showed $100 \%$ binding of both mitogens. A second SC.ID patient gave similin results.

E-rosetted lymphocytes from 2 scid patients were found to have surface immunoglobulin $(30-50 \%)$; r-rosetted cells from normal control had negligible amount (2.5\%). Tt is sugcrested that E-rosetting of S(II) $\mathrm{T}$ cells caused membrane topology changes revealing hidden immunoglobulin receptors.

153 MID LOBE SYNDROME, PERIBRONCHIAL LYMPHONODULAR HYPERPLASIA AND ELEVATED SERUM IgD. Hilaire J. Meuwissen, Ellen C. Moore, Catherine U. Kyong, Harvey Kausel, John Scott and Monica Bishop (Intr. by Hilda Knobloch). N.Y.S. Dept. of Health, Birth Defects and Kidney Disease Insts., Albany Med. Ctr. and Veteran's Admin. Hosp., Albany, New York.

We have studied six children with Middle Lobe Syndrome during the last 2 years. All had marked peribronchial lymphonodular hyperplasia, which obstructed the smaller bronchi and bronchioles. Within the peribronchial lymphoid tissue, lymphonodules were present, containing fully developed germinal centers. The lymphoid cap contained lymphocytes and plasma cells, and the bronchial epithelium overlying the nodules contained lymphocytes within vacuoles, strikingly similar to those seen in Peyer's patches. The epithelium often was eroded and destroyed. Contrast studies of the airways showed irregularities of the bronchial walls. Three of five patients had elevated levels of serum IgD. One patient had antibody deficiency and severe lymphonodular hyperplasia of the gut as well. All other patients had normal $B$ and $T$ cell and granulocyte functions and normal levels of complement. The disease process led to chronic lung disease in three patients. We suggest that Mid Lobe Syndrome is frequently caused by the process described above, and that this may lead to chronic lung disease. As many patients with dys- or hypogammaglobulinemia have lymphonodular hyperplasia of the gut, their lung disease may be caused or aggravated by lymphonodular hyperplasia of the bronchi. The elevated serum IgD we observed may be produced by peribronchial lymphoid tissues. Supported by PHS-GCRC Grant. \#010098.
154 ANTIBODY AND CELL-MEDIATED IRMUNE RESPONSE (CMI) TO CYTONEGALOVIRUS IN CHILDREN WITH RECURRENT RESPIRATORY INFECTIONS. A. Morag*, K.R. Beutner*, B. Morag*, J.M. Bernstein*, R. Diebel* and P.L. Ogra, Depts, of Peds., ENT and Microbiol., SUNY at Buffalo and N.Y. State Dept. of Health Laboratories, Albany.

The appearance of cytomegalovirus (CMV) specific CMI in circulating (CL) and tonsillar (TL) lymplocytes, and antibody activity in serum was studied in children who underwent tonsillectomy and adenoidectomy (T\&A) for recurrent tonsillitis, otitis or other respiratory infections, and in a matched group of normal children. The techniques of complement fixation, indirect hemagglutination and in-vitro lymphocyte transformation using inactivated CMV virus strains (AD-169, ADH-1-41, Davies) as the antigens were employed to study the specific immune responses. The prevalence of CMV antibody activity was 4-fold greater in T\&A subjects than observed in control population. Most subjects with detectable CMV antibody activity in serum manifested a specific CMI to CMV in $\mathrm{CL}$ and TL. The CMI activity in TL appeared to be $2-3$ fold higher than in CL. No CMI activity was observed in the $\mathrm{CL}$ of seronegative subjects. However, significant CMI activity was observed in the TL of many antibody negative subjects. Although CMI response to CMV were strain specific, several subjects elicited CMI activity to more than one strain of virus. These data suggest the developinent of CMV specific CMI in systemic and mucosal sites, which is independent of the antibody response in serum. In addition, these observations suggest the possibility of consecutive CMV virus infections in T\&A prone patients.

455 T AND B CELLS AND FUNCT MONAL RESPONSIVENESS IN HUMAN FETAL LIVER CELLS. Nalini Mukhopadhyay, John M. David Mancor, and Donald J. Fernbach (Intr. by Kenneth A. Starling), Baylor College of Medicine, Department of Pediatrics, Houston.

Liver cells were obtained from hysterectomy specimens of 7 dead human fetuses. Fetal age determined by crown-rump measurement ranged from 10-14 weeks. The 1 iver cells showed $0-2 \%$ I cells as detected by spontaneous sheep erythrocyte rosette formations (control range $55-70 \%$ ). Low numbers of $B$ cells bearing surface immunoglobul in were detected $(0-2.5 \%)$ while $5-10.5 \%$ of the liver cells bore a receptor for $C^{\prime} 3$. Blastogenic response for PHA, PWM, and mixed lymphocyte reactivity (MLR) to adult allogeneic lymphocytes were consistently negative, although they showed stimulatory ability in MLR for adult allogeneic 1ymphocytes (stimulation index 6.6 ). In cocultivation with lymphocytes obtained from a patient with $x$-linked severe combined immunodeficiency, the fetal liver cells increased the PHA responsiveness of the patient's lymphocytes 3-fold over control values. Liver cells stimulated with PHA released a factor in the supernatant medium which inhibited the blastogenic response of normal allogeneic 1 ymphocytes in the absence of cytotoxicity. These studies suggest that functional responsiveness may develop prior to detection of cell surface markers in human fetal liver cells.

156 STUDIES OF THE IMMUNE RESPONSE AND ANTIGEN PROCESSING

456 IN CONJOINED (SIAMESE) TWINS. Michael E. Norman, Burton Zweiman, Robert P. Lisak, Chester M. Zmi jewski, Jeffrey P. Harris, John M. Templeton, Charles E. Koop, Mary Ann South, The Children's Hospital of Philadelphia and the University of Pennsylvania School of Medicine, Philadelphia.

Thirteen-month old HL-A identical conjoined twins (thoracopagus-type), with a common circulation, presented a unique opportunity to study the immune response and antigen processing. After baseline studies antigens were given prior to surgical separation: [1] twin $\underline{C}$; a) topical DNCB on day -3, b) pneumococcal vaccines $\left(\mathrm{s} . \mathrm{c}_{0}\right)$ type 3 on day $\cdot 2$, type 8 on day -1 . [2] twin $A$; a) mumps (i.mo) on day -4 , b) influenza (s.c.) on day -3, c) skin grafts (SG) from 3 unrelated $H L-A$ and MLR typed donors $(B, H, N)$ on day -3 . SG-B was removed after 1 day, SG-H after 2 days, SG-N at rejection ( 15 days). Serum antibody responses, mumps, DNCB, and SG challenges were measured in both twins at appropriate times after separation. Both twins showed humoral immunity to both pneumococcal types and influenza, and delayed hypersensitivity (DH) to mumps. Twin $C$ but not twin $A$ showed positive DNCB challenge. SG from all 3 donors rejected as second set ( 4 days) by twin $A$, but oniy $S G-N$ was rejected in accelerated fashion ( 7 days) by twin $C$. Findings suggest that transfer of antigens and/or sensitized cells between twins occurred within 4 days for mumps $D H$, and within 3 days a) for humoral immunity, b) partially for al lograft sensitivity, but c) not for contact (DNCB) DH. These studies, feasible in humans only in conjoined twins, yield important new information about the comparative kinetics of developing systemic immunity to topically applied and injected antigens. 
457 CHARACTERISTICS OF SERUM AND SECRETORY IMMUNE SYSTEM IN SUDDEN DEATH SYNDROME IN INFANTS (SIDS). P.L. Ogra S.S. Ogra* and P.R. Coppola*, Depts, of Peds. and Microbiol., SUNY at Buffalo.

Employing the techniques of in-vitro tissue culture inoculation, indirect immunofluorescence and radial immunodiffusion, the autopsy specimens of blood, respiratory tract washings, bronchopulmonary tissue, spleen and thymus were examined for respiratory viruses, immunoglobulins, secretory component (SC), $\alpha$-fetoprotein (FP) and lactoferrin (LF) in infants with SIDS and in other infants (controls) with an identifiable cause of death. The levels of immunoglobulins, FP and LF in the serum of infants with SIDS were similar to those observed in control subjects. In some SIDS cases $r M$ immunoglobulin levels in serum were found to be slightly elevated. The pulmonary tissues of a few SIDS patients yielded respiratory syncytial virus and, no viruses were isolated from other subjects. Significantly, however, all patients with SIDS exhibited conspicuous absence or grossly decreased fluorescent antibody staining for secretory component in the bronchopulmonary epithelium. Although the levels of immunoglobulins in bronchial washings were unremarkable, little or no secretory (11S) $y \mathrm{~A}$ immunoglobulin was detected in SIDS secretions. These observations suggest a possible defect in the respiratory mucosal defense in patients with SIDS. The absence of secretory component in the bronchopulmonary mucosa may predispose to an increased or abnormal uptake of microbial and other macromolecular antigens by the respiratory tract, which nay result in local "anaphylaxis" like reactions. Such a mechanism may partly explain the pathogenesis of SIDS

458 IN VITRO STUDIES OF CELLULAR IMMUNITY IN SS HEMOGLOBINOPATHY. Olu O. Ogunye, John N. Sheagren and Warren Strober (Intr. by Roland B. Scott).

From the Depts. of Pediatrics and Medicine and the Ctr. for Sickle Cell Disease, Howard Univ. Col. of Med., Washington, D.C. and the Immunology Section, Metabolism Branch, National Cancer Inst., NIH, Bethesda, Maryland

To evaluate whether cellular immunity plays a part in the phenomenon of increased susceptibility to infections in SS hemoglobinopathy, we have determined the number of circulating T-lymphocytes by the E-rosette technique as well as circulating lymphocyte proliferative responses to non-specific $\mathrm{T}-c e l l$ mitogens in culture.

The mean nunber of $T$-lymphocytes (E-rosettes) in patients was $57+1.4$, whereas that of controls was $60+1.3$. (mean + SEM) These values are not significantly different $(p>.1)$. The mean response of peripheral lymphocytes to PHA (20 micrograms $/ \mathrm{ml}$ ) in patients was $19,638\left(\mathrm{cpm} / 10^{5}\right.$ cells $) \pm 3,696$, whereas the response of controls was $17,516+4,01 \overline{8}\left(\mathrm{cpm} / 10^{5} \mathrm{cells}\right)$. These responses were not significant $\bar{l} y$ different $(p>.1)$. Similarly, no differences between patients and controls were found at other PHA concentrations or with the use of the mitogens Concanavalin $\mathrm{A}$ or pokeweed at several mitogen concentrations.

These studies indicate that cellular immunity in patients with SS hemoglobin disease as measured by numbers of circulating $\mathrm{T}-1$ ymphocytes and $\mathrm{T}-1$ ymphocyte responses to mitogen stimulation is normal. Thus these functions do not appear to be an important component of the susceptibility to infection seen in SS hemoglobinopathy.

ANTI-COMPLEMENTARY ACTIVITY OF SERA FROM PATIENTS 459 WITH JUVENILE RHEUMATOID ARTHR ITIS (JRA), RHEUMATOID ARTHRITIS (RA), SYSTEMIC LUPUS ERYTHEMATOSUS (SLE)

AND NORMAL CONTROLS. Lauren M. Pachman, Sandra M. Baldwin, Shakuntala M. Gaekwar, Northwestern Univ. Med. Sch., Children's Mem. Hosp., Dept. of Ped., Chicago

Sera from patients with diseases involving antigen-antibody $(\mathrm{Ag}-\mathrm{Ab})$ complex formation (RA, JRA, SLE) may have anti-complementary activity which reflects clinical activity. To test this, sera samples were heat inactivated $\left(56^{\circ} \mathrm{C} \times 30^{\circ}\right)$, a known amount of normal human complement $\left(C^{\prime}\right)$ added, red cell hemolysis measured and the \% $\mathrm{C}^{\prime}$ fixed was calculated. Samples from 31 normal healthy children fixed $12.8 \%+1$. 9SE, while sera from 6 children with SLl fixed $37.5 \%+3.7(p<.0 \overline{0} 1)$. Sera from 66 with definite JRA (3- 7 samples/child) appeared to fall into three groups $(\mathrm{p}<.001)$ a) 32 fixed $13.9 \% \mathrm{C}^{\prime}+1.0$, b) 19 fixed a high amount of $\mathrm{C}^{\prime} 40.0 \%$ \pm 2.2 , and c) 15 fluctuated over time $24.4 \% \pm 2.0$. Successive freezing and thawing $(5 x)$ did not alter the $\% C^{\prime}$ fixed or the quantity of immunoglobulin detected in JRA sera. The $\% C^{\prime}$ fixed appears to be independent of the presence of rheumatoid factor (RF): sera from $50 \mathrm{RA}$ patients $(43+$ and $7-\mathrm{RF}$ ) fixed $54.3 \% \mathrm{C}+1.6$, while 89 normal adult sera fixed $17.7 \% \mathrm{C}^{\prime} \pm 1.7$ ( $\left.\mathrm{p}<.001\right)$. On $\overline{1} y 3$ JRA's in our population were RF positive. In linear studies of 15 patients with JRA ( 5 pauciarticular, 5 polyarticular and 5 systemic), clinical course was correlated with the $\% \mathrm{C}^{\prime}$ fixed and in some cases may reflect therapeutic response. It is speculated that the anti-complementary activity of sera from patients with other Ag-Ab complex diseases may be useful in predicting clinical course. Supported in part by a grant from the Ill. Chap.Arth. Fdn.
460 TRANSFER Factor therapy in agammaglobulineMIA. Photinis. Papageorgiou, Sarah Kaffe, Nieves M. Zaldivar and Philip R. Glade. Depts. Ped. CMDNJ, Rutgers Med. Sch., Piscataway, Suny, Mount Sinai Sch. Med., New York and Univ. Miami Sch. Med., Miami.

Recent studies indicate that $T-1$ ymphocytes are instrumental in the modulation of antibody production by B-lymphocytes. Transfer factor (TF) from healthy adults was administered to a 9 -year old boy with congenital agamaglobulinemia, who had been unsuccessfully treated with gamma globulin. Immunological evaluation prior to TF ( 14 days post IgG) revealed $140 \mathrm{mg} \%$ IgG, undetectable serum and secretory IgA, $35 \mathrm{mg} \%$ IgM. $B$-cell markers were $4.6 \%$ by EAC rosette and $7 \%(a, \gamma, \mu$, chain) by immunofluorescent staining. Pokeweed mitogen response of the lymphocytes was normal. After 2 doses of TF $\left(2 \times 10^{8}\right.$ lymphocytes per dose $), 70$ days post exogenous IgG, his serum IgG was $56 \mathrm{mg} \%$ and IgM $7 \mathrm{mg} \%$. Nine months post IgG ( 4 doses of TF) his IgG rose to $130 \mathrm{mg} \%$ and IgM became undetectable. At 10 months the IgG decreased to $40 \mathrm{mg} \%$ and IgM remained undetectable. Following another course of $T F$ the IgM increased to normal levels $(70 \mathrm{mg} \%)$. At 12 months the IgM decreased to $15 \mathrm{mg} \%$ and IgG remained low. During the first 9 months of TF therapy the patient was completely free of infections. Since then he has had several episodes of rhinitis and one episode of mild pneumonia.

7.1GRATION OF HUMAN NEUTROPHILS AGAINST GRAVITY: A NEW METHOD OF CHEMOTAXIS ASSAY. Byung H. Park, UCLA Med. Sch. Harbor Gen. Hosp. Dept. of Ped. Torrance, Ca. Chemotactic migration of human neutrophils is an important part of host defense mechanism and inflammatory response. The conventional assay of chemotaxis in vitro has been carried out largely by use of Boyden Chamber technique. In this method, the migrated cells are assumed to rema in attached at the lower surface of the filter. However, a variable portion of migrated cells was shown to be detached from the lower surface due to gravitational force. In order to remedy these inherent linitations, I devised a new, simple method of studying chemotaxis function of human neutrophils, in which the mobile cells migrate upward through the filter membrane, and thus the possibility of cell detachrient after migration is el iminated. $3 \times 10^{5}$ neutrophils in $0.1 \mathrm{ml}$ of hedium 199 with $20 \%$ plasmanate or autologous plasma were deposited on the surface of Millipore filter $(8 \mu$ pore size). The fluid was quickly absorbed by placing absorbent paper in the opposite site of the filter. The cells on the filter were quickly "sandwiched" betiveen 2 wet filters and placed in a blind well chamber with Medium 199 in the lower compartment. Then a chemotactic substance, E. coli culture filtrate, was introduced to the upper compartment. The entire chamber was incubated at $37^{\circ} \mathrm{C}$, water bath. for 3 hours. The filters were stained with hematoxylin. lligrated cells on the upper surface of filter averaged $115 \pm 20$ per high power field in 80 normal controls. This method would also distinguish true chemotactic migration from non-specific increase in random movement of neutrophils.

462 Diminished alternative COMPLEMENT PATHWAY (ACP) ACTIVITY AFTER SPLENECTOMY. Rutherford B. Polhill, Jr. Depts. of Ped, and Microbiology, Birmingham.

Splenectomized individuals and patients with sickle cell disease share a predisposition to overwhelming bacterial infection, defective particle trapping, and deficient antibody response to particulate antigen. Patients with sicklemia also have deficient serum ACP activity, resulting in inefficient opsonization when antibody is lacking. We have assessed hemolytic and opsonizing ACP activity in sera from splenectomized children. The hemolytic assay used depends upon ACP activation by unsensitized rabbit erythrocytes $\left(R_{a} E\right)$. Lysis of the $R a E$ occurred independently of early complement components. Cell density was monitored continuously by a simple, highly reproducible technique, and time required to lyse $\frac{1}{2}$ the $\operatorname{RaE}\left(t^{\frac{1}{2}}\right)$ was determined. The mean $t \frac{1}{2}$ with sera from 20 splenectomized children was $14.9 \pm 4.9$ (2SD) $\mathrm{min}$, and that of 30 matched controls, $11.3 \pm 2.0 \mathrm{~min}(\mathrm{P}<0.0001)$. Opsonization of zymosan was similarly deficient. Preliminary experiments with sera from 21 Hodgkin's disease patients suggest that the post-splenectomy deficiency is less severe in adults. Hemolytic ACP activity was most deficient in sera from 6 splenectomized children with thalassemia or cirrhosis, indicating a possible hepatic influence on this activity. These results suggest a splenic role in maintaining normal serum ACP activity. Greater dependence on ACP function might be expected in infants who have not yet acquired specific antibody, and this could account for their higher risk of infection after splenectomy. 

463 ADENOSINE DEAMINASE (ADA) ACTIVITY IN TISSUES OF ADA Bernard Pollara, Richard J. Pickering, \& Hilaire J. Meuwissen. N.Y.S. Kidney Disease \& Birth Defects Insts.* and Dept. of Peds., Albany Med. Col., Albany, N.Y. 12208.

Tissues obtained at necropsy from two female patients with CID and erythrocyte ADA deficiency were assayed for ADA activity. One patient had severe CID with typical bone lesions described in some ADA-CID patients. The other had residual $B$ cell function and no bone lesions. Using a modification of the Kalckar method for measuring ADA activity, low levels of enzyme activity, ranging from less than $1 \%$ to $10 \%$ of "normal" adult necropsy tissue, was found in spleen, liver, kidney, heart and abdominal muscles. Analysis of lymphocyte ADA activity in the severe CID patient with bone lesions stowed $3 \%$ of the level observed in normal lymphocytes. ADA activity was found in fibroblasts cultured from the skin of the patient without bone lesions. The specificity of the reaction was demonstrated using C14 adenosine as a substrate and thin layer chromatography for separation and identification of the nucleoside inosine. Our results correlate with those of Van der Weyden, et al. (BBRC 57:590, 1974) and further substantiate the hypothesis that ADA deficiency most likely results from a mutational event. The low ADA activity found in these patients militates against the gene deletion hypothesis. Supported in part by PHS Grant \#AI12241 and PHS-GCRC \$010098.

*Units of the New York State Department of Health.

MMIMUNOLOGIC STUDIES OF ATO PIC DERMIATITIS. Gary S. Rachelefsky, Sheldon C. Siegel, Gerhard Opelz, Paul I. Terasaki, and E. Richard Stiehm. University of California School of Medicine, Department of Pediatrics, Los Angeles. Immunologic abnormalities in atopic dermatitis (AD) have included elevated serum IgE, defective chemotaxis and low \% T-cells. Since none of these provide appreciation of the underlying immunologic abnormalities, detailed immunologic tests were performed on 37 children with AD. Most were on topical and none on oral steroids. Serum $\mathrm{IgE}$ levels (normal mean $75 \mathrm{I} . \mathrm{C} . / \mathrm{ml}+27 \mathrm{~S} . \mathrm{E}$.$) , \% \%$ and total B cells $(16+6$ and 480 $\left.\pm 60 \mathrm{cells} / \mathrm{mm}^{3}\right)$, $\%$ and total $\mathrm{T}$ cells $\left(56 \pm 2\right.$ and $\left.1720 \pm 150 \mathrm{cclls} / \mathrm{mm}^{3}\right)$ and lymphocyte response to graded doses of PHA were correlated with eosinophil count and clinical score. The latter was based on extent of erythema, vesiculation, crusting, excoriation, lichenification, pigmentation, pruritus and loss of sleep.

The IgE le vels $(692+189)$, eosinophil counts $(676 \pm 80)$ and total $(776 \pm 80)$ B cells were significantly $(p<.05)$ higher than normal and all correlated inversely $(\mathrm{p}<, 05)$ with clinical severity. B-cell $\%(19+6)$, \% $(52 \pm 14)$ and total $(2040 \pm 180) \mathrm{T}$ cells did not differ from normal. Eleven patients had low $\mathrm{T}$-cells $(<45 \%)$; they had varied clinical scores but high IgE levels. Lymphocyte response to P'HA was significantly $(p<.05)$ lower than normal at low PIIA dilutions.

The immunologic abnormality in $A D$ appears to be a subtle defect in T-cell (? repressor) function leading to increased B cells and resulting in increased $\mathrm{IgE}$ production. However, secondary alteration as a result of infection or chronic antigenic stimulation cannot be excluded.

465 A NEW MICRO-QUANTITATIVE, NITROBLUE TETRAZOLIUM TEST. Uma Raman and Ronald L. Poland, Dept. of Pediatrics, wayne State Univ. School of Medicine and the Children's Hospital of Michigan, Detroit, Mich. (Intr, by Adnan S. Dajani)

Conflicting data have appeared concerning the dye-reducing activity of the leukocytes of newborn infants with and without sepsis (Pediat, 45:125, 1970, J. of Pediat, 84:441, 1974, Clin. Med, $81: 16,1974)$. Since part of the problem seemed to be methodological, we have developed a microquantitative nitroblue tetrazolium dye reduction test. In this test, the patient's heparinized whole blood is mixed with an equal volume of $0.1 \%$ nitroblue tetrazolium dye in $0.15 \mathrm{M}$ phosphate-buffered saline - pH 7.25 (buffered saline alone is used for a control). The mixtures are incubated for $15 \mathrm{~min}$. at $37^{\circ}$ and then equilibrated to room temperature for $15 \mathrm{~min}$. at which time, $0.05 \mathrm{ml}$ of each is mixed with $1.0 \mathrm{ml}$ of $\mathrm{N}, \mathrm{N}$-dimethyl formamide and the formazan precipitate is extracted into the organic phase. The difference spectrum of these preparations (with and without NET dye) corresponds to the spectrum of pure formazan in the solvent with a peak of $525 \mathrm{~nm}$. The amount of dye extracted from whole blood into the organic phase is proportional to the amount of formazan added and to the O.D.525. The 0.D.525 of the NBT sample versus its blank is expressed in 0. D. units per 10,000 neutrophils $/ \mathrm{mm}^{3}$. This method is compared to that of Park, et a.l (Lancet, $\underline{2}: 532,1968$ ) using split. aliquots of 19 blood specimens. The correlation was close and linear $(\mathrm{N}=19, \mathrm{r}=0.94, \mathrm{p}=<.001)$. A prospective study using this test in newborn infants is ongoing.
SPECIFIC IMPAIRMENT OF CELL-MEDIATED IMMUNITY IN MOTHERS OF INFANTS WTTH CONGENTIAL CYTOMEGA LOVIRUS (CNIV) IN FECTION. M.Rola-Pleszczynski, L.D. Frenkel, D.A. Fuccillo, S.A.Hensen, M. M. Vincent, D. Reynolds, S. Stagno, J.A. Bellanti; Georgetown Univ. Sch。 of Med., Washington, D.C.; NIH; Microbiol. Assoe. Inc., Bethesda, Md。; Univ. of Alabama Sch. of Med。, Birmingham, Alabama.

A specific lymphocyte-mediated cytotoxicity to CMV was evaluated using a ${ }^{51} \mathrm{Cr}$-release microassay in 7 infants $(5-10$ months of age) with congenital CMV infection and 6 of their respective mothers together with 17 normal adult and infant controls. The indirect hemagglutination inhibition (IHA) antibody titers to CMV in the infected infants ranged from $1: 16$ to $1: 1024$. All of these infants had detectable specific immune release (SIR) of $51 \mathrm{Cr}$ ranging from $3.3 \%$ to $35.3 \%$ (mean $=16.3 \pm 4.4 \%$ ). The mothers of these infants demonstrated significantly elevated IHA titers to CMV (geom。mean titer $=1: 225$ ) as compared to 1:30 in controls $(t=3.28, p<0.005))$ but showed significantly depressed SLR $(2.8 \pm 0.9 \%)$ compared to normal seropositive controls $(22.9 \pm 3.1 \% ; \mathrm{t}=5.49, \overline{\mathrm{p}}<$ $0.005)$. An additional adult nulliparous woman with persistent CMV viruria was also found to have a depressed SIR to CMV $(5.7 \%)$. These data suggest that a specific impairment in cell-mediated immunity to CMV occurs in mothers of infants with congenital CMV infection and in some persistent CMV excretors and that this impairment may serve as a biologic marker of disease susceptibility.

467 LYMPHOCYTE-MEDIATED CYTOTOXICITY TO VIRUSES IN PATIENTS WTTH MULTIPLE SCLEROSIS: PRESENCE OF A BLOCKING FACTOR. M. Rola-Pleszczynski, M. Abernathy, M.M. Vincent, S.A. Hensen, J.A. Bellanti; Georgetown Univ. Sch. of Med., Washington, D. C.; Microbiological Associates, Bethesda, Md.

Lymphocyte-mediated cytotoxicity to virus-infected target cells was investigated in 14 patients with multiple sclerosis (MS) using a ${ }^{51} \mathrm{Cr}$-release lymphocytotoxicity microassay. Lymphocytes from patients or normal controls were incubated with ${ }^{51} \mathrm{Cr}$-labeled target cells persistently infected with either measles, SSPE or rubella virus and the culture supernatants were assayed for released $51 \mathrm{Cr}$, in the presence or absence of serum from normal measles immune individuals or from patients with MIS. In the absence of serum, specific immune release (SIR) was similar in both groups studied with all three virus strains. Serum from patients with MS, however, inhibited the SIR effected by normal or patient lymphocytes from measles or SSPE infected cells, but not from rubella-infected cells; normal immune sera of comparable titer did not significantly inhibit the SIR from any of the target cell lines with any of the lymphocytes tested. Partial characterization of this blocking factor by gel filtration with Sephadex G-200 revealed it to be associated with the 7S fraction. These data suggest a normal lymphocyte "killer" function in patients with MS, but present evidence for a serum blocking factor with specificity for measles and SSPE viruses.
468 HL-A W27 IN CHILDHOOD ONSET ARTHRITIS. J. G. Schaller $\frac{\text { H. D. Ochs, E. D. Thomas, R. J. Wedgwood. Univ. of }}{\text { Wash. Sch. of Med., Depts. of Ped. and Med., Seattle. }}$ HL-A antigen $\mathrm{W} 27$ has been associated with ankylosing spondylit is (AS), the spondylitis of bowel disease and psoriasis, Reiter's syndrome, and certain types of acute arthritis. It has also been claimed that W27 occurs frequently in juvenile rheumatoid arthritis (JRA).

HL-A antigens were determined in 96 well-studied patients with childhood onset arthritis, originally classified by accepted diagnostic criteria as having JRA. All patients had physical examinations, sacroiliac joint radiographs, and serologic studies. Six patients originally considered to have JRA had definite AS by the time of follow up. W27 (reported incidence in the normal population $4-8 \%$ ) was found with the following frequencies:

\begin{tabular}{|c|c|c|c|c|}
\hline Ankylosing & Paucia & cular JRA & Other & JRA \\
\hline Spondylitis & Male & Female & Male & Female \\
\hline $6 / 6$ & $8 / 12$ & $3 / 16$ & $3 / 18$ & $5 / 44$ \\
\hline
\end{tabular}

It is appaxent that $W 27$ occurred most frequently in patients with AS and in boys with pauciarticular arthritis. W27 occurred much less frequently in girls with pauciarticular arthritis, and in patients with systemic onset disease $(2 / 21)$, chronic iridocyclitis (2/13), seronegative polyarticular disease (3/22), and rheumatoid factor positive disease $(1 / 6)$. The correlation of $w 27$ with pauciarticular disease in boys probably indicates that many such patients have childhood onset AS rather than JRA, thus further differentiating the several syndromes we now call JRA. 
469 RESTORATION OF IMMUNITY BY TRANSPLANTATION OF THE THYMUS, William $\underline{\mathrm{T}}$. Shearer and $\underline{\mathrm{H}}$. James Wedner (Intro. by J. Neal Middlekamp), Washington Univ. School of Med., St. Louis Children's Hosp., Dept. of Ped., St. Louis, Mo. A 5-month-old female (74-9587) was admitted to hospital because of pneumonia and cyanosis of 3 days onset. Biopsy specimens showed florid P. carini infection of the lung, a dysplastic thymus, and paracortical lymphocyte depletion of a lymph node. Immunological evaluation demonstrated that the child's lymphocytes did not respond to stimulation with phytohemagglut in (PHA) and a complete absence of $\mathrm{T}$ cells as judged by surface staining specific for IgG and IgM. There was no response to skin tests with SKSD and monilial antigens. Serum immunoglobulins were normal for age but immunoelectrophoresis suggested an aberrant IgG band. Serological analysis demonstrated a lack of antibody response to polio, diphtheria and tetanus antigens despite prior immunizations. Following treatment with pentamidine isethionate, the child received one unit of human transfer factor and the thymus gland from the non-viable remains of a 13-week-old fetus obtained by a prostaglandin-induced therapeutic abortion。 72 hours post-thymus transplant (TTX) the patient's lymphocytes began to respond to PHA stimulation with increased DNA synthesis as measured by ${ }^{3} \mathrm{HTdR}$ incorporation which reached a maximum of $350 \%$ increase over controls 3 weeks post-TTx. Concomitantly the percent of peripheral B lymphocytes declined to normal levels of $30 \%$. A pronounced skin rash and eosinophilia $\left(12,000\right.$ cells $\left./ \mathrm{mm}^{3}\right)$ developed which gradually recrudesced. At 100 days post-TTx, the child was able to make an antibody response to diphtheria and tetanus antigens. The child continues to improve at 135 days post-TTx.

470 EFFICACY OF CORD BLOOD MONONUCLEAR CELLS IN ANTIBODYDEPENDENT CELL-MEDIATED CYTOTOXICITY TO TARGET CELLS INFECTED WITH HERPES SIMPLEX VIRUSES. Steven L. Shore, Henry Milgrom, Phyllis A. Wood and André J. Nahmias. Emory univ., Dept. of Ped,, and Center for Disease Control, Virology Division, At lanta.

We have recently described the phenomenon of antibody-dependent cell-mediated cytotoxicity (ADCC) for target cells infected with HSV, using immune serum with mononuclear cells (MC) from either immune or nonimmune individuals. Because a defect in ADCC could account for the newborn's increased susceptibility to HSV, cord blood specimens from normal newborns were evaluated for the presence of cytotoxic effector cells and 1ymphocyte-dependent antibody (LDA) to HSV infected cells. Ficoll-Hypaque purified MC from all 13 cord blood specimens tested showed significant cytotoxic activity in an $\mathrm{ADCC} \mathrm{Cr}^{51}$ release assay using $\mathrm{HSV}-1$ infected and HSV-2 infected Chang liver cells as targets. At an effector:target cell ratio ( $E: T$ ) of $30: 1$, the cord blood MC showed $30 \%$ (mean) less cytotoxicity than $M C$ from 10 normal adults. Since the cord blood specimens contained $86 \%$ (mean) more MC than the adult specimens, the total cytotoxic effector cell activity is actually comparable to that of adults. Indeed, when tested at an E:T of $60: 1$, cord blood cytotoxic activity was in the adult range. Studies of maternal-cord serum pairs demonstrated transplacental passage of LDA to HSV infected cells, confirming that the LDA is of the IgG class. These data imply normal function of ADCC in the newborn and are at variance with the report of McConnachie et al. (Pediaand are at variance with the report of McConnachie et al. (Pedia-
trics 52:795, 1973), which suggested a defect in the newborn's cytotoxic ceils in another ADCC system.

471 STUDIES OF THE MECHANISM OF IMPAIRED ANTI-STREPTOCOCCAL CARBOHYDRATE RESPONSES IN JUVENILE RHEUMATOID ARTHRITIS. Stanford T. Shulman and Elia M. Ayoub, Univ. of Florida Col. of Med., Dept. of Ped., Gainesville

Patients with Juvenile Rheumatoid Arthritis (JRA) manifest significantly depressed levels of antibody to group A streptococcal carbohydrate (A-antibody) when compared to age-matched controls. That this impaired A-antibody response is not related to a lower frequency of streptococcal infection is supported by the lack of significant differences in antibody responses to streptococcal extracellular protein antigens, anti-streptolysin 0 (ASO) and anti-desoxyribonuclease B (anti-DNAse B), between the JRA and control groups. The possibility of a generalized inability of JRA patients to respond to carbohydrate antigens was excluded by the finding of normal levels of isohemagglutinins. An alternate explanation for the impaired A-antibody response is a defect in intraphagocytic processing of the A-carbohydrate antigen. Enhanced degradation of group A to A-variant (AV) carbohydrate would result in low levels of A-antibody and in elevated levels of antibody to AV-carbohydrate (AV-antibody). This hypothesis was tested by determining serum AV-antibody levels using a radioimmune precipitin technique. The AV-antibody levels obtained for 66 JRA patients were significantly lower than those of a group of matched controls $(p<0.01)$. Thus, the low A-antibody levels observed in JRA cannot be the result of enhanced degradation of the A-carbodydrate antigen only. These data point to the existence of an alternate defect in the response of these patients to this bacterial antigen.
472 A NEW CLINICAL SYNDROME OF RECURRENT INFECTION ASSOCIATED WITH A COMBINED DEFICIENCY OF COMPLEMENT AND IMMUNOGLOBULIN. Otto $F$. Sieber, Jr., Robert $C$. Strunk and Vincent A. Fulginiti. Univ. of Arizona Col, of Med., Dept. of Ped., Tucson.

A 9 year old boy with recurrent pneumonia, septicemia, arthritis and staphylococcal granulomatous skin disease had been diagnosed as hypogamnaglobulinemic, and had received periodic gamma globulin injections without apparent clinical benefit.

On referral, he had low immunoglobulin levels ( $\operatorname{IgG}=68 ; \operatorname{IgA}=9$; IgM=11) and abnormal nitroblue tetrazolium (NBT) dye reduction suggestive of chronic granulomatous disease. The NBT test was corrected in the presence of normal serum. Other in vitro white blood cell function tests were normal.

Functional and immunochemical deterninations of whole complement, $\mathrm{C} 4, \mathrm{C} 2, \mathrm{C} 3, \mathrm{C} 5$, factor $\mathrm{B}$, and properdin were normal; however, functional activity of the properdin pathway ( $\mathrm{PH}_{50}$ ) by the method of Platt-Mills and Ishizaka (hemolysis of rabbit RBC in ethylene glycol bis-amino tetracetate buffer) was markedly diminished (patient=11; $N=18-25$ ). When the patient's serum was diluted by an equal amount of normal serum, a normal $\mathrm{PH}_{50}$ resulted. Thus, no evidence of a serum inhibitor was found.

In sumary, we describe 1) a combined deficiency in immunoglobulins and in the alternative (properdin) complement pathway 2) the relationship of an abnormal NBT response and complement function 3) the value of the $\mathrm{PH}_{50}$ as a diagnostic tool.

Monthly fresh plasma transfusions have decreased the frequency of infections and improved in vitro test results, further supporting a multiple immune factor deficiency.

\section{IMmunoglobulin E (IgE) IN ACUTE OTItis MEdia. Sloyer, John L., Jr., Howie, Virgil M.,

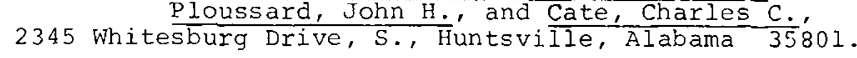

The concentration and specificity of IgE in middle ear fluids (MEF) and in sumultaneously drawn serum from infants and children with acute otitis media due to $\mathrm{zae}$ (HF) was studied by radioimmunoa ssay and indirect immunofluorescence. The mean serum IgE concentratior. from 34 patients under two years of age was $116 \mathrm{ng} / \mathrm{ml}$ and the mean MEF IgE was $239 \mathrm{ng} / \mathrm{ml}$. The increase in MEF IgE concentration over that of the serum level approached significance in the 7-12 month age group and was significant in the 13-18 month group ( $0<.05)$. The mean serum IgE concentration was approximately equal when compared by etiology (SP or HF) but was higher in the MEF from patients with SP otitis media. Of 131 patients whose serums and MEF were assayed by IFA, $58 \%$ had IgE specific antibody in at least one specimen, the most cominon of which (71\%) was MEF. The results indicate that the host may be responding to bacterial infection with local IgE antibody production. Whether such an immunological response is important in the immunopathology of acute otitis media remains to be firmly established.

474 LYMPHOCYTE SUBPOPULATIONS IN IMMUNODEFICIENCY DISORDERS, Ichiro Tsukimoto, Beatrice C. Lampkin, Kwan Y. Wong, Junichi Yata, Dept. of Ped., Univ. of Cincinnati Col. of Med., Children's Hosp. Res. Fndn., Cincinnati and Dept. of Ped., Univ. of Toho, Tokyo.

In 25 children with primary immunodeficiency the lymphocyte subpopulations were studied, using rosette formation with sheep erythrocytes (RFC) and responsiveness to phytohemagglutinin(PHA) as indicator for $T$-cells, complement receptor (CRL) and surface
immunoglobulin (Ig) as indicator for $B-c e l l s$. The results were:

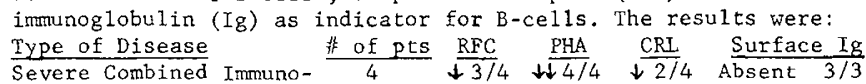
Deficiency (SCID)

Ataxia Telangiectasia

maglobulinemia (XaG)

$\begin{array}{llllllll}\text { Selective IgA Deficiency } 5 & \text { N } 5 / 5 & \text { N } & 5 / 5 & \text { N } & 5 / 5 & \text { N } & 2 / 2\end{array}$

$\begin{array}{llllllllll}\text { Acquired Common Variable } & 5 & \mathrm{~N} & 5 / 5 & \mathrm{~N} & 5 / 5 & \mathrm{~N} & 5 / 5 & \mathrm{~N} & 2 / 2 \\ \text { Ac } & 5 / 5 & \mathrm{~N} & 3 / 5 & \mathrm{~N} & 5 / 5 & \mathrm{~N} & 3 / 5\end{array}$ $\begin{array}{ccc}\text { Irmunodeficiency } & \downarrow 2 / 5 & \downarrow 2 / 5 \\ \text { The normal RFC but decreased PHA in one patient with SCID and one }\end{array}$ The normal RFC but decreased PHA in one patient with SCID and one tional and non-functional T-cells. Similarly, CRL was detected in all patients with XaG suggesting that CRL was found in both functional and non-functional B-cells. The absence of surface Ig in all patients with XaG suggests that the defect in this disorder lies in the transformation of the $\mathrm{B}$-cell into functioning cells. The variable presence of surface $\mathrm{Ig}$ in the other immunodeficient states may be the result of inability to synthesize Ig, 
475 IN VITRO LYMPHOCYTE RESPONSES IN PATIENTS WITH SELECTIVE IgA DEFICIENCY. David G. Tubergen, Ross E. Petty, and James T. Cassidy

Dept. of Ped. Ann Arbor Michigan.

Fourteen patients with selective IgA deficiency (serum IgA $<0.01 \mathrm{mg} / \mathrm{ml}$, salivary IgA undetectable, peripheral lymphocytes with surface IgA present), were studied. They ranged in age from $2-45$ years, with $50 \%$ under 15 years of age. Half had connective tissue disease, (juvenile rheumatoid arthritis 3 , rheumatoid arthritis 1 , ankylosing spondylitis 1 , dermatomyositis 1 , systemic lupus erythematosus 1); the rest had recurrent infections. In vitro responses to phytohemagglutinin (PHA) and pokeweed mitogen (PWM) were studied by micro-culture. Dose response curves were done for PHA $(0.05-50 \mu \mathrm{g} / \mathrm{ml})$ and a single dose $(5 \mu \mathrm{g} / \mathrm{ml})$ was used for PWM. Responses were measured by uptake of $3 \mathrm{H}$-thymidine after 3 (PHA) and 4 (PIM) day cultures. When values for PHA were expressed as the ratio of peak responses $(\mathrm{cpm})$ to background (cpm), the mean of the patient group was $70 \%$ of normal. Dose response curves were normal in $7 / 14$, but 7 patients had response curves below the $95 \%$ confidence limit for normals. PHA concentrations for maximal response were similar for normal and IgA-deficient groups. Although the range of normal values for PWM response was wide, PWM response in IgA-deficient patients tended to be lower than in normals. Depression of PWM and PHA responses was not correlated with disease category or occurrence of anti-IgA antibodies. These data indicate that selective IgA deficiency is not a homogeneous immunologic entity; some IgAdeficient individuals have abnormal in vitro responses to PHA.

476 LYMPHOID CHIMERISM WITH MILD GRAFT vs HOST REACTION FOLLOWING NON-MATCHED MARROW TRAINSPLAHTATION IN COKBINED IMMUNE DEFYCIENCY. David G. Tubcrgen and Ross $\frac{\text { E. Petty }}{\text { Dept. }} \frac{\text { (Intr. by Mason Barr) }}{\text { Ped., Ann Arbor, Michigan. }}$

Bept. of Pcd., Ann Arbor, Michigen transplantation with establishment of lymphoid chimerism and of in vitro lymphoid responsiveness was accomplished in a patient with combincd immune deficiency(CID)in spitc of a major HL-A difference and a positive mixed leukocyte culture.

A four month old male whose brother died with CID presented with diarrhca, candidiasis and skin rash: Laboratory studics showed normal. I\&A and $I_{g M}$ but low $I_{g G}(64 \mathrm{mg} \%)$, incrcased immunoglobulin bearing lymphocytes and $5 \%$ ' $\mathrm{l}$-ccll roscttes in peripheral blood. Lymphocytes did not rcspond to phytohemagglutin(PIA) or pokcwecd mitogen in vitro. Mother and paticnt differed at one HL-A locus and maternal cells reactca against paticnt's cclls in the one-way mixcd lcukocyte reaction. This reaction was grcatly diminished by maternal scrum. Bactcrial dccontamination was followed by an initial marrow transplant of $5 \times 10^{5} \mathrm{cells} / \mathrm{Kg}$. Cytoxan was given on days 2 and 3 post-transplant; mcthotrexate and maternal IgG wcre used 4 times to treat the mild GvH which began on day 10. In vitro responses to PHA were first scen on day 39 and reached ncarly normal lcvels on day 59. Lymphocyte chimerism was demonstratcd on day 46 . Aplastic ancmia devcloped and a sccond transplant of $10 \times 10^{6} \mathrm{cclls} / \mathrm{K} 8$ treated with anti-thymocyte slobulin was done on day 60 . Mild GvH followcd and up to day 87 requircd no trcatment. Improvement in marrow function has occurred. (Supported in part by NIH Grant AI 11009)

477 LYMPHOCYTE IN VITRO RESPONSE TO RUBELLA ANTIGEN AND PHYTOHEMAGGLUTININ (PHA) AFTER RUBELIA VACCINATION. Timo Vesikari and Elena Buimovici-Klein (Intr. by Louls $\frac{1}{2}$. Cooper). Col. of Physicians \& Surgeons of Columbia Univ., Roosevelt Hosp., Ped. Service, New York.

RA $27 / 3$ strain of live at tenuated rubella virus vaccine was given subcutaneously to 4 seronegative and 4 seropositive young female adults. Lymphocyte in vitro responses to rubella virus antigen and to PHA were assayed sequentialiy using a radioactive thymidine incorporation technique. Mild suppression of PHA response, detectable only with a suboptimal dose of mitogen, was seen in both seronegative and seropositive recipients of the vaccine. The seronegative vaccinees infially did not show lymphocyte response to rubella virus. Following vaccination highest lymphocyte stimulation with rubella was seen at day 21 , with decrease to normal immune level by day 43. One of the 4 seropositive vaccinees also showed temporarily increased lymphocyte reactivity to rubella after vaccination; in this case the in vitro response to control antigen was also enhanced simultaneously. The results suggest that specific cellular respons to rubella antigen develops independently from the suppressive effect of rubella vaccination on lymphocyte PHA-response.
478 ANTIGEN-ANTTBODY INTERACTION ON THE GUT SURFACE: Possible mechanism for control of allergic disease states. W. Allan Walker, Kurt J. Bloch and Kurt J. Isselbacher. Harvard Medical School, Mass. Gen. Hosp., GI Unit and Clinical Immunology Unit, Boston.

The gut is a potential site for the absorption of ingested antigens (allergens) and therefore might be of importance in the pathogenesis of local intestinal and systemic allergic disease states. We have studied the role of local antibodies in controlling the intestinal absorption of known allergens using everted gut sacs from immunized rats. After repeated intraperitoneal injection of protein antigens, everted gut sacs from immunized rats and controls were exposed to radiolabeled antigen in vitro. There was a significant decrease in absorption of antigen by gut sacs from immunized compared to control rats. Gut sacs from imm longed incubation. The mucous coat (glycocalyx) from gut sacs of immunized rats was analyzed on sucrose gradients; radioactivity was localized to the bottom of the gradient suggesting that antigen wras complexed to antibody in the mucous. These studies suggest that antigens become rapidly associated with antibodies present on the surface of the gut. Formation of antigen-antibody complexes prevents binding of antigen to and absorption by intestinal cells. Complexes in the mucous coat of the gut are readily degraded by local proteases and therefore less available for absorption. We suggest an additional role for antibodies present on mucosal surfaces, i.e., protection against absorption of ingested proteins which have escaped normal digestion in the intestinal lumen and may provide the basis for intestinal allergic states.
179 FETAL LIVER TRANSPLANTATION IN SEVERE COMBINED IMMUNODEFICIENCY (SCID): SUCCESS AND GRAFT-VERSUS-HOST (G$V-H)$ DISEASE. John K. Whisnant, Richard I. Schiff, Richard B. Gilbertsen, Marvin S. Platt and Rebecca H. Buckley. Duke Univ Sch. Med, Dept Ped, Durham, N.C.

Fetal liver transplantation is a possible alternate to bone marrow transplantation in infants with SCID. Two unrelated male infants with SCID had no histocompatible siblings. Both had lymphopenia, panhypogammaglobui inemia, cutaneous anergy, very low \#'s of T rosettes, elevated \#'s of C3 receptor and Ig-bearing B lymphocytes, absent in vitro lymphocyte responses to PHA, Con A, PWM, antigens and al logeneic cells, and normal RBC adenosine deaminase (ADA). At 11 months, infant \#1 was given $3 \times 108$ 1iver celis intraperitoneally from a 9 week fetus and at 13 months infant $\$ 2$ received $8 \times 10^{7}$ cells from an 8 week fetus. Both developed fever, a maculopapular exfoliative rash, alopecia, and slight liver enzyme elevations 7-10 weeks post-transpiant and had lymphocytic in filtrates on skin biopsy. Infant \#l also had diarrhea and eosino philia and remained unchanged immunologically except for an increase in $\mathrm{T}$ cell rosettes from 3.5 to $49 \%$. In contrast, infant \#2 had a disappearance of oral moniliasis by 8 weeks post-transplant and at 12 weeks showed an increase in $T$ cell rosettes from 1 to $30 \%$, a decrease in B cell rosettes from 38 to $20 \%$, normal in vitro lymphocyte responses to PHA and PWM, and a low response to Con A. These attempts at reconstitution 1) demonstrate that fetal liver transplantation in SCID may be accompanied by mild and transient $G-V-H$ and 2) confirm (in this the third successful case and the first ADA positive) that fetal liver can confer imminocompetence to some such infants. PROLONGED CNS VIRAL INFECTION WITH ECHO 30 IN AN
AGAMMAGLOBULINEMIC CHILD WITH INTACT CELL-MEDIATED IMMUNITY. John K. Whisnant, Edward L. Treadwell, T. Mohanakumar, Catherine M. Wilfert, and Rebecca H. Buckley. Duke Univ. Sch. Med, Dept. Ped., Durham, N.C.

Susceptibility to viral agents is considered unusual in patients with "pure" deficits in humoral immunity. We report here a prolonged $(>5$ mo) CNS viral infection in a 3 year old agammaglobulinemic girl. Studies showed panhypogammaglobul inemia, a normal lymphocyte count, normal (57\%) T cell rosettes, low $(5 \%)$ $B$ cell rosettes, few $(0.3 \%)$ surface Ig-bearing $B$ lymphocytes, normal delayed hypersensitivity and normal peripheral blood lymphocyte (PBL) responses to PHA, Con A, PWM and antigens. Initially, she had hepatomegaly and liver dysfunction which resolved over 7-2 months; serum was negative for HBsAg. Three months later she developed clinical meningoencephalitis with CSF pleocytosis and elevated protein. Symptoms lasted on ly 6 weeks but Echo 30 was isolated from CSF on 7 occasions over 5 months. The CSF eventually became virus-negative without specific antibody therapy. Throughout the virus infection $T$ cell function remained normal. The patient's PBL's exhibited a high degree of reactivity with an anti-human lymphoma-leukemia antiserum in cytotoxicity tests conducted at the time of her initial evaluation and periodically over the next 9 months. Coincident with the development of CSF virus-negativity, PBL reactivity with the anti-lymphomaleukemia antiserum disappeared. These observations may relate to the known high rate of malignancy amiong immunodeficient patients and suggest that prolonged viral infection could be etiologically related to such. 
481 ONTOGENY OF DIFFERENTIATION CAPACITY OF B LYMPHOCYTES IN MAN. L. Y. Frank Wu, Alfredo B1anco, and Alexander R. Lawton. Spain Immunology Labs., Depts. of Pediatrics and Microbiology, Univ. of Ala. in Birmingham, Birmingham, Alabama 35294.

The capacity of $B$ lymphocytes to differentiate into immunoglobulin-secreting plasma cells when stimulated by pokeweed mitogen (PWM) was examined using tissues from 9 autopsied fetuses, 9 cord blood specimens, and peripheral blood from 56 individuals aged 3 days to 71 years. Lymphocytes were cultured for 7 days with PWM, and cells containing cytoplasmic immunoglobulin enumer ated by direct immunofluorescence. Spleen cells from 2 fetuses aged 12-13 wks did not respond. Cultured cells from 5 fetuses aged $14 \frac{1}{2}-16 \frac{1}{2}$ wks, although similar to adult cells with respect to the frequency of lymphocytes bearing surface IgM, IgG, and IgA, generated plasma cells of all classes in very low frequency $(<1 / 1000)$. IgM-containing cells reached $20 \%$ of adult median frequency in cultures from 2 older fetuses (18-22 wks), while the proportion of IgG- and IgA-containing cells did not rise. A similar pattern was observed in neonates and infants up to $2 \frac{1}{2}$ months of age. Between 4 months and 5 years of age the IgM response reached the adult median of approximately $5 \%$ positive cells, while IgG and IgA responses remained lower than median adult values of $6 \%$ and $3 \%$ respectively. The highest responses were obtained in young adults. These observations indicate that the capacity of $B$ lymphocytes to respond to PWM with terminal differentiation matures with age, and at different rates for precursors of IgM, IgG, and IgA.

\section{INFECTIOUS DISEASE}

482 PUR PURA FULMINANS - AN AMPLIFIED DEFINITION OF THE SYNDROME

Arturo J. Aballi, M,D., Gungor Karayalcin, M.D. Fernando Costales, M,D., Dept. of Ped., Queens Hospital Center, Affiliation of the Long Island Jewish-Hillside Medical Center, Jamaica, New York.

Two cases of purpura fulminans (PF) coinciding with septice mias (Aerobacter aerogens and Diplococcus pneumonia) have been observed in our services in recent years. Both exhibited hemorrhagic necrosis of the skin and frank disturbances indicative of disseminated intravascular coagulation (DIC). Many cases of PF reported after 1964 developed typical symptoms at the time of fulminating active infections. The classical form of the syndrome occurs days or weeks after a relatively benign infection, not coincidental with it. However, hemostatic defects and pathological leslons are identical in both forms. Therefore, it is felt that the term PF should describe a hemorrhagic necrosis of the skin due to DIC, regardless of the mechanism involved. Preparatory and precipitating factors may vary considerably, but may lead to similar end results. This is comparable to what happens in different experiment models. We have triggered the Shwartzman Reaction by large doses of gram negative endotoxin given by slow intravenous drip, with and without antihistamines, and by small doses after preparation with cortisone or epsilon aminocaproic acid. Hemorrhagic necrosis of the skin was also produced by intradermal or subcutaneous injections of different substances. Illustrations of these experiments will be presented.

483

NEONATAL INFECTION BY HERPESVIRUS HOMINIS TYPE 2, A

COMPLICATION OF INTRAPARTUM FETAL MONITORING.

Garrett Adams, Dilip M. Purohit, Henrietta S. Bada and Billy F. Andrews, University of Louisville School of Medicine Department of Pediatrics, Louisville, Kentucky.

This report describes three neonates who acquired infection by Herpesvirus hominis type 2 ( $\mathrm{HVH}-2$ ) apparently as a consequence of abrasions received during intrapartum fetal monitoring with scalp electrodes. Electrodes were in place from 4 to 9 hours. Two babies were normal term babies; the third was inappropriately small for a 36 week gestation. All had caput succedaneum and had scalp abrasions at the site of electrode attachment. Vesicular lesions were noted at these sites between 4 and 10 days of age. Subsequently, new vesicles developed around the first lesions and at distant sites. HVH-2 was recovered from the vesicles in each instance even though the mothers denied knowledge of genital infections and no virus was recovered in their genital cultures. Lesions disappeared gradually in 3 to 5 days coincident with application of topical iododeoxyuridine (IUDR); however, in 7 months follow-up of the 2 term babies virus positive lesions recurred every 10 to 21 days for 4 months in one and still recur at this writing in the other baby. Recurrent lesions resolve spontaneously without therapy in the same time period as the treated ones, so a beneficial effect of topical IUDR has not been demonstrated. The third baby was the only baby with systemic infection. He recovered during treatment with adenosine arabinoside but was unfortunately lost to follow-up. Scalp abrasions from fetal monitoring must be considered a portal of entry for HVH-2 infection. 484 TOXOPLASMA GONDII: PLAQUE ASSAY IN A HUMAN CELL SYS Ahronheim (Intr. by David J. Lang), Dept. of Pediatrics, Duke Univ. Med. Ctr., Durham NC 27710

Species-specific virus-stimulated interferon (IF) protects some non-primate cell monolayers from destruction following infection by Toxoplasma gondii. A quantitative assay in a human cell system has not been heretofore published, nor has a role for IF been defined in human toxoplasmosis.

A semi-micro plaque assay has been developed, in which plaques of RH-strain T.gondii are formed in monolayers of human embryonic muscle fibrobTasts after 5 days incubation under tissue-culture maintenance medium containing $1 \%$ methylcellulose. Plaque counts in control wells equal the number of trophozoites in the inoculum as determined by direct counts.

Pretreatment of the monolayer with NIH reference human IF, in concentrations ranging from 0.01 to 10,000 units $/ \mathrm{ml}$, caused no reduction in $\mathrm{T}$.gondii plaque numbers, whereas parallel runs using vesicular-stomatitis virus (VSV) in an identical system yielded $50 \%$ reduction of VSV plaques at 10 units $/ \mathrm{ml}$ IF.

In this in-vitro assay, I.gondii infectivity appears to be insensitive to virus-stimulated human interferon.

\section{HEMOP OF IN-VITRO ANTA- Gerald $A$. Ahronheim (Intr. by Samuel L. Katz), Dept. Pediatrics, Duke Univ. Med. Ctr., Durnam NC 27710}

Conflicting statements exist regarding therapy of bacterial disease with combinations of "bactericidal" and "bacteriostatic" antibiotics. Little patient data and fewer in-vitro correlates have been published for Hemophilus influenzae type b (HITB); with the appearance of ampicillin-resistant strains in serious childhood disease, this question merits re-examination.

A micro-method utilizing the checkerboard technique has been adapted for work with HITB. Serial two-fold dilutions of antibiotics are made in two directions in 96-well plates, using MuellerHinton broth with $1 \%$ Difco Supplement $C$. Inocula of 10 and $10^{4}$ organisms $/ 0.025 \mathrm{ml}$ are used in a final volume of $0.10 \mathrm{ml} / \mathrm{well}$; endpoints are easily read by the appearance of a button of organisms at the bottom of growth-positive wells after 8-12 hours incubation in $5 \% \mathrm{CO}_{2}$. Bactericidal effect is determined by subculture to chocolate-agar plates.

None of the clinical isolates of HITB, tested with various combinations of antibiotics, showed antagonism between chloramphenicol and either ampicillin or penicillin-G. The expected additive effect was seen with pairs of penicillins.

In areas where ampicillin-resistant HITB disease has been recognized, it has been suggested that, until specific sensitivity testing has been accomplished, combined therapy with penicillin and chloramphenicol be initiated in serious disease. This study provides no in-vitro evidence to support the fear that combined therapy is potentially harmful. 
487

McIntosh). Univ. of Colo. Med. Ctr., Dept. of Ped. Denver and Ctr. for Disease Control, Bureau of Epidemiology, Atlanta.

Ongoing evaluations of the impact of measles vaccination in the United States have focused on the epidemioloay of measles, emphasizina morbidity, immunity levels, and vaccine usage as indicators of the status of measles control and high risk populations. Mortality data provides a more clearly defined data base in assessing these same parameters. Recorded measles deaths were evaluated for 1958-1963 to provide data on mortality prior to vaccine licensure, while 1965-1970 were analyzed to evaluate the impact of vaccine. Average annual measles mortality rates in 1968-1970 declined to 0.03 deaths per 100,000 population from the prevaccine rate of 0.23 in 1958-1963. Mortality rates were highest in children 6-11 months of age. Higher mortality rates were noted in places with less than 10,000 residents with a geographic clustering of states with high mortality rates in the southern part of the United States. Although the non-white population had higher mortality rates, this differential diminished when income levels were analyzed. Counties having a larger percentage of the population with incomes below poverty level had greater mortality rates. Case-fatality ratios were greatest in those under 1 and over 15 years of age. Althouqh measles mortality can only provide a retrospective analvsis, it focuses on those populations in need of attention in establishina priorities in future public health programs.

RAPID AMPICILLIN SENSITIVITY TESTING FOR HEMOPHILUS

488 INFLUENZAE。 Roger M. Barkin, Martha H. Roe, and of Colo. Med. Ctr. and The Children's Hosp. of Denver, Dept. of Ped. and Path., Denver, Colo.

Recent isolations of Hemophilus influenzae type $b$ resistant to ampicillin (MIC $>2 \mu \mathrm{gm} / \mathrm{cc}$ ) necessitate the development of a cillin sensitivity. Disc testing or MIC determinations usually give results 36-48 hours after the clinical specimen is obtained. To facilitate clinical management, a rapid agar dilution method specimens within 6-12 hours of admission. Chocolate agar biplates were made, one side having no additives and the other side containing $2 \mu \mathrm{gm} / \mathrm{cc}$ of ampicillin. Clinical specimens (CSF and blood culture broths) were streaked directly on both sides of the plates without subculturing and incubated at $37^{\circ} \mathrm{C}$ cillin (MIC $<2 \mu \mathrm{gm} / \mathrm{CC}$ ) grew only on the side of the biplate without ampicillin at concentrations ranging from $10^{2}-10^{5}$ orqanisms/ CC. Six ampicillin resistant strains (CDC) (MIC $>2 \mu \mathrm{mm} / \mathrm{CC}$ ) grew on both sides of the biplate at inocula concentrations ranging from $10^{2}-10^{5}$ organisms/CC. Sensitivity testing on the 28 strains by the chocolate aqar biplate method correlated with MIC's determined by agar plate dilution techniques.
MEASLES MORTALITY: A RETROSPECTIVE LOOK AT THE VAC-

CINE ERA. Roger M. Barkin. (Intr. by Kenneth rapid, dependable, and reproducible method of determining ampiwas developed permitting sensitivity determination on clinical in 10\% C0\%. 22 Hemophilus influenzae strains sensitive to ampi-

BOOSTER IMMUNIZATION WITH LIVE MEASLES VACCINE. JameS W. Bass, Gerald W. Fischer, , Scott B. Halstead, William Tripler Army Med. Ctr., Dept. of Ped., Honolulu 96819 A. Wiebe. Tripler Army Med. Ctr., Dept. Of Ped., Honolulu 96819. Recent reports of measles in patients who have been previously ity in such patients may not be lasting. Booster measles immunization was therefore studied in individuals having had previous measles or measles vaccine. In the measles prevaccinated group, the acute sera in $137 / 318$ had no HI antibody at a titer of $1: 10$. On retest 123 of these had measles neutralizing antibody at $1: 2$. In this group 77 had HI titers of $1: 2-1: 8$ and the remainder were without HI antibody. A screening test for measles HI antibody at a titer of $1: 10$ is therefore inadequate. Of 50 individuals with previous measles, 49 had positive neutralizing antibody and $\mathrm{HI}$ titers of $>1: 2$. Of the 49 immune, only one showed a $4 X$ HI antibody response. A decreased antibody response was noted in the 318 previously vaccinated children; ranging from $10 \%$ when vaccination was $<2$ years to $33 \%$ when vaccination had been $>8$ years prior Booster immunization in this group was $\geq 4 X$ in 106/318 ranging from $20 \%$ in those vaccinated $<2$ years prior to $63 \%$ in those vaccinated $>8$ years previously. In contrast to measles induced antibody, vaccine induced HI antibody declined with time and became increasingly responsive to booster immunization. In previously vaccinated children there were 14 without neutralizing antibody and 13 converted after reimmunization. These observations suggest that booster measles vaccination after primary immunization may reduce vaccine failures and favorably augment immunity to this disease.

PROLONGED RUPTURE OF MEMBRANES (PROM):VALUE OF 491 LABORATORY STUDIES IN ASSESSMENT OF INFECTION. D.S.Bisberg, D.V. Schidlow, and H.S. Dweck (Intr. by L. Gartner) Albert Einstein Col Med, Dept of Ped, Bronx-Lebanon Hos Ctr, NYC. A retrospective study of 141 babies of birth weight $790-4,791 \mathrm{~g}(31<$ $2,500 \mathrm{~g}$ ) and gestations of $28-44 \mathrm{wk}(25<37 \mathrm{wk})$ was done to determine the value of several laboratory tests in the assessment of infection in babies born after PROM ( $\geqslant 24 \mathrm{hr}$ ). Twenty-six of the babies (Group 1), 4 of whom died, had suspected or proven infection (by clinical signs and symptoms or positive systemic cultures, respectively)and received antibiotics. Thirty-two babies (Group II) had clinical signs and symptoms compatible with infection but explained by other diseases and did not receive antibiotics. The remainder, Group 11 (83), had no signs or symptoms suggestive of infection. Although a significantly $(p<0.05)$ greater proportion of babies from Groups I $(9 / 26)$ and II $(11 / 32)$ had $>10$ white blood cells (WBC) in the gastric aspirate compared to Group III (12/83), only the presence of funisitis in the histologic examination of the umbilical cord differentiated Group I(10/26)from Groups II (5/32;p <0.02) and III (12/83;p<0.005). The number of babies with WBC counts $>$ or $\left\langle 20\right.$ or $25 \times 10^{3}$; $\rangle$ or $\langle 500$ bands; or positive surface cultures (ear, axilla, inguinal) were similar among the groups.

These preliminary data suggest that funisitis is the most significant test in assessing the presence of suspected or proven infection in babies born after PROM. Furthermore, the significant number of babies with $>10$ WBC's in the gastric aspirate of Group I as well as II suggests that the presence of WBC's is not only related to infection, but may also be related to other disease stafes.

STREPTOCOCCAL PHARYNGITIS IN CHILDREN; A COMPARISON OF 489 FOUR TREATMENT SCHEDULES USING INTRAMUSCULAR BENIATHINE PENICILLIN. James W. Bass, Frank W. Crast, Clarence R. Knowles, Charles N. Onufer, Tripler Army Medical Center, Dept. of Pediatrics, Honolulu, HI 96819.

Four hundred children with streptococcal pharyngitis were treated by randomization with 4 treatment regimens: 100 each received a single injection of: 1) $600,000 \mathrm{U}$ benzathine penicillin (BZP); 2) 1.2 million U BZP; 3) 600,000 U BZP plus 600,000 U procaine penicillin (PP); and 4) 900,000 U BZP plus $300,000 \mathrm{U}$ PP. clinical response and severity of local reactions were judged in a double-blind manner at 24, 48 and 72 hours. Repeat throat cultures were taken at these visits and at 10,21 and 42 days. There was no difference in clinical response among the 4 treatment groups. The 2 regimens containing PP cleared the throat of streptococci in all cases within 72 hours, while those without did not. Both preparations containing PP had significantly reduced local reactions compared to the 2 doses of BZP alone. The incidence of treatment failure (pos. cult. at 10 days) plus relapse (pos. cul. at 21 or 42 days) in Group 1 was 21\%; Group 2, 11\%; Group 3, 15\%; and Group 4, 6\%. There was a significant increase $(P<.01)$ in treatment failures and relapses in patients in Group 1 compared to those in Group 4. No significant difference was noted comparing other treatment groups. The single injection of $900,000 \mathrm{U}$ BZP plus $300,000 \mathrm{U}$ PP resulted in a treatment response equal to 1.2 million U BZP alone, cleared the throat of streptococci much quicker and markedly reduced the incidence and severity of local reactions. This regimen appears to offer optimal injection therapy for strep throat in the pediatric age group.
SEROLOGIC AND EPIDEMIOLOGIC STUDIES OF $\underline{H}$. INFLUENZAE

492 MENINGITIS. Frederic W. Bruhn, Helen B.- Masters and Kenneth McIntosh. Univ. of Colorado Med. Ctr.,

Dept. of Ped Denver.

The family epidemiology of $\underline{H}$. influenzae type $B$ ( $H$. flu B) meningitis and carriage and the factors responsible for antibody response are not well understood. A serologic and epidemiologic study of $\mathrm{H}$. flu B meningitis was performed in 14 children ages 2 mos. to 3 years and their families. All patients were treated with ampicillin with bacteriologic cure. Antibody to the capsule was measured by precipitation of $I_{125}$ labelled purified polysaccharide. Of $11 \mathrm{child}$ ren from whom serial sera were obtained, 3 demonstrated no rise in antibody in response to meningitis, 5 demonstrated moderate rises which decreased in 4 of 5 instances to low $(<0.1 \mathrm{\mu g} / \mathrm{ml})$ or undetectable levels by $40-50$ days, and only 3 developed sustained, probably protective ( $>2$ $\mu \mathrm{g} / \mathrm{ml}$ ) levels. Nasopharyngeal (NP) carriage of $\mathrm{H}$. flu B late in the disease appeared immunogenic in two children, one of whom had not responded at a 71 to meningitis. Antibody response to disease was not correlated with age, days of illness before treatment, persistence of fever, NP carriage at the time of admission (4 of 11), or involvement of other systems, such as pleura or middle ear. It was, however, correlated with cerebrospinal fluid glucose on admission: 6 of 6 with values $<20 \mathrm{mg} \%$ had moderate temporary or no antibody response; 3 of 4 with values $>20 \mathrm{mg} \%$ had a sustained response. Of 17 households where other family members were tested, $\mathrm{H}$. flu B carriers were identified in 8 at the time of admission. 
493 ORAL DICLOXACILLIN AS A MODE OF THERAPY FOR

493 TREATMENT OF ACUTE STAPHYLOCOCCAL OSTEOMYELITIS (ASO). Yvonne J. Bryson, James D. Connor, Mark LeClerc and Samuel T. Giammona (Intr. by WilliamL. Nyhan), Dept. Ped., Univ. Calif., San Diego, La Jolla, CA and Children's Hosp., San Francisco. Standard Rx of ASO in infants and children includes use of a parenteral penicillin, requiring protracted hospitalization during the post-acute stage of disease for management of IV therapy. Morbidity during this period could be eliminated by Rx orally, if healing and frequency or recurrence were the same, or improved in comparison to standard IV therapy. Oral Diclox provides ease of management and reliable dose responses with satisfactory penetration to bone and joints. Fourteen children with ASO were treated with a regimen of an average 1 week course of IV semi-synthetic penicillin for the acute stage, followed by oral Diclox at $100 \mathrm{mg} / \mathrm{kg} /$ day for a minimum of 4 weeks and until 2 weeks beyond any positive clinical or lab finding. S. aureus was present in blood, pus or bone of $11 / 14$ and $75 \%$ of the isolates produced penicillinase. Serial Diclox serum concentrations revealed average peak at $1 \mathrm{hr}$. of $25 \mathrm{\mu g} / \mathrm{ml}$ and at $3 \mathrm{hrs}$., $12 \mathrm{\mu g} / \mathrm{ml}$. MIC's of 540 hospital-isolated penicillinase-producing $S$. aureus strains were $0.03-3$ $\mathrm{mg} / \mathrm{ml}$, average 1.6 to Oxacillin. All children had complete resolution mainder $₹ 1 \mathrm{yr}$. No adverse effects of Diclox have been detected. Average hospitalization was reduced to 14 days. In this preliminary study, Diclox in the post-acute course of ASO provided satisfactory management; an extended, larger study will provlde needed data on recurrence. with no recurrences; 7 are in follow-up $>2$ yrs., 4 at 2 yrs. and the re-

PARENTERAL TOBRAMYCIN AND CEPHALOTHIN IN THE TREAT496 MENT OF SUSPECTED SEPSIS IN NEUTROPENIC CHILDREN. Jesse D. Cohen, Thomas D. Miale (Intx. by Elia M. Ayoub), Dept. of Ped.; Univ. of Florida Col. of Med., Gainesville. A study of the efficacy of parenteral tobramycin, a new aminoglycoside antibiotic, in the treatment of probable septicemia in neutropenic children with various malignant diseases was undertaken. Seventeen episodes of suspected sepsis in 13 neutropenic children with underlying malignant processes including acute leukemia (10), Wilms' tumor (2) and Ewing's sarcoma (1) were included in this study. Initial blood and urine cultures yielded 3 positive respectively for E. coli., Proteus and Eikenella, an HB-1 organism. The patients were treated with parenteral tobramycin and cephalothin for 1-80 days while receiving chemotherapy for their malignancies and were followed closely for renal, hepatic, bone marrow and auditory toxicity. Of the 17 episodes of probable septicemia, 10 resulted in marked clinical improvement, 3 remained unchanged after a minimum of 5 days of therapy and 4 ended fatally. Of the 4 children who died, 3 had negative cultures while the fourth died with E. coli. sepsis less than 6 hours after hospital admission. Considerable individual variation in serum tobramycin and cephalothin levels was found among these patients. No evidence of renal, hepatic, or bone marrow toxicity was found either during or after antibiotic therapy. Only one patient showed audiometric evidence of a mild bilateral conductive hearing loss following therapy. These results support the safety and efficacy of broad spectrum antibiotic coverage with parenteral tobramycin and cephalothin in neutropenic children with malignancies and suspected sepsis.

HSV2 REPLICATION AT $41^{\circ} \mathrm{C}$ : DEPENDENCE ON HOST CELL

494 AGE AND TYPE: Patricia J. Chesney, Allen Soneji, Melvin I. Marks. McGill Univ., Montreal Children's Hosp. Research Inst, Montreal, Quebec.

The impaired ability of the newborn animal to develop a fever in response to infection, may contribute to its susceptibility to HSV2. Studies of newborn animals have demonstrated the beneficial effect of elevated body temperature on the outcome of a herpesvirus infection. HSV2 replication is impaired in vitro at temperatures over $39^{\circ} \mathrm{C}$. The effect of elevated temperature on HSV2 replication in newborn and adult mouse tissues and in fetal human brain was examined. Primary monolayers were infected at .01PFU/ce11. Cultures were incubated at $41^{\circ} \mathrm{C}$ for 40 hours and subsequently maintained at $37^{\circ} \mathrm{C}$. Control cultures were incubated at $37^{\circ} \mathrm{C}$ only. Viral titers were determined using a methyl cellulose plaque assay in PRK cells. Mouse brain, liver, lung, skin, kidney and spinal ganglia and human fetal brain were examined. Control cultures of all tissues were destroyed by HSV2 within 48 hours. Following incubation at $41^{\circ} \mathrm{C}$, HSV replication at $37^{\circ} \mathrm{C}$, was inhibited to varying degrees for different tissues. Fetal human brain showed the least degree of inhibition. Newborn mouse fibroblasts showed the greatest degree of inhibition. A significant percentage of adult mouse brain cultures never produced virus. These results suggest that HSV2 may replicate well or not at all at elevated temperatures depending on the age of the animal and tissue type infected.

THE TRANSMISSION OF CYTOMEGALOVIRUS (CMV) IN BLOOD

495 TRANSFUSION: A MURINE MODEL. Kwok- ing Cheung, Helen M. Smith, David J, Lang. Dept. of Pediatrics, Duke Univ. Med. Ctr., Durham, North Carolina.

CMV infections are frequently recognized in connection with pregnancy, organ transplantation and associated immunosuppressive therapy, neoplasms of the reticuloendothelial system and multiple transfusions or perfusion. All of these conditions may evoke responses associated with reactions to a homograft. We have proposed that latent CMV is activated by the host response to foreign antigens. Taking advantage of the existence of defined inbred strains of mice we have developed an experimental model to test this hypothesis of the mode of transmission and activation of CMV in relation to blood transfusion. IInbred mice $\left(\mathrm{C}_{3} \mathrm{H}\right.$ and n liver, spleen and salivary gland. After 2-3 months active infection was no longer detectable by in vitro assay of organ suspensions. Blood from these mice was transfused into uninfected allogenic and isogenic hosts. It was found that CMV was transmitted by blood which was virus free by conventional tissue culture assay as well as by co-cultivation techniques employing purified suspensions of lymphocytes. Virus appeared earlier in the salivary glands of mice after heterologous transfusion. Transfusion of blood from uninfected animals was followed by rapid activation of CMV in both heterologous and homologous recipients. All virus isolations correlated with the appearance or rise in titer of neutralizing antibody. "This experimental model demonstrates that latent murine CMV persists in blood and stimulation.
NEONATAL SKIN CARE AND THE PREVENTION OF

497 STAPHYLOCOCCUS AUREUS (STAPH) COLONIZATION. William F. Coyer (Intr. by James W. Bass). Department of Pediatrics, Tripler Army Medical Center, Honolulu, Hawaii.

A comparison of Ivory soap bathing, Ivory soap plus umbilical Isopropyl alcohol application, umbilical Triple Dye application, total body pHisoHex bathing, and umbilical Neosporin powder application was performed to determine the relative effect of each in controlling neonatal staph colonization. Infants were infants received daily inpatient care with the assigned study agent. Anterior nares and umbilical gutter cultures were obtained at the time of hospital discharge. Colonization was defined as culture positivity at either site. Overall Staph colonization was significantly decreased with Neosporin (see Table below). Neosporin powder or Triple Dye resulted in less umbilical colonization than the other study groups $(P=<001)$, and the use of Neosporin powder or pHisoHex resulted in less anterior nares colonization than the Ivory soap or Ivory soap plus Isopropy 1 alcohol groups $(\mathrm{P}=<.001)$.

\begin{tabular}{|c|c|c|c|c|}
\hline Study Agent & No. & Cult. Pos. & Col. \% & $\mathrm{P}$ \\
\hline Ivory & 49 & 36 & 74 & $<.001$ \\
\hline Ivory \& Isopropyl Alcohol & 52 & 36 & 69 & $<.001$ \\
\hline Triple Dye & 270 & 110 & 41 & $<.005$ \\
\hline pHisoHex & 283 & 114 & 40 & $<.005$ \\
\hline Neosporin Powder & 271 & 74 & 27 & - \\
\hline
\end{tabular}
randomized at the time of admission to the Newborn Nursery. All 
FACTORS RELATEN TO THE OUTCOME. OF SEPTIC ARTHRITIS IN

499

CHILDRFN. Howard S. Faden, Charles $\underline{B}$. Smith (Int. by f Utah Affil. Hosps., Dept. of Med., Salt Lake City, Utah.

The charts of 24 children with the diagnosis of septic arthritis were reviewed to ascertain the importance of host, microbiologic and therapeutic factors on the clinical outcome. Twenty children had satisfactory results (no residual functional or radiographic abnormalities) and four children had unsatisfactory results.

\begin{tabular}{lcc} 
& Satisfactory & Unsatisfactory \\
\cline { 2 - 3 } *Intensive antibiotic Rx & $11 / 20$ & $3 / 4$ \\
Arthrotomy & $14 / 20$ & $4 / 4$ \\
Intra-articular antibiotics & $7 / 20$ & $2 / 4$ \\
Symptoms present $\geq 7$ days & & \\
$\quad$ before Rx & $6 / 20$ & $3 / 4$ \\
Hip joint & $4 / 20$ & $3 / 4$ \\
Osteomyelitis & $4 / 20$ & $3 / 4$ \\
S. aureus & $7 / 20$ & $2 / 4$
\end{tabular}

*Appropriate antibiotic administered parenterally for 10 or more days.

Differences in therapy or etiologic bacterial agents did not affect the clinical results. The data suggest that hip joint disease, concomitant osteomyelitis, and the presence of symptoms for 7 or more days before therapy were associated with unsatisfactory results.

EARLY DIAGNOSIS OF NEONATAL BACTEREMIA BY BUFFY-COAT

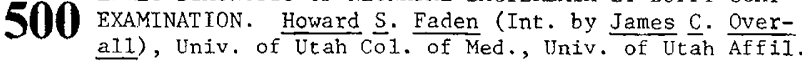
Hosps., Depts. of Med. and Ped., Salt Lake City, Utah.

The value of examination of the buffy-coat smear for the diagnosis of neonatal bacteremia was investigated. Simultaneous blood cultures and EDTA-anticoagulated blood samples were obtained from 86 neonates in a newborn intensive care unit. One $\mathrm{ml}$. of anticoagulated blood was centrifuged in a Wintrobe tube. The leukocyterich layer (buffy-coat) was smeared and stained with methylene blue, Gram's and Wright's stains. Of the 86 infants, 15 had positive blood cultures; of these 15,8 had positive buffy-coat smears. Organisms were demonstrated in the buffy-coat smears of $2 / 4$ infants with $\underline{E}$. coli bacteremia and $2 / 4$ infants with $\underline{S}$. aureus bacteremia. Single infants with $S$. pneumoniae, enterococcus, and Clostridium perfringens bacteremia had positive buffy-coat smears. One infant with S. aureus and Citrobacter bacteremia had both organisms demonstrated on the buffy-coat smear. Organisms were not seen on buffy-coat smears from 3 infants with blood cultures positive with $\underline{s}$. epidermidis and $I$ infant with a blood culture positive with diphtheroids. of the 15 positive blood cultures, 10 had bacterial growth in both aerobic and anaerobic bottles, and all 8 of the positive buffy-coat smears were from this group.

Four of 5 bacteremic infants who died had positive buffy-coat smears while 1 of 8 bacteremic infants with negative buffy-coat smears died, suggesting that positive buffy-coat smears correlated with severe illness. Our results indicate that buffy-coat examination, a procedure that takes less than one hour, is of value in the early diagnosis of neonatal bacteremia.
502

IMMUNOPOTENTIATION AND ANTIVIRAL CHEMOTHERAPY IN EXPERIMENTAL HERPESVIRUS ENCEPHALITIS. GeraId W. Fischer, John K. Podgore, Melvin W. Balk, and James W. Bass. Tripler Army Medical Center, Dept of Pediatrics, HonoluTu, HI 96819.

The high mortality of herpesvirus encephalitis may be related to an altered or impaired host immunologic response. Levamisole (LMS), a recently discovered immunopotentiator, used alone or in combination with standard antiviral chemotherapy, might theoretically be a valuable treatment modality for herpesvirus encephalitis. Ten-day-old Wistar rats were challenged IP with $10^{2} \mathrm{PFU}$ of Herpes hominus Type 2. LMS ( $3 \mathrm{mg} / \mathrm{kg} / \mathrm{dose}$ ) or adenine arabinoside $(\operatorname{Ara}-\bar{A}), 500 \mathrm{mg} / \mathrm{kg} /$ dose, were given in varied regimens. Survival in animals treated with LMS was $59 / 146(41 \%)$ while only $3 / 147(1 \%)$ of placebo treated controls survived $(p<.005)$. Treatment delay up to 24 hours after virus challenge did not alter the survival. Addition of Ara-A to the LMS regimen decreased the survival to $10 / 79$ or $13 \%$. Although the protective effect of LMS was partially inhibited by Ara-A, death was delayed in the LMS Ara-A groups when compared to controls. (Survival at 8 days; $0 \%$ in controls, $50 \%$ in LMS and Ara-A). Ara-A therapy alone was no better than placebo. No in vitro antiviral activity was demonstrated with LMS at $100 \frac{1}{\mathrm{~g}} / \mathrm{ml}$. Immunopotentiation may offer a unique treatment regimen for herpesvirus encephalitis, since treatment with levamisole in this model significantly enhanced survival. That Ara-A inhibits the beneficial effect of LMS in this model may be an important clue to both the mechanism of action of LMS and the immunologic response to herpesvirus invasion.

MMEGALOVIRUS INFEC

503 TION WITH A NEW CHEMOTHERAPEUTIC AGENT-PHOSPYONOACETIC ACID. Lowell A. Glasgow, Earl R. Kern, and James C. Overall, Jr., Dept. of Ped., U. of Utah Col. Med., SLC, Utah. Cytomegatovirus (CMV) is a common virus infection of humans with protean clinical manifestations. Although a number of antiviral substances have been utilized in man and experimental animals, no effective mode of therapy has been developed. In our murine CMV (MCMV) model infection IUDR, Ara $C$ and Ara $A$ have had negligible therapeutic effect. Phosphonoacetic acid (PAA) has been shown to be effective against herpesvirus hominis infection in tissue culture $(12-25 \mathrm{ug} / \mathrm{ml}$ minimal inhibitory conc.) as well as in animals, and is now entering clinical trials in man. MCMV was sensitive to $12-25 \mathrm{ug} / \mathrm{m} 1$ of PAA in mouse embryo cells. In mice infected IP with MCMV, a significant reduction in mortality was achieved in animals treated with $500 \mathrm{mg} / \mathrm{kg} /$ day, even when therapy was initiated after target organs were seeded, and MCMV replication was in progress. The untreated control animals had a final mortality of $70 \%(21 / 30)$, while those receiving PAA $2 \mathrm{~h}$ post infection had a final mortality of $10 \%(3 / 30)$, at $24 \mathrm{~h} 7 \%(1 / 15)$ and at $48 \mathrm{~h} 33 \%(5 / 15)$. Delineation of the specific effect of PAA on the pathogenesis of the virus infection indicated a striking suppression of MCMV replication in the liver $\left(>10^{5}\right.$ to $\left.<10\right)$ $\mathrm{PFU} / \mathrm{gm}$ ) with a lesser effect in kidney, spleen and lung. In summary, a new antiviral compound, PAA, has been shown to: (1) inhibit MCMV replication in vitro, (2) protect mice from a lethal MCMV infection, and (3) significantly inhibit MCMV replication in the liver thereby preventing death of the MCMV-infected animals.

504 EXPERIMENTAL HSV ENCEPHALITIS: EFFECTS OF TREATMENT WITH PHOSPHONOACETIC ACID. John F. Griffith and James F. Fitzwilliam. Duke Univ. Med. Ctr., Dept.

of Ped., Durnam, N.C.

The effects of phosphonoacetic acid (PAA) on survival and brain virus concentration were examined in young mice with experimental herpes simplex virus (HSV) encephalitis. Infection was established by direct intracerebral inoculation of 10 LD50 of type I HSV and parenteral therapy was begun 24 hours later and continued for four days. One half the LD50 of PAA was used. The concentration of virus per gram of brain tissue was determined at 48 hour intervals following the inoculation of virus.

A11 the untreated infected animals developed encephalitis and died by the seventh day after virus inoculation, compared to only $33 \%$ of those treated with PAA. The concentration of virus in the brains of treated animals was significantly less than in the untreated controls. Twenty four hours after beginning therapy it was $102 \mathrm{PFU} / \mathrm{gm}$ of brain tissue in the treatment group compared to $10^{5}$ in the untreated controls. This difference was maintained during the four day treatment period. In contrast to the untreated infected control group, the PAA treated animals maintained a stable weight and developed a brisk leukocytosis. There were no deaths or toxic effects attributable to the drug.

This is the first demonstration of the use of PAA in experimental encephalitis. These data indicate that it has antiviral activity in vivo, is well tolerated and probably deserves continued study to determine its clinical effectiveness. 
505 THE RETURN OF BOSTON EXANTHEM. Caroline B. Hall, James $\mathrm{D}$. Cherry, Hlford H. Hatch, Donald B. NeIson and Harland $S$. Winter. Unfv. Rochester, Rochester, NY, and UCLA, Ca, Dept Peds; State Lab Hygiene, Virus Section, Madison, Wis.; and CDC, Fnteric Virology Branch, Atlanta, Ga.

Exanthem due to ECHO 16 viral infection (Boston exanthem) was orlginally observed in 1951 and 1954. Since then, symptomatic and asyraptomatic Infection with FCHO 16 virus has been rare. During the summer and fall of 1974 we isolated ECHO 16 virus from 37 persons residing in $10 \mathrm{different}$ states. Ten of the patients were studied by the authors; in the remaining instances spectmens were referred for study. Of the total group the rajority had fever, $49 \%$ had rash and $35 \%$ had either encephalitis or meningitis. Exanthem occurred most commonly in children under the age of 3 yrs whereas encephalitis was mainly noted in adults. Seven of the 10 observed children had rashes and in 4 the illnesses suggested roseola infantum. Colncident with the occurrence of ECHO 16 infections in Rochester was an increase in the reporting of roseola suggesting more widespread EC10 16 disease. Thirtythree of the viral strains were recovered in primary monkey kidney tissue culture and 4 strains were recovered in diploid lung fibroblasts. In the 17 yrs prior to 1974 on 1 y 2 ECHO 16 viral strains were 1solated from spectmens submitted to the Wisconsin State Lab of Hyglene and only 14 strains were identified from 1967 to 1973 at the Enteric Virology Branch of the CDC. If ECHO 16 virus follows an ecological prevalence pattem similar to that of 2 other exanthem producing enteroviruses (Coxsackle A16 and ECHO 9 viruses) it is reasonable to predict that widespread epidemic Boston exanthem will occur in 1975.
506 ENDOTRACHEAL INTUBATION AND INFECTION. H. Harris, D. irtschafter, $G$. Cassady. Univ, of Ala, in B'ham., Division of Perinatal Medicine, Birmingham, Alabama. Fifty-five neonates who had never received antibiotics were prospectively assigned in random fashion to antibiotic or no-antibiotic groups before intubation. Colonization was assessed by na sopharyngeal and endotracheal cultures at intubation and each day while intubated. Systemic infection was monitored by blood cultures initially, usually with CSF and suprapubic urine cultures, and then every 3 days. Colonization and systemic cultures were obtained at time of extubation or death. Both groups were similar in birth weight and gestational age with no differences in black/white, male/female, or outborn/inborn ratios.

Colonization before intubation ( $43 \%$ ) was more common in neonates $>12 \mathrm{~h}$. postnatal age $(p<.005)$. Ultimate colonization, in those not colonized initially, tended to be more common in untreated infants with duration of intubation $>72 \mathrm{~h} .(\mathrm{p}<.10)$. Infection before intubation was present more commonly in babies $>12 \mathrm{~h}$. old $(32 \%$ vs $4 \% ; \mathrm{p}<.01)$ and was intimately related to prior colonization; $39 \%$ of those already colonized at intubation were concurrently infected vs $0 \%$ of uncolonized $(p<.001)$. A continuing relation between colonization and infection was observed; $43 \%$ of those subsequently colonized became infected vs $0 \%$ of those who remained uncolonized $(p<.025)$. In all but one patient, the infecting organism was one of the colonizers. Infection was found more commonly in untreated patients $(47 \%$ vs $19 \% ; \mathrm{p}<1.10)$.

These data suggest a high incidence of colonization in babies $>12 \mathrm{~h}$. old requiring intubation and a close relation between prior or subsequent colonization and ultimate systemic infection.

EPATITIS B ANTIGENEMIA \& ANTIBODIES AND SCABIES IN

507 KOREAN ORPHANS. Ruth C. Harris, Kyung S. Park, and Dav1d J. Gocke (Intr. by Edward C. Curnen, Jr.) Columbia Univ. Col. of P\&S, Dept. of Ped., N.Y.; Yonsei Univ. Col. of Med., Dept. of Ped., Seoul, Korea and Rutgers Med. Sch., Dept. of Med., New Brunswick, N.J.

In a survey of potentlally adoptive infants and children, abandoned within the preceding 6 mo, and of school children from homes in a low socio-economic group in Korea, the incidence of Hepatitis B Antigenemia $\left(\mathrm{HB}_{\mathrm{S}} \mathrm{Ag}\right.$ ) and Antibodies (AntiHB $\mathrm{B}_{\mathrm{S}}$ ) was high.

\begin{tabular}{|c|c|c|c|c|c|c|c|c|}
\hline \multicolumn{2}{|c|}{ Children } & \multirow{2}{*}{$\begin{array}{c}\text { Number } \\
113\end{array}$} & \multirow{2}{*}{$\begin{array}{l}\text { Agc* } \\
6-13 y\end{array}$} & \multicolumn{5}{|c|}{$\begin{array}{l}\text { Children with Positive Testss } \\
\mathrm{HB}_{\mathrm{S}} \mathrm{Ag} \text { AntiHB }\end{array}$} \\
\hline School & (1) & & & 17 & 6 & 23 & 20 & \\
\hline \multirow[t]{4}{*}{ Orphans } & (2) & 27 & $<6 \mathrm{~m}$ 。 & 1 & 8 & 9 & $33)$ & \\
\hline & & 27 & $7-23 \mathrm{~m}$. & 1 & 5 & 6 & $22\}$ & 21 \\
\hline & (3) & 55 & $2 m-8 y$, & 3 & 5 & 8 & $14 J$ & \\
\hline & (4) & 29 & $10 m-11 y$ & 6 & 9 & 15 & 52 & \\
\hline
\end{tabular}

(1) School children from families with low income (2) Orphans (4) Orphans with scabies, from orphanages and foster homes.

\section{* Age when tested.}

The $\mathrm{h} 1 \mathrm{gh}$ incidence of antibody in infants under 6 mo. reflects early exposure. The much higher incidence $(p=<.005)$ of antigen and antibody in orphans with scabies compared with those without scabies is being studied for vector relationshlp.
508 PREDOMINANT MEDICAL PROBLEMS OF ADOPTED OVERSEAS CHILDREN, Ruth C. Harris, Columbia Univ. Col. of
P\&S, Dept. of ped., N. Y.

Pediatric foresight about probable infections of children from underdeveloped countries will expedite improved health in these children and prevent disabilities in adopting families. Surveys of 700 families in 1972 and over 900 families in 1974 adopting infants and children from Asian countries show that $83 \%$ of these children have acute illness during the initial 6 mo. Persistent problems include ear (25\%) and upper respiratory infections (28\%), while diarrhea occurs during the first month in $42 \%$. Parasitic infestation $(7 \%)$, shigella, salmonella, virus infection and milk intolerance have been associated complaints. Skin infections have baffled local physicians, many of whom have failed to recognize that persistent eczematold impetiginous rashes were associated with scabies $(1-5 \%)$ until the entire family was similarly affected. Lice $(8 \%)$ has also been a poorly recognized family occurrence. Molluscum contagiosum, staphlococcal boils, styes and conjunctivitis (?trachoma) are other common disorders.

Intestinal $f l u$, hepatitis or infectious mononucleosis has occurred in about 30 families within the first 4 months of the child's arrival. A rare family has had salmonella, shigella or ameblasis in several members. Such infectious in no way diminish the adoptability of these children. It is incorrect to blame the new arrival for all family illnesses, but awareness and special care to prevent spread of infection is mandatory.

509 REAPpRAISAL OF KANAMYCIN USAGE IN NEONATES. Jorge $B$. Univ. of Texas Health Sci. Ctr. at Dallas, SouthUniv of Texas Heal

western Medical School, Dallas Kanamycin resistance of $\frac{E}{1}$ coli strains from neonates was first
observed in our nurseries in 1968 after 6 years of routine usage. The percentage of resistant strains increased to $71 \%$ in 1971 at which time kananycin usage was restricted. Subsequently this percentage decreased so that on $1 y$ 10\% are currently resistant to kanamycin. Surveillance studies in 6 additional North Arerican nurseries confirm this change in kanamycin susceptibilities of $\mathrm{E}$. coli. When kanamycin was again used routinely in our nurseries for 7 months in 1974, reemergence of resistance was not observed. Serum kanamycin dose-response curves were determined in 65 babies treated with either 7.5 or $10 \mathrm{mg} / \mathrm{kg}$ doses given every 12 hours. Peak serum levels varied with dosage, birthweight (BW) and chronologic age. Mean peak serum values after $10 \mathrm{mg} / \mathrm{kg}$ were 20 to $25 \mu \mathrm{g} / \mathrm{ml}$ in low $(<2000 \mathrm{~g})$ and normal $(>2000 \mathrm{~g})$ BW babies. By contrast, mean peak values after $7.5 \mathrm{mg} / \mathrm{kg}$ were 17 to $22 \mu \mathrm{g} / \mathrm{ml}$ in 1 ow $\mathrm{BW}$ and 12 to $15 \mu \mathrm{g} / \mathrm{ml}$ in normal BW babies. The distribution volumes and plasma clearances of kanamycin explained in part these pharmacokinetic differences. Serum half-life values correlated indirectly with gestational and chronologic ages. Kanamycin levels in cerebrospinal fluid specimens from 21 infants with meningitis were 0.5 to $12 \mathrm{\mu g} / \mathrm{ml}$ after $7.5 \mathrm{mg} / \mathrm{kg}$ doses.

These studies show: 1) E. coli strains from neonates are again susceptible to kanamycin and 2) Kanamycin dosages in neonates should be increased in order to consistently achieve peak serum levels in the therapeutic range.

DIAGNOSIS OF HEMOPHILUS INFLUENZAE B (H. FLU) AND

510 OTHER MENINGITIDES: GRAM STAIN (GS), LATEX AGGLUTINATION (LA) AND COUNTERCURRENT IMMUNOELECTROPHORESIS (CIE). David L. Ingram, Richard J. O'Reilly and Patricia J. Pond. Univ. N. C., Chapel Hill, Sch. of Med., Dept. Ped. and Mern. Sloan-Kettering Cancer Ctr., N.Y.C. (Intr. by Floyd W. Denny).

The diagnostic accuracy and utility of 3 tests were evaluated using initial cerebrospinal fluid samples from 132 children with suspected meningitis. Culture diagnoses were collated with GS, and LA and CIE tests using rabbit antisera to $\underline{H}$. flu, meningococcus Group $C(M C)$ and pneumococcus 82 types $(\mathrm{Pn})$ with these results:

\begin{tabular}{|c|c|c|c|c|c|c|c|c|}
\hline \multirow[b]{2}{*}{ CULTURE DIAGNOSIS } & \multirow[b]{2}{*}{ (No.) } & \multirow[t]{2}{*}{ GS $(+)$} & \multicolumn{3}{|c|}{$\mathrm{LA}(+)$} & \multicolumn{3}{|c|}{$\operatorname{CLE}(+)$} \\
\hline & & & H. flu & $\mathrm{MC}$ & $\mathrm{Pn}$ & H. flu & $M C$ & $P n$ \\
\hline H. influenzae $b$ & (35) & 26 & 29 & 1 & 1 & 27 & 1 & 0 \\
\hline $\bar{M}$ Mningococcus & ( 6$)$ & 4 & 0 & 1 & 0 & 0 & 0 & 0 \\
\hline$c$ & ( 3 ) & 2 & 0 & 2 & 0 & 0 & 2 & 0 \\
\hline Pneumococcus & ( 5$)$ & 3 & 0 & 0 & 1 & 0 & 0 & 2 \\
\hline Other bacteria & (4) & 3 & 0 & 0 & 2 & 0 & 1 & 0 \\
\hline Sterile & (79) & 2 & 2 & 1 & 12 & 0 & 2 & 0 \\
\hline
\end{tabular}

In $\underline{H}$. flu meningitis at least 1 test was positive in $32 / 35$ cases: LA detected 5 and CIE 4 among the GS (-) group; LA missed 2 and CIE 3 among the GS (+) group. The LA test, while most sensitive, showed more false $(t)$ and/or cross-reactions than did CIE, especially in non- $\underline{H}$. flu cases. However, the rapidity and simplicity of LA using stable reagents which were perfected may outweigh greater specificity of CIE in the diagnosis of bacterial meningitis 


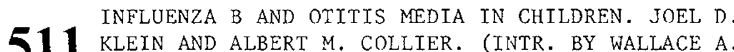
CLYDE, JR.) UNIV. OF NORTH CAROLINA, DEPT. PED.,

CHAPEL HILI.

An outbreak of Influenza virus Type B infections occurred in the Frank Porter Graham Day Care Center during Feb. - Apri1 1974. During this period there were 61 children enrolled in the Center (37 females \& 24 males), eighty percent black, with ages ranging from 3 months to 10 years. During the epidemic there were 27 isolations of Influenza $B$ virus from 20 children. One-half of these were obtained from children who were well at the time of culturing. Hemagglutination inhibition titers were determined against Inf. A/England/42/72 $\left(\mathrm{H}_{3} \mathrm{~N}_{2}\right)$, Inf. B/Victoria/98926/70 and $B$ Hong Kong/5/72 antigens. Five additional children with serologic evidence of Influenza $B$ infection were detected. Attack rates for most age groups approximated the $40 \%$ rate for the entire population. Clinical and microbiological data from this study were compared to those obtained in the center during the same 3 months in 1973. An increase in otitis media was seen during the 1974 study, especially during March which coincided with the peak of the Inf. B outbreak. There was also an increase in the incidence of fever $>38^{\circ} \mathrm{C}$ in the 1974 group. Intensive continuous microbiological surveillance for bacteria, viruses and mycoplasmas failed to reveal predominance of any other potential pathogen to account for these clinical findings. The analysis permitted by the nature of this study design revealed several unexpected findings: high attack rate unrelated to age; common inapparent infections; and frequent otitis media.

RISK OF ACQUIRED CYTONEGALOVIRUS (CMV) INFECTION IN

512 Eli Gold and Case Western Reserve Univ. Sch. of Med. at Cleveland Metropolitan General Hospital, Cleveland, Ohio.

A prospective study of 81 cytomegalovirus (CMV) negative infants born to mothers with cytomegaloviruria during pregnancy identified a cohort of 21 infants ( 11 females, 10 males) who acquired cytomegaloviruria early in life. Maternal viral excretion during the final month of pregnancy did not differ between the infected and uninfected groups. Two of 18 of the infants who became infected and none of the control infants studied had CMV positive placentas. All cord bloods were positive for CMN complement fixing (CF) antibody and 2 of 19 infants who became infected had elevate 1 cord $\gamma M$ antibody. Sixteen infants began excreting virus before 14 weeks of age. One infant was breast fed and none received blood transfusions. Virus excretion, as in congenitally infected infants, was invariably of high titer and usually present throughout the first year of life. Clinical symptoms coinciding temporally with the acquisition of infection occurred in 7 infants (33\%). Three infants had lower respiratory tract infections, 2 requiring hospitalization; 3 developed mild hepatosplenomegaly, 1 associated with a transient rise in serum transaminases and 17\% atypical lymphocytes; 1 developed extensive lymphadenopathy accompanied by a diffuse maculopapular rash. Physical exams at 1 year were normal, with mean I.Q. values comparable to the control group. In summary, $25 \%$ of our selected study group became infected, and a considerable proportion developed clinical symptomology. Currently all infants are doing well.

513 PERSISTANCE OF ANTIBODY FOLLOWING PRIMARY IRMUNIZATION 513 WITH MEINLNGOCOCCAL A AND C POLYSACCHARIDE VACCINES AND RESPONSE TO BOOSTER DOSES. Martha L. Lepow, Ronald Gold, Irving Goldschneider, and L.C. Gotschlich, UCONN Health Center and Rockefeller University, Dept. of Ped. \& Path. Farmington, $\mathrm{CT}$ and New York, NY.

500 infants and 1500 children less than 10 have been injected with 2-4 doses of meningococcal $A$ or $C$ vaccines at intervals of 3 mo. to 3 years.

Results with Group $C$ vaccine in infants indicate a maturation in primary antibody response during the first two years and a lower booster response in infants than in older children. Age, dose and spacing of injections are critical. A mean anti-C concentration of $2 \mathrm{Mg} / \mathrm{ml}$ can be achieved by primary immunization at 1 year of age. This level was associated with protection against disease in Brazil. Anti-C concentration returns to baseline 1-3 years after primary immunization. Little natural immunization occurs in childhood.

Antibody concentrations against Group $\Lambda$ polysaccharide gradually increase with age presumably as a result of exposure to crossreacting antigens. Age and number of boosters are critical factors in response of infants to Group $\Lambda$ vaccine. Significant primary and booster responses occur by 7 months of age. Antibody levels persist at $1 / 3$ to $1 / 2$ peak value for 18 months following booster. Variability of response to different lots of A vaccine correlates with molecular weight of vaccine. The minimal protective concentration of anti-A is not known.
514 WHITE CELL COUNT IN NEONATAL INFECTION. Barbara Manroe,,$\frac{\text { Arthur Weinberg, and Charles }}{\text { (Intr. }}$. Ry Chester Fink). Southwestern Med. Sch. at Dallas, Dept. of Ped.

Although the total white blood cell count is not helpful in diagnosing neonatal sepsis, the absolute neutrophil count, which includes both mature and immature forms, the band count, and the presence of toxic granulations may be useful indicators aidina in the diagnosis of neonatal infection. These parameters were investigated in 99 infants, all but 5 of whom had abnormalities of their white counts as defined in the recent literature. The babies were divided into groups by gestational age, weight, and 4 categories which reflect culture results and the clinical course. A course compatible with sepsis was defined by the presence of 3 or more factors and included lethargy, hypoglycemia, hyperbilirubinemia, poor feeding, failure to gain weight, abnormal temperature regulation, tachypnea, and tachycardia. Group I included 37 infants with positive blood cultures and a clinical course compatible with sepsis. Group II included 7 babies with pneumonia, urinary tract infection or necrotizing enterocolitis and who had significant cultures, but none obtained from the blood. Group III included 50 babies with clinical evidence of sepsis as defined, but no positive blood cultures. Group IV included 5 babies with positive cultures, but a normal clinical course and white blood count. These data suggest that the white blond count as defined above may be a useful tool in supporting the diagnosis of neonatal infection. PROSPECTIVE STUDY OF INFANTS WITH HIGH FEVER: Paul
L. MCCarthy and Thomas F. Dolan, Jr. (Intro, by Howard A. Pearson) Yale Univ. Sch. of Med. Yale-New Haven Hospital, Dept. of Ped. New Haven.

Selected laboratory studies were assessed prospectively in 125 children $\angle 24$ months of age with fever $\geq 104^{\circ} \mathrm{F}$. seen consecutively in the Yale-New Haven Hospital Emergency Room. One hundred and twenty four children had $C B C^{\prime} s, 104$ had sedimentation rates, 122 had blood cultures, 105 had chest x-rays, 57 had lumbar punctures, 42 had urine cultures and 22 had rectal cultures. Ninety-three children were examined on at least two occasions. Thirteen blood cultures were positive and 2 were associated with bacterial meningitis. Sixteen chest $x$-rays had infiltrates. Eleven CSF exams were abnormal and 3 grew bacterial pathogens. Three urine, 2 rectal and 1 wound drainage culture grew bacterial pathogens. These 46 abnormal studies occurred in 41 children. The source of fever was not clinically apparent in 27 of these 41 children and selected studies, especially chest $x$-ray ( 9 instances), blood culture ( 11 instances), CSF exam ( 3 instances) and urine culture ( 3 instances) were essential in making the diagnosis. The complete blood count and sedimentation rate were of limited value in assessing these children. Blood culture, chest $x$-ray and, often, CSF exam and urine culture will frequently lead to a diagnosis in infants with high fever without an obvious source.

OHERAPY FOR MENINGITIS DUE

516 TO GRAM-NEGATIVE ENTERIC BACTERIA. The Cooperative Neonatal Meningitis $\frac{\text { Study }}{\text { Dept. }}$ of Ped., Univ. of Texas H. McCracken, Jr., Coordinator, Dept. of Ped., Univ. of Texas

19 institutions from North and South Anerica have participated for 40 months in a collaborative study designed to evaluate intrathecal therapy of infantile meningitis caused by enteric bacteria. Infants have been randomly assigned to parenteral therapy with ampicillin and gentamicin (group A) or to parenteral therapy plus intrathecally administered gentamicin (group B). 110 patients were distributed randomly between the 2 therapy groups. 17 infants were greater than 30 days of age. E. Coli was the most common etiologic agent ( $70 \%$ of cases).

Time to sterilization of spinal fluid cultures was similar for the 2 groups. Uncorrected mortality rates were $33 \%$ (group $A$ ) and $27 \%$ (group B). Death of 2 group $B$ infants was unrelated to meningitis giving a corrected rate of $24 \%$. Uncorrected/corrected mortality rates for 77 patients with $E$. coli meningitis were $33 \% / 33 \%$ (group A) and $23 \% / 19 \%$ (group B). Resu1ts of 12 month followup examinations reveal that $73 \%$ of group $A$ and $38 \%$ of group $B$ patients are normal and $27 \%$ and $44 \%$ of group $A$ and $B$ babies, respectively, have moderate to severe neurologic residua. Among $E$. coli patients $59 \%$ of group $A$ and $36 \%$ of group $B$ babies are normal 12 mon ths after illness. Moderate to severe neurologic handicaps were detected in $24 \%$ and $36 \%$ of group $A$ and $B$ infants respectively.

The current data from our ongoing collaborative project indicate that the somewhat lower mortality rate with intrathecal therapy may be offset by the higher rate of serious sequellae. 
517 AITUMATED SIMMMARY REPORTS FROM A PEDIATRIC BACTERIOLOGY LABORATORY. Gilbert $W$. Mellin, Marion E. Hosmer. Columbia University College of Physicians and Surgeons, Columbia-Fresbyterian Medical Center, Department of Pediatrics, New York, N.Y.

More than 30,000 cultures are requested each year from the services of the Babies Hospital - The Children's Medical and Surgical Center of the Columbia-Presbyterian Medical Center. The primary purpose of this information is for patient care. The Babies Hospital laboratory data system has developed over a ten year period to make laboratory data files accessible for automated data processing. The summary reporting system relys on daily input directly dey punched from the laboratory reports and entered into the system through a remote terminal. When cultures are received in the laboratory, the time, date and acquisition number are stamped on the requisition. The acquisition number and date then become the report-link while the patient's unit number is the patient-link. Initial information, including name, birthdate, location, clinical impression and source, are entered and the culture renorted as pending. when the report is completed the results are entered and a summary report is generated through the facilities of the Columbia University Computer Center. A new summary is generated each time there is activity in a patient's file. Secondary uses of this data bank are clinical research, epidemiologic surveillance, antibiotic sensitivity patterns and laboratory management.

518 SAFETY OF PERTUSSIS RE-IMMUNIZATION IN ADULT HOSPITAL WORKERS: EXPERIENCE WITH 1000 SUBJECTS. Steven Minton, Calvin C. Linnemann, Jr. and John C. Partin. Dept. of Ped. (Children's Hospital Research Foundation) and Dept. of Medicine, University of Cincinnati, Cincinnati, Ohio.

A small epidemic of whooping cough occurred in Cincinnati in the spring of 1973 with significant spread among young adult house staff and nurses all of whom gave histories of prior complete vaccination. In an attempt to halt the spread among adults, $0.25 \mathrm{ml}$ (2 protective units) of aluminum phosphate absorbed killed monovalent pertussis vaccine was given in two groups of about 500 individuals each. The population, which consisted largely of nurses and patient-related employees, were provided with reaction forms $91 \%$ of which were collected within 2 weeks. No central nervous system complications occurred. One patient had an anaphylactic reaction requiring 1 day hospitalization and $20(2 \%)$ had reactions serious enough that they spontaneously consulted a physician; these reactions included: acute urticaria 2; sterile abscess 2; high fever and flu-like symptoms 5; joint swelling and erythema multiforme 1 ; erythema and arm swelling with axillary adenopathy 10 . Virtually all patients reported slight to moderate local reactions at the sight of injection and when questioned, $22(2.2 \%)$ subjectively rated these severe. "Feverishness" was reported by $10 \%$ and an additional $7.8 \%$ recorded their own fever; $6 \%$ reported fevers greater than 103.

Conclusion: No central nervous system complications occurred using this monovalent pertussis vaccine in 1000 adults; troublesome local or systemic reactions occurred in about $10 \%$.

519

TRANSFUSIONAL CYTOMEGALOVIRUS INFECTION. Gilles R. G. Monif, Univ. of Florida Col. of Med., The J. Hillis Miller Health Center, Dept. of Obstetrics \&

Gynecology, Gainesville, Florida.

The fact that the cytomegaloviruses in certain host populations (more specifically, those undergoing homograph transplantation or immunosuppressive therapy) may contribute to significant morbidity and mortality underscores the need to evaluate whether or not blood products containing the virus can be prospectively identified and excluded from utilization in such critical patient populations. Two hundred and eight patients who received a total number of 897 units of blood were monitored. A complement-fixing antibody titer of $1: 8$ as determined by microtiter technique was observed in $14.8 \%$ of the blood units. If the minimal threshold titer was decreased to $1: 4$, an incidence of $18.5 \%$ sero-positivity was identified. A total of 133 individuals in this study were sero-susceptible. Seventy-nine serosusceptible individuals did not receive blood containing complement-fixing CMV antibodies. In this patient population, 3 instances of sero-conversion were identified. Among the 54 serosusceptible individuals who received at least one unit of blood whose complement-fixing titer to the AD-169 strain of cytomegalovirus was $1: 8$ or greater, 13 sero-conversions were observed. If one restricted serological observations only to individuals receiving a unit of blood with a titer of 1:32 or greater, among the eight sero-susceptible individuals 3 were observed to have 8-fold or greater rise, 2 experienced 4-fold rise, and 3 no evidence of infection. In the 6 individuals with pre-existing titer in this category, 3 exhibited a 4 -fold antibody rise.
NOSOCOMIAL INFECTION WITH A KANAMYCIN-GENTAMICIN

520 RESISTANT KLEBSIELLA IN A NEWBORN INTENSIVE CARE UNIT Ellen F. Monkus, George w. Counts, Timothy J. Cleary, and Jenny Casto (Intr. by William $\underline{\text { W. }}$ Cleveland), Univ. of Miami Sch. of Med., Dep. of Ped, and Path., Miami, Florida.

Analysis of gram-negative bacteremia in the nurseries of a Regiona 1 Perinata 1 Center reveals the emergence of kanamycin-gentamicin resistant strains in the Newborn Intensive Care Unit (NB-ICU) JAN- APR- JUL- $O C T$ -

Klebsiella sens. $* \begin{array}{llll}\text { MAR } & \text { JUN SEP } & \text { DEC } \\ 0 & 2 & 4 & 2\end{array}$ *sensitive to genta.

E. coli

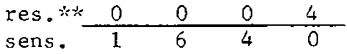

Other gram- res. \begin{tabular}{lllll} 
& 0 & 0 & 0 & 2 \\
\hline 2 & 2 & 5 & 6
\end{tabular} resistant to kana. and genta, sensitive neg. rods res. 0 o 000 to chloro. and poly. $\mathrm{B}$ Hospital surveillance first revealed kanamycin-gentamicin resistant gram-negative rods (Proteus rettgeri) in September, 1973; subsequentiy, organisms with kanamycin-gentamicin resistance have been recovered sporadica11y from other adult patients(Proteus(10), K1ebsiella(7), Serratia(12), and Herellea(2)). In late 1974 systemic infection with kanamycin-gentamicin resistant organisms reached epidemic proportions in the NB-ICU, with 6 cases and 3 deaths. Survey on $12 / 18$ of the 10 infants in the NB-ICU showed: 7 with resistant strains ( $3 \mathrm{Klebsiella,} 3 \mathrm{E} . \mathrm{coli}$, and 1 mixed) from rectal swab; 2 (1 Klebsiella, 1 mixed) in the nasopharymx. Transfer of antibiotic resistance may have occurred. It is not clear whether the multiresistant bacteria in the NB-ICU developed there de nove or came from other sites in the hospital.

521 ACQUISITION OF CYTOMEGALOVIRUS (CMV) IN INFANTS RECEIVING EXCHANGE TRANSFUSION. George A. Nankervis, Mary L. Kumar, Ashir Kumar and Eli Gold, Dept. of Metropolitan General Hospital, Cleveland, Ohio.

A prospective study of newborns requiring exchange transfusion was undertaken in order to better define the possible risk of transmission of CMV via blood. Buffy coat, urine \& saliva specimens for viral culture and serum specimens for CMN complementfixing (CF) antibody were obtained from 23 infant-mother pairs. Buffy coat from the donor blood bag was cultured, and CMV CF titers measured. Throat, urine $\&$ buffy coat cultures were obtained from infants at $6 \& 12$ weeks post transfusion. A matched control group of non-transfused infants $\&$ their mothers was included in the study. Five of the 23 study infants (21\%) developed positive cultures for $\mathrm{CM}$. Three infections occurred in 11 infants who were sero-positive at birth \& received blood from seropositive donors. No infection occurred in the 6 sero-positive infants receiving blood from sero-negative donors. Of 6 infants sero-negative at birth, 2 received sero-positive blood with 1 in fant subsequently developing viruria \& rising CF titer. Four sero-negative infants received sero-negative blood, with 1 infant developing viruria \& a titer rise. Infants with evidence of CMV infection were asymptomatic. All control baby \& donor cultures were negative. One mother of a negative study baby had cytomegaloviruria. Although CMN infection in infancy is naturally acquired by routes other than blood transfusion, exchange transfusion enhances the likelihood of acquired infection, particularly in infants receiving sero-positive blood.

522 FAILURE OF AN INTERFERON INDUCER IN THE TREATMENT OF 522 A MODEL NEONATAL AREOVIRUS INFECTION. James C. Overall Jr. Dept. of Ped. U. of Utah Col. Med., SLC, Utah In order to determine the efficacy of an interferon inducer (poly $I: C$ ) in the treatment of viral infections in newborn as compared with adult animals, we developed a model infection in sheep using Semliki Forest virus (SFV), a group A arbovirus. We previously reported that, following IV inoculation with SFV, newborn lambs exhibited higher titers of virus and interferon but lower levels of antibody in the serum when compared with adult sheep. Pretreatment with poly I:C $(0.1 \mathrm{mg} / \mathrm{kg}$ IV) $8 \mathrm{hrs}$ prior to IV inoculation with SFV prevented detectable viremia in 4 of 6 aduits (viremia occurred in 8 of 9 untreated adults) in contrast to none of 5 newborns (viremia in 5 of 5 untreated newborns). In addition, mean peak viral titers were significantly reduced in the adult (from $10^{3} .77$ to $101.62, P=.05$ ), but not the neonata (from 106.91 to 105.15, P $>.18$ ) animals. Peak neutralizing antibody titers were reduced in the adults with no detectable viremia (mean of 575) in comparison with the untreated adults (mean of 4378) $(\mathrm{P}=.001)$. In contrast, antibody titers were higher in treated (mean of 5300) as compared with untreated newborns (mean of 276) $(P=.004)$. Poly $I: C$ induced comparable levels of serum interferon ( 50 to 500 units) in both the adult and neonatal animals. The results suggest that interferon inducers, despite the capacity to induce interferon in newborn animals, may fail to effectively treat neonatal viral infection. A logical, but as yet unproven, explanation is that immature animals are less sensitive to the antiviral effects of interferon than mature animals. 
USEFULNESS OF MICROSEDIMENTATION RATE (MSR) IN THE

523 DIAGNOSIS OF SYSTEMIC NEONATAL INFECTION. Charles L. Paxon Jr., Pat Norgren, Carl E. Hunt. Univ, of Minnesota, Dept. of Ped., Minneapolis, Minnesota. (Intro. by Rolf R. Engel, Minneapolis).

Most laboratory tests utilized for the early detection of systemic bacterial infection in the older child are not practical in the neonate due to the large sampling volume required. Total poly morphonuclear leukocyte count (TPC), platelet count (PC) and MSR, al1 suggested as being useful in the early diagnosis of neonatal systemic infection, have been prospectively evaluated in 93 neonates: normal term infants (Group I); premature infants without RDS (Group II); 46 premature infants with RDS, 27 without (Group III) and 19 with (Group IV) documented systemic bacterial infection. A positive bacterial culture was obtained from the blood, CSF, and/or lung in all Group IV infants. TPC and PC were not helpful in identifying those with systemic infection.

\begin{tabular}{|c|c|c|c|c|}
\hline & Group & Group & Group III & Group IV \\
\hline Number of Pati & 27 & 20 & 27 & 19 \\
\hline Suspected Infect & 1 & 6 & 18 & 19 \\
\hline Documented Infec & 0 & 0 & $\overline{0}$ & 19 \\
\hline MSR: Range (Mean) 30 & $0-10(2)$ & $0-10(3)$ & $0-7(2)$ & $10-58(26)$ \\
\hline 60 Min. & $0-12(4)$ & $1-22(7)$ & $0-13(7)$ & $20-60036$ \\
\hline
\end{tabular}
60 Min. $0-12(4)$ (1-22(7) Group ( $P<001$ ). Without exception, positive bacterial cultures were subsequently obtained from a systemic site whenever the MSR was $>10$ at 30 minutes and/or $>22$ at 60 minutes. The MSR provides a rapid and reliable method for identifying those patients in whom systemic bacterial infection can be documented.
CULTIVATION OF PNEUMOCYSTIS CARINII IN VITRO.

524 Linda L. Pifer and Walter T. Hughes. Infectious Diseases Service, St. Jude Children's Research Hospital, Memphis, TN

Pneumocystis carinii, the causative agent of diffuse alveolar disease and interstitial pneumonitis, has not heretofore been cultivated in vitro or in vivo. We have accomplished the isolation and cultivation of this organism from both human and murine sources. Embryonic chick epithelial lung (CEL) cells were prepared from 14-day-old embryonated eggs by successive treatments with pronase. Cultures of epithelial aggregates were established in Medium-199 containing 10\% fetal bovine serum. Inocula were prepared from infected rat and human lungs. Passage at 3-day intervals over a period of 12 days resulted in an increase in numbers of cyst forms from $2.7 \times 10^{5}$ to 2.3 $X 10^{7}$. In the reproductive cycle a vegetative cell, designated $T$-form ("trophozoite"), attaches to the host CEL cell, probably for the transport of nutrients, and then detaches. The parasite does not enter the host cell. Sporozoites form within the detached T-form until a maximum number of 8 develop within the mature cyst. Excystment occurs through single or multiple sites in the cyst wall after which the released T-form seeks out and attaches to a new host cell. We have demonstrated the cultivation of $P$. carinii in vitro by direct observation of the reproductive cycle in the Sykes-Moore chamber, serial passage with increase in the number of cyst forms and by the cytopathic effect of the organism on the CEL cell culture, determined visually and by isotopic and glucose utilization studies. The isolated agent was identified as $P$. carinii by characteristic tinctorial and morphologic features and immunologically by indirect fluorescent antibody reaction to $P$. carinii antisera. Also, specific antisera inhibited growth of the organism in culture.
526 RECURRENT CYTOMEGALOVIRUS INFECTION IN ADULT FEMALES,

Alford. Dept. Ped., Univ. of Ala. in Birmingham. s of young mothers with prior immunity to CMV and of comparable age and parity were restudied during the pregnant and nonpregnant state for virological evidence of recurrent infection by tissue culture inoculation of cervical and throat swabs, colostrum, and urine specimens. The mean interval following initial examination was 27 months and an average of 1.3 specimens from each site per woman were processed satisfactorily. virus was recovered from 9 of 25 women who had previously delivered congenitally infected infants. Isolation rates from genital secretions, urine, and throat swabs were $4 / 24,5 / 25$ and $2 / 6$ women respectively. Active infection was also common (10/53) in females with a history of previous viral excretion, 12 of whom had previously infected their offspring at delivery. Site specific recovery rates were genital secretions (9/53), throat $(2 / 20)$, colostrum $(1 / 9)$ and urine $3 / 41)$. In contrast, recurrences developed in only 3 of 49 seropositive women in whom we could not demonstrate viral shedding previously. Two manifested viruria, and one shed virus from the cervix. In the 2 former groups, excretion was more common amongst women whose children were shedding virus in the oro-pharynx. These data suggest that recurrent CMV infection is more common in females with documented previous such episodes as well as those with past infection transmissable to the fetus. The causal relationship between maternal recurrences and excretion of virus by the offspring remains to be determined.
527 NASOPHARYNGEAL CARRIAGE OF HEMOPHILUS INFLUENZAE,

527 TYPE B, AND SERUM ANTIBODY LEVELS TO CAPSULAR POLYSACCHARIDE. Sarah H. Sell and Linda J. Duke. Vanderbilt Univ. School of Med. Dept. of Ped. Nashville. Intro by D. T. Karzon.

It has been postulated that clinical infections with Hemophilus influenzae, type $b$ (flu $b$ ), usually begin in the nasopharynx (NP). To determine whether naturally acquired serum antibody (ab) would prevent NP carriage, polyribophosphate(PRP)-binding power of serum was measured by radioimmuno-assay(RIA) in a group of normal children followed longitudinally during their first 3 years of life with periodic NP cultures and serum samples. Flu b was identified 34 times in 19/72(26\%) children. In 8/19 (42\%), there were multiple isolates with consecutive positive cultures obtained 3 days to 6 months apart. The PRP-binding power fluctuated, with temporary peaks ranging up to $2400 \mathrm{ng} / \mathrm{ml}$. Some of the peaks appear to be unrelated to flu b recovery and may be related to other cross-reacting antigens, such as pneumococcus. No detectable $a b$ was found in $7 / 34$ isolations while $25-125 \mathrm{ng} / \mathrm{ml}$ were present in $16 / 34$ and $>125 \mathrm{ng} / \mathrm{ml}$ in $6 / 34$. The highest ab level prior to first identification of flu b was $5125 \mathrm{ng} / \mathrm{ml}$ in $4 / 19 ; 25-125$ $\mathrm{ng} / \mathrm{ml}$ in 10/19; and no detectable ab in 3 children. In 2 infants, ages 5 and 7 months, flu b was detected in presence of declining maternal $a b$, each at $36 \mathrm{ng} / \mathrm{ml}$. It is concluded that flu $b$ can inhabit the NP of young children in presence of serum ab levels up to $>5000 \mathrm{ng} / \mathrm{ml}$ and there appears to be little relationship between serum $a b$ level and NP carriage of flu $b$.
525 A CANDIDATE STRAIN FOR LIVE VIRUS VACCINAStanley A.Plotkin, Max Just, Toru Furukawa, N. Zygraich, and C. Huygelen, Wistar Inst., Children' Hosp., Philadelphia; Kinderspital, Basel, Switzerland and R.I.T. Lab., Rixensart, Belgium.

The incidence of intrauterine CMV infection in developed countries is about 1\%. At least $10 \%$ of the infected neonates suffer brain damage, and the figure may be higher. Although the immunology of CMV infection is incompletely understood, it seemed prudent to begin efforts to develop a vaccine, A strain of HCMV, called Towne, was isolated from the urine of a congenitally infected child, and passaged in WI-38 human fibroblasts. After 125. passages lots were prepared and tested for the properties of the virus in comparison with low-passage virus. Antisera against the Towne strain also reacted with other CMV antigens. When used as a CF antigen, Towne compared favorably with the AD-169 strain. Trypsin sensitivity proved to be a marker of high-passage in that low-passage fresh isolates were sensitive, while high-passage CMV was not. The high-passage Towne was tested in human volunteers, with the following results: 1) There was no significant clinical or subclinical reaction; 2) No virus excretion was detected; 3) Virus administered intranasally did not infect; 4) Virus administered subcutaneously induced seroconversion.
SEVERE STAPHYLOCOCCAL SEPSIS IN ADOLESCENTS. Stanford

528 T. Shulman, Alvin H. Felman, and Elia M. Ayoub, Univ. of Florida Col. of Med., Dept. of Ped., Gainesville

Staphylococcal sepsis in older children, in the absence of underlying systemic disease or drug abuse, is very unusual. In the past three years, ten previously healthy children, 8-15 years old, presented with life-threatening staphylococcal septicemia. Extensive pulmonary involvement was present in nine, while osteomyelitis and/or pyoarthritis was found in six. Two children died of pulmonary complications. Coagulase-positive Staphylococcus aureus was isolated from blood of all eight patients cultured and from the following sites: joint fluid (4), bone (4), pleural fluid (2), urine (1), and pericardial fluid (1). All organisms recovered were methicillin-sensitive; the isolates from one patient only were penicillin-sensitive. One patient had a known antecedent focus, a furuncle on the lip. None had history of antibiotic therapy, evidence for malignancy, humoral and cellular immunological deficiency or drug abuse. Except for two siblings, the patients lived in widely-scattered geographic areas of north Florida. The recent rise in incidence of staphylococcal sepsis particularly in this age group was supported by the finding that only two infants and no adults presented with similar disease during the same pexiod. Review of hospital records for the preceding 5 years did not reveal similar cases of severe systemic staphylococcal infection in older children. Although no direct evidence for a causal relationahtp is available, the occurrence of these cases corresponds temporally with the implementation of the restrictions on the use of hexachlorophene in neonatal units and by the general public. 
529 CENTRAL NERVOUS SYSTEM CANDIDIASIS William Singer,

529 and Floyd Gilles, (Intr. by Wm. Berenberg), Childrens Hospital Medical Center, Boston, Massachusetts 02115. Fungi are unusual central nervous system pathogens in the neonatal period when gram negative bacterial infections predominate. Seven cases of unsuspected neonatal candida meningoencephalitis were encountered in our recent pediatric autopsies. All infants were critically ill and 5 were premature. All received hyperalimentation and broad spectrum antibiotics. Four had bowel perforation with peritoneal candidiasis and 6 had candida pneumonia. one patient had candida oomphalitis following exchange transfusion. Although candida organisms were cultured from 1 or more noncutaneous sites, in 6 patients the positive cultures were not considered significant and the infants went untreated. No evidence of candidiasis was noted on premortem cerebrospinal fluid analysis and only 1 patient had focal neurologic symptoms.

The premortem diagnosis of central nervous system candidiasis was complicated by the predominance of deep parenchymal lesions, microabscesses, cerebritis and vasculitis which may not be reflec ted by cerebrospinal fluid abnormalities. Fungi were not isolated premortem from the cerebrospinal fluid in those cases in which plexitis, meningitis and ventriculitis were found, up to 1972 the isolation of fungi from blood cultures was limited by laboratory techniques.

We suggest that unsuspected candida meningoencephalitis may undermine the intensive effort put forth in treating potentially salvageable neonates. In patients who are receiving hyperalimentation or have bowel perforations, non-cutaneous candidiasis is an indication for systemic antifungal therapy.

530

ENZYMATIC MICROASSAY OF CHLORAMPHENICOL. A. L

Smith, I. R. Rosenbera, D. H. Smith, anc: B. B.

merson. Children's llosnital Medical Centex, Dept.

of Med., Boston, Mass.

Chloxamphenicol acetyl transferase (CAT) was partially nurified from osmatic shockates of P. Escherichia coli $\$ 21218$ by del filtration and DFRE chromatocraphy. This enzyme, which appears to be different from nreviously renortea CAT, will also acetvlate dichloro [B-hydroxy-a (hydroxymethyl)-phencthyl] acetomide. p-methylsul fony lphenyl-2-dichloroacetamide-1, 3-propanediol as well as chloramphenicol (CM). Usino ${ }^{14} \mathrm{CH}_{3}$-acetylCoA and takino advantace of the pronerty of acetylchloramnhenicol to bind to cellulose nitrate, CAT was used to quantitate CM in $10 \mathrm{HI}$ aliquots of serum. The test recuixes $30 \mathrm{~min}$. incubation, and the standard curves are linear to $50 \mathrm{\mu a} / \mathrm{ml}$. Citrate, socium fluoride and the sodium salts of oxalate and citrate did not effect the quantitation of $\mathrm{CM}$ at concentrations to $10 \mathrm{x}$ those used in clinical laboratory prescrvatives. Likewise, penicillin G, Penicillin V, iethicillin, Oxacillin, Nafcillin and cloxacillin at $100 \mathrm{ur} / \mathrm{ml}$; Strentomvcin, Kanamvcin, Buteriosin, Neomycin, Amikacin and rentamicin at $50 \mathrm{ur} / \mathrm{ml}$; and polymyxin and pifamnin at $50 \mu \mathrm{g} / \mathrm{ml}$ did not have any sicnificant effect on the ruantitation of ci: Furthermore, chloramhenicol glucuroride is rot an inhibitor of or a substrate for CP.? This test, offers a convenient and rapid means to monitor blood c: concentration, and should be particularly useful in certain hich risk patients.

531

IMPLANTATION TO PREVENT INFECTION IN NEONATAL

ICU. Katherine Sprunt, Grace Leidy and Winifred Redman,

N.Y.C.

Implantation of the nasopharynx with a strain of alpha hemolytic $(\alpha)$ streptococcus has been used for control of infection in neonates at unusually high risk of superinfection secondary to abnormal colonization with potential pathogens (bacterial overgrowth) for the following reasons: earlier observations with serial cultures of the pharynx of over 40 adults and children demonstrated that bacterial overgrowth occurred as $\alpha$-streptococci were suppressed by antibiotics. When $\alpha$-streptococci persisted (because of antibiotic resistance) overgrowth did not occur. In a recent study of 134 neonates, all infections occurred in those with bacterial overgrowth: each infection was caused by the overgrowing strain. No infection occurred in infants with "normal" throat cultures $(\alpha$-streptococ$\mathrm{ci}$ the predominant organism in concentrations $>10^{4} \mathrm{cfu} / \mathrm{ml}$ ).

Implant strains with desired characteristics were selected from the pharynx of normal newborns. One strain has been implanted in the nasopharynx of 5 neonates at unusually high risk of infection from abnormal colonization. In 4 infants a single dose of the implant strain resulted in rapid appearance of pharyngeal $\alpha$-streptococci and a concomitant decrease in proportion of enteric pathogens, In 3 patients this reversal occurred within two days of implantation. No untoward effects were noted. The implanted strain has not yet been detected $>25$ days after implantation.
532 ESPIRATORY DISEASE IN DAY CARE CENTERS WITH DIFFERENT POLICIES REGARDING SICK CHILDREN. KATHERINE STRANGERT. DEPT. PED. KAROLINSKA HOSPITAL. STOCKHOLM, SWEDEN. (INTR. BY GERALD W. FERNALD, DEPT. PED. UNIV. N.C. CHAPEI HILL).

In Sweden, as in the USA, the problem of whether sick children in day care centers ought to be dismissed to minimize the total rate of infection remains unsolved. A clinical study was performed in 118 children ( 6 mos. -2 yrs.) from 14 Swedish day care centers (Sept.-Nov. 1973). In one center, but not in the others, 20 children were encouraged to attend regardless of health status and in these children weekly nose and throat swabs, monthly feces, urine and blood specimens were obtained for microbiological studies. Viruses were isolated from $12 \%$ of the throat swabs, $40 \%$ of the feces, $22 \%$ of the urine specimens and 15 cases of significant CF titer rises were found (coxsackie virus B5, adeno 1,2,5, respiratory syncytial, influenza $A, B$, parainfluenza 2 , Herpes simplex, cytomegaloviruses). (rroup A streptococci were cultured from 7 children, $\underline{H}$. influenzae from 4 and pneumococci $(6,19,9)$ from all 20. Most of the pathogens were found in children with no or very mild symptoms. Rhinitis was found in $69 \%$ and cough in $9 \%$ of the observed days in these children compared to $35 \%$ and $17 \%$ in those of the other centers. In the first group 0.5 febrile illness/child was found and in the second group 1.5 febrile illnesses/child. The inclusion of children with respiratory symptoms in a day care center did not increase the incidence of respiratory disease with the exception of afebrile rhinitis.
NEUTROPHIL (PMN) OXIDATIVE METABOLISM AND BACTERICI-

533 DAL FUNCTION IN SICKLE CELL DISEASE (SCD). Ronald G. Strauss, Richard B. Johnston, Jr., Hernan Moreno, Joyce E. Lehmeyer, and Bernard B. Keele, Jr., Children's Hosp. Research Fndn., Cincinnati, and the Univ. of Ala., Dept. of Ped. Bacterial infection is the most frequent cause of death in SCD. Functional hyposplenia and deficient serum alternative complement pathway opsonization have been described in SCD and could possibly explain this finding. In addition, it has been suggested that defective postphagocytic oxidative metabolism like that seen in chronic granulomatous disease exists in SCD. To explore this possibility, postphagocytic oxidative functions were studied in PMN from apparently well patients and matched controls. Hexose monophosphate shunt activation (oxidation of g1ucose- $-1-1{ }^{4} \mathrm{C}$ ), $\mathrm{H}_{2} \mathrm{O}_{2}$ generation (oxidation of ${ }^{1{ }^{4}} \mathrm{C}$-formate), and nitroblue tetrazolium reduction were equivalent in PMNs from controls and 23 patients, including 7 with frequent infections. The mean rate of superoxide anion generation by PMNs from 14 patients was $4.49 \pm .24$ (SEM) n moles/min, and that of controls was $4.39 \pm .23$ $\mathrm{n}$ moles/min. The phagocytosis-associated burst of luminescence believed to depend on singlet oxygen formation reached a mean peak of $14.6 \pm 1.0$ (SEM) $\times 10^{4} \mathrm{cpm}$ in PMN from 10 patients compared to $13.6 \pm .7 \times 10^{4} \mathrm{cpm}$ in control cells. The killing of $\underline{\mathrm{s}}$. aureus was also equivalent by PMNs from 7 patients and controls. Thus, it would appear that the oxidative metabolic events associated with PMN phagocytic bactericidal activity are normal in SCD and that a defect in these events cannot explain the propensity to bacterial infections in this disease.

UBERCULOUS MENINGITIS IN CHILDREN DURING THE ISO-

534 NIAZID ERA. Ciro v. Sumaya, Merlin Simek, Margaret H. D. S Smith, Michael F. Seidemann, Gregory $\frac{\text { S. Ferris }}{\text { UClA Sch. Med., }}$ Wallace Rubin. UClA Sch. Med., Dept. Ped., Los Angeles, Tulane Sch. Med., Dept. Neurol., New Orleans.

The records of 59 children with tuberculous meningitis seen at Charity Hospital since the addition in 1952 of isoniazid (INH) to the antituberculous drug regimen are reviewed. During hospitalization 14 children died. At discharge 21 had complete clinical recovery, 24 had minor or major sequelae. No meningitis relapses were documented.

Follow-up of 21 long-term survivors revealed 13 (62\%) with moderate to severe neurologic and social disabilities. No intracranial calcifications were detected in the 18 survivors tested. In general recovery and sequelae were directly related to the time of starting therapy and the severity of the meningitis at the time of admission. The reduction in case fatality rate (sequelae rate remained the same) between $1952-1961(24 \%)$ and 1962 $1972(12 \%)$ is associated with the use of a higher dose of INH and the incorporation of steroids into the therapeutic regimen.

Investigation of the household contacts of 14 patients revealed 24 siblings $(49 \%)$ and 28 adults $(47.5 \%)$ with a positive tuberculin skin test; 5 siblings $(10.2 \%)$ and 13 adults $(22.0 \%)$ had a chest roentgenogram compatible with tuberculosis. The reduction of this disease lies not so much with newer chemotherapeutic measures but mainly with public health measures of casefinding, utilization of chemoprophylaxis or posstble BCG and adequate follow-up care of adult sources and childhood contacts. 
535 ANAEROBES AND CHILDHOOD INFECTIONS. Muthayipalayam Uiv. Sch. Med., Detroit.

Recent interest in anaerobic infections prompted a prospective survey of their. frequency in sick children in a metropolitan area. Over a 14 month period, 96 anaerobes were recovered from 74 specimens obtained from 64 patients. Bacteroides were the most frequently isolated anaerobes (34), followed by Costridia (18), Prorionibacteria (17), Peptostrertocccei (8), and Fusoinceteria (5). An anaerohe was isolated singly from 28 specimens, two or more anaerobes coexisted in 3 specimens, but most specimens (43) yielded facultative orcanisms alond with one or more anaerobes. Most patierts (56\%) were over 5 years of age; only 148 were newborns. Blood was the commonest source of anaerchic bacteria (318); however, these accounted for only $2.8 \%$ of all isolates from blood cultures during the same period. Furthermoxe, of 22 children whose blood cultures yielded anaerobes, these organisms were definitely responsible for clinical illness in only 2 instances and may have heen significant in 3 others. Intraabdominal sources were responsible for $24 \%$, soft tissue infections for $21 \%$, and the respiratory tract for $10 \%$ of all anaerobes recovered. Except in blood cultures, anaerobes almost invariably coexisted with facultative bacteria. These findings suggest that while anaerobes are definitely significant pathogens under certain conditions, they play a minor role as a cause of septicemia in children and their relative importance in the pathogenesis of childhood infections remains to be defined. 538 Ushanalini Vasan, Dai M. Lim, Robert M. Greenstein, John R. Raye (Intr. by Martha L. Lepow)

UConn. Health Center, Dept. of Pediatrics, Farmington, Ct.

In the neonate, examination of the gastric aspirate (GA) for polymorphonuclear leucocytes (PINS) has become an accepted means for assessing the risk of infection following prolonged rupture of membranes (PROM). It has been assumed that the presence of increased numbers of PMNs is evidence for an active inflammatory response of fetal tissues. This study attempts to determine whether these PMN are of fetal or maternal origin by nuclear sexing of male gastric aspirate leucocytes following staining with quinacrine hydrochloride.

The GA cells from 18 male infants who were born after 12 or more hours of PROM were examined for positive Y-chromosome fluorescence $(\mathrm{Y}+)$. 16 female GAs were similarly studied. In male infants $84.8 \%$ of the gastric epithelial cells and $1.5 \%$ of the PMNs showed $\mathrm{Y}+$. The values in females were $1.2 \%$ and $0.2 \%$ respectively. This sex difference for epithelial cells was highly significant $(p<.001)$. There was no significant sex difference in $\mathrm{Y}+$ for $\mathrm{PMNS}(\mathrm{p}>05)$. Persistance of $\mathrm{Y}+$ in peripheral blood PMNs of male newborns was confirmed following test incubations in $\mathrm{GA}$, amiotic fluid, and solutions of varying pHs.

The results indicate that the gastric pMNs studied were genetically female cells, irrespective of the sex of the baby. These data suggest that the PMN in GAs of infants with PROM are of maternal origin and are not evidence of fetal participation in an inflamatory response.
536 THE INDIRECT HBT TEST--DETECTION OF BACTER

536 IAL MENINGITIS. James $K$. Todd and Peggy Tolliver. (Intr. by Kenneth McIntosh). Univ. of Colo. Med. Center and the Children's Hosp. of Denver, Dept. of Ped., Denver, Colo.

The routine (direct) nitroblue tetrazolium (NBT) test initially thought to be a sensitive and specific method of detecting patients with bacterial infections has been shown to have many false positive and negative reactions, possibly due to the nonspecific underactivity or overactivity of the patient's neutrophils. An indirect NBT test has been developed using neutrophils from normal donors to test patient body fluids for the presence of bacterial products. It routinely is able to detect the presence of $\geq 20$ nanograms/ml. of endotoxin and $\geqslant 10^{5}$ bacteria/ml. (Haemophilus influenzae) as well as gram positive organisms and products. The cerebrospinal fluid of $49 / 55$ children with untreat ed bacterial meningitis $(45 / 51$ gram negative, $4 / 4 \mathrm{gram}$ positive) gave positive indirect NBT tests while fluid from 7 patients with viral meningitis and 31 controls were negative. Although relatively insensitive, the indirect NBT test appears to be a specific method of detecting both gram negative and gram positive bacter ial products in patient body fluids. Modifications which increase its sensitivity are being investigated.

537 SUBCULTURE TECHNIQUE. James $K$. Todd and Martha H. Roe. (Intr. by Kenneth McIntosh). Univ. of colo. Med. Ctr. and The Children's Hosp. of Denver, Dept. of Ped. and Path., Denver, Colo.

The earliest detection of positive blood cultures, and subsequent rapid identification and antibiotic sensitivity testing of the isolated organisms is one of the most important functions of the pediatric clinical microbiology laboratory. Most hospitals rely on visual detection of growth in blood culture bottles and/or on subcultures done after 24 to 48 hours of incubation to detect positive cultures. This standard method was compared to an early subculture technique which initially subcultured the bottles at 4 to 14 hours (average $8 \mathrm{hrs}$.) after innoculation with blood from pediatric patients. 3,435 blood cultures were evaluated. The contamination rate was low $(1.8-2.8 \%)$ and true bacteremia was identified in $3.8-4.8 \%$ of blood cultures. $85 \%$ of clinically significant positive cultures were detected within 24 hours of blood drawing by the early subculture technique. Blood culture contaminant organisms rarely grew on early subculture plates $(p<0.01)$. Compared to the standard 24 48 hour stain and subculture technique, the 4-14 hour subculture allowed earlier detection, identification and antibiotic sensitivity testing of organisms in clinically significant positive blood cultures.
GROUP B BETA HEMOLYTIC STREPTOCOCCAL (GBS) COL-

539 ONIZATION OF NEWBORNS: ACQUISITION, PERSISTENCE A ND EFFECT OF TRIPLE DYE CORD CARE. Ellen R. Wald, Merrill J. Snyder, Ronald L. Gutberlet. (Intr. by Raymond L. Clemmens). Univ. of Maryland Med. Sch., Dept. Ped., Balto., Md.

Following an outbreak of GBS neonatal infection, GBS colonization and its modification by special cord care were assessed.' The umbilicus and pharynx of 378 full term newborns were cultured to detect GBS carriers at birth and discharge from the hospital. At birth $5.8 \%$ of infants were colonized with GBS. 218 infants received no special cord care and 160 had daily triple dye cord care. Prior to discharge $7 \%$ of neonates in the untreated group became colonized with GBS, while only $2.9 \%$ of the treated group became carriers. In the untreated group, 3 of 9 infants colonized at birth were no longer carriers at discharge; however, in the treated group, 10 of 13 babies colonized at delivery were GBS negative at discharge. These data indicate that triple dye was effective in decreasing the prevalence and duration of GBS colonization when compared to the control group.

A survey of 238 infants from 1 to 7 weeks of age showed similar GBS colonization rates with age. Benzathine penicillin treatment of 6 colonized infants did not eradicate the organism. Followup of 24 colonized babies, up to 3 months, revealed that most have persistence of GBS. Perineal cultures of mothers of prolonged carriers were also positive for GBS. These data suggest that after early nosocomial acquisition of GBS colonization, there is a prolonged carrier state in the infant associated with maternal GBS colonization and not eradicable by benzathine penicill in therapy. RECOVERY OF ANTIGENICALLY CHARACTERIZED "WILD" POLIOVIRUS 2 FROM AN IMMUNIZED INFANT. Catherine $M$. Wilfert, James H. Nakano, Carlos R. Abramowsky, Pearay t. Ogra: Duke Univ. Med Ctr. Uurkam, N.C Ctr. for

A heal thy 6 month old male infant received his only dose of trivalent oral poliovaccine on $5 / 14 / 73$. On $5 / 30 / 73$ he was admit ted with myocarditis and severe congestive heart failure (CHF). Supportive therapy resulted in clinical improvement and discharge from the hospital. He remained asymptomatic and stable for an additional two weeks after which he was readmitt
in CHF. He expired on the day of admission. in CHF. He expired on the day of admission.

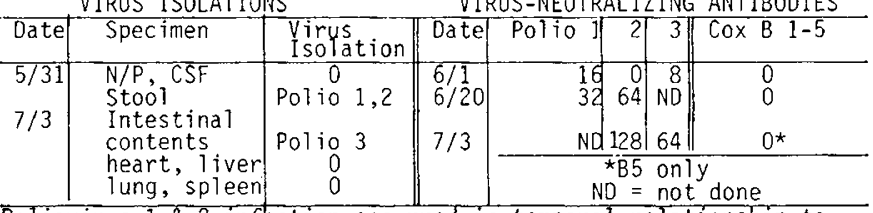

Poliovirus $1 \& 2$ infection occurred in temporal relationship to the myocarditis. Poliovirus 3 was isolated and an antibody rise occurred at the time of his exacerbation and demise. No poliovirus was isolated from and no antigen was detected by immunopolio 2 was antigenically characterized as wild type. The iso-
lates of polio 1 and 3 were vaccine-like. The antigenic characterization as wild type 2 virus may be a result of 1$)$ vaccine virus reversion in the patient, 2) reversion in vaccine recipients in the community and subsequent contact with the patient or 3 ) acquisition of wild type virus from the environment. 
PNEUMOCOCCAL OPSONINS IN SPLENECTOMIZED

541 CHILDREN. Jerry A. Winkelstein and George Hopkins Univ. Sch. Of Med., Dept. of Ped., Baltimore. Splenectomized children have an increased incidence of pneumococcal (Pn) sepsis. Children with Sickle Cell Disease (SCD) also have an increased incidence of Pn sepsis and have been shown to have both a functional splenectomy and decreased Pn serum opsonins. These observations suggest that splenectomy could lead to a decrease in Pn serum opsonins.

Accordingly, Pn serum opsonins were measured in children who had been splenectomized for a variety of reasons. Test serum, pneumococci and normal phagocytes were incubated together for $30 \mathrm{~min}$ at $37^{\circ}$ and the percentage of cells that had ingested organisms determined. Pn serum opsonizing activity was $40 \pm 12$ (S.D.) in 24 splenectomized children and $42 \pm 14$ in 23 control children. Functional titers of $\mathrm{C} 3$ and $\mathrm{C} 5$, important opsonins, were $3,400+1,400$ and $26,000+8,474$ respectively in splenectomized children, and 2,900t 1,284 and $21,000 \pm 6,363$ respectively in controi children. Two of the splenectorized children had had sepsis and their pn serum opsonizing activity was normal over a wide range of serum concentrations.

Thus, splenectomy per se does not decrease Pn opsonins and it is unlikely that the functional splenecto my in children with SCD is causally related to their low Pn serum opsonins.

\section{Live influenza A $\left(\mathrm{H}_{3} \mathrm{~N}_{2}\right)$ ts-I [E] VACCINE IN CHILDREN}

542 Peter $F$. Wright, Tatsuhiko Shinozaki, Jul iette $M$. Thompson, David $\bar{T}$. Karzon. Vanderbilt Univ. Sch. of Med. Dept. of Ped. Nashviile

Influenza $A\left(H_{3} N_{2}\right)$ ts-1 $[E]$, a temperature sensitive mutant found safe and effective in adults, was evaluated in seronegative children aged 14-32 months to determine safety, antigenicity and communicability during primary infection. 18 vaccinees and 6 unvaccinated controls were studied in 5 double-blind trials conducted in a day care setting in which the children were observed for 19 days surrounding vaccine administration.

Fol lowing intranasal administration of varying doses of vaccine $\left(10^{8.8}-10^{5.3}\right.$ TCID 50$) 14$ of 18 vaccinees shed virus for $5-10$ days (mean 8 days) with peak shedding of $106.3 \mathrm{TCID} 50$ per $\mathrm{ml}$ of nasal wash. In some instances loss of the ts marker was seen late in the course of virus shedding. There was no transmission of vaccine virus to the 6 controls. 16 of 18 vaccinees had an antibody response as determined by hemagglutination-inhibition(HAI), anti-neuraminidase(ANAB) or neutralizing antibody assays. No signs or symptoms could be related to vaccine administration;an equal incidence of intercurrent illness occurred in controls and vaccinees.

The trials are an opportunity to examine local and systemic host responses to a primary influenza $A$ infection. The low infective dose and extensive virus shedding in children compared to HAI negative adults:l)implicate children as major transmitters of natural influenza infection,2)demonstrate the necessity of evaluating future live influenza vaccines in seronegative children and 3) verify the biologic importance of $A N A B$.

VIREMIA: A POSSIBLE ETIOLOGICAL ROLE IN HIGH TEMPERA543 TURES IN CHILDREN. Terry Yamauchi, James A. Gammon, David $T$. Imagawa, and Miye Y. Nishita. (Intr. by Delbert A. Fishex).

Twenty-nine children between the ages of 9 months and 15 years were studied in the Acute Care Clinic at this hospital because of $103^{\circ}$ or greater temperatures and no obvious source of infection. No child had received antibiotics for at least two weeks prior to this evaluation. Every child had viral cultures of throat, stool and buffy coat. Twenty-six children also had their cerebrospinal fluid (CSF) cultured for pathogenes. In addition, each child had bacterial cultures of blood, throat and urine.

Viruses were recovered from 8 children (28\%). Five children had viruses isolated from their throats, 3 from feces, 1 from CSF and 4 from the blood. Some children had viruses cultivated from multiple sites. Paramyxovirus was recovered from the blood and pharynx of 2 siblings. One of these children developed parotitis 5 days after culturing. In the other child the only symptom was the initial febrile episode. Both children demonstrated a fourfold or greater rise in mumps neutralizing antibody titer in convalescent sera. Picornaviruses were retrieved from buffy coat cultivation in 2 additional children. One child was carrying the picornavirus in his throat and stool as well as blood.

While the discovery of paramyxovirus or picornavirus in the blood has been previously reported, the association with high fever is unique. The possibility that the temperature elevation was secondary to the viremia and similar to that seen with bacteremia cannot be ruled out in this study.

\section{METABOLISM}

54. CONGENITAL HYPERAMMONEMIA TREATED WITH $x$-KETO ANA-

544 LOGUES OF ESSENTIAL AMINO ACIDS, Mark L. Batshaw, Saul Brusilow, and Mackenzie Walser,

Med. Inst., Dept. Ped. and Dept. Med., Baltimore.

A 13-year-old girl with mental retardation, seizures, 2
trokes, episodes of vomiting and lethargy and elevated plasma ammonia, glutamine and alanine had $<15 \%$ hepatic carbamyl phosphate synthetase activity. Despite protein restriction $(0.5 \mathrm{~g} /$ $\mathrm{kg} /$ day), elevated values were obtained of morning plasma ammonia (mean $0.05 \mathrm{mM}$; normal<0.03 mM), glutamine (mean $1.1 \mathrm{mM}$; normal< $0.7 \mathrm{mM}$ ), alanine (mean $1.0 \mathrm{~mm}$; normal $<0.3 \mathrm{~mm}$ ). $\alpha$-Keto analogues of five essential amino acids (val, leu, ile, met, phe) were administered IV as an $8.5 \mathrm{~g}$. mixture of the sodium salts. Plasma $\mathrm{val}$, leu, ile, phe, met, tyr, and alloisoleucine increased sharp$1 y$ at the end of the four hour infusion (mean $+0.12+0.03 \mathrm{~mm}$ ). Alanine decreased from $1.2 \mathrm{mM}$ to $0.6 \mathrm{~mm}$ following infusion and ammonia decreased from 0.05 to $0.03 \mathrm{mM} 20$ hours post-infusion. Ketoacids given p.o. for ten days led to a significant reduction in plasma ammonia, glutamine, and alanine compared to control periods: $0.025+0.002 \mathrm{mM}, 0.74+0.07 \mathrm{mM}$ and $0.50+0.06 \mathrm{~mm}$ respectively. Urinary urea $\mathrm{N}$ excretion decreased and $\mathrm{N}$ balance improved. Seizures became less frequent and she became more alert. On longterm ketoacid management ( 3 months) an amino acid imbalance developed requiring essential amino acid supplementation. Subsequently the ammonia, glutamine and alanine fell to normal or near normal levels and the child has shown catch-up weight gain and continued palliation of symptoms. Ketoacids upon transamination to the corresponding amino acids reduce hyperammonemia indirectly and may be effective in treating urea cycle enzyme deficiencies.

IN

545 REYE'S SYNDROME. Joseph V. Baublis and Robert J. Ann Arbor, Mi

Cell cultures were prepared from liver and brain tissue of two patients with Reye's Syndrome who died during an outbreak of influenza $B$. These cultures did not hemadsorb, and have not shown a tendency to develop spontaneous cytopathology. Confluent monolayer cultures of these celts were incubated overnight with equimolar amounts of leucine-C $\mathrm{C}^{4}$ and ornithine- $\mathrm{H}^{3}$ in order to compare the incorporation of an urea cycle amino acid with one not directiy involved in this pathway. The ornithine/legucipe utilization ratio was expressed as the ratio of counts $\mathrm{H}^{3} / \mathrm{C}^{14}$ measured in the Trichloroacetic acid precipitate of cell extracts. Patient brain and liver cell cultures showed ratios which were similar to that obtained with a human foreskin fibroblast line. When cell cultures were incubated with a medium containing serum from a patient with Reye's Syndrome, a significant reduction in ratio was observed. This effect was not observed with medium containing normal human serum, and was not removed by overnight dialysis against tissue culture medium. Although tissue cultures derived from patients with Reye's Syndrome do not appear to possess a transmissible defect in ornithine utilization, serum from a patient in the acute stage of syndrome appears to possess a nondialyzable substance capable of interfering with ornithine incorporation.

546 POSTMORTEM VITREOUS CONCENTRATION (PV'C) OF NA, K, CL, CA \& MG IN SUDDEN INFANT DEATH SYNDROME (SIDS)

\& Alumenfeld \& Robert L. Catherman, Columbia Univ. Col. of Phys. \& Surg. N.Y.C., Dept. of Ped. \& Path., and office of the Med. Examiner, Phila,Pa. (Intr. by Robert W. Winters)

This study was performed to determine the PrC of $\mathrm{Na}, \mathrm{K}, \mathrm{Cl}, \mathrm{Ca}$ and $\mathrm{Mg}$ of 27 infants $8 \mathrm{~d}-8 \mathrm{mo}$., dying of SIDS, as the PYC can reflect abnormal antemortem serum concentration. The results were compared with electrolyte PV'C from patients of similar age known to be healthy prior to acute traumatic death or from patients with normal serum electrolytes prior to nontraumatic death.

\begin{tabular}{|c|c|c|c|c|c|}
\hline & $\mathrm{Na}(\mathrm{meq} / \mathrm{L})$ & $\mathrm{K}($ meq $/ \mathrm{L})$ & $\mathrm{C} 1$ (meq / L ) & $\mathrm{Ca}(\mathrm{mg} / \mathrm{d} 1)$ & $\because g(m g / c$ \\
\hline $\mathrm{ge}(\mathrm{mo})$ & $\vec{x}(\mathrm{~N})$ & $\bar{x}(N)$ & $\bar{x}(N)$ & & $\frac{\bar{x}}{\mathrm{x}}(\mathrm{N})$ \\
\hline et & $140(1)$ & $8.5(1)$ & $118(1)$ & $8.1(1)$ & $5.5(1)$ \\
\hline $1<2$ & $136(5)$ & $13.0(5)$ & 128 & $6.2(5)$ & $4.9(5)$ \\
\hline $2<3$ & $135(6$ & 12.5 & 119 & $6.2(5)$ & $4.6(5)$ \\
\hline $3<4$ & $134(7)$ & $11.3(4)$ & 121 & $6.1(7)$ & $3.7(7)$ \\
\hline $.4<6$ & 1421 & 10.9 & 127 & $6.0(4)$ & $4.3(4)$ \\
\hline $6<7$ & $132(2)$ & 12.53 & $119(2)$ & $5.9(3)$ & $3.9(3)$ \\
\hline $8<9$ & $144(1)$ & $11.7(1$ & $132(1)$ & $5.2(1)$ & $3.3(1)$ \\
\hline-8 & & & & & \\
\hline
\end{tabular}

$\begin{array}{llll} & & \end{array}$ of infant age, and there is no significant difference in the mean $\mathrm{PVC}$ of $\mathrm{Na}, \mathrm{K}, \mathrm{Cl}, \mathrm{Ca} \& \mathrm{Mg}$ in SIDS and control patients. SIDS is most probably not due to a chronic inbalance of the electrolytes studied or to a condition causing such an inbalance. 
TREATMENT OF REYE'S SYNDROME BY "EARLY DLAGNOSIS/

547 PROMPT EXCHANGE TRANSFUSION" IN 1973-74 EPIDEMIC.

Robert C. Bobo, William K. Schubert, John C. Partin. Children's Hospital Research Foundation, Cincinnati, Ohio.

It has been claimed that all treatments are equally ineffective in Reye's Syndrome. During the December 1973-March 1974 epidemic, an epidemic of homogenous severity throughout the midwest, 349 cases were collected by the Center for Disease Control with $40 \%$ mortality. During this epidemic, 27 cases were treated in Cincinnati by "Early Diagnosis/Prompt Exchange Transfusion". of these, 17 were severe (Grades 3,4), proven by liver biopsy and treated by exchange transfusion. The $10 \mathrm{mild}$ (Grades 1,2) cases were not exchanged. Survival was $100 \%$, significantly different than national survival for the same epidemic $\left(x^{2}=>10 ; p=<.005\right)$. During the same epidemic, 10 of 12 patients treated by IntensiveSupportive measures and Elective-Tracheal-Intubation survived (DeVivo et.al., in Reye's Syndrome, Grune-Stratton, New York,1975 in press) and $9 / 11$ cases treated by peritoneal dialysis died (Samaha, ibid.). Assuming equal sampling, survival after Intensive-Supportive care was significantly better $\left(X^{2} \Rightarrow 10 ; p=<.005\right)$ than overall survival in the epidemic. Peritoneal dialysis was not better $\left(x^{2}=.008\right)$

Conclusion: Treatment by the "Early Diagnosis/Prompt Exchange Transfusion" method was associated with significant reduction in mortality during the 1973-74 influenza B-associated Reye's Syndrome epidemic; the Intensive-Supportive care method also improved survival; peritoneal dialysis appears not to improve survival.

Supported in part by U.S.P.H.S. Grant \#RR-00123.

SERUM AND URINE ACTIVITIES OF LYSOSOMAL HYDRO548 LASES IN JVENILE DIABETES MELLITUS (JDM). Fred Bomback, Sachiko Nakagawa, Selma Kumin and Harold M. Nitowsky, Dept. Ped, Albert Einstein Col. Med., New York.

Although increased serum activity of several lysosomal hydrolases has been demonstrated in patients with diabetes, the relation of these changes to severity or complications of the disease remains obscure. Studies have been carried out on the activities of $\beta-D-N-$ acetylhexosaminidase (hex) $\beta-$ galactosidase ( $\beta$-gal), $\alpha$-glucosidase $(\alpha$-glu and acid phosphatase (AP) in serum and 24 hour urine from patients with JDM and age-matched contrds An assessment of severity of microangiopathy was made by clinical and laboratory studies for signs of retinopathy, neuropathy and nephropathy. In JDM with mild or no microangiopathy, the most consistent findings were a striking increase (10 to 35 fold) in urinary AP with little or no in crease in serum activity; a moderate increase in total urinary hex involving both $\mathrm{A}$ and $\mathrm{B}$ isozymes, with qualitatively similar changes in serum hex activity; minor increases in serum and urine $\beta$-gal and urine $\alpha$-glu activities. One patient with marked microangiopathy showed a 15 and 8 fold increase in total urine hex and $\beta$-gal respectively, but only a 2 fold increase in AP. This pattern differs from that shown by other patients. These findings suggest that a generalized increase in activity of lysosomal hydrolases does not occur in JDM. Instead the changes appear to be selective, and may bear some relation to the fundamental derangement or pathogenesis of complications in this disorder. (Supported by Grants from NIH (GM 19100) and N. Y. Diab. Assoc.)

METHODS TO INCREASE THE RELIABILITY OF BLOOD GLUCOSE

549 BY THE DEXTROSTIX SYSTEM. David R. Brown, Peter A. Woodbridge and Robert A. Ulstrom. Department of Ped iatrics, University of Minnesota Hospitals, Minneapolis, Minn. nitiated a clinical and laboratory study to improve this system.

Improved reliability was obtained utilizing the Eyetone Metering System (ET/DS), simulated blood glucose standards, serum rather than whole blood and shortening the duration of the glucose oxidase reaction. Simultaneously drawn capillary tube blood samples were evaluated for glucose concentration by the ET/DS system and a standard Hexokinase (HK) method.

Determinations on standards: hypoglycemic (N=100) ( $\bar{x}=50 \mathrm{mg} \%$; S.D. $=4.4 \mathrm{mg} \%) ;(\mathrm{N}-159)$ normoglycemic $(\overline{\mathrm{x}}=124 \mathrm{mg} \%$; S.D. $=4.5 \mathrm{mg} \%)$; $(\mathrm{N}=100)$ hyperglycemic $(\bar{x}=224 \mathrm{mg} \%$ S.D. $=6.3 \mathrm{mg} \%)$. These standard solutions demonstrate the reproducibility of fresh Dextrostix.

\begin{tabular}{|c|c|c|c|c|c|c|c|c|}
\hline \multirow{3}{*}{ Whole } & \multicolumn{3}{|c|}{ ET/DS } & \multicolumn{2}{|c|}{$\underline{\mathrm{HK}}$} & \multirow{3}{*}{$\underline{r}$} & \multirow{2}{*}{ prob. } & \multirow[b]{2}{*}{$\delta x \cdot y * *$} \\
\hline & Time & $\overline{\bar{y}}$ & S.D. & $\overline{\mathrm{x}}$ & S.D. & & & \\
\hline & Blood & \multicolumn{2}{|c|}{$(\mathrm{mg} \%)$} & \multicolumn{2}{|c|}{$(\mathrm{mg} \%)$} & & & (mg\%) \\
\hline & *60 Sec. & 97 & 47 & 105 & 41 & 0.89 & $<.001$ & 20 \\
\hline \multirow[t]{3}{*}{ Serum } & $55 \mathrm{Sec}$. & 112 & 46 & 100 & 39 & 0.95 & $<.001$ & 13 \\
\hline & $50 \mathrm{sec}$. & 114 & 52 & 105 & 40 & 0.90 & $<.001$ & 19 \\
\hline & $45 \mathrm{sec}$ & 99 & 36 & 102 & 35 & 0.98 & $<.001$ & 6.4 \\
\hline
\end{tabular}

We conclude increased reliability and correlation of ET/DS with the HK method may be obtained utilizing serum, a reaction time of 45 secs due to more rapid penetration by serum, and standard glucose reference solutions.
550 THE ROLE OF LACTATE AS A MAJOR METABOLIIC SUBSTRATE

550 IN THE OVINE: FETUS. Laurence I. Burd, M. Douglas Jones, Jr., Michael A. Sinmons, Edgar L- Makowski, Giacomo Meschia, and Frederick C. Battaglia. Division of Perinatal Modicine, univ. of Colorado Medical Center, Denver.

In chronic sheep preparations studied after recovery from surgical stress, umbilical venous (v) and arterial (a) whole blood lactate and pyruvate concentrations were measured by enzymatic methods using I-lactate dehydrogenase. In 40 samples drawn from 11 animals the mean umbilical venous lactate concentration of $2.21 \pm .15 \mathrm{SEM} \mathrm{mM} / \mathrm{L}$ exceeded the arterial concentration by $.16 \mathrm{mM} / \mathrm{L}(\mathrm{P}<.001)$. In 23 samples drawn from 6 animals a mean pyruvate concentration of $.25 \pm .08 \mathrm{SEM} \mathrm{mM} / \mathrm{L}$ was found in both vessels. The lactate-oxygen quotient ( $3 \mathrm{x}$ $v$-a lactate/v-a oxygen) was .25 with 95 percent confidence limits of .18 to .34 in 7 animals where simultaneous samples were obtained for determination of lactate and oxygen contents. Thus, lactate uptake by the umbilical circulation is sufficient to represent a major metabolic fuel of the ovine fetus. Previous studies from this laboratory permit the conclusion that 100 percent of the ovine fetal oxygen consumption can be accounted for by the aerobic metabolism of glucose, amino acids and lactate in the proportions of $2: 1: 1$ in late gestation.

551 REDUCTASE. I an J.Putler; Neil A.lloltzman; Seymour Kaufman; Stephen H.K'oslow; Allan Krumholz; Sheldon Milstien. Depts Ped. and Neurol.,Johns Hopkins Univ.Sch.Med., $\overline{B a 1 t ., M d}$; Lab. Neurochem., NIMH, Bethesda,Md; Lab. Preclin. Pharm., St.E1iz.Hosp.,D.C.

The onset of seizures and psvchomotor regression in a child with early treated phenyliketonuria (PKU) prompted a study of his phenylalanine metabolism. An open liver biopsy at 14 months had less than $1 \%$ dihydropteridine reductase (DHPR) activity and $25 \%$ phenylalanine hydroxylase activity compared to controls. DHPR activity was undetected in his cultured skin fibroblasts and brain (biopsy) tissue, whereas control tissue showed abundant activity.

DHPR is responsible for the regeneration of tetrahydrobiopterin, a cofactor essential in the enzymatic hydroxylation of phenylalanine, tyrosine and tryptophan. Thus a deficiency of DHPR might reduce production of dopamine, norepinephrine, and serotonin. Assay of neurotransmitter metabolites in CSF following oral probenecid did show subnormal levels. Disturbed neurotransmitter function may be responsible for the neurologic deterioration in this infant, which occurred despite excellent dietary control, suggesting need for another therapy. Assay of DHPR in cultured skin fibroblasts may be advisable in the initial diagnosis of PKU, at least until the frequency and course of this variant disorder is established.

TYPTORTNC AN ANTMAT MODEL FOR THE SUDDEN TNFANT DEATH

552 SYNDROME (SIDS: CRIB DEATH): I. CLINICAL OBSERVATIONS. Joan L. CaddelI (Intr. by Arthur E. McElfresh) Dept. Ped. and Path., St. Louis Univ. Sch. of Med., St. Louis, Mo. 63104.

Sudden, unexpected death is a cardinal feature of acute magnesium $(\mathrm{Mg})$ depletion in the young, rapidly growing animal. Other signs were compared with terminal findings in SIDS infants. 35 to $40 \mathrm{Gm}$ Wistar rats were fed $0.5,20$, or $40 \%$ casein and 0 or $150 \mathrm{mg}$ $\% \mathrm{Mg}$. By day 4 the $0 \mathrm{Mg}$ groups fed 0.5 protein had no erythema; the $20 \%$, slight; and the $40 \%$, marked erythema and often tremors. These last rats developed seizures within 10 seconds of an auditory stimulus; seizures were more difficult to elicit in the $20 \%$ rats, and the low protein group and ald rats fasted 24 hours were resistant to convulsions. From the erythematous phase through convulsions there is marked distortion of the EKG, often with spiking of $\mathrm{T}$ waves. Following seizures, $\mathrm{X}$-rays sometimes reveal hazy lung fields.

Although none of the signs are specific, there is striking similarity between the syndrome of Mg deficiency and of SIDS. (Lancet 2: 258, 1972). A report of SIDS with prolonged seizures, dyspnea, spiked $T$ waves in the EKG (New Eng. J. Med. 289: 261, 1973) is similar to the sequence in $\mathrm{Mg}$ deficiency. Sensitivity to auditory stimuli was noted in one SIDS victim (Lancet 1: 216, 1974). The most striking similarity is the ability of the stricken $\mathrm{Mg}$ deficient animal or a near SIDS child to recover, appear well for a time, and then suddenly die. Supported by NIH I R0I AMI 6219-0l and Mr. Ward Melville, Stony Brook, L.I., N.Y. 
553 EXPLORING AN ANIMAL MODEL FOR THE SUDDEN INFANT DEATH SYNDROME (SIDS: CRIB DEATH): II. BIOCHEMICAL CHANGES. Joan L. Caddell, George E. Gantner, Jr. and Max Jellinek (Intr. by Arthur E. McElfresh). Dept. Ped., Path., and Surg., St. Louis Univ. Sch. Med., St. Louis, Mo. 63104 The baby rat with acute magnesium $(\mathrm{Mg})$ deficiency was studied as an animal model for SIDS. Male wistar rats weighing 35 to 40 Gm were fed laboratory chow or purified diets containing either 20 or $40 \%$ casein, with 0 or $150 \mathrm{mg} \%$ added $\mathrm{Mg}$. On day 7 , half of the rats in each group were subjected to auditory stimuli for one minute. All animals were then immediately anesthetized with ether and cardiac blood was obtained percutaneously. Most of the rats were still alive at the time of sampling. Plasma was analyzed on a Technicon SMAC computer-controlled clinical analyzer, pH was determined on a radiometer, and potassium on an atomic absorption spectrophotometer. Statistical analysis was made using the Student's t test.

Comparing the plasma values of the Mg deficient rat post convulsion with the purified control values, significant increases were found in the mean values of: potassium, phosphorus, uric acid, LDH, SGPT, and the microhematocrit. Some protein values were markedly elevated. Significant decreases were noted in glucose and sodium. Calcium values were variable. No significant difference was noted in the mean $\mathrm{pH}$. These changes nearly fit the pattern noted in SIDS, except that marked acidosis was noted in SIDS. (Lancet 2: 258, 1972; Amer. J. Clin. Path. 54: 270, 1970; J. Pediat. 63: 290, 1963). Supported by NIH I ROI AMI 6219-01 and Mr, and Mrs. Robert Caddell of Hilton Head Island, S. C.

554 EXPLORING AN ANTMAL MODEL FOR THE SUDDEN INFANT DEATH L. Caddell, Henry Pinkerton, and Olga M. Blair (Intr. by Arthur E. McElfresh). Dept. Ped. and Path., St. Louis Univ. Sch. Med., St. Louis, Missouri 63104.

The appearance of the young rat dying of acute magnesium ( $\mathrm{Mg}$ ) depletion (J. Biol. Chem. 96:519, 1932) is similar to the infant dying of SIDS in several respects: pallor or cyanosis in a wellnourished rat, with other variable changes. Comparison was made between $50 \mathrm{~cm}$ male wistar rats fed diets containing $18 \%$ casein with or without Mg (J. Nutr. 103: 1385, 1973); and between groups of Mg deficient rats dying: (1) spontaneously, (2) of ether following audiogenic seizures, or (3) of ether without known seizures. Comparison was then made between control rats killed with ether or strychnine and those dying of Mg deficiency. Seizures from any cause could produce petechiae and some pulmonary inflamation; most striking pulmonary congestion followed $\mathrm{Mg}$ deficiency seizures. Lung weight increased significantly following seizures from $\mathrm{Mg}$ deficiency $(P<0.0025)$, but not from strychnine. Light microscopy revealed pulmonary congestion, hemorrhage, focal atelectasis, and mononuclear cell accumulation in alveolar walls, findings which are analogous to changes typically seen in uncomplicated SIDS (H.P.). More acute $\mathrm{Mg}$ deficiency in $35 \mathrm{Gm}$ rats fed $40 \%$ casein, $0 \mathrm{Mg}$, revealed either myocardial hemorrhage or calcification. It is suggested that increased capillary permeability may play a role in the pathogenesis of the pulmonary and cardiac lesions. Supp. by NIH I ROI AMI 6219-0l and Dr. and Mrs. Paul B. King, Jr. of Mountain Lakes, New I'ersey.

555 MAGESIUM LOAD EVALUATION OF INFANTS DURTNG THE FIRST SIX MONTHS OF LIFE. Joan L. Caddel1, Paul A. Byrne, Roland A. Triska and Arthur E. McElfresh. Dept. and Path., St. Louis Univ. Sch. Med., St. Louis, Mo. 63104.
Parenteral magnesium (Mg) load evaluation of 255 infants from 2 days to 6 months of age was made, with informed parenteral consent, using the test ( $J$. Pediat. 83: 129, 1973) with postload periods of 32 and 24 hours for neonates and 1-6 month-old infants, respectively. Most were studied for neuromuscular hyperirritability or other signs compatible with $\mathrm{Mg}$ deficiency; some with unrelated problems served as controls. Premature neonates retain. ed more than $40 \%$ of the load, the arbitrary limit of normal, unless Mg had been given to them or to their mothers prior to delivery. Of 64 infants $1-6$ months of age, 26 with low retention presented either with minor signs or signs otherwise explained, as by infection. A group of 12 who retained $72 \%$ were normal or small at birth; amply fed on demand; grew at accelerated rates, increasing from the 50th to the $88 \mathrm{th}$ mean percentile by 10 weeks, when they were "fat, hungry, jumpy babies" (R.T.), exempiifying the $\mathrm{Mg}$ deficiency syndrome of growth. Seven infants of $8.2 \pm 1$ weeks had had one or more sudden, transient episodes that included apnea; gasping; tonic or tonic-clonic fits; flaccidity; and often pallor, cyanosis, eye signs, sweating, or tearing. Despite preload $\mathrm{Mg}$ in 2, the group retained $88 \%$ of the load. Mg appeared to be specific therapy in high retention groups. The signs resemble premonitory signs of SIDS (Lancet 2: 258, 1972), suggesting a

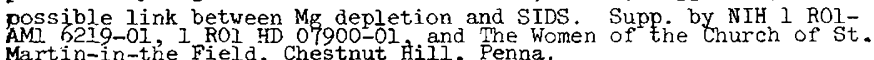

556 PARENTERAL MAGNESTUM LOAD TESTS IN POSTPARTUM AMERICAN WOMEN. Joan L. Caddell, FuIton L. Sajer, and Dept. Ped., Ob.Gyn. and Path., St. Louis Univ. Sch. Med., St. Louis, Mo. 63104

The magnesium (Mg) status of 185 low to moderate income urban American mothers was assessed in the immediate postpartum period by an intravenous Mg load test. Pre- and postload collections of urine were each made for approximately 24 hours because pilot studies revealed significant diurnal variation in $\mathrm{Mg}$ excretion. The Mg load provided 0.4 to $0.5 \mathrm{mEq}$. of $\mathrm{Mg} / \mathrm{Kg}$ of estimated lean weight. The mean retention was $51 \% \pm 2.2$ (S.E.M.). When patients retaining more or less than an arbitrary limit of $40 \%$ of the $\mathrm{Mg}$ load were compared, no differences in mean age, weight, or parity were found. The high retention group reported a diet lower in $\mathrm{Mg}$ and had a significantly lower plasma $\mathrm{Mg}$. Retention of over $90 \%$ of the $\mathrm{Mg}$ load was found in immature multiparas (less than 17 years) and in young mothers of twins. Multiparous women over 30 years had the longest interval since the previous pregnancy and the lowest retention values. Most of the primiparous patients had met the $M g$ requirements of a singleton pregnancy and rejected most of the load, but 6 primiparous women with active labor over 18 hours had significantly higher retention of the load than the other 70 primiparas (P<.005). No other symptoms or complications of pregnancy could be correlated with the Mg load values. Previously reported postpartum Thai women with high dietary Mg had significantly lower $\mathrm{Mg}$ retention. The high retention groups found represent the same categories of mothers with highest incidence of crib death. Supp. NIH I ROI-AMI 6219-01, I R01 HD 07900-01.
557 CHOLESTEROL BALANCE STUDIES IN NORMAL, HETEROZYGOU TYPE II AND HOMOZYGOUS TXPE II CHILDREN. GUY A. Carter, William E. Connor, and Ashim K. Bhattacharyya (Intr. by Ronald M. Lauer), Univ, of Iowa Hospitals, Depts. of Peds, and Int. Med., Iowa City, lowa.

The principal question in the pathogenesis of familial hypercholesterolemia is why these individuals develop excessively high plasma and tissue levels of cholesterol. This study was designed to evaluate the differences of cholesterol metabolism in children with normal, high and excessively high plasma cholesterol levels.

All children were admitted to the Clinical Research Center, and given an eucaloric cholesterol-free diet. All stools were collected for each one week study period and analyzed for total neutral and acid sterols. B-sitosterol was used as an indicator of sterol degradation.

The mean neutral sterol excretion of normals ( $N=6$ wks) was 7.04 $\pm 2.26 \mathrm{mgm} / \mathrm{kg} / \mathrm{day}$, of heterozygotes ( $\mathrm{N}=19 \mathrm{wks}) 8.41+2.44 \mathrm{mgm} / \mathrm{kg} /$ day and homozygotes ( $\mathrm{N}=4$ wks) $5.84+0.55 \mathrm{mgm} / \mathrm{kg} / \mathrm{day}$. These differences were not significant. There was a significant difference between the acid sterol excretion of homozygotes compared to both normal and heterozygotes. The bile acid excretion of homozygotes was $1.53+0.41 \mathrm{mgm} / \mathrm{kg} /$ day compared to normals of $3.33+0.94 \mathrm{mgm} / \mathrm{kg} /$ day $(p<0.01)$ and heterozygotes of $4.20+1.93 \mathrm{mgm} / \mathrm{kg} / \mathrm{day} \quad(p<0.025)$ There was a decreasing fractional clearance rate for cholesterol in the order normal > Heterozygous type II > Homozygous type II. These data are consistent with the hypothesis that the cholestero1.

558 LIN SECRETION AND DEFECTIVE GLUCONEOGENESIS.RUSSell W.Chesney, Bernard S.Kaplan, Eleanor Colle and Keith N. Drummond. McGill Univ., Dept.of Ped., Montreal, Canada.

A 5 yr old girl with growth failure,glycosuria, aminoaciduria, phosphaturia, renal tubular acidosis and rickets had ketonemia,ketonuria and elevated plasma lactate (1.7-3.5 $\mathrm{mM} / \mathrm{L})$, but no hepatomegaly or hyper- or hypoglycemia.Studies were done to determine the cause of the lactic acidosis.Urine and serum ketones were acetoacetate and $\beta-O H$ butyrate.An oral glucose tolerance test revealed negligible insulin release, serum glucose of $181 \mathrm{mg} / \mathrm{dl}$ at $69^{\prime}$,plasma lactate of $>1.8 \mathrm{mM} / \mathrm{L}$, no fall in plasma alanine, and el evated plasma glucagon levels. Intravenous glucose and i.m. or i... glucagon did not stimulate insulin release. Serum glucose did not increase after oral alanine, glycerol, fructose or galactose, but alauine, fructose and galactose appeared in the urine.Plasma lactate rose after each of these gluconeogenic substrates was given. Serum free fatty acids were normal. Insulin $(0.4 \mathrm{u} / \mathrm{kg}$ i.v.) caused a fall in serum glucose and a rise in growth hormone and lactate. After a $14 \mathrm{hr}$ fast i.v. glucagon $(0.1 \mathrm{mg} / \mathrm{kg})$ led to no rise in glucose or lactate, but, after feeding, glucagon led to a rise in serum glucose.Glucose feeding seemed to improve growth.

These studies show that increased glycolysis and lactate production with decreased gluconeogenesis contribute to the acidosis in this patient with Fanconi syndrome, and may decrease energy available for proximal tubular function. These findings have not previously been reported in the Eancons syndrome, but are similar to those seen in glycogenosis. 
CARBOHYDRATE ABNORMALITIES IN THALASSEMIA MAJOR.

559 Gertrude Costin, Maurice D. Kogut, Carol B. Hyman, Jorge A. Ortega, USC School of Medicine, Childrens Hospital of Los Angeles, Department of Pediatrics, Los Angeles. To assess carbohydrate metabolism in Thalassemia blood glucose (BG) and insulin (IRI) were measured after oral glucose (G) (1.75 $\mathrm{gm} / \mathrm{kg})$ and $\mathrm{IV}$ tolbutamide $(\mathrm{T})(20 \mathrm{mg} / \mathrm{kg})$ in 10 patients aged $9-18$ who received hypertransfusion treatment (Hytr $\mathrm{Rx}$ ) for 5 yrs.

Pretreatment mean fasting blood glucose(FBG) level was significantly > normal $(p<0.001)$; mean fasting IRI was significantly< normal $(p<0.005)$. Mean BG levels were normal but mean IRI levels were significantly < normal following $G$ and $T$.

Mean FBG levels were significantly $>\operatorname{normal}(p<0.001)$ after $1-5$ yrs of Hytr $R x$ and $>$ pretreatment values $(p<0.01)$ after the 3 rd to 5 th yr. Mean fasting IRI and mean IRI levels following $G$ and $T$ were significantly $>$ pretreatment values $(p<0.02)$ and similar to normal following $G$ after $1-5$ yrs of Hytr Rx. Mean IRI levels af ter $T$ were significantly $>$ normal $(p<0.05)$ after the 1 st and 5 th yr of Hytr $\mathrm{Rx}$. Despite the improved insulin responsiveness, diabetic GTT was present in $2 / 10$ patients after 1 yr, $3 / 10$ after 2 yrs, $4 / 10$ after 3 yrs, $4 / 8$ after 4 yrs, and $4 / 7$ after 5 yrs. Two patients required insulin treatment.

The studies suggest that 1) improvement in insulin responsiveness may be due to increased oxygenation of the $\beta$-cell, 2) diabetes may result from genetic predisposition, increased pancreatic hemosiderosis and/or peripheral insulin resistance due to alterations of insulin receptors in specific target tissues by hemosiderosis.

560 SLOPE OF THE IN VIVO $\mathrm{CO}_{2}$ EQUILIBRATION CURVE IN NEWBORN BABOON, RALPH B. DELL, HISAYO O. MORISHIMA AND WENDELL NEIMANN. COLLEGE OF PHYSTCIANS \& SURGEONS, COLUMBIA UNIVERSITY, DEPARTMENT OF PEDIATRICS, NEW YORK.

The in vivo $\mathrm{CO}_{2}$ equilibration curve relates blood acid-base status to steady-state, acute hypercapnia and defines the expected acid-base response to acute hypercapnia. Previous work in this laboratory has shown that in the adult dog the slope $\left(\Delta \log { }^{P_{C O}}{ }^{\prime}\right.$ $\triangle \mathrm{pH})$ of the in vivo $\mathrm{CO}_{2}$ equilibration curve decreases when $\mathrm{ECF}$ volume is increased (due to a redistribution of $\mathrm{HCO}_{3}^{-}$between blood and interstitial fluid) and increases when plasma $\mathrm{HCO}_{3}$ falls. However, in no case did the slope become as steep as the slope of the curve for blood equilibrated in vitro. The slope of the in vivo $\mathrm{CO}_{2}$ equilibration curve was estimated in newborn baboons to determine whether the slope in a newborn animal differs from the slope in an adult with similar body fluid compartment volumes and acid-base status. At a normal initial plasma bicarbonate concentration $(18-20 \mathrm{mEq} / 1)$ the slope of the curve is similar in both newborn and adult. But, as the animal became more acidotic and inftial plasma $\mathrm{HCO}_{3}$ concentration fell, the slope increased much more rapidly in the newborn than in the adult so that at an initial $\mathrm{HCO}_{3}$ of $13.5 \mathrm{mEq} / 1$ the slope of the in vivo curve was as steep as the in vitro curve. These results are quite different from results in the adult and are in keeping with clinical observations in the human newborn. Such a steep slope can occur only if there is considerable movement of $\mathrm{HCO}_{3}$ from ICF to ECF and suggests that combined metabolic and respiratory acidosis is associated, in the newborn, with considerable intracellular acidosis and further suggests the need for large amounts of $\mathrm{HCO}_{3}$ in treatment.

PAL RESPONSE TO A KETOGENIC DIET

561 IN TWO BROTHERS WITH PYRUVATE DEHYDROGENASE DEFICIENCY Rena E. Falk, Stephen D. Cederbaum, John P. Blass, Frank J. Pruss, Robert E. Carrel. (Intr. by Solomon A. Kaplan) UCLA Sch. of Med., Los Angeles. Depts. of Ped., Psych., Biol. Chem. Two brothers aged $111 / 2$ and $21 / 4$ had psychomotor and growth retardation from infancy. Minor respiratory infections (URIs) led to protracted weakness, ataxia, and ophthalmoplegia. The older had intermittent hyperalaninuria, particularly with URIs.

On a normal diet, both boys had elevated levels of blood pyruvate 2 hours post prandially $(1.6-1.7 \mathrm{mg} / \mathrm{d} 1 ; \mathrm{n} 1<1.2)$. During URIs, values rose to $>3.0 \mathrm{mg} / \mathrm{d} 1$. Lactate:pyruvate ratios were normal. Within 30 hours on a $65 \%$ carbohydrate diet, both boys dramatically deteriorated clinically while blood pyruvate rose to $>6.0 \mathrm{mg} / \mathrm{dl}$. On a $55-65 \%$ fat diet, their post prandial blood pyruvates fell. After 1 month on the high fat diet, the older boy has improved clinically, subjectively.

Cultured skin fibroblasts from the older boy oxidized $\left[1-{ }^{14} \mathrm{C}\right]-$ pyruvate more slowly than did controls. Cells from the parents oxidized it at rates intermediate between the patient's and control cells. Palmitate and glutamate were oxidized normally by all 3 lines. Enzymatic studies in progress suggest deficiency in the pyruvate dehydrogenase complex (PDH). The available data support autosomal recessive inheritance of the abnornality.

Clinical and experimental studies from several laboratories Indicate that the brain can utilize ketone bodies during ketosis and thus bypass a deficiency of the PDH complex. The long term value of a ketogenic diet in these patients is under study. MINE IN CHILDREN WITH FAMILIAL HYPERCHOLESTEROLEMIA. J.Ramsay Farah, Peter 0 . Kriterovich and Catherine A. Neill, Johns Hopkins Univ. Dept. of Ped. Baltimore, Ma.

Thirteen children with familial hypercholesterolemia(Fh) were treated with cholestyramine in a metabolic unit to relate the decrease in the plasma concentrations of cholesterol(C) and low density lipoprotein cholesterol (LDL) to drug-dosage, pretreatment levels of $\mathrm{C}$ and LDL and body weight. All children were on a diet low in cholesterol ( $200 \mathrm{mg} /$ day) with a polyunsaturated to saturated fat ratio of 2 . In 8 children with a mean $(+1 \mathrm{SD}) \mathrm{C}$ and LDL of $281 \pm 35$ and $224 \pm 36$, a dose of 1 gm/day was begun and increased by $1 \mathrm{gm} /$ day to $16 \mathrm{gm} /$ day, given twice daily. In $7 / 8$ children the LDL fell below normal(170mg/100ml)on an average dose of $6 \mathrm{gm} /$ day (range 3 to 11 ), a response directly proportional to the pretreatment LDL and not correlated with body weight. Their LDL continued to fall and reached a plateau at a mean IDL of 130 \pm 14 on an average dose of $11 \mathrm{gm} / \mathrm{day}$ (range 8tol4). The drug( 16 $\mathrm{gm} /$ day) failed to lower LDL below normal in one patient who had the highest LDL(290). These results were used to predict the dose required to normalize LDL in 5 other children. Predicted amounts were: LDLr200, $4 \mathrm{gm}$; LDL 290 , $16 \mathrm{gm}$ or more. Within 5 days, the LDL in each $12 \mathrm{gm}$; LDL 290,16 gm or more. Within 5 days, the LDL in each
child fell below normal. The appropriate dosage of cholestyramine in a child with $\mathrm{FH}$ is related to pretreatment levels of LDL. Losage appears predictable and should be individualized. A nadir of LDL exists, after which additional cholestyramine is ineffective. The drug can be conveniently and effectively given twice daily.

PARASYMPATHETIC CONTROL OF INSULIN SECRETION

563 IN CHILDHOOD OBESITY. R. H. Fiser and G. A. Bray Rock, Arkansas and U.C.L.A., Torrance, California.

Recent reports have emphasized the importance of the autonomic nervous system in obesity: Vagotomy prevents the hyperinsulinemia and development of obesity in ventro-medial nucleus lesioned rats and hypothalamic obesity in man. The present studies were

designed to assess parasympathetic control of insulin release in 5 obese and 5 lean adolescents. Plasma glucose and insulin concentrations were measured after: 1) an oral glucose load (OGTT), 2) computer simulated glucose load (SGTT) to match OGTT, and 3) OGTT after prior atropinization, (vagolytic) (OGTT+A).

The obese individuals demonstrated hyperinsulinemia $(94+12$ $\mathrm{uU} / \mathrm{ml}$ mean $+\mathrm{SE}$ versus $10+3$ in the lean, $\mathrm{p}=.001)$. Mean peak $\Delta$ glucose values were similar in both obese and lean individuals. Insulin changes in contrast were:

$\begin{array}{lrcc} & \text { OGTT } & \text { SGTT } & \text { OGTT+A } \\ \text { * Obese } & 690 \pm 99 & 457 \pm 83 & 358 \pm 81 \\ \text { *Lean } & 3755 \pm 1300 & 583 \pm 123 & 1317 \pm 154\end{array}$

*Values represent mean $\pm \mathrm{SE}$ peak $\Delta$ responses from control.

These data suggest an enhanced insulin response to glucose stimulation in lean children with relative similar responses to oral and intravenous glucose stimulation in lean and obese children. The failure of the obese children to suppress insulin release after atropine to the same degree as the lean indicates altered parasympathetic tone. This enhanced parasympathetic tone may contribute to the hyperinsulinemia in childhood obesity.

564 RELATIONSHIP TO CYCLIC AMP. Robert $\mathrm{H}$. Fiser, Paisl $\mathrm{R}$ Williams, Mark A. Sperling, William oh, and Delbert Fisher. Univ. of Arkansas Sch. of Med., Univ. Hosp., Dept. of We have previously reported an obtunded glucagon response to alanine stimulation in fetal and neonatal sheep. To explore the mechanisms responsible, we have studied the response of glucagon and glucose to alanine infusions after fasting and theophylline administration in 10 newborn ( $2-3$ days) and 4 infant ( 3 months) lambs.

The plasma glucagon response was minimal in the newborn lamb after either a 4 or 24 hour fast $(32 \%$ and $38 \%$ ). Although plasma glucose fell, basal glucagon levels remained unchanged with fasting $(121 \pm 31$ vs. $101 \pm 15 \mathrm{pg} / \mathrm{ml})$. In contrast, a brisk rise in glucagon ( $114 \%)$ was seen in infant sheep after alanine administration. In 4 newborns, theophylline produced no changes in basal glucagon or glucose levels but when infused concurrently with alanine, produced a marked augmentation of glucagon response $(117 \pm 10$ to $245 \pm 32 \mathrm{pg} / \mathrm{ml})$. Glucose values during fasting and after alanine infusions were similar in the newborn and infant lambs.

These data suggest a progressive maturation of pancreatic islet alpha cell glucagon secretion in the sheep during the post-natal period. The blunted glucagon response observed in the newborn is related to immaturity of the glucagon secretion mechanisms rather than deficient synthesis of the hormone. This immaturity may be related to impaired synthesis and/or enhanced degradation of cyclic AMP or to diminished responsiveness to cyclic AMP. 
565 Penicillamine Therapy: Nine-year Follow-up in Two Male Siblings with Wilson's Disease. A. Garnica,M.D., O. Rennert, M.D., C. Tiwary, M.D.*, Department of Pediatrics, University of Florida, Gainesville, *Department of Pediatrics, University of Nebraska.

The results of a nine year follow-up of penicillamine therapy in male siblings, ages sixteen and eleven years, are reviewed. Both patients were ascertained when an older sibling died with cirrhosis, and they were placed on a regimen including low copper diet, penicillamine, and pyridoxine at ages seven and two years. Subsequent yearly follow-up included clinical evaluation, serum copper and ceruloplasmin, urine copper EEG, and assessement of renal and hepatic function. Growth and development has been normal; both patients are above the fiftieth percentile for height and weight; the older of the two is undergoing normal pubertal changes. EEG in both patients was mildly abnormal, but has not changed significantly. Serum copper and ceruloplasmin have been consistently low and urine copper high. Hepatic functions and renal functions have remained consistently normal, except for mild impairment of urine concentrating ability in both siblings, and mild hypoalbuminemia in the older, who at diagnosis demonstrated evidence of hepatic disease. The above observations confirm the efficacy and safety of long term penicillamine therapy in children with Wilson's Disease.
NUTRIENT INTAKES AND LINEAR GROWTH OF POOR CHILDREN 566 George G. Graham \& Hilary M. Creed. Johns Hopkins (Internat. HIth.), Baltimore, and Instituto de Investigacion Nutricional, Lima, Peru.

Earlier studies in Peru revealed significant correlations between various socio-economic factors, notably disposable income, and the linear growth rates of previously malnourished children and their siblings. In 26 families, 7-day individual dietary weighings were carried out and nutrient intakes determined. Despite calorie intakes consistently below, and protein intakes consistently above those recommended by the FAO-WHO Expert Committee for children of their height age, linear growth rate was significantly correlated with \% dietary protein calories $(r=0.40, t=2.11)$ and \% dietary protein from animal sources $(r=0.68, t=4.51)$; also with calorie intake as $\%$ of FaOWHO recommended $(r=0.51, t=2.90)$ and prote in intake as $\%$ of same $(r=0.62, t=3.91)$. Per capita expenditure for food was significantly correlated with various indices of dietary adequacy, including \% fat calories. It would appear that FAOWHO have overstated calorie needs and, more important, grossly understated protein needs of children. Unaware of official advice, poor people, given more money, buy better food, not more of the same, with resultant benefit to their children.

Supported by NIH grant AM-09980 and a fellowship from the Drummond Trust.

ABSENCE OF CORREILATION BETWEEN CORD SERUM CHOLESTEROL

567 AND DEVELOPMENT OF HYPERCHOLESTEROLFMIA DURING CHILDHOOD. Harvey Grossman, Ralph E. Kauffman, and Antoni

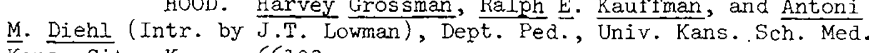
Kans. City, Kans. 66103

Recent studies have suggested that measuring total cholesterol (chol.) concentration in cord blood of infants at birth may be useful in identifying those infants at risk for later manifestation of type II familial hyperlipoproteinemia. This prospective study was designed to test the correlation between cord serum total chol. levels at birth and serum total chol. levels at 3-4 years of age. Cord serum total chol. concentration was determined in 587 consecutive infants born at the Univ. of Kans. Med. Ctr. duri.ng 1970-71. The mean cord chol. concentration was $89 \mathrm{mg} / \mathrm{dl}$. The values were normally distributed about the mean with S.D. of $22 \mathrm{mg} / \mathrm{dl}$. $18(3 \%)$ of the infants had values $\geq 2 \mathrm{~S} . \mathrm{D}$. above the mean. 82 (14\%) had values $\geq 1$ S.D. above the mean. Follow-up chol. determinations were obtained at 3-4 years of age in 38 of the 82 infants with high cord chol. levels and in 38 infants randomly selected from those with cord chol. levels within 1 S.D. of the mean. The infants were matched for sex, race, and birthweight. No correlation was found between cord serum total chol. concentration and occurrence of hypercholesterolemia at 3-4 years of age. It is concluded that cord serum total chol. concentration is not a useful screening tool to identify children at risk for familial type II hyperlipoproteinemia.
568 RECURRENT MYOGLOBINURIA AND MUSCLE CARNITINE PAIMITYLTRANSFERASE DEFICIENCY, James $J$ Herman, Mar 1 a Gumbinas, and Henry L. Nadler. Northwestern Univ. Med. Sch., Children's Mem. Hosp., Peds, Chicago, III.

$A$ I5-year old boy had two years of recurrent attacks of myalgia, muscle cramps without weikness, and myogloblnuria after periods of normal activity for his age. There was marked elevation of creatinine phosphokinase, serun glutamic oxalacetic transaminase, and aldolase enzymes. His lactic acid exercise tolerance test was compatible with idfopathic paroxysmal myoglobinuria, and his electranyography was consistent with a nonspecific myopathy. The microscopic and ultrastructural examinations of his muscle blopsy were also consistent with a non-specific myopathy; muscle phosphorylase $A$ and $B$ were normal as were glycogen content and phosphofructokinase. Since abnormal lipid metabolism has been shown to be associated with myoglobinuria, enzymes related to carnitine metabolism were investigated. His wuscle carnitine palmityltransferase, as weasured by a colorimetric method and a radloactive assay, was markedly decreased ( $2.7 \mathrm{uM} / \mathrm{min} / \mathrm{mg}$ protein) when compared to normal controls and ischemic normal controls (13.2 uM/min/mg protein and $14.5 \mathrm{uM} /$ andmg protein respectively). The patient's muscle carnitine acetyltransferase $(13.4 \mathrm{um} / \mathrm{min} / \mathrm{mg}$ protein) was $60 \%$ of normals $(25.5 \mathrm{uM} / \mathrm{m} 1 \mathrm{n} / \mathrm{mg}$ protein). Palmityl Col synthetase was normal in patient's wuscle as were his muscle and erum carnitine levels. Therefore, it appears that idiopathic myoglobinuria is associated with a marked deficiency of carnitine palmityltransferase in contrast to the other two enzymes in carnitine metabolism. Thus, contrast to the other two enzymes in carnitine metabolism, Thus,
this deficiency may be related to the episodes of myoglobinuria. 569 "PROTEIN CONSERVATION WITHHOUT DECRRASED GLUCONEROGENESIS IN MALNOURISHED INEANTS", Douglas Kerr, Michael
Stevens, Hazel Robinson, and Dovid Picou IIntr. by M. Klaus) Univ. Of the West Indies, Tropical Metabolism Research Unit, Kingston, Jamaica and Case hestern Reserve Univ., Univ. Hosp., Dept. Ped., Cleveland, 0.

Utilization of energy reserves and glucose production while fasting were estimated in 5 malnourished $(M)$ infants compared with tinemselves after recovery (R). After a standard diet, caloric utilization, estimated from hourly $\mathrm{O}_{2}$ consumption, remained around $70 \mathrm{cals} / \mathrm{kg} / \mathrm{d}$ in $\mathrm{M}$ and 80 in R while fasting for 24 hours. utilization of carbohydrate decreased and fat increased in both cases (estimated from the R.Q.) until fat provided 958 and $92 \%$ of total calories in $M$ and $R$ respectively. Protein utilization, estimated from $N$ excretion, was initially low in both cases but increased 2 fold in $R$, reaching a maximm of $8 \%$ of total calories. By contrast, there was little increase in M, reaching a significantly lower maximum of only $5 \%$ of calories. Plasma gluoose was not lower in M. Gluoose production due to gluconeogenesis, measured after glyoogen depletion by dilution of constantly infused $\mathrm{U}-13 \mathrm{C}$-gluoose, was $3.0 \mathrm{mg} / \mathrm{kg} / \mathrm{min}$ in $\mathrm{M}$ and 2.7 in $\mathrm{R}$ (not significantly different). Glycerol from fat could account for approximately $20 \%$ of this in both cases; gluooneogenic amino acids fram protein could account for $10 \%$ in $M$ and $20 \%$ in $R$. The remaining majority of gluoose production must be due to recycling of lactate, pyruvate, and alanine. It is concluded that prolonged undemutrition in infants usually results in conservation of endogenous protein witinout fasting hypoglyœemia since the majority of gluooneogenesis is due to the cori cycle.

570 NEONATAL INSULIN RESPONSE TO NASOGASTRIC (NG) GLUCOSE INFUSION. Katherine C. King, Peter A.J.Adam. Case Western

In order to determine whether glucose-induced pancreatic insulin secretion is enhanced by enteric hormones, normal fasting newborn infants were studied during continous 1 -hr IV or NG infusions of glucose at rates of 6 and $12 \mathrm{mg} / \mathrm{kg}$.min starting $2 \mathrm{hrs}$ or 3 days after birth. Blood glucose and plasma insulin levels were compared with those of normal infants infused with isotonic saline, normal adults infused with glucose, and infants of gestationally diabetic mothers (IGDM) infused with glucose.Results at $60^{\prime}$ of infusion in the 3-day old infants are presented (mean + SEM): Infants Infusion (mo/kg min) (n)

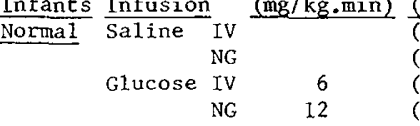

IGDM Glucose NG $12 \quad(4)$

(4) $71 \pm 4$

$\begin{array}{cc}71 \pm 4 & 5 \pm 2 \\ 66 \pm 4 & 6 \pm 2 \\ 103 \pm 5 & 8 \pm 1 \\ 101 \pm 13 & 7 \pm 3\end{array}$

$101 \pm 13$

$25 \pm 6$

In adults $N G$ infusion doubled the plasma insulin response to IV glucose. At 3 days of age, IV and NG infusions raising blood glucose to about $100 \mathrm{mg} \%$ had negligible effects on plasma insulin. In contrast, NG glucose infusion in the IGDM stimulated a marked plasma insulin response even though the blood glucose was lower than in normal infants. This response differs from the normal glucose:insulin relationship observed previously in IGDM infused continously with IV glucose. In conclusion, gastrointestinal absorption of glucose in the normal newborn is not associated with enhanced insulin responses as in IGDM or normal adults. 
PHYSIOLOGICAL ABNORYALITISS FOLLOWING BRATN DRATH.

571 Richard E. Kravath, Nathan Litman (Intr. by Laurence Finberg). Albert Einstein Col. Med., Montefiore Hosp. and Ked. Ctr., Dept. Ped., Bronx, New York.

We have observed several physiological peculiarities in patients with brain death who were on mechanical ventilators. From January 1972 through October 1974 brain death was diagnosed 29 times, using modified Harvard criteria,representing $18 \%$ of the pediatric deaths in our hospital. The 29 charts were reviewed for this report. 52\% had spinal reflexes present at the time of diagnosis of brain death which represents the now generally accepted modification of the Harvard criteria. 52\% of the brain deaths were due to trauma, $31 \%$ to anoxda and $7 \%$ to bacterial meningitis. $41 \%$ served as organ donors. All were hypothermic, $52 \%$ required pitressin for diabetes insipidus and 38\% required vasopressors, as expected. Diabetes insipidus requiring pitressin was seen in $89 \%$ of those with anoxia as a cause of brain death, compared with $53 \%$ of those with trauma, which may be explained by more diffuse damage with anoxia. Of particular interest is that none of the 9 children under 3 years of age required vasopressors, as did 55\% of those older,possibly representing a shift of neural control with age. Also noteworthy was hyperglycemia in all with glucose ranging from $110-1300 \mathrm{mg} / 10 \mathrm{ml}$. Hypocalcemia was observed in $53 \%$ of those tested and hypercalcemia was seen in one patient with a range of calcium in serum from $6.3-13.1 \mathrm{mg} / 100 \mathrm{ml}$. These levels reflect major disruptions in homecstatic control of carbohydrate and mineral metabolism which remain to be elucidated. Patients with brain death offer a unique opportunity to study endocrine, neurophysiologic \& metabolic functions in absence of brain function.

572 Ingeborg Krieger, Children's Hosp. of Mich. Detroit. Isovaleric acidemia was diagnosed in a 5 year old black female after 8 attacks of ketoacidosis and leukopenia. Pl. glycine was 1.98 and $2.10 \mathrm{mg} \%$ between and lower during attacks, (normal $1.30 \pm 0.34 \mathrm{mg} \%$ ). Oral loads of isoleucine, threonine, and valine were well tolerated; vomiting occurred 2 hours after $125 \mathrm{mg} / \mathrm{kg}$ leucine and continued for $Q$ hours. Pl.isovaleric acid (IVA) was $5.6 \mathrm{mg} \%$ (normal $0.05 \mathrm{mg} \%$ ). Excretion of IVA as the con= densation product with glycine (IVG) increased slightly, from 6.3 before to $9.9 \mathrm{mg} / \mathrm{mg}$ creatinine after the load. When this load was repeated with $250 \mathrm{mg} / \mathrm{kg}$ glycine no vomiting occurred, pl.IVA at 2 hours was only $0.93 \mathrm{mg} \%$, and urinary IVG increased by $372 \%$ to $26.3 \mathrm{mg} / \mathrm{mg}$ creatinine (determinations of IVA and IVG were done by Dr. K.Tanaka, Yale Univ.) The patient was then placed on a diet with a leucine content equivalent to the single load; $1 / 3$ the glycine dose that prevented vomiting was given with each of 3 meals. Glycine caused an increase of serine, alanine, glutamine, and branched chain amino acids. During $h$ months of treatment $1 i=$ near growth exceeded that of the preceeding $h$ months by $50 \%$. How ever, infections precipitated 3 more attacks, each started 14 hours after the last glycine dose. Because of vomiting, glycine was given rectally and ahorted these attacks. $175 \mathrm{mg} / \mathrm{kg}$ glycine did not cause a rise of $\mathrm{pl}$. glycine during attacks, presumably because it condensates to form IVG. The same dose caused a rise to $3.54 \mathrm{mg} \%$ when the patient was well. Conclusion: clinical and lakoratory responses showed that detoxification of IVA can be enhanced by providing an excess of glycine.

FURTHER EVIDENCE OF A LINK BETWEEN UREA CYCLE AND 573 SHORT CHAIN FATTY ACID METABOLISM. Ingeborg Krieger, of Mich. and Wayne State Univ. Sch. of Med. Detroit, Mich.

Diagnosis of ornithine transcarbamylase deficiency in this 24 month old black female was based on 1) hyperammonemia, $6.9 \mu \mathrm{g} / \mathrm{ml}$ in response to unrestricted protein intake, 2) orotic aciduria in the patient and her asymptomatic mother (419 and 136 ymoles) day), and 3) evidence of $x$-linked dominant trasnmission, lethal in a male sibling. Liver biopsy, which may cause fatal hyperamo= nemia, was not done. P1. propionate was 30 times normal (deter= mined by Dr. Claude Bachmann at UCSD). Leukocyte propionyl COA carboxylase was normal (determined by Dr. Y. E. Hsia, Yale Univ.) Hyperammonemia was consistently associated with elevations of pl. lysine, up to $7.95 \mathrm{mg} \%$ (normal $2.63 \pm 0.68 \mathrm{mg} \%$ ) and of gluta= mine, up to $6.18 \mathrm{mg} \%$ ( normal $2.01 \pm 0.83 \mathrm{mg} \%$ ). Homocitrulline was excreted during these attacks. Metabolites of lysine: saccha= ropine, pipecolic acid, and $\varepsilon-\mathrm{N}$-acetyl-l-lysine were not elevated in urine. A lysine load did not cause an increase of bl.ammonia. CONCLUSION: Elevation of lysine and glutamine was probably due to the need for ammonia fixation with $\alpha$-ketoglutarate which, conse= quently, is not available for formation of saccharopine from $1 \mathrm{y}=$ sine. In turn, lysine may serve in a compensatory urea cycle: lysine-homocitrulline-homoarginine. The connection between abnor= malities of ureacycle and propionate metabolism remains unex= plained. Whereas hyperammonemia has been observed in disorders of short chain fatty acid metabolism, this appears to be the first demonstration of a link in the reverse direction.
574 TYPE V HYPERLIPOPROTEINEMIA: A BIOCHEMICAL DEFECT OF CHILDHOOD? Feter O. Kwiterovich and J. Ramsay Farah, (Intr. by Cather

Dept. of Ped. Familial type V hyperlipoproteinema(HLp)previously considere a disorder of adulthood, was ascertained in P.N., a loyr. old girl who presented with markedly creamy plasma, hyperchylomicronemia, hyperprebetalipoproteinemia, plasma triglyceride(TG) and cholesterol concentrations of 6,880 and $490 \mathrm{mg} / 100 \mathrm{ml}$, and lipemia retinalis. Her parents and $8 \mathrm{yr}$ old sister had type IV HLp. Following 60 units $/ \mathrm{Kg}$ of I.V.heparin, the plasma l)lipoprotein lipase(LPL),2)hepatic triglyceride lipase(HTGL) and 3)histaminase (H) in P.N. at 5,10 , and $15 \mathrm{~min}$. respectively were: 1$) 3.1 ; 2.4 ; 2.9$ $\mathrm{umol} / \mathrm{ml} / \mathrm{hr}$; 2)15.1;16.9;15.5 umol $/ \mathrm{ml} / \mathrm{hr} ; 3) 18.0 ; 16.5 ; 15.0 \mathrm{pmol} / \mathrm{hr}$. Her sib also had low LPL increased HTGL and normal H. Both parents had low-normal LPL and normal HTGL; the father had low H. A four $\mathrm{hr}$ glucose tolerance test in P.N. revealed hyperinsulinism(185.8 $\mathrm{mu} / \mathrm{ml}$ ) at $30 \mathrm{~min}$, normal glucose and growth hormone levels. The effect of various diets, each given for 5 days to P.N. in a metabolic unit, was studied. With $40 \%$ fat, $40 \%$ carbohydrate(CHO) and $20 \%$ protein, TG decreased from 6,880 to 490 . On $20 \%$ fat, $85 \%$ CHO, the TG increased within $24 \mathrm{hr}$ to 900 , then gradually fell to 304. With a high fat diet $(64 \%)$ a rapid increase in TG to 2630 was followed by adaptation to a TG of 380 . With $30 \%$ fat, $50 \%$ CHO TG fell to 220 ; P.N. required the addition of medium chain triglycerides ( $15 \%$ of calories) to lower TG to normal (110). The biochemical findings of familial type V HLp, including low LPL, hyperinsulinism, CHO and fat intolerance can present in chilähood. Severe hyperlipidemia can be effectively treated. GLUCONEOGENIC PRECURSORS IN THE FASTING BABOON NEONATE. Lynne L. Levitsky, John B. Paton and David E. Fisher, Dept. Ped., Univ. Chicago at Michael Reese Hosp. Med. Ctr, Chicago, Ill. (Intro. by S. P. Gotoff)

The role of the splanchnic bed in neonatal carbohydrate homeostasis was studied quantitatively in 6 fasting baboon infants. Substrate concentrations in the inferior vena cava above and below the level of the hepatic vein were measured and blood flows were determined by the radioactive microsphere method in order to calculate hepatic vein substrate levels and splanchnic extraction (SE) and production rates.

$\begin{array}{lcccc}\text { Gluconeogenic substrate: Lactate } & \text { Glycerol } & \text { Alanine } & \text { Pyruvate } \\ \mathrm{SE}(\mu \mathrm{M} / \mathrm{min} \pm \mathrm{S} . \mathrm{E} .) & 42 \pm 25 & 2.7 \pm .9 & 1.1 \pm .5 & .34 \pm .3\end{array}$

Glucose production was $20 \pm 8.5 \mu \mathrm{M} / \mathrm{min}$ or $.73 \pm .28 \mu \mathrm{M} / \mathrm{min} / \mathrm{gm}$ liver. Total gluconeogenic equivalents were $23 \pm 13 \mu \mathrm{M} / \mathrm{min}$ of which $88 \%$ could theoretically be accounted for by extracted lactate. Alanine extraction could account for $<3 \%$ of ongoing glucose production. Arterial lactate was $2.6 \pm .4 \mathrm{mM} / \mathrm{L}$ and alanine was $.329 \pm .025 \mathrm{mM} / \mathrm{L}$ in contrast to animals studied at 6 wks of age [Lactate $2.4 \pm .4 \mathrm{mM} / \mathrm{L}$, alanine $\left..107 \pm .023 \mathrm{mM} / \mathrm{L}\left(p_{<}<.002\right)\right]$. Arterial glucose levels were equivalent $(3.1 \pm .2$ vs $2.9 \pm .5 \mathrm{mM} / \mathrm{L}$ at 6 wks). There was no evidence for deficient peripheral mobilization or hepatic extraction of alanine. However, it is suggested that lactate may be quantitatively much more important than alanine in neonatal hepatic gluconeogenesis.

576 GENETIC HETEROGENEITY IN DIABETES (DM): STUDIES IN GONADAL DYSGENESIS (GD). Barbara M. Lippe, Mark A. Sperling, liCla Sch. of Med., Dept. of Ped, Los Angeles, and Harbor Gen. Hosp., Torrance, California.

Hyperglucagonemia and inappropriate plasma glucagon responses to oral glucose have been found in DM and suggested as a characteristic of genetic DM. Patients with GD have been shown to have high incidence of carbohydrate intolerance but the relationship of this finding in patients with a known chromosomal abnormality to genetic DM is unclear. We therefore studied the plasma glucose, insulin and glucagon responses during an oral glucose tolerance test (OGTT) in 15 subjects with GD ranging in age from 4-20 years. GD patients were classified as being normal (Group A) or variably abnormal (Group B) on the basis of glucose responses to OGTT. Group A $(n=9)$ had mean plasma glucose +SEM at 1 and 2 hours of $136+7$ and $110+6 \mathrm{mg} \%$ as compared to Group $B \quad(n=6)$ of $225+$ 25 and $165+17 \mathrm{mg} \%$. Insulin levels at these 2 times were $70+16$ and $64+16 \mu \mathrm{U} / \mathrm{ml}$ for Group $\mathrm{A}$ and $115+29$ and $181+43 \mu \mathrm{i}, \mathrm{mi}$ for Group $B$ $(\mathrm{p}<0.01$ at both times). Mean glucagon levels were higher initially in both groups than measured in normal subjects in the same assay; $A=187+37$ and $B=242+43 \mathrm{pg} / \mathrm{ml}$. However, glucagon was suppressed by $62+14 \mathrm{pg} / \mathrm{ml}$ in Group $A(p<0.05)$ while there was actually a small $\mathrm{rise}$ in glucagon in Group $\mathrm{B}$. Thus the DM of children with GD is characterized by non-suppressibility of glucagon, but unlike juvenile DM, there is high endogenous insulin. These studies suggest 1) a possible relationship between the chromosomal disorder and early development of DM, 2) hyperglucagonemia may contribute significantly to the evolution of the DM, and 3 ) there is heterogeneity in pancreatic endocrine function in DM. 

577 LACK OF GLUCONEOGENESIS FROM ALANINE AT BIRTH. Michael at Cleveland Metropolitan Gen Hosp, Cleveland

The glycemic response to alanine, infused IV at $8.3 \mathrm{mg} / \mathrm{kg} . \mathrm{min}$ for $30^{\prime}$, was evaluated in normal 1 -hr-old infants to determine whether gluconeogenesis is stimulated by birth. Blood alanine, glucose, lactate, pyruvate and plasma glucagon were compared in 4 pairs of infants infused with alanine or isotonic saline, and in 4 adults infused with alanine. The results in infants at $30^{\prime}$ of infusion are summarized as follows (mean \pm SEM):

$\begin{array}{lccccc}\text { Infusion } & \frac{\text { Alanine }}{(\mathrm{mM})} & \frac{\text { Glucose }}{(\mathrm{mg} \%)} & \frac{\text { Glucagon }}{(\mathrm{ng} / \mathrm{ml})} & \frac{\text { Lactate }}{(\mathrm{mg} \%)} & \frac{\text { Pyruvate }}{(\mathrm{mg} \%)} \\ \text { Saline } & 0.27+0.02 & 52+7 & 0.49+0.26 & 12+2 & 0.9+0.1\end{array}$

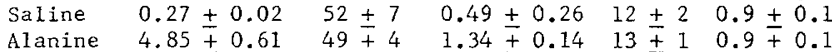

Infusion of alanine raised blood alanine levels $18-f o l d$, but the alanine disappeared rapidly postinfusion. Raising blood alanine did not increase blood glucose, lactate or pyruvate, even though plasma glucagon rose 2.7 -fold. This failure to raise blood glucose contrasts with the marked glycemic effect described by Pagliara in children with ketotic hypoglycemia. In the 4 adults fasted overnight, there was a slight incremental rise of blood glucose $(6 \mathrm{mg} \%)$, but a $50 \%$ rise in blood lactate and pyruvate. In conclusion, gluconeogenesis from alanine apparently is not established normally in utero or immediately after birth. Since blood lactate, pyruvate and glucose do not rise, while pancreatic glucagon responds to alanine, metabolism of the glucogenic amino acid seems to be diverted from the gluconeogenic pathway in the human fetus and in the newborn.

THE FALLACY CF GOOD DIABETIC CONTROL CRITEFIA. John I.

578 Malone, Arlen Rosenbloom, A. Grgic, F.T. Weber, $\overrightarrow{\text { Vept. }}$ Tampa, and Univ. of Florida, Gainesville, Fla. Intr. by L. Barness Diabetic control implies romalization of metabolism with periodic deteminations of plasma glucose (PG) and urine glucose (UG) corcentrations. The utility of these tests depends upon metabolic stability that allows random sampling to reflect the general metabolic state and the ability of the UG to reflect the PG. Random UG's were tested by 220 children and checked. The children's results varied from the techricians' in $49 \%$ of the urines tested. PG $30 \mathrm{~min}$. prior to second voided urines regative for glucose ranged up to $381 \mathrm{mg} \%$. FG $>200 \mathrm{mg} \%$ were found in $13 \%$ of children with negative UG. In 54 cases where 24 hour urine collections were verified by creatirine excretion, $12(22 \%)$ had good control on the basis of UG $<0.5 \mathrm{mg} / \mathrm{kg} / 24 \mathrm{hr}$. These 54 children had 108 fasting (F) PG of which 28 ( $26 \%$ ) were $<110 \mathrm{mg} \%$ but only half of these nomal FPC were in children with low UG. Two hour post prandial (PF) FG $<150 \mathrm{mg} \%$ were found in $22 \%(24 / 108)$ of the samples from this group. Normal same day FPG and two hour PP-PG occurred in the same child in only $10 \%$ of paired PG. The unreliable patient urine tests, the inconsistent correlation between UG and PG, and the variability of an individual's controi as reflected by different test results indicate that little correlatior exists between these criteria of control and normal metabolism.

77 FAVORABLE LONG-TERM MANAGEMENT OF VITAMIN BI2 579 RESPONSIVE METHYLMALONIC ACIDEMLA (MM-emia). Grant Morrow III and H. Eugene Evans. The Univ. of Arizona Depts. of Ped. and Psychiatry, Tucson, Arizona. Med. Ctr, Depts. of Ped. and Psychiatry, Tucson, Arizona.
M.G. is a 4 year old male admitted at 11 months of age in

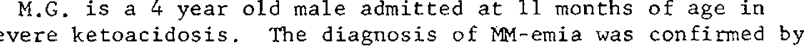
finding excessive urinary methylmalonate $(350-450 \mathrm{mg} /$ day) and defective in vitro enzyme activity. Hydroxycobalamin

(1 mg/week I.M.) plus a low protein diet (0.9-1.2 grams $/ \mathrm{kg} /$ day) have maintained methylmalonate excretion at less than $150 \mathrm{mg} /$ day and permitted adequate physical growth. His height and weight are in the 5 th percentile whereas his head size has been in the 50 th percentile. After 2 years of therapy, the generalized osteoporosis noted originally has resolved. Several febrile illnesses have occurred without development of ketoacidosis.

Psychological evaluations at 3 and $310 / 12$ years revealed normal mental development on the Stanford-Binet Intelligence scale. Perceptual, visual-motor coordination and psycholinguistic abilities were within normal values according to the BeeryVMI and Illinois psycholinguistic evaluation tests. There is no evidence of perceptual or cognitive deficits.

Treatment in a patient with vitamin $B_{12}$ responsive MM-emia can result in nomal physical and intellectual development.
580 THE EFFECT OF GLUCOSE UPON PLASMA ALANINE, MUSCLE PROBORN GUINEA PIG. Edward S. Ogat Jean Harrah, Malcolm A. Holliday. Univ. of California San Francisco, Dept. of Ped. and Cardiovascular Research Institute.

During starvation in adult mammals, net muscle protein catabolism provides alanine for gluconeogenesis. Giving glucose suppresses net protein catabolism - primarily muscle. Because starvation or glucose infusion occurs in $i 11$ newborns, we compared the effects of infusing glucose to continued starvation in newborn guinea pigs fasted either 30 or 96 hours after birth. After a 96 hour fast, a 12 hour glucose infusion increased plasma glucose and liver glycogen. Infusing glucose suppressed plasma alanine and urea production $(20.1 \pm 5.9 \mathrm{vs} .36 .5 \pm 9.0 \mathrm{mg} / 12$ hours $/ 100 \mathrm{gm})$. After 30 hours starvation, urea production was low and giving glucose did not suppress it $(16.5+5.9$ vs. $18.0+3.1 \mathrm{mg} / 12$ hours $/ 100 \mathrm{gm})$ or plasma alanine. Thus, the 96 hour fast resulted in increased net muscle protein catabolism and alanine mobilization for gluconeogenesis, which a glucose infusion suppressed. However, a 30 hour fast did not. To determine if this was due to increased protein synthesis or decreased protein catabolism, we determined fractional muscle protein synthesis rate using a ${ }^{34} \mathrm{C}$ leucine incorporation technique. After 96 hours starvation, infusing glucose did not modify fractional muscle protein synthesis rate $(18.5 \pm$ 5.2 vs. $19.0+5.0 \% / 24$ hours). The observation that glucose infusion reduced net muscle protein catabolism but did not stimulate synthesis rate may be due to expended substrate reserve in this usually rapid-growing animal.

\section{1}

EFFECTS OF ISOLEUCINE CONCENTRATION IN GROWTH MEDIA ON THE SELECTION OF HUMAN FIBROBLAST CELI LINES WITH ALTERED ISOLFUCINE UPTAKE. Elaine F. Otto and Richard E. Hillman, Washington Lniv. Sch. Med St. Louis Children's Hosp., Dept . of Ped., St . Louis, Mo.

We previously described two fibroblast cell lines with altered isoleucine uptake. To study the culture conditions necessary for selection of cell lines with altered transport systems, fibroblasts cultured from a patient with a defect in isoleucine metabolism were grown in a basic medium containing four different concentrations of isoleucine. Sixteen sub-cultures (four at each isoleucine level) were passed serially and isoleucine transport studied longitudinally with respect to cell passage. Although altered isoleucine uptake and altered cell morphology were more common at higher isoleucine concentrations the appearance of cell lines with altered isoleucine uptake seemed more related to the rate of cell growth than to the actual isoleucine concentration in the medium. Apparently stable cell lines have been selected which lack the very low affinity-high capacity transport systems seen in normal cell lines, and other lines have been selected which lack the high affinity-moderate capacity transport system seen in normal cell cultures. These latter cell lines allow kinetic analysis of a very high affinity-low capacity transport system whose presence in normal cell lines was suggested by previous studies, but whose kinetics could not be studied in the presence of the other transport systems. Thus growth of cells in selective media can produce changes in amino acid transport, some of which can be exploited to characterize the complexities of multiple transport systems.

582 REYE'S SYNDROME: SIMILARITY OF MITOCHONDRIAL CHANGES IN LIVER AND BRAIN. John C. Partin, Jacqueline S. Partin, William K. Schubert, and Robert L. McLaurin The Children's Hosp. Research Fndn. Dept. of Ped and Div of Neurosurg. Dept. of Surg., Univ. of Cincinnati Med. Sch., Cincinnati, Ohio.

Craniectomies to relieve cerebral hypertension were performed in a child suffering from severe Reye's Syndrome, 48 and 72 hours after the onset of encephalopathy. Cerebral biopsies were obtained for electron microscopy. The child survived and recovered good brain function. The biopsy findings, therefore, appear to reflect the organelle pathology of the brain at a scvere yet reversible stage in the disease process.

The cardinal ultrastructural changes in the brain in Reve's syndrome are universal injury on neuron mitochondria, extensive myelin bleb formation, moderate astrocyte swelling and deglycogenation, and the presence of a wide variety of neuronal intranuclear inclusions. The mitochondrial injury consists of matrix disruption with moderate but not massive matrix swelling, which resembles the unique mitochondrial changes seen in the liver parenchyma in Reye's Syndrome. The brain organelle pathology does not resemble that seen in other human hepatic encephalopathies or in experinental ammonia intoxication in primates.

The ultrastructural changes indicate a specific cytotoxic injury to mitochondria of neurons like that in the hepatocytes, and this injury seems to occur simultaneously in both organs.

Speculation: The same agent may be injuring both organs simultaneously. Supported in part by U.S.P.H.S. Grant RR-00123. 
583

METABOLIC STUDIES OF A FAMILY WITH PROBABLE GLUTAMATE FORMIMINOTRANSFERASE DEFICIENCY Hansen iatrics, University of British Columbia, Vancouver, B.C. and Rikshospitalet, Oslo, Norway. (Intr. by S. Israels)

We will describe two siblings who excrete massive amounts (up to $3.89 \mathrm{~m}$ moles $/ 24 \mathrm{hr}$. ) of $\mathrm{N}-\mathrm{f}$ ormiminoglutamic acid (FIGLU) in their urine. This unusual compound was isolated from urine, purified, and firmly identified as FIGLU by combined gas chromatographymass spectrometry. The patients presumably have a deficiency in activity of the hepatic enzyme, glutamate formiminotransfer ase. Our patients had normal serum folate levels, had no hematological abnormalities, were not mentally retarded, and had evidence of mild cerebral dysfunction. Speech development had been delayed in both children, the mother had also had delayed speech, the remaining younger sister had a small hand-strabismus and a maternal aunt is profoundly mentally retarded. None of these relatives excrete FIGLU. We doubt, therefore, whether there is any connection between the FIGLU excretion and the mild brain dysfunction in our patients. Administration of pharmacological doses of folic or folinic acid produced a decrease in excretion of FIGLU.

This disorder may represent a further example of metabolic exror which does not result either in accumulation of a toxic metabolite or in failure to synthesize a crucial metabolite, and which, therefore, produces no clinical disease syndrome.
586 RESPONSES IN CHILDHOOD CHEMICALSE (G) AND INSULIN (IRI) RESPONSES IN CHIDHOOD CHEMICAL DIABETES (CD). Arlan
$\mathrm{L}$. Rosenbloom, Lawrence $A$. Wheeler, Univ. of Florida Col. of Med., Dept. of Ped., Gainesville.

Plasma $G$ and IRI were analyzed during 4 hour oral glucose tolerance testing (OGTT) in 63 patients with abnormal OGTT and $93 \mathrm{SA}$ matched controls. Control tests (101) showed significant positive correlation of fasting and 4 hour post-ingestion $G$ with SA ( $p<.001$ ) but mean area under the $G$ curves did not vary between SA groups I (18-60 mos) II (70-131 mos) or III(132+ mos). The absence of differences at other sampling times permits uniforto diagnostic criteria. IRI was positively correlated with $S A$ at all testing times and mean levels differed significantly between each SA group at every sampling time; mean areas under the IRI curve also differed significantly between SA groups as did mean IRI/G area

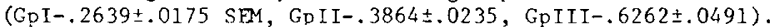
Patient tests (110) were separated into normal (N), borderline(B) and chemical diabetic(C) for each SA group. IRI means exceded controls for each test type in each SA group at all times; onefourth of these differences were significant. IRI also increased within each SA group from $N$ to $B$ to $C$ tests. Mean IRI areas and IRI/G areas were higher than controls; difference was greatest with the most abnormal(C) test type in each SA group. A subgroup oped overt diabetes in $1-3$ years. In contrast to the apparent relative $I R I$ inefficiency with maturation and with $C D$, they had exceptional responsiveness to their low levels. Variable involvement of $\alpha$ - and $\beta$-cells or changing receptor affinity might explain these paradoxical observations.
584 C-PEPTIDE SECRETION IN INSULIN REQUIRING JUVENILE DIABETES, R. S. Pildes, L. Grajwer, D. Horwitz and A. H. Rubenstein, Univ. of II1., Univ. of Chicago and Cook County Hosp. Depts. of Ped, and Med. Chicago, lllinois. Proinsulin is converted to insulin and $C$-peptide in the pancreatic B-cells; the latter two peptides are secreted in equimolar concentrations. Thus, measurements of $C$-peptide immunoreactivity (CPR) may provide a means of assessing residual pancreatic function in insulin treated diabetes. Thirty five pts. with a mean (+S.E.) age of $13.4+.6$ yrs who had juvenile diabetes mellitus for a mean of $4.8 \pm .3$ yrs were included in this study. Glucose and CPR were measured in the fasting state and $1 \mathrm{hr}$. after $\mathrm{lgm} / \mathrm{kg}$ (maximum, 50gms) of oral glucose. Pts. were assigned to adequate or poor control groups prior to knowledge of CPR values. 25 of the 35 (71\%) pts. had evidence of endogenous B-cell function, i.e. $C P R>.5 \mathrm{ng} / \mathrm{ml}$. Six had values $>2$. $0 \mathrm{ng} / \mathrm{ml}, 7$, between $1.1-2.0 \mathrm{ng} / \mathrm{ml}$ and 12 , between $0.51-1.0 \mathrm{ng} / \mathrm{ml}$. A significantly $(p<.05)$ greater no. of pts. With diabetes of $<5$ yrs. duration $(19 / 21)$ had CPR levels $>.5 \mathrm{ng} / \mathrm{ml}$ than those with diabetes $>5$ yrs duration $(6 / 14)$. Only $1 \mathrm{pt}$. Showed a rise in CPR after the glucose load. No significant correlations were seen between $C P R$ values and fasting plasma glucose, $\triangle$ glucose, dose of insulin or the no. of hospitalizations. Although growth hormone (HGH) values were higher in pts. in the poor control group, there was no correlation between $\mathrm{HGH}$ and $C P R$. Pts. With $C P R>2.0 n g / m l$ showed adequate control, those with $C P R<2$. Ong $/ \mathrm{ml}$ fell into both adequate and poor control groups. Thus, while high CPR may facilitate good control, low CPR and hence absent $B$-cell reserve need not be associated with poor control.

PERITONEAL DIALYSIS IN THE INITIAL TREATMENT OF

585 MAPLE SYRUP URINE OISEASE (MSUD). Kenneth R. Rettig, Ange lo M. DiGeorge, Iraj Rezvani, Alan B. Gruskin and Linda Hiner, St. Christopher's hospital for Children, Dept. of Ped., Temple Univ. Sch. Med., Philadelphia, Pa.

Classical MSUD was diagnosed in three infants at 9,14 and 10 days of age. In each instance the infant was symptomatic and the initial treatment consisted of intravenous fluid therapy and attempts to feed a formula free of branched-chain amino acids (BCAA). Inspite of these measures, rapid clinical deterioration occurred and there was no significant reduction in the markedly elevated levels of branched-chain amino acids. Peritoneal dialysis lasting 12-24 hrs. was performed at 4, 1 and 2 days after diagnosis, respectively (Table). Marked clinical improvement and significant reduction of serum BCAA occurred in each instance. In one infant, an exchange transfusion following the dialysis failed to further reduce the levels of BCAA. These data augment the scant published reports and clearly demonstrate the value of peritoneal dialysis in early management of MSUD. Although this therapeutic maneuver has been utilized in the past only for desperately ill infants, it should be seriously considered for all infants with markedly elevated levels of BCAA even in the absence of advanced symp toma to logy.

\begin{tabular}{|c|c|c|c|c|}
\hline & & Serum Leuc & (mg.\%) & \\
\hline atier & $\begin{array}{l}\text { Pre- } \\
\text { Dialysis }\end{array}$ & $\begin{array}{l}\text { Post- } \\
\text { Dialysis }\end{array}$ & $\begin{array}{l}\text { Pre- } \\
\text { Exchange }\end{array}$ & $\begin{array}{l}\text { Post- } \\
\text { Exchange }\end{array}$ \\
\hline 7 & 26.6 & 76.6 & $\cdots$ & - \\
\hline 2 & 45 & 23.0 & 23.0 & 22.1 \\
\hline 3 & 42.6 & 27.0 & -- & $-\infty$ \\
\hline
\end{tabular}

BETA-METHYLCROTONIC ACIDURIA, P-OHPHENYLLACTIC ACID-

587 URIA IN A PATIENT WITH LACTIC ACIDOSIS. K. Roth, R. Cohn, P. Dodd, and S. Sega1. U. Pa. Sch. Med., Children's Hosp. of Phila., Phila., $\mathrm{Pa}$

To date, beta-methylcrotonic acid(MCA) has been demonstrated in the urine of three patients. This compound is an intermediate in the degradative pathway of leucine, and is further metabolized by a biotin-dependent carboxylase. One of these patients was also found to excrete $p$-OHphenyllactic acid(PHPLA).

We have seen a patient with transposition of the great arteries in whom we documented a severe lactic acidosis (lactate $14.9 \mathrm{mM}$ pyruvate $0.6 \mathrm{mM}, \mathrm{pH} 7.15)$. Despite the fact that a satisfactory atrial balloon septostomy was performed, the anion gap acidosis persisted. Gas liquid chromatographic analysis of urine confirmed high lactate excretion and suggested the presence of several un usual organic acids not found in normal infant controls. Mass spectral analysis confirmed the identity of two major peaks as MCA and PHPLA.

The presence of MCA in urine has been considered pathognomonic of a defect in $\mathrm{CO}_{2}$ fixation in the leucine pathway. Our patient amino acids and intravascular coagulopathy. With such profound liver dysfunction and lactic acidosis, selective derangement of certain enzyme functions is not unlikely. Our data suggest that the presence of MCA in urine may not be diagnostic of an inborn error but may be encountered in certain acquired states. Further studies are underway to estimate the activity of the carboxylase in tissues from this patient, in order to clarify the discrepant data in the literature. WITH DIABETES MELLYTUS, Albert $J$, Schneider, Thelma R. Schneider, and Richard J. Doisy (Intro, by Frank A. Oski) S.U.N.Y. Upstate Med. Ctr., Dept. of Peds, Syracuse,N.Y. Our experiences with 3 established diabetics and 6 virgin diabetics are summarized in the table. M.P. had been insulin resistant (150-3000 U/d) for 18 months, but control was achieved with IV insulin. D.S. was in moderate ketoacidosis. J.P. presented with the double handicap of hypoglycemia and ketoacidosis. The new cases had 2-hour glucose tolerance tests followed by IV insulin.

\begin{tabular}{|c|c|c|c|c|}
\hline Pt. & $\begin{array}{l}\text { Age } \\
\text { (Yrs) }\end{array}$ & $\begin{array}{l}\quad \text { Infusion } \\
\text { Glucose } \\
(\mathrm{gm} / \mathrm{kg} / \mathrm{h})\end{array}$ & $\begin{array}{l}\text { Rate } \\
\text { Insul in } \\
(\mathrm{U} / \mathrm{kg} / \mathrm{h})\end{array}$ & $\begin{array}{l}\text { Rate of fall in Blood } \\
\text { Sugar }(\mathrm{mg} / \mathrm{dl} / \mathrm{h})\end{array}$ \\
\hline M.P. & 16 & $\begin{array}{l}.3 \\
.3 \\
\end{array}$ & $\begin{array}{c}<.2 \\
.2-.24 \\
\end{array}$ & $\begin{array}{r}0 \\
50\end{array}$ \\
\hline D.S. & 15 & $\begin{array}{r}0 \\
.1\end{array}$ & $\begin{array}{l}.09 \\
.07 \\
\end{array}$ & $\begin{array}{r}110 \\
26 \\
\end{array}$ \\
\hline J.P. & 5 & .3 & .08 & 530 \\
\hline $\begin{array}{l}\text { New } \\
\text { Age } 2 \\
\end{array}$ & $\begin{array}{l}\text { Diagno } \\
11 \text { yrs }\end{array}$ & $\begin{array}{c}\text { Cases } \\
0\end{array}$ & .05 & $\begin{array}{l}80-240 \text { first hour } \\
40-135 \text { thereaf ter }\end{array}$ \\
\hline
\end{tabular}

Several points deserve emphasis: 1) attempts to induce symptomatic hypoglycemia were barely successful; 2) subcutaneous insulin should be given at or prior to discontinuation of the IV insulin; 3) the almost linear drop in blood sugar with or without IV glucose permits precise control of therapy without hazard to the patient; 4) IV insulin was given either in a Metriset or by pump without the use of albumin. 
GAMMA-GLUTAMYL TRANS PEPTIDASE (GGTPase):INVESTIGATIONS

589 IN NORMALS AND PATIENTS WITH INBORN ERRORS OF SULFUR METABOLISM. J.D.Schulman, A.D.Patrick, S.I.Goodman, F.Tietze, and J.Butler. NICHD and NIAMDD, NIH, Bethesda; Inst. of Child Health, London, England; and Univ. Colorado Sch. Med., Dept. of Pediatrics, Denver.

We report here selected properties of human GGTPase in leukocytes, cultured fibroblasts, liver, kidney, spleen, placenta, and urinary protein from normal and mutant individuals. GGTPase (Meis ter, Science 180:33,1973)may function as an enzymatic mediator of amino acid transport associated with the"glutathione cycle!"Cystine is the most potent amino acid activator of human GGTPase (Km 1.3$1.6 \mathrm{mM}$; activity stimulation at $2 \mathrm{mM} 140-340 \%$; leukocytes-placenta resp.). GGTPase is present in lysosomal fractions of human leukocytes, and concentrated in rat liver lysosomes prepared by Triton WR-1339 flotation; lysosomal and whole cell GGTPase has pH optimum 8-9 with no acid pH component. Sucrose density gradient profiles, DEAE-cellulose elution patterns, kinetic and heat inactivation studies revealed no abnormalities in lysosomal or whole cell GGTPase in cystinosis (cystine or gly-gly acceptors). Assay of the enzyme in a cystinuric kidney, and in urine from il cystinurics revealed normal activity (same acceptors). A patient has been discovered with massive glutathionuria ( $\mathrm{gm} / 24 \mathrm{hrs}$ ) and extreme reduction of GGTPase in cultured fibroblasts ( $<5 \%$ control mean) with cystine, gly-gly, or methionine acceptors, and $\gamma$-glutamyl-nitroanilide substrate. Ongoing studies of this patient and derived cells and tissues should permit for the first time a critical evaluation of the possible role of GGTPase in human amino acid transport and its importance in glutathione metabolism.

HYPERALIMENTATION AND PERITONEAL DIALYYSIS DURING 590 ACUTE METABOLIC DECOMPENSATION IN MAPLE SYRUP URINE DISEASE Vivian E. Shih, John T. Herrin, Arline M. Erickson, Neuroloay

In classical maple syrup urine disease, death usually results from irreversible fluid-electrolyte and amino acid (AA) imbalance. Proper management of such crises is crucial to survival. Our Datient developed marked keto-acidosis, seizure and coma at 18 months, despite prior good control on synthetic diet since neonatal period. Vigorous fluid and electrolyte therapy corrected the ketoacidosis, but severe hyperleucinemia and coma persisted. Peritoneal dialysis (PD) resulted in rapid fall in blood leucine and prompt improvement of neuroloqical status. However, complications and metabolic relapse necessitated a second dialysis. Hyperalimentation (HyAl) with glucose, fat emulsion and special AA mixture was required for 5 weeks. The AA formula provided $1-3 \mathrm{Gm} A A / \mathrm{kg} / 24^{\circ}$, specifically excluding branched-chain $A A^{\prime} \mathrm{s}$. Supplemental alanine at $100-200 \mathrm{mg} / \mathrm{kg} / 24^{\circ}$ was needed to restore markedly depressed alanine levels to normal. Leucine, isoleucine and valine supplements were added to maintain normal blood leveis. $P D$ and HyAl were important contributing factors in this patient's recovery from nrolonged metabolic decompensation. We suggest that HyAl tailored to the special nutritional requirements be instituted in any patient with a metabolic disorder, who is intolerant to oral feedings, particularly in catabolic states.

$\mathbf{5 9 1}$ EFFECTS OF PYRIDOXINE ON NEONATAL HYPOPHOSPHATASIA.

591 C.Sia, R.Wapnir, M.Soka l, R.G.Harper, S. Intizar \&.F. Lifshitz, Dept. of Ped., North Shore Univ. Hosp., Manhasset, \& CornelT Univ. Med. Col., N.Y., N.Y.

In vivo and in vitro effects of pyridoxine (PY) in a newborn with hypophosphatasia and his parents were studied. Pyridoxal $\mathrm{PO}_{4}$ $(P P)$ is a co-factor of the enzyme 0 -phosphorylethanolamine phospho-lyase that catalyzes phosphoethanolamine (PEA). The patient had poor bone mineralization, deformities, low serum alkaline phosphatase (AP) levels (0-15 ImU), high urinary PEA excretion (162-318 McMole/day), seizures and respiratory distress. He was continuously treated with anticonvulsants,antibiotics and oral phosphate $(1-3 \mathrm{gm} /$ day). Parents were asymptomatic but showed increased urinary PEA excretion (184-316 McMole/day) with serum $A P$ levels of 20-35 ImU. The patient and his parents were given PY $10-200 \mathrm{mg} /$ day for up to 4 months. Gradual decrease in frequency and severity of the patient's seizures and increase in skull density were noted. Serum AP rose to 20 ImU with no decrease in urinary PEA excretion. The parents had $25-69 \%$ reduction of urinary
$P E A$.

The effect of PP in vitro was tested on the sera of the patient, his parents and controls before and after PY treatment. Prigr to therapy, neither the baby nor the parents had AP activity in the serum vS PEA. Activity was maximally stimulated at to-5 M PP. Controls required 10-3 M PP for a comparable effect. Addition of PP elicited no response when the AP substrate was pnitrophenol-PO . On the basis of these results, the possible beneficial effects of pyridoxine therapy on hypophosphatasia deserves further investigation.
ACCUMULATION ON INOSINE TRIPHOSPHATE IN RBC - A DOM-

592 INANTLY INHERITED BIOCHEMICAL TRAIT. Christian Soder, J. Frank Henderson and Ernest McCoy, Cancer Research Unit and Dept. of Ped., Univ. of Alberta, Edmonton, Alberta.

RBC from $5 \%$ of a normal population accumulate relatively high concentrations of inosine triphosphate (ITP) (>70 nmoles/ $10^{10} \mathrm{cells} / 2 \mathrm{hr}$ ) when incubated with $14 \mathrm{C}$ hypoxanthine. The exact pathway of synthesis of ITP and its role in RBC nucleotide metabolism have not been defined. However, ITP accumulation seems to be inversely related to the activity of a pyrophosphohydrolase. This report shows that the ability of RBC to accumulate ITP at high rates appears to be inherited in a dominant manner.

Nine index subjects detected during a screening program for inborn errors of purine metabolism had ITP accumulation in the range of 80 to 240 nmoles $/ 1010 \mathrm{RBC} / 2 \mathrm{hr}$. A total of 72 individuals in nine families of these index cases were studied, including three generations in three families and two generations in six families. Thirty-four family members accumulated $>70$ nmoles ITP $/ 10^{10} \mathrm{RBC}$ in $2 \mathrm{hr}$. At least one member in each generation of each family accumulated high amounts of ITP (71 to 260 nmoles $/ 10^{10} \mathrm{RBC} / 2 \mathrm{hr}$ ) compared to 7 to 61 nmoles ITP $/ 10^{10} \mathrm{RBC} /$ $2 \mathrm{hr}$ in those with usual rates of ITP accumulation. Pedigrees will be presented to show that in each family the ability to accumulate ITP at increased rates is inherited in a dominant manner. This biochemical trait should be useful in future genetic studies.
593 PLYTIENAATE METABOLISM IN SKIN FIBROBLASTS OF A Solomons, Department of Pediatrics, iniversity of Colorado Medical Center, Denver, Colo.

Skin fibroblasts from a 14 year-old female patient with Type I primary hyperoxaluria and severe calcium oxalate nephrolithiasis were incubated in vitro with U-C.-14 sodium glyoxalate for 3 days. Electrophoretic analysis of the cell pellet showed that conversion of glyoxalate to oxalate occurred in both the patient's fibroblasts and in normal cells which were similarly maintained as monolayer cultures. Under basal conditions, the patient's oxalate production expressed as $14-\mathrm{C}$ oxalate per cell was 2.3 fold greater than that of the control cells $(p<0.21)$. Exposure to sodium glycollate $\left(10^{-4} \mathrm{M}\right)$ increased oxalate production in the patient's and the normal cell lines by 2.0 and 1.8-fold respectively. These results are consistent with the proposed genetic block in Type I oxalosis, and the excessive production of oxalate induced in normal humans and rats when ethylene glycol or glycollic acid is ingested. The fibroblast model offers a means of identifying patients and possibly the carriers of this usually-fatal recessive disorder and may become useful for intrauterine diagnosis. 
595 A PROPOSEO ROLE FOR GALACTOSE IN NEONATAL GLUCOSE Almorris Lynch, and Ronald A. Chez, (Intr. by C.U. Lowe) Lab. Biomed. Sci. and Preg. Res. Br. NICHD, NIH, Bethesda, Maryland.

While galactose is present in large amounts in infant diet, $i$ ts role in carbohydrate regulation is poorly understood. We tested the effect of galactose on glycogen metabolism and found a different mode of activation of glycogen synthetase (GS) in isolated perfused near-term monkey and adult rat liver. In adult fasted rat liver, GS in the active form (\% I) was proportional to perfusate glucose $(100-500 \mathrm{mg} \%)$. Over this glucose range, added galactose increased \% $\mathrm{I}$ by 1.5 -fold. This maximal response was elicited by galactose $\geq 75 \mathrm{mg} \%$. Insulin did not modify the effects of glucose or galactose. In nearterm newborn rhesus monkey liver, \% I was 1 low $[4 \% \pm 1(S E M)]$ and not proportional to perfusate glucose up to $600 \mathrm{mg} \%$. Addition of either insulin $\left(10^{-7} \mathrm{M}\right)$ or galactose $(300 \mathrm{mg} \%$ ) to glucose (300 $\mathrm{mg} \%$ ) increased $\% 12$-fold $(7.8 \pm 2,8 \pm 1)$. The combination of insulin, galactose, and glucose increased \% by $10-$ fold $(40 \pm 5)$. Phosphorylase was consistently low in both the rat and the monkey series.

Since neonatal hypoglycemia is generally associated with depletion of liver glycogen, and since galactose but not glucose increased GS in the neonate, we propose that galactose may be uniquely important for neonatal liver glycogen synthesis and secondarily for glucose homeostasis.

596 ADRENERGIC MODULATION OF HORMONAL RESPONSES TO ARGI NINE IN NORMAL ANO DIABETIC CHILDREN. MarK A. Sperling and S. Voina. UCLA Sch. of Med., Harbor Gen. Hosp., Dept. of Ped., Torrance, California.

Plasma growth hormone (GH) and glucagon (GLU) concentrations are both inappropriately elevated in juvenile diabetics (JDM). To evaluate the potential contribution of the adrenergic nervous system in mediating this hypersecretion, plasma GH and GLU responses were measured by specific radioimmunoassay, in JDM and control subjects following intravenous arginine (ARG), $0.5 \mathrm{Gm} / \mathrm{kg}$, given alone, or in conjunction with a or $\beta$ adrenergic blockade. Each subject had all 3 tests, and the blocking agent (phentolamine 2-4 mg plus $0.4 \mathrm{mg} / \mathrm{min}$ or propranolol $3-5 \mathrm{mg}$ plus 0.08 $\mathrm{mg} / \mathrm{min}$ ) was infused for 1 hour commencing 15 min prior to the ARG infusion. Basal GH levels were not significantly different in the 2 groups, but the peak GH response of $34 \pm 7 \mathrm{ng} / \mathrm{ml}$ (mean \pm SEM) was significantly greater in JOM than controls, and suppressed to $10 \pm 4 \mathrm{ng} / \mathrm{ml}$ with a blockade $(p<0.05)$; $B$ blockade had no effect. Basal GLU concentrations in JDM $(107 \pm 17 \mathrm{pg} / \mathrm{ml})$ were not significantly different from controls $(147 \pm 31 \mathrm{pg} / \mathrm{ml})$ despite the fasting hyperglycemia of the former $(212 \pm 45 \mathrm{mg} / 100 \mathrm{ml}$ vs. $77 \pm 5 \mathrm{mg} / 100 \mathrm{ml})$. Neither basal nor maximally stimulated GLU concentrations $(368 \pm 26 \mathrm{pg} / \mathrm{ml})$ were significantly affected by $\alpha$ or $\beta$ blockade in JDM or controls, al though the glycemic response was significantiy augmented by each blocker in both groups. The results suggest that 1) sympathetic overactivity via $\alpha$ receptors contributes to the GH hypersecretion in JDM, 2) neither the $\alpha$ nor 8 adrenergic receptor alone modulates basal or ARG stimulated GLU secretion in normal and diabetic children.

IN VIVO ANALYSIS OF BONE MINERAL CONTENT (BMC) IN 597 FULL TERM AND PRETERM INFANTS. Jean J. Steichen, Arun Pramanik and Reginald C. Tsang, Univ. of Cincinnati, Col. of Med., Dept. of Ped.

Photon absorptiometry is a precise $(2 \%)$ and accurate method for in vivo bone mineral analysis (5\% error compared to bone ash analysis) far superior to previous photodensitometric techniques (20\%-70\% error). There have been no reports of in vivo BMC analyses in infancy. BMC was measured at $1 / 3$ and $1 / 10$ of the distance from the dista1 left radius in 25 full term and 11 preterm 32-36 weeks gestation infants with the Norland Cameron Bone Mineral Analyzer adapted for infants. For full term infants (age day 3 ) at the $1 / 3$ site, BMC was $0.144 \pm 0.055 \mathrm{gm} / \mathrm{cm}$ (mean $\pm \mathrm{SD}$ ), bone width (BW) was $0.649 \pm 0.43 \mathrm{~cm}$ and $\mathrm{BMC} / \mathrm{BW}$ (the best measurement for bone density) was $0.259 \pm 0.051 \mathrm{gm} / \mathrm{cm}^{2}$. There was no difference between black and white infants. BMC/BW was significantly lower in female $(0.239 \pm 0.059)$ than in male infants $(0.28 \pm 0.048 \mathrm{t}$ test, $p=0.05)$. BMC measurements at the $1 / 10$ site were undetectable in $90 \%$. In preterm infants, (ages 3 days -3 weeks) at the $1 / 3$ site $\mathrm{BMC}$ was $0.094 \pm 0.04$ and $\mathrm{BW}$ was $0.439 \pm_{0} 0.254 ; \mathrm{BMC} / \mathrm{BW}$ was $0.227 \pm 0.024$ $\mathrm{gm} / \mathrm{cm}^{2}$, significantly lower than that for term infants ( $t$ test, $p<0.05)$. Measurements of BMC were undetectable at the $1 / 10$ site in $95 \%$. In 4 additiona 1 preterm infants $28-34$ weeks gestation $=4$ weeks of age BMC was unmeasureable even at the $1 / 3$ site. Photon absorptiometry is a convenient noninvasive quantitative method for in vivo neonatal bone mineral content analysis, and offers the potential for in vivo study of bone growth in the developing infant.
598 NEONATAL HYPOGLUCOSEMIA ASSOCIATED WITH INTRAPARTUM FETAL HYPERGLUCOSEMTA IN DIABETIC PREGNANCIES. J. Sumers, P. Bailey, G. Cassady. Division of Perinatal Medicine, Univ. of Ala. in B'ham., B'ham., Ala.

Serum glucose levels were obtained on umbilical venous blood (UV glu) from 81 living products of diabetic pregnancies as an index of late intrapartum fetal glucosemia. Severity of diabetes was class $A$ in 67 , class $B$ in 8 , class $C$ in 3 and class $D$ in 3 . Neonatal hypoglucosemia (serum glucose $<30 \mathrm{mg} \%$ ) occurred in $15 / 81$ infants (19\%).

Fetal hyperglucosemia (UV glu > $150 \mathrm{mg} \%$ ) was associated with subsequent neonatal hypoglucosemia. In $8 / 17(47 \%)$ with UV glu > $150 \mathrm{mg} \%$ neonatal hypoglucosemia was found; UV glu $\leq 150 \mathrm{mg} \%$ was associated with neonatal hypoglucosemia in only $7 / \overline{6} 4$ cases (11\%) $(\mathrm{p}<.001)$.

These data indicate the need for prevention of intrapartum maternal (and consequent fetal) hyperglucosemia in diabetic pregnancies in order to diminish the risk of neonatal hypoglucosemia. The clinical utility of UV glu $>150 \mathrm{mg} \%$ in predicting a greater than 4 fold increased risk of neonatal hypoglucosemia is emphasized.

ACID-BASE AND EIECTROLYTE BALANCE IN INFANTSS OF DIABETIC MOTHERS. VAGINAI DELTVERY VERSUS CAESAREAN SECTION. Bertil Thalme, Karin Edström, Jlf Broberger, Lars Engström \& Gïnter Kretzschmar, Dept.of Ped.\& Obst. Karolinska Hospital, Stockholm, Sweden.

The acid-base and electrolyte balance of 30 women was studied at delivery and in their infants during the first $48 \mathrm{hrs} .18$ women were diabetics, 10 of these were delivered vaginally (DM ) and 8 by elective caesarean section ( $\mathrm{DM}_{\mathrm{cs}}$ ). 12 healthy women waere vaginally delivered (HM). The infants of diabetic mothers received active infusion therapy.

At birth the DM and their infants (IDM ${ }_{\text {Naf }}$ ) had a more pronounced metabolic acfiosis than the DM and veteir babies (IDM ). HM and their infants (IHM ).

vepter birth no significant acid-base and electrolyte balance between IDM and IDM. The plasma potassium level remained lower in IDM Zf:

The study stresses the importance of adequate management of diabetes in pregnancy in combination with active intravenous therapy during delivery and to the infant in the immediate neonatal period. The slightly larger metabolic acidosis seen in combination with vadinal delivery suggests that this mode of delivery should not be attempted uncritically in diabetic women.

60 CYSTINE DEPLETION OF CYSTINOTIC FIBROBLAST

600 BY CYSTEAMINE (MERCAPTOETHYLAMINE, MEA). Oshima, william Rhead, and Jerry A. Schneider. Univ. of Calif., San Diego, Sch. of Med., Dept. Ped., La Jolla, $\mathrm{Ca} .92037$

Fibroblasts cultured from patients with nephropathic cystinosis contain over 100 times more free (nonprotein) cystine than control fibroblasts. Methods to deplete these cells of free-cystine include growth in cystinefree medium and treatment with ascorbic acid, which require about $72 \mathrm{hrs}$. for a 50-70\% reduction, and dithiothreitol which requires toxic concentrations to effect $100 \%$ depletion. MEA removes all the freecystine from cystinotic fibroblasts under conditions which do not alter the morphology of these cells (but do decrease their cloning efficiency). In normal medium 1.0mM MEA removes $90 \%$ of the cystine from cystinotic cells in 2 hrs. and $0.1 \mathrm{mM}$ removes $60 \%$ in $24 \mathrm{hrs}$. In a cystine-free medium, 1.0mM MEA removes $100 \%$ of the free-cystine from cystinotic fibroblasts in $1 \mathrm{hr}$. and $0.1 \mathrm{mM}$ removes $90 \%$ in $2 \mathrm{hrs}$. Cystamine, the disulfide of MEA, is equally effective at these concentrations. After labelling cystinotic fibroblasts with $35 \mathrm{~s}$ cystine and then treating them with $1.0 \mathrm{mM}$ MEA for $1 \mathrm{hr}$. , no $35 \mathrm{~S}$ was found as cystine. Other $\mathrm{S}$ metabolites were unchanged and no $35 \mathrm{~S}$ was found as cystine-cysteamine mixed disulfide. 

601 THE QUANTITATIVE DETERMINATION OF SHORT-CHAIN $\left(\mathrm{C}_{2}-\right.$ ANALYSIS OF para-BROMOPHENACYL ESTERS. Richard M. Thompson and Barbara G. Belanger (Intr. by Ira K. Brandt), Dept. of Ped., Indiana Univ. School of Med., Indianapolis:

In order to more easily detect short-chain organic acidopathies such as propionic acidemia, it was necessary to prepare a stable, quantitatively-extractable derivative which had properties suitable for GC and GC-MS analysis. The p-bromophenacyl ester derivative was chosen because it coula be formed in aqueous solution at slightly acidic pH. A small aliquot of serum $(0.5-1 \mathrm{ml})$ or urine $(2 \mathrm{ml})$ is adjusted to $\mathrm{pH} 5-6$ with dil. $\mathrm{H}_{2} \mathrm{SO}_{4}$ and diluted with two volumes of $95 \%$ ethanol (the serum protein ppt. is separated by centrifugation at 10,000 rpm). An excess $(25-50 \mathrm{mg})$ of p-bromophenacyl bromide is added and the resulting solution heated to $90-100^{\circ} \mathrm{C}$ for $\mathrm{l} \mathrm{hr}$. The ethanol is removed in vacuo and the derivatives extracted into ethyl acetate. GC analysis is carried out on an SE-30 column (temp. prog. $2^{\circ} \mathrm{C} / \mathrm{min}$ from $100^{\circ} \mathrm{C}$. Caproic acid $\left(\mathrm{C}_{6}\right)$ can be added as an internal standard prior to the $\mathrm{pH}$ adjustment. The esters of $\mathrm{C}_{2}, \mathrm{C}_{3}, \mathrm{C}_{4}$, iso- $\mathrm{C}_{4}, \mathrm{C}_{5}$, and iso $\mathrm{C}_{5}$ are clearly separated by GC. The mass spectra contain intense ions at $\underline{m} / \underline{e} 183$ and 185 due to the pbromobenzoyl group and which would be suitable for ion monitoring. An acylium ion characteristic of each short-chain acid is also observed at lower mass: $C_{2}$ (m/e 43$), c_{3}$ ( $\left.\underline{m} / \underline{e} 57\right), C_{4}$ and iso- $C_{4}$ ( $\underline{m} /$ e 71$)$, and $C_{5}$ and iso- $C_{5}^{-}(\underline{m} / \underline{e}$ 85). The technique is useful at concentrations at least down to the millimolar level.

602 CONVERSION OF PHENYLPYRUVIC ACID (PPA) TO PHENYLACETIC ACID (PA) DURING EXTRACTION OF URINARY ORGANIC ACIDS FROM PHENYLKETONURICS. Richard $M$. Thompson, Barbara G. Belanger, Rebecca S. Wappner, and Ira K. Brandt, Dept. of Pediatrics, Indiana Univ. School of Med. Indianapolis.

Organic acid metabolites are usually separated from physiologic fluids either by solvent extraction or ion-exchange chromatography prior to GC and GC-MS analysis. Extraction of acidified, salt-saturated urine or deproteinized serum is preferable for the rapid screening of large numbers of specimens and is sufficiently quantitative for the analysis of aromatic acids. Indeed, routine screening is carried out in our laboratory by ether extraction of $\mathrm{NaCl}-s a t u r a t e d$, acidified ( $\mathrm{pH} \mathrm{O}-\mathrm{l}$ ) urine or serum, followed by GC analysis of the TMS-derivatives on $9^{\prime}-10 \%$ OV-17 columns. Free $\alpha$-ketoacids, however, are normally not quantitatively recovered by solvent extraction. Therefore, oxime or 0 alkyloxime derivatives are prepared prior to extraction in order to improve recoveries. TheTMS-ether-TMS-ester derivatives of these oximes have excellent GC and GC-MS properties. Using these principles, we have shown that one $\alpha$-ketoacid, PPA, is at least partially decarboxylated and converted to PA by acidification and extraction of urine from PKU's. This conversion is eliminated by oximation prior to extraction. The PA/PPA ratio without oximation ranged from .36 to 3.1; with oximation .04 to .24 . In aqueous solution PPA ( $\mathrm{Na}$ salt, no PA present) was quantitatively converted to PA within minutes by acidification to $\mathrm{pH} 1$.

A SCREENING METHOD FOR DETECTING HYPERLIPIDEMIA (HL)

603 SURFACE TENSION (ST) MEASUREMENT OF THE SERUM LIPID EXTRACT ( $L$ ext.) Chandra M. Tiwary, John Riedler, Dinesh 0. Shah and Kashinath D. PatiT. (Intr. by C. Anqle.) Univ. of Nebr. Med. Ctr., Dept. Ped. and Statistics, Omaha, and Univ. Fla., Dept. Chemical Enqineering, Gainesville.

Identification of $\mathrm{HL}$ children is a prerequisite for prevention of premature atherosclerosis. Therefore a quick, convenient and reliable method for screenina such subjects is needed. For this purpose we describe a physical method based on surface tension lowering property of the lipids. ST was measured on the serum $L$ ext. from a total of 25 serum samples obtained from normal ( 12 samples), Type II (11 samples), and Type IV (2 samples) subjects. HL typing was established by triglyceride/cholesterol ratio; ultracentrifugation and 1 iponrotein electrophoresis. The serum total lipid, estimated by summation method, ranged from 400 to $2071 \mathrm{mg} / 100 \mathrm{ml}$. Lipids from the serum were extracted by chloroform-ethanol $(95 \%)$ mixture $(7: 11 \mathrm{~V} / \mathrm{V})$ and the supernatant was used for ST determination. 15-35 ST measurements at different aliquots $(10-350 \cdot \mathrm{mcl})$ were made on each serum $L$ ext. sample. An inverse relationship between the ST and serum total lipids was observed. The best correlation $(r=.95)$ between serum total lipids and ST was obtained with $40 \mathrm{mcl}$ of serum $L$ ext. The method is rapid and the results are highly reproducible. The reagents (distilled water, chloroform and ethanol) are inexpensive and the apparatus is portable. The technique can be learned in a few hours and is adabtable for automation.
NEONATAL MAGNESIUM (Mg) HOMEOSTASIS IN INFANTS OF DI-

604 ABETIC MOTHERS (IDM). Reginald C. Tsang \& David R. Brown. Univ. of Cincinnati Col. of Med., Dept. of Ped.

In a prospective study of 56 IDM and their mothers, neonata 1 serum $\mathrm{Mg}$ levels (birth,12, 24, $48 \& 72$ hours) were at least once $\leq 1.5 \mathrm{mg} \%$ (hypomagnesemia, mean $-2 \mathrm{SD}$ for control infants) in 21 infants and at least twice in 9 infants. Lower postnatal serum Mg was related to greater severity of maternal diabetes, younger mothers, mothers of lower gravidity and less gestational maturity. Serum $\mathrm{Mg}$ was not related to maternal diuretic use, race, toxemia, mode of delivery, labor duration or birth asphyxia. Serum $\mathrm{Mg}$ from 12 to 72 hours was significantly related to maternal serum Mg at delivery $(r=0.43$ to $0.64, p-0.01$ ). When serum $\mathrm{Mg}$ was $1.5 \mathrm{mg} \%, 64 \%$ of concurrent serum $\mathrm{Ca}$ was $\leq 7 \mathrm{mg} \%$ and $72 \% \leq 8 \mathrm{mg} \%$. Jitteriness was not related to low serum $\mathrm{Mg}$, with or without low total or ionized $\mathrm{Ca}$. Low serum $\mathrm{Mg}$ was not correlated with simultaneous blood sugar or hypoglycemia. Higher serum Mg at 24 hours was related to higher serum ionized $\mathrm{Ca}$ at 72 hours $(r=0.42, p-0.05)$ and the increase in parathyroid (PTH) levels from $24-48 \mathrm{HR}$ to $72 \mathrm{hrs}$. ( $r=0.48, p<0.05)$. Higher serum $\mathrm{Mg}$ at $72 \mathrm{hrs}$. was associated with greater PTH function in the first 2 days of life (calculated as serum PTH increases in response to serum Ca falls). Thus, significant imbalances occur in neonatal magnesium homeostasis in infants of diabetic mothers the respective roles of maternal disease and neonatal parathyroid function in the $\mathrm{Mg}$ homeostasis of such infants deserves further study.
605 Warshaw, Mary L. Terry, Dept. of Ped., Yale School Medicine, New Haven, Conn.

It has become increasingly recognized that the developing brain can oxidize alternate substrates such as ketone bodies and branched chain amino acids. We have investigated fatty acid (FA) oxidation in developing rat brain and have also measured activities of palmityl-CoA synthetase and palmitylcarnitine transferase, enzymes important for the activation and mitochondrial transport of CoA esters of FA. Oxidation of palmitate-1-14C by rat brain homogenates increased from $0.13 \mathrm{~nm} / \mathrm{mg} / \mathrm{hr}$ at 1 day of age to 1.1 $\mathrm{nm} / \mathrm{mg} / \mathrm{hr}$ by 22 days of age. Biphasic peaks were seen at 10 and 22 days of age. Activity then declined to adult levels which were $0.3 \mathrm{~nm} / \mathrm{mg} / \mathrm{hr}$. Rates with palmityl-CoA were somewhat higher. Similar changes in oxidative rates were observed when decanoate, acetyl-CoA and acetate were substrates. The specific activity of acetyl-CoA oxidation increased from 1.0 to $9.5 \mathrm{~nm} / \mathrm{mg} / \mathrm{hr}$ and showed the biphasic pattern seen with palmitate. During this period, increased cytochrome oxidase activity of homogenates indicated a $25 \%$ increase in brain mitochondrial number. Palmityl-CoA synthetase activity of fetal and newborn brain was $1.5 \mathrm{~nm} / \mathrm{mg} / \mathrm{min}$. This decreased to $0.8 \mathrm{~nm} / \mathrm{mg} / \mathrm{min}$ by the time of weaning. Palmityl carnitine transferase activity increased slightly following birth and remained stable until 21 days of age when a $50 \%$ reduction to adult levels was seen. Changes in FA oxidation by postnatal brain probably reflect increases in mitochondrial number, responses to suckling and weaning and to energy requirements during the brain growth spurt. The data also suggest that FA may be
alternate substrates in the developing brain.

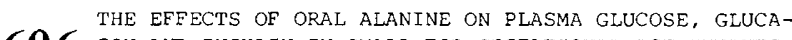
606 UCLA Sch. of M.R. Williams*, R.H. Fiser, M.A. Sperling, and W. Oh. priate for gestation age (AGA) infants were studied during the first four days of life to determine the effects of oral alanine feeding $(500 \mathrm{mg} / \mathrm{kg})$ on glucose balance. During the first 24 hours of life, the SGA infants had a significantly higher piasma alanine concentration $(\mathrm{p}<.05)$. Oral alanine feeding resulted in an expected increment in plasma alanine concentration. The table below shows a significant increase in plasma glucagon (GN) in both AGA $(n=13)$ and SGA $(n=14)$ infants following the alanine loading. Plasma glucose (G) and insulin (I) levels also increase significantly in AGA infants but not in SGA group.

\begin{tabular}{|c|c|c|c|c|c|c|c|c|c|}
\hline & \multicolumn{3}{|c|}{ Glucagon $\mathrm{pg} / \mathrm{ml}$} & \multicolumn{3}{|c|}{ Insulin $\mathrm{uU} / \mathrm{ml}$} & \multicolumn{3}{|c|}{ Glucose mg : } \\
\hline & $\overline{0}$ & $30^{\prime \prime}$ & $60^{\prime \prime}$ & 0 & $30^{\prime \prime}$ & $60^{\prime \prime}$ & 0 & $30^{\prime \prime}$ & $60^{\prime \prime}$ \\
\hline AGA & $133 \pm 21$ & $\star 233 \pm 37$ & * $245 \pm 42$ & $10 \pm 1$ & $13 \pm 1$ & 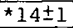 & $52 \pm 2$ & $\star 61 \pm 4$ & $6 \mathrm{l} \pm 5$ \\
\hline$S G A$ & $208 \pm 42$ & $\star 269 \pm 57$ & $\star 307 \pm 64$ & $15 \pm 2$ & $15 \pm 2$ & $15 \pm 1$ & $55 \pm 4$ & $52 \pm 4$ & $\overline{50 \pm 4}$ \\
\hline
\end{tabular}

*Significant difference by paired test ( $p<.05), M \pm S E M$ In 25-96 hours old infants (both $A G A$ and SGA) no significant changes were noted in plasma $G N, G$ or I concentrations following alanine feeding. The data indicate that gluconeogenic amino acid (alanine) appropriately produce a significant increase in hepatic $G$ output in well nourished (AGA) infants but not in malnourished (SGA) infants. The latter may reflect decreased glycogen stores, an inefficient gluconeogenic enzyme system, or possible increased glucose utilization in the SGA group. 
607 A NEW INBORN ERROR OF TRYPTOPHAN METABOLISM. Paul W. K. Wong, Phillip Forman and Parvin Justice. Abraham

A 6-year Jld male with photosensitive, scaly, red rash; cerebellar ataxia; and stunted physical growth was investigated. There was no hyperaminoaciduria nor indicanuria. Thin layer chromatography showed a striking lack of urinary tryptophan (T) metabolites. Two oral ( $T$ ) loading tests $(100 \mathrm{mg} \mathrm{L-tryptophan} / \mathrm{Kg})$ in the patient ( $P$ ) and controls (C) showed no significant difference in plasma $(T)$ (determined every 2 hrs for $8 \mathrm{hrs}$ ) and urinary ( $T$ ) and 5-hydroxyindoleacetic acid (determined on 6-hourly urine for 24 hrs) indicating normal intestinal and renal absorption of $(T)$. Urinary Kynurenine (KYN) and $\mathrm{N}$-methylnicotinamide (NMN) were markedly reduced following loading ( $\mathrm{u}$ mole $/ \mathrm{Kg} / 6 \mathrm{hr}$ )

\begin{tabular}{|c|c|c|c|c|c|}
\hline Time ( & -s) & $0-6$ & $6-12$ & $12-18$ & $18-24$ \\
\hline$\overline{K Y N(P)}$ & $1 \mathrm{st}$ & 0.00 & 2.25 & 0.48 & 0.00 \\
\hline & $2 n d$ & 3.79 & 0.00 & 0.00 & 0.00 \\
\hline (c) & lst & 9.25 & 27.52 & 0.91 & 0.00 \\
\hline & 2nd & 16.54 & 8.77 & 0.00 & 0.00 \\
\hline $\operatorname{NMN}(\mathrm{P})$ & $1 \mathrm{st}$ & 0.17 & 0.46 & 0.46 & 1.16 \\
\hline & $2 n d$ & 0.29 & 0.41 & 0.34 & 0.46 \\
\hline (c) & lst & 0.70 & 1.51 & 1.15 & 0.92 \\
\hline & 2nd & 0.84 & 1.33 & 0.64 & 0.70 \\
\hline
\end{tabular}

Urine was treated with purified urease and chromatographed. The (C) showed marked excretion of formylkynurenine which was absent in the patient's urine, indicating a previously undescribed metabolic block at tryptophan pyrrolase. A sensitive method for assaying this enzyme using methylene-C14- $(T)$ has been developed for studying liver tissue, WBC and cultured fibroblasts.

\section{MORPHOGENESIS}

A NEW PHENOTYPE OF THE CEREBRO-HEPATO-RENAL SYNDROME

608 (CHRS) OF ZELLWEGER IN SIBLINGS. Robert C. Bobo, Cadams. Ohio.

Two male siblings in a sibship of three males showed strikingly similar physical, histological and laboratory findings. Both children had macrocephaly, scaphocephaiy and unusual facies which was, however, distinctly different from the usual Down's like facies usualiy seen in CHRS. Both had hypotonia, the first worse than the second, and multiple other similar congenital anomalies. Soon after birth they manifested failure to thrive, marked developmental retardation and seizures. Laboratory findings on both revealed severe hypoproteinemia and markedly elevated liver enzymes. A chromium-51 labeled albumin study was normal in the first sib and both had normal 72 hour fecal fat collections. The second sib also has laboratory evidence of hypothyroidism. Pneumoencephalography revealed cerebral atrophy in both. The first child died at 6 and $1 / 2$ mos. of age from aspiration after progressive physical deterioration. The second boy started on a similar dowhill course soon after birth. A massive pericardial effusion developed at 3 mos. of age requiring a pericardotomy. At 19 mos. of age he is still alive and seems somewhat improved but still has marked elevation of liver enzymes with progressiveIy worsening cirrhosis on subsequent liver biopsy. Autopsy specimens from the first child (brain, kidney, liver) and biopsies of the brain and liver from the second shows the characteristic histological findings of the CHRS.

NUTRITIONAL SUPPLEMENTATION OF THE PREGNANT MOUSE AS 609 A METHOD OF REDUCING THE INCIDENCE OF DRIGG-INDUCED CLEFT PALATE. Robert L. Brent and K.T. Szabo, Jef ferson Med. Col. and Smith Kline \& French Lab., Phila., Pa.

We have reported that the oral administration of certain tranquilizers to pregnant mice resulted in cleft palate in the offspring and that the increased incidence of cleft palate occurred in those pregnancies exhibiting a marked reduction in maternal food and water intake (Iancet, 1974, i, 565). We, therefore, hypothesized that maternal nutritional deprivation might be an important factor in the induction of cleft palate following the administration of certain tranquilizers and possibly other drugs. In order to test this hypothesis we duplicated the previous experiments and included several groups of mothers that were both force-fed and drug treated from the 10th to 13 th day of pregnancy. Haloperidol (20 mg/kg/day p.o.) and Perphenazine $(25 \mathrm{mg} / \mathrm{kg} /$ day p.o.) administered to pregnant mice produced $43.6 \%$ and 100 cleft palates, respectively. In the force-fed group treated with the same dose of these drugs the incidence of cleft palate was 18.68 and 20.88 respectively. The mechanism of the beneficial effect of force-feeding could include changes in matemal and embryonic nutrition or the alteration of drug metabolism and excretion. Regardiess of the eventual explanation for the teratogenesis, our data again emphasize the fact that the application of teratogenic studies in the mouse to the human may sometimes be inappropriate. (Supported by NIH Grant HD 630)
610 HERPESVIRUS HOMINIS AS A CAUSE OF ABNORMAL HUMAN EM-

BRYONIC KIDNEY GROWTH IN ORGAN CULTURE. JOhn F.S. Crocker, Judy Sparling, Kenneth Easterbrook, Juan Embil, Depts. of Pediatrics and Microbiology, Dalhousie University Canada.

Few microrganisms have been shown to be teratogenic but recent interest in Herpesvirus hominis as a possible teratogenic agent has led to experimental assessment of its effects in rabbits and hamsters. Lapinleimu et al (Lancet 1, 1127, 1974), have reported a high seroconversion to herpesvirus during pregnancy in mothers who deliver children with congenital anomalies.

Fifty pairs of human embryonic kidneys of 5 to 12 weeks gestation were maintained in whole organ culture by our previously described technique, with Herpesvirus hominis type 1 and 2 added to our regular media ( $1 \mathrm{ml})$. One kidney of each pair served as a control and was exposed to identical culture medium without virus.

Cultures were maintained for between 24 and $120 \mathrm{hrs}$ to study the time sequence of viral infectivity. Organs were then examined for the presence of virus by electron microscopy and histochemical staining. Histological studies of virus-infected kidneys showed either: 1) complete organ death, or 2) virus localization in undifferentiated cells, plus disorganization of architecture in differentiated areas. Control organs showed normal organization.

This study shows that Herpesvirus hominis type 1 and 2 are capable of causing either complete organ death or disturbed embryonic architecture in whole human kidneys in organ culture.

PALMAR CREASE VARIANTS IN NEWBORNS. Hanna Dar and Harold M. Nitowsky, Dept. Ped. , Albert Einstein Col Med., New York.

Studies of palmar crease patterns (PCP) in newborns suggest variant forms are useful as signs of early intrauterine developmental disturbances. A system for classification of PCP was developed from observations on 500 newborns. Variant patterns were classified into three groups: a) simian creases and variants, b) Sydney lines and variants, c) other unusual patterns. Each group was composed of approximately 30 subtypes, schematically represented and numbered for easy categorization. In a study of 300 consecutive infants admitted to an intensive care nursery, PCP were evaluated independently of any clinical assessment. Several groups were found to have an increased frequency of variant PCP as compared with normal fullterms. The differences were most striking for simian crease variants. Compared to the $8 \%$ normal infants with variant patterns, the frequency was $35 \%$ in infants with congenital malformations; $68 \%$ in infants with chromosomal abnormalities; $29 \%$ in babies born to addict mothers on methadone; and $18 \%$ in prematures. Newborns with other complications (e.g. sepsis, hyperbilirubinemia, maternal diabetes) did not differ significantly from normal fullterms. These findings suggest that variant PCP may provide a useful objective criterion for possible fetal developmental abnormalities. From these studies, we recommend a standard form be utilized to identify variant PCP as part of the routine newborn examination. (Supported by NIH Grant GM 19100).

TERATOGENIC EFFECTS OF RETTNOIC ACID IN MONKEYS. $612 \frac{\text { Alan G. Fantel }}{\text { and Benjamin C. }}, \frac{\text { Thomas H. Shepard, }}{\text { Moffett, Depts. of }} \frac{\text { Laura L. Newell }}{\text { Ped. and Ortho- }}$ dontics, Univ, of Wash., Seattle, Wash.

In the development of a teratogenic protocol for inducing cleft palate in viable newborn monkeys, we have been able to delineate a set of malformations which we refer to as the "retinoic acid syndrome".

To date, 11 pregnant Macaca nemestrina of known gestation have received $10 \mathrm{mg} / \mathrm{Kg} /$ day of retinoic acid (vitamin A acid) in corn oil by gavage from days 20 to 45 . From the treated pregnancies, 3 normal fetuses have resulted, two of which aborted before term. In addition, two animals aborted during the embryonic stage and a single macerated embryo was recovered. A major concomitant of this treatment is abortion or fetal death followed by intrauterine retention ( 6 of 11). Placental separation is considered likely as maternal vaginal hemorrhage has been frequently observed.

4 animals have presented with palatal clefts and two of these as well as two with normal palates have demonstrated other common malformations of skeletal, visceral and external systems. In addition to 1 cleft hard palate and 3 cleft soft palates, we have seen 3 cases of severe auricular malformation including failure of helix formation, 3 cases of open eye, 2 of marked kyphosis, 1 of which also showed scoliosis, 2 animals with missing digits and other digital defects, and one with severe curvature of the radius. Although specific osseus defects are frequently found in this syndrome, there appears to be no effect on the rate of osseus maturation. 
613 SHORT STATURE FROM SKELETAL DYSPLASIAS:

Murray Feingold, Tufts Univ. Sch. of Med. Dept. of ped., Boston.

A study was undertaken to help differentiate the normal short child from an infant or child with a skeletal dysplasia involving the limbs or vertebrae. The distance between the anterior superior iliac spine (A) and the tibial tuberosity (C) and the distance between the anterior superior iliac spine (A) and the position of the tip of the middle finger with the arm fully extended on the thigh (B) were ascertained in 200 normal infants and children. The percent of $A B$ in relationship to $A C$ was then determined. The $A B / A C$ percentages were: infants, 45-55\%; 1-2 years, 50-55\%; 2-3 years, 55-608; 4-1l years, 60-65\%. A 3 month old infant with an $A B$ distance of $7 \mathrm{~cm}$. and an $A C$ distance of $14 \mathrm{~cm}$. would be considered normal $(50 \%)$. Measurements were then obtained on patients with various types of skeletal dysplasias. Patients with achondroplasia have an $\mathrm{AB} / \mathrm{AC}$ percentage ranging between 25 and 35 in contrast to patients with various types of spondyloepiphyseal dysplasia whose percentages were between 65 and 75. Besides serving as a guide in differentiating the normal short patient from those with skeletal dysplasias, these measurements are also useful in distinguishing between some of the various types of skeletal dysplasias.

horacic Dys-

614 trophy. A.Garnica, M.D., R. Fennel1, M.D., W.

Donnelly,M.D.*, J. Frias, M.D., Dept. Pediatrics, *Dept. Pathology, Univ. Fla., Gainesville, Fla.

A six year old male referred because of renal failure, short stature, peculiax hands and feet, relative narrowing of the upper thorax, and a past history of hospitalization for cholangitis was diagnosed to have asphyxiating thoracic dystrophy. In addition to the classical chondrodystrophic skeletal changes and the renal abnormalities (glomerulosclerosis and tubular atrophy) the patient had significant hepatosplenomegaly. Liver function tests (SGOT, SGPT, LDG, alkaline phosphatase, prothrombin time, bilirubin, BSP retention, and protein electrophoresis) were all norma1. HAA, alpha-1-antitrypsin, and quantitative immunoglobulins were within normal limits. Microscopic examination of liver biopsy demonstrated enlargement of portal areas associated with periportal fibrosis and bile duct proliferation. A similar lesion has been described in four other patients with asphyxiating thoracic dystrophy, suggesting that hepatic involvement in this disorder may occur more frequently than previously recognized.

615 MONOSOMY AND TRISOMY IN SIBS FOR A PORTION OF CHROMOSOME 1Q. Kenneth L. Garver and Angela M. Ciocco. Children's Hosp., Dept. of Ob-Gyn, Ped., and Path. Pittsburgh, Pennsylvania. (Intr. by Thomas K. Oliver, Jr.)

A family will be described in which two siblings, CF and RF, had multiple congenital abnormalities. Chromosome studies showed that they had a partial monosomy of the long arm of chromosome 1 due to an interstitial deletion [46, XX, del (1) (q25q32)]. Their older sister, AF, who was less severely affected, was trisomic for the same portion of the long arm of chromosome 1 due to an interstitial insertion into the short arm of chromosome 1. Their mother was a balanced carrier with transposition of 19 into Ip $[46, X X$, ins (1) $(p 32 q 25 \rightarrow 32)]$.

The proband CF weighed 4' $7 "$ at birth and had multiple anomalies including micrognathia, low set rotated ears, protuberant eyes with increased distance between the palpebrai fissures, thick neck, bilateral clinodactyly, short toes and fingers and broad thumbs. RF was born 14 months after CF and weighed 4' $15^{\prime \prime}$ at birth and resembled her in many respects. An older sib, AF, was considered normal by her parents, had lymphedema at birth involving her hands, feet and legs. When examined by us at the age of $41 / 2$ years, she was quite large for her age and had hypertelorism and epicanthal folds. These aberrations are most likely the result of a meiotic crossover so that CF and RF received their mother's deleted long arm and the normal short arm, resulting in monosomy for that portion of the long arm. AF received the abnormal short arm and the normal long arm and was therefore partially trisomic for lq.
616

PROMINENT LATERAL PAIAATINE RIDGES: DEVEIOPMENTAL AND CLINICAL SIGNIFICANCE. James $W$. Hanson, M. Michael Cohen, Jr., and David W. Smith, Univ. of Washington Sch. of Med., Dept. of Ped., Seattle.

Prominent lateral palatine ridges due to thick submucosal tissues have been noted by us in a variety of dystiorphic conditions. Such ridges have been observed in infants with neurological disorders associated with hypotonia (e.g., the PraderWilli and Down syndromes) as well as hypertonia (e.g., the Menkes, Smith-Lemli-Opitz, and 18 Trisomy syndromes). These palatine ridges have also been noted in children with structural anomalies such as an unusually narrow palate (e.g., Apert syndrome) or a small or displaced tongue (e.g., Noebius or Robin anomaly). In normal fetuses these structures are quite prominent, but they are less readily apparent in term infants. A survey of the normal post-term babies suggests that these ridges usualiy become progressively flattened out, reaching an adult configuration during infancy. These observations lead us to hypothesize that these ridges are normal in the developing fetus. As the tongue becomes well developed and functional it plays a role in molding these lateral palatal prominences. In disorders in which there is a deficit of tongue thrust, on the basis of functional or stmetural abnormalities, these ridges may become unusually prominent. Accordingly, prominent lateral palatine ridges are interpreted as one clue to a long-standing deficit of neurological function or structural anomaly which limits tongue thmist into the hard palate in early life.

TIE FETAL HYUANTOIN SYNDROA正. James W. Hanson, and David W. Smith, Univ. of Washington Sch. of Med. Dept. of Ped., Seattle.

During the past 6 years evidence has accumulated through epidemiologic studies that hydartoin anticonvulsants have a serious teratogenic potential. Nimerous dysmorphic features have been reported but most reports focus on individual defects of major medical significance. We report 5 unrelated patients who share a broad multisystem pattern of abnomalities suggesting a specific Fetal Hydantoin syndrome. Craniofacial features include a broad depressed nasal bridge with short upturned nose, epicanthic folds, hypertelorism, ptosis, strabismus, minor ear abnormalities, and wide mouth with prominent lips. Limb defects iniude hypoplasia of distal phalanges and nails, finger-like thumb, and alterations of palmar creases and dermal ridges. Growth deficiency is usually of prenatal onset and appears to be permanent. Mental deficiency of mild to moderate degree has been a frequent feature. Ilajor defects such as cleft lip and/or cleft palate and cardiac defects, associated with Dilantin in the past, are less frequent. Recognition of the broader syndrome may serve to identify affected children with greater precision. This symdrome has been misdiagnosed in individual cases as Coffin-Siris syndrome and as Noonan syndrome. Its frequency among infants of women taking hydantoins remains unknown.

618 GENETIC DISORDERS WITH NEGATIVE FAMILY HISTORY. 618 L. B. HOLMES, MASS. GENERAL HOSPITAL, BOSTON, MA.

Often the pediatrician must recognize the affected infant as having a genetic disorder without the history of similarly affected relatives to aid in recognizing the genetic etiology. We have found this true in evaluating all clinically apparent genetic disorders in 18,000 consecutive newborms over the past 3 years. The reason why most affected infants are the first to be affected in their families is inherent in the three types of underlying genetic abnomalities: multifactorial inheritance, single mutant genes and chromosome abnormalities.

157 infants had congenital malformations due to multi-

factorial inheritance: 26 meningomyelocele-anencephaly, 9 cleft lip and palate, 4 cleft palate, 55 hypospadias, 10 congenital hip dislocation, 20 club foot, and 33 cardiac anomalies. Only 5 of the 157 infants had affected first degree relatives (parents or sibs).

65 newborns had clinical disorders due to single mutant genes. Only 1 of 6 infants with an autosomal recessive disorder had an affected sib. 5 of 8 with serious autosomal dominants had an affected parent, the remainder presumably representing spontaneous mutations. 12 of 49 infants with hereditary polydactyly had an affected parent.

Only 1 of 27 infants with a clinically recognizable chromosome abnormality had a similarly affected sib.

The parents of the affected newborn rely on the pediatrician to recognize that their infant has a genetic disorder and to properly inform them. 
619 X-LINKED RECESSIVE CLIFT PALATE. L. B. HOLMES,

Only one family with $\mathrm{x}$-1inked recessive cleft palate has been reported (Pediatrics $46: 123,1970$ ). We found another family with four affected males in an evaluation of congenital malformations among 18,000 consecutive newborns. The propositus has an affected half-brother, maternal uncle and matemal great uncle. All affected males have only a cleft soft palate and no other anomalies. The carrier female has no craniofacial anomalies. This maternal ancestry is an Indian tribe in Eastern Canada. The other reported family is from a presunably unrelated British Columbia Indian tribe.

Fach of the other 4 infants with isolated cleft palate identified in this survey of newborns had no affected relatives. No phenotypic heterogeneity was evident. This is in contrast to the fact that 2 of the 11 infants with cleft lip and palate had associated major anomalies without any specific diagnos is.

The propositus with $X$-linked recessive cleft palate is 1 of 57 infants anong 18,000 newborns who had isolated malformations due to single mutant geres.
ALPHA-FETOPROTEIN (AFP) AND $\beta$-TRACE PROTEIN ( $\beta$ TP) IN

622 PRENATAL DIAGNOSIS, Aubrey Milunsky, James N. Macri, Robert R. Weiss, Elliot Alpert. Harvard Med. Sch., State Univ. of New York, E.K. Shriver Ctr., Mass. General Hosp., and Nassau County Med. Ctr., Depts. Pediat., Med., Animal Research, obstetrics \& Gynecology, Boston, Mass. and New York.

Assays for AFP represent a valuable but non-specific technique for prenatal diagnosis of neural tube defects (NTD). Since $3 T P$ is present in the amniotic fluid (AF) of fetuses with open NTD it might provide a more specific diagnostic test. Therefore $28 \mathrm{AF}$ samples from fetuses with NTD, and 8 other 'abnormal' samples (Trisomies 18 and 21 , unbalanced $D / G$ translocation, fetal death, hydropic fetus) were assayed for AFP (electroimrunodiffusion) and STP (Ouchterlony double diffusion).

In NTD samples AFP values were elevated in all $10<24$ weeks gestation and falsely positive in 6 'abnormal' AF's. Normal/nondetectable values were noted in 18 ITD samples $>24$ weeks gestation and 2 'abnormal' $A F^{\prime} s$. In contrast $T P$ was present in 9 and uncertain in 1 NTD sample $<24$ weeks gestation and falsely positive in 1 and uncertain in 4 abnormal AF's. After 24 weeks gestation in NTD samples $3 \mathrm{TP}$ was falsely negative in 5 and uncertain in $1 \mathrm{AF}^{\prime} \mathrm{s}$. Hence whi le 3TP may also be non-specific, further quantitative refinements are needed to more accurately define the abilities of the ?TP assay. These studies will be viewed against the background of our experience with AFP assays in $>1,000$ cases, concentrating on the problems and pitfalls, especially with regard to false negative and false positive results.
THE AICARDI SYNDROME. Alasdair G.W. Hunter, J.Raymond

620 for Sick Children, Depts. Of Genetics and Ophthalmology, Toronto, Canada (Intr. by A. Sass-Kortsak).

This paper describes 3 cases of the Aicardi syndrome. The syndrome is characterized by absence of the corpus callosum, early onset of myoclonic seizures, pathognomonic retinal lacunae and a universally poor prognosis. Frequently noted findings are scoliosis (with or without segmentation and fusion anomalies), cortical heterotopia, and an electroencephalogram characterized by a markedly periodic rhythm and independent activity of the two hemispheres.

21 cases have appeared in the European literature since Aicardi first described the syndrome in 1965, but these are the first to be reported from North America. We have seen 3 cases in 2 years and feel this readily recognizable syndrome may be relatively common.

All cases, including ours, have been female and all have been sporadic. These findings are best explained by an $\mathrm{X}$-1inked dominant gene lethal prenatally in hemizygous affected males, and a genetic lethal in heterozygous affected females. Central nervous system and ophthalmologic abnormalities, myoclonic seizures, absence of the corpus callosum, and vertebral anomalies have been seen in other syndromes thought to be due to $\mathrm{X}-1$ inked dominant genes, with prenatal lethal expression in the male (Incontinentia pigmenti, Goltz syndrome, Type I Oral-facial-digital syndrome).

621 CONGENITAL ANOMALIES ASSOCIATED WITH TISSUE BANDS, Jeannette Is rael, Donald W. Day and George F. Smith Rush Medical Col., Dept. Ped., Chicago

Congenital annular bands producing physical anomalies are usually considered to be a rare event; however, the extent of the physical defects they are capable of producing may be tremendous. Four patients with various degrees of physical anomalies produced by annular bands will be presented. The physical anomalies in these patients range from minor amputations of the fingers to major anomalies of the head, face, fingers and toes. The etiology and how these bands arise to produce the anomalies are far from settled. Two of the most widely held views on the production of these bands are the concepts of Streeter and Torpin. Streeter suggested that the anomalies were produced by either macerated sheets of epidermis or residual strands of defective tissue intrinsic to the developing fetus. Torpin, on the other hand, favored the hypothesis of rupture of the amnion leading to the development of amniochorionic strands which produced pathological changes. The present report deals with the etiology and pathophysiology of the condition in light of our own case material, using light and electron microscopy studies. These data will be correlated with previously published information on human and animal studies.
BIRTHWEIGHT DOUBLING: A FRESH LOOK, Charlotte G.

623 Neumann (Intr. by Solomon Kaplan) UCLA Sch. of Med., Dept. of Ped., Los Angeles.

Infant birthweight doubling time long used as an index to assess normal physical growth of the infant is widely quoted as being achieved between 5 and 6 months. The purpose of this study was to determine the time of birthweight doubling and to identify important related variables. 357 normal infants with birthweights between 2500 and $4100 \mathrm{gms}$ all born and followed in the same hospital were studied for age and length at the time of birthreight doubling. Doubling age was determined from calculation of the rate of daily weight gain between two known ages and extrapolating the age (in days) of birthweight doubling assuming a steady daily rate of weight gain. Length at the age of birthweight doubiing was similarly calculated. Bresst fed infants (those nursed for 3 months) doubled their birthweights significantly later than bottle fed infants: 124 days vs. 113 days (pe.05). Early introduction of soilds (mean 1.9 months) correlated highly with bottle feeding as did late introduction of solids (mean 3.9 months) with breast feeding. Boys doubled their birthweights significantly earlier than girls 111 days vs. 129 days ( $p<.0001$ ) and were in the 75 th Harvard percentiles for both mean height and weight. Harvard percentiles for mean weight and length at time of weight doubling for breast fed infants were both j0th percentile: for bottle fed infants weight was 75 th percentile and length was 50 th percentile. Thus bottle fed infants appear to have weight gains in excess of length gain and may be developing early obesity. In light of $\mathrm{fat}$ cell number hyperplasia, due to early overnutrition closer supervision of early feeding practices is indicated.

Q H. Qazi, C. Ganapathy and George Kassner. Depts. of Fed. and Radiol., State Univ. of New York, Dowrstate Med. Ctx., Brookiyn, N.Y.

Crossed asymetry is not an infrequent finding in individuals with isolated congenital hemihypertrophy. To our knowledge it has not been reported in Russell-silver syndrome.

We have observed a six year old male with characteristic features of Russell-silver Syndrome (short stature, pseudohydrocephaly, triangular face, cafe'au-lait spots, bilateral clinodactyly and syndactyly of 2 nd and 3 rd toes and sexual precocity). The most striking finding was the crossed asymmetry: the right arm and hand and the left leg and foot wexe larger than the opposite structures. Radiographs demonstrated enlargment of bones and soft tissues in the affected regions. The chromosome analysis was normal $(46, X Y)$ and the dermatoglyphic patterns were similar on both sides. Intravenous excretory urograms on three occasions have shown a prominent bulge of the lower lateral margin of the left kidney.

Because of the crossed pattern of asymmetry - more typical of isolated hemihypertrophy than of the Russe11-silver syndrome the unusual configuration of the left kidney was alarming. Absence of change on serial urograms and normal sonographic characteristics have provided reassuring evidence that the renal bulge may be an innocent variation of renal lobation and for the moment renal arteriography has been deferred. 
TREATMENT OF HUMAN ACHONDROPLASIA. Thomas H. Shepard,

625 Judith G. Ha1l, M. Michael Cohen, Jr. and Benjamin C. Graham. Depts. of Ped and Radiology and Oral and Maxillo-Facial Surgery, Univ. of Wash., Seattle, Wash.

A defect in oxidative phosphorylation involving site III of the terminal electron particle (ETP) system was shown in the ac/ ac dwarf rabbit. Subsequently a similar defect in oxidative phosphorylation of human dominant achondroplastic muscle was found by Mackler et al. (Arch. Biochem. Biophys. 159:885, 1973). The human mitochond $x$ ia exhibited an absence or inactivity of site I where ADP is phosphorlated to ATP. Other mitochondrial functions were intact.

Since the organ affected in achondroplasia is the endochondral growth plate which has no blood supply and consequently a very reduced concentration of oxygen one might ask "is the organ specificity related to a combination of 1 imited oxygen substrate with a partial defect in oxidative phosphorylation"? Insufficient amounts of ATP could lead to reduced cell multiplication in the growth plate.

To safely test this low substrate hypothes is two achondroplastic children were placed in oxygen tents at $40 \%$ during their hours of sleep for 5 month periods. Measurements of height were made in triplicate by three observers and the radiologic measurements were repeated at half year intervals.

Although not fully completed no effect of oxygen on growth rate or health has been observed. The standard error of standing height measurements by separate observers was found to be very small but some unexplained variability in monthly velocity was found.

626

DUHAMEL ANOMALY AND MONOZYGOTIC TWINNING: AN ASSOCIATION BETWEEN TWO MALFORMATIONS. David $W$. Smith, Catherine Bartlett, and Lyle $M$. Harrah, Univ. of Washington Sch. of Med., Dept. of Ped., Seattle.

The ituhamel anomaly encompasses degrees of a presumed single early primary defect in caudal mesoderm varying from imperforate anus and other single defects of the sirenomelia complex to sirenomelia itself. Included within this non-random spectrum are previously recognized associations such as the VATER association, Cauda1 Regression syndrome and Rokitansky syndrome. Our studies indicate that a distinct association exists between the Iuhamel anomaly and monozygotic twinning. The most severe degree of the Duhamel anomaly, sirenomelia, is 150 times as common in monozygotic twins as in dizygotic twins or singletons and the indi vidual features such as imperforate anus, renal agenesis, and bicornuate uterus are 8 or more times as common. The frequency of monozygotic twinning in cases of sirenomelia is $7 \%$ and in patients ascertained as having individual features of the Duhamel anomaly it was found to be $4 \%$. This excessive incidence of various components of the Duhamel anomaly in monozygotic twins partially accounts for their enhanced frequency of associated malEormation, which is twice as great as in dizygotic twins or singletons. The following hypothesis could account for this nonrandom association: the primary abnormality in germinal center or disc morphogenesis which results in monozygotic twinning is also liable to upset the early organization of the primitive streak and thereby the nigration of the early cauda1 mesoderm, giving rise to varying degrees of the Inuamel anomaly.

627 SHIFTING LINEAR GROWTH RATES DURIING INFANCY. David W. Smith, William E. Truog, Mary Ann Sedgwick Harvey, James E. Rogers, Lawrence J. Greitzer, Alfred L. Skinner, and John J. McCann, Univ. of Washington Sch. of Med., Dept. of Ped. Seattle.

A percentile linear growth chart, from the 3 rd to 97 th percertile, was derived from longitudinal data on 92 normal full-term infants of well-to-do families. The growth rate was $15 \%$ faster than the Boston Stuart standards for 3-9 months. Individual shifting in eariy growth rate was common with $23 \%$ crossing one percentile line, 30\% crossing 2 lines and 9\% crossing 3 or more. of those moving up, 57\% did so by 3 months and $70 \%$ by 6 months. Only $5 \%$ of those shifting downward did so by 3 months with $92 \%$ slowlng between 3 and 18 months. To better determine the timing and nature of these shifts a search was made for otherwise normal full-term infants who were <loth percentile at birth and moved up 3 percentile lines ( 18 babies) and those >90th percentile who moved down 3 lines ( 23 babies). Those shifting upwards had accelerated growth soon after birth and had achieved a new "channel" at a mean age of 11.5 months. Those shifting downward did not decelerate until after the first 3-6 months. These data plus the correlation coefficients relating parental data to infantile linear growth are compatible with the following statements: Birth length relates predominantly to maternal size. During the first 2 years the baby's genetic potential, as indicated by mean parental stature, not uncommonly results in shifting rates of growth. Those "catching-up" usually do so in early infancy whereas those "lagging-down" tend to do so in mid-to-late infancy.
6Y OYSCHONDROSTEOSIS - REPORT OF A POSSIBLE HOMOZYGOUS INFANT, Annemarie Sommer, Ann Rogers and Stella B. Kontras, Ohio State Univ., Coll. of Med., Dept. of Ped., Children's Hospital, Columbus.

A newborn male infant weighing $2.91 \mathrm{~kg}$. and being $45.5 \mathrm{~cm}$. long was referred for evaluation of short extremities. The physical examination was within normal limits except for short forearms, ulnar deviation of the hands and short legs. Roentgenological evaluation showed bilateral aplasia of the proximal one half of the fibula and mild dysplasia of the proximal tibial metaphyses; absence of the distal femoral and proximal tibia epiphyses corresponding to a bone age of less than 36 weeks; aplasia of the distal two thirds of the ulna and mild dysplasia with shortening and bossing of the radius bilaterally. The findings were consistent with mesomelic dwarfism.

The parents who were cousins, were both short $\left(4^{\prime} 10^{\prime \prime} \& 5^{\prime}\right)$ \& had short forearms with limitation of motion at the elbows and wrists. X-rays showed the Madelung deformity in both parents \& at least 6 other family members spanning 3 generations.

Although the expression of the dyschondrosteosis gene may be veriable, the radiological findings in our patient did not readily allow classification under any of the well known mesomelic brachymelias including dyschrondrosteosis. In view of the documented consanguinity, affected parents and deformities which appeared to be more severe than would be expected from the effects of a single dyschrondrosteosis gene, it is postulated that the patient represents an example of homozygosity for dyschondrosteosis.
COFS SYNDROME. Rawatma1 B. Surana, Juan R. Fraga,

629 Stanley M. Sinkford (Intr. by Melvin E. Jenkins). Howard Univ. Col. of Med., Dept. of Ped, and D.C.

General Hosp., Dept. of Ped., Washington, D.C.
Recently Pena and Shokeir (Clin. Gen. 5, 295, 1974) reported ten cases of a new syndrome from Canada, for which they coined the term Cerebro-Occulo-Facio-Skeletal (COFS) syndrome.

We report a similar case in a black female infant who was born at term to a 33-year-old mother and a 40-year-old father. During the second and third trimesters of pregnancy the mother received Propoxyphene Hydrochloride (Darvon) for frequent headaches. The Apgar score at one minute was $l$ and the general condition of the infant remained very poor until her death at 31 hours of age. The infant remained very poor until her death at 31 hours of age. The $27 \mathrm{~cm}$. The infant had microcephaly, palpebral fissures turned outwards and upwards, large and low set external ears, upper lip overlapping lower, prominent nasal bridge, flexion contractures at elbows, wrists, hips and knees, bilateral talipes equino var us deformity of feet with second toes overriding great toes. Roentgenograms of limbs revealed multiple fractures and severe degree of osteoporosis. At autopsy, on gross, infant had small brain and lungs with a few greyish nodules in 1 iver parenchyma.

Chromosome analys is on peripheral blood by G-banding technique was $46, \mathrm{XX}$. The family history is negative for similar findings or consanguinity.

On reviewing the literature it seems that cases reported by Neu et al (Ped., 47, 610, 1971), Lowery et al (J. Ped. 79, 282, 1971), Pena and Shokeir and one by us probab1y belong to either a single syndrome or to closely related syndromes.
630 FURTHER HETEROGENE ITY IN THE ORAL-FACIAL-D IGITAL

SYNDROMES. Philip L. Townes, Beverly P. Wood and Joseph V. McDonald. Univers it of Rochester School ot Medicine and Dentistry, Division of Genetics, Rochester, N. Y. The Oral-Facial-Digital syndrome (OFD) described by PapillonLeage and Psaume is characterized by hypertrophic lingual and buccal frenuli, lateral clefts of tongue and jaws, medial cleft of upper lip and anomalies of digits. It is a well delineated $\mathrm{X}$-linked dominant trait with fetal lethality in males. In a study of two sibs with oral, facial and digital anomalies somewhat similar to those reported by Mohr and Clausen, Rimo in and Edgerton, proposed that the Mohr syndrome constituted an OFD Type IX. A distinguishing feature of Type $I X$ is the presence of bilateral polysyndactyly of the halluces. We have studted two unrelated fernale patients both having all of the major features of OFD I. In addition both patients have polysyndactyly of the halluces (one unilateral, one bilateral). Although unilateral involvement has been reported in a small number of OFD patients, bilateral polysyndactyly has not been previously reported. Its cccurrence prompted review of OFD I and II classif ication with the finding that there are significant differences between the two families which resulted in the original OFD Type II classification and that the few cases that have been subsequently reported or retrospectively cited represent a heterogeneous group of disorders; in some instances having only bilateral polysyndactyly. We conclude that bilateral hallucal polysyndactyly is not a sine qua non of OFD II and that OFD II represents a heterogeneous group of disorders rather than a distinct clinical entity. 


\section{NEONATOLOGY}

IN VIVO EFFECT OF INTRALIPID INTRAVENOUS F.EEDI.JG OiV

631

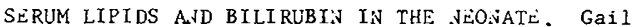
Andrew, George Chan, David Schiff, Depts. of Pediatrics, and obstetrics \& Gymecology, University of Alberta, Edmonton.

Intravenous alimentation with a $10 \%$ fat emulsion (Intralipid) 1a used as a calorie source in premature infants. The present study assessed whether infants are able to metabolize intravenous lipid without adverse effects. 18 infants, from 28-38 weeks gestation, in the first 48 hours, recelved Intrallpid, in a dose of $1 \mathrm{gm} / \mathrm{kg}$ for 4 hours. 7 infants (33 weeks and 7 infants $>33$ weeks gestation were appropriate weight for gestational age (AGA); 4 infants of 32-39 weeks gestation were small for gestational age (SGA). At set intervals, reserve bilirubin binding capacity $(R B C)$, red blood cell bound bilirubin ( $R B C-B R$ ), bilirubin, triglycerides (TG), free fatty acids (FFA), lipoproteln lipase activity, were determined. No significant change in $R B C$ or RBC$B R$ was found in $A G A$ infants. The change in $T G$ was greater in AGA infants $<33$ weeks $(192+51)$, compared to those $>33$ weeks $(80+26)$ $(p<0.05)$. The peak $F \bar{F} A$ levels appeared later in infants $<\overline{3} 3$ weeks. The decrease in RBC was greater in $S G A$ vs. AGA babies $(-8.9+2.3$ vs. $0.3+0.5, \mathrm{p}<0.01)$, and this was associated with a greater increase in TG $(606+113$ vs. $136 \pm 32, p<0.001)$ and FFA $(3.11+0.94$ vs. $0.93+0.12, p<0.05)$. In 2 cases, free bilirubin was noted at peak lipemia with FFA/albumin molar ratio of 16 and 20 respectively. The utilization of infused fat in AGA infants <33 weeks gestation and SGA infants is poor in the first week of life. Redistribution of bilirubin by FFA may be potentially hazardous to the jaundiced SGA infant.

632 OUTCOHE OF HIGH RISK NEVBORNS RECBIVING INTENSTVE CARE Billy F. Andrews ana sofia $\mathrm{H}$. Franco Univ. of Louisvilie sch. of ned., Louisville Gen. Hosp., Dept. of Fed., Louisville.

Neonatal intensive care and comprehensive health care following hospitalization are currently subjects of great interest. The value of both, from our results, as well as from other centers, is becoming available. our neonatal mortality has fallen from $2 \gamma$ to $13 / 1000$ live births (28 days) or with infants unaer $1000 \mathrm{gm}$. excluded to 7/1000. Our first 1166 patients tested with the Denver Developmental screen: ing Test (DDST) had 95\% passing: of 399 prematures 380 passed; of 365 high risks 345 passed; of 402 of low socio-economic status 384 passed. Subsequent psychological testing on 308 of our oldest patients revealed that of 96 prematures 89 had I.2.'s over 70 , of 100 high risks 91 had I.Q.'s over 70 , and of 112 of the low socio-economic group 109 had I.Q.'s ovex 70. of the 1166 children studied, only 27 had convulsive or recognizable neuromuscular problems. of the 27,8 were prematures, 16 were high risks, and 3 had had no detectable problems during the neonatal period. It is encouraging that prematures and high risks have not shown great differences from their socio-economic contemporaries.

BLOOD TRANSFUSION (BT): POSSIBLE POTEIJTIATING

633 RISK FACTOR IN RETROLENTAL FIBROPLASIA (RLF). Jacob V.Aranda, Tina E.Clark, Robert Maniel lo and Eugene $\bar{w}$. Outerbridge, (intro. by Eleanor Colle) MCGill University-Montreal Children's Hospital Research Institute and Roche Developmental Pharmacology Unit, McGill University, Montreal.

All 8 children in our hospital who have developed severe (Grade 3+) cicatricial RLF had received an exchange BT, suggesting that BT may increase the risk of RLF. To further assess this possibility, the records of 68 premature and full term neonates who had frequent $\mathrm{PaO}_{2}$ determinations and fundoscopic examinations during and after oxygen therapy were reviewed. The infants were divided into 2 groups according to whether or not they had received a BT of approximately $10 \%$ or more of their blood volume. Of the BT group, 8 had received 1 or more exchange $B T$. The incidence of retinopathy (retinal vasoconstriction or active RLF) is compared below.

$\begin{array}{lccc}\text { No transfusion } & \text { Normal } & \frac{\text { Retinopathy }}{25} & \frac{\text { Total }}{26} \\ \text { With transfusion } & 25 & 17 & 42 \quad p<.05 \\ \text { This suggests that BT may increase the risk of RLF }\end{array}$ in the newborn. A possible explanation is the shift in oxygen-hemoglobin affinity caused by transfusion with adult erythrocytes in the newborn infant, thus increasing oxygen availability to the retinal vessels and tissues.
634 RETROLENTAL FIBROPLASIA AND EXCHANGE TRANSFUSIONS. Bard, H., Cornet, A., Orquin, J. and Doray, B. H., Perinatal and Ophthalmology Services, Hôp. Sainte-Justine, Dept. of Pediatrics, Univ. of Montreal, Montreal, Que.

In order to determine if by exchanging high oxygen affinity red cells (fetal) for low oxygen affinity red cells (adult) during the neonatal period by an extrange transfusion (ET) the risk for the development of retrolental fibroplasia (RLF) is increased, 15 infants with the cicatricial phase of RLF had their neonatal intensive care (NIC) charts reviewed. 8 of the infants had at least one ET during their NIC. Two groups of newborn infants who received NIC during the same 14 year period were then compared. The number of newborns who had an ET and later devel oped cicatricial RLF was very significantly greater than the number of newborns who did not have an ET and developed cicatricial RLF $\left(X^{2}=27.1, P<0.001\right)$. It thus appears that an $E T$ during oxygen therapy may be an important additional etiological factor in the development of RLF. This may be due to the exchange of red cells containing mainly fetal hemoglobin by red cells with adult hemoglobin. The latter having a more efficient oxygen delivery ability. Levels of oxygen that are considered safe after ET during the neonatal period may have to be reevaluated in face of a lower affinity of oxygen that is provided by adult red cells.

LLMBAR EPIDLRAL ANALGESIA WITH BLPIVACAINE IN

635 LABOUR. CONTINLOLS FETAL HEART MONITORING AND DRLG CONCENTRATION IN MATERNAL, FETAL AND NEONATAL BLOOD. Patrick Belfrage, Anita Berlin, Nils Raabe \& Bertil Thalme. Dept. of Obstetrics \& Pediatrics, Karolinska Hospital, Stockholm. (Intro. by Charles D. Cook).

Lumbar epidural analgesia with bupivacaine was given to 33 women for labour. Serial determinations of $\mathrm{pH}$ and bupivacaine were made in fetal scalp blood and maternal venous blood as vell continuous monitoring of the fetal heart rate. At birth and during the next 20 hours the bupivacaine concentration was determined in maternal and neonatal blood. Fetal scalp blood pH was within normal limits. A temporary decrease of the normal baseline fetal heart irregularity was seen in 6 cases. The fetal and neonatal drug concentrations were low and about $1 / 4$ of the maternal levels. The $\mathrm{UA} / \mathrm{LV}$ drug concentration ratio increased with time after injection and approached one after about 60 minutes. During the first 20 hours an initial rapid decline followed by a slower fall was observed in the neonatal drug concentration. All babies were in good condition during the period of the study.

SPHEROCYTOSIS: AN INDEX OF SEVERITY IN ABO-HEMO-

636 LYTIC DISEASE (HD). Ines A. Bonacossa and Juan $J$. Gershanik, Dept. of Ped., Confederate Memorial Medical Center and L.S.U. School of Medicine in Shreveport, Louisiana (Intr. by J. A. Little)

Spherocytosis has been reported as a characteristic hematologic finding in ABO-HD. However, few data support this widelv accepted contention.

We performed a prospective study of cord blood spherocyte counts in 100 infants of blood groups $A$ or $B$, born to group 0 mothers. Neonates of Rh incompatible mothers were excluded. Spherocytes were defined as red cells whose diameter was les than $7 \mu$ with no central pallor and spherical in shape (axis $1: 1)$. One thousand red blood cells were counted. $A B O-H D$ was defined as (1) jaundice with a total bilirubin of at least $10 \mathrm{mg} / 100 \mathrm{ml}$ within 60 hrs. after birth and, (2) the demonstration of anti-A or anti-B antibodies in the cord serum by the indirect Coombs and a heat elution antibody test.

There were 14 infants who met our criteria of ABO-HD (group I) and the remaining 86 were used for comparison (group II).

There was no significant difference in their mean birth weights. Mean spherocyte count of group I $(9.8 \%)$ was significantly greater $(\mathrm{p}<0.001)$ than group II $(4.7 \%)$. In group I the spherocyte count and early rise of bilirubin $(>10 \mathrm{mg} / 100 \mathrm{~m} 1)$ were highly correlated $(r=-0.83, p<0.001)$.

our findings suggest that the spherocyte count is a valuable tool in the diagnosis and prognosis of $A B O-H D$. 
637

REDUCED THROMBUS FORMATION WITH SILICONE RUBBER (SILASTIC) UMBILICAL ARTERY CATHETERS. Stephen J. Boros, Theodore R. Thompson, John W. Reynolds, Charles W. Jarvis, and Hugh J. Williams. Children's Hospital, St. Paul, Minnesota and Dept. of Ped., Univ. of Minnesota, Mpls., MN.

The complications associated with umbilical artery catheterization are well known. Catheter related thrombus formation has been observed in $90-95 \%$ of infants with indwelling polyviny chloride (PVC) umbilical artery catheters. This report describes clinical experience with a radiopaque silicone rubber (silastic) umbilical artery catheter. Twenty infants, 10 with PVC umbilical artery catheters and 10 with silastic umbilical artery catheters, all positioned at the aortic bifurcation, had pull-out aortograms performed at the time of catheter removal. Thrombi were observed in nine of the 10 infants $(9 \mathrm{C} \%$ ) with PVC umbilical artery catheters and in one of the 10 infants $(10 \%)$ with silastic catheters. The incidence of lower extremi ty vasospasm associated with the two catheters was not significantly different. Aortic pressure tracings recorded through silastic catheters were accurate, however, slightly damped. Autopsies were performed on five additional infants who expired with indwelling silastic umbilical artery catheters. None of the catheters, nor their surrounding tissues, showed evidence of thrombus formation on either gross or microscopic examination. It is our experience that radiopaque silastic tubing can be used as an umbilical artery catheter and appears to have the advantage of being less thrombogenic than the standard PVC tubing currently in general use.

"PREDICTED" NEONATAL MORTALITY RATE BASED ON BIRTH

WEIGHT AND GESTATIONAL AGE, Joseph V Brazie, Lula O.
Lubchenco, Univ. Colorado Med. Ctr. and Children's Hosp., Division of Perinatal Med., Denver

The "predicted" mortality rate can be calculated for neonatal populations, based on birth weight and gestational age. Utilizing the mortality rates for $250-$ gm birth weight/1-wk gestational age subgroups developed for babies born during 1956-1968 at the Univ. Colorado Med. Ctr. (J. Pediat., 81:4, 1972), the "predicted" neonatal mortality rate is calculated by the following formula:

$\frac{\sum\left[\left(\begin{array}{l}* \text { live births in each } \\ 250-g m / 1-w k \text { subgroup }\end{array}\right) \times\left(\begin{array}{l}\% \text { mortality rate } \\ \text { for each subgroup })\end{array}\right)\right.}{\text { total live births }} \times 10=\begin{gathered}\text { "Predicted" } \\ \text { neonatal } \\ \text { mortaly } \\ \text { rate/1000 LB }\end{gathered}$

The "predicted" mortality rate reflects the risk due to the birth weight/gestational age characteristics; whereas, the "observed" mortality rate reflects all factors. With these calculations, one can more critically evaluate the outcome of various populations. For example, during the years 1956-1968, the "predicted" and "observed" mortality rates in the UCMC reference neonatal population was $23 / 1000$. In 1972-1973, the "predicted" mortality was $16.6 / 1000$ and the "observed" rate was $13.6 / 1000$. This indicates an improvement in the risk of the population, based on birth weight/gestational age distribution (23 to 16.6), as well as an improvement in outcome related to other factors (16.6 to 13.6$)$.

639 FAILURE OF EXCHANGE TRANSFUSTON TO ALTER COURSE OR OUTCOME OF RDS AND VERY IOW BIRTY WEIGHT INFANTS.

S. A. Bustamante and K. E. Scott, (Intr. by R. B. Goldbloom). Department of Pediatrics, Dalhousie University and Grace Maternity Hospital, Halifax, Nova Scotia.

Exchange transfusion (E.T.) has been proposed for treatment of premature infants with respiratory distress syndrome (RDS) and those with very low birth weight (VLBW), since $E . T$. is known to increase $P_{50}$ and oxygen unloading from blood to tissue. Possible benefits of $\mathrm{E}$. $\mathrm{T}_{0}$ on the course and outcome of RDS and VIBW might also be related to other factors associated with mortality in these conditions, such as blood pressure (B.P.), total protein (T.P.) and $\mathrm{pH}$.

Forty prematures with severe RDS or birth weight $1250 \mathrm{gm}$ or less were assigned to either E.T. or control group by emvelope randomization. The groups were matched evenly for gestational age, weight and sex. Initial B.P., pH, hemoglobin and cesarean rate were similar in both groups. "The T.P. of the E.T. group was initially lower than the control.

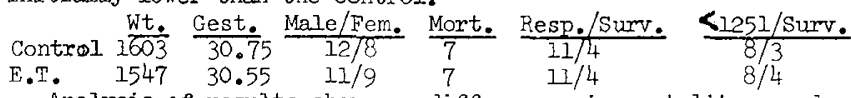

Analysis of results shows no differences in mortality, need for axtificial ventilation or respirator survivors, though there was an elevation in B.P., T.P. and pH after E.T. These elevations lasted approximately one day and made no clinical difference between the E.T. group and the controls.
640 VARTANCE IN ALBUMIN LOADING IN EXCHANGE TRANSFUSIONS George Chan, David Schiff, Depts, of Pediatrics, and obstetrics \& Gynecology, University of Alberta,

Edmonton .

Albumin loading prior to exchange transfusions has been used routinely in many newborn centres as an adjunct in the more efficient removal of bilirubin. The purpose of the present study is to compare the variance of albumin primed with non-albumin primed exchanges. 14 hyperbilirubinemic infants requiring exchange transfusions were given salt poor human albumin ( $1 \mathrm{~g} / \mathrm{kg}$ body weight) one hour before the exchange. The pre- and postalbumin values are respectively: bilirubin $16.7 \pm 1.4,17.1 \pm 1.2$, p 0.5 ; red cell bilirubin $1.33+0.15,0.96+0.16, \bar{p} 0.10$; reserve bllirubin capacity $4.8+1.1,6 . \overline{7}+0.8, p 0 . \overline{2}$; saturation point $21.4+1.2,23.7+1.0$, p $\overline{0} .2$; albumin $3.2+0.1,3.5+0.1$, p 0.1 ; biliz̄ubin-albumin molar ratio $0.82+0.0 \overline{5}, 0.82+0.04, \mathrm{p} 0.5$. The pre, post and 4 hour post exchange bilirubin concentrations are followed in these infants as well as in 29 exchanged jaundiced babies receiving no albumin. There is no statistical difference between the two groups: pre exchange $14.8+0.9$ vs. $16.5+1.3$, p 0.3 ; post exchange $7.3+0.4$ vs. $6.7+0.5, \bar{p} 0.3 ; 4$ hours post exchange $11.6 \pm 0.6$ vs. $12.1+1.2, p 0 . \overline{5}$, for the non and albumin primed infant $\bar{s}$ respectivel $\bar{y}$. The amount of bilirubin removed is slightly higher in the primed than its non-primed counterpart $(26.0+2.5$ vs, $23.4+2.1, p 0.4)$. The above data indicates that albumin infusion of $\mathrm{l} \mathrm{g} / \mathrm{kg}$ offers little, if any, advantages in the treatment of hyperbilirubinemia and in the removal of bilin rubin.

FOAM STABILITY TEST ON GASTRIC ASPIRATE AND THE DIAG641 NOSIS OF RESPIRATORY DISTRESS SYNDROME (RDS). Richard M. Cowett, Edward J. Unsworth, David O. Hakanson, John R. Williams, and William oh. Brown University Program in Medicine, Women $\&$ Infants Hospital of Rhode Island, Department of Perinatal Medicine, Providence, R.I.

The lecithin sphingomyelin ratio $(L / S)$ and foam stability test (FST) in amiotic fluid (AF) are reliable indices for the prediction of fetal lung maturity and the diagnosis of RDS. If $\mathrm{AF}$ were unavailable, gastric aspirate (GA) collected at birth may be utilized as an alternative biological fluid for the FST. GA was collected in 57 infants within ten minutes after birth and the FST and L/S were performed independently by different investigators and correlated with the incidence of RDS. Twenty-seven paired $A F$ were also analyzed for comparison with GA in reference to FST, L/S, and $\mathrm{pH}$. Similarity of mean GA and $A F \mathrm{pH}(\mathrm{GA}=7.71$; $\mathrm{AF}=7.81$ ) suggest that $\mathrm{GA}$ represents $\mathrm{AF}$ swallowed immediately prior to birth. Of the 45 infants with positive FST on GA, one developed RDS. Eighteen paired AF also demonstrated positive FST. Of those with positive FST on GA, 16 of 19 had L/S greater than 2.0. In six infants with intermediate FST on GA and paired AF. three developed transient respiratory distress. In six infants with negative FST on GA and paired AF, all developed RDS. Both $\mathrm{GA}$ and $\mathrm{AF}$ in three of these infants had L/S of less than 1.0. These data indicate that GA FST is a reliable parameter for the assessment of fetal lung maturity and is useful in the prediction and diagnosis of RDS at time of birth in infants when ammiotic fluid is unavallable.

642 arTery CATHETER PLACEMENT. R.M. Cowett, G. Peter, D. O Hakanson, L.Stern, and W.Oh. Brown Univ. Program in Med., Women \& Infants Hosp. of Rhode Isiand, Providence, R. I. To determine the risk of "cannula sepsis" from indwelling umbilical arterial catheters(UAC) and the related indication for prophylactic antibiotics, 90 catheterized neonates with respiratory distress were divided into antibiotic(A) treated (Penicillin $50,000 \mathrm{u} / \mathrm{kg} /$ day and Kanamycin $15 \mathrm{mg} / \mathrm{kg} /$ day) and non-treated(NA) groups. Four of 76 peripheral venous(PV) and $1 / 70$ UAC blood cultures obtained at UAC insertion within 24 hours of 1 ife were positive. The infant with a positive UAC culture also grew the same organism (H.influenza) from the PV blood and was clinically septic. The other PV isolates were probable contaminants. PV and catheter blood (CB), and catheter tip(CT) cultures obtained on UAC removal (see Table) and review of $\frac{\text { Group }}{\mathrm{A}} \frac{\mathrm{PV}}{2 / 31} \frac{\mathrm{CB}}{0 / 28} \frac{\mathrm{CT}}{2 / 41}$ monstrated no cases of "cannula NA $1 / 20 \quad 10 / 21 * 4 / 29$ monstrated no cases of "cannula
sepsis." Although the number of (\#A of pos. culture/\# of cases) positive $C B$ cultures were significantly higher among NA than $A$ group $(p<.005) *, 7 / 10$ isolates were non-pathogenic. The inclderiee of positive blood cultures was not related to duration of UAC. Umbilical and nasopharyngeal colonization with gram megative becteria and Kanamycin-resistant Klebsiella species did not differ among the $A$ and NA groups. The data suggest that UAC is a satisfactory sampling site for blood culture on catheter insertion soon after bixth, that UAC even of prolonged duration appears to be unrelated to risk of infection, and the prophylactic antibiotics are probably not indicated for UAC placement. 
BIIIRUBIN BINDING OF CORD SERUM ALBUMIN, ADULT SERUM

643 ALBUMIN AND COMMERCIAL CRYSTALITNE ALBUMIN. Marilyn L. Cowger and Jung J. Lee, Albany Med. Col., Dept. of Ped., and State Univ. of New York, Dept. of Chemistry, Albany, NY There is some controversy concerning the ability of newborn albumin to bind bilirubin as efficiently as adult albuein. Inasmuch as adult albumin has been utilized for most binding studies and since albumin concentration is used clinically to estimate the ability of the newborn to bind bilirubin it is important to determine whether there are major intrinsic differences between the two. The bilirubin binding of cord serum albumin (CSA) and adult serum albumin (ASA) purified by a chromatographic technique was compared to Sigma crystalline human serum albumin (HSA). On a dry weight basis CSA showed a $6 \%$ lower UV absorption than ASA or HSA, but this difference disappeared following dialysis. The dialyzed preparations were compared with and without bilimuin using absorption, fluorometry and analytical ultracentrifugation. All 3 techniques showed the proteins to have only minor differences. CSA-bound bilirubin had an appreciable absorption at $490 \mathrm{~nm}$ (suggesting a bound lipid). Protein-bilimubin mixtures of the 3 were scanned in the ultracentrifuge using the $460 \mathrm{~nm}$ and $280 \mathrm{~nm}$ maxima. The extent of bilirubin binding was identical at bilirubin:protein ratios of $1: 1$ and $2: 1$. Not until a ratio of $3: 1$ was there any discemible difference with binding decreasing in the order of ASA $>\mathrm{HSA}>\mathrm{CSA}$. This difference barely exceeded the instrumental error. A bilirubin binding curve of CSA from a scanner study showed initial breaks on the curve at bilirubin:protein ratios of 0.5 and 1.0 . The break at 0.5 was very small.

EFFECT OF EARLY FEEDING IN LARGE FOR GESTATIONAL

644 AGE (LGA) BABIES. O. Chaivorarat and H.S. Dweck (Intr. by L. Gartner). Albert Einstein Col. of Med., Dept. of Ped., Bronx-Lebanon Hosp. Ctr., New York City.

Twenty term LGA babies were randomly assigned to early (E) or late (L) feeding groups and fed $20 \mathrm{cal} / \mathrm{oz}$ formula every $3 \mathrm{hr}$ starting at 3 and 12 $\mathrm{hr}$, respectively. The $\mathrm{L}$ group had a greater mean birth weight $(4,252 \pm$ $292 \mathrm{~g}$ vs $4,1010 \pm 172 \mathrm{~g}, \mathrm{E} ; \mathrm{p}<0.05)$. Heel stick samples for plasma glucose (glucose oxidase) were obtained prior to, as well as 30 and $90 \mathrm{~min}$ after, each feeding time for up to $21 \mathrm{hr}$ old. Prolonged fasting in the $\mathrm{L}$ group resulted in a progressive decline in mean glucose; significantly lower than the $E$ group at $12 \mathrm{hr}$ after birth (L $49 \pm 8.3$ vs $E 64 \pm 14.6 \mathrm{mg} \%$; $\mathrm{p}<0.02$ ). Mean 9 and $12 \mathrm{hr}$ fasting values of the $\mathrm{L}$ group were also significantly lower than the mean $3 \mathrm{hr}$ fasting glucose (pre-initial feed) of the E group. Mean of all glucose values from birth to $12 \mathrm{hr}$ old wos significantly higher $(p<0.001)$ in the $E$ group compared to the $L$ group. Both groups had higher mean glucoses $30 \mathrm{~m}$ in after the first feeding compared to the fasting samples. While each group had a similar proportion of glucose values $<40 \mathrm{mg} \%$ before their first feeding (E $4 / 29$ vs $L 16 / 91$ ) and fewer such values after their first feeding (E $4 / 144$ vs $L 4 / 77)$, the $L$ group had more than twice as many glucose values $<40 \mathrm{mg} \%$ during the first 12 hrs (E 8/116 vs L 16/91; $X^{2}=5.68, p<0.02$ )

These data suggest that early feeding of LGA infants at $3 \mathrm{hr}$ intervals tends to raise plasma glucose while low glucose values ( $<40 \mathrm{mg} \%$ ) ore more likely to occur when feeding is delayed.

645

RETINOPATHY OF PREMATUFITY: OBJECTYVE EVALUATICN OF INCIDENCE, NATURAL HISTORY ANL RESOLUTION BY FUNDUS PHOTOGRAPHY AND INTRAVENOUS FEUORESCEIN ANGIOGRAPHY. J.S. Curran, S.J. Cantolino, W.C. Edwards, T.C. Van Cader. Univ. Seventy-five premature infants weighing less than $2000 \mathrm{gms}$. managed with aortic blood gas monitoring were investigated by fundus fluorescein angiography and indirect ophthalmoscopy. Serial studies were performed to demonstrate small vessel changes in the fundi of infants from 680-1970 gms. Angiographic changes of retinopathy of prematurity were demonstrated in $46.6 \%$ of patients and all but one were reversible.

\begin{tabular}{crcccc}
\hline WEIGHT & AGA-\# & PTS & ABN ANGIO & SGA- $\$$ PTS & A.BN ANGIO \\
\hline 750 gms. & 1 & $1 / 1$ & 1 & $0 / 1$ \\
$751-1000$ gms. & 6 & $3 / 6$ & 9 & $4 / 9$ \\
$1001-1250$ gms. & 11 & $6 / 11$ & 9 & $5 / 9$ \\
$1251-1500$ gms. & 15 & $9 / 15$ & 3 & $2 / 3$ \\
$1501-1700$ gms. & 12 & $5 / 12$ & 0 & 0 \\
$1701-2000$ gms. & 8 & 0 & 0 & 0 \\
\hline
\end{tabular}

The "healing" process was characterized by an angiographic

barrier in the temporal retina proceeding to sinusoidal vessel

formation \pm hemorrhage and finally barrier penetration and vascu-

larization of the peripheral retina without residual over $17 \mathrm{mths}$.

Strict correlation of occurrence of vascular changes to acrtic $\mathrm{P}_{\mathrm{a}} \mathrm{O}_{2}$, need for ventilation, vitamin $\mathrm{E}$ levels, exchange transfusion or jaundice could not be demonstrated. Changes were more common in multiple births. Angiographic evidence will be presented documenting retinopathy of prematurity as a common developmental and non-specific reactive process of the immature retina.
646 DISTRIBUTION OF BLOOD FLOW WITH ACUTE ALKALOSIS AND ACIDOSIS. Donald V. Eitzman and Richard L. Ped., Gainesville. Bucciarelli. Univ. of Fla. Col. of Med., Dept. of

Distribution of blood flow was studied with radioactive spheres in 13 goats at the time of birth (post C-section) and in 10 goats age $1-37$ days. Injections were made 10-15 minutes after production of the experimental condition. Pressure was measured directly in the left atrium, femoral artery, and pulmonary artery. At the end of the experiment, individual organs and the entire carcus were ashed and counted. Resistance was calculated and cardiac output was checked by the Fick Principle. In all animals with acidosis, created by three techniques (hypoxia, lactic acid infusion and stress), there was an increased blood flow to the brain of $11 / 2$ to 3 times control values. The distribution of flow to the other organs during acidosis was variable with increased flow to the kidney and heart. However, respiratory acidosis produced decreased flow to the kidneys. The acidosis did not seem to have any uniformly deleterious effect on distribution of flow or cardiac output. Alkalosis, metabolic or respiratory, produced minor changes in distribution of flow and total cardiac output. The one consistent effect of increased flow to the brain with acidosis, regardless of the etiology, was essentially unexplained, but may contribute to the increased incidence of intracranial hemorrhage in acidotic low birth weight infants with either metabolic or respiratory disease.

647 aid For NEONATAL STATUS EVALUATION. W.D. Ekert (Intr. by D. Vidyasagar). Kinderklinik, Kreiskrankenhaus, D-703 Boblingen, Elsa-Branastromstr. 22, West Germany.

Method: A cardiorespirogram is recorded via 3 skin electrodes. It comprises two traces: 1) The cardiogram, a recording of beat to-beat heart rate 2 ) The respixogram, a respiratory trace recorded using the impedance method which is largely proportional to breathing volumes. Patient sample: Cardiorespircgrams were recorded from more than 350 children. These were for the most part premature infants and neonates who needed intensive care or observation. Recordings taken for purely diagnostic reasons lasted $80 \mathrm{~min}$. Cardiorespirograms from seriously ill children were often recorded over several days without interruption. Older children monitored were primarily patients with cerebral illnesses, poisoning or other serious illnesses. Cardioresoirograms were systematically recorded from 100 healthy neonates. We also monitored at random older healthy children, children with anemia, hypoxemia hypercapnia, acidosis, alkalosis, shock, heart failure, poisoning and various cerebral illnesses. Results: We found that highly abnormal cardiorespirograms were particularly common in cases of cerebral illnesses. Specific factors, such as blood gas variations or heart failure only affect the cardiorespirogram if the condition of the infant deteriorates noticeably. The cardiorespirograph is therefore an excellent aic for timely status evaluation. Furthermore, we attempted to devise a scheme for evaluating the cardiorespirogram. It appears feasible to apply a points nostic conclusions to be drawn.

CHEMORECEPTOR FUNCTION AND SLEEP STATF TN APNEA. $S$. Allen Fagenhol $z$, Kathleen $0^{\prime}$ Connel1, Daniel C.Shannon. Harvard Med.Sch., Mass.Gen. Hosp.,Dept.of Ped., Boston.

Sleep apnea has been associated with ventilatory unresponsiveness to $\mathrm{CO}_{2}$, so-called Ondine's curse. 10 infants beyond the immediate newborn period (3-11 weeks) with idiopathic frclonged sleep apnea $(>20 \mathrm{sec})$ and otherwise normal pulmonary function and 14 unaffected siblings of sudden infant death syndrome (SIDS) cases were compared to 15 age-natched controls. During natural sleep EEG, EKG, eye movements, impedance pneumogram, tidal volume by nasal pneumotachygraph and end-tidal $\mathrm{PCO}_{2}$ were recorded. The steady-state ventilatory response to $5 \% \mathrm{CO}_{2}$ in air and the transient response to $100 \% 02$ were determined in quiet and in rapideye movement (REM) sleep. The following were calculated: $\mathrm{VE}$ (ml/ $\mathrm{kg} / \mathrm{min}), \mathrm{f}(\mathrm{min}-1), \mathrm{PCO} 2(\mathrm{~mm} \mathrm{Hg})$, slope $(\mathrm{ml} / \mathrm{kg} / \mathrm{min} / \mathrm{mm} \mathrm{Hg} \mathrm{CO} 2)$, intercept $\left(\mathrm{PCO}_{2}\right.$ at $\left.\dot{\mathrm{V}}_{\mathrm{E}}=300\right)$, and $\Delta \dot{V}^{\prime} \mathrm{EO} 2\left(\%\right.$ change in $\dot{V}_{\mathrm{E}}$ on $30 \mathrm{sec}$ $100 \% 0_{2}$ breathing).

\begin{tabular}{|c|c|c|c|c|c|}
\hline \multicolumn{6}{|c|}{ Controls } \\
\hline & $V_{E}$ & & & & ept $\Delta$ \\
\hline & $211^{+} 62$ & $30.0+6.2$ & $35.4+2.2$ & & $36.4+1.9 \cdot-3$ \\
\hline & $233+87$ & $33.7+6.2$ & $34.8+2.1$ & 54 & $37.7+4.0$ \\
\hline
\end{tabular}

Each measurement for the infants with apnea and the SIDS siblings did not differ significantly ( $p>.02)$ from controls. It appears that prolonged sleep apnea in ycung infants may coexist with normal central and peripheral chemoreceptor function. 
INFLUENCE OF SLEEP STATE ON THE CONTROL OF VENTILA-

649

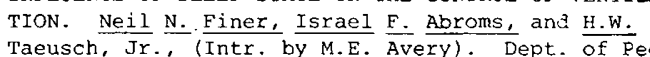
and Neurol., Harvard Medical School, Boston.

The response of premature and full-term infants to an endexpiratory airway occlusion is considered a measure of vagal and central control of respiration. We investigated the effect of sleep state on the occlusion response of 10 full-term infants (age $=32$ hrs., weight $=3200 \mathrm{gms}$. gest $=40$ weeks). The occlusions were pexformed with a mask and pneumotachograph and state was determined by continuous clinical observation, EEG, EOG, and chin EMG. An average of 17 single breath occlusions were obtained on each infant in each sleep state during the study period (2lihrs.).

Significantly greater percent increase in the inspiratory time (Ti) of the occluded breath compared to the $\mathrm{Ti}$ of the control breath was observed in Non-REM sleep compared to REM sleep $(+7.138$ vs. $-11.84 \%$ ) and there was a significant increase in the $\mathrm{Ti}$ of the occluded breath in Non-REM versus REM sleep (p<.01). There was no change in either the clinical state or EEG during an occlusion. Respiratory rate was significantly faster in REM compared to NonREM sleep (62/min vs. 50/min.)

State is an important variable in the interpretation of a newborn's response to an airway occlusion, and does not change during an occlusion. The greater percent increase of the occluded $\mathrm{Ti}$ observed in Non-REM sleep probably indicates increased responsiveness to vagal afferent input. There appears to be little if any volume related vagal input in REM sleep, hence the increased respiratory rate observed in this sleep state may be centrally mediated.

FAMILY CENTER:A COMPREHENSIVE PROGRAM FOR PREGNANT

650 DRUG-DEPENDENT WOMEN AND THEIR NEWBORNS. Loretta P. Finnegan, James F. Connaughton and Jacob Schut (Intr. by Maria Delivoria-Papadopoulos), Philadelphia General Hospital (PGH), Depts.of Ped.\& Obstet. Univ.Penna.Sch. of Med., Phila.,Pa.

A comprehensive program was developed at $\mathrm{PGH}$ in an effort to reduce the morbidity and mortality commonly seen amongst addicts and their passively addicted newborns. The program includes $O B$, psychosocial and addictive care for mothers in a hospital outpatient setting. To evaluate its success, 4 groups of patients who delivered at PGH were studied:Group $1(\mathrm{~N}=62)$ heroin dependent women, no prenatal care;Group $2(\mathrm{~N}=75)$ non-drug-dependent women, no prenatal care; Group $3(\mathrm{~N}=124)$ methadone maintained women with prenatal care (aver. 8 visits) and psychosocial counseling; Group 4 ( $N=75$ nondrug dependent women with prenatal care(aver.9 visits). OB complications occurred more in Group 1 than in any othex group. The difference in low birth weight infants $(<2500 \mathrm{gms})$ in Groups $1-4$ was significant: $478 ; 9.48 ; 17.88 ; 17.38(P<0.05)$. Mean birth weights for the groups were $2514,3011,2868,3044$ gms. Mean gestational age for Group 1 was 37 wks.and in Groups $2-4,39.5$ wks.Significant neonata problems (aspiration pneumonia, asphyxia neonatorum, congenital syphilis and sepsis) were seen more in Group 1 than 3. Withdrawal symptoms occurred in $95 \%$ of infants in Group 1 and in $90 \%$ of Group 3.Severe neonatal abstinence was less in Group 3 in comparison to Group 1 ( 158 vs 28\%). Neonatal mortality was 4.88 in Group 1 and $2.4 \%$ in Group 3. Average hospital stay in days for infants in Groups $1-4$ was: $27 ; 5.5 ; 17 ; 5.5$. These data suggest that administration of methadone with medical-psychosocial care for pregnant drug-dependent women reduces perinatal morbidity and mortality.

651 JAUNDICE. L.P.Finnegan, D. Reeser, J.F.Connaughton, and J.Schut. (Intr. by M.Delivoria-Papadopoulos)Phila.Gen. Hosp., Dept. of Ped.Univ. of Penna.Sch. of Med., Philadelphia,Pa.

Previous studies suggest that infants born to heroin addicted mothers have low levels of bilirubin(B) in the first 3 days of life.The present study evaluated the effect of maternal methadone usage on neonatal jaundice. Two groups were studied 1) 24 infants (S) born to methadone dependent mothers (avex.dose-27.5 mg/day) 2) 24 control infants (C) matched for birth weight (BW) and gestational age (GA). Mean BW was (S) 2752; (C) 2772 gms. Mean GA- 37.5 wks $S$ and $C$. Total serum $B$ determinations were done in the first 4 days of life. Infants predisposed to hyperbilirubinemia were excluded (e.g.hemolytic disease, sepsis). Of $24 \mathrm{~S}$ infants, 23 manifested symptoms of abstinence; 12 of 15 received phenobarbital or paregoric by the 2nd day of life.Jaundice occurred in 19 of $24 \mathrm{~S}$ infants and 18 of $24 \mathrm{C}$ infants. Mean total serum $\mathrm{B}$ levels of the $S$ and $C$ groups were not significantly different in the first 3 days of life.The difference at 96 hrs. was significantly greater in $C$ group than in $S(9.7$ vs 7.1$) p<0.01$. Determination of the mean peak total serum $B$ in $S$ group was $8.6 \mathrm{mg}$ and in $C$ group 9.7 $\mathrm{mg} z(p<0.10)$. The average age for peak $B$ in $S$ group was 59 hrs.; in $C$ group it was 71 hrs. $(p<0.05)$. B remained greater than $8 \mathrm{mg} \%$ for 2.3 days in both groups. Methadone significantly decreased 1) mean serum B level at $96 \mathrm{hrs}$. and 2) peak level and time of occurrence. Different pharmacological actions of heroin and methadone or the use of treatment medications for neonatal abstinence may be responsible for differences in the effect upon neonatal jaundice.
THE REGULATION OF RESPIRATORY PATE IN THE PREMATURE

652 InfANT. Ivan D. Frantz, III, Saul M. Adler, and Bradley T. Thach. Department of Pediatrics, Harvard Medical School, Boston, MA. (Introduced by M.E. Avery)

We have examined the effects of carbon dioxide and vagally mediated lung stretch-receptor afferents on respiratory rate in thirteen normal infants with gestational ages from 28 to 36 weeks at 3 and 10 days of postnatal age. All measurements were made with an infant face mask and pneumotachograph with the infant sleeping in a thermoneutral environment. The infants had an average resting respiratory rate of $51 \pm 13$ (SD) breaths/min which did not change with gestational age, postnatal age or steady state breathing of $\mathrm{CO}_{2}$ in air, although minute ventilation increased from $300 \mathrm{ml} / \mathrm{min} / \mathrm{kg}$ in air to $800 \mathrm{ml} / \mathrm{min} / \mathrm{kg}$ in $4 \% \mathrm{CO}_{2}$. Respiratory rate, unaffected by pulmonary stretch receptor influences, was examined by measuring the duration of inspiration following occlusion of the airway at FRC. The occluded inspiratory time $(0.75 \pm 0.27 \mathrm{sec})$ was greater than unoccluded, $10.51 \pm$ $0.11 \mathrm{sec}$ ) and was not influenced by gestational or postnatal age or $\mathrm{CO}_{2}$. During $\mathrm{CO}_{2}$ breathing tidal volume increased from 2 to 4 times resting levels with no decrease in inspiratory time. The vagus was known to be active because a $24 \%$ increase in inspiratory time was found following airway occlusion. We conclude from these studies that the respiratory center of the premature infant is under a maximal degree of vagal influence in the tidal volume range studied and that $\mathrm{CO}_{2}$ does not affect respiratory rate either through volume activation of lung stretch receptors or central stimulation.

653 THE USE OF CONTINUOUS NEGATIVE PRESSURE (CNP IN HYALINE MEMBRANE DISEASE (HMD): LATE CRITERIA. Juan J Sch. of Medicine in Shreveport, Louisiana (Intr. by J.A. Little). Application of distensibility pressures have apparently shown improved survival in HMD. Criteria for placement varies.

We performed a prospective 1 year trial of CNP in infants with $\mathrm{HMD}$ weighing $>\mathrm{I} \mathrm{Kg}$ and meeting the criteria of a $\mathrm{PaO}_{2} \leq 45 \mathrm{~mm}$ of $\mathrm{Hg}$ in $\mathrm{FiO}_{2}$ of 1.0 .

We studied 17 infants. Mean birth weight was $1885 \mathrm{gm} \mathrm{(1106-}$ 3175). Mean gestational age was 33.3 wks. (29-38). Mean age of placement in CNP was 27 hrs. (15-52). Duration of CNP therapy varied from 45 minutes to $240 \mathrm{hrs}$. Maximum pressures applied ranged from -7 to $-21 \mathrm{~cm} \mathrm{H} \mathrm{H}_{2} \mathrm{O}$ with a mean of $-13 \mathrm{~cm}$. Mean $\mathrm{PaO}_{2}$ before $\mathrm{CNP}$ was $32.8 \pm 8.9 \mathrm{~mm} \mathrm{Hg}$, and rose to $59.5 \pm 33.4$ within 40 minutes following application of CNP, a significant difference $(\mathrm{p}<0.005) . \quad \mathrm{PaCO}_{2}$ changes were not significant.

We successfully treated 8 infants with CNP alone. IPPB was required for 9 . Five died, 4 weighing less than $1.5 \mathrm{Kg}$. Two died of pulmonary hemorrhage, two had intracranial bleeding and one had both.

During the study, there were 11 additional infants who met the early criteria for CNP of a $\mathrm{PaO}_{2}$ of $<60 \mathrm{~mm} H$ in $\mathrm{FiO}_{2} \geq 0 \mathrm{f} 0.7$. (Fanaroff et al, J.Ped. 82:921,1973). They survived without requiring mechanical assistance and had no apparent sequela to the high $\mathrm{FiO}_{2}$. If these 11 infants are added to the surviving 12 infants of the CNP study, the survival rate would be about $80 \%$.

This study suggests that criteria for placement in CNP may reed further evaluation.

THE ROLE OF EXCHANGE TRANSFUSION IN THE MANAGEMENT

654 OF INFANTS WITH SEVERE RESPIRATORY DISTRESS SYNDROME (RDS): Michael A. Gottuso, Margaret L. Williams, Roger J. Shott and Frank A. Osk1. S.U.N.Y., Upst Center, Department of Pediatrics, Syracuse, New York.

Exchange transfusion (Ex. Tx.) as an adjunct to the management of infants with severe RDS was compared with conventional support alone or support plus the use of fresh frozen plasma $(15 \mathrm{ml} / \mathrm{Kg})$ in a group of 71 infants; 25 with birth weights of less than 1000 grams and 46 with welghts 1001 to 1960 grams. Of th1s group, 30 recelved Ex. Tx. at a mean age of 9.75 hours and 18 survived $(60 \%)$, whtle of the 41 managed with support alone or support plus plasma, there were only 6 survivors $(14.6 \%)$ (p <.002). In the group under 1000 grams, 6 of 11 exchanged infants 1tued $S$ or more days while 0 of 14 in the support groups survived. Measurements of $\mathrm{PaO}_{2}, \mathrm{FiO2}$, pH, f1brinogen and partial thromboplastin times before and after Ex. Tx. or at comparable periods in the plasma and support groups failed to demonstrate a consistent improvement in coagulation factors that could be responsible for increased survival. In infants receiving Ex. Tx., a rise in $\mathrm{Pa}_{2} 2$ could be demonstrated followIng Ex. Tx. when infants with comparable $\mathrm{FiO}_{2}$ 's were examined. Early Ex. Tx. appears to decrease mortality in infants with RDS. This effect cannot be attributed to improvement in coagulation alone but suggests that improvement in pulmonary perfusion or ventilation may also contribute to the effect. 
655 Alvin Hacke1, philip Sunshine, John D. Johnson, Stanford Univ. Sch. Med., Depts. Anesthesia and Peds. Stanford, Cal.

In 1971 a prospective study was initiated to evaluate various physiological indicators and the effect of factors involved in the transport mechanism on the survival of critically ill infants transported to Stanford Medical Center (SMC). From January 1972 through June 1974,462 infants under 1 month of age were transferred. The overall survival at 48 hours after transport in 1972,1973 and the first six months of 1974 was $80,85 \& 91 \%$. Survival at discharge from SMC was $63,73 \& 80 \%$ for the same time perlods. Analysis of survival by interhospital distance and mode of transport revealed insignificant differences. Of significance was the difference in survival for the variables: core temperature and arterial $\mathrm{pH}$ on arrival at SMC.

\begin{tabular}{|c|c|c|c|c|}
\hline Factor & $\#$ Infants & & vival & \\
\hline & & $\underline{1972}$ & $\underline{1973}$ & 1974 \\
\hline Temperature $<35.5^{\circ} \mathrm{C}$ & 54 & 37 & 38 & 67 \\
\hline Temp. $\geqslant 35.5^{\circ} \mathrm{C}$ & 406 & 68 & 78 & 83 \\
\hline $\mathrm{pH}<7.2$ & 87 & 33 & 46 & 44 \\
\hline $\mathrm{pH} \geqslant 7.2$ & 294 & 74 & 79 & 85 \\
\hline
\end{tabular}

These results indicate survival of infants transferred while critically 111 need not be affected by the transport mechanism, whereas body temperature and arterial pF at the time of arrival signlficantly influence survival.

THE HIGH-RISK PERINATAL REGISTRY: A PILOT

656 PROGRAM FOR PREVENTION OF PERINATAL DISEASE. R,G.Harper, S.Sokal, M.Sokal, and V.F. Mastrota, Depts.of Ped.\& ob.-Gyn.,No.shore Univ. Hosp. Manhasset,N.Y.\& Depts. of Ped.\& Ob.-Gyn.,Cornell Univ.Med.Col.,N.Y.,N.Y. (Intr. by Fima Lifshitz).

A High-Risk Perinatal Registry has been established to identify women who delivered stillborns or infants who died in the neonatal period and to prevent subsequent reproductive failures among these women. Using death certificates as a reporting system, all women who experienced perinatal loss in Nassau County in 1973 were identified. Abstracts of 342 perinatal records at 12 hospitals were compiled and reviewed by a panel of perinatologists and recommendations made for management during subsequent pregnancies. Recommendations included fetal monitoring, delivery at a perinatal high-risk center, elective premature cesarean section, and genetic counseling. The Registry success depends on the participation of the private practitioners: strategy for County-wide professional support was evolved to insure practitioner participation. Co-sponsorship was obtained from the local obstetrical and pediatric societies, cooperation from the State Health Department and local hospitals and local department of health endorsement. The High-Risk Perinatal Registry could serve as a prototype for a prospective approach to the prevention of perinatal disease.

657

IMPACT OF FETAL AND NEONATAL INTENSIVE CARE ON

MORBIDITY AND MORTALITY IN TWIN PREGNANCY.

Sze K. Ho and Paul Y.K. Wu, Dept. of Ped., Univ. of

So. Calif. Sch. of Med. and LAC-USC Medical Center.

Previous reports on twin pregnancies involved patients studied in an era when fetal monitoring and neonatal intensive care were either non-existent or in their infancy. This report is based on 177 pairs of twins born during the last 2 years at our institution with active fetal and neonatal intensive care units. Intrapartum fetal monitoring occurred in $36 \%$ of deliveries. Incidence of cephalic presentation in the first and second twin was $79 \%$ and $59 \%$ respectively. Midforceps or C-section were required in $29 \%$ of deliveries. Pre-term delivery occurred in $54 \%, 55 \%$ were $<2500$ gm. b.wt., and incidence of S.G.A. was $24 \%$. Fetalplacental wt. ratio was similar to that of singletons. Apgar scores, at 1 and 5 mins., were lower in breech deliveries and in second twins. Admission to special care nurseries was required in $51 \%$ as compared to an overall of $9 \%$ in all live births. Neonatal complications were: RDS $(29 \%)$, hypoglycemia $(10 \%)$, hypocalcemia (4\%), hyperbilirubinemia,>10 mg/100 $\mathrm{ml}(12 \%)$. Perinatal mortality was $107 / 1000$, which was 4.5 times higher than the overall incidence at our hospital, but lower than previous reports on twin pregnancies. Ninety-four percent of neonatal deaths were infants $<37$ weeks gestation, and the incidence of mortality by weight group was not higher than for the general nursery population. Since the outcome is directly related to prematurity, methods for prevention of premature labor should reduce the risks of twin pregnancy.
658

NEW TRANSPARENT INSULATING INFANT GOWN TO MAINTAIN THERMAL STABILITY Jean F. Hobbs, Nellie MacKuanying, Arthur I.Eidelman and Keith M. Schneider (Intr. by

Lawrence M.Gartner) Dept. Ped.,Albert Einstein Col. of Med. Bx. The need to develop adequate infant coverings for use in areas where exogenous sources of heat are unavailable or inadequate is recognized.Swaddling wraps of foil or double-layex air-pocket plastic are cumbersome and interfere with observation and monitoring. A practical single-layer transparent polyethylene gown was therefore designed to provide insulation. The gowned infants are easily handled and observed; breath and heart sounds are clearly audible by stethescope through the material. Adhesive strips permit close fit and reseal after insertion of catheters or administration of medications. Thermal effectiveness was tested in delivery rooms with mean ambient temps of $68^{\circ} .30$ full-term healthy newborns were randomly assigned to 3 groups.Group 1 was placed under radiant heaters servocontrolled at skin temp of $99^{\circ}$ to achieve core temp of $98.6^{\circ}$; group 2 was dressed in the polyethylene gowns; group 3 was gowned and placed under the heaters.Rectal and abdominal skin temps were recorded at 5 min intervals. Mean rectal temps over 30 min ranged as follows:

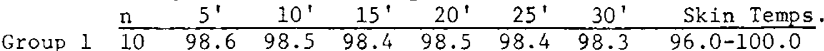

$\begin{array}{lllllllll}\text { Group } 2 & 10 & 97.8 & 97.6 & 97.8 & 97.7 & 97.8 & 97.9 & 95.4-95.7\end{array}$

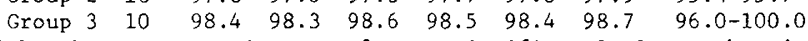
Although core temps in group 2 were significantly lower than in groups 1 or 3 (P<.01) gowned babies were maintained consistently within the range of thermoneutrality. Servocontrolled heating of infants in gowns did not result in hyperthermia.

A TRANSPORTER FOR ALL SEASONS, Leonard Indyk,

659 Robert Moffat, Alvin Hackel, (Intr. by Philip Sunshine) Coll. of Physicians and Surgeons of Columbia Univ., Dept. of Ped., New York; Stanford Univ. Sch. of Engr. and Med., Depts. of Mech. Engr., Anesth., and Ped., Stanford, Calif.

Critical design elements of an infant transport unit include provision of a neutral thermal environment (NTE), complete visibility of the infant, and easy accessibility without loss of heat, to allow continuous intensive care under difficult environmental conditions. We report here the results of an evaluation of the Stanford Mark III transporter using testing procedures which have been used in a previous evaluation of two commercial infant transporters (Indyk, L., Clin. Res. 20, 280(1972). The tests were run in a controlled environment raom at the NASA Ames facility. The temperature of a blackbody in the transporter was used to monitor the NTE. The transporter provides radiant heat by use of a transparent, open ended, semi-cylindrical, retractable heated cover. Results of the tests are as follows: 1) At external temperatures down to $9^{\circ} \mathrm{C}$, the unit was able to provide an NTE. 2) A simulated battery failure at $12^{\circ} \mathrm{C}$ resulted in a fall of $3 \mathrm{C}^{\circ}$ in 3 minutes. When power was restored, it took 7 minutes to attain the NTE. 3) With the heat shield retracted to provide an access slit $4^{\prime \prime} \times 20^{\prime \prime}$ alongside the infant (in addition to the open ends), NTE was maintained with external temperatures down to $14{ }^{\circ} \mathrm{C}$. 4) The battery lifetime under continuous power drain (temperature below $9^{\circ} \mathrm{C}$ ) was 120 minutes. These results are far superior to those previously reported for the commercial transporters.

660 TRANSCUTANEOUS MEASUREMENTS OF ARTERIAL PO2 IN SICK NEWBORNS. Leonard Indyk and L. Stanley James Coll. of Physicians and Surgeons of Columbia Univ., Depts. of Ped. and Anes., New York.

The ability to monitor continuously the arterial oxygen tension $\left(\mathrm{PO}_{2}\right)$ in sick newborns represents an important advance in the technology of intensive care medicine. A non-invasive method has been developed by Huch, Huch and Lubbers using a surface electrode which produces local hyperthermia. It has been tested by them in a number of different classes of well newborns and adult patients. We have had the opportunity to test the electrode on 6 sick newborns (850-3600 grams) in our neonatal ICU. Serial measurements have been made with the electrode placed on the chest for up to 6 hours with no lass of the clinical usefulness of the data due to drift. The electrade is a modified Clark cell which is affixed to the skin with an adhesive ECG ring. Calibration of the electrode is done in two water baths and takes about 3 minutes. Following initial placement on the skin, thermal equilibrium is attained within 15 minutes. Thereafter, the transcutaneous reading of the $\mathrm{PO}_{2}$ correlates within $5 \%$ of the arterial $\mathrm{PO}_{2}$ measured by gas analysis of an umbilical artery sample over a range of 40 to $130 \mathrm{mmHg}$. We have observed it to respond to very small changes in $\mathrm{Fi}_{\mathrm{O}} 2$ in infants on nasa! CPAP, within 30 seconds of the adjustment. The availability of continuous $\mathrm{PO}_{2}$ values by use of this non-invasive method should reduce the need for prolonged catheterization with the attendant problems and should further be of great benefit in starting and maintaining an infant on assisted ventilation. 
661 REFLEX ACTIVITY IN PHOTOTHERAPY TREATED JAUNDICED INFANTS. Kathy J. Kazmaier, Richard E. Butcher, William J. Keenan, Neil K. Edwards, and Harry D. Atherton. (Intr. by James M. Sutherland). Univ. of Cincinnati Col. of Med. Dept. of Ped. Neurologic performance of jaundiced infants treat
ed with phototherapy was compared with that of jaundiced infants not treated and nonjaundiced infants. Behavioral examinations were completed every 12 hours on 43 full-term jaundiced infants, 17 of whom were treated with phototherapy, and 12 nonjaundiced in fants. Three measures of non-nutritive sucking (NNS) activity were evaluated: (1) rate (sucks per second), (2) \% abnormal wave forms, and (3) \% tremorous activity during the pause between bursts of sucking. Significant intercorrelations (all $\mathrm{p}<.001$ ) among these measures suggest $a$ unitary and quantifiable reflex pattern which correlated with levels of serum bilirubin in our previous investigations. Records of hyperbilirubinemic infants demonstrate a disorganized pattern of NNS activity with rate being slower than that of nonjaundiced infants $(t=3.6, p<.005)$. Within the treated group NNS rate did not improve significantly from pre- to post-treatment records $(t=1.6, n s)$. Treated and untreated infants both improved slgnificantly by the termination of the study but did not differ in amount of improvement in NNS rate from time of peak bilirubin to termination $(t=1.6, n s)$ despite a greater fall in serum bilirubin in the phototherapy group $(t=3.7, p<.001)$. The failure of the phototherapy group to demonstrate a greater improvement in wNS could be related to a failure of phototherapy to ameliorate the neurotoxicity associated with hyperbilirubinemia, undetected dehydration, or a direct of fect of phototherapy upon the measured reflex.

NON-SIGNIFICANCE OF ACIDOSIS IN THE DEVELOPMENT OF

662 HYLLINE MEMBRANE DISEASE (HMD). John D. Kenny, James M. Adams, Anthony J.S. Corbet and Arnold J. Rudolph, Dept. of Ped., Baylor Col. of Med., Houston.

Acidosis at birth is believed to increase the incidence of HMD. To test this hypothesis, blood was collected from the umbilical artery at birth from 108 unselected premature infants and analyzed for $\mathrm{pH}, \mathrm{PaCO}_{2}$, standard bicarbonate and lactate. Gestational age was assessed by historical and physical data and the infants were followed until a definitive diagnosis was made of HMD ( 33 infants), Type II RDS (16) or the absence of respiratory distress (59). Infants without RDS demonstrated a positive correlation between Apgar score and gestational age $(\mathrm{P}<0.01)$ and a negative correlation between $\left[\mathrm{H}^{+}\right]$and gestational age $(P<0.01)$. HMD was more frequent $y i$ th decreasing gestational age $(P<0.001)$. Infants with HMD had lower Apgar scores than infants without RDS ( $\mathrm{P}<0.001$ ); however this could be due to lecreased mean gestational age in the HMD group. The values for $\left[\mathrm{H}^{+}\right]$in the three groups were: HMD $72.3 \pm 4.3 \mathrm{nM} / \mathrm{L}$ (mean $\pm \mathrm{SE}$ ), Type II RDS $63.1 \pm 2.4$ and no RDS $67.7 \pm 1.9$, however these values did not differ significantly $(P>0.2)$. There were no significant differences for $\mathrm{PaCO}_{2}$, standard bicarbonate or lactate in the three groups, eg. lactate in the HMD group was $4.95 \pm 0.53 \mathrm{mM} / \mathrm{L}$, Type II RDS $4.34 \pm 0.33$ and no RDS $4.53 \pm 0.33$ $(\mathrm{P}>0.4)$

We conclude that acidosis at birth does not contribute to the development of HMD even in infants of more advanced gestational age.

663 SUCCESSFUL TREATMENT OF "PERSISTENT FETAL CIRCULATION" WITH TOLAZOLINE. Sheldon B. Korones and Fabien G. Eyal (Intr. by Robert [. Summitt). Dept. of Ped., Univ. of Tennessee Ctr. for the Health Sciences, Memphis.

Five infants with severe hypoxemia refractory to respiratory therapy responded to intravascular tolazoline, $2.0 \mathrm{mg} / \mathrm{kg}$. All had normal chestfilm, EKG and no signs of heart disease. All were term infants (2350-4200 gms.), normal vertex deliveries. Four were meconium stained;Apgars were $\geq 6$. Severe tachypnea, cyanosis and transient mild retractions appeared within 3 hours after birth. Admission $\mathrm{PaO}_{2}$ wass 30 torr in 3,52 and 60 in 2 respectively; $\mathrm{PH} \leq 7.18$ in $4,7.28$ in $1 . \mathrm{PaCO}_{2}$ was $\leq 50$ torr in 4,61 in 1 .

Sustained correction of base deficit occurred after $1-2$ doses of alkali. Four babies responded transiently to respiratory therapy; 1 infant did not. Tolazoline was given when $\mathrm{PaO}_{2}$ was $<50$ torr ( $<30$ torr in 3 ). All were nor motensive. Pre-and postductal blood gas samples were taken 15 minutes after tolazoline. $\mathrm{PaO}_{2}$ rose to $>160$ torr in 3,144 in 1 and 51 in another. $\mathrm{PaCO}_{2}$ fell by $5-21$ torr in 4 , no change in 1 . Systolic blood pressure fell $5-20 \mathrm{~mm} \mathrm{Hg}$ in only 3 babies, returned to previous levels 15 minutes later. Hypoxemia did not recur. Tolazoline was repeated 6 times in 1 baby and once in 1 other, when $\mathrm{PaO}_{2}$ had fallen to 50-70 torr while decreasing respiratory therapy. After injection $\mathrm{PaO}_{2}$ rose to $<1605$ times and to 120 in 2 .

All survived. All breathed spontaneously in $\mathrm{F}_{i} \mathrm{O}_{2} 0.40$ with in $3-40$ hours. They breathed in room air 15-136 hours after tolazoline. Tolazoline maybe life saving for infants with hypoxemia due to persistent fetal pulmonary circulation.
TRENDS IN THE USE OF ELECTIVE ABORTION IN A GROUP OF

664 BROOKLYN HOSPITALS. Jonathan T. Lanman and Schuyler
G. Kohl. The Population Council, New York and Dept. of Obstetrics and Gynecology, Downstate Med. Ctr., New York.

The New York State abortion law was liberalized on 1 July, 1970. Major changes in the outcome of pregnancy appeared when a $3 \frac{3}{2}$ year period before change in the law (Period A) was compared with a one year period following (Period B). We wondered whether or not these changes represented a transient surge of high use. Analysis of an additional $1 \frac{1}{2}$ year period (Period C: July 1971December 1972) shows that high use of abortion and the associated changes have continued and even increased, particularly among nonwhite, ward patients. The number of pregnancies terminated in hospital (by delivery or by spontaneous or elective abortion) rose by $51 \%$ in Period $\mathrm{B}$ and fell by $8 \%$ in Period $\mathrm{C}$. Elective abortions rose from a rate of $30.9 / 100$ pregnancies terminated in hospital in Period $B$ to 33.6 in Period C. Corresponding figures among white, private patients were 17.9 and 10.5 ; for non-white, ward patients, 37.9 and 46.8. Spontaneous abortions among all patients declined by $20 \%$ in period $\mathrm{B}$ and by a further $26 \%$ in Period C. The fraction of births made up of immature (500-1000 gm) infants, which had fallen by $36 \%$ in Period $\mathrm{B}$, rose by $11 \%$ in Period $\mathrm{C}$ but remained $27 \%$ below the fraction for Period $A$. We believe these data reflect largely a change from the use of illegal abortion, sometimes inadequately performed, in Period A, to abortions performed in hospital. The sharply higher and rising use of elective abortion by non-white, ward patients we believe reflects their relatively poor use of other contraceptive means and deficient community family planning resources.

A COMPUTER ASSISTED LOG FOR PERINATAL PATIENTS Sherry Loo, Bedford Bonta, Joseph Warshaw, Yale University School of Medicine, New Haven, Connecticut

Record keeping and long term surveillance of patient information is a major problem facing the regional perinatal center. Such records should reflect frequency of problems encountered, pa. tient disposition, utilization of facilities, and basic demographic, seasonal and medical profiles of such units. We have developed a basic computer-assisted system for maintaining and accessing a $\log$ of admissions to our Special Care Unit. This allows ready access to patient records for chart review of particular problems, provides regular basic mortality and morbidity figures for current and cumulative review, facilitates review of utilization of the unit and management trends, and has the capacity for expanded functions without deletion or duplication of data already on file. 20 items regarding labor, delivery, maternal and patient profile and patient disposition are included in the data base for each patient. 90 different problems are currently being tracked, but a greater number of diagnoses are extractable by selection of various combinations of problems. 21 major diagnostic and therapeutic procedures, including various types of surgery are also being monitored. All data is entered on two sides of a single, coded check sheet. Monthly summaries are easily and inexpensively obtained at a computer-time cost of approximately $\$ 1.00 / \mathrm{mo}$. Immediate access to cumulative data is more expensively available via a terminal located in the unit. Physician time spent in record keeping and retrieving is minimized, making the costeffectiveness of the system considerable.

66 DEVELOPMENT OF A MASS SPECTROMETER SYSTEM FOR CONTIN-

666 UOUS IN VIVO BLOOD GAS ANALYSIS. Sady Matalon, Arnold S. Leonard, Carl E. Hunt. Univ. of Minnesota, Depts. of Physiol., Surg., and Ped., Mínneapolis, Minnesota. (Intro. by Rolf R. Engel, Minneapolis).

A mass spectrometer system for continuous in vivo blood gas analysis has been developed and experimentally evaluated. The system consists of (1) a quadrupole mass spectrometer capable of continuously measuring the partial pressure of any gas with a molecular weight of 2-99 and (2) an in vivo blood gas catheter consisting of a semi-permeable membrane mounted on the tip of a 13inch long stainless steel cannula (SRI). Partial pressure of $\mathrm{O}_{2}$ $\mathrm{CO}_{2}$ and any three other gases can be monitored continuously and simultaneously. This system has been evaluated experimentally in dogs. The catheters were inserted in a femoral artery and advanced to the thoracic aorta. Following calibration with an IL $\mathrm{pH} /$ Gas Analyzer, $\mathrm{PaCO}_{2}$ was varied over a range of $12-50$ and $\mathrm{PaO}_{2}$ over a range of 43-498 mmHg. $\mathrm{PaCO}_{2}$ and $\mathrm{PaO}_{2}$ were continuously monitored for as long as 30 hours and multiple blood samples were obtained for simultaneous IL measurements. There was good correlation between in vivo and IL measurements, with $\mathrm{r}=.975$ for $\mathrm{PO}_{2}$ and $\mathrm{r}=.85$ for $\mathrm{PCO}_{2}$. There was no loss of correlation even after 30 hours. In addition to the obvious benefits of continuous $\mathrm{PaO}_{2}$ and $\mathrm{PaCO}_{2}$ monitoring in patients with critical cardiorespiratory insufficiency, the capability of also monitoring other blood gases (i.e. $\mathrm{PaN}_{2}$ ) will permit detailed assessment of ventilation-perfusion inequalities whenever simultaneous end-tidal measurements are also available. 
667 EFFECT OF PHOTOTHERAPY ON THROMBOPOIESIS. HaTOId M. Maurer, Melvin J. Fratkin, Joyce C. Haggins, Nancy $B$. Pediatrics and Radiology, Richmond, Virginia.

Incorporation of 75 selenomethionine $\left({ }^{75} \mathrm{SeM}\right)$ has been used to evaluate platelet production and survival in rabbits receiving conventional phototherapy. ${ }^{75} \mathrm{SeM}$ appears in circulating platelets after its initial incorporation into megakaryocytes. Partially shaved New Zealand white rabbits, caged individually in plexiglas "isolettes", were illuminated under phototherapy units $(820 \mathrm{wt}$ lamps) continuously for $96 \mathrm{hrs} .6$ rabbits received blue light (F20 $T 12 / B), 6$ received Daylight $(F 20 T 12 / D)$, and 6 received no therapy. Cumulative light dose at $460 \mathrm{~nm}$, determined with a Beckman light monitoring badge at $24 \mathrm{hr}$. intervals, was: (Joules/ $\mathrm{cm}^{2} /$ day) blue, 92.6; Daylight, 56.4; no therapy, 2.3. In vivo incorporation of $75 \mathrm{SeM}$ into platelets was measured serially after $I . V$. injection of isotope. In controls, peak ${ }^{75} \mathrm{SeM}$ incorporation into circulating platelets occurred 3 days after injection and platelet life span was 6.5 days. In the blue light group, incorporation of
$75 \mathrm{SeM}$ occurred earlier (peak, day 2), platelet uptake of 75 Se was significantly increased, and life span was shortened to 5.4 days. In the Daylight group, time of peak incorporation and life span were similar to controls but ${ }^{75}$ SeM uptake was increased to levels seen with blue light. Serial mean platelet counts, however, were not significantly different among the 3 groups. These observations suggest that phototherapy increases the rate of platelet production and shortens platelet life span in direct proportion to irradiance at $460 \mathrm{~nm}$.
EFFECT OF PHOTOTHERAPY ON PLATELET COUNTS IN LOW

668 BIRTH WEIGHT (LBW) INFANTS. Harold M. Maurer, NanCy B. MCWilliams, Cheryl Hunter. Dept of Pediatrics, Medical College of Virginia, Richmond, Virginia.

In a companion abstract submitted to this society, we report studies which show that conventional phototherapy stimulates platelet production and turnover, and shortens platelet life span in rabbits, in direct proportion to irradiance at $460 \mathrm{~nm}$ (blue light> Daylight). In this study, the effect of phototherapy on platelet counts in $L B W$ infants was determined. Infants weighing $<2000$ gms were randomized at 24 hrs +12 of age to receive either Daylight phototherapy (8 20 wt lamps, F20T12/D) continuousIy for $96 \mathrm{hrs}$. or no therapy as part of a cooperative longitudinal study. platelet counts were performed daily in both groups. Infants with positive Coombs tests or sepsis were excluded. Daily Mean platelet count $\times 10^{3} / \mathrm{mm}^{3}+S E M$

Control (26) $\frac{\text { Initial }}{215+16} \frac{1}{210+14} \quad \frac{2}{214+15} \quad \frac{3}{228+17} \quad \frac{4}{228+16} \quad \frac{5}{231+14}$ $\begin{array}{lllllll}\text { Treated (31) } 218+10 & 209+14 & 219+16 & 208+15 & 208+16 & 230+18\end{array}$ Mean platelet counts were decreased on days 3 and 4 in the treated group, but differences were not significant. However, in 12 of the treated infants platelet counts fell below $150,000 / \mathrm{mm}^{3}$. whereas this occurred in only 3 of the controls. After phototherapy was stopped, platelet counts sharply rebounded to normal. These results are in accord with our findings in rabbits which suggest that phototherapy increases platelet turnover. We recommend that platelet counts be monitored during phototherapy, especially when they are initially $<200,000 / \mathrm{mm}^{3}$.

THE EFFECTS OF CONTINUOUS PROLONGED ILLUMINATION ON

669 NEWBORN PRIMATE RETINA. Alphonse E. Leure-Dupree, M. Jeffrey Maisels (Int by Nicholas M. Nelson). Penn State Univ. Col. of Med., M. S. Hershey Med. Ctr., Dept. Ophthal., Anat., Ped., Hershey, Pa.
A recognized potential hazard of phototherapy in the newbor A recognized potential hazard of phototherapy in the newbor fluorescent light. Previous animal studies are of questionable relevance to the human. The eyes of 3 newborn stumptail monkeys (Macaca arctoides) were continuously exposed to 400 foot candles of cool white fluorescent light for 3 to 7 days. No mydriatics were used. The eyelids of the right eye (control) were sutured closed and the eye was patched.

Fundoscopic examination of the experimental eyes was normal. The enucleated eyes were examined by light and electron microscopy. In all 3 animals electronmicroscopic observations of the experimental (left) eye showed substantial degeneration of the photoreceptors and diffuse changes in the pigment epithelium which included swelling of the mitochondria. However, there were no observable alterations in the smooth endoplasmic reticulum. The laminated disc of the photoreceptor in the experimental eye often appeared tubular and disorganized and was separated from the villous process of the pigment epithelium. The inner layer of the retina appeared structurally unaltered and Bruch's membrane and the choroid were unremarkable and similar in appearance to the control eyes which appeared normal. The extent of damage correlated well with the duration of exposure. These studies suggest that the eyes of infants undergoing phototherapy should be securely patched.

670 CLOSTRE MECHANISM OF THE UNBILICAL VESSEIS by W.W. Yeyer, J.Lind, and A.C.Yao, Path. Inst. Univ. of Hainz, Wermany and Dept.Ped. Karolinska Inst. Stockholm Sweden The normal structure and changes in the umbilical vessels during their occlusion were studied in 50 noral deliveries. $10 \mathrm{~cm}$ sements of the urbilical cords were obtained at different time intervals after birth by a double-blade clamp. The umbilical ve-nous wall consists of concentricaliy arranged sheets of circular smooth musculature overlapping $\in$ ach other as series of cylinders of increasing size as seen in the cords double-clamped immediately after delivery. In the early circumscribed contractions of the vein, the fuscular sheets become folded and transformed into bunjles of tightly packed muscle cells. The bundles form diaphragnatic inden tation which narrows the iumen. A similar rearrangement of the musculature occurs with the circumscribed contractions of the arteries. Hovever, in the arteries the indentations protruding in the lumen consist of both the circular and longitudinal muscle layers. The contractions of the ambilical vessels considerably reduce the blood volume transported through them. This resizts in a lowering of blood pressure in the vessels distal to the flow oistruction and a reduction in wall tension. This leads to a progressive narrowing and shortering of the vessels due to their elasticity and contractility. And this shortening represents a secondary phenomenon and does not prove the conclusion that the closure of the umbilical arteries is primarily aue to the contraction of the longitudinal arterial musculature.

671 VERY LOW BIRTH WEIGHT INFANT. Stephen D. Minton, Reginald C. Tsang, David R. Brown \& William J.Keenan. Univ, of Cincinnati Col. of Med., Dept. of Ped.

Little is known about physiologic effects of phototherapy in small premature infants. In this prospective study, the incidence of apnea and bradycardia was recorded in a $820 \mathrm{gm}$. infant of 28 weeks gestational age during nineteen $4-\mathrm{hr}$. periods of phototherapy alternating with nineteen $4-h r$. periods without phototherapy. Phototherapy was begun at $18 \mathrm{hrs}$. of age for hyperbilirubinemia. Recurrent episodes of apnea and bradycardia began 2 hrs. 1ater. The mean number of episodes of apnea (no respiration $>20 \mathrm{sec}$.) and bradycardia (heart rate $<80$ beats/min.) per hr. with phototherapy $(2.14 \pm .314$, mean \pm 1 SEM) was twice the number of episodes without phototherapy $(0.87 \pm .142 ; t$ and paired-t, $p<0.01)$. The incidence of apnea and bradycardia did not change with age during the period of study, nor was it different between day (8am to $4 \mathrm{pm}$ ) and night (8pm to $4 \mathrm{am})$. There was no evidence of habitauation or col litioning. Analysis of available rectal (RT), skin (ST) and isolette temperature(IT) showed no effect of RT but did show that ST and IT were higher with phototherapy (ST 96.88 \pm $0.177^{\circ}$, IT $96.26 \pm 0.16^{\circ} \mathrm{F}$ ) than without phototherapy (ST $96.45 \pm$ $0.21^{\circ} \mathrm{F}$, IT $95.83 \pm 0.21^{\circ} \mathrm{F} ; \mathrm{p}<0.05$, paired-t; $\mathrm{p}>0.10$, Student's $\mathrm{t}$; ST and IT), suggesting pissible temperature-related effects as $a$ factor in phototherapy-aggravated apnea and bradycardia. It is suggested that, for small premature infants susceptible to apnea and bradycardia, these problems may be worsened by phototherapy.

672 SYSTEM IN THE NEWBORN. V.G. Muraligopal, I.J. Weinfeld, B. Zanini, L.A. Cabal and W.S. Tsai (Intr. by J. Hodgman), Dept. of Pediatrics, LAC-USC Med. Ctr., Los Angeles. Continuous instantaneous heart rate (HR) was studied in 67 neonates from I minute of life throughout the first hour. The group was comprised of 28 normal term (1 min Apgar 7), 26 depressed term (Apgar :6), 16 prematures ( $\leq 37$ wks;:2500 gms), of which 13 had Apgar 7 and 3 Apgar $\leq 6$. The initial HR in the normal term neonate was $175 \pm 28 \mathrm{bpm}$; rose to a peak of $189 \pm 12$ by $1.2+1.2$ min of age; fell by $20 \mathrm{bpm}$ from the peak in $5.6 \pm 3$ min and $\overline{f e}] 1$ $40 \mathrm{bpm}$ in $19.2+14.0 \mathrm{~min}$. The normal prematures had similar initial HR rising to a lower peak ( $p .01)$ in the same time period with slower decline $(p, .01)$. The depressed infants had a lower initial HR $(p .01)$, peaks later $(p .01)$ and had a slower rate of decline $(p .01)$. The depressed prematures had a slower response than the depressed term infants ( $p .01)$. V-shaped drops in HR (rapid fall in HR dropping 10-50 beats, lasting 5-10 seconds with a slower return to baseline NHR) were observed with greater frequency in term infants when compared to prematures $(p .02)$ and between normal vs. low Apgar term neonates $\left(p^{<} .01\right)$. We postulate that the initial rapid rise to peak, rapid decline to baseline HR and $V$-shaped drops are indicative of a mature autonomic system. In the premature and depressed infants there appears to be a dampened reactivity of the autonomic system with prolongation of sympathetic activity. This may be caused either by immaturity or asphyxia during labor. Further studies will be required to evaluate the potential prognostic value of these observations. 
673 IMMENOLOGIC MRASUREMENT OF METHADONE IN URINE: ITS Nathenson. Albert Einstein Col. of Med., Montefiore Hospital \& Med. Ctr., Dept. Pediatrics, Bronx, New York.

The observations that methadone withdrawal in the neonate may be protracted, recurrent or of very late onset suggested the need for a laboratory technique to help monitor the withdrawal state, along with clinical assessment. The identification of methadone in urine, utilizing a hemagglutination inhibition titration fulfills that need. Sixteen newborns whose mothers used methadone during pregnancy constituted the study group. Spot urines for methadone analyses were collected in the first days and then weeks following birth and detailed clinical observations were made for comparison. Titers of methadone in urine ranged from $1: 1280$ to $1: 2(\cong 3.0 \mathrm{ug} / \mathrm{ml}$ to $0.005 \mathrm{~kg} / \mathrm{ml})$. The following relationships were found: the amounts of methadone detected in the urine at the outset did not correlate with the drug dosage in the mother, the time of onset of syraptoms of withdrawal or the degree of symptoms observed; the most rapid rate of decline in titer occurred in the first few days and in most instances methadone was absent by about 14 days of life (range 7 to 21 days); the fall in urinary excretion was constant and progressive; a fall in titer to low levels $(1: 4$ or less) was consistent with abatement of symptoms and there was no recurrence of withdrawal or late onset in any infant once these levels were attained. The titration of methadone in urine is a useful adjunct in the management of neonatal withdrawal, particularly with regard to determining the length of time affected infants require medical observation.

THE EFFECT OF INTRAUTERTNE (IU) ANEMTA ON THE 674 OXYGEN AFFINITY (P50) OF FETAL BLOOD. M.Orzalesi and W.W.Hay, (Intr.by Charles D. Cook) Yale Univ. Sch。of Med。, Dept. of Ped。, New Haven, Conn, , USA and Univ. of Naples $2^{\text {nd }}$ Med.Sch., Chair of Neonatology, Naples, Italy。

The effect of IU anemia on the fetal blood $P_{50}$, the red cell concentration of 2,3-Diphosphoglycerate (DPG) and the relative proportions of adult ( $\mathrm{HbA}$ ) and fetal ( $\mathrm{HbF}$ ) hemoglobins was studied at birth in 18 infants with Rh incompatibility, with (8) and without (10) IU anemia (i.e.Hb<13g/100m 1). The $P_{50}$ and DPG content of cord blood were significantly $(p<.001)$ higher in anemic infants and correlated inversely $(r=\ldots 90)$ with the $H b$ concentration. The rise in $\mathrm{P}_{50}$ was due to an increase of the "Effective DPG Fraction"(EDPGF), i.c. the Function: DPG ( $\mu$ Moles/1 RBC) $\times[\mathrm{HbA}(\%)+0.4 \times \mathrm{HbF}(\%)]$. There was a linear correlation $(r=.95)$ between $P_{50}$ and EDPGF with a slope similar to that previously described in normal neonates (Pediatrics, $48: 857 ; 1971$ )。 These results indicate that the changes in blood $\mathrm{P}_{50}, \mathrm{DPG}$ content and EDPGF in fetuses subjected to ancmia are similar to those described in anemic children and adults. Supported by NIH Grant FR 00125 and CNR Grant 72.00273/ 43.

THE EFFECT OF AGAR FEEDING ON SERUM BILIRU-

675 BIN LEVELS OF LOW BIRTH WEIGHT INFANTS. M.Orzalesi, P.G.Savignoni, S.Nodari, L.Conca G.Marzetti, and S.Tambucci (Intr. by C.D. Cook). Inst. of Pediatrics - Univ of Rome Med. Sch. - Rome - Italy.

Early feeding with Agar can prevent neonatal jaun dice in full term infants, but some doubt remains concerning the beneficial effects of Agar in premature in fants. The present controlled trial was therefore performed on 32 infants with a birth weight lower than $2500 \mathrm{~g}$ and a negative direct Coomb's Test. After initial stratification according to gestational age and serum bilirubin level at 24 hours of age, the infants were randomly assigned to either the treatment or control group. Agar was started at $24 \mathrm{~h}$ and continued for 6 days at a dose of $100 \mathrm{mg} / \mathrm{kg} / \mathrm{f}$ eeding $(700 \mathrm{mg} / \mathrm{kg} / \mathrm{day}$ ). Blood hematocrit and serum bilirubin levels were measured at least daily, for the first 8 days. Statistical analysis of these measurements showed no significant differences between the 2 groups in the daily and maximal serum bilirubin levels. It is concluded that Agar fceding has no appreciable effects on hyperbiliru binemia of prematurity.

Supported in part by CNR contract $N^{\circ} 72.00273 / 43$.

\section{6}

WITHDRAWAL. THE CONSERVATIVE TREATMENT OF NEONATAL NARCOTIC

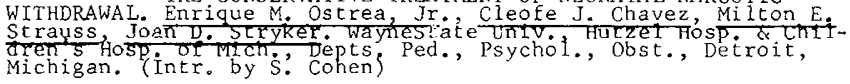

There are no established guidelines as to which infant un-

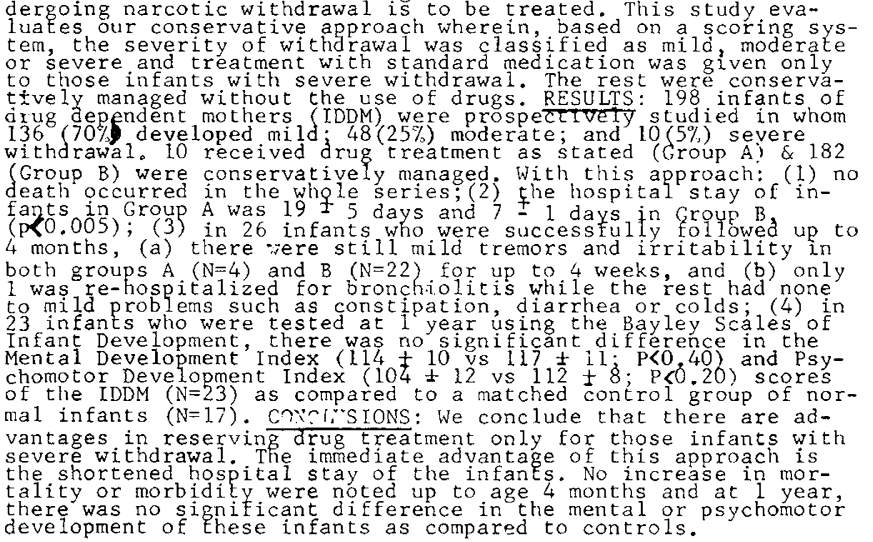

development of these infants as compared to controls.

677 The Effect of Intraamniotic Sodium Bicarbonate $\left(\mathrm{NaHCO}_{3}\right)$ on the Asphyxiated Fetus. John B. Paton, David E. Fisher, Ycai Roan and C. W. DeLannoy. Dept. of Ped. Michael Reese Med. Center, Chicago, Ill. Intro, by S. P. Cotoff.

The use of intraamniotic $\mathrm{NaHCO}_{3}$ to treat human fetal acidosis has been reported(Am. J. Obstet. rynec. 112, 834, 1972). To evaluate the effect of intraamniotic $\mathrm{NaHCO}_{3}$ on fetal asphyxia under controlled conditions, fetal acid-base status, umbilical blood flow (UBF) and organ blood flow distribution were studied in 18 baboons Fetuses asphyxiated without bicarbonate(group 1) showed a fall in UBF, metabolic acidosis and an increase in \%cardiac output (\%CO) to heart and brain. When amniotic fluid was partially exchanged for $\mathrm{NaHCO}_{3}$ (group 2) the fall in UBF and increase in \%CO to heart was similar to group 1 , however, the $\% \mathrm{CO}$ to brain did not increase in response to asphyxia. The metabolic acidosis was compensated in group 2 and post-asphyxia $\mathrm{HCO}_{3}$ was higher; fetal oxygenation showed better recovery than group 1. In group $3 \mathrm{NaHCO}_{3}$ exchange with amniotic fluid was performed but the fetus was not asphyxiated. $\mathrm{HO}_{3}$ levels in groups 2 and 3 were similar throughout but the volume of $\mathrm{NaHCO}_{3}$ necessary to maintain amniotic fluid volume was 2-3 times greater in group 2. Although respiratory movements were observed, swallowing as a mechanism contributing to the acute transfer of $\mathrm{HCO}_{3}$ to the fetus could not be demonstrated. $\mathrm{HCO}_{3}$ exchange occurs across the amnion and depends on an intact umbilical circulation for rapid transfer to the fetus and mother. Fetal asphyxia due to impaired maternal uterine circulation is not beneficially modified by intraamniotic $\mathrm{NaHCO}_{3}$; fetal and neonatal recovery may be compro-
mised by the resultant hypernatremia and the failure to improve cerebral blood flow.

COMPUTER-ASSISTED INTENSIVE CARE. Paul H. Perlstein, Neil K. Edwards, Harry D. Atherton, James M. Sutherland. Univ. of Cincinnati Col. of Med., Dept. of Ped.

A minicomputer has been programmed to aid in the intensive care of high-risk neonates. The computer provides 24 -hour on-line access to physiologic and environmental data and, using well-defined constraints, controls 6 incubators in 2 separate nurseries. The incubator control algorithm limits fluctuations in air temperature and protects the infant against escape from neutral thermal conditions. The mortality rate for 105 infants (birth wt. 400-3670gms) cared for using the computerized system was significantly reduced $\left(x^{2}=3.905 ; \quad p<.05\right)$ when compared to that of 105 matched high- $x$ isk infants cared for using standard non-computer-assisted techniques in the same nursery setting:

\begin{tabular}{lcc}
\multicolumn{1}{c}{ type of care } & died & total \\
computer-assisted & $24(22.8 \%)$ & 105 \\
standard & $37(35.28)$ & 105
\end{tabular}

The intergroup survival rates for the 14 pairs of infants weighing less than $800 \mathrm{gms}$ in this series were not different. The survival rates were different for the 91 pairs of infants weighing more than 800 gms $\left(x^{2}=6.787 ; p<.01\right)$. Although during the first week of life the daily mean caloric intake for standard care infants $[90.71 \mathrm{cal} / \mathrm{kg} / \mathrm{d} \pm$ (S. E.) 6.29$]$ was significantly higher than for the computer-assisted infants[56.83 cal $/ \mathrm{kg} / \mathrm{d} \pm(\mathrm{S} . \mathrm{E}) 5.18].(\mathrm{p}<001)$, the mean daily weight change for infants in the computer-assisted group did not statistically differ from that for infants in the standard care group, demonstrating the caloric sparing influence of the computerized system. 
MILK PROTEIN QUALITY ANO QUANTITY: BIOCHEMICAL AND

679 GROWTH EFFECTS IN LOW BIRTH WEIGHT INFANTS (LBWI). N. Raiha, D. Rassin, K. Heinonen \& G. Gaull. Dept. Ped., Univ. Helsinki; Dept. Ped. Res., N.Y. State Inst. Res. Ment. Retdn., Staten Island, and Dept. Ped., Mt. Sinai Sch. Med., CuNY.

Controversy about protein (prot) intake for LBWl has concerned quantity only. In this study 105 well LBWI ( $\leq 2100 \mathrm{~g})$ were grouped by gestational age $\left(T_{1}=28-30\right.$ wks; $\left.T_{2}=31-33 ; T_{3}=3<-36\right)$ and randomly assigned to pooled breast milk (BM) $(0.8-1.0 \%$ prot; whey prots:caseins, $60: 1: 0)$ or to one of four isocaloric formulae varying in quantity and quality of prot: $F_{1}=1.5 \%, 50: 40 ; F_{2}=3.0 \%$, $60: 1+0 ; F_{3}=1.5 \%, 13: 82 ; F_{4}=3.0 \%, 18: 82$. Caloric intake was 117 cal $/ 150 \mathrm{mI} / \mathrm{kg} /$ day for $\mathrm{F}_{1-4}$ and $\mathrm{BI}$ was fed at $170 \mathrm{ml} / \mathrm{kg} / \mathrm{day}$ to attain equal caloric intake.

No differences between feeding groups were found in weight gain or linear growth from time of regaining birth weight to discharge. BUN, total prot, and urine osmolality correlated directly with quantity of diet prot $\left(F_{4}, F_{2} \gg F_{3}, F_{1}, B M\right)$. Some sinall infants on $\mathrm{F}_{4}$ had prolonged metabolic acidosis. Prot quantity and quality affected amino acid levels: phe, tyr, met, thr, leu, ileu and val in plasma and urine and cystathionine in urine vere strikingly increased on high prot, especially $F_{4}$. Some small $F_{4}$ infants' plasma tyrosine remained 10-100 times that of BM-fed for several weeks. In general, plasma and urine amino acids were lowest on $B M$ (except taurine, which was highest for longer on BM). Biochemical parameters of $F_{1}$ resembled $B M$ most closely.

We suggest that previously recomended prot intakes for LBWI are probably too high, because protein quality has not been evaluated systematically.

VESICO-PUSTULES AND SCALY-COLLARED PIGMENTED MACULES

680

IN THE NEONATE, R.S. Ramamurthy, M. Reveri, N.B. Esterly, 0. Fretzin and R.S. Pildes, Chicago Med.Sch., Univ. of 111., Univ. of Chicago, Cook County Hosp, Michael Reese Hosp., Depts. of Ped. and Pathology, Chicago, 111.

The frequent observation of hyperpigmented macules and vesicopustular skin eruption in the neonate in our nursery led us to study the nature of these lesions. Lesions were seen in 23/515 $(4.4 \%)$ black and $1 / 145(0.6 \%)$ white consecutive births. Sex ratio was $1: 1$. The lesions were present at birth and involved the forehead, cheeks, neck, lower back and occasionally the trunk and extremities. The most frequent lesions were pigmented macules $(0.2 \mathrm{~mm})$. The vesicopustules present at the time of birth were superficial, ruptured easily, leaving a brown macule with a collarette of scale. Smear of the vesicle fluid showed predominance of polymorphs with occasional eosinophils. No organisms were found on smear or culture. Skin biopsies from 5 infants with the vesicopustules demonstrated subcorneal vesicle formation with acanthosis, edema and a mixed inflammatory infiltrate. A variable degree of edema, capillary dilatation and polymorphous infiltrate was noted in the dermis. No virus particles were seen on electron microscopy ( 3 infants). In 8 infants biopsies from hyperpigmented macules showed only hyperkeratosis and increased melanin in the epidermis. The lesions disappeared over a period of 3 weeks to 3 months. The clinical and histological findings were distinct from those of erythema toxicum and caused considerable concern on the part of physicians and parents of ten leading to work up for pustulosis. Etiology is unknown but the pigmented lesions could represent the healing stage of an intrauterine skin eruption.

THE ASSOCIATION BETWEEN MATERNAL DIABETES AND THE

681 RESPIRATORY DISTRESS SYNDROME. Marie F. RObert, Raymond $K$. Neff, John P. Hubbel $1, H$. William Taeusch, and Mary Ellen Avery, Dept. of Ped., Harvard Medical School. Dept. of Biostatistics, Harvard School of Public Health, Boston.

Cohorts of 815 diabetic and 10,153 non-diabetic pregnant women who delivered at Boston Hospital for Women from 1958-1968 were evaluated to investigate the disputed relationship between respiratory distress syndrome (RDS) in infants and manifest maternal diabetes (Am. J. Obstet. Gynecol. 111:826, 1971; Lancet. ii:577, 1973). The risk of RDS for an infant of a diabetic mother is 45 times the risk of RDS for an infant of a non-diabetic mother $(p<0.00001)$. After controlling for potentially confounding factors (gestational age, route of deliverv, presence and type of labor, birthweight, sex of infant, Apgar scores at one and five minutes, presence of hydramios, maternal anemia, and maternal age), the relative risk was found to be 6.2 $(p<0.00001)$.

Diabetes per se is an important predisposing condition from 31 to 38 weeks gestation regardless of route of delivery or presence of labor. Preliminary analysis suggests that with increasing severity of maternal diabetes, there is also an increased risk of RDS.

682 DISPLACEMENT OF BILIRUBIN IN CORD SERA BY GENTAMICIN AND CARBENICILLIN. David N. Schaffer, M. Jeffrey Maisels, Cheston M. Berlin (Intr. by Nicholas M. Nelson). Penn State Univ. Col. of Med., M. S. Hershey Med. Ctr., Dept. of Ped., Hershey, Pa.

The emergence of kanamycin resistant $\mathrm{E}$. coli has made the use of gentamicin essential in the treatment of neonatal sepsis. Recent studies have suggested that therapeutic levels of gentamicin in newborn serum may interfere with bilirubin-albumin (B/A) binding. We have studied the potential displacing effect of gentamicin on albumin-bound bilirubin by measuring the binding of bilirubin to red blood cells and mitochondria.

Red cell bound bilirubin was determined in whole cord blood at $\mathrm{pH} 7.4$ and $\mathrm{B} / \mathrm{A}$ molar ratios of 0.45 - 1.51 (serum bilirubin concentrations 14.9 - $50 \mathrm{mg}$. \%). No increase in red cel1 binding of bilirubin occurred with serum gentamicin levels of $0-20 \mathrm{\nu g} / \mathrm{ml}$. (toxic levels $>12 \mu \mathrm{g} / \mathrm{ml}$.). Lowering $\mathrm{pH}$ to 7.1 with $\mathrm{B} / \mathrm{A}$ molar ratio of 1.37 produced no change. Similar results were obtained with carbenicillin. In two very low birth weight infants, the addition of $20 \mu \mathrm{g} / \mathrm{ml}$. gentamicin to pre-exchange sera (bilirubin concentrations 11.7 and $13.0 \mathrm{mg} . \%-\mathrm{B} / \mathrm{A} 0.43$ and 0.47 ) produced no change in red cell binding.

Studies using rat liver mitochondria and cord sera at $\mathrm{pH} 7.4$ showed no effect of gentamicin on bilirubin binding to the mitochondria (B/A 0.93 and 1.04). These data suggest that therapeutic levels of gentamicin and carbenicillin do not displace bilirubin from albumin. MIDE. Seetha Shankaran and Ponald L. Poland, Dept. of Ped., wayne State Univ. Sch. of Med. and the Crilaren's Hospital of Michigar, Detroit, Mi.ch. (Intr. by Sanford N. Coberi)

Furosemide, a sulforamide ciuretic, ras beer. recommerced for clinical use in the newborn infant (Peciat. Clin. N. Amer. 18:1011, 1971 and Arch. Dis. Child. 46:520, 1971). It is extensively bound to plasma proteins (Clin. Chem. 20:152, 1974). We therefore studied the effect of furosemide on the albumin-bilirubin complex using two methods. Aliquots of whole blood from 20 icteric in:fants were diluted in phosphate buffer alcng with varyirg concentrations of furosemide $(5,10,20 \mu \mathrm{g} / \mathrm{ml})$ and the red cells and globulins were separated, washed and resuspended in human alburin solution. Bilirubin concentraticns were measured and the fraction of total bilirubin not on albumin was then calculated. In each case, the addition of furosemide resulted in more bilixubin bound to cells and globulin (cisplacement). The disflacirg power of furosemide $(20 \mathrm{jg} / \mathrm{ml})$ was 22 to 838 of that of sulfizoxazcle (Gartrisin) $200 \mu \mathrm{g} / \mathrm{ml}$. Secord, the hydroxyberzazobenzoic acid (EBREA) dye binaing test of Porter and waters (J. Lab. Clin. Med. 67:660, 1966) was performed using the sera of eight jaundiced newborn infants. The binding capacity varied from 55 to 848 of the control (human albumin). With the adaition of furosemide to a firal concentration of $2 \mu \mathrm{g} / \mathrm{ml}$, the dye binding capacity of the sera were recuced by up to $33 \%$ (miean $=138$ ) of the drug-free aliquots. As furosemide concentrations increased from 0.6 to $80 \mathrm{\mu g} / \mathrm{ml}$, there was a continuous decrease ir dye-binding capacity. Thus, furosenide may increase the incidence or severity of bilirubin neurotoxicity in cases of neonatal jatrdice.

THEOPHYLLINE TREATMENT FOR LOW-BIRTF WEIGHT INFANTS

684 WITH APNEA. Daniel C. Shannon, Felicita Gotay, Israel M. Stein, Nark C. Rogers, I. David Todres, Fergus $M$ E. Moylan. Harvard Med.Sch., Mass.Gen.Hosp., Depts. of Ped. and Anesth., Boston.

Seventeen low-birth weight $(\bar{x} 1.40 \mathrm{~kg})$ infants with recurrent apnea and cyanosis in the first week of life were selected for study. Cardiac and pulmonary disease, CNS hemorrhage, septicemia and hypoglyceria were excluded by appropriate tests. Apnea was detected and accurately quantified by a 13 hour respiratory recording using impedance pneumography. Nursing observations coupled with on-line alarm systems detected only $39 \%$ of severe apneic episodes ( $>20 \mathrm{sec}$.duration) as compared with the pneumogram technique. Theophylline, given as a $10 \%$ alcchol elixir by nasogastric tube every 6 hours $(1.5-4.0 \mathrm{mg} / \mathrm{kg}$ ) yielded 2 hour serum concentrations from 6.6 to $11.0 \mu \mathrm{g} / \mathrm{m} 1$ which completely controlled apneic spells exceeding $20 \mathrm{sec}$.duration, markedly reduced those of $10-19 \mathrm{sec}$.duration $(28.5$ to 5.6 spells per 13 hours, $p=0.002$ ) and eliminated bradycardia. When theophylline was discontinued in 5 infants, severe apnea and bradvcardia recurred within 48 hours requiring reinstitution of therafy. Then theophylline administration yielded 2 hour serum concentrations over $13 \mu \mathrm{g} / \mathrm{ml}$, excessive tachycardia $(>180 / \mathrm{min})$ and vomiting were observed. Serum half-life was over thice as long (19 hours) in those with toxicity requiring reduced dosage to achieve a serum concentration of $6-11 \mu \mathrm{g} / \mathrm{m} 1$. Acid-base status was normal (PCO2 $37 \mathrm{~mm} \mathrm{Hg}, \mathrm{pH} \mathrm{7.38)}$ and was not altered by therapy. Growth in weight was comparable to expected growth for birth weight. 
68 EFFECT OF IMMEDIATE TREATMENT OF THE HIGH RISK

685 NEWBORN IN A TRANSITIONAL INTENSIVE CARE UNIT. Raymond Stark and L. Stanley James, College of Physicians and Surgeons, Columbia University, Dept. of Ped., N.Y.C.

The impact of a transitional neonatal intensive care unit (TN) within the delivery suite, on the hospital course of neonates has been studied over a 12 month period during which the TN was open first for 8 hours, then 16 hours, and finally 24 hours per day. During this time 213 infants were treated in the $T N$, and subsequently went to the Neonatal Intensive Care Unit (NICU) where 199 remained for greater than 24 hours. Another 159 infants went directly from the delivery room to the NICU where 137 remained for greater than 24 hours. The mortality in the two groups who remained in the NICU for longer than 24 hours was $5.0 \%$ and $8.8 \%$ respectively.

The percentage of infants who remained in the NICU for 24 hours or less was $6.6 \%$ in the TN group and 13.8 in the group who came directly from the delivery room. Thus a period of care and observation in the TN is not only associated with a Jower mortality in the infants at highest risk, but also reduces the number of unnecessary transfers of infants in a lower risk category, resulting in the avoidance of unnecessary use of ICU bed space and of parental anxiety and expense.

686 THE CARDIOVASCLLAR EFFECTS OF APNEA IN PRETERM Dept. of Paediatrics, Univ. of Toronto and the Research Inst., The Hosp. for Sick Children, Toronto, Ontario, Canada.

We have studied the cardiovascular events during spontaneous apnea in four newborn preterm babies whose gestation varied from 27 to 34 weeks. Aortic blood pressure was continuously recorded via umbilical artery catheters. Peripheral blood flow was measured in the leg using a mercury-in-rubber strain gauge. Respiration was monitored with a pneumotachograph, impedance pneumograph or by means of chest magnetometers. Bradycardia frequently followed apnea sometimes as soon as two seconds after its onset and cardiac acceleration could also occur within two seconds of return of breathing. There were no consistent changes in mean blood pressure during apnea but the pulse pressure was sometimes increased. Periodic breathing was not generally reflected by any cardiovascular changes. Apnea led to a marked reduction (up to $88 \%$ ) in peripheral blood flow indicating a corresponding rise in peripheral vascular resistance. We could detect no changes in cardiovascular parameters preceding apneic episodes and conclude that the cardiovascular events are chemoreceptor responses secondary to the apneic asphyxia.
THE EFFECT OF EPIDURAL ANALGESIA ON MATERNAL, FETAL

688 AND NEONATAl ACrD-BASE BALANCE. Bertil Thalme, Patrick Belfrage \& Nils Raabe. Dept. of Pediatrics \& obstetrics, Karolinska Hospital, Stockholm. (Intr.by Charles D. Cook).

Twenty-four full-term primiparae and their babies were studied. Twelve received lumbar epidural analgesia with bupivacaine and 12 conventional obstetrical analgesia with meperidine, chlorpromazine, nitrous oxide and prilocaine. Fetal and maternal blood was sampled twice during labour and at delivery and neonatal blood four times during the first two hours after birth. $\mathrm{Ph}, \mathrm{P}_{\mathrm{CO} 2}, \mathrm{BD}_{\mathrm{Hb} 5}$, glucose and lactate were determined on all samples. The newborn infants were placed in incubators after birth and several clinical parameters recorded (activity, rectal temperature, heart and respiratory rates, Silverman score, reflexes).

Epidural analgesia to the mothers resulted in a lower degree of metabolic acidosis, mainly due to lower lactate values, than conventional obstetrical analgesia.

The clinical and blood chemical parameters recorded in this study indicated no harmful effects on the newborn infants after epidural analgesia to the mothers.

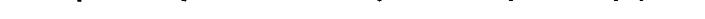

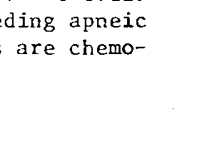


EFFECT OF THEOPHYLLINE ON PRIMARY APNEA OF PREMATUR:-

691 TY: A PRELIMINARY REPORT, Ricardo Uauy, Donald

Shapiro, Barbara Smith, Joseph Warshaw, Dept. Ped. Yale Univ. Scir. Med, New Haven, Connecticut

8 premature infants with severe primary apnea (at least two episodes requiring bag and mask ventilation) were treated with theophylline (ElixophyllineR) at a dose of $4 \mathrm{mg} / \mathrm{kg} \mathrm{q} 6^{\circ} \mathrm{p.o}$. as an alternative to assisted mechanical ventilation. The average birth weight for the group was $1356+114$ gms and the gestational age was $30.5+.73$ wks. The results were:

\begin{tabular}{|c|c|c|c|}
\hline & & & No. episodes \\
\hline & Total No. & No. Daily & Requiring \\
\hline & Apneic episodes & Apneic episodes & Bagging \\
\hline Pre theophylline & 73 & 8.13 & 2.87 \\
\hline During & 5.1 & 0.79 & 0.12 \\
\hline $\mathrm{p}$ & $<0.025$ & $<0.005$ & $<0.005$ \\
\hline
\end{tabular}
$\mathrm{P}$
Subsequent to therapy, no infants required respirator therapy. Blood glucose measured by DextrostixR was $45-90 \mathrm{mg} \%$ prior to and during treatment. There were no changes in blood pressure which could be attributed to the drug. There was a rise in heart rate from 152.5 beats/min to $167.5(p=<0.001)$. The rate of weight gain appeared to decrease while on therapy. In this initial trial, there was no attempt at randomization of patients for comparative purposes. Nevertheless the response to therapy seems sufficiently dramatic that a preliminary report is warranted.
694 PREDICTIVE RISK FACTOR FOR PNEUMOTHORAX IN NEWBORNS John F. Vogt, Luis A. Caba 1 and Bernardino Zanini USC Med. Ctr,, Dept. of Peds., Los Angeles, California.

Pneumothorax (PNT) is a serious complication of assisted vencilation (AV). To ascertain factors that may be predictive in infants at risk of PNT we studied 16 newborns (mean BW $1288 \mathrm{Gm}$, mean GA 29.6 wks) with $\mathrm{HMD}$, a 11 of whom underwent AV with the Baby Bird Respirator. On the basis of median $\mathrm{PCO}_{2}$ value prior to $\mathrm{AV}$ these babies were divided into 2 groups. Median $\mathrm{PCO}_{2}$ in G-I $(n=9)$ was $>50$ and in $G-I I$ was $<5$. There was no statistical difference in BW, GA, Apgar, Resuscitation, BP, Temp., age AV began, initial PIP, PEEP, Rate, Maximum PIP/PEEP or PIP at time of PNT. 5 of 7 babies in G-II experienced apnea prior to AV as compared with 1 of 9 in $G-I$. As compared to G-I, infants in G-II (low $\mathrm{PCO}_{2}$ group) developed interstitial emphysema earlier $\left(p^{=} .014\right)$, PNT occurred earlier $(p=.01)$, and the time difference between the attainment of the maximum PIP used and the development of PNT was shorter $(p=.036)$. The rate of increase in PIP tended to be more rapid in infants in G-II.

Infants in G-II appear to show better pulmonary compliance as evidenced by lower $\mathrm{PCO}_{2}$ prior to AV. Our data seems to support the hypothesis that the lungs of these infants in G-II are more compliant and during AV they are more distensible and more vulnerable to positive airway pressure. It is reasonable, therefore, to believe that with the more judicious use of positive pressure this complication of $\mathrm{AV}$ may be reduced in this group of infants.
NOISE CHARACTERISTICS OF RESPIRATORY DFVICES. Dharma-

692 puri Vidyasagar, Maurice F. Joseph and Lee R. Hamilton. Depts. of Ped. and Otolaryngology, University of Illinois Abraham Lincoln School of Medicine, Chicago, I1.

There is an increasing concern regarding the environmental hazards in the neonatal IrU. Te studied the noise produced by the respiratory devices using a Brüel \& Kjaer noise level meter in a quiet room. An endotracheal tube with a finger cot tied to the end served as a simulated lung, was connected to the respirator to be tested and was placed in the isolette. All respirators were tested with settings of: 1)respiratory rate of 302 ) peak pressure of $30 \mathrm{cmH}_{2} \mathrm{O} 3$ ) I.E. ratio of $1: 2$. Two CPAP devices, fregory and Carden's were studied similarly at $5 \mathrm{cmH}_{2} \mathrm{O}$ pressure. Results were: FREQUENCY $\left(\mathrm{H}_{Z}\right)$

\begin{tabular}{|c|c|c|c|c|c|}
\hline & MAI & BOURN & BABYBTRD & GRFCORYY & CARDFN \\
\hline A scale & 58 & 51 & 45 & 64 & 71 \\
\hline 125 & 54 & 61 & 56 & 51 & 51 \\
\hline 250 & 49 & 50 & 45 & 43 & 45 \\
\hline 500 & 43 & 44 & 33 & 39 & 56 \\
\hline $\mathrm{IK}$ & 54 & 47 & 32 & 37 & 63 \\
\hline $2 \mathrm{~K}$ & 48 & 39 & 38 & 40 & 62 \\
\hline $8 \mathrm{~K}$ & 39 & 25 & 29 & 64 & 60 \\
\hline
\end{tabular}

The data suggests: 1$)$ noise levels between the three respirators was not very different 2) noise in all respirators was high at low frequencies and low at high frequencies 3) CPAP devices tend to have relatively higher levels of noise at all frequencies. Since it is known that high noise levels of long duration have sessment of all devices used in the care of the sick neonate and b) auditory follow-up of these infants.

SCIEREMA NEONATORIM: EFFECTS OF EXCHANGES TRATS-

693 FUSION AND ASSOCLATION WITH LACTIC ACIDEMIA, Nadarasa Visveshwara, Vichien Lorch and Charles R. Bauer. (intr. by William W. Cleveland). Univ. of Miami School of Medicine, Dept. of Ped., Miami, Florida

Six neonates developed marked Sclerena Neonatorum associated with sepsis in three, These infants were all premature (30-35 weeks) with weights between 1000 and 2020 grams. Four of the infants were treated with a two volume exchange transfusion using fresh whole blood. Al1 4 survived. The remaining 2 were not exchanged and died. Lactic Acid levels were followed on 4 of the 6 infants as a reflection of the degree of tissue hypoxia. Two of the 4 infants exchanged had lactic acid levels of $110 \mathrm{mg} \%$ and $124 \mathrm{mg} \%$ prior to exchange. Post-exchange, diminution of the sclerema occurred at levels of $80 \mathrm{mg} \%$ and $75 \mathrm{mg} \%$ and disappearance occurred at levels of $18 \mathrm{mg} \%$ and $24 \mathrm{mg} \%$ respectively. The 2 infants that were not exchanged were similarly followed and the appearance of sclerema was just noted at levels of $82 \mathrm{mg} \%$ and 108 $\mathrm{mg} \%$. Blood pressures were maintained in the normal range in all infants. $\mathrm{P}_{\mathrm{a}} \mathrm{O}_{2}$ levels varied markedly and could not be related directly to the appearance of sclerema. This data suggests that exchange transfusion may be therapeutic in the correction of conditions such as tissue hypoxia, acidosis, etc. and can result in the disappearance of sclerema. Furthermore, it suggests that lactic acid levels are more valuable than $\mathrm{PaO}_{2}$ in determining deterioration, with approximately $80 \mathrm{mg} \%$ being the level at which sclerema appears or begins to clear.

* and severe hyaline membrane disease in the remaining three.
69 NEONATAL HYPERVISCOSITY: INCIDENCE AND EFFECT OF

695 PART IAL PLASMa EXCHANGE TRANSFUSION, Frederick $\mathrm{H}$. Wirth, Karen $E$. Goldberg, Lula $O$. Lubchenco, Univ. Colorado Med. Ctr., Division of Perinatal Med., Denver

During 1974, all inborn infants were screened for polycythemia during the first 4 hours after birth. $12.6 \%$ of the UCMC population had peripheral hematocrits $>70 \%$ (104 of 829 consecutive births). Of these $104,34 \%$ showed central polycythemia (Hct $>63 \%$ ). The incidence of hyperviscosity was $5 \%$.

22 infants with marked hyperviscosity were randomly assigned to receive early partial plasma exchange transfusion (within 8 hour after birth) or to receive conventional treatment. 2 in the latter group developed neurologic symptoms and were treated. 10 infants with normal blood viscosity served as controls. The Brazelton Neonatal Behavior Assessment was done at 6,24 and 72 hours and at 2-3 weeks of age. Infants with hyperviscosity in the first 4 hours showed lip cyanosis, lethargy, poor feeding and labile color changes with slow recovery. Hyperviscous infants who were not exchanged became more irritable by the $3 \mathrm{~d}$ day, showed paucity of movement, tremulousness and sustained clonus. They were rarely in an alert state and had difficulty in orienting to visual or auditory stimuli. At 2 weeks of age, symptoms persist ed and disturbed sleep was reported. Hyperviscous infants who received early partial plasma exchange transfusion differed from the control infants at 3 days of age, but were not as symptoma$t$ ic as the untreated group. By 2-3 weeks of age, the exchanged group was indistinguishable from the control group.

696 EFFECT OF PHOTOTHERAPY ON NITROGEN, ELECTROLYTE AND WATER BALANCE IN PRE-TERM INFANTS. Paul Y.K. Wu
and Amod S. Moosa. Dept. of Ped., Univ. of So. Calif. Sch of Med and LAC-USC Medical Center.

During phototherapy infants are frequently noted to pass loose stools. This may affect absorption of nutrients, and electrolyte and water balance. In order to investigate this, 24-hour metabolic balance studies were performed on 5 pre-term male infants ( $\overline{\mathrm{m}} \mathrm{b}$. wt. $1725 \pm 152 \mathrm{gm})$. Each infant was studied twice, once during phototherapy ( $\mathrm{m}$ age $3.8 \pm 0.5$ days) and once 48 hours after cessation of phototherapy. The results were compared to results of balance studies from 5 comparable infants without phototherapy.

When compared to the post-phototherapy period and nonphototherapy infants, during phototherapy mean intestinal transit time was decreased by $50 \%$, stool water loss was increased threefold and weight gain was less. Total $N$ retention and $\mathrm{N}$ retention as percentage of intake were decreased. Similarly, $\mathrm{Na}, \mathrm{K}$, and $\mathrm{Cl}$ retentions were decreased. There were no differences in serum osmolarity, electrolytes, serum water and rate of urinary output in all the infants during the study periods. The observed differences in these balance studies, as well as previous studies on insensible water loss, underscore the need for careful attention to fluid and caloric supplement during phototherapy. 
FACTORS AFFECTING VARIATIONS IN IRRADIANCE FROM

697 PHOTOTHERAPY LAMPS. Paul Y.K. Wu, Dept. of Ped. Univ. of So. Calif. Sch. of Med., LAC-USC Med. Ctr. Photobiologic responses and side effects of phototherapy may vary according to irradiance from light sources. A spectra (Mode T PR-1000) Photometer/Radiometer with filters to separate the following bands: $355-442,415-500,505-580,650-740$, above $770 \mathrm{~nm}$, was used to measure irradiance from a commercial phototherapy lamp inside an incubator (Ohio-304-3224-900), $8 \mathrm{~cm}$. above the mattress. Line voltage was controlled by a Variac (W20MT3A) autotransformer and recorded with a Rustrack recorder. Irradiance measurements were made at varying line voltages from 90 to 140 volts. Results at 124 volts were:

\begin{tabular}{|c|c|c|c|c|c|c|}
\hline Band Width $(\mathrm{nm})$ & Total & $355-442$ & $415-500$ & $505-580$ & $650-740$ & $>770$ \\
\hline $\begin{array}{l}\text { Energy }\left(\mu \mathrm{w} / \mathrm{cm}^{2}\right) \\
1 \text { Tayer Plexiglas }\end{array}$ & 727 & 237.77 & 259.13 & 342.80 & 35.99 & 3.24 \\
\hline $\begin{array}{l}\text { Energy }\left(\mu \mathrm{w} / \mathrm{cm}^{2}\right) \\
2 \text { layer Plexiglas }\end{array}$ & 686 & 217.46 & 233.70 & 316.65 & 31.75 & 2.80 \\
\hline
\end{tabular}

Irradiance was found to be maximum in the center of the area covered by the lamp and decreased progressively towards the periphery by as much as $30 \%$. Continuous variations in line voltage ( $1-5$ volts) were observed from wall outlet. Irradiance was found to vary linearly with change in line voltage. Since variations in line voltage may occur from place to place, and at different times from the same out Tet, these variations may affect the efficiency and side effects of phototherapy.
700 OF RENAL FUNCTIONAL MATURATION COMPARED IN THE HUMAN NEONATE. Billy S. Arant, Jr. (Intr. by James $\mathrm{N}$. Etteldorf), Univ. of Tenn. Ctr. Health Sci., Dept. of Ped., Memphis. The IP of renal functional maturation were described from clear ance studies performed in 53 healthy, normoglycemic infants within 48 hours of birth whose gestational ages(CA)were 25-4l wh and whose weights $(\mathrm{W})$ were $780-4150 \mathrm{Gm}$. Creatinine clearance $\left(\mathrm{C}_{\mathrm{cr}}\right), \mathrm{glu}$ cose $\left(U_{G} V\right)$ and alpha amino nitrogen $\left(U_{N} V\right)$ excretions and fractional reabsorption of glucose(TRG), phosphate(TRP) and $\operatorname{sodium}\left(\mathrm{T}_{\mathrm{Na}}\right)$ were determined for each period. $C_{c r}$ at birth increased with $C A$ and $W$ $(\mathrm{r}=0.70)$. From $25-34$ wk and $780-2500 \mathrm{Gm}, \mathrm{C}_{\mathrm{cr}}$ increased gradually from $0.22+0.16 \mathrm{ml} / \mathrm{min}(\mathrm{mean}+\mathrm{SD})$ to $0.57+0.21$. Thereafter a more rapid increase to $2.52+1.6 \overline{4}$ was noted at 40 wk. Mean values for $\mathrm{U}_{\mathrm{G}} \mathrm{V}(5.75 \mathrm{mg} / \mathrm{min} / 100 \mathrm{ml} \overline{\mathrm{G}} F \mathrm{~F})$ and $\mathrm{U}_{\mathrm{N}} \mathrm{V}(1.45 \mathrm{mg} / \mathrm{min} / 100 \mathrm{ml} \mathrm{GFR})$ were greatest, while $\operatorname{TRG}(8 \%)$ and $\mathrm{T}_{\mathrm{Na}}(97 \%)$ were least in the youngest and smallest infants, with values for $38-40$ week infants $\left(U_{G}, V^{0} 0.30+\right.$ $0.15 ; U_{N} V 0.44+0.28 ;$ TRG $99.7+0.3 ; T_{N a} 99.3+0.32$ ) achieved by $34 \mathrm{wk}^{-}$ and $2500 \mathrm{Gm}$, a period of morphological importance. $\mathrm{T}_{\mathrm{G}} / \mathrm{C}_{\mathrm{cr}}$ did not change $(0.56+0.1$.$) . TRP was always \geqq 85 \%$. In 8 of the smallest infants studied similarly 3-8 times at weekly intervals to observe E maturation, a P similar to IP was noted except that changes occurred earlier (32 wk) and at a lesser $\mathrm{W}(1500 \mathrm{Gm})$. TRP decreased and $\mathrm{U}_{\mathrm{N}} \mathrm{V}$ increased when oral feedings were introduced. Therefore, maturational patterns of $I$ and $E$ renal functions are similar. How ever, since the EP was noted to occur earlier than the corresponding GA or $W$ in the IP, it is suggested that physiological demands upon the E kidney hasten functional maturation.
698 PERINATAL MORTALITY: FIVE YEAR RESUlts AT THE LACUSC MEDICAL CENTER. Carl Yaeger and Richard H. Paul (Intr. by Paul K. Wu), Dept. Ob-Gyn and Pediatrics, LAC-USC Medical Center, Los Angeles, California.

Perinatal mortality was studied for five years, 1970 through 1974. There were 50,669 births during this time and 14,038 of these patients were evaluated with intrapartum electronic monitoring. Perinatal mortality fell from a rate of more than 40 per 1000 to 20 per 1000 over this period. The fall in the fetal death rate was largely attributable to a reduction in intrapartum fetal deaths. The neonatal death rate fell progressively over the five years in both full-term and low birthweight infants. It was of particular interest to note that there was a significant improvement in mortality in the low birthweight infants who were monitor ed during labor. The incidence of fetal monitoring rose from 18 percent to 33 percent over the five years. Indications for selective monitoring were those classicaliy associated with "hignrisk" maternal or fetal findings. The unmonitored patients were presumed to be "normal" or "low-risk". In spite of this selection bias, the monitored patients had a lower incidence of intrapartum death. It was of particular interest to note the significantly lower death rate in the monitored low birthweight infants as contrasted with those who were unmonitored. It seems reasonable to assume that intrapartum fetal monitoring provides useful information which, when properly used, can play a significant role in reducing perinatal mortality.

\section{NEPHROLOGY}

699 NEONATAL RENAL PAPIJLARY AND CORTICAL INECROSIS (RPCN) Sudhir K. Anand, James D. Northway, John A. Smith, (Intr. Ira K. Brandt), Indiana Univ. Sch. Med., Indiana Univ. Hosp., Dept. Ped. and Radiology, Indianapolis.

RPCN in the newborn infants in past has been generally fatal. This report describes 3 children who survived neonatal RPCN and have been followed from 23 to 57 months. The etiology consisted of exsanguination and shock in \#1; exsanguination and asphyxia neonatorum in \#2 and \#3. At onset kidneys were normal' on palpation in all 3 patients. Patient \#I had anuria for 6 days whil $\epsilon$ other 2 had hematuria. The highest BUN's recorded were 76,43 and $73 \mathrm{mg} \%$ respectively. Renal biopsy in \#l at $I$ month of age showed very severe meduliary tubular atrophy and replacement with fibrous tissue. Initial excretory urogram at I month of age in \#3 showed mild enlargement of both kidneys with bizzare appearin $\varepsilon$ calyces containing sloughed papillae.

At follow up \#1 and \#2 were hypertensive whereas \#3 had norma] blood pressure. All 3 patients had elevated BUN's. Creatinine clearance and maximum ability to concentrate urine was decreased in \#1 and \#2. Peripheral plasma renin activity was normal in \#I and increased in \#2. These studies have not yet been completed in \#3. Excretory urograms in all 3 patients showed dilated bizzare appearing calyces and renal cortical atrophy. These radiological findings during follow up of $\mathrm{RPCN}$ are similar to those found in renal dysplasia, segmental hypoplasia or so-called chronic pyelonephritis.

This suggests that young children who present with scarred shrunken kidneys with or without hypertension may have had neonatal RPCN.
SIMILARITIES OF RENAL BIOPSIES IN ANAPHYLACTOID

701 PURPURA (AP) ANID BERGER'S DISEASE (BD). Dinyar Bhathena and Nancy H. Holland. Depts. of Pathology and Ped., Univ. of Kentucky Med. Ctr., Lexington, Kentucky.

Similarities in histologic, immunohistochemical and ultrastruc. tural characteristics were observed in consecutive renal biopsies of 3 children with AP (age 5-14 years) and 4 with BD (age 9-19 years). Except for a teenage girl with $A P$, all patients were male The usual reason for referral was recurrent or persistent gross hematuria. Cne 5 year old boy with AP had severe multisystem disease but otherwise symptoms were mild or absent in both patient groups.

By light microscopy lesions in both diseases ranged from minimal, diffuse or local mesangial cell proliferation to focal tuft necrosis with crescents. Mesangial deposits of IgA considered diagnostic of $B D$ were also found by fluorescence microscopy in the biopsies of each case of AP. Mesangial deposits of IgG, $B\rceil C$, occasionally fibrin and rarely IgM were seen in both diseases. Ultrastructurally in all biopsies examined electron dense mesangial deposits were seen at the junction of the mesangium and lamina densa and occasionally extended into the mesangial stalk.

These observations invite speculation that $A P$ and $B D$ have a common pathogenesis, possibly involving glomerular deposition of qualitatively similar antigen-antibody complexes or immunoglobulin aggregates with subsequent activation of complement and the coagulation cascade. The differences may be quantitative with AP representing cases in which imune deposits affect a wider range of target-organ tissues.

702 SUANETHIDINE THERAPY IN CHRONIC HYPERTENSION OF 2 CHILDHOOD. Trevor Brock lebank, Joseph Mor, Barbara Dept. of Ped. and St. Louis Children's Hosp., St. Louis, Mo. 40 children, ages 3.5 to 20 yrs (mean $11.6 \mathrm{yrs}$ ), with chronic hypertension secondary to a variety of renal lesions have been treated with guanethidine and a low salt diet for control of blood pressure. The average period of follow-up on guanethidine was 21 months. In 20 of the patients guanethidine was used only after other hypertensive drugs had proven unsatisfactory. Cood blood pressure control was achieved in 17 patients using guanethidine alone given as a mean single daily cose of $0.6 \mathrm{mg} / \mathrm{kg}$ body wt. In 9 , taking a mean dose of $1.2 \mathrm{mg} / \mathrm{kg}$ body wt., 1 t was necessary to add a diuretic. In the remaining 14 a second hypertensive drug was required, apresoline being given as a single evening dose to provide control at night. Few children complained of symptoms which were attributable to guanethidine and in no case was it necessary to stop the drug. 4 had diarrhea, 5 postural hypotension and 2 complained of both these symptoms. Several children who felt weak and noted easy fatigability had a marked fall in blood pressure when examined during and after exercise. This troublesome complaint improved when the dose of guanethidine was reduced.

It is concluded that guanethidine has an important role in the treatment of chronic hypertension of childhood. The possibility of hypotension with exercise should be recognized. When controlling children on guanethidine the measurement of blood pressure during exercise enables one to minimize this complication. 
703 EFFECTS OF $1 \alpha$-HYDROXYCHOLECALCIFEROL $\left(1 \alpha-0 \mathrm{H}-\mathrm{D}_{3}\right)$ IN

703 CHILDREN WITH RENAL OSTEODYSTROPHY. Salvador Castelis, Florence Shai, Bernard Gauthier, Hector F. Deluca, Seyed-Ebrahim Hashemi and Churku Mohan Reddy, S.U.N.Y., Downstate Medical Center, Dept. of Pediatrics and Medicine, Brooklyn, N.Y. and Univ. of Wisconsin, Dept. of Biochemistry.

Renal osteodystrophy is considered to be due to a deficiency of the kidney to produce 1,25-Dihydroxycholecalciferol $\left(1,25-(\mathrm{OH}) \mathrm{D}_{3}\right)$ the active form of Vitamin D. The synthetic $1 \alpha-\mathrm{OH}-\mathrm{D}_{3}$, an efficient mediator of $\mathrm{Ca}$ absorption, exhibits a more sustained action than $1,25-(\mathrm{OH}){ }_{2} \mathrm{D}_{3}$ in animals, is probably hydroxylated by the liver and is cheaper to produce. Four renal osteodystrophy patients, one due to renal tubular acidosis, and three due to end state kidney disease requiring hemodialysis were admitted to the metabolic ward. After 1 week equilibration, three weeks of control metabolic $\mathrm{Ca}, \mathrm{P}$ and $\mathrm{N}$ balance were followed by three weeks of daily $2 \mu \mathrm{g}$ of $1 \alpha-\mathrm{OH}-\mathrm{D}_{3}$ orally. Ca balance increased during the treatment period by $79-460 \%:-104$ to $+237 \mathrm{mg} / \mathrm{d}$ during control period to +166 to $+538 \mathrm{mg} / \mathrm{d}$ during the treatment period. The increase resulted from net gastrointestinal absorption of $\mathrm{Ca}$. There were no significant changes in $\mathrm{P}$ and $\mathrm{N}$ balance. Basal serum $\mathrm{Ca}$ increased from the control range of $8-9 \mathrm{mg} \%$ to a range of $10-10.5$ $\mathrm{mg} \%$. Serum levels of parathyroid hormone (PTH) by immunoassay decrease from 576 to $392 \mathrm{pg} / \mathrm{ml}$ and from 1374 to $654 \mathrm{pg} / \mathrm{ml}$ in two patients. No changes in serum alkaline phosphatase were noted during the short period of therapy. The data indicates that $1 \alpha-$ $\mathrm{OH}-\mathrm{D}_{3}$ increased intestinal $\mathrm{Ca}$ absorption and decrease serum PTH levels. These observations suggest a role for $1 \alpha-\mathrm{OH}-\mathrm{D}_{3}$ in the managment of renal osteodystrophy. (NIH Grant RR-318)

704 CANDIDA ENDOCRINOPATHY SYNDROME WITH MEMBRANOPROLIFERATIVE GLOMERULONEPHRITIS (GN) . Russell W. Chesney, Har-
vey J.Guyda, Keith N.Drummond and Sean $0^{\prime}$ Regan.McGill Univ.-Montreal Children's Hosp. Research Inst., Depts.of Nephrology \& Endocrinology, Montreal, Canada.

A boy developed chronic mucocutaneous candidiasis at age 3 . Acute nephritis appeared at age 10 with $C_{3}=40 \mathrm{mg} / \mathrm{dl}$. Renal biopsy showed a lobular proliferative $\mathrm{GN}$ with basement membrane thickening. $C_{3}$ levels remained decreased $(\langle 50 \mathrm{mg} / \mathrm{dl})$. At 13 yrs the serum calcium was $\langle 7 \mathrm{mg} / \mathrm{dl}$ and phosphorus $>10 \mathrm{mg} / \mathrm{dl}$. PTH ( $450 \mathrm{u})$ increased renal phosphate clearance twelvefold.Serum 8 am cortisol was 5 $\mu \mathrm{gm} / \mathrm{dl}$ and failed to increase after ACTH stimulation.Gastric and thvroid autoantibodies were found.Candida albicans infection persisted. PHA stimulation, T and B cell count and MIF were normal.Serum anti-Candida antibodies were also found.

At 14 yrs acute nephritis developed with hematuria, $2.4 \mathrm{gm}$ proteinuria/day, $\mathrm{C}_{3}=40 \mathrm{mg} / \mathrm{dl}, \mathrm{C}_{4}=26 \mathrm{mg} / \mathrm{d} 1$. Renal biopsy again showed a lobular proliferative GN with chronic interstitial inflammation. Glomerular IgG and $C_{3}$ but not $C_{4}$ were seen on immunofluorescent microscopy. EM showed dense intramembranous deposits.Glomerular deposition of Candida antigen was detected by immunofluorescent staining with FITC-labelled antibody to the patient's Candida only after citrate elution. There was also $\mathrm{C}_{3}$ and anti-Candida antibody staining of some tubule basement membranes.

These data suggest that chronic Candida infection led to an immune complex form of chronic GN.Activation of the alternate complement pathway also appears to be present.

705 URINARY LDH ISOENZYME V (LOH-V) IN EXPERIMENTAL PYELONEPHRITIS. Robert J. Cunningham, and Hugo F. Carvajal (Intr. by Luther 8 . Travis), University of Texas Med. Br., and Shriners Burns Institute, Dept. Of Ped., Galveston, Texas.

Abnormally high levels of LDH- $V$ have been reported in the urine of patients with pyelonephritis and its measurement has been used to differentiate kidney from bladder infections. (Ped. Res., 8:180, 1974). To further validate such investigations, urinary LDH isoenzyme determinations were performed in 9 Sprague Dawley white albino female rats with surgically induced pyelonephritis and in 10 sham controls. Fasting $24 \mathrm{~h}$ urines were collected from each animal one day preoperatively and on days 1,4 and $n$ postoperatively. On the 10 th postoperative day the rats were sacrificed and their infection documented histologically and by means of kidney tissue cultures. In addition to LOH isoenzymes, the following urinary variables were also measured: Volume, osmolality (OSM), leukocyte excretion rate (LER), and sodium and potassium concentrations (Elect.). On days 1, 4 and 9 the pyelonephritis group disclosed urinary LOH-V levels 6-10 times higher than sham controls $(p<0.05)$. Likewise, urine OSM was lower $(1045$ vs 1400$)$ and LER higher $(14 \times 105 / \mathrm{h}$ vs $61 \times 105 / \mathrm{h})$ in pyelonephritis than sham controls $(p<0.05)$. No differences in volume or Elect. concentration were demonstrated between groups. These studies offer additional proof to the contention that pyelonephritis results in the excretion of large quantities of $L D H-V$ in the urine.
706 RECURRENT URINARY TRACT INFECTIONS (UTI's).

Robert S. Fenne11, III, Sandra G. Wilson, Norman D. Pryor, Car1 D. Sorgen, Eduardo H. Garin, R. Dixon Walker, George A. Richard (Intr. by Elia M. Ayoub), Univ. of Florida Col. of Med., Dept. of Ped., Gainesville.

Family members of children with long histories of recurrent UTI's were screened for the presence of bacteriuria. The affected children were participating in a home culture program using a semi-quantitative screening device (Bacturcult ${ }^{R}$ provided by Wampole Laboratories). The family members' urines were first cultured at home by one of the parents. Suspicious cultures were confirmed by pour plate technique independently in clinic. Approximately $20 \%$ of 141 families participating in the program screened other family members for a total of 98 primary relatives. No positive urines were noted in 24 fathers and 20 brothers. However, 3 out of 25 mothers (11.5\%) had positive urines confirmed independently in clinic. More significantly, 5 out of 28 female siblings (all under age 20 years) had positive home cultures confirmed independently in clinic for a rate of $17.8 \%$. Four out of the 5 children subsequently had IVP's and VCU's; 2 out of the 4 had significant reflux. Most of the 5 female children were asymptomatic. Enuresis or urgency were the only reported symptoms. Female siblings of children with recurrent UTI's should be screened for the presence of asymptomatic bacteriuria.

THE RESPONSE OF STEROID RESISTANT NEPHROTIC SYNDROME

707 TO NITROGEN MUSTARD THERAPY, B.P. Fine, R. MUNOZ, Jersey Med. Sc.S. Uy, M. Fathalla, and A. Ty, Dept.

Despite corticosteroid therapy some children with the idiopathic nephrotic syndrome either fail to respond initially or become nonresponsive after frequent relapses. The reports of treatment of these pts with cyclophosphamide have shown varied responses. We treated 4 early and 4 late nonresponders with another alkylating agent, $\mathbb{H N}-2$ (nitrogen mustard), $0.1 \mathrm{mg} / \mathrm{kg} /$ day times 4 days I.V.. The average age of these pts was 7.5 years (range 5-10). The renal biopsy showed minimal changes in 5 , structural changes in 2 , and no biopsy was done in 1. All with minimal change (and the one with no biopsy) responded within 2 weeks to $\mathrm{HN}-2$ with complete clearing of the proteinuria and the serum biochemical abnormalities. Four of these pts are still in remission (from 13-47 months). The other two relapsed after 4 and 6 months; both remitted after a second course of $\mathrm{HN}-2$ and then relapsed after 7 months. The two pts with structural lesions were early nonresponders. One had focal proliferative lesions and responded to $\mathrm{HN}-2$ with complete clearing of clinical symptoms and serum biochemical abnormalities; however, the proteinuria persisted. This pt remains in a partial remission 24 months later. The other pt had membranoproliferative changes and responded to $\mathrm{HN}-2$ on 2 occasions, each lasting 2 months. The advantages of $\mathrm{HN}-2$ thexapy are: low toxicity, ease of administration, and apparent response of some pts who are resistant to corticosteroids. These observations suggest that $\mathrm{HN}-2$ has a role in the therapy of idiopathic nephrotic syndrome of childhood.

CHANGES IN PROXIMAL TUBULAR FUNCTION \& STRUCTURE DUE

708 TO LEAD POISONING. B.P. Fine, A. Barth, R. Munoz, M. Fathalla and M. Lyons, Dept. of Ped. \& Path., New Jersey Med. Sch., Newark, N.J. (Intr. by F.C. Behrle)

Lead poisoning causes proximal renal tubular dysfunction as indicated by aminoaciduria and glycosuria. Since the glycosuria quickly subsides after chelation therapy, it appears to be different from the toxicity caused by other heavy metals. To measure the functional recovery after therapy three adult dogs were fed a diet containing lead acetate, and seven control dogs were fed a similar diet containing sodium acetate instead of lead. After 3 months the cumulative positive balance of lead was $16.6,5.1$, and $8.8 \mathrm{mg} / \mathrm{kg}$ of body weight in the 3 experimental dogs. The difference was due to the quantity of food ingested. The whole blood lead levels rose from $\angle 5$ to $40 \mathrm{ug} \%$. The glucose $\mathrm{Tn} /$ GFR was $2.45 \pm 0.22$ for the controls and $1.39,1.69$, and 1.84 for the experimental dogs. After 5 days of intramuscular EDTA, $12.5 \mathrm{mg} / \mathrm{kg}$, and BAL, $4 \mathrm{mg} / \mathrm{kg}$, the $\mathrm{Tm} / \mathrm{GFR}$ was $1.62,3.04$, and 2.93 respectively. These changes were due to increases in $\mathrm{Tm}$ values; the GFR values only changed slightly. There were cytoplasmic myelin figures, electron dense deposits, and loss of basilar infoldings in the proximal tubule cells after lead poisoning which diminished after therapy. The mitochondria were normal. These results indicate that proximal tubular dysfunction and structural changes are rapidly reversed by chelation therapy. 
CYCLOPHOSPHAMIDE (CY) THERAPY DURING ACTIVE DISEASE

709 AND DURING REMISSION IN RELAPSING NEPHROTIC SYNDROME. Eduardo H. Garin, Robert S. Fennell, William H. Donnelly, Norman D. Pryor, Car1 D. Sorgen, R. Dixon Walker, George A. Richard (Intr. by Elia M. Ayoub), Univ. of Florida Col. of Med., Dept. of Ped., Gainesville.

Twenty-three children with relapsing, steroid sensitive (SS), minimal change idiopathic nephrotic syndrome (MCHINS) were treated with an 8 week course of $\mathrm{CY} 2 \mathrm{mgs} / \mathrm{kg} / \mathrm{day}$. Patients were followed from 6 months (m) to $63 \mathrm{~m}$ with an average of $29.5 \mathrm{~m}$. Patients were divided into 2 groups. Group I included 15 patients who received $C Y$ after remission with prednisone $(2 \mathrm{mgs} / \mathrm{kg} /$ day) was achieved. Group II included 8 patients who received both drugs during relapse. Four patients in Group I relapsed, all within $6 \mathrm{~m}$ after $\mathrm{CY}$ was discontinued. Three patients in Group II relapsed, all within $8 \mathrm{~m}$ after $\mathrm{CY}$ was discontinued. In these 7 patients the frequency of subsequent relapses was similar to that noted before treatment with $\mathrm{CY}$. An association could not be demonstrated between these relapses and the age and sex of the patients or the duration of the disease before $C Y$ was begun. Life table analysis suggests that $74 \%$ of the patients in Group $I$ and $63 \%$ of the patients in Group II will remain in remission 4 years after discontinuation of $\mathrm{CY}$. An 8 week course of $\mathrm{CY}$ appears to prolong the duration of the remission in relapsing, SS, MCHINS. CY given during relapse appears at least as effective as when given after remission has been achieved with prednisone. 710 CHILDREN WITH HENOCH SCHONLE IN PUR PURA. JOSeph Giangiacomo and Cheng-Chang Tsai. (Intr. by A.E. McElfresh). Cardinal Glennon Mem. Hosp. for Children and St. Louis Univ. Sch. of Med., Dept. of Ped. and Path., St. Louis, Missouri.

Twelve patients between 1.5 and 12 years of age with Henoch Schonlein Purpura (HSP) were studied prospectively. Males predominated 2 to 1 over females. All patients had the characteris tic rash, $66 \%$ had joint involvement, and $50 \%$ had abdominal pain with or without melena. Urinalysis was abnormal in $41 \%$. During the acute illness $3 \mathrm{~mm}$. biopsies of the hemorrhagic skin lesions and uninvolved skin were examined by immunofluorescent against human IgG, IgA, IgM, $\mathrm{C}_{3}-\mathrm{C}_{4}$ complement and fibrinogen. Two patients had percutaneous kidney biopsies, which were also examined by the same technique.

The immunofluorescent pattern of involved skin showed granular deposition of IgA and $\mathrm{C}_{3}$ complement in dermal capillaries and interstitium of all twelve patients. The uninvolved skin reacted less intensely and only in dermal capillary walls to IgA and $\mathrm{C}_{3}$ The glomerular mesangium consistently fluoresced with IgA $3+$ and less to $\mathrm{C} 3$ and $\mathrm{IgG}$.

The immunofluorescent pattern of the skin is quite characteristic of HSP and it would appear that primarily IgA is involved in the mediation of the cutaneous vasculitis as well as the glomerulonephritis. There was no correlation of the pathologic changes of the skin with the degree of renal involvement as assessed by urinary protein excretion rate and glomerular filtration rate.

CHANGES IN RENAL RENIN SECRETOKY RATE (RRSR) AFTEH

711 ANGIOGRAYHY. Alan B. Gruskin, lictor $\mathrm{H}$. Auerbach, Iain F.S. Black and David W. Sapire. St.Christopher's Hosp. for Children, Dept. Fed. remple urif, Mied. Sch., Phila., Pa In order to investigate the mechanism of reduced renal blood flow (RBF) following anglocardlography, 22 determinations of RFSR were performed in 11 children with congenital heart disease. RRSR was calculated by multiplying the renal venoarterial differences in immunoreactive plasma renin activity ( $P R A$ ) by the mean renal plasma flow. RBF was determined following renal artery injections of $133 \mathrm{xe}$. Plasma flow was derived from the hict, and mean RBF. Prior to and $9-25$ minutes after injection of $1.2-3.3 \mathrm{ml} / \mathrm{kg}$ of cor trast media, RRSR was measured. Before angiography, arterial PRA averaged ?.I $\mathrm{ng} / \mathrm{dl}$ in cyanotics and $5.5 \mathrm{ng} / \mathrm{dl}$ in acyanotics. No significant differences in renal hemodynamics attributable to angiography were found between the 6 cyanotic children and the 5 children with acyanotic lesions. then evaluated as a group, injections caused the following changes: Hict. fell $8.4 \%$, mean fir decreased 26\%, arterial and renal vein PRA fell $38 \%$ and $35 \%$ respectively, and RRSR decreased $58 \%$. Decreases in RRSR after anglography might result from the effect of increased BF and intravascular volume on renal baroreceptors. Simultaneously, by actine as an osmotic diuretic, contrast media would increase sodium concentration at the macula densa. This effect would decrease nephron $\mathrm{RBF}$ by increasing intranephron release and consumption of renin. Accordingly, after anglography, the decreases in both REF and RRSR may be explained by the known physiologic factors involved in renin regulation. Supp. by $\mathrm{NIH}$ Grants HEI2651, RR75, and RR5624.
SODIUM BALANCE AND PERIPHERAL RENIN ACTIVITY (PRA) IN

712 CHILDREN. Linda B. Hiner, $H$. Jorge Baluarte, Mary L. Cote, Joseph Girone, Edward Rothstein, Ruth Schiller, Neil Schlackman, Howard Thomas, and Alan B. Gruskin, St. Christopher's Hospital for Children, Dept. Ped., Temple Univ. Med. Sch., Philadelphia, Pa.

PRA varies in response to changes in sodium ( $\mathrm{Na}$ ) intake in order to maintain body $\mathrm{Na}$ balance. Knowledge of $\mathrm{Na}$ input or output in Na balance is necessary for clinical interpretation of PRA. This study was designed as the first step to establish a pediatric nomogram relating PRA to $\mathrm{Na}$ excretion. Ninety-two normotensive caucasian children ages 3 to 17 years on ad lib $\mathrm{Na}$ intake provided $24 \mathrm{hr}$. urine specimens for determination of $\mathrm{Na}$ excretion. The $4 \mathrm{hr}$. upright PRA was determined. PRA declined with age $(.07 \mathrm{ng} / \mathrm{ml} / \mathrm{hr} / \mathrm{yr} ; \mathrm{p}=.04)$. Sodium excretion corrected for body surface area (BSA) did not vary with age. Thus, the decline in PRA with age is not related to an increase in $\mathrm{Na}$ intake. Only 5 and 2 children had $\mathrm{Na}$ excretion of less than 100 and $50 \mathrm{mEq} / 24 \mathrm{hr} /$ $1.73 \mathrm{~m}^{2}$, respectively. Although PRA tended to be inversely related to absolute $\mathrm{Na}$ excretion, $\mathrm{p}=0.07$, this tendency disappeared when $\mathrm{Na}$ excretion was corrected to $1.73 \mathrm{~m}^{2}, p=0.35$. Since so few children on ad lib diets excreted less than $50 \mathrm{mEq} / 24 \mathrm{hrs}$. $\mathrm{Na}$, the level at which definite increases of PRA occur, completion of the nomogram will require studying children on sodium restricted diets. We have studied the PRA of 7 children with "essential" hypertension on ad lib Na diets. Their PRA values do not differ from those of the 92 normal children. Supported by NIH Grants HE 12651, RR 75, and RR 5624 .

1 FAILURE TO DEMONSTRATE TRANSPLACENTAL PASS713 AGE OF A NATRIURETIC HORMONE. JOhn K. Hurley, Stanley E. Kirkpatrick, Paul T. Pitlick, William F. Friedman and Stanley A. Mendoza.

Sodium excretion of the fetal lamb (110-137 days
gestation) was studied serially in chronically instrumented animals. Cl4-inulin and I 131 -iodohippurate were used to estimate glomerular filtration rate (GFR) and renal plasma flow (RPE). The mean fetal GFR was $1.93 \pm$ 0.15 (S.E.) $\mathrm{ml} / \mathrm{min}, \mathrm{n}=79$ while the $\mathrm{RPF}$ was $2.62+0.40 \mathrm{mI} /$ min, $n=54$. Neither value changed significantIy with increasing gestational age. A saline load to the fetus caused substantial increases in both GFR $\bar{c}=0.69+0.15$ $\mathrm{ml} / \mathrm{min}, \mathrm{n}=10, \mathrm{p}<.001)$ and sodium excretion(0.24 To 0.54 megNa/15 min, $n=24, p<.005)$. A similar increase in GFR $(69-106 \mathrm{ml} / \mathrm{min}, \mathrm{n}=14, \mathrm{p}<.005)$ and a more marked natriuresis ( 1.17 to 15.03 meqNa/15 min, $n=39, p<.001$ ) was observed in the mother after receiving a comparable saline load. Of great interest was the absence of a change in fetal sodium excretion after maternal saline loading $(\bar{L}=-0.06+0.10$ meqNa/15 min, $n=16, p>.5)$. Thus, the fetus is capabie of responding to a fetal sodium load by a natriuresis. However, since the fetus did not respond to a maternal saline load, no evidence was found for a maternal natriuretic hormone capable of crossing the placenta. 714 CHRONIC HYDRONEPHROSIS (CHN). Robert A.Hutcheon, Keith N.Drummond and Bernard S.Kaplan.McGill Univ.-Montreal Children's Hosp. Research Inst., Dept. of Nephrology, Montreal, Canada Nine children who had $\mathrm{CHN}$ and metabolic acidosis were studied
determine whether the acidosis was a consequence of chronic renal failure alone or was also due to a distal renal tubular defect in $\mathrm{H}^{+}$secretion. Determination of the urine minus blood $\mathrm{pCO}_{2}$ concentration in alkaline urine - (U-B) $\mathrm{PCO}_{2}$ - is a simple test of distal tubular ability to excrete $\mathrm{H}^{+}$(Halperin et al, J.Clin. Invest. 53:669,1974). Patients with dRTA(Type I) have low $(U-B) \mathrm{pCO}_{2}$ values.

Seven boys and 2 girls, aged 2-17 yrs, with CHN had dR'TA as evidenced by hyperchloremic metabolic acidosis and urine $\mathrm{pH}>6.0$. Three had colonic/ileal ureteral conduits and 4 children had cutaneous ureterostomies. One boy had reimplanted ureters and 2 girls with major reflux have not been operated on. Serum creatinine concentrations were $1.0-3.9(\bar{x}=2.2) \mathrm{mg} \%$.

When given an oral $\mathrm{NaHCO}_{3}$ load of $1-3 \mathrm{mEq} / \mathrm{kg}$ to raise the urine $\mathrm{pH}$ to $>7.2$, the $(\mathrm{U}-\mathrm{B}) \mathrm{pCO}_{2}$ values were: 9 children with $\mathrm{CHN},\langle 12(-1$ to 12); 4 children with idiopathic dRTA, $\langle 12(0$ to 12$) ; 8$ normals, $>35$ ( 35 to 58 )

This is the first study documenting dRTA in many patients with CHN.Thus the metabolic acidosis seen in these patients is contributed to by an inability of the distal nephron to secrete $\mathrm{H}^{+}$as well as by other known causes of acidosis in patients with chronic obstructive nephropathy. 

715 HYPERTENSION IN PEDIATRIC RENAT TRANSPLANT PATIENTS. Iowrie, M. Topor, and W.E. Grupe. Children's Hospital Medical Center, Boston, Mass. 02115

To learn which children may be at risk for developing hypertension (HT) with its attendant morbidity a retrospective analysis was undertaken of the prevalence and etiolocy of $\mathrm{HT}$ in 52 renal homografts (TX) in 49 patients followed from 1 week to 3 and $1 / 2$ yrs. HT is defined as BP above 2 SD for age-approoriate norms. Rejection $(R)$ was associated with $H T$ in 29 , resolving after reversal of acute $R$ and continuing with chronic $R$. HT heralded recurrence $(\mathrm{RC})$ of original disease in 3. Nine had transient $\mathrm{HT}$ with high-dose steroids. Four had $\mathrm{HT}$ related to arterial disease, post-TX HT in one resolved when his native kidneys were removed. one $3 \mathrm{yr}$. old, nephrectomized (NX) pre-TX, had HT with no demonstrable cause. Only 7 have never had HT after the initial postoperative period.

Etiology of renal failure may influence development of post-TX HT. In 13 with renal dysplasia, only 3 had ore-TX HT; post-TX 4 have never been HT and 3 others only transiently with steroids. of 5 with obstructive uropathy, 3 have been normotensive and 2 only transiently HT with steroids. In contrast, all 6 with membranoproliferative $G N$ have had HT ore- and post-TX (with $R$ or $R C$ in 5). Other nephritides had similar natterns. Thus, our experience suogests that patients with glomerulonephritis are especially prone to have post-TX HT, whereas those with structural or concenital abnormalities are at lower risk.

\section{ARTERIOLAR, GLOMERULAR AND PERITUBULAR CAPILLARY CHANGES DURING DEVELOPMENT. E. John, C.M. Edel- mann, Jr. and A. Spitzer. Albert Einstein College of Medi-} cine, Department of Pediatrics, New York.

The functional behavior of the developing kidney is considered to reflect the morphological characteristics of its component nephrons. In an attempt to quantitate differences in vascular structures, kidneys of 1 week and 6 to 10 week old puppies were fixed "in vivo", infused at normal arterial pressure via the renal artery with silicone - rubber (Microfil, MV), removed, cleared, sliced and photographed at the same magnification (116X). This allowed for measurements of afferent and efferent arteriolar and glomerular diameters in subcapsular (SC) and juxtamedullary (JM) areas:

\begin{tabular}{cccccc}
\multicolumn{3}{c}{ Glomerular diameter $(\mu)$} & \multicolumn{4}{c}{ Afferent/efferent ratios } \\
1 week & $6-10$ wks. $\quad p \quad$ & 1 week & $6-10$ wks. $p$ \\
$100.3 \pm 9.3^{*}$ & $130.6 \pm 16.8<.02$ & $1.2 \pm .2$ & $1.1 \pm .2$ & $<.10$ \\
$166.7 \pm 5.7$ & $151.6 \pm 12.4<.02$ & $1.7 \pm .3$ & $0.9 \pm .1$ & $<.01$ \\
$<.001$ & $<.02$ & & $<.10 \quad<.10$
\end{tabular}

The capillary loops were more loosely arranged, and the postglomerular vessels were fewer and more difficult to fill in the younger group. Thus, the kidney of the newborn puppy is characterized by a higher degree of glomerular heterogeneity than that of the older animal. The difference in diameter between afferent and efferent arterioles tends to be higher in the younger than in the older animals, particularly in the juxtamedultary nephrons.

$* \bar{x} \pm S . D$.

717 HYPERURICEMIA IN THE HEMOLYTIC UREMIC SYNDROME(HUS). Bernard S.Kaplan and Peter D.Thomson(Intr.by Keith $\mathbb{N}$. Drummond) Witwatersrand Univ. Transvaal Mem.Hosp. Dept.Paed., Johannesburg, South Africa and McGill Univ.-Montreal Children's Hosp. Research Inst., Dept. of Nephrology, Montreal.

Hyperuricemia was found in each of 18 infants with HUS in whom serum uric acid concentration was measured.On admission the urate levels ranged from $5.6-29 \mathrm{mg} \%(\bar{x}=16.6 \mathrm{mg} \%)$. The highest concentrat ions recorded were on admission or were the first values obtained in 17 infants. The serum uric acid level of the 18 th infant rose from 5.6 to $27.4 \mathrm{mg} \%$ despite peritoneal dialyses.

BUN, serum creatinine and uric acid concentrations were compared

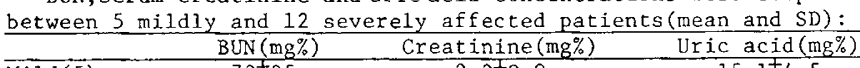

\begin{tabular}{lccc} 
& BUN $(\mathrm{mg} \%)$ & Creatinine $(\mathrm{mg} \%)$ & Uric acid(mg\%) \\
\hline Mild (5) & $73^{ \pm} 25$ & $2.2 \pm 0.8$ & $15.1^{ \pm 4.5}$ \\
Severe(12) & $120.4 \pm 42$ & $5.2 \pm 2.0$ & $19.9 \pm 4.4$ \\
P value & $<0.05$ & $<0.01$ & $>0.5$
\end{tabular}

value $<0.05$ the

Since none of the mild cases had oliguria and had significant-
y lower BUN and creatinine levels but similar uric acid concentrations, it seems that the hyperuricemia was due to factors other than renal failure alone,e.g., lactic acidemia.Measurement of serum uric acid, lactate and creatinine levels was $16.4,30,13.5 \mathrm{mg} \%$ on Day 1 and $9.2,16.0$ and $2.0 \mathrm{mg} \%$ on Day 4 in one patient.

Since hyperuricemia per se can cause acute renal failure and permanent renal damage we speculate that the very high levels found in these infants may aggravate their underlying nephropathy.Hyperuricemia may thus be an important indication for early peritoneal dialysis in patients with HUS.
CADAVERIC RENAL ALLOGRAFT TRANSPLANTATION FOR CHILDHOOD CYSTINOSIS. Mohammad H. Malek, Maur i ce D. Kogut Alfred $J$. Pennisi and Richard N. Fine. Univ. of So. Calif. Sch. of Med., Dept. of Ped. and Childrens Hospital of Los Angeles, Dialysis and Transplant Program, Los Angeles, Calif.

Four cystinotic cadaver renal al lograft recipients, (200-2,030 days posttransplant) were studied. Renal function was normal in 3 , (serum creatinine $<0.8 \mathrm{mg} \%$ ) and I had evidence of chronic rejection (CR), (serum creatinine $5.4 \mathrm{mg} \%$ ). There was no clinical and/or biochemical evidence of recurrence of the Fanconi Syndrome. Urinary aminoacid, phosphate, sodium and potassium excretion were normal. There was no glycosuria. Growth and rehabilitation were satisfactory. Cystine levels were elevated in the peripheral white blood cells (WBC), cultured fibroblasts and allograft tissue in all recipients. Polarized and electron microscopy revealed an abundance of cystine crystal deposition in the allograft interstitium. A few glomeruli revealed mesangial cystine deposition. The tubular epithelial cells were spared.

Although the patient with CR had the highest level of cystine in the graft tissue and $W B C$, no relationship between cystine deposition and graft function was evident. It is hypothesized that cystine deposition in the graft results from migrating WBC.

Although renal transplantation is the treatment for renal failure resulting from cystinosis, it does not correct the primary "enzymatic" defect leading to cystine deposition.

The children, singly and in a group, were actively involved in the decision to participate in this investigation. Informed consent and follow up were documented on video tape.

T19 METHYL PREDNISOLONE THERAPY OF THE NEPHROTIC SYNDROME ASSOCIATED WITH MESANGIAL PROLIFERATIVE GLOMERULO NEPHRITIS. Bruce M. McDonald, Paul T. McEnery, Clark D. West, A. James McAdams. Dept. of Ped. Univ. of Cincinnati Co1. of Med., Children's Hosp., Cincinnati, Ohio.

Five patients aged $2-15$ years with renal biopsy evidence of mesangial proliferative glomerulonephritis and nephrotic syndrome have been treated with 3-5 intravenous doses of 0.5 to $1.0 \mathrm{gm}$ of methyl prednisolone on alternate days. All patients had previously been treated with daily prednisone and 4 of the 5 were unresponsive after 4-7 weeks of therapy, while the fifth patient relapsed frequently on alternate day prednisone. The response to methyl prednisolone was monitored by measurement of urine protein, transferrin and albumin and serum albumin levels. Three of four steroid resistant patients and one with frequent relapses diuresed promptly (2-7 days) and the urine became protein free (2-8 days). Three of the prednisone resistant patients and the patient with frequent relapses were also treated with Cytoxan following remission with methyl prednisolone. Follow-up for 2-7 months indicates that methyl prednisolone does not produce a prolonged remission; two patients relapsed shortly after treatment. Creatinine clearances were normal in 3 of 5 patients prior to therapy and reverted to normal in the other 2 after therapy with methyl prednisolone. Methyl prednisolone may have a place in producing remissions in nephrotic patients with mesangial proliferative glomerulonephritis resistant to therapy with oral prednisone. Similar treatment of 2 patients with focal glomerulosclerosis was ineffective.

720 RETIAL GLYCOSURIA, AN EARLY SIGN OF FOCAL SEGMENTAL GLOMERULÓSCLEROSIS (F.S.G.). Melinda ! McVicar, Ramon Exeni, Myron Susin, North Shore Univ. Hosp., Depts. Peds. \& Path., Manhasset, $N_{c} \overline{Y .}$, and Comell Univ. Med. Col. Dept. Peds. New York. New York (Intr. by Fima Lifshitz). The purpose of this report is to describe 2 girls with nephrotic syndrome (N.S.) who had renal glycosuria and other renal tubular defects as early signs of F.S.G. Rapid progression to chronic renal insufficiency occurred in both and was associated with interstitial fibrosis and atrophic fubules characteristic of F.S.G.

Within 7 months of onset of N.S., glucose excretion went up to 6.3 $\mathrm{gm} / 24 \mathrm{hr}$ in one patient and was intermittently 3-4t on random urine specimens in the other. Urinary alpha-amino nitrogen to creatinine nitrogen ratio was 1.8 and 2.6 respectively (normal $=.55+.33$ ), indicating hyperaminoaciduria. Spontaneous blood $\mathrm{pH}$ of 7.28 associated with urine $\mathrm{pH}$ 7.23 was diagnostic of the renal tubular acidosis associated with episodes of glycosuria in one patient. The other child was tested by $\mathrm{NH}_{4} \mathrm{Cl}$ loading and the lowest urine $\mathrm{pH}$ was 6.23 with simultaneous blood $\mathrm{pH} 7.22$. Maximum urine osmolality for this patient was $426 \mathrm{mOsm} / \mathrm{Kg}$. Thick, mucouslike globules, $1-2 \mathrm{~cm}$ in diameter, were frequently passed in her urine. Hydrolysis and amino acid analysis of this substance demonstrated that $35 \%$ of the dry weight was protein. Hydroxyproline, a major constituent of collagen, was not present. Carbohydrates contributed $15 \%$ of dry weight, most of which was glucose, galactose and manose.

These cases demonstrate that renal glycosuria and other tubular defects may be early signs of F.S.G. and should suggest the presence of this lesion in the nephrotic child. 
721 DICARBOXYLIC AMINOACIDURIA: A NEW INBORN ERROR OF GLUTAMIC AND ASPARTIC ACID TRANSPORT WITH GENETIC HETEROGENEITY. Serge B. Melançon, Louis Dallaire, Bernard Lemieux, Pierre Robitaille and Michel Potier. Pediat. Research Ctr., Ste-Justine Hosp., Univ. of Montreal and Sherbrooke.

A defect in renal transport of the dicarboxylic aminoacids glutamic and aspartic acids is described in four unrelated children of french-canadian ancestry. The two younger subjects, EL and $C D$ are apparently healthy males whose daily renal loss of glutamic acid ranges from $0.2-0.7 \mathrm{~g}$ with approximately one-tenth as much for aspartic acid. The other two children, $\mathrm{CP}$ and $\mathrm{BC}$ are females who excrete between $0.014-0.150 \mathrm{~g} / \mathrm{day}$ of glutamic acid and approximately one-third as much aspartic acid. All patients have normal plasma and CSF concentration of the respective dicarboxylic aminoacids.

\begin{tabular}{|c|c|c|c|c|}
\hline PATIENT & AGE & MEAN RENAL & CLEARANCE & $\left(\mathrm{ml} / \mathrm{min} / 1.73 \mathrm{M}^{2}\right)$ \\
\hline & (years) & Asparate & Glutamate & Inulin \\
\hline EL & $9 / 12$ & $883(3)$ & 260 (3) & 106 \\
\hline$C D$ & $15 / 12$ & 467 (4) & 167 (4) & 52 \\
\hline$C P$ & 3 & $82(4)$ & $9(4)$ & 109 \\
\hline $\mathrm{BC}$ & 10 & $140(4)$ & 12 (4) & 147 \\
\hline Controls & & $t R-8.8$ & $0.1-2.4$ & $127 \pm 32$ \\
\hline
\end{tabular}

While the high clearance values in the two older girls support the evidence of an incomplete reabsorption from the glomerular filtrate, the excretion of dicarboxylic acids well in excess of the inulin clearance values in the younger two boys indicate renal secretion.

722 Intrarenal Blood Flow in the Baboon Fetus. Eddie $\mathrm{S}$ Moore, Maurina B. Galvez, John B. Paton, David E. Fisher and Richard E. Behrman. Dept. of Ped. Univ. f Chicago at the Michael Reese Med. Center, Chicago, Il.

The purpose of the present study was to investigate intrarenal blood flow in the primate fetus. Control studies were performed on eight (8) near-term ( $>140$ days gestation) baboon fetuses and three ( 3 ) immature ( $<120$ days) baboon fetuses. Renal blood flow (RBF) and intrarenal flow to the inner cortex (I.C.) and outer cortex (o.C.) were measured by injection of radiolabeled carbonized microspheres into the fetal circulation after exteriorization of the fetus. The effect of acute systemic acidosis produced by infusion of lactic acid and the effect of phenobarbital infusion on intrarenal flow was also studied in eight (8) and four (4) near-term fetuses respectively. The following flows in $\mathrm{ml} / \mathrm{min} / \mathrm{gm}$ of tissue were obtained:

\begin{tabular}{|c|c|c|c|c|}
\hline & RBF & O.C. & I.C. & O.C./I.C. \\
\hline Near-Term & $\overline{3.11}$ & $\overline{6.96}$ & $\overline{6.75}$ & 1.03 \\
\hline Imnature & 2.00 & 3.15 & 3.84 & 0.82 \\
\hline Acidosis & 3.26 & 6.10 & 6.98 & 0.97 \\
\hline Phenobarbital & 1.47 & 2.74 & 3.72 & 0.74 \\
\hline
\end{tabular}

These studies demonstrate that distribution of intrarenal blood flow in the primate fetus parallels patterns of kidney growth. In early gestation there is a preponderence of I.C. flow where growth of kidney tissue is most rapid. In late gestation when 0.C. growth accelerates, there is a redistribution of flow to this area. In late gestation, acute acidosis or changes in RBF induced by phenobarbital results in a redistribution of fetal intrarenal blood flow with a reversal of the O.C./I.C. ratio.

72 PAH Uptake by Cortical Kidney Slices in Suckling Rats.

723 Eddie s. Moore, Jonathan B. Rubenstein, Margarita Ocampo, Elizabeth Lyons, Ellis E. Chung and Eduaxdo Cevallos. Dept. of Ped. Univ. of Chicago at the Michael Reese Med. Center, Chicago, I1.

The influence of age and of body and kidney size on maturation of renal function in early life was studied by measuring PAH uptake by rat kidney slices. Newborn infant rats were separated in to unequal litter sizes within the first 24 hours of life. At 15 days of age, $0.3 \mathrm{~mm}$ kidney slices from control and penicillin treated rats were incubated in PAH media for $15,30,45$ and 60 minutes. Slice to media (S/M) PAH ratios were obtained as follows:

\begin{tabular}{|c|c|c|c|c|}
\hline & \multicolumn{4}{|c|}{ S/M RATIO } \\
\hline$\frac{\text { Control }}{\text { Sma11. }}$ & $\frac{15 \mathrm{~min} .}{2.41^{\star}}$ & $\frac{30 \mathrm{~min} .}{4.64}$ & $\frac{45 \min }{6.48}$ & $\frac{60 \mathrm{~min}}{6.53}$ \\
\hline Large & $3.92 *$ & 5.28 & 7.85 & 5.42 \\
\hline Penicillin & & & & \\
\hline Sma11 & 2.49 & 4.42 & $9.99 *$ & $13.53 *$ \\
\hline Large & 2.42 & 5.82 & 7.59 & $10.57 *$ \\
\hline
\end{tabular}

* Statistically Significant

Although the rats had statistically different body and kidney weights, the difference between mean control S/M ratios was only significant at 15 minutes incubation. After treatment with penicillin for three (3) days, smaller rats had a significantly higher S/M ratio than the small controls at 45 and 60 minutes, but on$1 y$ at 60 minutes for the large rats. These data suggest that spontaneous maturation of PAH transport is age dependent. Smaller rats appear to have a greater capacity for induced maturation.

\section{4} ABNORMAL RED CELL PHOSPHOLIPIDS(PL) IN THE HEMOLYTIC UREMIC SYNDROME (HUS)ASSOCIATED WITH DECREASED SERUM TOCOPHEROL LEVELS.Sean O'Regan, David K.Melhorn, Russe11 W. Chesney, Bernard S.Kaplan and Keith N.Drummond McGill Univ.Montreal Children's Hosp.Research Inst., Dept. of Nephrology, M(*.ttreal, Canada and Case Western Reserve Univ., Rainbow Babies\&Childrens Hosp., Dept. of Ped.Hematology,Cleveland, Ohio.

Two children with HUS had low serum $\alpha$-tocopherol levels $(0.23$ $\mathrm{mg} / \mathrm{dl}$ and $0.36 \mathrm{mg} / \mathrm{d} 1$ vs. 0.7 to $1.4 \mathrm{mg} / \mathrm{dl}$ control). Analysis of red cell PL patterns by thin layer chromatography revealed a decrease in total PL content. The percentage of phosphatidyl ethanolamine( $\mathrm{PE}$ ) was decreased ( 19 and 6 vs. 29 control)and the percentage of sphyngomyelin(SM) was increased( 50 and 46 vs. 26 control). Simultaneous analysis of red cell membrane PL of the mother of one patient revealed similar abnormalities. Though in one case correction of red cell PE and SM ratios took place after a single p.o. dose of 500 units of vitamin E, a striking decrease in total red cell PL content $\left(3.05 \times 10^{-10} \mathrm{mg} / \mathrm{cell}\right.$ vs. $4.2 \times 10^{-10} \mathrm{mg} / \mathrm{cell} \mathrm{con}-$ trol)was observed associated with an increase in phosphatidyl choline percentage, $\alpha$-tocopherol activity was not detectable in the serum.

Vitamin E, because of its antioxidant properties, is active in the maintenance of normal red cell membrane PL composition. Thus the association of depressed $\alpha$-tocopherol activity with red cell membrane PE depletion is suggestive of lipid peroxidation. Hemolysis in some cases of HUS may be due to massive membrane Iipid peroxidation in association with low serum vitamin $E$ levels. Intrarenal coagulation may b secondary to thromboplastin release from hemolysing red cells.
725 MEASUREMENT OF GLOMERULAR FILTRATION RATE BY FLUORESCENT EXCITATION OF NON-RADIOACTIVE IOTHALAMATE. Sheldon Orloff, Pierre Guesry, Leon Kaufman, Sybil Swann and Malcolm Holliday. Univ. of California San Francisco, Dept. of Ped.

Measurement of Glomerular Filtration Rate (GFR) is known to be a valuable way to follow patients with renal disease. Past methods have been inaccurate (Creatinine clearance), involve frequent large blood samples (double exponential model), or have used rad ioactive materials (single exponential model). We present a method that is reproducible, easily measured, involves no radiation, and therefore is readily applicable to small children in an outpatient setting. We show that the clearance of nonradioactive meglumine Iothalamate (Conray 60) following a single injection and assayed by Fluorescent Excitation Analys is is a good GFR indicator. The ratio of the clearance of Conray (Ccon) to the clearance of inulin ( $\mathrm{C}_{\mathrm{IN}}$ ) using a constant infusion technique in rats (Ccon: $\left.\mathrm{C}_{\mathrm{IN}}\right)$ was $0.937(\mathrm{R}+0.944)$; the same as the clearance of $\mathrm{I}^{125}$ Iothalamate (CIO) to clearance of inulin ratio reported earlier. In a group of 10 children and one adult, the single injection clearance ratio of $\mathrm{C}_{\mathrm{IO}}$ :Ccon using the double exponential decay curve was $1.0(R+0.992)$. Further we show that deriving Ccon from the single late exponential curve (involving only three or four samplings) yields good agreement with the GFR determination by the two exponential model, the ratio having a correlation coefficient of 0.98 . Single exponential clearances using radioactive isotope have a lower coefficient of variation than other usable clinical techniques (C1in.Sci. 1969,37,169). The present method should provide a clinically acceptable, reproducible method for measuring and following GFR in a child with renal disease.

726 FATAL HEMOLYTIC-UREMIC SYNDROME IN SIBLINGS. Qutub H. Qazi and Edward J. Schutta, Depts, of pediatrics, Downstate Med. Ctr. and Brookdale Hospital Med. Ctr.

Brooklyn, N.Y.

Although hemolytic-uremic syndrome has been described in pairs of siblings, the genetic basis for the disease has been questioned because of simultaneous occurrence of the condition in affected members. We have observed a family with fatal hemolyticuremic syndrome in two brothers who were affected two years apart. The parents are unrelated and have a six year old healthy daughter. J.M. was born in December 1970 and died in May 1971. G.M. was born in February 1973 and died in August 1973. The onset and the course of the disease were almost identical in both infants. The clinical picture included gastroenteritis, hemolytic anemia, progressive renal involvement and hypertension. Autopsy of G.M. showed renal changes consistent with the diagnosis of hemolytic-uremic syndrome.

To our knowledge, this is the 3 rd example of the nemolyticuremic syndrome occurring separately in siblings. The etiology of hemolyitc-uremic syndrome may very well be multiple, but occurrence of disease in siblings provides strong evidence for the genetic predisposition of hemolytic-uremic syndrome. 
72 EARLY MORPHOLOGIC ABNORMALITIES OF THE KIDNEY IN

727 MEDULIARY CYSTIC DISEASE. Jack S. Resnick and Robert L. Vernier, Dept. of Ped., Univ. of Minnesota Medical School, Minneapolis, Minnesota.

Medullary cystic disease (M.C.D.) is inherited as an autorecessive or dominant nephropathy whose etiology is unknown, but may be due to a toxic factor or metabolic abnormality that results in secondary cyst formation. Two children from separate families affected with recessive M.C.D. have been studied with renal biopsies before the onset of significant renal insufficiency $\left(\mathrm{Ccr}=72 \mathrm{cc} / \mathrm{min} / 1.73 \mathrm{~m}^{2}, 60 \mathrm{cc} / \mathrm{min} / 1.73 \mathrm{~m}^{2}\right)$ at a time when concentrating defects were apparent. Routine histology in both revealed scattered dilated tubules (distal), of ten surrounded by mononucleax infilisate, containing an unusual foamy, pale staining ( $\mathrm{H} \& \mathrm{E}, \mathrm{AZO}$ and PAS + ) material with atrophic or degenerating tubular cells. This material was also detected in the renal interstitium and was PAS + after amylase and hyaluronidase digestion and negative for lipids. Fluorescence microscopy in 1 patient showed focal GBM granules containing IgG, C3, and C4. The tubular material was + for albumin, fibrin, IgG, IgA, C3 and Tamm-Horsfall protein. Electron microscopy (E.M.) of one patient revealed thickened, laminated tubular basement membrane, amorphous interstitial material and thinning of tubular cytoplasm. E.M. of the other patient is similar. These findings are consistent with the hypothesis that M.C.D. is caused by accumulation of a toxic substance in distal tubules with tubular cell damage, necrosis, inflammation and secondary cyst formation.
728 ESSENTTAL HY:ERT NSIION IN THE YUUNG: HEMOUYNAMIC UTU-

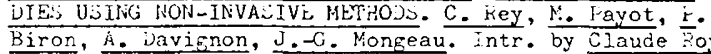
University of Riontreal, Ste-Justine tospital for Children, jept. of iediatrics.

iwenty three young patients (from 4 to 24 years, average 15.9 years) sufforing from essential iabile hypertension were studied by non-invasive means and compared with 43 normal subjects of similar ape and to 9 patients suffering from stable hypertension of renal parenchymal origin. Cardiac index, stroke volume, total peripheral resistance, mean velocity of circumferential fiber shortening, mean and maximal velocity of left ventricular posterior wall (mean and maximal $d l / d t$ ) were measured.

Using an "inciex of kinetism" (the ratio of cardiac index to 1/5th of the perioheral resistance) the natients with essential labile hypertension could be separated into two rroups of anproximately equal size and age: the hyperkinetic patients (index of kinetism > 1) more characterized by a high cardiac index, stroke volume and mean and maximum $\mathrm{dl} / \mathrm{d}$ t and a normal peripheral resistance the hyperresistant (index of kinetism < l) showed an increase in peripheral resistance with other parameters being normal. All but one of 11 patients re-examined after a 2 to 3 ronths perioc stayed in the same group. It is postulated that, contrary to current be lief, essential labile nypertension may be characterized even at an early age, by either a hyperikinetic or a nyperresistant state suggesting perhaps more than one etiology.
730 TREATMENT OF MERCURIAL DIURETIC INTOXICATION WITH BAL AND PERITONEAL DIALYSIS. RObillard, J.E., Rames, L. and Roberts, R, J。 Dept, of Pediatrics and Pharmacology, University of lowa, lowa City, lowa 52242.

A 3 month old girl $(3.4 \mathrm{~kg})$ with congenital heart disease (TGA) died in renal failure subsequent to the administration of mercaptomerin for treatment of congestive heart failure. A total of 62 $\mathrm{mg}$ of mercaptomerin was given $(7 \mathrm{mg} / \mathrm{kg} /$ day on each of 3 days) during a 7 day period. At the time of admission but prior to compiete anuria the blood and urine mercury $(\mathrm{Hg})$ levels were 456 $\mathrm{mg} / 100 \mathrm{ml}$ and $19.3 \mathrm{mg} / \mathrm{L}$ respectively. Subsequently, peritoneal dialysis and intramuscular injections of BAL (12 mg g $6 \mathrm{hr}$ ) were begun. The mean calculated dialysance of $\mathrm{Hg}$ over the next 3 day period was $0.37 \mathrm{ml} / \mathrm{min}(0.07-0.54 \mathrm{ml} / \mathrm{min})$. Despite a marked reduction in $\mathrm{Hg}$ blood levels $(15 \mu \mathrm{g} / 100 \mathrm{ml})$ the patient died on the 12 th day still oliguric and on dialysis. Tissue levels of $\mathrm{Hg}$ ( $\mu \mathrm{g} / 100 \mathrm{gm}$ of tissue) included brain 29 (normal (N) $=10$ ), liver $1201(\mathrm{~N}=30)$, kidney $2324(N=275)$, lung $489(N=10)$, heart $95(N=15)$.

These data indicate that despite a significant reduction in $\mathrm{Hg}$ blood levels during the period of treatment with BAL and peritoneal dialysis, very high levels of $\mathrm{Hg}$ persisted in various tissues.

731 SERUM IMMUNOGLOBULINS AND THE NEPHROTIC SYNDROME. Alan M.Robson, Thomas G.Cleary, Barbara R.Cole and Joseph Giangiacomo. Wash. Univ. Sch, of Med., Dept. of Ped. and st. Louis Children's Hosp., St. Louis, Mo.

Serum IgG and IgA levels in 37 children with untreated minimal change nephrotic syndrome (MCNS) averaged $18.5 \%$ and $59.8 \%$ of normal; in contrast serum IgM levels were consistently elevated (av $243 \%$ norma 1). IgG and IgA were similarly reduced in 36 nephrotic patients with chronic nephritis (CGN) but IgM levels varied. Steroid administration to MCNS patients resulted in progressive increases in IgG and IgA levels but IgM levels remained high. In 28 patients in remission from MCNS and of $f$ all therapy for a mean of 2.3 yrs, IgG and IgA levels were reduced $(74.6 \%$ \& $69.5 \%$ normal, $p<.005$ ) but IgM values averaged $208 \%$ of normal ( $p<.001$ ). Nephrotic patients with CCN had increased glomerular permeability to large mol.wt. PVP-125I; in contrast those with MCNS had normal permeability to these molecules.

The low IgG levels in nephrotic patients may contribute to the increased incidence of infection in these patients. However, the results challenge the traditional concept that the low serum Igc levels are due to increased glomerular permeability with loss of immunoglobulins through the kidney. The nigh IgM, low IgG and low IgA levels in MCNS, found even after prolonged remission off therapy, suggest that these patients may have a basic abnormality of their immune mechanisms. These changes could be related to the etiology of MCNS and account for such patients developing MCXS rather than the more usual irmune complex disease as a consequence of infection. Although the present study suggests such an explanation it cannot confirm it.

\section{2} HYPERSENSITIVITY TO FOODS IN STEROID-DEPENDENT NEPHROSIS (SDN). Douglas H. Sandberg, Rawle M McIntosh, Charles W. Bernstein, Bruce Deling and Jose Strauss. Univ. of Miami Sch. of Med., Dept. of Ped., Miami, Fla. and Univ. of Colorado Sch, of Med., Dept. of Ped and Med Denver, Colo.

Although allergens have been suggested as etiologic agents in childhood nephrosis, the role of these agents in etiopathogenesis of the disease has not been definitely established. To evaluate this relationship 6 children with steroid-dependent minimal change nephrosis were studied to establish the relationship of sensitivity to foods to etiology and pathogenesis of their renal disease. Morphologic, ultrastructural and immunohistologic studies were performed on renal tissue from 5 patients. Immunoglobulin and complement component levels were determined during remission and exacerbation induced by administration of food allergens. Introduction of specific foods (e.g., cows milk, wheat, etc.) was accompanied by increase in protein excretion to as much as $29 \mathrm{gm}$ per $24 \mathrm{hr}$, decrease in serum immunoglobulins not related to urinary losses, alteration in complement component concentrations which in some patients suggested activation of the classical pathway. Exclusion of these antigens from the diet was associated with decrease in protein excretion to normal or nearly normal levels and reversal of the serum immunologic abnormalities These preliminary observations support a role for food hypersensitivity in exacerbation of nephrosis in some patients, possibly by immune cornplex or IgE mediated mechanisms, food allergen activation of the complement system or gastrointestinal autologous 
733 LONG TERM FOLLOW-UP OF POSTSTREPTOCOCCAL GLOMERULONEPHRITIS IN CHILDREN. Robert $G$. Schacht, Mohammed Sajiad Iqbal and David S.Baldwin (Intr. by Joseph Dancis) New York University Medical Center. Depts. of Ped. and Med., New York.

A study has been made of the natural history of acute poststreptococcal glomerulonephritis in 76 children, three of whom had two distinct episodes. Three patients progressed to terminal uremia within six months. Observations are available in $46 \mathrm{pa}$ tients 2 to 17 years after onset. Persistent proteinuria is present in $20 \%$, hypertension in $11 \%$ and elevated serum creatinine in $15 \%$. At least one of these clinical abnormalities is present in $30 \%$. Glomerular filtration rate as measured by inulin clearance is reduced to less than $100 \mathrm{ml} / \mathrm{min}$. in $33 \%$. Partial or complete sclerosis of some glomeruli is demonstrable in four out of ten patients in whom late renal biopsies are available. Exaggerated natriuresis, an altered renal response to rapid infusion of hypertonic saline, was observed in 13 of 18 independent of blood pressure level. Complete resolution occurred only in those with mild clinical and histologic features at onset, while persistent evidence of disease was observed following either mild or severe initial manifestations and was not related to age. The present study provides evidence that irreversible renal damage, which may prove to be progressive, occurs in over 30 percent of children with poststreptococcal glomerulonephritis.

73 IDIOPATHIC PERSISTENT MICROSCOPIC HEMATURIA (IPMH)

734 M. Schoeneman, H. Boichis, J. Earon, C.M. Edelmann, JT., A. Spitzer and T. Greifer. Albert Einstein College of Med., Department of Pediatrics, New York.

The history, clinical signs, and histological findings of patients presenting with IPMH suggest that this is a highly heterogeneous group, making it difficult to predict the course in the individual patient. 52 patients with IPMH were studied in an attempt to find clinical and histological variables with prognostic significance. Isolated hematuria was found in 23 patients, 5 of whom also had a family history of hematuria. None of these showed deterioration of renal function over a follow-up period of 1 to 17 years. Of the remaining 29 patients, 12 had deafness and/or cocular abnormalities and a family history of deafness and/or renal disease, whereas 17 patients had only a family history of deafness and/or renal disease. 11 of these 29 patients developed renal insufficiency over a period of observation ranging from 2 to 15 years. The likelihood of progression was higher for patients with deafness $(p<.01)$, ocular abnormalities $(p<.01)$, proteinuria $>1 \mathrm{gm} /$ 24 hrs. $(p<.01)$ and for those with foam cells and interstitial fibrosis on renal biopsy $(p<.01)$. Among patients with isolated hematuria, those with a family history of deafness developed renal insufficiency in a significantly higher percentage $(p<.01)$ than those without such history. The outcome was not related to the sex of the patient or the line of inheritance. The severity of renal disease in the family did not seem to correlate with that of the patient. The rate of deterioration of renal function with age was similar among siblings.

735

URETERIC STONES IN CHILDREN.Samue1 B. Sheps, Bernard S. Kaplan and Keith N.Drummond. McGill Univ., Dept.of Ped. Montrea1, Canada.

Information on the non-surgical outcome of ureteric stones in children is scarce, hence indications for surgical versus medical management are unclear. This study was done to determine our experience with, and to clarify our approaches to this problem.

Between 1955-1974,22 children with ureteric calculi were seen; this represents $60 \%$ of patients found to have documented urolithiasis. Mean age was 10.5 yrs; 18 children had pain, 4 had gross hematuria, duration of symptoms being $1 \mathrm{hr}$ to 14 mos.Microscopic hematuria occurred in 19 , while only 2 had positive urine cultures. Radiopaque calculi were seen on IVP in 16; the remainder were documented by surgery or passage.Predisposing factors to stone formation were:cystinuria(3), infection(3), hypercalciuria(3), hyperuricemia(1);12 were idiopathic.Stones were passed in $20(90 \%)$ with medical management(hydration, alteration of urine $\mathrm{pH}$ and analgesia); this occurred within one week in most patients. In 2 patients lithotomy was performed:the first during, but incidental to, ureteric reimplantation; and the second because it was thought that the stone would not pass.Six patients had recurrence;none of these were idiopathic.Most stones contained Ca-oxalate and none of the stones which passed exceeded $5 \mathrm{~mm}$.

Therefore,although surgical intervention is well documented as being the predominant mode of therapy for ureteric stones in children,our experience stands in marked contrast, and suggests that most of these stones should be managed conservatively.
736 SERUM PROSTAGLANDIN E LEVELS IN THE NEONATE. Richard $\frac{\text { L. Siegler, }}{\text { William Jubizonald } \mathrm{H} \text {. Crouch, }} \frac{\text { Margaret W. Miller, }}{\text { (Intr. by Lowel }} \frac{\text { A. Glasgow). Dept. of }}{\text { A. }}$ Ped. and Med., Univ. of Utah Col. of Med., Univ. and VA Hosp., Salt Lake City.

$\mathrm{Pl}$ asma prostaglandin $\mathrm{E}$ (PGE), renin activity (PRA) and aldosterone levels were measured by radioimmunoassay in cord blood $(n=6)$ and at 2-3 days of age $(n=7)$. PGE and PRA were determined in an additional group of infants 3-6 months of age $(n=4)$. The following results were obtained (mean $\pm \mathrm{SD}$ ).

\begin{tabular}{|c|c|c|c|}
\hline & PGE $(\mathrm{ng} / \mathrm{ml})$ & $\mathrm{PRA}(\mathrm{ng} / \mathrm{m} 1 / \mathrm{hr})$ & Aldosterone (ng/dl) \\
\hline cord blood & $1.78 \pm 1.05$ & $9.3 \pm 4.8$ & $84.1 \pm 51.0$ \\
\hline 2-3 days age & $0.15 \pm 0.14$ & $11.1 \pm 5.7$ & $106.3 \pm 61.9$ \\
\hline 3-6 months age & $0.34 \pm 0.24$ & $7.5 \pm 4.6$ & \\
\hline normal adults & $0.53 \pm 0.20$ & $1.1 \pm 0.5$ & $14.2 \pm 4.0$ \\
\hline
\end{tabular}

Neonatal PRA and aldosterone values were significantly higher ( $<0.001)$ and PGE levels significantly lower $(p<0.001)$ than the normal adults. While our numbers are small, this preliminary data suggests that contrary to adults, neonates fail to secrete PGE in response to the high angiotensin levels that result from increased PRA. These inappropriately low PGE levels correlate with the observation that the renomedullary body, presumably the site of $P G$ production, is absent during the first month of life. It is also tempting to speculate that these alterations may be responsible for some of the functional peculiarities of the neonatal kidney.

737 TREATMENT OF NEPHROCENIC DIABETES INSIPIDUS (NDI). Carl D. Sorgen, $\frac{\text { Robert S. Fennell, }}{\text { Norman D. Pryor, Eduardo H. Garin, }}$ (Intr. by Elia M. Ayoub), Univ, of Florida Col of Med., Dept. of Ped., Gainesville.

Fourteen patients with NDI were followed for a period of up to 115 months $(\mathrm{m})$ with an average of $42 \mathrm{~m}$. Twelve of 14 had onset of symptoms under 2 m of age and 11 of 14 had a history of fever and dehydration. After diagnosis, all patients were begun on a $2 \mathrm{mEq} /$ $\mathrm{kg} \mathrm{Na}, 2 \mathrm{gm} / \mathrm{kg}$ protein, ad-1ib fluid diet, and $4-5 \mathrm{mg} / \mathrm{kg}$ hydrochlorothiazide or $40-50 \mathrm{mg} / \mathrm{kg}$ chlorothiazide regimen. Further episodes of fever and dehydration were rare during the therapy period. Growth rate improved in 5 of 12 patients, remained unchanged in 2 and declined in 5 of 12 patients. Eight of 12 patients gained weight, 3 of 12 lost weight and 1 was unchanged. All IVPs showed faint visualization. Of 9 initially normal, 3 became abnormal and 2 remained normal. Of 3 abnormal IVPs, 1 remained abnormal during the follow-up period. Initial and follow-up clearances in 10 patients were normal. Six of 6 tubular reabsorption of phosphate determinations were normal. No evidence of metabolic acidosis was found in any of the 14 . with the exception of concentrating ability, renal function tended to be intact. Gains with respect to weight and avoidance of dehydration were noted. However, therapy for this disease may not be optimal with respect to height gain, and of note, 5 of the 14 patients ended up in foster homes.

738 PERIPHERAL PLASMA RENIN ACTIVITY (PRA) IN NORMAL CHILDREN, Phil ip Stalker, Theodore Kotchen, Jane M. and Community Med., Univ. of Ky. Med. Ctr., Lexington, Ky.

A) though peripheral PRA has been measured in the neonatal period (Kotchen, T., J. Pediat. 80:938, 1972), normal values for infants and children are not well documented. Specimens for PRA assay were collected in 91 normal children (2 mo. to 18 yrs.) at the time of admission to the hospital for elective surgery or during routine clinic visits. All were afebrile, normotensive and considered well children. PRA was measured by the radioimmunoassay method of Haber. During infancy, PRA was elevated compared to normal adult standards $(p<0.01)$ aithough lower $(p<0.01)$ than PRA in newborns (NB). Values gradually declined during the first 6 yrs. After this age values remained constant at a level found to be normal for adults in our laboratory. The correlation between age and PRA was significant $(p<0.0069)$

Age (Years)

No.children

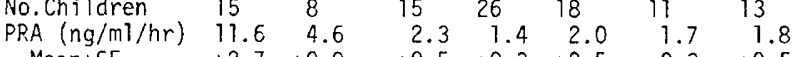

$\begin{array}{llllll}\text { Mean } \pm \text { SE } & \pm 2.7 & \pm 0.9 & \pm 0.5 & \pm 0.3 \quad \pm 0.5 \quad \pm 0.3 \quad \pm 0.5\end{array}$

PRA in 7 children with acute hypertensive encephalopathy secondary to chronic pyelonephritis was markediy elevated (13.2 to $22.0 \mathrm{ng} / \mathrm{ml} / \mathrm{hr}$ ) but returned to normal values for age after control of the hypertension. Diagnostic and therapeutic decisions based on PRA determinations must take into account the higher normal levels in pediatric patients. 
739 THE COMPLEMENT REACTIVE FACTOR(S) IN SERUM FROM C. Frederic Strife, Enrique H.Vallota, Clark D. West, University of Cincinnati College of Medicine and Children's Hospital, Dept. of Peds., Cincinnati.

The presence of anticomplementary factors in the sera of 49 patients with acute post-streptococcal nephritis (AGN) was determined by the breakdown of $\mathrm{C} 3$ upon incubation of AGN serum with normal human serum (NHS). In serum from $50 \%$ of the patients a heat labile $\left(50^{\circ} \mathrm{C}\right.$ for $30 \mathrm{~min}$ ) complement reactive factor( $s$ ) (CRF) was detected. The reaction was divalent cation dependent and maximum activity was manifest at a $1: 1(\mathrm{v} / \mathrm{v})$ ratio of AGN: NHS. The presence of CRF correlated with the clinical onset of AGN. Within 4 days of clinical onset $64 \%$ of 22 initial serum samples had significant $\mathrm{CRF}$, producing an average of $50 \%$ breakdown of C3. The transient nature of the CRF was demonstrated in 8 patients in whom serial determinations of CRF showed a rapid decrease, usually within 10 days of clinical onset. A CRF was present in one patient prior to the development of hypocomplementemia. The initial serum sample, collected 1 day prior to the onset of hematuria, contained high levels of CRF associated only with a diminished properdin level. Five days later, the serum C3 and C5 levels were a1so markedly depressed yet the CRF had almost disappeared. No correlation was observed between the CRF and serum $\mathrm{Clq}, \mathrm{C} 4$ or properdin levels. The results suggest that transient anticomplementary activity can be present early in $A G N$ and that this activity may be present in association with a low serum properdin level prior to any other complement alteration.

$\mathbf{7 4 0}$ THE TREATMENT OF THE HEMOLYTIC UREMTC SYNDROME (HUS). Peter D. Thomson and Bernard S.Kaplan. (Intr.by Keith $N$. Dept.Paed., Johannesburg, South Africa and McGill Univ.-Montreal Children's Hosp. Research Inst., Dept of Nephrology, Montreal, Canada.

In 1969 when the results of treatment of patients with HUS were analysed, the mortality rate was thought to be unacceptably high.Patients were divided into 2 groups prospectively from 1969: mildly affected(MA) infants who were not anuric; severely affected (SA) infants who were anuric for $>24 \mathrm{hrs}$. All SA infants from 196974 were managed with early and frequent peritoneal dialysis. Water and electrolyte disturbances and hypertension were meticulously controlled in all MA and SA patients;none were given steroids, heparin or streptokinase.

The results of therapy in 40 consecutive patients with HUS (TMH, Nov.' 69--Aug. '74) were analysed and compared with those of 60 patients treated from $1960-69$ :

\begin{tabular}{ccccccc} 
& Total no. & MA & SA & Deaths & \% mortality \\
\hline $1960-69$ & 60 & 34 & & 0 & 0 \\
& & & 26 & 20 & $77\}$ & 33 \\
\hline $1969-7 l_{r}$ & 40 & 18 & & 0 & 0 \\
& & & 22 & 2 & $9\}$ & 5 \\
\hline
\end{tabular}

deaths of SA infants; 2) no deaths in MA infants.

Thus, any claims for using heparin and/or streptokinase should be measured against these improved results with active symptomatic treatment, and potentially hazardous measures should be avoided unless a lower mortality rate can be shown.

741 REIAL MANIFESTATIONS GF PERIHATAL ASPHYXIA James D. Thullen, Avroy A. Fanaroff, Sudesh P. Makker School of Medicine, Department of Pediatrics, Cleveland, Ohio

There is little information available on the effects of asphyxia on the neonatal nephron. Clinical and chemical parameters of renal function were studied in 17 asphyxiated term infants during the first weeks of 1 ife. All had low 1 minute Apgar scores and fetal distress was present in 15 infants. Birthweights ranged from $2410-4590$ grams (mean 3880 grams), gestational age 38-43 weeks (mean 40.2 weeks). Urine volume was reduced in $16 / 17(94 \%)$ and bloody in $13 / 17(76 \%)$. Seven infants had gross hematuria; another 6 had microscopic evidence of bleeding. Proteinuria and cylinduria were present in all infants. BUN was elevated in $8(56 \%)$. Hyponatremia occurred in 6 infants. This appeared to be due to reduced free water clearance; inappropriate $A D H$ secretion was not documented. Creatinine clearance, when measured, was always low ( 6 patients). Histologically, acute tubular necrosis and medullary hemorrhage was present in 2 out of 3 infants who died.

Asphyxia is the most common cause of hematuria encountered in lour nurseries. The altered renal furction following perinatal asphyxia may result in acute renal failure. Clinically, this necessitates judicious fluid management since provision of usual fluid requirements can produce fluid retention, water intoxication, electrolyte disturbances, and cardiac overload. Recistribution of the cardiac output, reduced renal blood flow and intrarenal shunting with asphyxia may be responsible for the renal dysfunction.
742 LATE NON-RESPONSIVENESS TO STEROIDS IN CHILDREN Spitzer, C.M. Edelmann, Jro, and I. Greifer, Albert Einstein College of Medicine, Department of Pediatrics, New York Children with the nephrotic syndrome (N.S.) who fail to respond to steroid therapy are known to have a poorer prognosis than those whose proteinuria ceases. However, little is known about the histology and clinical course of nephrotics who initially respond but who later become steroidresistant (late non-responders). We have reviewed the records of $195 \mathrm{chil}$ dren with N.S. and have found among them 10 such patients, Resistance to steroid therapy occurred from 2 to 24 months after the onset of the N.S. All 10 patients were found to have minimal lesions on renal biopsy. Seven patients received courses of cyclophosphamide; a remission occurred in all instances. Remissions have lasted for a period ranging from 9 months to over 4 years. Five of these seven patients have subsequently relapsed, and in 3 of them all relapses have responded to prednisone. The other 2 had a recurrence of steroid-resistance, but in both instances a second course of cyclophosphamide induced a complete remission. Of these 2 patients, 1 has had no further relapse while the other has relapsed but has again become responsive to prednisone. Three patients were not treated with cyclophosphamide. One of them remitted after further treatment with intermitfent prednisone; the second remitted after 36 weeks of azathioprine; the third died from sepsis 4 months after the onset of steroid-resistance. The 9 surviving patients have been followed for a mean of 4.7 years, and all are doing well and have normal renal function.

743 SERUM IgE LEVELS IN HENOCH-SCHONLEIN PURPURA SYNTorrance, CA. DROME. Carl W. Trygstad and Douglas C. Heiner Dept. of Ped. UCLA Sch. of iled. Harbor Gen. Hosp.,

Serum IgE levels were determined in 37 children (age 3-16) who had Henoch-Schonlein Syndrame (HSS) and in 31 children with other renal disorders 10 of whon: had minimal change nephrotic syndrome. In 26 children with HSS acute and convalescent sera were available; in one child samples were available before and after developing the disease. Serum IgE levels were detemined using the double antibody radioimunoassay described by Gleich et al. The mean normal adult level in our laboratory is 63 , range $10-400$ international units $/ \mathrm{ml}$. Levels were converted to logarithms to the base of 10 for calculations. The geometric mean value for children with HSS was $103 \pm 31$, 1 S.D. and for children with other renal disorders was $143 \pm 32$, not significantly different. Paired values for acute and convalescent sera from children with HSS did not differ. Values were similar in children with HSS regardless of whether or not they had renal involvement. In the patient studied before, during and after the onset of the disease serum IoE levels remained norrial despite the development of renal involvment. In 10 children with minimal change nephrotic syndrome the ige level was not elevated as suggested by others and did not differ significantly from children with other renal disorders. We conclude that serum Ig[ levels are not aitered during the course of itSS.

74 GENTAMICIN THERAPY AND SERUM LEVELS IN PATIENTS WIT AND WITHOUT RENAL IMPAIRMENT. Leonard B. Weiner and Lilian L. Poe (Intro. by Frank A. Oski), S.U.N.Y. Upstate Medical Ctr., Dept. of Pediatrics, Syracuse, N.Y.

A study was undertaken in an attempt to determine if the currently employed dosage schedule of gentamicin (GM) provides optimum serum levels $(4.0-8.0 \mathrm{mcg} / \mathrm{ml})$. Twenty-one patients were evaluated, 10 patients with normal serum creatinine levels (Gp.A) and 11 patients with values in excess of $3.0 \mathrm{mg} \%$ (Gp. B). All patients had been on GM for at least $72 \mathrm{hrs}$. and received an initial dose of $4-5 \mathrm{mg} / \mathrm{kg}$ for the first $24 \mathrm{hrs}$. Patients in Gp. B then received $1.4 \mathrm{mg} / \mathrm{kg} /$ dose at intervals calculated from the following formula: hours between dose $=$ creatinine $x 8$. Specimens were obtained immediately before and 2-3 hrs. after a single IM or slow IV infusion dose. Three additional 2-3 hr. post-dosage serum specimens were obtained on all 21 patients after comparable subsequent doses. GM levels were assayed by agar diffusion, utilizing impregnated paper discs. Mean GM levels on patients in GP. A was $1.3 \mathrm{mcg} / \mathrm{ml}$ with a range of $<0.5 \mathrm{mcg} / \mathrm{ml}$ to $5.0 \mathrm{mcg} / \mathrm{ml}$. In $\mathrm{Gp}$. B the mean level was $2.3 \mathrm{mcg} / \mathrm{ml}$ with a range of $<0.5 \mathrm{mcg} / \mathrm{ml}$, to 8.8 $\mathrm{mcg} / \mathrm{ml}$. In Gp. A only 1 post-dosage determination in each of 2 patients was in the therapeutic range (11\%). In Gp. B 4 values in 1 patient and one in each of 2 patients were greater than $4.0 \mathrm{mcg}$ $\mathrm{m} 1$ ( $14 \%)$. No sample drawn prior to GM administration was within the therapeutic range. These results suggest that previously accepted dose schedules for patients with or without renal impairment may not provide adequate serum levels for therapeutic efficacy and emphasize the need for frequent monitoring of GM serum levels in patients regardless of renal function. 
745 MITOCHONDRIAL GRANULES IN RENAL PROXIMAL TUBULES OF UREMIC AND NON-UREMIC CHILDREN. Joanne D. Whitney, Joseph R. Goodman, and Carolyn F.Piel, Dept. Ped. Univ. California at San Francisco, San Francisco, CA 94143.

Electron-dense, calcium-rich granules in the mitochondria of several tissues (gut, bone, etc.) have been correlated with calcium transport under the influence of such hormonal agents as Vitamin $D$, parathyroid hormone, and calcitonin. Since chronic renal disease is associated with faulty Vitamin $D$ metabolism and elevated levels of parathyroid hormone and calcitonin, we used diagnostic percutaneous renal biopsy to study calcium granulation in the proximal tubule of 11 uremic and 12 non-uremic children as an index of calcium reabsorption. Mitochondria, cut from at least 10 electron micrographs of each sample from different sections of these tubules, were weighed. In the uremic children, there were 4.6 to 15.5 and in the non-uremic children 23.4 to 32.9 granules per gram of mitochondria. Granulation in uremics was similar to patterns obtained from the renal mitochondria of chickens made rachitic by Vitamin $D$ deprivation while non-uremics resembled those of chickens treated with Vitamin D. Hinimal granulation found in proximal tubules of these uremics raises the question of whether there is decreased reabsorption of calcium in this area, and suggests that the known conservation of calcium in uremia results from distal tubular reabsorption.

746 EFFECT OF HYPOXIA ON RENAL FUNCTION IN PUPPIES. M. H. Winterborn, W. A. Primack, C.M. Edelmann, Jr., and A. Spitzer. Albert Einsteín College of Medicine, Department of Pediatrics, New York.

There is reason to believe that the kidney of the newborn is better adapted than that of the older animal to withstand hypoxia and the ensuing acidosis which occur in RDS. In order to test this hypothesis moderate hypoxia $\left(\mathrm{p}_{a} \mathrm{O}_{2} 41 \pm 2\right)$ without hypocapnia $\left(\mathrm{paCO}_{2} 47 \pm 5\right)$ was induced in unanesthetized $1-4$ day old $(n=4)$ and $10-20$ day old $(n=5)$ puppies.

After 4 hours of hypoxia, the metabolic acidosis produced in the older puppies $\left(\mathrm{pH} 7.01 \pm .07\right.$, st. $\left.\mathrm{HCO}_{3} 9.6 \pm 1.7\right)$ was greater $(\alpha<.02)$ than in the newborn ( $\mathrm{pH} 7.2 \pm .04$, st. $\mathrm{HCO}_{3} 16 \pm 0.5$ ). Significant but similar decreases occurred in both groups in GFR $(39 \pm 14 \%)$, TRP $(39 \pm 16 \%)$ and urine $\mathrm{pH}$ $(10.7 \pm 3.3 \%)$. However, whereas $F E_{N a}$ increased $6.3 \pm 1.6$-fold in the older puppies, the increase was only $3.6 \pm 1.4$-fold in the newborn. Similarly fractional water excretion increased to a greater extent in the older puppies. The direct correlations found in the older animals between increases in $\mathrm{H}+$ and $\mathrm{Na}+$ excretion $(\mathrm{r}=0.64, \propto .01)$ and between $\mathrm{H}+$ and $K+$ excretion $(r=0.52, p<.01)$ and the inverse correlations found in the newborn ( $\mathrm{Na}+, r=-0.76, p<.001 ; \mathrm{K}+, r=-0.59, \propto<.01)$ suggest a greater distal delivery of $\mathrm{Na}+$ in the former group. Thus, in response to hypoxia, the 1-4 day old animal appears less liable than the 10-20 day old to develop acidosis and hyponatremia or to become dehydrated.

747 INTPALTERINE EXPOSURE TO NARCOTICS ON RENAL FUNCTION. A.Munire Yucecglu, Iradj Amirlak, Sook Ja Lee, Edward Tassermar and Kurt Lange. New York Medical College, Penal Service, Depts. of Ped. \& Med., New York, N.Y.

penal function was investigated in 45 infants born to drugaddicted mothers and in 46 normal control infants. 24-hour urine specimens obtained within the first 48 hours after birth were analyzed for urea clearance, $\mathrm{NH}_{4}^{+}$and titratable acid excretions, total osmolar concentration of the urine and electrolyte excretion pattern. Except for urinary sodiun excretion, both groups of infarts had comparable results. Neonates of mothers addicted to Methadone or Nethadone and Heroin combined, had higher urinary sodium excretion than that observed in the contrcls.

\begin{tabular}{lllll} 
& \multicolumn{2}{l}{ Urinary Sodium Excretion } & \\
\cline { 2 - 3 } & $\frac{\text { Heroin }}{27(59 \%)}$ & $\frac{\text { Her. }}{7(64 \%)}$ & $\frac{\text { Meth. }}{2(8 \%)}$ & $\frac{\text { Methadone }}{2(20 \%)}$ \\
$\geq 10 \mathrm{mEq} / \mathrm{L}$ & $\frac{19}{46}(41 \%)$ & $\frac{4}{11}(36 \%)$ & $\frac{22}{24}(92 \%)$ & $\frac{8}{10}(80 \%)$
\end{tabular}

While Heroin alone does not seen to alter the sodium excreticn of the neonate, Methadone alone or Heroin increases the sodium excretion significantly. Although the number of cases in each group is small, this functional alteration occurred without other functional derangements of the kidney; we therefore believe that this observation strongly suggests the possibility that intrauterine exposure to narcotics interferes with the renal sodium reabsorption at the tubular leve 1 .
748 NEPHROGENTC DIABETES INS IPIDUS - TYPE II: DEFECT DISTAL TO THE ADENYLATE CYCLASE STEP DOna 1d Zimmerman and Orville C. Green. Northwestern Univ. med. School and The Children's Mem. Hosp, Dept. Ped., Chicago, III A $28 / 12$ year-old female with severe nephrogenic diabetes insipidus (NDI) but with a negative family history of defective urine concentration, was studied for responses to water loading, antidiuretic hormone $(\mathrm{ADH})$ and tolbutamide. She responded to ADH administration ( 0.05 units intravenous ly) with an $89 \%$ increase in urinary cyclic AMP concentration but with no change in urinary osmolality $(55 \mathrm{mosm} / \mathrm{Kg}$ ). Intravenous tolbutamide increased urinary cyclic AMP concentration by $42 \%$. These results are compared with those in a 7 year-old female with nephronophthisis and a 4 year-old male with classical $\mathrm{X}-1$ inked NDI. The parents of this patient both showed good urinary concentration after a 12 hour thirst, with mother's urine concentration to $808 \mathrm{mOsm} / \mathrm{Kg}$ and father's urine to $795 \mathrm{mosm} / \mathrm{Kg}$.

This patient's disease seems to differ from classical NDI both biochemically and genetically. Biochemically, while the defect in classical nephrogenic diabetes insipidus appears to blunt the cyclic AMP response to ADH, this response is intact in the patient reported here. Genetically, if the disease of this patient is heritable, its mode of inheritance seems different from the $X-1$ inked recessive mode of classical nephrogenic diabetes insipidus. We have therefore called classical X-linked NDI a Type I form, and this newly described defect a Type II form of NDI.

This study supported in part by a Grant (MOL RR00199-10) from the GCRC Branch, DRR, USPHS.

\section{NEUROLOGY}

749 CLINICOPATHOLOGIC CORRELATES IN FAMILIAL DYSAUTONOMIA Felicia B. Axelrod, Joseph Dancis \& John Pearson N.Y. Univ. Sch. of med.,Dept. of Ped. E Path., N.Y. Pathological reports of familial dysautonomia have been negative, contradictory or representative of too limited an experience for interpretation. Based on an experience with 5 post-mortem examinations and 6 sural nerve biopsies, we now report consistent and distinctive pathological lesions.

Post-mortem examination has revealed dorsal root and paraspinal sympathetic ganglia to be small, depleted of neurons, with minimal changes suggestive of a degenerative process. The ciliary ganglia, representative of the parasympathetic system showed no qualitative pathology and no abnormalities could be detected in brain with current techniques. The papillae of the tongue were generally snall or rudimentary with sparse representation of taste buds and depletion of submucosal neurons.

Quantitative studies on the predominantly sensory sural nerve revealed a reduction in total fascicular area. Non-myel inated fibers were considerably diminished in number with complete absence of the larger fibers. A deficiency in catecholamine containing axons was demonstrated by fluorescent techniques.

Familial dysautonomia is primarily a developmental disease of the autonomic and sensory nervous systems. The findings are consistent with the hypothes is of a trophic substance controlling the development and maintenance of those systems.

750 POWER SPECTRUM OF EEG DURING SLEEP IN TERM AND PRETERM INFANTS. Victor Chernick, Rima Childiaeva, and Viktor Havlicek. Depts. of Pediatrics and Physiology, Univ. of Manitoba, Winnipeg, Canada.

We have analyzed the characteristics of the neonatal EEG during various stages of sleep in preterm and term newborn infants using fast Fourier transform and power analysis. Sleep state was assigned by clinical observation of the infant. Normal full-term infants ( 37 weeks g.a.) showed significantly higher power in $0.1-12.5 \mathrm{~Hz}$ frequencies during quiet sleep when compared to REM sleep. In preterm infants ( $<38$ weeks g.a.) there was no significant power difference between quiet and REM sleep. The EEG of quiet sleep in preterm infants is characterized by periodicity since large amplitude waves (bursts) alternate with low voltage activity (flats). From 32-37 weeks bursts showed a moderate increase in power of most frequencies with the greatest increase $(2.3 x)$ in delta 2 band $(1.5-3.5 \mathrm{~Hz})$. However, flats had a large increase $(45 x)$ in power of the integrated EEG $(1.5-25 \mathrm{~Hz})$. Since bursts are considered to be subcortical in origin and flats cortical, these findings suggest significant cortical maturation from $32-37$ weeks. It is suggested that analysis of the EEG power spectrum in preterm infants during quiet and REM sleep may be useful for the early detection of delays in brain maturation.

(Supported by Dept. Health and Soc. Dev. (Canada), Mrs. James A. Richardson Fdn., Inc, and the Children's Hosp. Res. Fdn., Inc.) 
751 C.A.T. SCAN IN NEUROGENETIC DISORDERS. Donald W. Day, George F. Smith, Jeannette N. Is rael and Richard Penn, Rush Medical Col lege, Depts. Pediatrics and Neurosurgery, Chicago.

knowledge in the field of neurogenetic disorders recentiy has been expanded by computerized axial tomography (C.A.T.). This new radiologic technique united a narrowly collimated $x$-ray source, scintillation detector crystals and a computer to produce a transverse axial tomographic image of a narrow slice of CNS architecture. We have used this noninvasive, atraumatic procedure to evaluate the cerebral architecture of 31 children with variably severe neurogenetic disorders. Our patients presented with the following symptoms: enlarged head (13), unusual cranial shape (7), mental + developmental retardation (15), seizures (5), and microcephaly (2). Ages range from 2 weeks into adolescence. Anong the 3l C.A.T. exams, $78 \%$ have demonstrated abnormalities of the cerebral architecture. These findings include: dilatation and/or abnormal shape of the ventricles: with clinical signs of hydrocephalus (8) and wi thout such signs (11); evidence of cortical atrophy (1); porencephalic cysts (2); extracerebral, intracranial cysts (2); gross asymmetry of CNS architecture (3). In $8 \mathrm{children,} \mathrm{additional} \mathrm{neurologic} \mathrm{proced-}$ ures (brain Scan, PEG or angiogram) were performed. In each case these procedures collaborated the CAT findings. The benign nature of this procedure permits repeat follow-up CAT exams to evaluate the stability of the pathologic or therapeutic process.

752 COMFUTERIZED AXIAL TOMOGRAPHY IN THE DETECTION OF INTRACRANIAL METASTASES IN CHILDREN. Paul G. Dyment, A. David Rothner, Paul M. Duchesneau, Meredith A. Weinstein. Depts. of Ped. Hematology-Oncology, Fed. Neurology, and Neuroradiology. Cleveland Clinic Fndn., Cleveland, Ohio (Intr. by William M. Michener)

Computerized Axíl Tomography (C.T.) represents a major advance in diagnostic neuroradiology. It utilizes a digital computer to measure variations in $x$-ray absorption produced by different brain tissues. X-ray beams pass through the skull in successive planes producing sectional tomograms of the brain substance without the necessity of injecting the child with radioisotope. The resulting scans resemble transverse cross sections of neuroanatomic specimens and are permanently recorded on black and white polaroid films. C.T. has reliably been used here and elsewhere to diagnose primary and metastatic brain tumors, brain abscesses, infarcts, hemorrhages, subdural and epidural hematomas, porencephalic cysts, and focal and diffuse cerebral atrophy. It is accurate, almost painless, and only requires sedation in young children.

Cerebral metastatic disease is unusual in childhood, but during the past 6 months 4 children with this complication were seen at this institution. Three of them had a C.T. and the metastases were demonstrable in each of them. Their primary tumors were osteogenic sarcoma, rhabdomyosarcoma, and hepatocarcinoma, and all patients expired within 6 months of the onset of this complication.

C.T. helps in the evaluation of intracranial disease, and it can frequently replace carotid angiography or pneumoencephalography.

753 CENTRAL NERVOUS SYSTEM SEQUELAE TO MECHANICAL

753 VENTILATION IN INFANTS WETGHXNG LESS THAN 1501 GRAMS AT BIRTH. Pamela Fitzhardinge, Karen Pape, Charles Net1ey, Pau1 Swyer. (Research Inst., Hosp. for Sick Children, Univ. of Toronto, Toronto, Canada.)

In the 28 month period from January 1,1970 to April 30 , 1973, 640 infants weighing less than 1501 grams were referred to the intensive care unit. Positive pressure mechanical ventilation either via nasotracheal tube or face mask was used in the management of 299 or $47 \%$. Seventy-five $(25 \%)$ of the ventilated infants survived the neonatal period. All but 2 of the survivors (i.e. 35 boys, 38 gir1s) have been followed prospectively; 14 for a minimum of 1 year, 59 for longer than 2 years. Major neurological defects occurred in 14 boys $(40 \%)$ and ? girls (18\%) as follows: 7 with shunted hydrocephalus, 7 with hemiplegia, 3 with spastic quadriplegia and 4 with spastic diplegia. Bayley Developmental scales done at 1 year of age averaged 93.5 for the mental developmental index and 81.3 for the psychomotor developmental index. Twenty-nine children $(40 \%)$ at 2 years of age appeared to be severely handicapped either by a major neurological defect and/or by retarded intellectual development. An analysis of the neonatal course showed a marked association between intracranial hemorrhage or seizures and severe central nervous system sequelae ( $p<.001$ ).

(Supported by Ontario Provincial Health Research (rant - PR 137.)
754 METABOLIC ACTIVITY OF ISOLATED BRAIN CAPILLARIES.G.W.
Goldstein*, J.s.Wolinsky, and I.Diamond, Depts. Neurol. and Pediatrics, Univ.California at San Francisco,

San Francisco, CA 94143 .

Endothelial cells in brain capillaries are sealed together by tight junctions which exclude small molecules from interstitial space, suggesting movement of substances between blood and brain through endothelial cells whose properties may influence permeability of the blood-brain barrier. Heretofore, methodologic difficulties have prevented study of brain capillaries in vitro. We have isolated capillaries from disrupted rat brain by density flotation in $25 \%$ bovine serum albumin. Electron microscopic examination reveals intact vessel segments which are free of contamination by glia, neurons, mi tochondria and nerve endings.Histochemically, alkaline phosphatase and $\gamma$-glutamyl transpeptidase may be' located primarily within capillaries. These enzymes were enriched 15- and 20-fold, respectively, compared with a cerebral cortex homogenate. Choline acetyltransferase, thought to be localized primarily in neuronal elements, was reduced 40-fold. Isolated capillaries accumulated ${ }^{14} \mathrm{C}-2$-deoxyglucose by a temperature-dependent process that is linear with time and saturable with substrate. Production of lactate and ${ }^{14} \mathrm{C}-\mathrm{glucose}$ was linear to $90 \mathrm{~min}$.Alterations in capillary function seem to contribute to the cerebral edema that occurs in many acute childhood illnesses. By using capillaries isolated from animals with encephalopathies, in vitro study of metabolic basis of abnormal brain capillary permeability should now be possible.
755 GLUTARIC ACIDURIA DUE TO GLUTARYL-COA DEHYDROGENASE DEFICIENCY : INHIBITION OF BRAIN GLUTAMATE DECARBOXY LASE BY GLUTARIC, B-FYDROXYGLUTARIC, AND GLUTACONIC ACIDS. Stephen I. Goodman, Oddvar Stokke, and Paul G. Noe (intr. by Donough O'Brien). L'niv. Colo. Med. Ctr., Dept. Ped., Denver. Glutaric aciduria is a disoxder of lysire, trytophan, and hydroxylys ine metabolism characterizec by intermittent metabolic acidemia, dystonia, athetosis, and mental retardation. We have shown that it is due to a recessively (autosomal) interited deficiency of glutaryl-COA dehydrogenase, the enzyme(s) which catalyze the dehydrogenation of glutaryl-COA to glutaconyl-COA ana decarboxylation of the latter to crotoryl-COA. A.bnormald quantities of glutaric, E-hydroxyglutaric, and glutaconic acids are found in the urine of these patients.

The nature of the movement disorder prompted study of the effects of abnormally excreted metabolites on brain glutanate decarboxylase, an enzyme implicated in the pathogenesis of Huntington's chorea. Glutamate cecarboxylase activity was exaninec in rat and rabbit brain acetone powders, stabilized with pyricoxal phosphate and glutathione. The preparation produced approximately equimolar amounts of ${ }^{14} \mathrm{CO}_{2}$ and ${ }^{14} \mathrm{C}$-GABA from $\left[1,5-{ }^{14} \mathrm{C}\right]-$ glutamate, was inhibited 93 pexcent by $10^{-4} \mathrm{M}$ aminooxyacetic acic, and had a $\mathrm{K}_{\mathrm{m}}$ clutamate of $2.86 \times 10^{-3} \mathrm{M}$. Glutarate, B-hydroxyglutarate, and glutaconate were competitive inkibitors of the enzyme, $\mathrm{K}_{\mathrm{i}}$ 's being $1.33 \times 10^{-3} \mathrm{M}, 2.51 \times 10^{-3} \mathrm{M}$, and $7.5 \times 10^{-4} \mathrm{~N}$ respectively. Inhibition of enzyme in fresh brain homogenates was also demonstrated. This inhibition may explair. the neurological accompaniments of this syndrome.

756 SWEAT GLAND REACTIVITY TO LOCAL AFPLICATION OF HEAT IN FAMILIAL DYSAUTONOMIA. Marvin Green. (Intr. by Miriam Lending) Dept. of Peäiatrics, New York Medical college, New York.

Hyperhidrosis is a commonly observed clinical sign in dysautonomia, though its cause is uncertain. One explanation is an erhanced excitatory state of autonomic centers. Since responsiveness of sweat glands to local application of heat is at least in part due to a central reflex, increased glandular reactivity to heat might elucidate the cause of excessive sweating in this disease. Stimulation tests were performed on seven surjects with dysautonomia and on eight control subjects. Environmental temperature and relative humidity ranged from $23.0 \mathrm{C}-24.5 \mathrm{C}$ and $288-$ 358 respectively. Body temperature and temperature of the test site (volar forearm) were comparable for both groups. Heat was applied with a hollow copper disc through which thermo-regulated water flowed. Disc temperatures of $41.5 \mathrm{C}$ and less were applied for a maximum of up to 10 minutes. For each subject the minimal duration of heating at the lowest temperature capable of evoking sweating was determined (threshold). Thresholds were lower. in all subjects with dysautonomia, ( $P<.01$, Fisher exact test). The results suggest that hyperhidrosis in dysautonomia may be due to an increased excitatory state of sudorific centers. These tests do not appear to be helpful in detection of the heterozygote since one control subject, the father of a child with dysautonomia, had the highest threshold.

Supported by the Dysautonomia Foundation, grant 48-132-3

Submitted to J. Appl. Physiol., November, 1974. 

757 Maureen Hack, Simón Miranda, Robert Fantz (Intr. by Marshall Klaus) Case Western Reserve Univ. Dept. of Pediatrics \& Psychology, Cleveland.

The emergence of visual function in the preterm neonate is of special interest when planning the infant's environment. To explore the earliest visual capabilities of the preterm neonate, we studied pattern discrimination responses in 20 preterm infants ranging in age from $31-34$ weeks and compared them with 24 term newborns. We used the $V$ isual Preference Technique (Fantz, Science 140:296,1963) which measures differential fixation of pairs of patterns by using corneal reflection as the criterion of fixation. The stimuli were patterns varying in size and number of black squares on a white background.

In all infants, strong visual preferences were found for the larger size and the larger number of details, indicating responsiveness to these two important stimulus variations. However, when the black-white ratio was held constant, no preferences were found with increasing detail. Pattern vision was found as early as 31 weeks in some infants. The preferences of the preterm group were similar to those of term infants. There were, however, individual differences in the age of the earliest discriminative response. Also, in the infants followed serially, there was a gradual increase in the indices of visual attention (cessation of suckirg, widening of the eye, and active versus passive examination of the stimulus).

These observations reveal the very early functional development of the visual system and suggest that further explorations are relevant.

758 THYMIDINE KINASE AND HERPES SIMPLEX ENCEPHALITIS Milo D. Hilty, $\frac{\text { Virginia Merchant, Ralph E. Haynes }}{\text { M. Col. of GA, Dept. of Ped., Augus ta, GA and Ohio }}$ State Univ., Col. of Med., Children's Hosp., Dept. of Ped., Columbus, Ohio (Intro. by Bruce D. Graham)

The diagnosis of herpes simplex encephalitis (HSE) is difficult to establish unless the virus is recovered from brain tissue or cerebrospinal fluid (CSF). Since herpes simplex virus (HSV) is rarely recovered from the CSF, we have elected to determine if thymidine (TdR) kinase, an early enzyme in the HSV replicative cycle is present in the CSF of patients with HSE. Quantitative TdR kinase determinations were performed on CSF from three neonates with HSV-2 encephalitis, four adul ts with HSV-l encephalitis and 17 control patients with non-HSV central nervous system infections. The mean CSF TdR kinase activity (picomoles of TdR phosphorylated/min/ 106 mononuclear cells) for children with HSV-2 encephalitis was $341+149$ S.E.M. and $6 \pm 3$ S.E.M. for children with non-HSV encephalitiss. The mean TdR kinase activity for adults with culture proven HSV-1 encephal itis was $4.25+3.32$ S.E.M. and $0.31+0.10$ S.E.M. for adults with non-HSE. In both neonates and adults, mean levels of TdR kinase activity were higher in CSF of patients with HSE established by recovery of virus, than in CSF from appropriate control patients with non-HSV central nervous system infections.

These results indicate that TdR kinase is induced in CSF mononuclear cells by HSV during the course of HSE. The detection of TdR kinase in CSF may be useful as a non-invasive, rapid approach to the diagnosis of HSE.

759 CEREBRAL METABOLISM IN THE SHEEP: A COMPARATIVE STUDY OF THE ADULT, THE LAMB, AND THE FETUS. M. Douglas Jones, Jr., Laurence 1 . Burd,

Edgar L. Makowski, Giacomo Meschia, and Frederick C. Battaglia. Division of Perinatal Medicine, University of Colorado Medical Center, Denver.

The cerebral uptakes of glucose, oxygen, lactate, pyruvate, acetoacetate and $\beta$-hydroxybutyrate were compared in the adult, the fetal and the newborn sheep. Beginning one to two days after surgery, samples were withdrawn simultaneously from an artery and from the superior sagittal sinus, for up to two weeks. At all ages, glucose was the only substrate measured which was taken up in significant quantity relative to oxygen. No positive arteriovenous differences for ketone bodies were found. The glucose-oxygen quotients were $0.98<1.03<1.08$ in adults, $0.92<0.99<1.06$ in lambs, and $0.92<0.98<1.03$ in fetuses. These quotients were not significantly different. There was a significant $(P<0.05)$ arteriovenous difference for lactate in the adult $(-0.031 \mathrm{mM})$, but not in the lamb or fetus. A significant $(P<0.05)$ arteriovenous difference for pyruvate was found in the adult $(-0.013 \mathrm{mM})$ and fetus $(-0.020 \mathrm{mM})$. The findings of a glucoseoxygen quotient of approximately one and the lack of significant lactate production in fetal sheep brain are inconsistent with the hypothesis, suggested by some investigators, that anaerobic glycolysis is important to steady-state fetal brain metabolism.
760 CHANGES IN CSF IgG AND ALBUMIN DURING THE COURSE OF EXPERIMENTAL ALLERGIC ENCEPHALITIS AND HERPES VIRUS ENCEPHALITIS. Neil Lombardi Edward F. Rabe, Martin Flax, and Te W. Chang, Tufts Univ. Sch. Of Med., New England Medical Center Hospital Dept. of Ped. Neuro., Boston, Mass.

The differential diagnosis of virus vs. post-infectious encephalitis in childhood is often complicated by similar course, CSF findings and the difficulty of virus isolation. In order to determine if a differentiation could be made on the basis of quantitative CSF IgG measurements, experimental allergic encephalitis and Herpes virus encephalitis was produced in each of two groups of rabbits and serial measurements were made of CSF and serum IgG and albumin simultaneously throughout the course of the diseases, using the technique of electro-immunodiffusion. Statistically significant elevations in CSF of both albumin and IgG occurred during EAE, peaking the fourth week. However, there was also a statistically significant increase in the ratio of IgG to albumin, suggesting a simultaneous breakdown in the blood-brain barrier and the local production of IgG within the CNS during the course of EAE. No significant changes in either IgG or albumin occurred in the group with Herpes encephalitis.Pathologic examination of the brain and spinal cord of each animal was made at the end of the experiment to confirm the nature of the disease produced in each animal.

INTRACEREBELLLAR HEMORRHAGE AS A POSSIBLE COMPLICATION OF MASK APPLIED MECHANICAL VENTILATION IN THE LOW BIRTH WEIGHT INFANT. Karen Pape, Dawna Armstrong, and Pamela Fitzhardinge. Research Inst.,Hosp. for Sick Children, Univ. of Toronto, Toronto, Canada. (Intr. by A. Sass-Kortsak.)

In the past 2 years we have noticed at necropsy a previously undescribed type of hemorrhage in the brains of the very low birth weight infant. This hemorrhage is extensive and is located in one or both cerebellar hemispheres. It may occur singly or be associated with subependymal plate bleed ing and intraventricular hemorrhage. In the last year, 9 of 34 autopsies on infants under 1500 grams have shown this type of intracerebellar hemorrhage. review of their neonatal course revealed that these 9 had all received intermittent positive pressure ventilation via a face mask. The cerebellar lesions were not present in any of the 12 babies ventilated by endotracheal tube alone. Face mask ventilation has become increasingly popular over methods requiring endotracheal intubation because of the reduction in infection and chronic lung disease. It is the practice in our unit to attach the mask snugly to the head with a velcro band across the occiput. Application of these masks for more than 2 days in the very small infant causes extensive head molding with compression of the occipital area. It is possible that such occipital molding accompanied by the buffeting effect of positive pressure ventilation may be related to the development of the intracerebellar bleeding. It is recommended that a less traumatic method of attachment be devised before the face mask is used in these small infants.

762 REDUCED DEFORMABILITY OF ERYTHROCYTE MEMBRANES FROM PATIENTS WITH DUCHENNE MUSCILAR DYSTROPHY. Alan K. Percy and Michael E. Miller. Charles R. Drew Postgrad. Medical School, Dept. of Pediatrics, Los Angeles, California 90059. A generalized membrane defect has been hypothesized as the underlying genetic basis for Duchenne type muscular dystrophy (DMD). Erythrocyte membranes have been implicated in DMD by the recent observations of Matheson and Howland(Science 184:165,1974) who inave demonstrated surface deformation with scanning electron micros copy. In order to evaluate the functional characteristics of intact ery throcyte membranes in DMD, we have studied membrane deformability by the technique of cell elastimetry. Through the use of a micro-manipulator, individual erythrocytes or polymorphonuclear leukocytes (PMNS) have been directed to the orifice of smal1 pore glass pipettes (internal diameter $1-2 u$ and $3-5 u$ respectively). Upon application of carefully measured negative pressure to the pipette, the "deformability" of the cell membrane is expressed as the amount of negative pressure required to draw the cell into the pipette. The assay is highly reproducible and resulted in the following observations:1)The deformability of PMNS from DMD patients was comparable to that of PMNS from control subjects, 2) Whereas exythrocytes from control subjects were easily deformable, those from DMD patients could be deformed on $1 \mathrm{y}$ at negative pressures 10-15 times control values. We conclude that the observed rigidity of the OMD erythrocytes provides the first evidence of a functionally significant memb rane disorder in DMD and correlates well with previously described anatomical and chemical alterations in this disorder. 

763 TRANSPORT OF LOM BIRTH WIIGHiT INFANTS AND Anselo Ferrara, Sarvesh Visam, Sonhie Pierog, (Intr. by Jose Gtr., Dept.Ped; Jewish llosn.dyed.Ctr. Brooklyn, Dent. Ped. From $4 / 71$ to $4 / 74$ the NYC Infant Transport Service transferred 3099 infants to 16 NJ.Cus. Overall survival was $81 \%$. Autopsies (including connlete neuronatholorical exam) of low birth weirht ( $<2500 \mathrm{rm}$ ) non-survivors vere reviewed for intracranial bleeding. Controls vere autopsies of non-transported non-survivors cared for in the same vicus, matched for weight. Elininated from the study were congenital anotalies, diagnosed bleeding dist orders,breech presentations, and those dying beyond one week of age. At :ICU "A", autopsics of 17 inborn and 15 transported infants were reviewed. At NICU "B", 28 inborn and 31 transported autopsies were sinilarlv reviewed. There was no statistically significant difference in intracranial bleeding between transported and non-transported infants in all weipht categories. $C$ HI SOUARE VALUES OF TRANSPORTED \& INBORN NEONATES

\begin{tabular}{|c|c|c|c|}
\hline & All Weights & $\angle 1000 g \mathrm{~m}$ & $1001-2500 \mathrm{gm}$ \\
\hline NICU "A" & 0.122 & 0.0 & 0.1200 \\
\hline Transported & not & not & not \\
\hline vs I aborn & significant & significant & significant \\
\hline$\overline{\mathrm{NICU}} " \mathrm{~B} "$ & 0.0859 & 0.2920 & 1.3110 \\
\hline Transported & not & not & $\begin{array}{l}\text { not } \\
\text { sionificant }\end{array}$ \\
\hline vs Inborn & significant & $\operatorname{signit} x \mathrm{c}$ ant & \\
\hline
\end{tabular}

764 SERUM NERVE GROWTH FACTOR LEVELS IN FAMILIAL DYSAUTONOMIA. John G. Rogers, David C. Siggers, Samuel H. Boyer, iouise Margolet, Henry Dorkin, Snailesin P. Banerjee, Eric M. Snooter, (Introduced by Barion Childs) Jonns Hopkins Univ., Johns Hopkins Hosp., Dept. of Ned., Baltimore and Stanford Univ., Sch. of Med., Stanford.

Anatomical and physiological similarities exist between recessively innerited familial dysautonomia (SD) and mammals immunosympatnectomised by treatment with antibodies to mouse nerve growth factor (NGF). We proposed that FD might accrue from a molecular abnormality of $\mathrm{NGF}$. We used three properties of $\mathrm{NGF}$ to test tinis: stimulation by $B$ NG $F$ of neurite growth in explanted chick dorsal root ganglia (Bioassav); chain-specific antigenicity of the $\alpha, \beta$, and $\gamma$ NGF subunits measured by radioimmunoassay; and binding of 2.55 derivative of 3 NGT to a microsomal fraction of rabbit cervical. ganglia.

We tested 8 nomal subjects and 8 dysautonomics from $8 \mathrm{kin}$ dreds. Bioassay was the same in all. When sera were pretreated with antibody to whole $V G F$, FD sera were less easily innibited than that of normals. Radioimmuroreactive levels of $\alpha$ and $Y$ NGF (cross reacted with the appropriate goat anti-mouse NGF subunit) were the same for all; B NGF levels were $2 \frac{1}{2}$ times greater in $F D$ than in normals and heterozygotes. Binding assay showed FD serum displaced 1.35 times more $2.55 \mathrm{NGF}$ than normals.

The parallel rise of function (binding assay) and imunoreactivity, suggests that the elevated levels of $B$ WGF in FD is secondary, rather tian a primary molecular defect. However a primary lesion in NGF or one of its subunits is not excluded.

765

AGENESIS OF THE CORPUS CALLOSUM DIAGNOSIS BY COMPUTERIZED AXIAL TOMOGRAPHY. A. David Rothner, Paul M. Neurology and Neuroradiology. Cleveland Clinic Fndn. Cleveland, Ohio (Intr. by William M. Michener).

Computerized axial tomography (C.T.) is a revolutionary noninvasive neuroradiologic technique that enables the brain to be electronically displayed in sections that resemble neuroanatomic specimens. These displays are permanently recorded on black and white polaroid film. The technique utilizes a digital computer to measure variations in $x$-ray absorption through different tissues.

$C$. T. has been used to accurately visualize primary and metastatic brain tumors, hydrocephalus, cerebral abscesses, subdural and epidural hematomas, infarcts, hemorrhages and diffuse and focal atrophy.

Agenesis of the corpus callosum (A.C.C.) may be partial or complete. Associated anomalies may include midline structural defects (absence of the septum pellucidum, holoprosencephaly) or more generalized malformations (polygyria, macrogyria, gray matter hetertopias). Symptoms may include seizures, hydrocephalus, mental retardation and/or focal neurologic deficits and usually appear in early childhood.

Two patients with delayed development and seizures are presented. Both had A.C.C. diagnosed utilizing the C.T. and confirmed by angiography and oneumoencephalography.

This safe, rapid, new technique is useful in the study of numerous intracranial conditions and will allow the diagnosis of certain congenital anomalies to be made without risk to the patient.
766 EFFECTS OP METHYLPHENIDATE ON CHILDREN WITH HYPERACTIVE BEHAVIOR. Richard J. Schain and Carol L. Reynard, UCLA Sch, of Med., Dept. Ped., Los Angeles. Ninety-eight hyperactive children, ages 6 to 12 years, have been treated with individually titrated doses of methylphenidate or placebo under double blind conditions for a 16 week period. Only one of the 48 children receiving placebo was judged to be a drug success at the end of the trial period. 74 of 94 children receiving methylphenidate (79\%) were judged to be drug successes at the end of the study. This judgment was based upon behavioral rating scales (Conners) filled out by teachers and parents at the beginning and end of the study. The main behavioral alteration observed was increased ability to focus and sustain attention. A wide range of dosage $(0.2-1.9 \mathrm{mg} / \mathrm{kg})$ was required for optimum drug effects. Drug failures were due to either the appearance of withdrawn, apathetic behavior (6 cases) or lack of evidence of drug effectiveness ( 14 cases). Two obese children were total nonresponders even though $\mathrm{mg} / \mathrm{k} g$ dosage was equivalent to children categorized as drug successes. The most dramatic responses were seen in children characterized as exhibiting developmental hyperactivity, i.e. hyperactive behavior in the absence of evidence of other neurological or cognitive disturbances. These observations provide further evidence that administration of graded doses of a central stimulant agent to children designated as 'hyperactive' often results in behavioral changes felt to be favorable by parents and teachers. The question of the effect of this type of intervention on longterm academic success and social adaptation remains to be evaluated.

767 ANALYSIS OF COGNITIVE EFFECTS OF WITHDRAWAL OF SEDATIVE ANTICONVULSANTS IN EPILEPTIC CHILDREN. Richard J. Schain, Joan P. Riehl and Joseph Ward. UCLA Sch. of Med., Dept. Ped., Los Angeles.

Phenobarbital and related sedative anticonvulsant drugs have often been observed to interfere with cognitive functions in children. The present study evaluates effects on cognitive stvle of replacement of phenobarbital or primadone (Mvsoline) with carbamazepine (Tegretol) in 9 epileptic children. The tasks utilized were the Matched Faniliar Figures Test (MFFT-Kagan) and the Children's Embedded Figures Task (CEFT-Witkin). At the end of the 4 month replacement period, large improvements in performance were seen in 6 of the 9 subjects. Degree of improvement correlated with initial deficit estimated from normative data. A mean $42 \%$ reduction in errors on the MFFT and a mean $50 \%$ increase in correct responses in the CEFT occurred on retesting after replacement of drugs. By contrast, only a $3 \%$ increase occurred in WISC IQ scores during this time perid. Eight of the 9 children appeared perceptibly orighter and less hyperactive after drug replacement. Seizure control was equally effective with carbamazepine as with sedative anticonvulsants. Plasma carbamazepine levels ranged from 4.7 to $13.0 \mathrm{ug} / \mathrm{ml}$ (mean-7.7ug $/ \mathrm{ml}$ ) In two instances, urticarial rashes required discontinuance of carbamazepine. These studies indicate that measures of cognitive style may be used to judge effects of anticonvulsants on cognition. Improvement of cognitive performance occurs in some children when carbamazepine is substituted for phenobarbital or primadone.

768 EFFECTS OF PHENOBARBITAL VS. UNDERNUTRITION UPON EARU BRAIN GROWTH. Richard J. Schain and Xathv S. Watana-
be*, UCLA Sch. of Med., Dept. of Ped., Los Angeles. Male wistar rats of birth weight between 6 and 7 grams were as signed to litters of 4 animals shortly after birth. Six of these litters (24 animals) received daily subcutaneous injections of 0.06 $\mathrm{mg} / \mathrm{g}$ of phenobarbital from dav 3 to day 20 of life. Initial sedation was produced but tolerance to sedative effects developed within a few days. Six litters of 4 animals each (controls) and six other litters containing 16 animals each ('undernourished') were handled only for weighing pirposes. All animals were sacrificed at age 21 days. The mean body weights of the phenobarbital treated animals and the 'undernourished' animals were significantly smaller than the control animals (phenobarbital, -31\%; undernourished, $-40 \%$ ). The mean brain weights of phenobarbital and undernourished animals were also significantly smaller than control animals (phenobarbital $-12 \%$; undernourished $-7 \%$ ). Reductions in cerebellar size were greater than total brain reductions. Comparison of undernourished animals to phenobarbital animals revealed that body weight reduction was significantly greater in undernourished animals while brain weight reduction was significantly greater in phenobarbital animals. Calculation of brain weight-body weight ratios indicate that control and undernourished aninals will fit on a single exponentially decreasing curve where the horizontal axis is body weight. Brain weight-body weight ratios of phenobarbital treated animals lie below the curve. These data indicate that nonhypnotic doses of phenobarbital selectively retard brain growth in a manner that is at least partially independent of nutrition. 
768 CSF AMINE METABOLITES IN CHILDREN WITH MINIMAL BRAIN DYSFUNCTION (MBD) -EVIDENCE FOR ALTERATION OF BRAIN DOPAMINE. Bennett A. Shaywitz, Donald J. Cohen, Malcom B. Bowers, Jr. Yale Univ. Sch. of Med. (Intr. by Howard A. Pearson)

Minimal brain dysfunction (MBD) is one of the most pressing problems in present day pediatrics, estimated to affect $8-10 \%$ of the school-age population. Evidence from epidemiologic investigations as well as the response to amphetamine-type medications suggest that the syndrome of $M B D$ is intimately related to brain catecholamines-evidence however that is by necessity circumstantial and inconclusive. We have approached the problem by utilizing the technique of oral probenecid loading to measure CSF concentrations of 5-hydroxyindole acetic (5-HIAA) and homovanillic acid (HVA), the major metabolites of serotonin and dopamine respectively. Within the last two years we have incorporated six children with symptoms of MBD into our ongoing study of CSF amine metabolites in neuropsychiatric disorders of children. Our results (table-mean ${ }^{+} S E M$ ) indicate

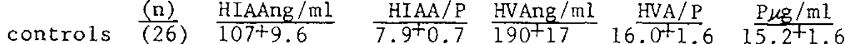

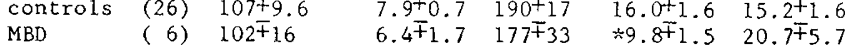
comparable values for 5-HIAA, 5-HIAA/P (robenecid), HVA and P(robenecid). However, children with MBD have significantly lower concentrations of $\mathrm{HVA} / \mathrm{P}$ (robenecid) $(\mathrm{p}<0.05, \mathrm{t}$-test). This data suggests a reduced turnover of brain dopamine in children with $\mathrm{MBD}$ and is the first direct evidence demonstrating disordered brain catecholaminergic function in this perplexing disorder. $768 \mathbf{6}$ (MBD) IN DEVELOPING RATS - "THRESHOLD" BRAIN DOPAMINE CONCENTRATIONS AFTER 6-HYDROXYDOPAMINE (6-OHDA). Bennett A. Shaywitz and Robert D. Yager Yale Univ. Sch. of Med. (Intr. by Howard A. Pearson)

We have produced an experimental model in developing rats that is similar to the clinical syndrome of $M B D$. This model is effected by the administration of 6-OHDA to 5 day old rat pups, resulting in a rapid and permanent depletion of brain dopamine to $38.0 \%$ of controls. For the first 2 weeks of postnatal 1 ife both 6-OHDA treated rat pups and their littermate controls show little spontaneous movement. Between 2-3 weeks of age activity increases dramatically, but by 4 weeks has declined again, to remain stable until it declines again in senescence. (Table) 6OHDA treated rat pups are significantly more active at both 15

Spontaneous Activity (\% of total activity) mean $\pm_{S E M}$

controls $\frac{8 \text { days }}{24.5^{+2.6}} \quad \frac{15 \text { days }}{58.4+3.0} \quad \frac{22 \text { days }}{63.1+2.6} \quad \frac{26 \text { days }}{46.6+3.2}$

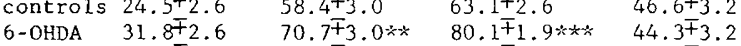
days $(p<0.01)$ and at 22 days $(p<0.001)$, (t-test). The inverse correlation between brain dopamine concentration and hyperactivity is evident. Reduction of brain dopamine concentrations below $55 \%$ of controls results in significantly greater activity at 2-3 weeks of age. Reduction of brain dopamine to only $70-90 \%$ does not alter activity, while brain dopamine concentrations 55$65 \%$ of normal has variable effects. Thus, there appears to be a critical threshold for brain dopamine concentrations that determines the development of hyperactivity.

769 A SIMPLE DIAGNOSTIC TEST FOR DIFFERENTIATION OF SUBACUTE NECROTXZING ENCEPHALOMYLLOPATHY (SNE, Leigh's Disease), THIAMINE ( $B_{1}$ ) DEFICIENCY (Wernicke's Disease) AND ALLIED CNS DISORDERS. Jean Holowach-Thurston, Richard E. Hauhart, and David B. McDougal, Jr. Dept. Pediat, Wash U. Med School, St. Louis, Mo

In $B_{1}$ deficient mice brain pyruvate (PYR) and $x$-ketoglutarate $(x \mathrm{KG})$ dehydrogenase activities were reduced and brain PYR and $x$ KC greatly 1ncreased (Holowach et a1., 1968, J. Neurochem. 15, 621). If such cerebral metabolite changes occur in human $B_{1}$ deficiency, they might be reflected in CSF. The validity of this hypothesis was tested. PYR concentration in the CSF of 167 normal children was $94 \pm 2 \mu \mathrm{M}, x \mathrm{KG}$ was $2.35 \pm 0.07 \mathrm{\mu M}$; In a child with CNS disease associated with dietary restrictions for allergy, PYR and $x$ KG in CSF were $287 \mu \mathrm{M}$ and $18.0 \mu \mathrm{M}, 3$ and $8 \mathrm{x}$ controls, respectively. Thiamine diphosphate (TPP) in RBC was undetectable and only 2.06 $\mathrm{nM}$ in CSF. Control values were $179 \pm 23 \mathrm{nM}(\mathrm{n}=3)$ in $\mathrm{RBC}$ and $9.8 \pm 0.9 \mathrm{nM}(\mathrm{n}=4)$ in CSF. PYR and $\alpha$ KG levels in CSF and TPP values in blood and $C S F$ were normal $10 d$ after $B_{1}$ therapy was started. Signs of CNS involvement disappeared, confirming the diagnosis of $B_{1}$ deficiency. By contrast, in a case of suspect SN with enzyme-inhibiting factor present in urine, PYR and $\alpha$ KG levels in CNS did not differ from controls. Measurements of PYR and $\alpha$ KG in CSF are proposed as a simple, rapid afd in differentiating Wernicke's disease and hereditary enzymatic defects of PYR oxidation from SNE due to reduced thiamine triphosphate levels secondary to inhibition of TPP:ATP-phosphotransfexase by the CooperI tokawa-Pincus enzyme inhibitor.

\section{0} "NEUROLOGICAL SEQUELAE IN BATTERED BABIES" Lawrence $\mathrm{G}$. Tomasi \& $\underline{\mathrm{N}}$. Paul Rosman

Dept. Ped., Boston U.S ch.of Med.,Boston City Hospital Introduced by Charles $\underline{C}$. Lobeck

The recognition of the Battered Child Syndrome has lead to an understanding of many previously unexplained neurological symptoms in infancy. The frequency of intracranial injury in battered infants is unknown, but of the multitude of injuries described, it is the most severe with respect to both survival and prognosis for normal development.

of 112 battered infants admitted to Boston City Hospital from $1971-1973,48(43 \%)$ had sustained a significant head injury. Seven patients $(15 \%)$ had neurological sequelae at discharge, and all seven had presented in coma. The findings in these seven patients on admission were subdural hematoma (5), skull fracture(4) and refractory seizures(3). At discharge, neurological sequelae included seizures(4), severe developmental delay(4), hemipares is (3), EOM palsies(3)and blindness(1). None of the remaining 41 battered infants demonstrated neurological abnormalities at discharge, despite the fact that 9 of the 41 had presented with seizures. In all 9 , subdural taps were negative. Each of the 32 remaining infants had a cerebral concussion, 14 of which had associated skull fractures.

Thus, significant head injuries are seen frequently in battered infants and coma is an ominous sign of future neurological disability. Post-traumatic seizures and cerebral concussion are common presenting symptoms but do not necessarily indicate a poor prognosis. Follow-up is necessary to determine the overall incidence of neurological sequelae in child abuse.

\section{VOICEPRINT ANALYSIS IN NEUROPATHOLOG Y DIAGNOSIS}

771 Henry M. Truby, Carol S. Shear, and William L. Nyhan.

Univ. of Miami, Sch. of Med., Dept. of Ped., Miami, Florida; Univ. of Cal. at San Diego, Sch. of Med., Dept. of Ped., La Jolla, California.

We have differentially examined sound spectrograms of the vocalizations of children with Cornelia de Lange Syndrome (Type II), and of children with other neuropathologies, in order to validate sound-spectrographic analysis as a supplementary diagnostic procedure in the detection and/or corroboration of neurophysiological abnormality.

Theoretically, acoustigraphic analysis of vocalization reflects both neurophysiologic and neuropathologic idiosyncrasy, and certain pediatric researchers have even variously hypothesized that there are differentiating voiceprint correlates for specific neuropathologies. If so, classical clinical diagnoses of neuropathology should be confirmable on the basis of relevant sound-spectrographic criteria.

Evaluation of some 350 sound spectrograms for 20 de Lange children confirms that there are clear sound-spectrographic correlates for two major vocalization types--the easily recognizable monotonous, intermit tent grunting and the raspy, bark-like crying (de Lange patients being predominantly non-verbal). Broadband turbulences are apparent as another consistent acoustigraphic-pattern criterion. In a few instances, additional voiceprint patterns are recognizable.

We are thus finding positive confirmation of the reliability of voiceprint criteria in identifying de Lange syndrome and other specific developmental abnormalities. Ultimate clinical application of this technology would be in providing preliminary diagnostic indices in early infancy.

THE NEUROLOGIC OUTCOME OF INFANTS EVALUATED FOR UN-

772 BOUND BILIRUBIN, Frederick H. Wirth, Karen E. Goldberg, Lula $O$. Lubchenco, Univ. Colorado Med. Ctr., Division of Perinatal Med., Denver

Twenty-four newborns with hyperbilirubinemia, gestational ages between 34 and 41 weeks, were tested for the presence of unbound bilirubin, using the Hart Sephadex gel technique. The peak bilirubin levels were between 11 and $26 \mathrm{mgm} \%$. Except for 1 infant with respiratory distress and mild acidemia, the infants otherwise had uncomplicated neonatal courses.

A developmental history, neurologic and developmental evaluations, including the Bayley Scale of Infant Development and the $A$. Milani-Comparetti reflex examination were done at 15 to 21 months of age. There was no relationship between the presence of unbound bilirubin during the neonatal period and the findings of gross abnomalities in motor development or neurologic outcome. However, 15 of the 24 infants $(62 \%)$ had abnomal neurologic findings. Thirteen of the 15 showed a uniform pattern of neurologic abnormalities consisting of hypotonia of the proximal musculature, marked head $\mathrm{lag}$ and incomplete Landau reactions.

The ratio of total serum bilirubin to total serum protein (the "Odell number") was predictive of neurologic outcome in infants without iso-immunization (p $<.05)$. 


\section{ONCOLOGY}

COMPARISON OF FOUR SCHEDULES OF CHEMOTHERAPY DURING REMISSION OF CHILDHOOD ACUTE LYMPHOCYTIC LEUKEMIA (ALL). Rhomes J.A. Aur, Joseph V. Simone, Manuel S.Verzosa, H.O. Hustu, Larry F. Barker, and Donald P. Pinkel.

Since all drugs used to prolong remission of ALL are not equally effective, the dosage of a better agent often must be reduced because of the added toxicity of less effective agents. The purpose of this study was to determine whether it is better to give fewer agents in relatively high dosage, or a greater number of agents in necessarily lower dosage. After inducing remission with prednisone, vincristine and asparaginase, patients received cranial irradiation and I'T methotrexate (MTX). Then patients were randomized into one of 4 groups: A - MTX 40-80 $\mathrm{mg} / \mathrm{m}^{2} / \mathrm{wk}$ IV; B - MTX $20-30 \mathrm{mg} / \mathrm{m}^{2} / \mathrm{wk}$ IV + mercaptopurine $50 \mathrm{mg} / \mathrm{m}^{2} / \mathrm{d} \mathrm{PO}$ C - As in group B + cyclophosphamide $200 \mathrm{mg} / \mathrm{m}^{2} / \mathrm{wk} \mathrm{IV;D} \mathrm{-} \mathrm{As} \mathrm{in} \mathrm{group} \mathrm{C} \mathrm{+}$ arabinosyl cytosine $50 \mathrm{mg} / \mathrm{m}^{2} / \mathrm{wk}$ IV over $4 \mathrm{hr}$. These were starting dosages to be adjusted to tolerance. From Jan. 72.74, 164 patients attained remission, received CNS therapy and were randomized. The frequency of relapse and complications differed by groups. In Group A (MTX alone), 14 of 20 patients relapsed and 9 developed leukoencephalopathy without evidence of CNS leukemia or infection, so randomization to this group was discontinued. In Group B, 7 of 49 patients relapsed and there was one case of Pneumocystis carinii pneumonia (PCP). In Group C, 12 of 48 patients relapsed and there were 3 cases of PCP. In Group D, 5 of 47 patients relapsed and there were 16 cases of PCP with 3 deaths in remission. Infections and interruption or decrease of chemotherapy were directly related to the number of drugs used. The results so far indicate: (1) In this study MTX $40-80 \mathrm{mg} / \mathrm{m}^{2} / \mathrm{wk}$ IV was prohibitively toxic to the CNS and failed to produce longlasting remissions; (2) The frequency of relapse was least with the 2-and 4-drug regimens; and (3) The frequency of life-threatening immunosuppression was directly related to the number of drugs given.

774 THE PLATELET COUNT IN MALIGNANCIES PRESENTING AS JUVENILE RHEUMATOID ARTHRITIS. M. Sharif Badri, Thomas D. Miale, and Elia M. Ayoub, Univ. of Florida 1. of Med. Dept. of Ped., Gainesville

Children with malignancies may present with clinical manifestations of Juvenile Rheumatoid Arthritis (JRA). Six patients who presented to our rheumatology clinic with polyarthritis and normal peripheral blood smears, were found to have acute lymphocytic leukemia (ALL) on bone marrow examination. To determine if any other criteria could have suggested the diagnosis of ALL, the initial clinical and laboratory data on these patients were compared with six age and sex-matched patients with JRA. No difference in the clinical manifestations, including type of fever, arthritis, organomegaly, radiological findings, ESR, peripheral WBC and differential were noted between the two groups. The only significant difference encountered was in the initial peripheral platelet count. Patients with JRA had platelet counts ranging from $240,000-375,000 / \mathrm{mm}^{3}$ (mean $=313,000$ ) whereas patients with leukemia had counts ranging from 85,000 to 230,000 (mean 154,000). These means were significantly different $(p<0.001)$. Further analysis revealed that five of the six patients with JRA had platelet counts $>250,000$ while all six patients with ALL had initial platelet counts $<250,000 / \mathrm{mm}^{3}$; in fact, five of the patients with ALL had platelet counts $<200,000 / \mathrm{mm}^{3}$. The difference in the distribution of the platelet counts above or below the 250,000 level was also significant $(p<0.01)$. These findings indicate that a platelet count of $<250,000 / \mathrm{mm}^{3}$ in a patient presenting with "JRA" should suggest the probability of underlying malignancy and constitute a strong indication of a bone-marrow examination.

775 LEUKEMIA IN CHILDREN WITH WILMS' TUMOR. Edward S. Baum, Hahng Lee, $\frac{\text { Allen D. Schwartz, Northwestern }}{\text { Univ. Sch. }}$ Dept. of Ped., Chicago, Illinois.

Five children developed leukemia following therapeutic control and apparent cure of Wilms 'tumor. To our knowledge this is the first documentation of leukemia occurring in a group of children who have been treated for Wilms' tumor or any common non-lymphomatous childhood neoplasm. All 5 of the cases received radiation therapy; 3 received Actinomycin D. Two cases of acute lymphocytic leukemia, 2 cases of acute myelogenous leukemia and 1 case of $\mathrm{Ph}$ chromosome positive chronic myelogenous leukemia developed. Four of the leukemia cases were diagnosed within a 15 month period. Using overall survival statistics for patients with Wilms' tumor and risk figures for leukemia, less than 1 case of leukemia would have been expected to occur in a population of survivors of Wilms' tumor during this period of time. The age range ( 5 to 17 years) and histologic types of leukemia in these patients do not represent a typical group of leukemic children. A latent period preceeds the onset of radiation-induced leukemia and may account for the older ages of the children in the series. Non-lymphocytic leukemia is most commonly reported in those who develop the disease following exposure to drugs or radiation. Therefore, the authors believe that radiation therapy played the major etiologic role in the development of leukemia in these children. The oncogenic effect of Actinomycin $D$ or the possibility that these children were leukemia-prone is difficult to determine. Either or both may have been contributing factors.
776 THE INCREASED EFFECTIVENESS OF CANCER CHEMOTHERAPEUTIC AGENIS WHEN COMBINED WITH SIMELTANEOUS MICROWAVE
IRRADIATION. Robert L. Brent and Devora Hami, Jefferson Medical Col.. Stein Research Ctr., Phila., Pa. 19107

our laboratory has been interested in the effects of microwave radiation upon embryonic development and has reported that exposure to microwave radiation can raise the temperature of the embryos and under certain circumstances produce interruption of pregnancy in the rodent. Since the rate of metabolism varies somewhat at different temperatures and since microwave radiation permits one to control the temperature of exposed tissues, it might be possible to alter the effectiveness of drugs present in hyperthermic tissues. Since the embryo is a rapidly growing tissue and we have significant experience in controlling the temperature of the embryo, we utilized the rat embryo as the test organism. Methotrexate and 5 fluorouracil were administered to pregnant rats at an ID/10 to LD/30 dose and immediately exposed to microwave radiation for 30 minutes so that the temperature of the embryos were maintained at $42{ }^{\circ} \mathrm{C}$ or $430 \mathrm{C}$. The results of these experiments indicate that the 1D/50 dose of these drugs was significantly lowered in the hyperthermic entryos. These studies indicate that microwave radiation may be utilized to increase the biological effectiveness of the drug in the irradiated tissue and therefore be utilized in cancer chemotherapy or in other situations where the local increase in effectiveness of a drug may be of benefit to the organism. (Supported by NIH Grants $\mathrm{HD} 630$ and HD 370; AEC Contract 3268; NIH Contract 70-2306)

COMPARISON OF ALLERGIC REACTIONS TO L-ASPARAGINASE DERIVED FROM ESCHERICHIA COLI AND FROM ERWINIA CULTURES. Charles T. Dellinger, Thomas D. Miale, (Intr. by Elia M. Ayoub), Univ. of Florida Col. of Med., Dept. of Ped., Gainesville.

Despite its proven efficacy in resistant childhood leukemia and other malignancies, L-asparaginase has been limited to investigational use. This is largely due to serious toxicity, principally life-threatening anaphylaxis. The availability of L-asparaginase derived from two antigenically-distinct bacterial sources (E. coli and Erwinia) has reduced the frequency of anaphylaxis among children treated with this drug at a dose of

10,000 Int.U. $/ \mathrm{m}^{2} /$ wk. IV.

The frequency of anaphylaxis associated with the use of each of these two asparaginases in childhood leukemia was examined. A retrospective analysis revealed that 11 of 31 children had anaphylactic reactions, $9 / 27$ with E. coli and $3 / 10$ with Erwinia L-asparaginase. Thus, no differences in the overall incidence of anaphylaxis was observed. An increasing frequency of anaphylaxis was noted with repeated use of each form of asparaginase. The incidence of anaphylaxis per dose increased from $3.3 \%$ with the first dose to $32 \%$ by the fifth. The most marked increment in incidence of anaphylaxis was encountered with the fourth dose of either asparaginase. These data suggest that the alternate use of E. coli and Erwinia asparaginase may minimize the incidence of anaphylaxis.

AGGRESSIVE CHEMOTHERAPY FOR STAGE IV NEUROBLASTOHA. $\frac{\text { Jerry Z. Finklestein, }}{\text { Irwin Bernstein, Martin Klemperer, Robert Hittie and }}$ Denman Hammond. Children's cancer Study Group (CCSG) LoS Angeles. Combination chemotherapy with cyclophos hhamide (CTX) $750 \mathrm{rig} / \mathrm{m}^{2}$ $\times 1$, imidazole carboxamide (DTIC) $250 \mathrm{mg} / \mathrm{m}^{2}$ a.d. (days 1-5) and vincristine (VCR) $2 \mathrm{mg} / \mathrm{m}^{2} \times 1$ (day 5) was administered I.V. to 75 children with stage IV neuroblastoma. Courses of therapy were to be repeated at 21-day intervals. Complete or partial regression of measurable disease was experienced by 45 of 61 evaluable patients. This response rate of $73 \%$ was greater than that obtained by CCSG in previous nationwide studies. Twenty of the 45 responders had complete regression of all measurable disease. The median survival (34l days) for all evaluable patients is similar to that observed in previous studies. However, the survival of children who enjoyed a complete response has improved with a greater than $40 \%$ survival rate at 18 months. Because of toxicity, drugs were administered at the percentage of protocol dose as follows: CTX-75.1\%, VCR-79\%, and DTIC-77.1\%. The major side effect was myelosuppression. Pneumonitis was experienced 22 times in 18 children. Seven patients expired during their lung disease; two were tumor free. Etiological factors included Pneumocystis carinii (2), cytomegalic inclusion disease (1), staphylococcal pneumonia(1), interstitial pneumonia (1), possible pulmonary hemorrhage (1), and E. coli and viral infection (1). Three children with proven Pneumocystis carinii recovered. The improved response rate and survival seen with this aggressive combination chemotherapy must be balanced with significant toxicity. 

779 THE CARCINOEMBRYONIC ANTIGEN (CEA) ASSAY: PROGNOSTIC Patrick F. Bray, James $T$. Wu, and Gary C. Lang. Division of Pediatric Neurology, Departments of Neurology and Pediatrics, University of Utah College of Medicine, Salt Lake City, Utah

Plasma carcinoembryonic antigen (CEA) levels were measured at the time of diagnosis and sequentially during treatment in 14 children with histologically proven neural crest tumors. Clinical cure appeared established in 11 patients, 8 of whom had CEA levels at diagnosis below $4.0 \mathrm{ng} / \mathrm{ml}$ while 2 others had values of 4.0 and $5.0 \mathrm{ng} / \mathrm{ml}$. Clinical recovery was associated either with sequential CEA levels consistently within the normal range $(<2.5 \mathrm{ng} / \mathrm{ml})$ or with sequential CEA levels eventually falling into this range. All cases showed a decline in catecholamine excretion which often preceded a decline in CEA levels. Fatalities occurred in 3 cases in whom the CEA level at diagnosis was $4.0 \mathrm{ng} / \mathrm{ml}$ or higher. In these fatal cases sequential CEA levels remained elevated above $2.5 \mathrm{ng} / \mathrm{ml}$ in association with persistently elevated catecholamine excretion. The results suggest a possibly significant prognostic role for the CEA assay in the management of neural crest tumors. Levels at diagnosis below $4.0 \mathrm{ng} / \mathrm{ml}$ appear to indicate a favorable prognosis while values above this level call for a guarded prognosis.

780 CONOYLOMATA aCCUMINATA: EVIOENCE FOR MULTICELLULAR ORIGIN. J.M. Friedman and Philip J. Fialkow (Intr. by $C$. Ronald $5 \cot t$ ), Univ. of Washington, Depts. of of Med. and Genetics, and VA HoSp., Seattle.

In an attempt to gain insight into the mechanisms by which human malignancies of viral etiology could arise, we have used a system of naturally occurring cell markers to study the development of the benign viral "neoplasms", condylomata accuminata. These cauliflower-like clusters of many verrucous subunits are caused by papova virus infection of the epidermis. A viral neoplasm could develop in one of two ways: The virus might spread from cell to cell, transforming cells which have become infected, producing a tumor of multicellular origin. Alternatively, the tumorigenic change could be due to a rare event, e.9., one or more uncommon mutations, in which case the resulting neoplasm would develop as a clone from a single cell. This question has been approached by studying 834 verrucous subunits from 4 condylo mata accuminata in two females heterozygous at the $x-1$ inked $G-6-1 D$ locus. Since this locus is subject to $X$-chromosome inactivation, heterozygous females are mosaics of two cell populations, one producing G-6-PD type A, the other, G-6-PD type B. All four condylomata accuminata contained both types of enzyme. Furthermore, 5\% of the smallest individual subunits also had double enzyme phenotypes. Thus, these benign viral "neoplasms" have multicellular origin. In contrast, Burkitt lymphoma, a malignancy of putative viral etiology, has unicellular origin. Knowledge of the factors which underlie this difference may be important for understanding the mechanisms by which clinically apparent viral malignancy develops in man.

781 "GRAFT VS. LEUKEMIA" (GVL) In TWO PATIENTS With ACUTE LYMPHOBLASTIC LEUKEMIA (ALL) FOLLOWING BONE MARROW TRANSPLANT (BMT). L.A. Furman, J.R. Humbert, C.S. August, S.L. Rusnak, D.C. Peakman, H. Morse and J.H. Githens. University of Colorado School of Medicine, Denver, Colorado.

Animal experiments suggest that GVL reactions may eliminate leukemic cells in a host after BMT. We have observed two boys with ALL who developed marrow relapses following BMT but whose marrows later became normal during severe graft vs. host reactions (GVIR). At the time of BMT both patients ( $p t s$. ) had marrow and testicular relapses resistant to all drugs including Methotrexate (Mt $x$ ). Each pt. received $120 \mathrm{mg} / \mathrm{kg}$ cyclophosphamide, 1000 rads total body X-ray (XR), testicular XR and a graft from a nistocompatible sister (Seattle protocol). Both were given Mtx after the BMT and one, pt. A, received antithymocyte globulin for his GVHR. Both pts. had normal and female cells in their marrows. 15-21 days after the BMT. Both pts. showed marrow relapses 25-35 days after the BMT with predominately male cells (65 and $68 \%$ by $Y$ fluorescence). Florid GVHR (documented by skin biopsies) then occurred in both pts. Two and four weeks after relapse, their marrows were morphologically normal with a marked decrease in male cells ( 34 and $16 \%$ fluorescent $Y$ ). The normal marrow persisted in pt. A until his death from interstitial pneumonitis on day 103. A normal marrow persists in pt. B on day 70 . Since neither pt. received any effective antileukemic chemotherapy following the BMT, we conclude that GVL may have been responsible for the disappearance of the leukemic marrow cells.
782

RESULTS OF MULTIDISCIPLINARY TREATMENT OF EMBRYONAL RHABDOMYOSARCOMA OF DIFFERENT ANATOMICAL SITES.

Fereshteh Ghavimi, Philip R. Exelby, Giulio J. D'Angio and M. Lois Murphy. Memorial Sloan-Kettering Cancer Center.
Depts. of Pediatrics, Surgery and Radiation Therapy. New York.

From 1970-1974, 47 consecutive, previously untreated patients from 4 months to 21 years of age, with primary tumors of head and neck, trunk, intra-abdominal, genitourinary or extrenities, were treated by: surgical removal (S) of the tumor, followed by chemotherapy (Ct) and also by radiation therapy (Rt) for patients with yross or microscopic residual disease. Rt was given in 4500-7000 rads range. All patients received $C t$ for two years, consisting of sequential administration of dactinomycin, adriamycin, \& vincristine plus cyclophosphamide, with obligatory periods of rest.

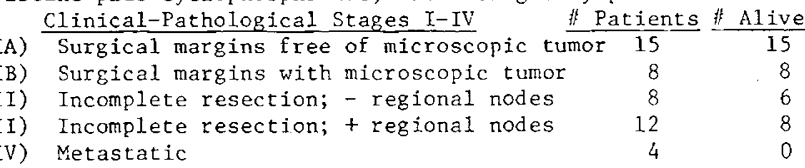

Thus, $37 / 47$ patients are tumor-free for a median of $22+$ months (range $4+$ to $51+$ months). Life table analyses show that the twoyear survival rate of $80 \%$ observed in this group is superior ( $\mathrm{p}<0.05$ ) to that of $37 \%$ noted among 108 patients treated in this Center between 1960-1970. All 15 patients with stage IA treated by $S$ and $C t$ are free of local and systemic disease without Rt and its associated morbidity, especially when combined with $\mathrm{Ct}$. Supported by NCI Grants \#CA-05826-13 and \#CA-08748

92 ADRIAMYCIN CARDIOMYOPATHY IN RABBITS: DIGITALIZED VS. NON-DIGITALIZED; SINGLE VS. DIVIDED DOSES. Angela C. Gilladoga, Frederick S. Philips, Stephen S. Sternberg, Corazon Manuel, and M. Lois Murphy. Memorial SloanKettering Cancer Center. Depts. of Pediatrics, Pathology, and the Pharmacology Laboratory. New York, N.Y.

Dose limitation is at present the only way to prevent adriamycin(Adr) cardiomyopathy(CMY). There are experimental reports indicating that pretreatment with digitalis or giving $\mathrm{Adr}$ in livided doses may prevent CMY by decreasing cardiac $A d r$ uptake. The objective of this study was to confirm these observations. Groups of 6 male albino rabbits were treated with Adr at 4week intervals totaling $11-15 \mathrm{mgs} / \mathrm{kg}$. At each course, Group $\mathrm{I}$ was given $2-3 \mathrm{mgs} / \mathrm{kg} \times 1$; Group II, $2-3 \mathrm{mgs} / \mathrm{kg} \times 1$ one hour after $0.03 \mathrm{mg} / \mathrm{kg}$ digitoxin; Group III, $0.4-0.6 \mathrm{mg} / \mathrm{kg}$ daily $\mathrm{x} 5$. Survivors in Groups $I(4 / 6)$, II $(4 / 6)$, and III $5 / 6)$ were sacrificed at 28 weeks. Groups I and II showed progressive weight loss with 4 deaths; 6 of 8 survivors had abnormal electrocardiograms(ECG) and elevated BUN, whereas Group III gained weight, had only mild ECG changes, normal BUN, and one unrelated death. On microscopy, myocardial and renal damage were seen in all three groups with no significant difference in severity. Heart lesions were present only in left ventricle with focal distribution. We also found no decrease in $\mathrm{Adr}$ uptake on cardiac tissue assay in rabbits and rats pretreated with maximal doses of digitalis.

We conclude that digitalis and divided-dose schedules do not prevent Adr CMY although the latter showed less severe clinical disturbance.

Supported by NCI grant CA-05826-13, CA-08748 and Pardee Fndn.

78 MOTILE AND PLASMA CELL ANTIGEN BEARING LYMPHO-

784 CYTES IN CHRONIC LYMPHOCYTIC LEUKEMIA. Armond S. Goldman, Nick S. Harris and Jack B. Alperin, Departments of Pediatrics, Surgery and Internal Medicine, The University of Texas Medical Branch and Shriners Burns Institute, Galveston, Texas.

Recently, it has been reported that the relative frequency of motile forms of human blood lymphocytes can be increased 4 to 5 fold by removing the cells from plasma. By using this procedure, we studied lymphocyte motility in eleven patients with chronic lymphocytic leukemia (CLL) and examined the occurrence of motile forms in relation to the frequency of $T$ and $B$ lymphocytes. Motile forms were identified by interference contrast microscopy. T lymphocytes were determined by SRBC binding (E-rosettes) and immunoglobulin (Ig) bearing lymphocytes were visualized by fluorescent microscopy ofter staining with FITC-conjugated antisera to human immunoglobulins. In addition, blood lymphocytes with plasma cell surface antigens were identified by indirect immunofluorescence by using a rabbit antisera against human plasmacytoma cells (Nature 250:508, 1974). The relative frequency of motile forms of blood lymphocytes in CLL patients was $10 \pm 3 \%$ (normal, mean $\pm \mathrm{SD} 45 \pm 5 \%$ ). A similar decrease in $\mathrm{E}$-rosetting lymphocytes was found $(11 \pm 5$ in CLL; $53 \pm 8$ in normals). The deficiency in motile forms was not found to be consistently related to an increase in Ig bearing lymphocytes but was inversely related to the total number of B lymphocytes because of an increase in plasma cell antigen bearing lymphocytes (mean $43 \%$ in CLL; $12 \%$ in normals). It therefore appears that motile forms in $\mathrm{CLL}$ are restricted to normal T lymphocytes. 
785 LYMPHOBLAST SURFACE MARKERS AND TERMINAL TRANSFERASE IN ACUTE LYMPHOCYTIC LEUKEMIA. Martha F. Greenwood, Mary S. Coleman, John J. Hutton, I. Tsukimoto, Beatrice C. Lampkin and Phillip HolTand. Depts. of Ped. and Med., Univ. of Ky. Med. Sch., Lexington and Children's Hosp., Cincinnati.

Blood lymphoblasts from children with ALL were examined for surface markers, terminal deoxynucleotidyl transferase (TdT), an enzyme normally associated with thymocytes, and surface morphology by scanning electron microscopy (SEM) at the time of diagnosis and throughout the induction period. Results prior to therapy are:

\begin{tabular}{|c|c|c|c|c|c|c|c|}
\hline Patier & $\mathrm{WBC} / \mathrm{cmm}$ & Blasts & SRBC & $E A C_{m o}$ & $\frac{1}{1 g}$ & TdT & SEM \\
\hline & 29400 & $\frac{\%}{95}$ & $\frac{\%}{88}$ & $\frac{\%}{14}$ & $\%$ & u/108 cells & smooth \\
\hline 2 & $\begin{array}{l}294,000 \\
286,000\end{array}$ & 98 & 2 & 24 & 14 & 210 & 90 \\
\hline 2 & 123,000 & 96 & 3 & 31 & 6 & 98 & 81 \\
\hline 4 & 80,000 & 88 & 86 & 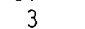 & 6 & 9 & 88 \\
\hline 5 & 159,000 & 80 & 93 & 4 & 4 & 50 & 91 \\
\hline Formal & lymphocy & & $78 \pm 6$ & $15 \pm 6$ & $24 \pm 7$ & $<.01$ & $<4$ \\
\hline
\end{tabular}

TdT values were markedly elevated in all patients. With effective induction therapy, TdT activity declined to $<2 u / 108$ leucocytes and surface markers and ul trastructure returned toward normal. Additional patients off chemotherapy after 3 years continuous remission had TdT values and surface markers within the normal range. These studies indicate the heterogenous nature of lymphoblast surface markers and initial TdT activities. The continued presence of TdT in the absence of blood smear lymphoblasts late in remission induction suggests that TdT assays may prove more sensitive than morphologic criteria in monitoring disease activity.

786 FACTORS IN DELAY IN DIAGHOSIS OF MALIGNANCY IN ADOLESCENTS, Jonathan Horwitz:; I. Ronald Shenker: and Phil ip Lanzkowsky, Sch. of Med., Health Sciences Ctr. State Univ. Of II.Y. at Stony Brook, Long Island Jewish-Hillside Med. Ctr., Dept. of Ped., New Hyde Park, N.Y.

We have prospectively investigated the reasons for delay in diagnosis of 14 consecutive adolescents with malignant disease. of these, 7 had malignancies of the hemonoietic system, 3 of the lymphoid system and 4 had solid tumors. The mean duration of delay from onset of symptoms to diagnosis was 10.3 weeks. Most patients attributed symptoms to minor illnesses, trauma or overwork at school. One patient and no parents admitted being worried about cancer. The mean delay from onset of symptoms to reporting to parents was 5.4 weeks, the mean delay of parents seeking medical advice was 3.5 weeks and the mean delay of doctors making a diagnosis was 1.4 weeks. 12 of the primary physicians

\begin{tabular}{|l|l|l|l|l|}
\hline Delay & Range(weeks) & Mean(weeks) & S.D. Median(weeks) \\
\hline
\end{tabular}

Patient

Parent

Doctor

Total

referred the ir patients for consultation and in 11 cases the

primary physician performed laboratory tests. The common initial oiagnosis made by the primary physician was an infective process and 5 of 14 prescribed antibiotics. In 11 patients the total delay was over one month. The major delay in cancer diagnosis in this sample of adolescents is the patient and parent and these findings emphasize the need to improve adolescent and parent can-

787 VINCA ALKALOIDS AND NEUTROPHIL FUNCTIONS: IN VITRO DEMONSTRATION OF AN ANTI-PHAGOCYTIC EFFECT. James R. Humbert, Patricia D. Hecht, and Christine H. Thoren. (Intr. by Dr. John H. Githens) Univ. Colo. Med. Ctr., Depts. Biophysics/Genetics and Pediatrics, Denver.

Vincristine (VCR) disrupts microtubular structures which participate in the phagocytic process by neutrophils (PMN's). The effect of VCR was thus investigated in 51 separate experiments of normal human PMN's incubated for 2 h hours with VCR at concentrations of $5 \times 10^{-6} \mathrm{M}$ to $2 \times 10^{-3} \mathrm{M}$. Following the first halfhour of incubation, PMN's were mixed with Staphylococcus aureus 502A for another 2 hours. Phagocytosis and bactericidal power of PMN's were then simultaneously studied by Tan's method. Results were expressed as percent of viable intracellular (IB) or extracellular bacteria (EB), compared with the original bacterial inoculum and correlate respectively to kactericidal power and phagocytosis.

values $(X \pm$ SEM $)$ in 14 studies with saline incubation were IB $1.9+0.5$, EB $7.0 \pm 1.2$. IB values were not significantly altered at VCR concentration of $5 \times 10^{-5} \mathrm{M}$ or less, but were abnormally high at concentrations of $2 \times 10^{-4} \mathrm{M}(41.3 \pm 9.8)$, $5 \times 10^{-4} \mathrm{M}(54.5 \pm 9.6)$ and $2 \times 10^{-3} \mathrm{M}(93.5 \pm 14.6)$. The same effect coula be produced with vinblastine at concentrations 10 times lower. Time course studies revealed that the toxic effect of VCR was undetectable after 60 minutes, but present after 90 minutes of incubation. These data indicate that vinca alkaloids at high concentrations inhibit phagocytosis of PMN's, but do not affect bactericidal power in these leukocytes.
788 AMINO-IMIDAZOLE-CARBOXAMIDE EXCRETION IN CHILDREN ITH ACUTE LYMPHOBLASTIC LEUKEMIA: THE EFFECT OF CHEMOTHERAPY. Helen Johnson, Charles A. Lapin, Grant Morrow III, and James J. Corrigan Jr., Dept. of Ped., University of Arizona, Tucson, Arizona.

Children with acute lymphoblastic leukemia (ALL) have been reported to have increased excretion of amino-imidazolecarboxamide (AIC) in their urine. The urinary level has been correlated with bone marrow disease status. Using our modification of the method of Skibba, et al (3ioch. Med. 3:150 1969) 60 determinations for AIC were performed on urine from 19 children with ALL and compared with that from 6 normal children and 2 leukemic patients off therapy. The effect of chemotherapy on urinary AIC was studied comparing patients on Vincristine and Prednisone $(\mathrm{V} \because \mathrm{P}$ ) with those on 6-Mercaptopurine, Methotrexate and Cyclophosphamide (6MP, MTX, CTX). Children on V : P were found to have significantly higher levels of AIC (Mean + S.E. = $2.73 \pm 0.34 \mathrm{mg} / \mathrm{M}^{2} /$ day) than those on $6 \mathrm{MP}$, MTX, CTX $(1.0 \overline{3} \pm 0.0 \mathrm{l})$; and were higher than both the controls $(1.00 \pm 0.36)$ and those off therapy $(1.05+0.09)$. Ten children who had sequential levels also demonstrated consistently elevated AIC levels on $V \&$ $\mathrm{P}$ as compared with their levels on 6MP, MTX, CTX. AIC levels of children on $V \&$. P were compared during periods of relapse 2.57 $\pm 0.45)$ and remission $2.84 \pm 0.52$ ) and were not significantly different. The data suggest that chemotherapy as well as disease status must be considered in evaluating AIC levels in children with ALL.

789 DEFECTS OF COMPLEMENT ACTIVATION IN ACLTE LYMPHOBLASTIC L.ELKEN:IA (ALL). David K. Kalwinsky, Joan R. Urmson, Ann E. Stitzel and Roger E. Spitzer. Upstate Medical Center, SLAY, Dept. of Ped., Syracuse, X.Y

Serial studies of children with AlL reveal that at the time of diagnosis or relapse, there are demonstrable defects in the utilization of both the alternative and classical complement pathrays. As a measure of alternative pathway activity, in 15 of 16 children the addition of zymosan $(Z)$ or cobra venom factor ( $C \cap F)$ to serum results in the incomplete consumption of C3-C9. At rerission, this defect disappears and all sera react normally with 2 and all but one with CoF. In addition, quantitative levels of serum factor $B$ are initially elevated in $65 \%$ of patients. With remission, factor $B$ values fall to 3 standarc deviations below normal in $80 \%$ of the patients. Finally, at diagnosis, $20 \%$ nornal serum added to the patient's serur: eliminates the $Z$ defect in C3-C9 consumption; normal serum heated to $50^{\circ} \mathrm{C}$ or abscrbed with $z$ at $0^{\circ} \mathrm{C}$ or $17^{\circ} \mathrm{C}$ fails to do so. These data suggest that the defect in the alternative pathway may be due to a specific serum deficiency. Evaluation of the classical pathway shows that at diagnosis, the sera of 6 of 13 patients fail to consure $C 4$ with immune precipitate (INP). Quantitative values of $C 3$ and $C 4$ are elevated in these patients, but by the time of remission fall significantly. Only $20 \%$ of sera have an abnormal Ix.P assay wher. patients are in remission. These defects in both the alternative and classical complement systems suggest that complement mediated tumor clearance may play an important role in this disease.

(Supported in part by NiH grant AM 17376.)

790 aBNORMALITIES OF LYMPHOCYTE BLASTOGENESIS IN ACLTE LYMPHOBLASTIC LEUKE:IIA (ALL). David K. Kalwinsky, Joan R. Urmson, Ann E. Stitzel, and Roger E. Spitzer Upstate Medical Center, SUNY, Department of Pediatrics, Syracuse, N.Y. 13210.

Serial studies of children with AlL reveal that at the time of diagnosis there are defects in the ability of the patient's lymphocytes to react in vitro with phytohemagglutinin (PHA). A serum factor is also describec. that depresses the PHA response of normal t-1ymphocytes. In 6 of 12 patients at the time of diagnosis, tritiated thymidine uptake is diminished 5 fold compared to controls when lyrphocytes are cultured with PHA. All patients have a normal PHA response when remission is achieved; when one patient subsequently relapsed, thymidine incorporation then became abnormal. Further, in 6 of 12 patients, serum taken at the time of diagnosis depresses the in vitro response of control normal lymphocytes to PHA by $50 \%$ or more. In all patients, serum at remission has no effect on the control PHA assay. Supernatants from leukemic blasts in vitro contain a detectable inhibitor of the PHA response by 48 hours of culture. Control supernatants have no detectable inhibitor even when assaved serially through 144 hours. The serum inhibitor is unaffected by altering the PHA concentration from $1-15$ ug/mil of tissue culture and does not shift the peak of thymidine incorporation in kinetic studies. The disappearance in remission of both the abncrmal PHA test and the inhibitor of PHA blastogenesis suggests that these defects may play a role in the pathogenesis of ALL. (Supported in part by NIH grant AM 17376 and N.Y. Kidney Dis. Inst. C 71376.) 
791 ABNORMAL FOLATE BINDER IN ACUTE LYMPHOCYTIC LEUKEMIA Bart Kaman, Douglas Caston and Samuel Gross, Case Western Reserve Univ. Sch. of Med. and Univ. Hosp. Depts. Of Anat. and Ped. Cleveland, Ohio

Recent studies using radiochemical-ligand binding assays have identified both free and protein bound folate (FB) fractions in sera of normal individuals. Elevated FB levels have been described in pregnancy, in chronic liver and renal disease and in sporadic cases of malignant disorders. An investigation of both sera and CSF folate compartments was carried out in children with acute lymphocytic leukemia in order to evaluate the consequences of drug therapy and changing cell populations on folate metabol ism. Serial measurements were done on 46 patients. Sera and CSF levels of free folate were constant during all stages of disease, nor did they differ from normal controls. The mean sera $F B$ in newly diagnosed cases was $14.6 \mathrm{ng} / \mathrm{m} 1$; in remission, $2.5 \mathrm{ng} / \mathrm{ml}(p>.007)$; and in normal controls, $2.0 \mathrm{ng} / \mathrm{ml}$. Significant rise in $F B$ (mean, $9.4 \mathrm{ng} / \mathrm{ml}$ ) was a constant finding in relapse and usually heralded clinical expression by $1-4$ weeks. In CNS leukemia, FB was markedly depressed (mean, $0.14 \mathrm{ng} / \mathrm{ml}$ ). In remission, irrespective of the state of peripheral disease, the levels matched normal controls, i.e., 9.4 and $10.2 \mathrm{ng} / \mathrm{ml}$, respectively. Elevated levels of serum FB appear to serve as a predictor of relapse. In CNS leukemia a paradoxical response occurs: the FB essentially disappears. These striking differences probably indicate the nature of cell dynamics in 2 different body compartments and do not appear to be causally related to drug administration.

792 TRANSFUSION-INDUCED PARTIAL REMISSION INACUTE LEUKBMIA Joseph Kochen, Eva Radel, Gerald Nathenson (Intr. by Laurence Finberg) Albert Einstein Col. Med., Montefiore Hosp. \& Med.Ctr。, Dept。 Pediatrics, Bronx, New York。

Cytotoxic sera from normal individuals that appear specific for acute lymphoblastic leukemia (ALL) cells have previously been described and may explain the occasional remission after blood transfusion.We report a patient with ALL in relapse who repeatedIy experienced a precipitous decrease in leukocyte counts after infusions of plasma from a normal donor. The patient was a 9-yr. old boy who presented with typical disease in 1972. Renission was induced and maintained with conventional therapy. He relapsed in June 1974 and for the next two months showed little response to drugs. At the family's request, he received no further chemotherapy. After five weeks he was hospitalized in florid relapse with WBC $>$ $120,000 / \mathrm{mm}^{3}$. Because of epistaxis he was given unmatched platelet concentrates from 10 unrelated donors. In the next 5 days his $\mathrm{WBC}$ decreased to $8500 / \mathrm{mm}^{3}$ with marked clinical improvement oSerum from one of the donors was found to be cytotoxic to the patient's blasts, but not to his parents' lymphocytes, by a dye-exclusion test.Utilizing fluorescein conjugated antiserum, it was shown that incubation with the donor serum resulted in an uptake of immunoglobulin by the blasts. The patient was given a total of 12 infusions of this plasma during the next 50 days. On three other occasions $W B C$ 's $>100,000 / \mathrm{mm}^{3}$ fell precipitously. However, at no time was the blood free of blast cells. He received no other therapy, but re quired repeated blood and platelet transfusions because of epistaxis. He died of massive bleeding after platelet infusions became ineffective.Post-mortem exam showed massive infiltrative disease.

RELATIONSHIP OF LIVER AND SPLEEN SIZE TO INITIAL

793 WHITE CELL COUNT (WBC) IN ACUTE LYMPHOCYTIC LEUKEMIA (ALL). Phil ip Lanzkowsky and Ashok Shende*, Sch. of
Sciences Ctr., State Univ. of N.Y. at Stony 8 rook Med., Health Sciences Ctr. State Univ. of N.Y. at Stony Brook Hyde Park, N.Y.

Since liver and spleen size might be related to total leukemic load as reflected in the WBC count, an investigation was carried out to determine the relationship of organ size to WBC count. Fifty children aged one to 19 years with a diagnosis of ALL were studied. The organ size and WBC counts on initial examination were recorded. The liver size varied from 0 to $8 \mathrm{~cm}$. below the right costal margin and spleen from 0 to $9 \mathrm{~cm}$. below the left costal margin. The WBC count varied from 1,300 to $204,000 / \mathrm{mm} 3$. The data are indicated in the table below:

\begin{tabular}{|c|c|c|c|c|c|c|c|c|}
\hline \multirow[t]{2}{*}{ Organ } & \multirow[t]{2}{*}{$\begin{array}{l}\text { Initial WBC } \\
\times 103 / \mathrm{mm}^{3}\end{array}$} & \multirow[t]{2}{*}{$\begin{array}{l}\text { No. of } \\
\text { Patients }\end{array}$} & \multicolumn{6}{|c|}{$\begin{array}{l}\text { \% of Patients in each WBC Group } \\
\text { for different Organ sizes (cm) }\end{array}$} \\
\hline & & & & $0-2$ & $2-4$ & $14-6$ & $6-8$ & $8-9$ \\
\hline \multirow{3}{*}{-iver } & & 27 & 22 & 33.3 & 29.6 & 7.4 & 7.4 & 0.0 \\
\hline & 0.0 & & 33. & 33.3 & 22.2 & 11.1 & 0. & 0.0 \\
\hline & $>$ & 14 & 21.4 & 21.4 & 35.7 & 14.2 & 7.1 & 0.0 \\
\hline & & 27 & 37. & 29.6 & 25.9 & 3.1 & 3.1 & 0.0 \\
\hline & 10. & 9 & 22.2 & 44.4 & 11.1 & 0.0 & 11.1 & 11.1 \\
\hline & $>20.0$ & 14 & & 14.2 & 28.4 & 28.4 & 7.1 & 14.2 \\
\hline
\end{tabular}

There was no correlation between liver and spleen size and $W B C$ in this series, and these two factors appear to be independent variables.
794 COMPLICATIONS OF LAPAROTOMY AND SPLENECTOMY IN THE STAGING OF HODGKIN'S DISEASE (H.D.) IN CHILDREN. Phil ip Lanzkowsky, Gungor Karayalcin*, Ashok Shende*, and Robert Levy*. Dept. Of Ped. and Med., Sch. of Med., Health Sciences Ctr., State Univ. of N.Y. at Stony Brook and Long Island Jewish-Hillside Med. Ctr., New Hyde Park, N.Y.

Twenty-five cases of H.D. ( 15 males and 10 females) aged 5 to 17 years (mean 12.6) were studied from April 1970 to December 1974. Histology revealed: lymphocyt ic predominance (2), nodular sclerosis (12), and mixed cellularity (11). Pathologic staging revealed: $I A(3)$; $1 B(1)$; $11 A$ (5); $11 B$ (4); $111 \mathrm{~A}$ (6) and $111 \mathrm{~B}(6)$. Laparotomy altered staging in 12 patients ( 9 were staged up and 3 down). All patients received extended field radiation and 5 had recurrence of disease and were treated with combination chemotherapy. Twenty-three are alive without evidence of disease (2-56 months) and the two deaths were not due to H.O. but to hematobilia (post liver biopsy) and pneumococcal septicemia, purpura fulminans and disseminated intravascular coagulation ( 14 months post-splenectomy). Other complications included: Recurrent intestinal obstruction (1); intestinal obstruction (1); post-operative sub-phrenic abscess (1); streptococcal arthritis (1). Seventeen patients received penicillin prophylaxis post-operatively and the 3 with infection were amongst the 8 not receiving it. It is concluded that laparotomy and splenectomy in children is essential for accurate staging but carries significant risk and continuous antibiotic prophylaxis is recommended.
795 COMBINATION OF ORGAN IRRADIATION AND CHEMOTHERAPY IN ACUTE LYMPHOCYTIC LEUKEMIA (ALL) IN CHILDREN. Philip Lanzkowsky, Ashok Shende*, Isamett in Aral*", Gurbir S. Saluja*. Dept. of Ped., Sch. Of Med., Health Scjences Ctr., State Univ. of N.Y. at Stony Brook and Long Island JewishHillside Med. Ctr., New Hyde Park, N.Y.

Thirty consecutive children with ALL were treated from June 1971 to December 1974 with an induction regimen of VCR $(1.5 \mathrm{mg} /$ $M^{2}$ I.V. weekly $\times 5$ ) and PRED. ( $40 \mathrm{mg} / \mathrm{M}^{2}$ P.0. daily $\times 28$ ). Following remission, cranial irradiation (200 rads daily $\times 12$ ) and intrathecal MTX ( $12 \mathrm{mg} / \mathrm{M}^{2}$ every 3 days $\left.\times 5\right)$; liver, spleen and kidney irradiation $(120$ rads daily $\times 10)$; $6 \mathrm{MP}\left(50 \mathrm{mg} / \mathrm{M}^{2} \mathrm{I} . \mathrm{V}\right.$. daily); CPM (200 mg/M2 P.O. weekly); and MTX (20 mg/M2 P.O. weekly) were administered during maintenance. VCR $\left(1.5 \mathrm{mg} / \mathrm{M}^{2} \mathrm{I.V}\right.$. weekly $\left.\times 3\right)$ and PRED. ( $40 \mathrm{mg} / \mathrm{M}^{2}$ P.0. daily $\times 15$ ) were administered at $10-12$ week intervals. One patient died during induction and 1 required L-Asparaginase to achieve remission. Treatment was tolerated well with no deaths due to infections or drug toxicity. Patients vere subdivided into two groups (high and standard $r i s k$ ) based on initial WBC count and the results were analyzed by the Life Table nethod revealing:

$\begin{array}{lr}\text { (1) Combined high and standard risks } & \frac{\text { P Continuous Remission }}{74.7} \\ \text { (2) High risk (initial WBC> 25, 000/mm3) } & 42.9 \\ \text { (3) Standard risk (initial WBC }<25,000 / \mathrm{mm}^{3} \text { ) } & 87.9\end{array}$

Results of this regimen for standard risk ALL are encouraging whereas it is unsatisfactory for high risk ALL.

796 HOME CARE FOR THE DYING CHILD. Ida M. Martinson, John H. Kersey, Eleanor Dawson, Carol Neuburger, Mary 1 . Anglim, Barbara Veatch, Mary Weisensee, Mark E. Nes-

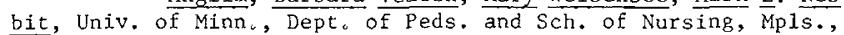
Minn.

Health professionals tend to encourage admission of the dying child to the hospital. During the past $21 / 2$ years, 8 families have been involved in a program that attempts to identify the feasibility and desirability of home care for the dying child as an alternative form of care for a family with a child with cancer. of the 8 children studied, 5 died at home and 3 in the hospital, one of whom was in the hospital for only 2 hours before death. This is contrasted to the previous 10 deaths from leukemia at this hospital; all occurred in the hospital with an average of 28.5 days of final hospitalization and at an average cost of $\$ 4,480$. The number of home visits ranged from 3 to 10 per child. These home visits were supplemented with an average of 10 phone calls per family, plus an occasional clinic visit. Total hours of direct professional involvement came to approximately 20 hours per family. The length of involvement for the family with this program was 5 days to 4 months; average length was 45 days. Estimated cost was $\$ 1,000$. per child. The following are required for this type of service: availability of the primary health professional 24 hours a day, parent understanding of the possible complications and appropriate interventions, and family instruction in injection for pain control. We conclude that a home care program for a dying child may be a viable alternative to terminal hospitalization. 
797 COMBINATION CHEMOTHERAPY OF UNUSUAL CHILDHOOD TUMORS。 Nancy $B_{0}$ McWilliams, Harold M. Maurer and Jexry $L$.

Pediatrics, Richmond, Virginia.

Certain malignant tumors in children are of sufficient rarity that treatment regimens are not well established.

In the past 27 months, 7 children, with unusual tumors, have been treated with combination chemotherapy. All had stage 3 or 4 disease at diagnosis. Except for patients \#1 and \#4, all received radiation therapy. All achieved complete response. Pertinent data are listed below:

\begin{tabular}{|c|c|c|c|c|c|}
\hline \# & Age & Histology & $i^{\circ}$ site & $R x$ & Outcome \\
\hline $\bar{z}$ & $\overline{9 y}$ rs. & Leimyosarcoma & Mesentery & $\overline{D, V}, C$ & $\overline{N E D 2}$ y $x s$. \\
\hline ? & 7 yrs. & Mesenchymoma & Liver & $D, V, C$ & Recurrence \\
\hline 3 & 9 yrs. & Mesenchymoma & Liver & $D, V, C$ & Recurrence \\
\hline & 9 mos. & Teratoma & $\begin{array}{l}\text { Sacrum } \\
\text { Coccyx }\end{array}$ & $D, V, C, A$ & NED 4 mos。 \\
\hline & 27 mos. & Teratoma & $\begin{array}{l}\text { Sacrum } \\
\text { Coccyx }\end{array}$ & $D, V, C$ & NED $6 \mathrm{mos}=$ \\
\hline & rs。 & $\begin{array}{l}\text { Mixed } \\
\text { Mesodermal }\end{array}$ & $\begin{array}{l}\text { Retro- } \\
\text { peritoneum }\end{array}$ & $D, V, C, A$ & NED $27 \mathrm{mos}$ " \\
\hline & 4 yrs。 & Embryonal Ca & Ovary & $D, V, C, A$ & NED $4 \mathrm{mos}$. \\
\hline
\end{tabular}

These encouraging results suggest that the 3 or 4 drug combination as outlined by the Intergroup Rhabdomyosarcoma Study is effective therapy in a variety of childhood neoplasms.

$D=$ Dactinomycin $\quad A=$ Adriamycin

$V=$ Vincristine $\quad N E D=$ No Evidence of Disease

$c=$ cytoxan

798 PULMONARY FUNCTION(PF) IN SURVIVORS OF WILMS' TUMOR (WT): ROLE OF RADIATION THERAPY (RT), Anna T. Meadows, George Polgar, Philip Littman, and Patricia F. Borns.

University of Pennsylvania Sch.of Med., Children's Hosp. of Phila.

Occult evidence of abnormal PF was sought in 33 long-term survivors of WT (4-20 yxs. median 8 yrs.) to ascertain impairment related to pulmonary disease, surgery, radiation or chemotherapy.

All but 4 patients (pts) received dactinomycin (AMD). RT was given to 10 pts (Group M) for pulmonarv metastases and to 5 pts (Group P) for prophylaxis. 18 pts had no RT to the lungs (Group N). Doses were similar in both treated groups ( $1400-1600$ rads).

There wexe no differences between expected normals and Group $N$ pts suggesting no adverse effect of AMD alone. Median PF values with significant differences were as follows (Wilcoxon 2 sample Ranks Test):

FUNCTION

Vital Capacity (VC)

Functional Residual

Capacity (FRC)

$\begin{array}{lllllll}\text { Capacity (FRC) } & 68 & 91 & 94 & .01 & .05 & \text { NS } \\ \text { Residual Volume (RV) } & 68 & 97 & 93 & .01 & .01 & \text { NS }\end{array}$

MEDIAN ( 8 NORMAL) SIGNIFICANCE-DIFF.

$\begin{array}{llllll}\frac{\mathrm{GP}}{67} & \frac{\mathrm{GP}}{79} \mathrm{P} & \frac{\mathrm{GP} N}{99} & \frac{\mathrm{M}-\mathrm{N}}{.01} & \frac{\mathrm{M}-\mathrm{P}}{\mathrm{NS}} & \frac{\mathrm{P}-\mathrm{N}}{.01}\end{array}$

$\begin{array}{llllllll}\text { Total Lung Capacity (TLC) } & 62 & 87 & 100 & .01 & \text { NS } & .05\end{array}$

$\begin{array}{lllllll}\text { One second Forced Expira- } & & & & & & \\ \text { tory volume }\left(\mathrm{FEV}_{1}\right) & 61 & 75 & 88 & .01 & .01 & .01\end{array}$

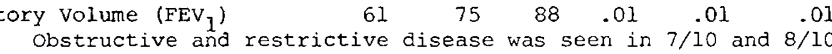

Group M pts, respectively. Restrictive disease was also present in Group $P$ pts with TLC and VC diminished compared with Group N and not different from Group $M$. These findings suggest caution in the use of prophylactic RT. Survivors should be followed closely.

79 EFFECT OF HUMAN LYMPHOCYTE PROLIFERATION INHIBITORY

799 FACTOR ON THE BLASTOGENESIS OF ALLOGENEIC LYMPHOCYTES. Nalini Mukhopadhyay, John M. Falletta, Anne Craig, and Donald J. Fernbach (Intr. by Kenneth A. Starling), Baylor College of Medicine, Department of Pediatrics, Houston.

Proliferation inhibitory factor (PIF) is released in vitro from antigen- or mitogen-stimulated human lymphocytes (Ly) and inhibits ly replication in the absence of cytotoxicity. PIF was detected in culture supernatants by preincubating human Ly with PHA in autologous plasma ( $P-S)$. Controls included cultures reconstituted with the mitogen (R-S) immediately before harvesting the supernate and cultures receiving no mitogen $(0-S)$. The presence of PHA in P-S and R-S was detected by a double sandwich immunofluorescent staining technique. Inhibition of proliferation as meas ured by a decrease in the uptake of thymidine ( $3 \mathrm{HT}$ ) was found to occur consistently in allogeneic target Ly grown in P-S. At a dilution of $1: 2$, the blastogenic response of Ly grown in $\mathrm{P}-\mathrm{S}$ was significantly lower than that of cells grown in $R-S(P=4.005)$. $\mathrm{P}-\mathrm{S}$ cultures to which Con $\mathrm{A}$ was added also demonstrated a depressed blastogenic response. After separation of human tonsil Ly on a BSA gradient, only cells from $T$ cell-rich fractions released PIF. Leukemic blasts obtained from 5 children with acute lymphatic leukemia (T cell leukemia-2; "nu11" cell leukemia-3) did not release PIF. Peripheral blood Ly from a patient with Bruton's agammaglobulinemia released PIF. While PIF may function by inhibiting the growth of "foreign" cells present during neoplastic transformation, failure of neoplastic lymphoid cells to release PIF suggests that this factor is not involved in the ineffective host response against these malignant cells.
800 CLINICAL STAGING IN CHILDHOOD ACUTE LyMPHOBLASTIC LEUKEMTA. Thomas F. Necheles, Uma S. Rai, Dana M. Whitten, Carol Crowley, William M. Davis, and Barbara Warren. (Intr. by Sydney $S$, Gelilis) Dept, of Pediatrics, N.E Medical Center Hosps, and Tufts University School of Medicine, Boston.

Forty children with acute Iymphoblastic leukemia were treated with a single regime consisting of induction with Vincristine and Prednisone, cranial irradiation combined with intrathecal Methotrexate, 6-Mercaptopurine and Methotrexate maintenance, and periodic ( $q 10$ weeks) reinforcement with Vincristine and Prednisone. A "standard risk" group was defined as those children between the ages of 2 and 8 years who had an initial WBC of less than $20,000 / \mathrm{cmm}$, who had no discernible nodes greater than $2.5 \mathrm{~cm}$. in diameter, whose spleen and liver were less than $3 \mathrm{~cm}$. below the costal margin, and who had no evidence of extra-nodal disease. All children who exhibited one or more parameters which did not fit these specifications were placed into the "poor risk" group. Overall survival for the entire group was $80 \%$ at 36 months. Five of the six deaths were in the "poor risk" group. The relapse rate was also significantly higher in the "poor risk" group; only $31 \%$ were in remission at 36 months versus $73 \%$ for the "standard risk" group. Furthermore, the rate of CNS relapse was much higher (6/17) in the "poor risk" group when compared with the "standard risk" groups $(1 / 23)$. We feel that these clinical parameters may reflect, to some extent, the total body "tumor" burden and may well identify those children where more aggressive therapy is indicated.

801 HIGH-DOSE CYCLOPHOSPHAMIDE AS ADJUNCTIVE THERAPY IN THE TREATMENT OF PRIMARY OSTEOGENIC SARCOMA. Thomas $F_{0}$. Necheles, Seymour Zimbler, John Munzenrider, and Henry Banks. (Intr. by Sydney S. Gellis). Dept. of Pediatrics, N.E. Medical Center Hospitals and Tufts University School of Medicine, Boston.

Six children (age less than 20 years) with primary osteogenic sarcoma have been treated with a combination of preoperative radiotherapy $(5 / 6)$, amputation, and cyclophosphamide. Cyclophosphamide has been given in a dose of $25 \mathrm{mg} / \mathrm{kg}$ every other day for a total of five doses. This cycle is repeated every 3 months for 7 cycles. Five out of 6 patients are surviving without evidence of metastases $26+$ to $159+$ weeks after diagnosis with two off all medication for 36 and 81 weeks. Side effects include severe alopecia and frequently a profound leukopenia lasting about 10 days after the final dose. The latter, however, has not been associated with serious infections. Hemorrhagic cystitis has not been a problem, probably because all patients receive 3.0 liters $/ \mathrm{M}^{2} / 24^{\mathrm{h}}$ intravenous fluids during, and 48 hours after, each course of drug. High-dose cyclophosphamide appears to be an active agent in the adjunctive treatment of osteogenic sarcoma and does not appear to be associated with some of the complications (thrombocytopenia, stomatitis) inherent in other regimens which have recently been proposed.

802 CHANGES IN T AND B LYMPHOCYTE POPULATIONS DURING THE COURSE OF ACUTE LYMPHOBLASTIC LEUKEMIA (ALL) IN CHILDREN. Rochelle G. Neiburger and Robert L. Baehner. Indiana Univ. Sch. of Med., Dept. of Ped., Indianapolis.

The percentage of $T$ and $B$ lymphocytes was determined in peripheral blood (PB) and bone marrow (BM) of children with ALL at diagnosis, during remission and at relapse. T cells were determined by sheep erythrocyte rosette technique; $B$ cells bv labeling with fluorescent anti-IgS, -IgA, and -IgM. Twenty newly diagnosed patients (pts) were studied. Mean blast percentage was 63 in $P B$ and 91 in BM. All samples showed decreased B cells. T cells were decreased in 11 , but normal in 9 pts. No difference in white blood count (wbc) between the two groups was observed. There was close correlation between $P B$ and $B M$ values. During remission $T$ and total $B$ values returned to normal ( 23 pts) but the distribution of $B$ cell subclasses was altered; IgM- and IgAbearing $B$ cells were slightly decreased and IgG-bearing cells were increased. At relapse 10 of $14 \mathrm{pts}$ had normal $\mathrm{T}$ and IgM-B cell values and the IgA- and IgG-B cell subclasses were Internediate between remission and diagnosis. Mean $P B$ and $B M$ blast cell number was significantly lower during relapse than at dlagnosis (PB $10 \%$ vs $63 \%$; BM $60 \%$ vs $91 \%$ ). These studies demonstrate that blast cells in ALL may either lack all immunologic markers or be associated with normal percentage of $T$ cells, independent of initial wbc. The immunologic markers return during remission. Ear1y relapse can be fdentified by a decrease in IgA- and IgG-B cells. Close correlation exists between the immunologic patterns of $P B$ and $B M$ cells in all phases of ALL and suggests that monitoring of PB markers will help identify early BM relapse. 
803 URIC ACID, RANTHINE AND HYPOXANTHINE EXCRETION (UXH) AS AN INDEX OF "REMISSION" STATUS. James A. Stockman, III, Marie J. Stuart and Frank A. Osk1. S.U.N.Y., Upstate Medical Center, Department of Pediatrics, Syracuse, N.Y.

In an attempt to more accurately assess tumor burden and response to therapy in children with acute lymphatic leukemia (AlL), dally 24-hour urine collections were obtained for 3 to 4 weeks during initial induction in 18 patients and during apparent remission in 17 patients. Vincristine (VCR) and prednisone (P) were employed for induction and perfodically during remission. UXH excretion averaged $933 \mathrm{mg} / \mathrm{M} 2 / \mathrm{day}$ in week 1; 607 in week 2; and 676 in week 3 (normal 184 265). Peak UXH occurred 2.6 to 3.2 days after VCR in weeks 1 to 3 . During apparent remission, mean UXH was significantly lower, mean 355 , but only 7 of 17 patients had values within the normal range. UXH did not correlate with the number of circulating blasts, correlate with initial liver and spleen size. UXH remained elevated during induction despite the presence of less than 5 per cent blasts in marrow $(M-1)$ and was found elevated prior to relapse despite $M-1$ marrow status. No peak UXH occurred following VCR and $P$ administration during remission suggesting that these agents may not be extremely useful in treatment procedure that appears to be a more sensitive index of success of remission therapy than currently employed morphologic criteria.

804 INCREASED ADRIAMYCIN (A) CARDIOTOXICITY IN LEUKENIC OHILDREN. R. Propper, B. Camitta, N. Jaffe, D. Traggis and S. Sallan (Intr. by D. Nathan), Sidney Farber Canc. Ctr., Children's Hosp. Hed. Ctr. and llarvard Med. Sch., Boston, Mass. 02115

Cardiotoxicity has been reported in $2-7 \%$ of 1cukemic children receiving $A$ at a cumulative dose usually above $550 \mathrm{mg} / \mathrm{M}^{2}$.

Since $1972 \mathrm{~A}$ has been administered to 102 leukemic patients at this institution in $30-40 \mathrm{mg} / \mathrm{M}^{2} \mathrm{IV}$ doses (adjusted for myelosuppression) every 3-5 weeks. Mrednisonc, 6-ip and vincristine were given concomitantly. Cardiotoxicity (defined as clinical, radiologic and EKC abnormalities) has been greater than reported elsewhere.

Sixty-two patients (age 1-17 years)received 300 to $600 \mathrm{mg} / \mathrm{M}^{2}$ A. Twelve (19\%) developed clinical evidence of cardiotoxicity at cumulative A dosages of $380-605 \mathrm{mg} / \mathrm{M}^{2}$. Seven (58\%) developed congestive failure. Three of these seven died in remission of cardiac disease. Toxicity appeared in some children while on $A$, while in others it developed up to 6 months after the last dose of A. Expressed as percent cardiotoxicity per accumulated A dose, these data indicate that $A$ in the above schedule has greater toxicity at lower dose levels than previously reported. ilore sensitive measures are needed for detecting cardiac abnormalities before overt A toxicity occurs. per cent marrow blasts, or serum urfc acid. UXH excretion did during maintenance of ALL. The measurement of UXH is a simple

806 COMBINATION CHEMOTYERAPY IN THE TREATMENT OF ADVANCED WIMS' TUMOR。 Eva Radel, Stuart Toledano and Joseph Kochen (Intr. by Laurence Finberg). Albert Einstein Col.Med., Montefiore Hosp. \& Med.Ctr.,Dept.Ped., Bronx, New York

The addition of chemotherapy to surgery and radiation has inproved the survival of children with Wilms' tumor.However, the 2year disease free survival of children with non-metastatic disease treated with either dactinomycin (DAC) or vincristine (VCR) is still not better than $70 \%$. It has been suggested and calculated that combined therapy with DAC and VCR might further improve survival to $90 \%$. We have treated 8 children with advanced wilms' tumor with surgery, radiation, and combination chemotherapy. Most patients received courses of $\nabla C R 1.5 \mathrm{mg} / \mathrm{M}^{2}$ weekly for 6 weeks and DAC $.075 \mathrm{mg} / \mathrm{kg}$ during the first week of the course, with courses repeated every 12 weeks for 2 years. One patient who developed a tumor in the other kidney after 3 courses of DAC received VCR and cytoxan. Therapy was tolerated well. Toxicity included nausea and vomiting on the days of DAC administration,mild to moderate alopecia, and mild to moderate leukopenia which necessitated stopping the DAC after 2 courses in one patient. One patient (group V) was 17 mos. old; the others were from 4 to 13 years old. Extent of disease (NWTS classification) was group II in one patient; group III in 3 patients (one with a congenital single kidney); group $I V$ in 3 patients, and group V in one patient. A 13-year-old girl with bone metastases had no response and died within 2 months. The other 7 patients are alive and well with no evidence of disease with a total duration of disease from 4 to 62 mos. (median 47 mos.) and no evidence of disease for a median of 31 mos. Five patients have been off therapy for 9 to 31 mos.

807 RELATIVE THROMBOCYTOSIS IN PATIENTS WITH NEUROBLASTOMA. Sreedhar P. Rao, Audrey K. Brown, and Maria L. Falter. Dept. of Ped., Downstate Medical Center, Brooklyn, N Y.

Patients with neuroblastoma most often present with widespread disease and traditionally are expected to have anemia and thrombocytopenia. Records of 19 patlents seen between 1957 and 1974 were revlewed with special reference to hemoglobin and platelet count prior to therapy. A surprising discordance in the effect of the disease upon the production of red cells as compared to production of platelets was found. Hemoglobin values were available in all 19 patients; direct platelet counts were available in 10 and indirect platelet counts in 9. Thirteen of the 19 patients had widespread tumor, with evidence of marrow metastasis in 10 . Of these 10, 7 showed anemia (hgb below $9 \mathrm{gm} \%$ ) while only one showed mild thrombocytopenia. In the remainder platelet counts were high normal to elevated. Among the 9 patients with hemoglobin below $9 \mathrm{gm}$, platelet counts were high normal in 4 , $(250,000-400,000)$ significantly elevated in $4(450,000-930,000)$ and only slightly decreased in one $(80,000)$. In 10 children who showed no anemia, platelet counts were high normal to increased. All the patients with elevated platelet counts had increased numbers of megakaryocytes in the marrow. These data suggest that neuroblastoma may have a specific stimulating effect on platelet production even in the presence of depressed red cell production. Thrombocytosis, particularly in the presence of anemia in a child with an $a b-$ dominal tumor suggests neuroblastoma.

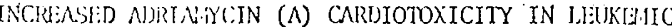

805 aliluris, R. Fropper, B. Camitta, N. Jaffe, D. Traggis and S. Sallan (Intr. by D. Nathan), Silncy Farber Canc. .Ctr., Children's Hosp. lícd. Ctr. and Harvard Med. Sch., Boston, Mass. 02115

Cardiotoxicity has been reported in $2-7 \%$ of lcukemic children recciving $A$ at a cumblative close usually above $550 \mathrm{mg} / \mathrm{M} 2$.

Since 1972 A has been administered to 102 leukemic patients at this institution in $30-40 \mathrm{mg} / \mathrm{M}^{2}$ IV closes (adjusted for myelosuppression) every 3-5 weeks. Prednisone, 6-MP and vincristine were given concomitantly. Cardiotoxicity (defined as clinical, radiologic and I:KG abnomalities) has been greater than reported elsewhere.

Sixty-two patients (age 1-17 ycars)received 300 to $600 \mathrm{mg} / \mathrm{M}$ A. Thelve (19\%) leveloped clinical quidence of cardiotoxicity at cumlative $A$ dosages of $380-605 \mathrm{mg} / \mathrm{M}^{2}$. Seven (58\%) developed congestive failure. Three of these seven died in remission of cardiac disease. Toxicity appeared in some children while on $\wedge$, while in others it developed up to 6 months after the last dose of A. Fxpressed as percent cardiotoxicity per accumulated A dose, these data indicate that $\Lambda$ in the above schedule has greater toxicity at lower lose lcvels than previously reported. More sensitive measures are needed for detecting cardiac abnormalities before overt A toxicity occurs. dren's Hosp. Med. Ctr., Depts. of Ped. and Card., Boston, Mass.
The clinical course of 5 children with hepatic hemanqioendo-

\section{Jominal tumor suggests neuroblastoma.}

Q0 HEPATIC HEMANGIOENDOTHELIOMA - HEMODYNAMIC OBSERVA-

808 TIONS AND TREATMENT. Albert P. Rocchini, Amnon Rosenthal and Alexandex S. Nadas, Harvard Med. Sch., Chil-
Med. Ctr., Depts. of Ped. and Card., Boston, Mass. thelioma (HH) seen at The Children's Medical Center since 1965 is reviewed with emphasis on the hemodynamic alterations observed at cardiac catheterization and the efficacy of prednisone in the treatment of this disorder.

All the patients presented with congestive heart failure (CHF) between the ages of 1 to 15 months. The hemodynamic abnormalities observed were: a rise in blood oxygen saturation at the level of the hepatic veins ( 24 to 278 ), an increased cardiac output ranging from 9.2 to $10.5 \mathrm{~L} / \mathrm{min} / \mathrm{m}^{2}$ with over fifty percent of this output being shunted through the arteriovenous malformation; an elevated right and left end diastolic pressure (8-10 mmHg and 9$15 \mathrm{mmig}$ respectively); a small gradient (probably secondary to increased flow) across the pulmonary outflow tract (4-6 mmllg); and mild elevation of pulmonary artery pressure (PAP) ranging from $35 / 16$ to $37 / 12 \mathrm{~mm} H \mathrm{~g}$. PAP diminished in response to oxyqen inhalation in one patient.

Four of the 5 patients were treated with prednisone. All 4 improved while on prednisone therapy; three are well 1 to 3 years after cessation of therapy. One patient died from unrecognized ClF following discontinuation of prednisone and another before prednisone was started. We conclude that prednisone in high doses $(2-4 \mathrm{mg} / \mathrm{kg} / \mathrm{day})$, digitalis and diuretics (for CHF) are an effective treatment regimen for infants with hepatic hemangioendothelioma. 
809 ADENOCARCINOMA OF VAGINA IN AN. INFANT: AN APPROACH TO ITS MANAGEMENT. Ashok Shende*, Philip Lanzkowsky Isamett in Aral $*$, Burton Bronsther: Sch. of Med., Health Sciences Ctr. of the State Univ. of N.Y. at Stony Brook and Long Island Jewish-Hillside Med. Ctr., Depts. of Ped. and Radiology, New Hyde Park, N.Y.

Only 17 cases of this disorder have been described. Eleven died within a year. Surgery (vaginectomy and hysterectomy) and pelvic radiation play a major role in treatment and adjuvant chemotherapy has been used in only 4 patients. Intracavitary radiation alone was used unsuccessfully in 2 cases. This report describes a new approach to management. A 7 month old female presented with adenocarcinoma of the right anteriolateral wall of the vagina. Treatment consisted of vaginal intracavitary cesium 137 applicators delivering a tumor dose of 3000-6000 raos in two sittings (at 0.5 and $1 \mathrm{~cm}$ depths) and Vincristine (VCR) $2 \mathrm{mg} / \mathrm{M}^{2}$ I.V. weekly $\times 6$, Act inomycin-D (ACT-D) $15 \mathrm{mcg} / \mathrm{kgm} 1 . V$. daily $\times 5$, Cyclophosphamide (CPM) $10 \mathrm{mg} / \mathrm{kgm} \mathrm{I.V.} \mathrm{daily} \times 5$ followed by Methotrexate (MTX) $20 \mathrm{mg} / \mathrm{M}^{2} \mathrm{P} .0$. once a week. Ten weeks following this therapy, subtotal hysterectomy and subtotal vaginectomy were performed. Multiple sections of vagina, uterus and para-aortic lymph nodes did not reveal tumor. Maintenance chemotherapy consisted of CPM P.O. alternate days, MTX P.O. weekly, and courses of VCR and ACT-D I.V. every 10-12 weeks. Patient is asymptomatic with no evidence of disease for 14 months. This regime of therapy is effective, tolerated well, and is not as mutilating as commonly recommended therapy.

810 PLATELET DYSFUNCTION IN VINCRISTINE TREATED PATIENTS. Peter G. Steinherz, Emily Schmalzer, Margaret HilNew York Hospital, Dept. of Ped., New York.

A recent report on the use of vincristine (VCR) in patients with refractory ITP prompted us to evaluate the function of the platelets ( $p 1)$ produced with this drug. White showed in vitro that VCR destroys $\mathrm{pl}$ microtubules but that clot retraction and pl aggregation (ag) were not affected at doses much higher than those used clinically. 14 children with ALL on $1.5 \mathrm{mg} / \mathrm{m}^{2}$ VCR every 4 weeks were examined on 25 separate occasions at different time intervals from their last VCR dose. Pl adhesion was measured by Salzman's method. Pl were tested for their ability to ag when stimulated with suprathreshold doses of ADP, epinephrine (EPI) and collagen (COL). The lowest concentration of ADP and EPI to give biphasic ag was determined. PI adhesion was abnormal in 6 of 10 patients. The ag results are shown. Even in

$\%$ of pat with abn ag after VCR the patients who had normal ADP

2 ds 1 wk 2 wks 3 wks 4 wks ag at 4 weeks, 4 out of 5 tested EPI $100 \quad 100 \quad 100 \quad 100 \quad 23$ had an elevated threshold for

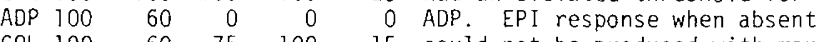

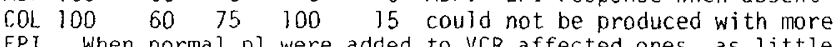
normal pl were added to VCR affected ones, as little as $15 \%$ normal pl reestablished the biphasic EPI ag. These findings suggest that VCR renders the $p l$ less sensitive to ADP. This is not an absolute defect. There is a difference between EPI and ADP produced second phase ag. Both the circulating pl and the megakaryocytes are affected.

811 FACTORS REIATING TO PROGNOSIS OF NEUROBLASTOMA UNDER 1 YEAR OF AGE. D. Traggis, N. Jaffe, G. F Vawter and R. F. Filler (Intr. by David Nathan), Sidney Farber Cancer Ctr., Children's Hosp. Med. Ctr. and Harv. Med. Schl., Boston, Mass. 02115.

The prognosis of 75 patients (pts) under 1 yr of age with neuroblastoma was evaluated by the following parameters: site of primary tumor, extent of disease at diagnosis, type of surgical procedure, pathology, chemotherapy (principally nitrogen mustand, vincristine and cyclophosphamide) and radiation therapy. Disease free survival was $51(66 \%)$ for more than 2 years but identical therapeutic maneuvers produced discordant results in pts with primary tumors located at different sites and extent of disease. Survival by primary site (total number presenting at that site in parentheses) was mediastinal 11 (11); neck 4 (6); aboominal $34(56)$ and central nervous system 2 (2). Twenty-nine of 33 Stage I and II survived whereas only 4 of 25 Stage III and IV survived (staging system by Evans et al. Cancer 27:374, 1971). In 19 of the above pts with varying combinations of localized primary tumor and involvement of the liver, bone marrow and/or skin, but negative skeletal survey, there were 18 survivors (958). These data indicate that certain anatomic sites carry a more favorable prognosis in pts under 1 yx of age undergoing treatment for neuroblastoma.

(Supported by Grant C-6516) .
812 BIOCHEMICAL EVIDENCE OF ONCORNAVIRUS LIKE PARTICLES IN THE BONE MARROW PRECEDING THE ONSET OF LEUKEMIA AND LYMPHOMA. Gerald J. Vosika, William Krivit, Jon M. Gerrard, Peter Coccia, Mark E. Nesbit, Jacqueline J. Coalson, B.J. Kennedy. Univ. of Mn., Dept. of Med.\& Ped., Minneapolis, Mn. Reverse transcriptase and oncornavirus like particles have been reported associated with cytoplasmic fractions from disrupted human leukemia cells, and in supernatent fluid from human leukemia cells cultured in vitro. These particles have a characteristic density in sucrose gradients associated with a high molecular weight RNA and a DNA polymerase which utilizes a RNA template and has defined synthetic template characteristics, Recently, 2 patients in whom malignancy was considered as a cause of their disease were investigated for such particles. The first, a $7 \mathrm{yr}$. old had an acute onset of fever and severe exythema nodosum. His bone marrow was hypercellular with normal morphology. Following in vitro culture of this bone marrow, analysis showed particles with the density of complete virus and viral cores respectively having a DNA polymerase with viral template preferences. Two months later a repeat bone marrow revealed myeloblasts containing Auer rods. The second patient was a $2 \mathrm{yr}$. old male with hepatosplenomegaly. A normal appearing bone marrow also revealed oncornavirus like particles. Autopsy 3 months later revealed a histiocytic lymphoma involving the bone marrow, lymph nodes, liver, brain and pericardium. These 2 cases represent the first observation of oncornavirus like particles in patients who subsequently developed malignancy. This or a comparable assay for oncornavirus may be useful in the diagnosis of suspected premalignant states.

813 FEVER, NEUTROPENIA AND INPECTION IN CHILDREN WITH ACUTE LYMPHOBLASTIC LEUKEMIA (ALL). Joel A. Wolk, James A. Stockman, III, and Marie J. Stuart (Intr. by Frank A. Oski), S.U.N.Y., Upstate Medical Center, Department of Pediatrics, Syracuse, New York.

In an attempt to determine the relationships between neutropenia, fever, infection, and disease status, 20 patients with ALL were observed for a period of 2087 patient days. During 1512 days of induction-remission, patients were neutropenic (granulocyte count $<1000 / \mathrm{mm}^{3}$ ) for 740 days. One febrile episode occurred per 24 neutropenic days while one per 14 days occurred during the non-neutropenic period. A cause for fever was found in 16 of 31 (51\%) of the neutropenic fevers and in 32 of $54(60 \%)$ of the non-neutropenic fevers. When counts fell below $200 / \mathrm{mm}^{3}$, fever occurred with a frequency of one per 15 days and in 4 of 7 episodes (55\%) a cause was found. Febrile episodes occurred with greater frequency in neutropenic patients during relapse averaging one per 7 days but was only one per 23 days in non-neutropenic patients in relapse. A cause for fever was found in only $8 \%$ ( 4 of 50 ) of episodes in neutropenic patients in relapse. In non-neutropenic patients in relapse, a cause for fever was identified in $44 \%$ of cases ( 4 of 9). Fever occurred once per 13 days with counts below $200 / \mathrm{mm}^{3}$ and a cause was found on each occasion. Fever during the course of neutropenia in patients in induction-remission has an identifiable cause in about $50 \%$ of episodes while fever in relapse is more likely due to the disease process until the granulocyte count falls below $200 / \mathrm{mm}^{3}$. IMFRA (INTERMITTENT METHOTREXATE AND FRACTIONAL RADIA
TION) CNS PROPHYLAXIS - ? A "CURE FOR CHILDHOOD LEUKEMIA"? W.W. Zuelzer, Y. Ravindranath, Basil Considine Ir., Child Res. Ctr. of Mich.; Children's Hosp. of Mich.; Harper Hosp.; Wayne State Univ. Sch. Of Med., Dept. of Ped., Detroit.

Prophylactic CNS radiation in childhood ALI was introcuced recently by Aur et al. Analysis of a previously studied group of 79 patients treated by us without CNS prophylaxis showed a continuars pattern of CNS relapses throughout the first 3 years, suggesting that de novo metastatic colonization of the cNs occurs at any time in the course of the disease. Such recurrences might not be preventable by initial prophylaxis alone. The dosage of 2400 rads, moreover, is apt to create iatrogenic problems. Therefore a protocol of repetitive low dosage radiation to the entire neuraxis was devised here in 1972, 100 rads are given when remission is achieved and at 10 week intervals thereafter, each time preceded by a diagnostic tap and intrathecal injection of a single dose of MTX. Comparison with an apparently identical group receiving identical supportive and chemotherapy, but no CNS prophylaxis, shows highly significant improvement with the incidence of relapse of any kind dropping from 52.78 to $10 \%(p<.01)$ at 22 months of actual observation $(n=25)$ and projected complete remissions of $83.3 \%$ at 3 years $(n=52)$. Relapse occurred predominantly in the "high risk" group (hyperleukocytosis and/or mediastinal mass), 5/16, compared to $22 / 25$ in the earlier group $(p<.005)$. Only 2 of the other 36 patients relapsed, compared to $16 / 47$ in the previous group. The data suggest a possible scheme for staging ALL as a prognostic aid. The therapy was well tolerated and the number of intercurrent deaths from infection did not change significantly. 
815 INHIBITION OF LYMPHOCYTE RESPONSE TO PHYTOHEMAGgLU815 IININ (PHA) BY SERUM OF UNTREATED ACUTE LYMPHOBLASTIC LEUKEMI A (ALL), Zusman, J., Nesbit,M.E., Duke Univ. Med. Ctr., Durham, Univ, of Minnesota, Minneapolis. (Intr.by F.S. Porter)

The occurence of anergy in cancer patients has been related to the presence of substances in the serum which inhibit lymphocyte response to PHA (Glasgow, A., et al, NE.JM 291:1263,1974). The purpose of this study was to define the kinetic characteristics of the inhibitory activity in ALL and to determine the effects of therapy.

The lymphocyte response to increasing doses of PHA was determined simultaneously in pooled human serum(PHS) and autologous serum (AS) in 60 normal controls and in 14 children with ALL with a peripheral $\mathrm{WBC}<20,000 / \mathrm{mm}^{3}$. The differences were not significant (p).05) for the controls at all concentrations of PHA nor for the ALL patients at optimal doses $(10 \& 20 \mu \mathrm{g} / \mathrm{ml})$. At suboptimal doses of PHA $(.25,15, \& 1 \mathrm{\mu g} / \mathrm{ml})$ the response in AS was significantly less than in PHS $(p<.05)$ for the ALL patients. $6 / 14(42.5 \%)$ had abnormal responses; $5 / 6$ were abnormal only at suboptimal doses but were in the normal range at optimal doses; one patient was abnormal at all concentrations. The inhibitory activity (IA) disappeared upon achieving remission. Al1 14 patients continue in their initial remissions for 12-24 months without reappearance of IA.

A dose-dependent IA $_{\mathrm{A}}$ of lymphocyte response to suboptimal concentrations of PHA was detectable in the serum of $42 \%$ of children with ALL. Th1s effect was obscured by higher concentrations of PHA. IA was specific for the relapse state.

\section{PULMONOLOGY}

816 VENTILATORY STABILITY OF THE PREMATURE INFANT. Saul M. Adler, Bradley T. Thach, and Ivan D. Frantz,
III. (Intx. by M.E. Avery) Dept. of Ped., Harvard Medical School, Boston.

The premature infant must appropriately adjust tidal volume $\left(V_{m}\right)$ under changins conditions that impose loads on inspiratory effort such as position, feeding, and nasal obstruction. The effective elastance of the respiratory system (E'rs) is a measure of its ability to resist change of $V_{T}$ with these elastic loads. $E^{*} x s$ was determined by measuring $V_{T}$ (using a face mask and pneumotachograph) and the inspiratory pressure (P) generated after occlusion of the airway at FRC $\left(E^{\prime} r S=P / V_{T}\right)$. Studies were done on the third day of life in 13 healthy premature infants breathing air, and $2 \%$ and $4 \% \mathrm{CO}_{2}$ in air. E'rs did not change with elevated $\mathrm{V}_{\mathrm{T}}$ during $\mathrm{CO}_{2}$ breathing.

gestational age, weeks (g.a.) 29-32(n=5) 33-36(n=8) 39-41 (n=ld) E'rs $\left(\mathrm{cmH}_{2} \mathrm{O} / 1\right) \pm \mathrm{SEM} \quad 793 \pm 58 \quad 540 \pm 384.18 \pm 36$ Specific E'rs + SEM $\quad 40 \pm 3 \quad 31 \pm 2 \quad 33 \pm 2$ (E'rs $x$ thoracic gas vol.) at 4 weeks of age and a specific E'rs of $31 \pm 3$. Premature infants have a greater ability to adjust $V_{\mathrm{T}} \overline{\text { with }}$ added elastic loads than age-matched term infants. Factors contributing to E'xs include the stiffness of the lung and chest wall, neural recruitment of intercostal muscles, and the force/length characteristics and geometry of the thoracic muscles. Correcting for thoracic size (specific E'rs) eliminated all differences except for the higher values found on day 3 in the 29-32 week group.

PULMONARY WATER AND PULMONARY BLOOD FLOW (PBF) IN 817 LAMBS WITH HYALINE MEMBRANE DISEASE (HMD). A.C. Allen, D.A. Stinsoil, H.M. MacDonald, E.A. Contreras, I.R. Holzman, and P.M. Taylor, Univ. of Pittsburgh Sch. of Med. Magee-Womens Hosp., Dept. of Pediatrics, Pittsburgh, Pa.

We tested the hypothesis that pulmonary edema plays a central role in the pathogenesis of ovine HMD. Lung weight(LWt), water content(LW) and extravascular chloride space(LECS) were measured in the left lung of 31 lambs delivered prematurely by C-section at 135-140 days' gestation and sacrificed at age 0-8.5 hr. of 26 lambs sacrificed beyond $15 \mathrm{~min}$ of age, 18 with moderate or severe $\mathrm{HMD}\left(\mathrm{P}_{2} \mathrm{CO}_{2}>70\right)$ had significantly higher LWt, LW and LECS than 8 with no distress or mild $\mathrm{HMD}\left(\mathrm{P}_{a} \mathrm{CO}_{2}<70\right)$. There was a stat istically significant direct correlation between $\mathrm{P}_{3} \mathrm{CO}_{2}$ and $\mathrm{LW}$.

In 14 additional lambs at 129-133 days' gestation, a precalibrated electromagnetic flow transducer with non-occlusive zero was implanted on the postductal portion of the common pulmonary artery. These lambs were returned to the amniotic cavity for $7 \mathrm{~d}$ and then delivered by C-Section. PBF was measured repeatedly during extrauterine life. Eight lambs were sacrificed at age 4.3$4.5 \mathrm{hr}$ and 6 lambs at $8.3-10.4 \mathrm{hr}$ and right LW measured. In both groups of lambs greater $\mathrm{LW}$ at death was associated with significantly higher PBF after the initial PBF surge which occurred during the first hour of life (previously reported, Pediat. Res. 8: 433, 1974). At age $4 \mathrm{hr}$ mean PBF for the 7 lambs with wetter lungs was $325 \mathrm{ml} / \mathrm{kg} / \mathrm{min}$ (range 262-417) contrasted with a mean $\mathrm{PBF}$ of $161 \mathrm{~m} 1 / \mathrm{kg} / \mathrm{min}$ (range 95-216) for the remaining 7. These data suggest that pulmonary edema related to high PBF is centra] in the pathogenesis of ovine HMD.
818 CARDIOPULMONARY PATHOPHYSIOLOGY IN BRONCHO PULMONARY DYSPLASIA. Ronald L. Ariagno*, John L. Watt*, Fe Dumpit* and June P. Brady. Dept. Ped. Children's Hosp. and Cardiovasc. Res. Inst. Univ. of Calif. San Francisco, California

We found increased wasted ventilation and reduced effective pulmonary blood flow ( $Q$ pc eff.) in preterm infants with bronchopulmonary dysplasia (BPD). We measured $\dot{Q p c}$ eff. using nitrous oxide, minute ventilation $\left(\dot{V}_{\mathrm{E}}\right)$ and end-tidal $\mathrm{CO}_{2}\left(\mathrm{P}_{\mathrm{ACO}}\right)$ using a pneumotachograph (Pediatrics, 1972) and arterialized $\mathrm{PO}_{2}$ and $\mathrm{PCO}_{2}$ in 7 preterm infants with BPD and 7 "normal "("N") preterm infants; mean birth weights were 1500 and $1150 \mathrm{gm}$ and mean gestational ages 32 and 30 weeks respectively. All infants with BPD had characteristic pulmonary roentgenographic findings and all had had severe IRDS, prolonged oxygen requirements and assisted ventilation (one had only CPAP and oxygen). We calculated $\mathrm{PAO}_{2}$ and venous admixture (VA). Results:

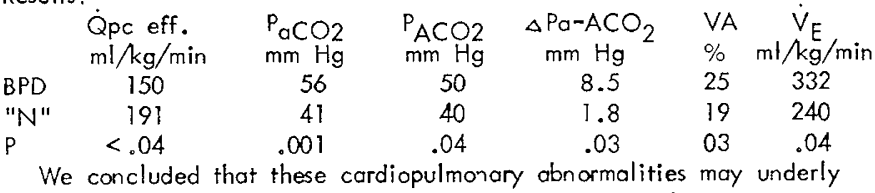
the chronic respiratory distress of bronchopulmonary dysplasia.
820 SERUM GLUCOCORTICOID LEVELS FOLLOWING BETAMETHASONE THERAPY TO PREVENT RESPIRATORY DISTRES SYNDROME (RDS). Philip L. Ballard, Roberta A. Ballard and Patricia Granberg, Cardiovas. Res. Inst. NHLI Sp. Ctr. Res.-Pulm. and Depts of Ped., Univ. of Calif. and Mt. Zion Med. Ctr., San Francisco

Antenatal betamethasone appears to reduce the incidence of RDS in premature infants. To evaluate the safety and optimal dose schedule of such therapy, we determined betamethasone and cortisol levels in 20 mothers and 43 newborns using the glucocorticoid radioreceptor and $C B G$-isotope assays. Women in premature labor (27-36 wks) received $2 \mathrm{IM}$ doses of 12 $\mathrm{mg}$ betamethasone acetate and phosphate $24 \mathrm{hrs}$ apart. Maternal betamethasone peaked at $75 \mu \mathrm{g} / 100 \mathrm{ml}$ cortisol equivalents at $1 \mathrm{hr}$, declined by holf at $6 \mathrm{hrs}$ and was $22 \mathrm{\mu g} / 100$ at $12 \mathrm{hrs}$. In cord bloods the steroid level peaked at $12 \mu \mathrm{g} / 100 \mathrm{ml} 4-8 \mathrm{hrs}$ after the first dose and was $5 \mu \mathrm{g} / 100 \mathrm{ml}$ at 24 hrs. The mean maternal:fetal gradient was 3:1 (range 1.6-4.1:1) in matched samples. Betamethasone was not detected in either maternal or cord blood samples obtained 2 days after the second dose. During the 2 days of treatment unbound glucocorticoid activity (cortisol plus betamethasone) in cord blood ranged from 4.0 to $8.6 \mu \mathrm{g} / 100 \mathrm{ml}$ cortisol equivalents compared with a mean of $2.8 \mu \mathrm{g} / 100 \mathrm{ml}$ unbound cortisol in a comparable group of 22 untreated infants. In untreated infants with RDS the level after birth averages $7.8 \mu \mathrm{g} / 100 \mathrm{ml}$. Thus we find that antenatal betamethasone administered by this dose schedule results in glucocorticoid levels in the physiologic stress range for not more than 3 days. This exposure should be safe for the premature infant and is apparently adequate to induce lung maturation. 
821 CYCLIC AMP (CAMP) AND SURFACTANT PRODUCTION: NEW Cynthia T. Barrett, Alex Sevanian and Solomon A.

Kaplan, UCLA Sch, of Med., Department of Pediatrics, Los Angeles. Recent evidence suggesting that cAMP is involved in surfactant production has raised the intriguing possibility that maturation of the fetal lung can be enhanced by agents which increase cAMP levels in the lung. We have shown that cortisone may increase surfactant production by this means; its injection into pregnant rabbits at a dose of $1.95 \mathrm{mg} / \mathrm{kg} /$ day from days $24-26$ of gestation results in an increase of lung CAMP levels, a decrease of cAMP phosphodiesterase (PDE) activity and increased activity in both the transmethylation and CDP-choline transferase pathways leading to lecithin synthesis. That CAMP may be an important factor in regulating lung lecithin synthesis is indicated by our results when we injected 6 does with $6.25 \mathrm{mg} / \mathrm{kg} /$ day of aminophylline, a potent inhibitor of PDE. Biochemical responses were similar to those obtained with cortisone. On day 27 we measured specific activities of ${ }^{3} \mathrm{H}$ methionine and ${ }^{14} \mathrm{C}$ chol ine incorporated into lecithin (cpm/mg lecithin), PDE activity ( $\mathrm{pM}$ cAMP hydrolyzed/mg protein), cAMP ( $p M / m g$ tissue). All are shown as mean \pm 1 S.D. $3 \mathrm{H}$ PDE CAMP

Control $\quad 6286 \pm 681 \quad 7747 \pm 1716 \quad 26.14 \pm 3.24 \quad .190 \pm .051$ Aminophylline $7458 \pm 555 \quad 9908 \pm 918 \quad 18.79 \pm 2.55 .385 \pm .111$ Cortisone $8306 \pm 557 \quad 11034 \pm 2724 \quad 18.20 \pm 2.42 .342 \pm .102$

Because agents affecting CAMP levels can increase surfactant, the way is now open to a search for means other than cortisone for enhancing pulmonary surfactant production.

822 APIEA ASSOCIATED WITH RESPIRATORY SYNCYTIAL VIRUS INFECTION. Frederic W. Bruhn, Stefan T. Mokrohisky and Kenneth McIntosh. "Univ. of Colo. Mejd. Ctr., Dept. of Ped. and Denver Genera Hospital, Denver.

A retrospective study of children under 6 months of age with respiratory syncytial virus (RSV) infection was conducted to determine the frequency of associated apnea. The study included 278 patients from Jan. 1, 1970 through Dec. 31, 1974 at the three University affiliated hospitals in Denver. RSV was recovered from all at the time of respiratory illness. 274 charts were available for review. Of the 263 patients where qestational age at birth was recorded, 71 were premature ( $\leqslant 37$ weeks). Apnea with RSV infection was recorded in 56 patients (observed in 47 by medical personnel), 4 of whom died. If these 56 patients, 29 had been premature at birth. Apnea was the presenting complaint in 41 . Apnea occurred in children 13 davs to 5 months old; it was commonest in infants $0-1$ month old ( 24 of 66 infected patients), and next most frequent in those $1-2$ months old (18 of 67). It usually responded to minimai resuscitatory measures al though 8 , patients required intubation. Spinal fluids obtained from 40 patients were normal. Bacterial cultures of blood yielded no pathogens. ATl 4 deaths were in otherwise compromised hosts. Hospital acquired infection occurred in at least 15 patients below 6 months of age $(5.5 \%)$ of whom 3 experienced apnea.

Apnea is a previouslv undocumented but common symptom of infection with RSV in young infants. Its possible relationship to sudden infant death syndrome is speculative.

823 Lack of Disaturated Lecithin (DSL) Stimulation in Cortisol Treated Lambs.

George Brumley, Carlyle Crenshaw, and John Knelson Duke University and NIEHS, Durham, N. C.

Twin lambs were infused with $0.5 \mathrm{mg} / \mathrm{hr}$ cortisol (C) and saline (S) respectively for $72 \mathrm{hrs}$., and sacrificed at 116 and 125 days. Serum cortisol levels in the " $\mathrm{C}$ " lambs approximated those seen during labor and stress $(18-20 \mu \mathrm{g} / 100 \mathrm{ml})$. At sacrifice the DNA content of the "C\&S" lamb lungs was not significantly different. Histological and pressure-volume comparisons showed the acceleration in maturation previously noted by DeLemos et al (Am. Rev. Resp. Dis., 102:459, 70). Analys is of whole lung lecithin (WLL) from the " $C$ " lambs showed an increase in specific activity (SA) at both gestational ages and increase in quantity at 116 days. In contrast DSL was reduced in both quantity and $S A$ in the " $C$ " lambs as compared to controls.

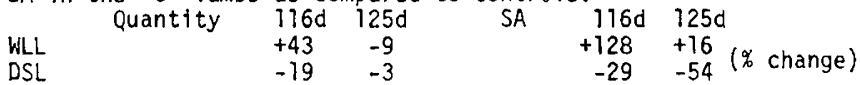

We have interpreted these resuits to indicate that: 1) there are two lecithin pools in the lung, 2) the pools are differentially effected by cortisol and 3) the histologic and pressurevolume maturational effects of cortisol do not appear to correlate with enhanced DSL synthesis.
824 THE RESPONSE OF NEONATES TO ADDED RESPIRATORY LOADS. M. Heather Bryan, Richard L. Knil1, S. Margaret L. Kirkpatrick, M. John O'Brien, and A. Charles Bryan. Dept. of Pediatrics, Univ. of Toronto and the Research Inst., The Hosp. for Sick Children, Toronto, Canada.

We have studied the ability of 10 newborn infants to compensate for added respiratory elastic loads. Elastic loads, (closed containers) were added to the breathing circuit for 5 breaths while changes in tidal volume $\left(V_{T}\right)$ and mouth pressure $\left(\mathrm{P}_{\mathrm{m}}\right)$ were measured. The response to the elastic load varied with the infants' behavioral state. When the infant was awake or in quiet sleep the load produced a predictable fall in $V_{T}$, but a progressive rise in $V_{T}$ and $P_{m}$ during subsequent treaths indicating load compensation. However during active (REM) sleep load compensation was ineffective, either due to a sustained decrease in $V_{T}$ or due to glottic closure. During intermediate sleep states load compensation is erratic. The natural load on the respiratory system is cortinually varying with nasal obstruction, abdominal distention, posture etc.. As infants spend most of their time in active or intermediate sleep, this inability to compensate for changes in respiratory load has an obvious clinical sigrificance. The mechanism appears to depend on monosynaptic reflex inhibition during active sleep.

85 NEOIHATAL HEART RATE VARIABILITY (NHRV) AS A PROGNOSTIC TOOL IN THE MAIAGEMENT OF RESPIRATORY DISTRESS SYNDROME (RDS). L. A. Cabal, B. Zanini, S. Y. Yeh and E. H. Hon (Intr. ty Paul K. Wu), Dept. of Pediatrics and Ob-Gyn, LAC-USC Med. Ctr., Los Angeles, California.

The relationships of NHRV with maturity, chronological age (CA). morbidity and mortality are unknown. 37 neonates were divided into Gp. 1, heaT thy term (10); Gp. 2, heal thy prematures (10); Gp. 3 , prematures with RDS--survived (9); and GP. 4, prematures with RDS--died (8). Instantaneous heart rate was monitored continuously by cardiotachometer in the first six hrs. of life and at 24,48 , 168 hrs. of age. The NHRV was analyzed as the heart rate range in beats per minute in 2-minute periods per hour of study.

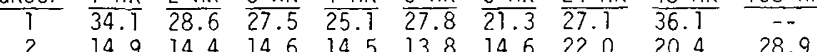
$\begin{array}{rrrrrrrrrr}2 & 14.9 & 14.4 & 14.6 & 14.5 & 13.8 & 14.6 & 22.0 & 20.4 & 28.9 \\ 3 & 16.0 & 8.8 & 9.4 & 7.5 & 10.1 & 8.7 & 7.6 & 12.0 & 15.6\end{array}$ $\begin{array}{llllllllll}4 & 5.1 & 4.9 & 4.2 & 5.3 & 6.9 & 6.7 & 4.6 & 5.4 & --\end{array}$ (p.01); significant differences were found between Gos. 1 and 2 (p.01 except in $24 \mathrm{th} \mathrm{hr.),2}$ and 3 (p.01 except in lst hr.), 3 and 4 ( $p .05$ except in 4 th and 6 th hrs.) at any given $C A$. The decreased NHRV in Gp. 2 as compared to GP. 1 can be related to the immaturity of the autonomic nervous system. The decrease or absence of NHRV in Gps. 3 and 4 as compared to Gp. 2 can be of value in predicting the outcome of prematures with RDS. Although NHRV awaits further clinical evaluation, these results suggest its diagnostic and prognostic usefuiness in the management of RDS.

826 DEMONSTRATION OF HUMAN LEUKOCYTE DEGRANUEATION INDUCED BY SERA FROM CYSTIC FIBROSIS PATIENTS AND CARRIERS. James H. Conover, Elaine J. Conod, Kurt Hirschhorn. Dept. Pediatrics, Mount Sinai School of Med., N. Y. The ability of sera from normals and cystic fibrosis (CF) patients and carriers to promote the release of lysosomal enzymes from sensitized human polymorphonuclear leukocytes (PMN's) was assessed through the measurement of release of 8-glucuronidase activity. The extent of lysosomal degranulation of human PMN's exposed to fresh untreated sera of each of five controls, seven $C F$ affected and eight carriers, expressed as the percentage of $B$ glucuronidase release, was $7.84( \pm .934)$ \& for control sera, 14.01 $( \pm 1.79)$ \% for $C F$ affected sera and 10.61 ( \pm 1.43$)$ \% for carrier sera The difference between $\mathrm{CF}$ affected and controls is significant as is the difference between $\mathrm{CF}$ affected and carriex individuals and between controls and carriers, although some overlap was observed Treatment of these sera with IM EACA gave values for $\beta$-glucuronidase release which are slightly reduced when compared to those obtained with fresh untreated samples due to reduction of enzyme activity by EACA. The pathophysiology of $\mathrm{Cr}^{\mathrm{C}}$ can be explained by excessive degranulation of exocrine glandular cells, resulting in inspissation of their ducts. The finding of degranulator molecules in $C F$ sera allows for a test of this hypothesis. That these molecules, related to anaphylatoxins, are present in excess in CF sera may be due to the deficiency of an enzyme which normally controls their level by inactivation. The exact nature of the degranulator and its relation to ciliary dyskinesia factor must await biochemical purification. 

$\mathbf{8 2 7}$ EFFECT OF POSITIVE-END-EXPIRATORY-PRESSURE (PEEP) ON BRANE DISEASE (HMD). Robert B. Cotton, Daniel $P$. Lindstrom, Keith S. Kanarek, $\frac{\text { Hakan Sundell }}{\text { Depts. of Mildred T. Stahlman. }}$ One mechanisn through which PEEP improves oxygenation in HMD is reduction of intrapulmonary shunts by improving ventilation: perfusion inequalities. To investigate the possibility that PEE? also acts by altering extrapulmonary fetal shunts, right and left ventricular outputs (RVO,LVO) and their distributions were measured in 5 premature lambs with HMD using a modification of the radioactive microsphere technique of Makowski et al (Circ. Res., 1968). This technique allows simultaneous quantitation of each ventricular output separately, as well as both right-to-left and left-to-right shunts. Flows were measured during artificial ventilation before and during $5-8 \mathrm{~cm} \mathrm{H} 20$ PEEP. During PEEP, svstemic flow (SF) increased as a result of either a diversion of part of LVO from the pulmonary circulation or an increase in LVO or both. RVo increased even though there was little change in pulmonary vascular resistance and was partly accounted for by the increase in venous return from the improved SF. The remaining increment in RVO resulted from reduced right-to-left shunting at the foramen ovale (FO). Pulmonary flow increased during PEEP unless there was a large diversion of LVO away from the ductal shunt. Right-toleft ductal shunting was minimal or absent before and during PEEP. These results indicate that PEEP favorably affects extrapulmonary shunting through fetal pathways by improving the forward flow of LVO and reducing right-to-left shunt at the Fo.
EFFECT OF POSITIVE END EXPIRATORY PRESSURE (PEEP)

830 Edmund A. Egan and Jack R. Hessler, Depts, of Ped. and Comp. Med., Univ, of Fla. Col. of Med, Gainesville.

Ventilation with PEEP to improve oxygenation can raise pulmonary resistance and theoretically increase Qs/Qt in an immature mammal with a potentially parallel circulation. Fourteen exteriorized fetal goats, 117-140 days gestation, were ventilated with $\mathrm{O}_{2}$ and PEEP of 7.5 and $15 \mathrm{~cm}$. $\mathrm{H}_{2} \mathrm{O}$ between control periods of 0 PEEP. Ventilation was adjusted to maintain minute volume and inspiratory pressure constant. Simultaneous samples were obtained from pulmonary artery (PA), pulmonary vein (PV), carotid artery (CA) and femoral artery (FA) blood, $\mathrm{PO}_{2}$ was measured, $\mathrm{O}_{2}$ content calculated, and Qs/Qt determined by the standard shunt equation.

Qs/Qt increased in 8 of 14 experiments at $7.5 \mathrm{~cm}$. PEEP and 7 of the 12 experiments at $15 \mathrm{~cm}$. PEEP. The analysis of Qs/Qt for each oxygenated sample, PV, CA and FA was grouped by response. The group of animals showing increase in Qs/Qt had increased ductus shunting significant at $P>.05$ (at $7.5 \mathrm{~cm}$.: 0 PEEP $=18 \%$, PEEP $=45 \%$; at $15 \mathrm{~cm} .: 0$ PEEP $=23 \%$, PEEP $=59 \%)$. No significant change occurred at other sites. Intrapulmonary shunting increased in only 1 , but foramen ovale shunting increased in half of each PEEP. The group of animals who did not increase Qs/Qt showed a decrease in intrapulmonary shunting significant at $P>.05$ (at 7.5 : 0 PEEP $=34 \%$, PEEP $=20 \%$; at $15 \mathrm{~cm}$. 0 PEEP $=34 \%$, PEEP $=24 \%$ ). No significant changes in $Q S / Q t$ occurred at the foramen ovale or the ductus. PEEP did produce an increase in extrapulmonary shunting of venous blood in some animals, but tended to increase PV oxygenation in all animals.
828 EFFECTIVENESS OF ATROPJNE SULFATE AEROSOL AS A BRON8 CHODILATOR IN ASTHMATIC CHILDREN. Gerd J.A. Cropp.

The motor innervation of human airways is primarily through parasympathetic efferent fibers in the vagus nerve. Since acetylcholine is the neurotransmitter at parasympathetic motor nerveendings, atropine can block the cholinergic receptor sites on bronchial smooth muscles by competitive inhibition. In order to evaluate the importance of parasympathetically mediated bronchial smooth muscle tone in asthma, we compared the bronchodilatory effectiveness of atropine sulfate with that of isoproterenol hydrochloride and placebo aerosols. Atropine $(0.07 \mathrm{mg} / \mathrm{kg})$ and isoproterenol (1.5mg) was evaluated in each of 23 perennial asthmatics (9-15 years of age); a placebo was tested in 18 of the 23 patients. Drugs were delivered by a specially designed aerosolizer during tidal breathing. Pulmonary functions (vital capacity, peak expiratory flow rate, forced expiratory volume in first second, maximum mid-expiratory flow rate, airway resistance, specific airway conductance and residual volume) were tested before and 10-15 minutès after administration of aerosol. All patients showed significant abnormalities in baseline pulmonary function tests. Placebo administration did not improve any of these abnormalities, however, atropine and isoproterenol brought about statistically highly significant and essentially the same improvements in all abnormal pulmonary functions. The identical effectiveness of both drugs suggests that they relaxed large and small airways to the same degree and probably acted at the same aizatomical sites, and that vagal discharges contribute importantly to bronchospasm in asthmatic childxen.
PRIMATE FETAL LUNG IN GESTATIONS COMPLICATEO BY MATERNAL GLUCOSE INTOLERANCE. Michael F. Epstein and Philip M. Farrell, NICHD, NIH, Bethesda, Md.

The effects of maternal glucose intolerance on primate fetal lung lecithin synthesis were studied in the Macaca mulatta. Ten monkeys in the first trimester were treated with streptozotocin (Stz, $47 \mathrm{mg} / \mathrm{kg}$ IV), a pancreatic $\beta$ cell cytotoxin, with resultant impairment of maternal insulin secretion and glucose clearance in response to IV GTT's. Ten pregnancies, delivered by C-section at 86-89\% term, showed changes similar to those seen in human diabetic pregnancies--fetal macrosomia and $B$ cell hyperplasia, hydramnios, and placental hyperplasia. De novo pulmonary leci thin synthes is via the two major pathways--choline incorporation (1) and phosphatidylethanolamine methylation (11)--was measured in fetal lung slices incubated with $2 \mu \mathrm{Ci}$ of $14 \mathrm{C}$-choline or $1{ }^{4} \mathrm{C}$-methionine and the amniotic fluid (AF) L/S ratio was determined. Five fetuses of normal, age-matched gestations were controls.

Results: Pathway I Pathway II AF L/S Ratio

$\begin{array}{llll}\text { Stz } & 800 \pm 90^{*} & 90 \pm 35 & 2.5 \pm 0.4 \% \\ \text { Normal } & 490 \pm 60 & 20 \pm 1 & 1.0 \pm 0.2\end{array}$

Pathway I activity (cpm/mg lung/hr) accounted for $90-96 \%$ of lecithin synthesis in both groups. Pathway 1 activity and AF L/S ratio were significantly $(* p<.05)$ greater in the Stz group; pathway II was not. Therefore, maternal glucose intolerance was associated with an increase in fetal lung choline incorporation into lecithin, reflected in an earlier increase in the $A F L / S$ ratio. This suggests that in the human it may be the timing and mode of delivery rather than maternal diabetes mellitus that results in the increased risk of RDS in the neonate.
(CPAP) APPLIED CONTINUOUS POSTTIVE AIRWAY PRESSURE (CPAP) APPLIED BY FACE-MASK. M. Douglas Cunningham, Thomas H. Pauly, and Nirmala S. Desai. (Intr, by Jacqueline A. Noonan). Albert B. Chandler Med. Ctr., Univ, of Kentucky, Dept. of Ped., Lexington.

Face-mask CPAP is a non-invasive means of assisted ventilation for infants with respiratory distress syndrome (RDS), but our observations suggest 1 imitations. 27 infants had clinical, laboratory, and $x$-ray evidence of RDS. Masks held in place by elastic paper bandage were connected to underwater pop-of $f$, aneroid manometer, and $500 \mathrm{ml}$ anesthesia bag for maintenance of CPAP. CPAF pressures at $1 \mathrm{hr}$. were $3-6 \mathrm{~cm}$ water. Nasogastric tubes for continuous stomach decompression were used. Average birth weight was $1,928 \mathrm{gm}$, and average gestational age was 34 wks. 2 groups of patients were identified. Group I consisted of 16 infants $(59 \%)$ successfully treated. Mean pre-CPAP pH was 7.29 , and $1 \mathrm{hr}$. post-onset of CPAP 7.28. Mean decrease of alveolar to arterial gradient $\left(\mathrm{A}-\mathrm{aDO} \mathrm{D}_{2}\right)$ was $-68 \mathrm{mmHg}$ following $1 \mathrm{hr}$. of CPAP therapy. Group II consisted of 11 infants who ultimately required mechanical ventilation. Mean pre-CPAP $\mathrm{pH}$ was 7.21 , and 1 $\mathrm{hr}$. post-onset of CPAP 7.17. Mean A-aDO2 decrease at $1 \mathrm{hr}$, was -36 mmHg. 7 infants abruptly developed profound apnea from 1 to $12 \mathrm{hrs}$. of treatment and required endotracheal intubation for resuscitation. Pneumothorax developed in 2 infants of each group 4 infants with a mean birth weight of $1,378 \mathrm{gm}$ died. The case fatality overall was $15 \%$. Persistent acidosis ( $\mathrm{pH}<7.28$ ), an unyielding $\mathrm{A}-\mathrm{aDO} 2$ gradient ( $<36 \mathrm{mHH}$ decrease) after $1 \mathrm{hr}$, and birth weight under $1500 \mathrm{gm}$, may portend early irreversible apnea and treatment failure.
NEONATAL RESPIRATORY DISTRESS FOLLOWING ELECTIVE

832 DELIVERY. A PREVENTABLE DISEASE? Fanaroff, A., Hack, M., Mendelawitz, B., Merkatz, I., Klaus, M. Depts of Pediatrics and Reproductive Biology. Case Western Reserve Univ. School of Medicine. Cleveland, Ohio Amniocentes is and tests of fetal pulmonary maturity are avaliable but not uniformly employed before elective delivery.

From Hov. 1973-April 1974, 19/245 (8\%) infants admitted to the Neonatal Intensive Care Unit were born after elective intervention ( 15 cesarean section and 4 vaginal inductions). All were white and $18 / 19$ were private compared to yearly admissions of white $(56 \%)$, private $(57 \%)$. 18/19 were admitted from the region via the transport service. Mean blith was $2.69 \mathrm{~kg}$. (range $1.5-$ $3.48 \mathrm{~kg}$ ) with 18 infants greater than $2 \mathrm{~kg}$. Pediatric gestational age from a physical and neurological evaluation ranged from 3239 weeks (mean 36.2 weeks) in contrast to obstetrical dating which ranged from $39-44$ weeks (mean 39 weeks). The obstetrical dating was 3 or more weeks greater than the pediatric age in 11 infants. No pricr documentation of pulmonary maturity had been obtained in any infant. Pulmonary disease included transient tachypnea (5) and respiratory distress syndrome (14). This comprised $12 \%$ of all resplratory distress observed during this period. 12 required CPAP or assisted ventilation. Mean hospitalization was 23 days (range 1-140), with est imated costs of $\$ 3,337$ per baby. Two infants died.

Respiratory distress following elective delivery remains a potent source of on-going perinatal morbidity. Regional programs must direct increased educational efforts to eliminate this preventable disease. 
833 FFFECT OF CONTINUOUS DISTEND TNG AIRWAY PRESSURE ON LUNG MECHANICS IN BRONCHIOLITIS. Tilo Gerhardt, Eduardo Bancalari, (Intr. by w. W. Cleveland) Univ. of Miami, Dept. of Ped., Miami.

Since small airway resistance is related to lung volume, we studied the effects of changing the latter by applying a continuous distending airway pressure (CDAP) in four infants with bronchiolitis. This was done by applying external negative pressure in three and continuous positive airway pressure in one. Respiratory rate, tidal volume, inspiratory and expiratory flow and esophageal pressure were measured before and during applica$t$ ion of a CDAP of $8-10 \mathrm{~cm} . \mathrm{H} 2 \mathrm{O}$. Compliance (CL) and resistance (R) were calculated from these values. Results were similar whether negative or positive pressure was used.

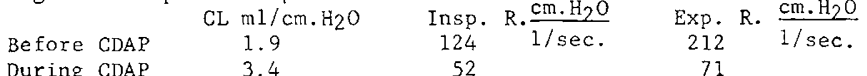
Clinical improvement occurred in all patients immediately after CDAP was applied, with a decrease in retraction and disappearance of wheezing. This correlates with the significant improvement in $\mathrm{CL}$ and reduction in pulmonary $\mathrm{R}$. The lower resistance may be explained by distension of small airways produced by the increased lung volume during CDAP. In addition, during CDAP patients make less active expiratory effort avoiding further airway collapse. The low CL in these patients is caused by their high airway resistance and frequency dependence and therefore the improvement observed with CDAP is probably related to the reduction in resistance. All these changes were reversed as soon as the CDAP was discontinued.

\section{4} EFFECT OF ENVIRONMENTAL TEMPERATURE ON RESPIRATORY CONTROL IN PREMATURE INFANTS. Tilo Gerhardt, Eduardo Dept. of Ped., Miami.

The Hering Breuer Reflex (HBR) has been reported to be active in premature infants and of importance in determining their respiratory rate and tidal volume and may have a stabilizing effect on the respiratory system when it is subjected to varying loads. Because temperature affects respiration in premature infants, airway occlusion at end expiration was performed in four prematures at different ambient temperature. Esophageal and mouth pressures and inspiratory time were measured before and after occlusion.

$\begin{array}{lllll}\text { Mean } & \text { Mean } & \text { Ambient } & \text { \% Increase } & \% \text { Increase } \\ \text { Wt. (g) } & \text { GA (wks) } & \text { Temp. OF. } & \begin{array}{l}\text { Insp. Time } \\ \text { Inter Occlusion }\end{array} & \begin{array}{l}\text { Insp. Pressure } \\ \text { After Occlusion }\end{array} \\ 1475 & 31.5 & 89.7 & 91 \% & 96 \% \\ & & 99.5 & 35 \% & 53 \%\end{array}$
After airway occlusion at high ambient temperature, the increase in inspiratory time and mouth pressure was significantly less, approximately half that at lower temperature. Some infants became apneic. This indicates a decrease in the strength of the HBR at higher temperatures, decreasing the possibility of adaptation of the respiratory system to increased loads. This may explain, in part, the increased incidence of apnea in prematures exposed to high incubator temperature.

$\mathbf{8 3 5}$ EARLY INTERVENTION IN THE RESPIRATORY DISTRESS SYNDROME (RDS). Boyd $W$. Goetzman and Willard J. Blankenship, Univ. California Sch. of Med., Dept. Ped., Sacramento Med. Cntr., Davis. Criteria for early intervention in the RDS (nasal prongs or continuous regative pressure) were developed by analyzing the arterial blood gas data for 51 consecutive patients with RDS who required only increased ambient oxygen during their hospital course. The following table indicates the percent of these patients whose $\mathrm{Pa} 02$ fell below $50 \mathrm{mmHg}$ in various ambient oxygen concentrations at several postnatal ages.

\begin{tabular}{|c|c|c|c|c|c|c|c|}
\hline $\mathrm{AGE}$ & $\mathrm{O}_{2}$ & 50 & 60 & 65 & 70 & 80 & 100 \\
\hline 24 hrs & & 31 & 20 & 12 & 8 & 4 & 0 \\
\hline $48 \mathrm{hrs}$ & & 43 & 35 & 20 & 18 & 10 & 0 \\
\hline 96 hrs & & 51 & 41 & 27 & 24 & 12 & 0 \\
\hline
\end{tabular}

If treating $10-12 \%$ of the patients who require only
increased ambient oxygen is acceptable, then the following criteria for early intervention in RDS may be used:

1). $\mathrm{PaO}_{2}-50 \mathrm{mmHg}$ in $65 \% 02$ during the first 24 hours of life; 2). $\mathrm{PaO}_{2}-50 \mathrm{mmHg}$ in $80 \% 02$ thereafter.

Consideration of birth weight and gestational age did not affect the above criteria. Analysis of $\mathrm{PaCO}_{2}$ data did not yield criteria different from those previously accepted for respiratory failure in the newborn. Neonatal centers must analyze their own patients' data to establish early intervention criteria if overtreatment is to be avoided.
836 EFFECTS OF ANTENATAL BETAMETHASONE IN PRETERM INFANTS. Patricia Granberg, Roberta A. Ballard, Philip L. Ballard, Frederick Berman. Depfs. of Peds. \& Obs., Mt.

Zion Med. Ctr. and Dept. of Ped., Univ. of Calif., San Francisco

Ten preterm infants without respiratory distress syndrome whose mothers received $2 \mathrm{IM}$ doses of $12 \mathrm{mg}$ betamethasone 24 hours apart 1-5 days prior to delivery we re evaluated during the neonatal period for possible adverse effects associated with glucocorticoid therapy. These infants (mean birth weight $1472 \mathrm{gm}$, mean gestational age, $32.2 \mathrm{wks}$ ) were matched with 10 untreated infants (mean $1462 \mathrm{gm}$ and $32.0 \mathrm{wks}$ ).

We found normal values at birth for serum electrolytes, hemogram, calcium, and $\lg M$ in each group. Time to tolerate $100 \mathrm{Cal} / \mathrm{kg}$ feeding and to regain birth weight in the treated group ( 4.3 and 10.8 days, respectively) was similar to that of untreated infants ( 5.5 and 12.4 days). There was no evidence of adrenal insufficency, or increased susceptibility to hyperbilirubinemia, infection, late acidosis or patent ductus arteriosus in the treated group. None of the treated infants had apneic episodes compared with 7 of 10 controls. To assess maximal suppression of fetal cortisol, plasma corticoid levels were determined by CBG-isotope assay in cord bloods of another group of infants $(n=19$ ) born 2-12 hrs following betamethasone treatment. The level in treated infants averaged $6.7 \pm 0.8(\mathrm{SE}) \mathrm{\mu g} / 100 \mathrm{ml}$ compared with $13.65 \pm 1.65$ ( $p<0.001)$ in a comparable control group $(n=22)$ and only 3 of the treated infants had values less than $3 \mu \mathrm{g} / 100 \mathrm{ml}$. Our studies suggest that the present dose and duration of antenatal betamethasone therapy is not associated with adverse effects for the premature infant during the neonatal period.

837 RDS: THE ABSENCE OF PHOSPHATIDYLGLYCEROL IN SURFACTANT. Mikko Hallman, Bernard Feldman and Louis Gluck. Univ. of Calif., San Diego, Sch. of Med., $\frac{\text { Dept. Ped. }}{\text { Ded }}$ Div. Perinatal Med., La Jolla.

Phosphatidylglycerol (PG), once thought to be phosphatidyldimethylethanolamine (PDME), is the second major surfactant phospholipid (PL) in the adult. Using two-dimensional thin layer chromatography, we studied the PL pattern of lung surfactant obtainad in serial samples by gastric aspiration, pharyngeal suction, tracheal aspiration or alveolar wash from 38 newborn babies. At term PG was present in fetal lung fluid and in amniotic fluid. Four hours after birth its content in surfactant was 2.5 to $12 \%$ of PL-P (22 "heal thy" newborn, gestational ages 28-40 weeks). Thereafter a gradual increase in $P G$ frequentily was seen. During a few postnatal hours the PL pattern was similar in parallel specimens obtained from gastric aspirates, pharyngeal suction and tracheal aspirates. In RDS, PG was absent $(0.0-0.7 \%$ of $P L-P, N=11)$ and phosphatidylinositol was elevated. During recovery gradual normalization of $P L$ pattern took place. In retained lung fiuid syndrome $P G$ was abnormally low $(0.5-1.7 \%$ of $P L-P, N=2)$. However, in infants with pneumonia $(\mathrm{N}=2)$ and fatal congenital heart disease $(i v=1)$ levels were within the normal range. Surfactant obtained from patients with moderate RDS had high amounts of disaturated lecithin ( $52 \%$ of total lecithin, control $64 \%$ ) and the sphingomyelin content was low ( $4.7 \%$ of PL-P, control $2.2 \%$ ). In RDS absence of $P G$ appears to be the most prominent defect in PL composition of the surfactant complex. This may be the principal deficiency in mild to moderate RDS. (Support:NIH HD04380, SCOR HL14169).

838 INITIAT RESPONSE TO CONTINUOUS POSITIVE AIRWAY PRES SURE (CPAP) AND THE COURSE OF RESPIRATORY DISTRESS SYNDROME. John $\mathrm{V}$. Hartline and Richard D. Zachman, Univ. WI Sch. of Med., Dept. Ped., Madison, WI

The effect of CPAP on the alveolar arterial oxygen gradient $\left(\mathrm{AaDO}_{2}\right)$ was analyzed in 69 patients treated with CPAP for RDS. CPAP was administered initially by face mask or nasal cannula in 66 patients. The mean gestational age was 32.4 weeks and the mean bixth weight was 1888 grams. Before CPAP, mean $\mathrm{F}_{1} \mathrm{O}_{2}$ was $75.9 \%$ and mean $\mathrm{P}_{3} \mathrm{O}_{2}$ was $48.3 \mathrm{~mm}$. Starting CPAP pressure averaged $6.9 \mathrm{cmi}{ }_{2} \mathrm{O}$ After CPAP, $\mathrm{AaDO}_{2}$ dropped an average of 28.4 . Among 33 patients who required CPAP only, the $\mathrm{AaDO}_{2}$ decreased by $54.3 \mathrm{~mm}$. Arong 36 eventually needing the respirator (CR), only a 2.0 average decrease was noted $(p<0.01)$. If the $\mathrm{AaDO}_{2}$ increased, $68 \%$ eventually needed assisted ventilation and $32 \%$ died. If an increasing gradient with CPAP was noted at less than $18 \mathrm{hrs}$. of age, $86 \%$ needed a respirator and $50 \%$ died. Only $40 \%$ needed assisted ventilation and $15 \%$ died when the $\mathrm{AaDO}_{2}$ narrowed. Survivors showed a mean decrease in $\mathrm{AaDO}_{2}$ of $38 \mathrm{mmHg}$ whereas nonsurvivors showed a mean insrease of 9.1 . ${ }^{2}$ Changes in $\mathrm{PaCO}_{2}$ were not predictive of the subsequent course.

Overall survival was $76.3 \%(55.6 \%$ in the $C R$ group). Those who died were younger ( 30.1 vs 32.8 wks, $p<0.01$ ), smaller ( 1260 vs $1916 \mathrm{~g}, \mathrm{p}<0.001$ ), more depressed at birth (Apgar 6.1 vs 7.7 , $\mathrm{p}<$ 0.01 ), and in need of CPAP earlier ( $7.9 \mathrm{vs} 17.1 \mathrm{hr}, \mathrm{p}<0.1$ ) than those who lived. 42 of the 53 survivors were spared the risk of endotracheal intubation.

Babies showing a widening of $\mathrm{AaDO}_{2}$ with CPAP are very likely to need assisted ventilation and to die. 
839 THE INFLUENCE OF ACUTE PERINATAL ASPHYXIA ON THE DEVDonnelly, H. Calderwood and S. Cassin, Univ. of Florida., Col. of Med., Depts. of Comparative Med., Ped., Path., Anesth. and Phy., Gainesville.

There is much clinical evidence that correlates perinatal asphyxia with an increased incidence and severity of H.M.D. The influence of asphyxia on the pathogenesis of H. M.D. was studied in 9 Macaca mulatta and $9 \underline{M}$. arctoides fetuses ranging from 139 to $16 \overline{\text { days }} \frac{\text { gestational age }(168 \text { term) }}{160110 w i n g ~ d e l i v e r y ~ b y ~ C a e-~}$ sarean section, 12 animals were asphyxiated for 5-6 minutes and 6 were not prior to being allowed to breath or ventilated. Arterial blood pressure, gases and $\mathrm{pH}$ were monitored for $3 \mathrm{hrs}$. after which time the animals were euthanatized. Nine of 12 asphyxiated animals developed pathology consistent with H.M.D. characterized by large right to left shunts determined from blood gas data on $F_{1} 0$ 1.0; decreased radiopacity of the lungs; low residual air volumes and small hysteresis determined on post mortem static pressure volume curves; and histopathologic hyaline membranes, atalectasi.s and edema. The 3 asphyxiated animals that did not develop H.M.D. were the only animals in the study of 150 days gestational age. Two of 6 animals not asphyxiated developed H.M.D. but the disease was less severe at the end of the $3 \mathrm{hrs}$. than all but one of the affected asphyxiated animals. It is concluded that in these animals, asphyxia did increase the incidence and severity of H.M.D. It is further concluded that these two species of nonhuman primates provide a suitable animal model for studying pathogenesis, prevention and treatment of H.M.D.

MEMBRANE OXYGENATOR TREATMENT OF SEVERE NEONATAL RESPIRATORY INSUFFICIENCY. Carl E. Hunt, R. Brad Pyle, PIRATORY INSUFFICIENCY. Carl E. Hunt, R. Brad Pyle,
William A. Neal, Frederick W. Johnson, William Helton, William G. Lindsay, Demetre M. Nicoloff. Univ. of Minnesota Hosp. Depts. of Ped, and Surg., Minneapolis, Minnesota. (Introduced by Rolf R. Engel, Minneapolis).

Extracorporeal membrane oxygenation (ECMO) has been utilized in two newborn infants, one with meconium aspiration (MA) and one with respiratory distress syndrome (RDS). A $0.8 \mathrm{M}^{2}$ Kolobow spiral membrane lung was used with veno-arterial perfusion. Maximum support with assisted ventilation and PEEP was required until ECMO was initiated (see Table). Neither patient survived. No lung \begin{tabular}{l|l|l|l|l|l|l}
\hline Patient & Age (hrs) & $\mathrm{FIO}_{2}$ & $\mathrm{PaCO}_{2}$ & $\mathrm{PaO}_{2}$ & ECMO Flow (cc/kg/Min) \\
\hline
\end{tabular}

\begin{tabular}{c|c|c|c|c|c}
\multirow{2}{*}{ MA } & 10 & 1.00 & 62 & 21 & None \\
\cline { 2 - 6 } & 13 & .21 & 35 & 68 & 76 \\
\hline \multirow{2}{*}{ RDS } & 16 & 1.00 & 50 & 31 & None \\
\cline { 2 - 6 } & 18 & .40 & 24 & 62 & 61 \\
\hline
\end{tabular}

recovery occurred in MA despite eight days of successful ECMO. In RDS, ECMO support could be maintained for 35 hours. Micruscopic sections of lung obtained at autopsy confirmed the clinical diagnosis in both cases. Adequate oxygenation was easily achieved with ECMO flows of $<50 \%$ of estimated cardiac output. No significant problems occurred related to the ECMO system or to hemolysis, bleeding or infection. The two major complications of pro1onged ECMO were renal failure and poor peripheral perfusion. Prolonged ECMO support is technically feasible in the neonate and may provide an important adjunct to the clinical management of severe cardiorespiratory insufficiency.

841 MUSCLE OXYGEN AVAILABLE $\left(\mathrm{O}_{2}\right.$ a) AS A CRITERION FOR CPPB EF.Er,TIVENESS. Robert $F$. Huxtable, Anthony $V$. Beran, kenneth G. Proctor, Donald R. Spering Intr. by T.L. Nelson) Univ. of CA, Col. of Med. Dept. of Ped. Irvine, CA.

As 1 ittle as $3-5 \mathrm{~cm} \mathrm{H} \mathrm{H}_{2} \mathrm{O}$ increase in proximal airway pressure (AP) applied to normal lung reduces cardiac output (Q). It is postulated that decreased pulmonary compliance (SCL) in IRDS acts as a barrier thus offsets this effect. Since $\dot{Q}$ is not routinely measured, severe reduction in $\dot{Q}$ could accompany regression of disease while AP is unchanged. This study was undertaken to determine whether $\mathrm{O}_{2}$ a could be used to detect changes in perfusion during CPPB. CPPB was evaluated on 10 normal $(\mathrm{N})$ rabbits $\left(\mathrm{SC}_{\mathrm{L}}=9.5\right.$ $\pm 1.8 \mathrm{cc} / \mathrm{g}$ at $25 \mathrm{~cm} \mathrm{H} \mathrm{H}_{2} \mathrm{O}$ ) and 10 pulmonary damaged (PD) rabbits $\left(\mathrm{SC}_{\mathrm{L}}=5.5 \pm 1.4 \mathrm{cC} / \mathrm{g}\right.$ at $\left.25 \mathrm{~cm} \mathrm{H} \mathrm{H}_{2} \mathrm{O}\right)$ produced by subjecting them to $100 \% 02$. AP was increased from $0-12 \mathrm{~cm} \mathrm{H} \mathrm{H}_{2} \mathrm{O}$ in $3 \mathrm{~cm} \mathrm{H} \mathrm{H}_{2} \mathrm{O}$ increments at $10 \mathrm{~min}$. intervals. $\mathrm{O}_{2} \mathrm{a}$ and $\mathrm{PaO}_{2}$ were monitored continuously. In $\mathrm{N}, \mathrm{O}_{2}$ a decreased at $3 \mathrm{~cm} \mathrm{H} \mathrm{H}_{2}$ Ap reaching $15 \%$ of control at 12 cm $\mathrm{H}_{2} \mathrm{O}$, at which pressure $\mathrm{PaO}_{2}$ decreased. $100 \% \mathrm{O}_{2}$ at this AP increased $\mathrm{PaO}_{2}$ to $450 \mathrm{~mm} \mathrm{Hg}$, while $\mathrm{O}_{2}$ a returned to $40 \%$ of control.

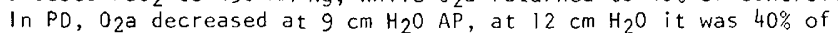
control at which pressure $\mathrm{PaO}_{2}$ decreased. $100 \% \mathrm{O}_{2}$ at this AP increased $\mathrm{PaO}_{2}$ to $330 \mathrm{~mm} \mathrm{Hg}$, but $02 a$ to $\geqslant 100 \%$ of control. These data indicate that with excessive AP, muscle hypoxia may exist during systemic hyperaxemia and that the low compliance lung exerts a protective effect on $0_{2} a$. Since changes in $\dot{Q}$ during CPPB

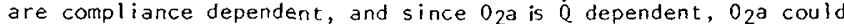
provide a means of selecting optimal AP during CPPB.
842 PREDNISONE THERAPY IN BRONCHOPULMONARY DYSPLASIA. J.M. Kagan and B.W. Goetzman, (introduced by W.J. Blankenship), Univ. of Cal. Sch. of Med., Dept. Peds., Davis. California.

The use of prednisone during the proliferative stage of bronchopulmonary dysplasia (BPD), which may occur as a sequela of therapy for hyaline membrane disease, has recently been described. As a result, six of our patients with BPD received a two week course of prednisone $(5 \mathrm{mg} / \mathrm{kg} / \mathrm{day})$. One patient received two courses of thcrapy. Blood gas criteria for acceptable improvement on prednisone were set prior to its administration, and the drug was continued for two weeks if the patient fulfilled these criteria within three days. In five of the six patients, response appeared dramatic both clinically and by blood gas analysis. However, retrospective analysis of alveolar-arterial oxygen difference and $\mathrm{PaCO} 2$ data for a two week period before and after initiation of prednisone revealed that only one patient can be shown to have made significant improvement as a result of prednisone therapy and two other patients had equivocal improvement. The fourth and $f$ ifth patient, who had appeared to improve dramatically can not be shown to have had a significant and lasting effect. Our experience suggests that prednisone therapy may be of value in some patients with BPD. Further clinical evaluation and elucidation of the mechanism of action of this agent appear to be indicated.

843 a RAPID AND SIMPIE OUANTITATIVe ASSAY FOr lung phosPHOLIPIDS, Padmani Karna and Ronald L. Poland, Dept. of Pediatrics, wayne State univ. School of Medicine and the Children's Hospital of Michigan, Detroit, Mich. (Intr. by George Polgar)

The dipalmitoyl derivatives of lecithin, phosphatidyl-dimethylethanolamine and cephalin along with bovine sphingomyelin and phosphatidylinositide are separated by thin layer chromatography on commercial silica gel plates (Baker-flex 1B2) using chloroform: methanol: water $(50: 25: 4\}$ and dried. The plates are then sprayed with 0.18 Rhodamine $6 \mathrm{G}$ dye, dried, scanned horizontally using a Turner fluorometer. Tracings of fluorescence are recorded and the area under the curve produced by scanning a phospholipid spot is proportional to the phosphorous content of the spot (by a modification of the Subba-Row method) and to the amount of standard phospholipid spotted. The method is accurate for as little as one microgram of phospholipid. The mean Rf values of sphingomyelin, lecithin, phosphatidyl-dimethylethanolamine and cephalin were $0.26,0.39,0.59$ and 0.70 respectively. Phosphatidyl-inositol did not stain with Rhodamine $6 \mathrm{G}$. With borate in the solvent system the spots were more compact but resolution suffered. Materials from tracheal aspirates obtained from newborn infants were extracted into chloroform after the procedure of Gluck (Pediatr. Res. $1: 237,1967)$ and chromatographed as above. Infants with severe hyaline membrane disease lacked a phosphatidyl-dimethylethanolamine spot. Further studies on the diagnostic value of this assay are ongoing.

THN MiTURATION IN FETAL RABBITS. E.H.Karotkin, W.J.Cashore, M.Kido, R.A.Redding, W.Douglas, L.Stern and W.On Brown Univ. Program in Medicine, Women \& Infants Hospital R'ode Island, Dept. of Perinatal Medicine, Providence, R.I.

It is known that steroid, thyroxine, \& heroin can induce fetal lung maturation. Aminophylline (A), betamethasone (BM), and phenobar bital( $\Phi)$ were tested for this phenomenon in 32 pregnant rabbits. The drugs were given I.M. to the does 5-10 days prior to term and pups delivered by C-section at 27-30 days gestation. Fetal lung maturity was assessed by pressure volume curves, phospholipid content (PLC) of lung tissue homogenate and alveolar washings, and electronmicroscopy (EM) of lung tissue for lamellar bodies. The lung homogenate and alveolar wash PLC in A and BM treated pups were significantly higher than the controls, whereas the $\phi$ treated groups had lower tissue and alveolar wash PLC. (see table) \begin{tabular}{l|l|l|l|l} 
Gest. & Lung Tissue PLC mg/gm dxy wt & Lung wash PLC ug/gm dry wt \\
\hline days) Control & A & BM & Control & B
\end{tabular}

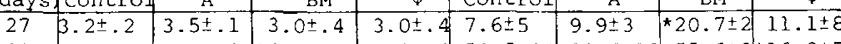

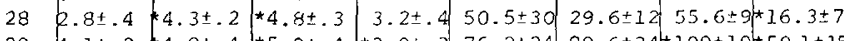

$294.1 \pm .2 * 4.8 \pm .4 * 5.9 \pm .4 * 3.0 \pm .3 \quad 76.2 \pm 24 \quad 89.6 \pm 34 * 109 \pm 10 * 50.1 \pm 15$ \begin{tabular}{ll|l|l|l|l|l}
30 & $4.5 \pm .4$ & $4.5 \pm .4$ & $4.0 \pm .4$ & $5.1 \pm .3109 .0 \pm 13 * 156 \pm 20 * 201 \pm 60$ & $83.4 \pm 17$ \\
\hline
\end{tabular} L+S.D., *values are significantly different from controls (p<.05) for the A and BM groups were lower than control at $27,28 \& 29$ days gestation ( $p<.01)$, and highex in the $\Phi$ treated groups at 28 days gestation (p<.01). EM data confirms the inhibitory effect of $\Phi$ on lung maturation. This data confirms that steroids induce fetal lung maturation as does Aminophylline. In contrast, Phenobarbital may inhibit lung maturation. 
845 SUCCESSFUL PROLONGED EXTRACORPOREAL MEMBRANE OXYGENATION OF A LEUKEMIC CHILD WITH VARICELLA PNEUMONIA. Thomas R. Kinney, Richard E. Hicks, L. Henry Edmunds, Russell C. Raphaely, J. Frederick Jones, Milton H. Donaldson Univ. of Penna. Sch. of Med., Children's Hospital of Phila., Depts. of Pediatrics(Oncology), Surgery, Anesthesiology, Penna.

A 5 year old boy with acute lymphoblastic leukemia (ALL) in continuous complete remission (CCR) for one year developed varicella pneumonia diagnosed by physical examination and roentgenogram. Progressive respiratory insufficiency developed. Despite maximal conventional respiratory support, his alveolar-arterial oxygen difference $\left(\mathrm{A}-\mathrm{aDO}_{2}\right)$ increased to 608 torr. Arterial oxygen tension $\left(\mathrm{PaO}_{2}\right)$ was below 65 torr on a fractional inspired oxygen concentration $\left(\mathrm{F}_{2} \mathrm{O}_{2}\right)$ of 1.0 for 58 hours (hrs.). Extracorporeal perfusion with a membrane oxygenator (ECMO) at a flow rate of 1.8 Titers/minute from veins to axillary artery was started and continued for 92 hrs. ECMO was stopped when $\mathrm{PaO}_{2}$ was 60 torr at $\mathrm{F}_{1} \mathrm{O}_{2} 0.7$ with ECMO flow at $10 \%$ of total cardfac output. Respiratofy support was withdrawn as his A-aDQ had decreased to 208 torr $\left(\mathrm{PaO}_{2}=475, \mathrm{~F}_{2} \mathrm{O}_{2}=1.0\right) 28$ days later. Ouring the next 7 months exercise tolerance continued to improve and ALL remains in CCR on chemotherapy. His chest $x$-rays remain abnormal and several bouts of bronchitis have occurred. This patient is the first survivor of life-threatening viral pneumonia treated by ECMO and the youngest patient to survive ECMO. This result suggests that severe viral pneumonia can heal in the presence of ALL and chemotherapy if respiratory function is supported and oxygen toxicity avoided.

FATTY ACID COMPOSITION OF TOTAL AND COLD ACETONE IN-

846 SOLUBLE LECITHIN. Robert V. Kotas and Robert D. Harlow. W. K. Warren Med. Res. Ctr., TuTsa, OK 74136

We have described sources of error in the Gluck technic of fatty acid assay which requires re-evaluation of changes in lung surfactant over gestation. Lipids extracted from the lungs of (A) 19 bunnies of 26 day gestation and (B) 36 two week old rabbits had Lecithin $(P C)$ isolated by thin layer chromatography and treated with cold acetone. Fatty acids were converted to their corresponding methyl esters by acid catalyzed transesterification for quantitation by hydrogen flame-gas liquid chromatography. Enzymereagent blanks were run concurrently and the peaks obtained subtracted from the runs containing added lipid. T=Total PC before cold acetone exposure. $\mathrm{AP}=$ Acetone insoluble lipid.

\begin{tabular}{|c|c|c|c|c|c|c|c|c|}
\hline & $14: 0$ & $16 \cdot 0$ & 76.1 & $78: 0$ & $18: 1$ & $78: 2$ & 0thers & $\%$ Sat. \\
\hline$\overline{A-T}$ & 7.5 & 63.1 & 2.8 & 4.7 & 76.7 & 7.5 & 3.7 & 69.3 \\
\hline$A-A$ & 2.1 & 69. & 1. & 5. & 14.1 & 5. & 2.5 & 76.5 \\
\hline$B-T$ & 1.5 & 56. & $T .5$ & 6.5 & 76.6 & 71.4 & 6.4 & 64.7 \\
\hline B-AP & 1.6 & 66. & 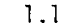 & 6.5 & 13.1 & 6.9 & 4.1 & 74.8 \\
\hline
\end{tabular}

More saturated fatty acids were found in the acetone insoluble fraction. There were no significant differences between fetal rabbits and neonatal bunnies. Cold acetone insoluble PC was free of highly unsaturated fatty acids with carbon number over 18. Cold acetone seems to be an inefficient way of separating saturated lipid from unsaturated lipids.
848 ASSESSMENT OF SMALL AIRWAYS DISEASE IN CYSTIC FIBROSIS. Jean-Guy Lapierre, Noe Zamel and Henry Levison. Dept. Of Peds., Univ. of Toronto, Res. Inst. Hosp. for Sick Children, Toronto, Canada.

The early pulmonary lesion in Cystic Fibrosis (CF) is small airways obstruction. We have recently shown that the volume at which maximal expiratory flow ( $v$ max) becomes density independent (Visovi) is a sensitive indicator of small airways disease. We studied 26 patients with CF of varying severity and measured lung volumes, forced expiratory volume in the first second 'FEV maximal expiratory flow rates (MMEF), and specific airways conductance (ȘGaw). We determined ymax at $25 \%$ and $50 \%$ of vital capacity $\left(\dot{V}_{25}, \dot{V}_{50}\right)$ and the Visov from their flow-volume curves while breathing alr and $80 \%$ Helium and $20 \%$ Oxygen. Eighteen patients had abnormal pulmonary function tests; of the remaining 8 , only 3 had normal Visov. We found also a signficiant correlation $(p<0.05)$ between $V$ isov and each of the other tests. Assuming that $V$ isoV is a measurement of small airways disease, our data show that progressive small airways disease results first in abnormal RV/TLC, $\hat{v}_{25}$, and MMEF, subsequently in $\dot{v}_{50}$ and later on in an abnormal ${ }^{2}$ G Gaw and $\mathrm{FEV}_{1} / \mathrm{VC}$. We conclude that Visot is useful in the detection of the early pulmonary lesion and could be used for monitoring the course of small airways disease in $\mathrm{CF}$. 
851 INTERMITTENT POSITIVE PRESSURE BREATHING (IPPB) USING NASAL PRONGS. M. Jeffrey Maisels, Maria J. Davi (Intr. by Nicholas M. Nelson). Penn State Univ. Co1. of Med., M. S. Hershey Med. Ctr., Dept. of Ped., Hershey, Pa.

Nasal prongs have been utilized to administer continuous positive airway pressure (CPAP) to infants with hyaline membrane disease (HMD). (Kattwinkel et a1. Pediat. 52:170, 1973.) We have used the same route to administer IPPB to 6 neonates using a time/flow ventilator. These infants had birthweight of $760-$ 2480 gms and gestational ages ranged from 27-34 weeks. Four had HMD, one pneumonia and one the Wilson-Mikity syndrome. The indications for IPPB with nasal prongs were persistent severe apnea with bradycardia, hypercapnia and hypoxemia in the face of adequate CPAP. Duration of IPPB with nasal prongs was 12 hours - 9 days and it was possible to maintain inspiratory pressures of $20-40 \mathrm{cms} \mathrm{H}_{2} \mathrm{O}$ with end expiratory pressures of $3-10 \mathrm{cms} \mathrm{H}_{2} \mathrm{O}$. Ventilatory assistance was achieved using slow rates (generally 10-20 breaths/minute) and intermittent mandatory type ventilation the infant breathing spontaneously between respiratory "breaths". Five infants who had IPPB for 14-60 hours all showed marked improvement and all survived. One infant with severe WilsonMikity disease who died had IPPB for 9 days without improvement of the hypercapnia or hypoxemia, although apnea was prevented. one infant developed bilateral pneumothoraces while receiving IPPB .

This technique is useful in the management of selected infants with respiratory insufficiency and avoids the hazards of endotracheal intubation.

852 ASSESSMENT OF THE TREATMENT OF RESPIRATORY OISTRESS SYNDROME (RDS) REQUIRING ASSISTED VENTILATION (AV) Houchang D. Modanlou, Robert F. Huxtable (Intr. T. L Nelson) Univ. of CA, Irvine, Col. of Med. Dept. of Ped., Irvine,CA

Earlier reports of RDS prognosis indicate best predicators are arterial pH and $\mathrm{PaO}_{2}$ while on $\mathrm{FiO}_{2}$ of 1 . We have retrospectively evaluated prognoses of 64 infants on AV with respect to birthweight, gestational age (GA), age AV started, $\mathrm{PaO}_{2}, \mathrm{pH}$, effective A-a gradient and inborn or transported. AV included Continuous Positive Airway Pressure and Intermittent Positive Pressure Ventilation, for at least 4 hours. $\mathrm{PaO}_{2}, \mathrm{PH}$ and $\mathrm{A}$-a were plotted against time. Intervals between values were $15 \mathrm{~min}$. to $4 \mathrm{hrs}$. Linear progress between values was assumed. Arbitrary baseline of $\mathrm{PaO}_{2}$ $50 \mathrm{mmHg}, \mathrm{pH} 7.20$ considered as lower limit of normal, and A-a 350 $\mathrm{mmHg}$ were chosen. By a computer simulated plotting process the percent of time that each patient spent below the baseline was recorded and the total area of the infant's progress graph that lay below the baseline was calculated. Similar plotting was done for A-a above the baseline of $350 \mathrm{mmHg}$. Comparisons (T-test) were made between infants that survived (38) to those that expired (26) on the basis of the above data. Significant differences $(p<0.05)$ were found between the two groups in regard to $G A(33+3$ vs $30 \div 3)$, age AV started $(18 \pm 14$ vs $13 \pm 15)$, percent of time below baseline for $\mathrm{pH}(4 \pm 6$ vs $52 \pm 30), \mathrm{PaO}_{2}(28 \pm 2)$ vs $\left.59 \pm 28\right)$ and above A-a $(42 \pm 32$ vs $83 \pm 20)$ and for total area below the baseline for $\mathrm{pH}(13 \pm 36 \mathrm{vs}$ $118 \pm 125)$ and $\mathrm{PaO}_{2}(108 \pm 119$ vs $183 \pm 110)$. This study reveals that the mortality rate on $A V$ is related to GA, age $A V$ started and time below baseline $\mathrm{PaO}_{2} 50 \mathrm{mmHg}$ and $\mathrm{pH} 7.20$. This approach may have most value in evaluating treatment protocols.

853

PNEUMUTHORAX IN IDIOPATHIC RESPIRATORY DISTRESS SYNDROME (IRDS): INCIDENCE AND EFFECT ON VITAL SIGNS, BLOOD GASES, AND pH. Edward S. Ogata, Joseph A. Kitterman, George A. Gregory, Roderic H.Phibbs, William H. Tooley. Univ. of California San Francisco, Department of Pediatrics and Cardiovascular Research Institute.

We determined the incidence of pneumothorax (PT) in 295 infants (mean birth weight $1917 \mathrm{gm}$ ) with IRDS treated by the same protocol. To assess the value of frequent measurement of vital signs, blood gas tensions, and $\mathrm{pH}$ in the recognition of PT, we analyzed these variables by the cumulative sum statistical technique. 49 infants (mean birth weight $1594 \mathrm{gm}$ ) developed PT (17\%). Infants treated wi thout assisted ventilation had the lowest incidence of PT $(2 / 58,3.5 \%)$, while infants treated with mechanical ventilation with end expiratory pressure (PEEP) as their primary therapy had the highest $(21 / 64,33 \%)$. We used CPAP as primary therapy in 173 infants. 119 did not require PEEP and the incidence of PT in this group was $14 / 119(12 \%)$. 54 infants were treated with PEEP after CPAP. Their incidence of Pr was $12 / 54(22 \%)$ At the time of the development of PT, we found significant falls in blood pressure $(28 / 49,57 \%)$, heart $x$ ate $(32 / 49,65 \%)$, respiratory rate $(29 / 33,67 \%)$ and pulse pressure $(24 / 45,55 \%)$. In 20 cases in which ventilatory settings were constant for at least 3 hours prior to $\mathrm{Pr}, \mathrm{Pa}_{0}$ fell significantly in 17 . There was no consistent pattern of change for $\mathrm{pH}$ or $\mathrm{Pa} \mathrm{CO}_{\text {. The }} \mathrm{risk}$ of $\mathrm{PT}$ increases with the severity of IRDS and with the more aggressive therapy it requires. Frequent measurement of vital signs and $\mathrm{Pa}_{2}$ offers an early indication of the development of PT.

Supported by PHS Grants HL-14201 and HD00397.
\$54 AN ISOLATED, VENTILATED, PERFUSED NEWBORN RABBIT LUNG PREPARATION TO BE USED AS A MODEL OF OXYGEN TOXICITY. Earl B. Olson, Ir. and Richard D. Zachman, Univ. WI Sch. of Med., Dept. Ped., Madison, WI.

Newborn rabbits are anesthetized, and ventilated. The pulmonary vasculature is perfused with a Krebs-Ringer Bicarbonate solution containing glucose and plasma expanders, and the lungs are isolated in a $37^{\circ} \mathrm{C}$ chamber at $100 \%$ humidity.

The success of a perfusion is evaluated on three levels: 1 Physical a. visual appearance, $\mathrm{b}$. the addition of $0.001 \%$ methylene blue identifies any regions of nonperfused vasculature. 2) Mechan. ical a. flow - a drop of greater than $20 \%$ indicates vascular failure, $b$. organ weight - an increase of more than $25 \%$ indicates interstitial fluid accumulation. 3) Biochemical a. the endogenous ATP concentration and $b$. the level of the enzyme choline phosphotransferase (CPT) are assumed to reflect biochemical integrity. Isolated, perfused, ventilated newborn rabbit lungs maintained for four hours with no changes in the monitored physical and mechanical criteria have an endogenous ATP level of $1.06 \pm 0.06^{*}$ nmoles

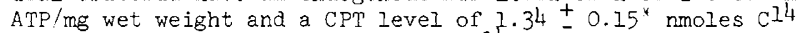
lecithin produced from CDP-choline $\mathrm{C}^{14} / \mathrm{mg}$ protein $/ 30 \mathrm{~min}$.

This isolated newborn lung preparation is a model for the study of the direct effects of high concentration of respired oxygen on the lung as an isolated organ. However, preliminary data indicates that there is no difference during the first four hours of perfusion in the monitored viability parameters of lungs ventilated with air versus lungs ventilated with $100 \%$ oxygen.

* $95 \%$ Confidence Limits

$\mathbf{8 5 5}$ TERBUTALINE IN THE TREATMENT OF STATUS ASTHMATICUS。 Leila M. Pang, Frank Rodriguez-Martinez, William Chernack, William J.Davis, Robert B. Mellins.

Columbia Univ. Co!。 of Physicians and Surgeons, Depts. of Ped. and Anesthesio! ogy, New York, NoY.

The effect of terbutaline, a new 8-adrenergic agonist on the pulmonary and cardiovascular systems was studied in ten patients $11 / 2$ to $13 \mathrm{I} / 2$ years of age with status asthmaticus. All patients were unresponsive to adrenalin 5.c., theophylline $i_{\circ} v_{.}$, and isoproterenol by inhalation. An indwelling arterial catheter was used to monitor blood pressure continuously and to follow arterial blood gases in 7 patients; the ECG was monitored continuously in all patients. Terbutaline, 0.01 to $0.04 \mathrm{mg} / \mathrm{kg}$ was given s.c. in multiple doses. Gross clinical improvement was apparent by 15-30 minutes in all but one patient. A decrease in systolic, diastolic and mean arterial blood pressure was significant $(p<0.05)$ only after the first dose of terbutaline with no significant change in cardiac or respiratory rates. Minor ST and + wave changes were noted in three. In 12 studies when the initial $\mathrm{PaCO}_{2}$ was $\geq 45$ torr, the mean decrease in $\mathrm{PaCO}_{2}$ after terbutaline was 4 torr $(S E=1.5)(P<0.05)$ and the mean increase in $\mathrm{PaO}_{2}$ was 4 torr $(S E=2.2)(P>0.05)$. Overall, terbutaline did not worsen ventilation/periusion abnormalities. The largest fall in $\mathrm{PaO}_{2}$ (10 torr) was accompanied by the largest rise in $\mathrm{PaCO}_{2}$ ( 8 torr); this patient was not only refractory to terbutaline s.c. but also to isoproterenol $i . v$. We conclude that terbutaline is an effective drug in the therapy of status asthmaticus with only modest effects on the cardiovascular system.

STUDIES ON PULMONARY ALVELAR PROTEINOSIS (PAP). Byung H. Park, Karlman Wasserman, Raymond Ajugwo, and Douglas C. Heiner, UCLA School of liedicine, Harbor General Hospital, Depts. Ped, and Med., Torrance, Ca.

Two brothers 10 and 11 years of age and 4 adults with PAP are followed at this hospital. Each has required bilateral lavage of degassed lungs on one or more occasion to remove proteinaceous fluid and insoluble lipid. This resulted in dramatic improvement, usually lasting one or more years, and pulmonary function tests showed an amelioration in restrictive defects and diffusion abnormalities. Each lung was lavaged with 10 or more instillations of 500-2000 ml saline accompanied by mechanical chest massage. The lst instillation resulted in the greatest removal of soluble protein whereas the 2nd and 3rd removed the most lipid, with successively less being recovered by additional instillations. A total of $40 \mathrm{gm}$ soluble protein, $6 \mathrm{gm} \mathrm{IgG}, 1.2 \mathrm{gm}$ $\mathrm{IgA}, 0.1 \mathrm{gm} \mathrm{IgM} \mathrm{and} 380,000 \mathrm{IU}$, or $0.8 \mathrm{mg}$, of IgE were removed from one adult (equal to $600 \mathrm{ml}$ plasma). After the 8 th instillation a plateau was reached yielding 1-2 $\mathrm{ml}$ plasma equivalent per instillation.

Radioimunoassays indicate that the IgG and IgE recovered in the alveolar washings have the same antibody specificity as plasma IgG and IgE suggesting simple diffusion into the lung rather than local manufacture. PAP may be a "protein-losing alveolopathy" analogous to protein losing enteropathy but with no ready mechanism for removing the accumulated protein and lipid, as can occur in the G.I. tract.

Supported by NIH Grant HL 16753-01. 
857 The Effect of Intermittent Positive Pressure Ventilation(IPPV) on the Circulation of the Infant Monkey. John B. Paton, David E. Fisher and Richard E. Behrnan. Dept. of Ped. Michael Reese Med. Center, Chicago, Ill. and Babies Hospital, New York, N.Y.

Changes in cardiac output and regional distribution of blood $f$ low were measured in four groups of infant monkeys given IPPV with different gas mixtures(air, oxygen, $8 \% \mathrm{CO}_{2}$-air, $8 \% \mathrm{CO}_{2}$-oxygen) and compared with a fifth group allowed to breathe air spontaneously. IPPV, wi.th a Bird Mark 8 ventilator, was with pressure-rate settings so that the air ventilated group maintained normal acidbase and oxygenation measurements. Cardiac output $(\mathrm{CO})$ and regional distribution of blood flow were measured by the radiolabeled microsphere method. Blood flow measurements are expressed as a percentage change from preventilation flow rates and the mean $\%$ change tabulated below.

\begin{tabular}{|c|c|c|c|c|c|}
\hline & Control & Air & Oxygen & $\mathrm{CO}_{2}$-Air & $\mathrm{CO}_{2}-\mathrm{O}_{2}$ \\
\hline Cardiac Output & -7.3 & $-\overline{33.4}$ & -25.8 & -12.7 & -11.4 \\
\hline Renal & -8.3 & -35.1 & -26.7 & -33.6 & -31.1 \\
\hline Coronary & -3.1 & -11.9 & -45.7 & +0.3 & +4.1 \\
\hline Cerebra1 & -5.9 & -27.8 & -20.7 & +20.6 & +20.6 \\
\hline
\end{tabular}

Renal blood flow decreased by about one-third in all ventilated animals while the change in cardiac output was less in those breathing $\mathrm{CO}_{2}$ mixtures. Hypercapnia increased cerebral circulation and $\mathrm{CO}_{2}-\mathrm{O}_{2}$ was no different than $\mathrm{CO}_{2}$-air. In contrast, ventilation stress and hyperoxia resulted in decreased $\mathrm{CO}$, renal, coronary and cerebral blood flows.

858

PULMONARY VASCULAR DYNAMICS IN CHRONIC PULMONARY DISEASE OF PREMATURITY. Douglas L. Roy, Elihu P. Rees, Thomas F. Ward (Int r. by Richard B. Goldbloom) Dalhousie Univ., Dept. of Pediatrics and Izaak Walton Killam Hospital for Children, Halifax, N.S., Can.

30 cardiac catheterizations (CC) were performed in 21 patients with chronic pulmonary disease of prematurity (CPDP). At birth gestational age (GA) of the group was 25 - 36 wks and weight ranged from $760 \mathrm{gm}-2640 \mathrm{gm}$. The GA of the group at CC was 30 - 51 wks. 8 infants, all with (a) a GA at CC of 36 wks or less, (b) weight at CC of $<2000 \mathrm{gm}$, and (c) left atrial oxygen saturations (LAO) of $<87 \%$, demonstrated 'normal' pulmonary arteriolar resistance (PAR). In 14 of 15 with (a) GA of 36 wks or over, (b) weight of $>2000 \mathrm{gm}$ and (c) LAO $<87 \%$, PAR was

elevated. Of this group 13 of 14 jemonstrated a decrease in PAR while breathing high oxygen, with PAR falling to normal in 6 of 13. In the remaining 7 , PAR did not fall to normal. In 2 , significant left-to-right shunt occurred during the hyperoxia study, though PAR remained slightly elevated. One patient had no shunt, but PAR remained elevated. The remaining 4 died of CPDP. 3 of 4 of this group had high PAR despite LAO of $>95 \%$ during hyperoxia test. In the last patient with elevated PAR, LAO remained low despite $100 \%$ oxygen.

Post mortem in three of four showed advanced intimal proliferation and medial thickening of pulmonary arterioles. We conclude that infants with CPDP whose GA is less than 36 weeks and weigh less than 2,000 gms are incapable of increasing PAR by hypoxia. No conclusions could be drawn as to factors leading to persistence of elevated PAR in those that died.

859 PROGNOSTIC IMPLICATIONS OF SEQUENTIAL CHANGES IN ACID-BASE AND BLOOD GAS PROFILE IN CHILDREN WITH PNEUMOCYSTIS CARINII PNEUMONITIS (PCP). Shyamal K. Sanyal, Walter $T$. Hughes, and Kenneth $S$. Harris. Dept. of Pediatrics, St. Jude Children's Research Hosp., Memphis, Tn

A prospective study was designed to detcrmine the prognostic significance of sequential changes in acid-base and blood gas profile in 55 children with PCP. Scrial arterial blood samples were obtained throughout acute and convalescent stages while the patients were breathing (i) room air, (ii) $100 \%$ oxygen for 15 minutes, and analyzed for $\mathrm{pH}, \mathrm{PaO}_{2}, \mathrm{PaCO}_{2}$, bicarbonate, $\mathrm{A}-\mathrm{a} \mathrm{DO}_{2}$, and venous admixture ( $\mathrm{R}-\mathrm{L}$ shunt). On admission, cach paticnt had hypoxia. Respiratory alkalosis was scen in 44 (compensated 31; uncompensated 13). Mean values for $\mathrm{pH}, \mathrm{PaO}_{2}, \mathrm{PaCO}_{2}, \mathrm{~A}-\mathrm{aDO}$ and $\mathrm{R}-\mathrm{L}$ Shunt werc $7.48 \pm 0.05,53.7 \pm 11.2,27.8 \pm 3.4,274.2 \pm 58.8$ and $18.9 \% \pm 2.4$. Subsequently all but one patient developed progressive hypoxia that required an increase in inspired oxygen concentration $\left(\mathrm{FIO}_{2}\right)$. Thirty-five patients who recovered spontancously did not require $\mathrm{FlO}_{2}>45 \%$. Improvement in arterial oxygenation started within 1.5 to 7 days (mean 4). Twenty-one other patients who needed mechanical ventilation developed progressive hypoxia, $\mathrm{A}-\mathrm{aDO}_{2}$ and $\mathrm{R}-\mathrm{L}$ shunt and nceded higher FIO. Early institution of mechanical ventilation ( $\mathrm{FIO}_{2} 50 \% ; \mathrm{R}-\mathrm{L}$ shunt $28 \%$ ) resulted in higher survival. Latc institution of ${ }^{2}$ ventilatory thcrapy $\left(\mathrm{FIO}_{2}>60 \%\right)$ was usually fatal with histologic and ultrastructural evidence of pulmonary oxygen toxicity. Thus, we conclude that sequential analysis of acid-base and blood gas profile in children with $P C P$ differentiates patients with moderate disease who recover spontaneously from those with severe disease who need early institution of meshanical ventilation to prevent a fatal termination or to minimize hazards of pulmonary oxygen toxicity.
860 A NEW APPROACH TO MANAGEMENT OF SEVERE HYPOXIA IN OLDER CHILDREN WITH DIFFUSE
PNEUMONITIS. S.K. Sanval, W.T. Hughes, and K.S. Harris. Dept. of Ped., St. Jude Children's Res. Hosp., Memphis, Tn.

In neonates with severe respiratory distress syndrome, continuous negative chest wall pressure (CNP) therapy has been shown to be an effective means of improving arterial oxygenation. Reported here is a simple device to apply CNP in older children and our experience with this mode of therapy in 15 children 4-20 yrs of age who developed progressively severe hypoxia due to $P$. carinii pneumonitis. An Emerson tank respirator was modified by disconnecting the bellows and attaching the tank to a vacuum pump to apply continuous negative pressure $\left(-1\right.$ to $\left.-18 \mathrm{~cm} \mathrm{H}_{2} \mathrm{O}\right)$ around the chest wall and lower parts of the body. An aneroid manometer was fitted to the tank to monitor the negative pressure within that was regulated on the basis of serial blood gas determinations. Before CNP therapy each patient had a respiratory rate $>70$ per minute, $\mathrm{PaO}_{2}<55$ $\mathrm{mmHg}\left(\mathrm{FIO}_{2} \geq 50 \%\right)$, and $\mathrm{PaCO}_{2}<40 \mathrm{mmHg}$. Within 24 hrs of CNP therapy $\mathrm{PaO}_{2}$ increased from pre-CNP mean of $53.4 \pm 16.8$ to $73.7 \pm 10.8$, respiratory rate decreased from $71.6 \pm 22.2$ to $55.7 \pm 19.1, \mathrm{FIO}_{2}$ from $51.6 \pm 5.9$ to $40.3 \pm 2.3$, $4-\mathrm{aDo}$, from $390 \pm 90.6$ to $280 \pm 103.4$ and intrapulmonary R-L shunt from $27 \% \pm 5.49$ to $24 \% \pm 3.3$. Six patients developed pneumothorax that was successfuliy decompressed. 11 patients survived. The other 4 patients, after initia improvement, developed progressive hypoxia and died. The survival pattern with CNP therapy contrasts sharply with our previous experience in 5 other patients with severe hypoxia due to PCP, received volume-controlled positive ventilation with PEEP and died. Since institution of CNP therapy, the mortality rate from PCP has decreased from 30.6 to $10.4 \%$. We conclude that CNP provides an effective means of improving arterial oxygenation in spontaneously breathing older children, minimizes exposure to high $\mathrm{FIO}_{2}$, and obviates the use of prolonged sedation, muscle relaxants and endotracheal intubation.

861 VAGAL CONTROL OF BREATHING INDEPENDENT OF LUNG INFLATION IN NEONATES. Mary M.K. Seshia, Rodney B. Boychuk, niv of Manitoba, Dept. of Ped. Winnipeg, Canada.

Chernick). Univ. of Manitoba, Dept. of Ped., Winnipeg, Canada. Wet of lung inflation on the control of respiratory rate and depth. They were given successive steady-state $\mathrm{CO}_{2}$ inhalations with different background concentrations of $\mathrm{O}_{2}$. Airway was occluded for one breath at end-expiration during the various levels of ventilation. Five preterm and 8 term infants also rebreathed $0_{2}$ from a bag and inspiratory elastic loads, ranging from $500 \mathrm{~cm} \mathrm{H} \mathrm{H}_{2} \mathrm{O} / \mathrm{L}$ to infinite load, were added at end-expiration. We measured inspiratory time $\left(T_{i}\right)$ and duration of the respiratory cycle (T) during steady-state, rebreathing and after loading. $T_{i}$ and $\mathrm{T}$ were statistically the same with varying inspired gases. $\mathrm{T}_{i}$ (mean $\pm \mathrm{S} . \mathrm{E} .=.45 \pm .033 \mathrm{sec}$ ) remained unchanged with increase in tidal volume $\left(V_{T}\right)$. T $(1.50 \pm .06 \mathrm{sec})$ did not change in 5 infants and increased linearly in 23 infants (slope=10.6 ml $/ \mathrm{sec}$ ) with increasing $V_{T}$. $T_{i}$ and $T$ of the first loaded breath were prolonged in relation to the preceding unloaded breaths $(p<0.01)$. This prolongation was similar during steady-state and rebreathing and was not affected by gestational age $(p>0.2)$. These findings suggest that in the neonate: 1) vagal control of breathing is independent of lung volume as reflected by a fixed $\mathrm{T}_{\mathbf{i}}$ with increasing $V_{T}$; 2) the duration of expiration determines $T$ and thus the respiratory frequency; and 3 ) unlike the conscious adult man, instantaneous frequency decreases with added elastic loads.

862 SEQUENTIAL PULMONARY FUnCTION STUdIES IN PREMATURE LAMBS WITH CONT!NUOUS POSITIVE AIRWAY PRESSURE (CPAP) DURING THE FIRST SIX HOURS OF LIFE. Thomas H. Shaffer and Maria Delivoria-Papadopoulos. University of Pennsylvania, School of Medicine, Depts. Of Physiology \& Pediatrics, Phila., PA The purpose of these studies is to compare lung mechanics and volumes of premature lambs delivered at $130-140$ days of gestation by cesarian section and ventilated by intermittent positive pressure (IPP) and CPAP. Following intubation and mechanical ventilation, indwelling arterial and venous catheters were placed. Seven lambs received IPP and five CPAP. During the first six hours of life, continuous measurements of intraesophageal and tracheal pressure, air flow, and tidal volume were made in all lambs. Determinations of lung compliance $\left(C_{L}\right)$, specific lung compliance $\left(\hat{C}_{L}\right)$, lung resistance $\left(R_{L}\right)$, specific lung conductance $(\hat{G})$, and functional residual capacity (FRC) were made at half hour intervals. For IPP lambs, mean values \pm SE were $c_{\mathrm{L}}=0.95 \pm 0.28$ $\mathrm{ml} / \mathrm{cm} \mathrm{H} 20 / \mathrm{kg}, \hat{\mathrm{C}}_{\mathrm{L}}=0.042 \pm 0.009 \mathrm{ml} / \mathrm{cm} \mathrm{H} 20 / \mathrm{FRC}, \mathrm{R}_{\mathrm{L}}=59.0 \pm 10.0 \mathrm{~cm} \mathrm{H} \mathrm{H}_{2} /$ $\mathrm{L} / \mathrm{sec}, \mathrm{G}=0.34 \pm 0.09 \mathrm{~L} / \mathrm{sec} / \mathrm{cm} \mathrm{H} \mathrm{H}_{2} \mathrm{O} / \mathrm{FRC}$, and $F R C=95.0 \pm 28.0 \mathrm{ml}$. For lambs with CPAP, values were $c_{L}=0.46 \pm 0.08 \mathrm{ml} / \mathrm{cm} \mathrm{H} \mathrm{H}_{2} 0 / \mathrm{kg}, \hat{\mathrm{C}}_{\mathrm{L}}=0.015 \pm$ $0.003 \mathrm{ml} / \mathrm{cm} \mathrm{H} \mathrm{H}_{2} \mathrm{O} / \mathrm{FRC}, \mathrm{R}_{\mathrm{L}}=77.0 \pm 13.0 \mathrm{~cm} \mathrm{H} \mathrm{H}_{2} \mathrm{O} / \mathrm{L} / \mathrm{sec}, \hat{G}=0.14 \pm 0.05 \mathrm{~L} / \mathrm{sec}$ $/ \mathrm{cm} \mathrm{H} \mathrm{H}_{2} \mathrm{O} / \mathrm{FRC}$, and $\mathrm{FRC}=170 \pm 55 \mathrm{ml}$. $\mathrm{P}_{2} \mathrm{CO}_{2}$ and $\mathrm{pH}$ were maintained within a physiologic range, while $\mathrm{P}_{a} \mathrm{O}_{2}$ varied from $29-440 \mathrm{~mm}$ at $\mathrm{Fi}_{i}=0.95$. These results indicate that $\hat{C}_{L} \quad(p<0.05), C_{L}$ ( $p<$
$0.15)$, and $G(p<0.10)$ decreased, while $R_{L}(0<0.20)$ and $F R C$ ( $<<$ $0.15)$, and $G(p<0.10)$ decreased, while $R_{L} \quad(p<0.20)$ and $F R C$ ( $p<$
$0.15)$ increased in lambs with CPAP as compared to those with IPP. While CPAP improved FRC, lung compliance decreased and airway resistance increased, suggesting that the work of breathing may
have increased. 

863 JPIATE DEPENDENT MOTHERS. Zarrin Shouraie, Loretta innegan, $\frac{\text { Dian S. Reeser, }}{\text { Maria Delivoria-Papadopoulos. Phila. General Hosp. }}$ Shaffer, and Maria Delivoria-Papadopoulos. Phila. General Hosp Infants born to opiate dependent mothers show an increased respiratory rate (f) during narcotic withdrawal, without specific pulmonary disease. In the present studies, serial measurements of lung compliance $\left(C_{L}\right)$, inspiratory $\left(R_{L} i\right)$ and expiratory $\left(R_{L_{e}}\right)$ pulmonary resistance, and minute volume $\left(\dot{V}_{E}\right)$ were made in 5 term infants born to opiate dependent mothers ( 2 heroin, 3 Methadone) and 5 term controls of comparable weight and gestation. Aside from the neonatal abstinence, the neonatal course was uncomplicated. Four of the 5 infants were treated with phenobarbital or paregoric on the second day of $1 \mathrm{ife}$. All infants were studied repeatedly during the first 3 days of life. Results are presented as mean \pm SD, and represent the average of measurements over the 3 day period. Values for the control group were: $C_{L}=2.83 \pm 0.28 \mathrm{ml} /$

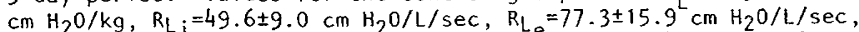
$\mathrm{f}=61.5 \pm 2.4$ breaths $/ \mathrm{min}$, tidal volume/weight (TV/wt) $=7.55 \pm 0.38 \mathrm{ml} /$ $\mathrm{kg}$, and $\dot{V}_{E}=1350 \pm 90 \mathrm{ml} / \mathrm{min}$. Values for the withdrawal infants at comparable times were: $C_{L}=2.11 \pm 0.29 \mathrm{ml} / \mathrm{cm} \mathrm{H} H_{2} 0 / \mathrm{kg}, R_{L_{i}}=40.4 \pm 6.2 \mathrm{~cm}$ $\mathrm{H}_{2} \mathrm{O} / \mathrm{L} / \mathrm{sec}, \mathrm{R}_{\mathrm{Le}}=47.9 \pm 7.1 \mathrm{~cm} \mathrm{H} \mathrm{H}_{2} \mathrm{O} / \mathrm{L} / \mathrm{sec}, \mathrm{f}=85.8 \pm 7.2 \mathrm{breaths} / \mathrm{min}$, TV/wt $=6.41 \pm 0.52 \mathrm{ml} / \mathrm{kg}$, and $\dot{V}_{E}=1550 \pm 60 \mathrm{ml} / \mathrm{min}$. These results show an increase in $f(p<0.01)$ and $\dot{V}_{E} \quad(p<0.06)$, a decrease in $C_{L} \quad(p<0.06)$, $R_{L_{e}}(p<0.08)$, and TV/wt $(p<0.06)$, and no change in $R_{L_{i}}(p<0.25)$. The significantly decreased compliance of the withdrawal infants in the presence of a persistently increased respiratory rate suggests an uneven pulmonary ventilation.

864 AMIIOTIC FLUIO ALPHA -ANTITPYYSIN AND SURFACE ACTIVE IATERIAL II PRETERM IIIFAIITS WITH THE RESPIRATORY DISTRESS SYWDROME. Allan D. Singer, Donald $W$. Thibeault, Calvin 3 . Hobel and Douglas C. Heiner, UCLA Sch. Med., Harbor Gen. osp., Torrance, Calif.

$A$ decreased surface active material in anniotic fluid (AF) can predict the fetus at risk to develop ROS. A decreased cord serum alpha -antitrypsin $(\alpha-A D$ is also predictive of a newborn developing FiDS. The amniotic fluid $\alpha]^{-A T}$ has not been studied throughout gestation. The of -AT was measured in AF throughout yes tation and related to AF fodr: stailility test (FST) in fetuses at risk to develop RDS. Ariniotic fluid $\alpha_{1}-A T$ and the FST were measured in 125 presnancies from 11 to 42 weeks gestation. Sixtyseven samples were obtained within 24 hours of delivery. itineteen infants developed RDS. The $x_{7}$-AT in AF was low in the first trimester and rose to maxiniur: levels at 25-35 weeks, thereafter decreasing. Aniniotic fluid a $-A T$ was linearly related to the total protein $(r=703 p<.001)$, but there was no relation between the ratio a $]^{-A T / T P}$ and gestational age. In infants with P.JS the ainniotic fluid $\alpha,-A T$ and $\alpha_{Y}-A T / T P$ were significantly elevated when compared to infants without RDS of similiar gestational age $(p<.001)$. The $\alpha$-AT concentration was not related to the FST. The eight infants with a low surfactant (negative FST) had ?DS regardless of the concentration of the a $j_{-A T}$. However, there were 9 fetuses who had an elevated amniotic fluid al-AT (>50n13. b) who had an intermediate or positive FST and 7 of these developed $K \mathrm{KO}^{\circ}$, one transient tachypnea. An elevated AF $\alpha]^{-A T}$ (above 50ing. $\not h$ ) is thus reredisfive of risk for RDS in infants with intermediate or

865 CORD SERUM PLASMINOGEN, AMNIOTIC FLUID SURFACE ACTIVt. 865 MATERIAL AND THE RESPIRATORY DISTRESS SYNDROME. Allan D. Singer, Donald W. Thibeault, Calvin J. Hobel and Douglas C. heiner, UCIA Sch. Med., Harbor Gen. Hosp., Torrance, CA. Low serum plasminogen (PL) in premature infants is reported to be a factor in the development of the Respiratory Distress Syndrome (RDS). The relationship of amniotic fluid (AF) surface active material to cord serum PL level has not been studied. Surfactant was assessed in $A F$ (collected within 24 hours of birth) by the foam stability test (FST) in 60 pregnancies of gestationa? ages 17 to 40 weeks. Cord PL was measured in these pregnancies using radial 1mmuno-diffusion. Cord PL was significantly related to cord total protein (TP): $\mathrm{mg} \% \mathrm{PL}=(1.22 \times \mathrm{gm} \% \mathrm{TP})-0.52 ; \mathrm{r}=0.75$, $\mathrm{p}<0.001$. Although the cord PL rose with increasing gestational age, the cord PL/Total Protein ratio was not related to gestattonal age $(r=0.085, p=>0.1)$. The cord PL was not closely related to the AF FST. With a negative FST (low surfactant) RDS occurred regardless of the level of the cord PL. However, there were 12 infants who had intermediate or positive FST and cord PL $\leq 4 \mathrm{mg} \%$. Eleven of these had either RDS (6) or resplratory distress without all classical features of RDS (5). PL was measured In a total of 129 samples of AF collected from 120 pregnancies of 11 to 42 weeks' gestation. It was detected in only 24 samples in a range of 0.2 to $2.6 \mathrm{mg} \%$. Neither cord nor AF PL is related to $\mathrm{AF}$ surfactant. Low cord $\mathrm{PL}<4 \mathrm{mg} \%$ and $10 \mathrm{w}$ cord Total Protein $<4.5 \mathrm{gm} \%$ are related to respiratory distress. 866 EFFECT OF $\mathrm{HCO}_{3}$-INFUSION IN SPONTANEOUSLY VENTILATING Univ. of Cincinnati Col. of Med., Dept. of Ped.

Previously we have shown that in newborn dogs. whose ventilation was mechanically fixed so that they could not increase $\mathrm{CO}_{2}$ excretion, infusion of $\mathrm{HCO}_{3}$-did not significantly alter $\mathrm{pH}$ but did raise $\mathrm{PCO}_{2}$. The present series of experiments was designed to study the effect of rapid infusion of $\mathrm{HCO}^{-}$on acid-base homeostasis in 24 spontaneously breathing anesthetized newborn dogs 1-20 days of age. Group I $(\mathrm{n}=11)$ breathed spontaneously without any ventila tory restriction. Group II $(n=13)$ breathed spontaneously against an increased airway resistance and dead space resulting in increased $\mathrm{PCO}_{2}$ and decreased $\mathrm{pH}$. After blood gases had stabilized for $30 \mathrm{~min} .7$ animals from group I and 8 animals from group II received $2 \mathrm{meq} / \mathrm{kg}$ of $\mathrm{HCO}_{3}^{-}(.5 \mathrm{meq} / \mathrm{ml})$ infused over a $2 \mathrm{~min}$. period. The other animals served as controls. Blood gases were measured at $1,3,5,10,15,30,45,60,90,120,150,180 \mathrm{~min}$. after the $\mathrm{HCO}_{3}^{-}$infusion. In group I pH rose by .07 units at $1 \mathrm{~min}$. then gradually fell and at $90 \mathrm{~min}$. was not significantly different from contro1 immediately stabilized at $4 \mathrm{meq} / \mathrm{L}$ for $2 \mathrm{hrs}$. $\mathrm{PCO}_{2}$ did not significantly change for $60 \mathrm{~min}$. and then slowly started to rise. In group II $\mathrm{pH}$ increased by only $.03-.04$ units and the rise remained lower than in group $I$ for $1 \mathrm{hr}$. BE followed the same pattern as tia group I. $\mathrm{PCO}_{2}$ rose by 10 torr at $1 \mathrm{~min}$. and then stabilized 6-7 torr above controls up to $90 \mathrm{~min}$. These results support the hypothesis that the $\mathrm{pH}$ raising effect of $\mathrm{HCO}_{3}$-Infusion is blunted in patients with ventilatory restriction because of the inability to adequately excrete $\mathrm{CO}_{2}$ produced from $\mathrm{HCO}_{3}{ }^{-}$.

CORD BLOOD ALPHA -AITITRYPSIV, A.MIIOTIC FLUID SURFAC-

867 TANT, AND THE RESPIRATORY DISTRESS SYNDROAE. JONald W. Thibeault, Allan D. Singer, Douglas C. Heiner and

Calvin J. Hobel, UCLA Sch, Ted, Harbor Gen. Hosp., Torrance, Ca.

A decreased anniotic fluid (AF) concentration of surface active material (SA:1) and elevated (AF) concentration of alpha 7 -antitryp$\sin \left(\alpha_{1}-A T\right)$ are useful in predicting the fetus at risk to develop ROS. The AF surface active material and $\alpha_{1}-\hat{A} T$ appear to be independent variables. It is known that decreased cord serum $\alpha_{1}-A T$ is related to the development of ROS. The relationship of cord $a]-A T$ to $A F \alpha_{1}-A T$ and $S A M$ is not known. Cord serum $\alpha_{1}-A T$ and total protein were measured in 66 infants ranging in gestational aye froin 17 to 40 weeks. In 3j of the presnancies, $A F$ was obtained within 24 hours of delivery and $i$ ts $\alpha$-AT level and Foari stability Test (FST) were measured. Cord $\alpha\}^{-A T}$ was not related to gestational age $(r=0.139 p>0.01)$. The presence of intrapartum complications was associated with a significant lowering of the cord al-AT and a 1-AT/TP at all Gestational ages. However, in the 7 infants with premature rupture of membranes $\geq 18$ huurs, the cord $\alpha_{7}$-AT vias normal or elevated. The cord $\alpha$-AT was not related to the FST. If the FST was nezative (low surfactant), ROS occurred regardiess of the level of cord $\alpha$ - AT. However, 10 infants with interinediate and positive FST had RDS; all had low cord $\alpha$ - AT ( $<0.2 \mathrm{gm} .0)$. Cord and AF $\alpha$ - $-A T$ concentrations were measured in 9 infants with RDS and intermediate or positive FST; 8 had low cord/FiF $\alpha_{1}-A T$ ratio compared to non RDS patients. Thus, a decrease of retal serum $\alpha_{1}-A T$ pricr to birth and/or an elevated AF $\alpha_{j}-A T$ is helpful in identifying infants at risk for KDS who have adequate A.F surfactant.

868 NEONA TAL LUNG PHOSPHORYLCHOLINE CY'PIDYLTRANSFERASEEFFECT OF LYSOLECITHIN ON ACTTVITY IN VARIOUS SPECIES Francis H. C. Tsao and Richard D. Zachman, Univ. of WI Sch. of Med., Dept. Peds., Madison, WI.

The control of lecithin biosynthesis in newborn lung tissue is being investigated indirectly by assaying enzyme activities responsible for its synthesis and degradation. Phosphorylcholine cytidyitransferase (CyT), the enzyme of the second step in the CDP-choline lecithin synthetic path, has not yet been studied in human or pig lung. CyT is present in lung homogenates of the rat, rabbit, pig and human nepnate. The enzyme is assayed by determining the conversion of $\mathrm{C}^{14}$ phosphorylcholine into cytidine-diphosphate-choline $\mathrm{Cl}^{14}$ in the presence of cytidine triphosphate and $\mathrm{MgCl}_{2}$. Delipidation of newborn lung homogenate with ethanol decreased CyT activity to $10-20 \%$ in pig and human, 50-70\% in rabbit and rat. The activity of delipidated CyT in pig and human lung could be stimulated 4-fold by addition of the ethanol extract and egg lysolecithin (0.2 - $0.4 \mathrm{mg}$ for maximum activity in the reaction mixture containing $2-5 \mathrm{mg}$ of protein), or boiled homogenate in pig, but had no significant effect in rabbit and rat. Lysolecithin specificity in relation to the activation of delipidated CyT activity in pig and human lung was also investigated. Lysolecithin-l-hexadecanoyl $\left(\mathrm{C}_{16}\right)$, a breakdown product of the predominate lung surfactant, stimulated the enzyme activity 4 -fold, as did egg lysolecithin, but lysolecithin-I-decanoyl $\left(\mathrm{C}_{10}\right)$ had no effect. Lysolecithin regulation of $\mathrm{CyT}$, especially lysolecithin-l-hexadecanoyl could play an important role in the control of lecithin biosynthesis in human and pig neonatal lung. 
869 CONTINUOUS MONITORING OF ESOPHAGEAL PRESSURE (PES): A SIMPLE TECHNIQUE AND ITS POTENTIAL USE. Ricardo Uauy and Etsuro K. Motoyama, Yale School of Medicine Dept. of Pediatrics \& Anesthesiology, New Haven, Ct. 06510. Continuous positive airway pressure (CPAP) breathing is an effective treatment for IRDS but no simple method has been available for the determination of the optimal CPAP. Information on pleural pressure can be obtained by the measurement of esophageal pressure (Pes) by means of a thin walled balloon or a fluid filled catheter in the esophagus. In our Newborn Special Care Unit a simplified technique has been used to monitor Pes continuously on all patients who are receiving CPAP treatment. A saline filled No. 5 French tube is placed in the lower third of the esophagus and the proximal end is connected to a pressure transducer and amplifier. Pes at the end expiratory plateau is monitored on an osciloscope screen as well as on a recording paper. Preliminary observations on infants with IRDS have proven the benefit of continuous Pes monitoring to assess the optimal CPAP. As CPAP was increased there was a clear step up in Pes at a certain level of CPAP at which $\mathrm{PaO}_{2}$ increased markedly. Additional uses of Pes measurements include: 1. Eva1uation of the efficacy of CPAP using the nasal airway technique; 2. Easy diagnosis of pneumothorax evidenced by a sudden rise in Pes; and, 3. Continuous monitoring of effective respixator treatment; asyncronous negative deflection of Pes signifies the patient's attempt to breathe against the respirator. Thus, continuous monitoring of Pes is a simple and effective tool in the management of the newborn on CPAP. (Supported in part by NIH
grant HLI4179).

870 LUNG ASPIRATE DIAGNOSIS IN PEDIATRIC PNEUMONIAS. Mark J. Merkens and Leonard B. Weinex (Intro. by Frank A. Oski), S.U.N.Y., Upstate Med. Ctr., Dept. of Pediatrics, Syracuse, New York.

This study was undertaken in an attempt to improve the rate of establishing an etiology in patients admitted to the pediatric service with the primary diagnosis of pneumonia. During a 24 month period 168 patients between the ages of 1 month and 15 years were evaluated. Of this group 59 patients had lung aspirates performed. An etiology was established in $32 \%(19 / 59)$. Etiologic agents included pneumococcus 6 , mycoplasma pneumoniae 9 , pneumocystis carinii $1, \mathrm{H}$. flu type B 1 , fusibacterium N.l and $H \cdot p a r a f l u$ in 1 patient. Despite the fact that $3 / 19$ patients had been on preceding antibiotic therapy for $>24$ hours their lung aspirates yielded $H$. flu, mycoplasma pneumoniae and $H$. paraflu. Positive lung aspirates occurred with greater frequency $(55 \%)$ in the 20/59 patients with underlying disorders, e.g. leukemia, immunologic disorders and CNS disease. Complications occurred in $5 \%(3 / 59)$ of patients and were limited to rapidly resolving asymptomatic, minimal pneumothorax. Sputum, $N-P$ or throat cultures conflicted with the lung aspirate isolation in $21 \%(4 / 19)$ of patients and concurred in $16 \%(3 / 19)$. In the remaining 109 patients an etiologic diagnosis was made by all other means in on $1 y$. $12 \%$. This study reemphasizes that the lung aspirate is a valuable diagnostic procedure and should be more commonly employed in the management of patients with pulmonary infiltrates. It is particularly useful in patients with chronic debilitating disease where prompt diagnosis may prove life saving.

871 THE EFFECT OF CONTINUOUS POSITIVE AIRWAY PRESSURE (CPAP) ON LUNG FUNCTION IN INFANTS WITH THE RESPIRATORY DISTRESS SYNDROME (RDS).

Henry Wiebe. John G. Brooks, George A. Gregory, University of California, Departments of Anesthesia, Pediatrics and the Cardiovascular Research Institute, San Francisco, California.

We have measured the effect of CPAP on lung mechanics, lung volumes, and arterial blood gases in 5 newborn human infants with RDS, during the first four or five days of life. Functional residual capacity (FRC) (helium rebreathing), transpulmonary pressure and respiratory flow were measured simultaneously on 200 occasions. Each infant was studied at 3 different levels of CPAP on each day: that selected clinically as most appropriate, and levels $2 \mathrm{~mm} \mathrm{Hg}$ higher and lower.

Measurements made after a $2 \mathrm{~mm} \mathrm{Hg}$ increase in CPAP were compared by chi square with those when CPAP had been lowered $2 \mathrm{~mm} \mathrm{Hg}$ (a $4 \mathrm{~mm} \mathrm{Hg}$ difference). There was a significant $(P<.05)$ correlation between a rise in CPAP and a rise in FRC, $\mathrm{PaO} 2, \mathrm{PaCO} 2$, and a fall in specific dynamic compliance $\left(\mathrm{SC}_{\mathrm{dyn}}\right)$, respiratory rate, minute ventilation, and tidal volume.

After the first two days of 1 ife, the average changes in FRC and $\mathrm{SC}_{\text {dyn }}$ with a $4 \mathrm{~mm} \mathrm{Hg}$ change in CPAP are larger, emphasizing the importance of precise regulation of aimway pressure during the recovery period of RDS.
872

"WET LUNG" SYNDROME IN PREMATURE INFANTS, T.F.Yeh, M. Reveri, L. Grajwer, R. S. Pildes, Depts of Ped., Cook County Hosp, and Univ. of III. Hosp. Chicago, iil.

Premature infants with wet lung syndrome have symptoms similar to that of RDS but lack the radiologic findings of RDS and pursue a different clinical course. To quantitate the severity of derangement in blood gas tension, acid base status and amount of shunting, 59 infants were studied prospectively. Mean $+S . E$. ges tational age was $33.4+3.5$ wks and birth wt. was $1.9+0.6 \mathrm{~kg}$. Apgar score was $6.5+0.4$ and RDS score at birth was $5,2+1, \overline{7}$. Six infants were born by $\overline{\mathrm{C}}$-section. At $0-4 \mathrm{hrs}$ of age, there was mild respiratory and metabolic acidosis; $\mathrm{pH} 7.26+0.01, \mathrm{pCO}_{2} 43.8+1.9$, base deficit, 7.1+0.9 p0, 85.1+7.4, $\mathrm{FiO}_{2} 44.3+2.1$ RDS score, 5. $1+0.2$. Fifteen $(15 / \overline{3} 4)$ infants had initial $\mathrm{pH}<\overline{7.25}$ and required $\mathrm{Fi}_{2}>0.4$. By $12 \mathrm{hrs}$. of age, the blood gases were normal. $\mathrm{A}^{-\mathrm{aDo}_{2}}$ and estimated $Q S / Q T$ were calculated in 22 infants who received $100 \% 0_{2}$ and did not have any clinically abnormal cardiovascular status. $\mathrm{A}-\mathrm{aDO} 2$ reached to $480.4+27.7$ at $8-12 \mathrm{hrs}$ of age and dropped to $391.6+31.1$ by $72 \mathrm{hrs}$ of age. The QS/QT was $27.8 \%$ during the first $24 \mathrm{hrs}$ and persisted at this level in spite of clinical improvement over the next $24 \mathrm{hrs}$. All infants tended to be easily hyperoxygenated, particularly after correction of acidosis. RDS scores were $>2$ for $48 \mathrm{hrs}$ and returned to normal at 72-96 hrs. All infants survived and none required any kinds of assisted ventilation. Since the respiratory symptoms are similar to RDS, clinical identification of this group of infants is important in order to avoid overzealous management.

873 DOES MATERHAL ADDICTICN PROTECT AGAINST THE DEVELOPMENT OF IDIOPATHIC PESPIRATORY DISTPESS SYNDROME? Jina Ja Yoon, Pita G.

Harper \& Sheela Padgonkar, Dept. of Ped. State iniv. of N.Y., Downstate l!ed. Center,Bklyn., \& Depts. of ped. \& Ob.-Cyn., N. Shore Univ. Hosp., Manhasset, \& Depts. of ped. \& Ob.-Gyn., Cornell univ. Med. Col., N.Y., N.Y. (Intr. by Fima Lifshitz).

To assess whether premature infants of addicted women have a decreased incidence of idiopathic respiratory distress syndrome (IPDS), the clinical course of 466 premature infants between 1000 and 2165 arams and less than 38 weeks aestational age were analyzed. Eiahty-nine of these infants were diaanosed as havino IPDS; 28 were korn to known addicts or developed the typical symptoms of drua withdrawal. The ircidence of IPDS for infants of non-addicted mothers was 19.6 . $(86 / 438)$; the incidence of IRDS for infants of addicted mothers was $10.7 \%(3 / 28)$. This difference was not statistically significant.

When infants with known factors which predisposed to or protected from IPDS were deleted from both groups, the incidence of IRDS for infants of nonaddicted women was $16.2 \div(36 / 222)$; while that for infants of drug addicted mothers was $11.7 \%(2 / 17)$. This difference was not statistically sianificant.

From this study, it appears that infants born to addicted mothers do not enjoy a statistically significant decreased incidence of IRDS. 